\title{
Asymmetric Intramolecular Dearomatization of Nonactivated Arenes with Ynamides for Rapid Assembly of Fused Ring System under Silver Catalysis
}

Tsubasa Ito, ${ }^{\dagger}$ Shingo Harada, ${ }^{*}{ }^{\dagger}$ Haruka Homma, ${ }^{\dagger}$ Hiroki Takenaka, ${ }^{\dagger}$ Shumpei Hirose,${ }^{\dagger}$ and Tetsuhiro Nemoto ${ }^{*}, \dagger, \dot{\dagger}$

†Graduate School of Pharmaceutical Sciences, Chiba University, 1-8-1, Inohana, Chuo-ku, Chiba 260-8675, Japan

¥Molecular Chirality Research Center, Chiba University, 1-33, Yayoi-cho, Inage-ku, Chiba 263-8522, Japan.

\section{E-mail : tnemoto@faculty.chiba-u.jp, Sharada@chiba-u.jp}

\section{Table of Contents}

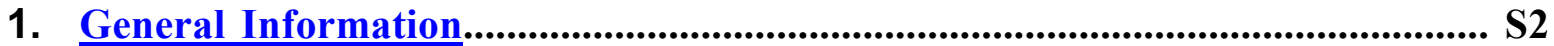

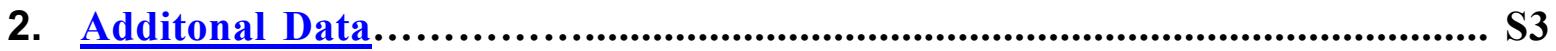

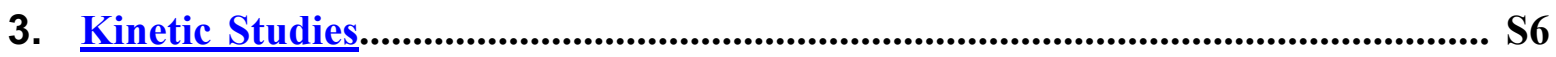

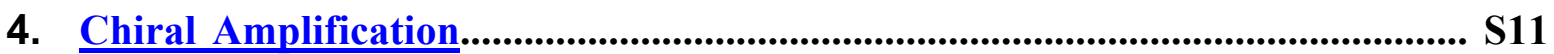

5. Synthesis and Characterization of Cycloheptatriene 2 and 3 ............................... S12

6. Synthesis and Characterization of Compounds 6................................................ S29

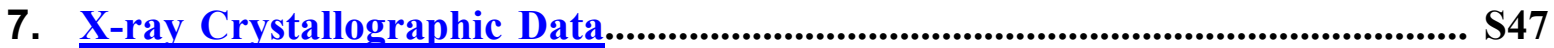

8. Synthesis and Characterization of Substrates............................................. S58

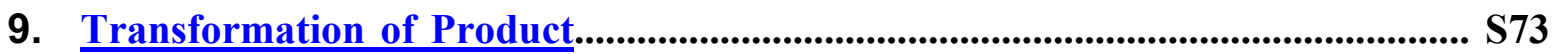

10. Computational Details..........................................................................................58

11. Charts of ${ }^{1} \mathrm{H}$ - and ${ }^{13}$ C-NMR Spectra.............................................................. S135

12. Chiral and Racemic HPLC Traces............................................................................ S255

13. References.................................................................................................................. S288 


\section{General Information}

NMR spectra were recorded on a JEOL ecs 400 , spectrometer. Chemical shifts in $\mathrm{CDCl}_{3}$ were reported downfield from TMS $(=0 \mathrm{ppm})$ for ${ }^{1} \mathrm{H}$ NMR. Data are reported as follows: chemical shift, multiplicity $(\mathrm{s}=$ singlet, $\mathrm{d}=$ doublet, $\mathrm{t}=$ triplet, quin $=$ quintet, $\mathrm{m}$ $=$ multiplet, and $b r=$ broad), integration and coupling constants in $400 \mathrm{~Hz}$. For ${ }^{13} \mathrm{C}$ NMR, chemical shifts were reported in the scale relative to the solvent signal $\left[\mathrm{CHCl}_{3}\right.$ (77.0 ppm)] or [acetone-d 6 (29.84 ppm)] as an internal reference. ESI mass spectra were measured on JEOL AccuTOF LC-plus JMS-T100LP. Optical rotations were measured on a JASCO P-1020 polarimeter. Infrared (IR) spectra were recorded on a Fourier transform infrared spectrophotometer, equipped with ATR. The enantiomeric ratio (er) was determined by HPLC analysis. HPLC was performed on JASCO HPLC systems consisting of the following: pump, PU-980; detector, UV-970; column DAICEL CHIRALPAK AD-H, DAICEL CHIRALCEL OX-3, DAICEL CHIRALCEL IA-3, DAICEL CHIRALCEL IG-3, DAICEL CHIRALCEL IH-3; mobile phase, $n$-hexane/i-PrOH. Melting points were measured with a SIBATA NEL-270 melting point apparatus. Column chromatography was performed with silica gel $60 \mathrm{~N}$ (spherical, neutral 40-50 mesh). Reactions were conducted in dry solvent. Other reagents were purified by the usual methods. 


\section{Additional Data}

Optimization of reaction conditions for the [4+2]-cycloaddition using isolated 2.
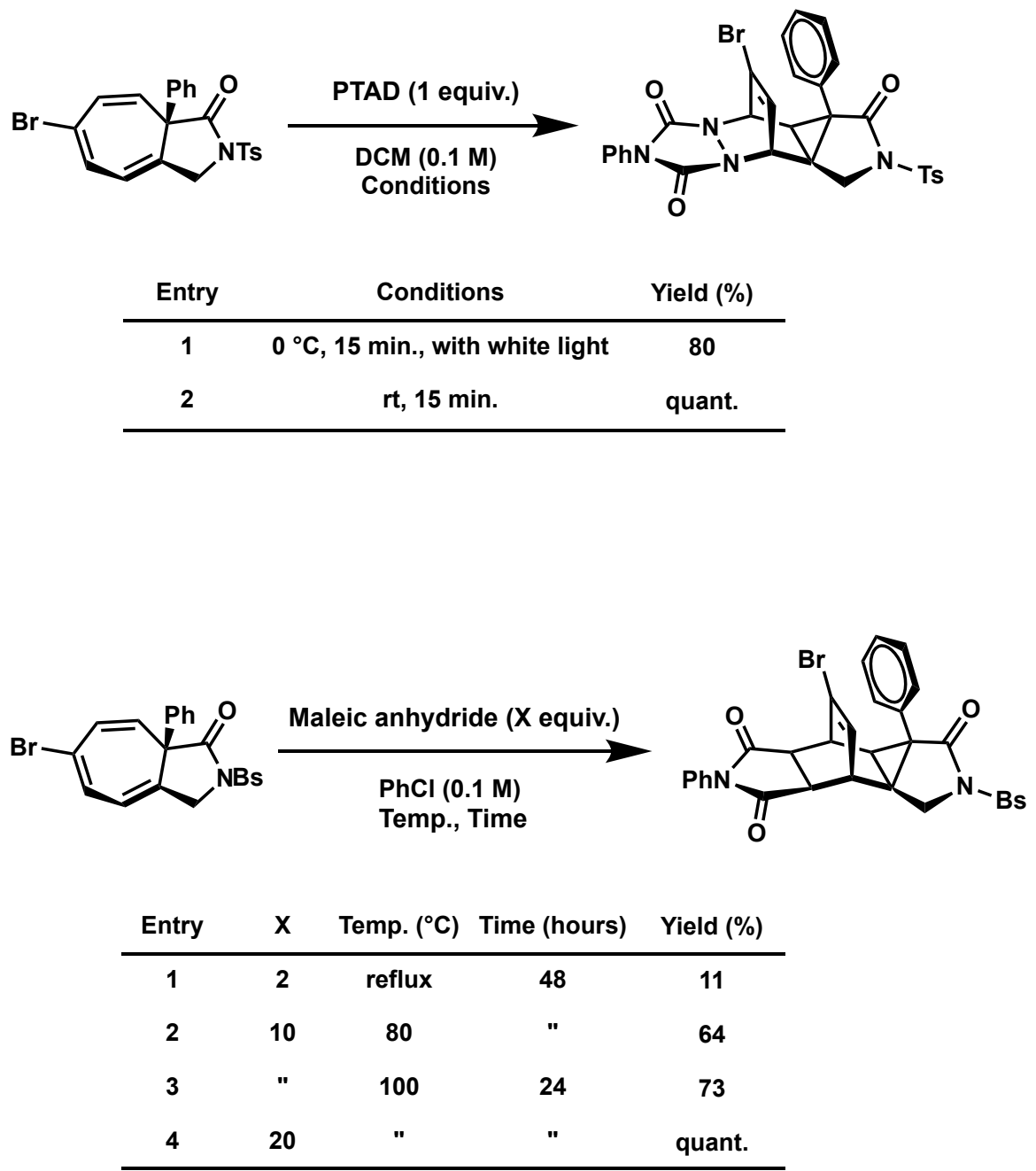
Detailed optimization of reaction conditions.<smiles>[As]N(C#Cc1ccccc1)Cc1ccccc1</smiles>

$1 X$

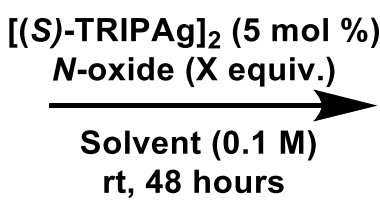

rt, 48 hours

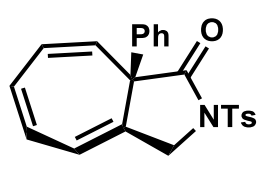

$2 \mathrm{X}$<smiles>O=C(C(=O)N([As])Cc1ccccc1)c1ccccc1</smiles>

$3 x$

\begin{tabular}{|c|c|c|c|c|c|c|}
\hline Entry & Solvent & $N$-oxide & $1 X(\%)$ & $2 \times(\%)$ & $3 \times(\%)$ & er \\
\hline 1 & $\mathrm{PhCl}$ & 8-methylquinoline $\mathrm{N}$-oxide & 0 & 82 & 16 & $91: 9$ \\
\hline 2 & $"$ & pyridine $N$-oxide & 79 & 16 & trace & $87: 13$ \\
\hline 3 & $"$ & 4-methoxypyridine $\mathrm{N}$-oxide & 64 & 22 & trace & $80: 20$ \\
\hline 4 & $"$ & 4-methylpyridine $\mathrm{N}$-oxide & 68 & 29 & trace & $88: 12$ \\
\hline 5 & $"$ & quinoline $N$-oxide & 69 & 28 & trace & $88: 12$ \\
\hline 6 & $\mathrm{Et}_{2} \mathrm{O}$ & 8-methylquinoline $\mathbf{N}$-oxide & 0 & 60 & 20 & $91: 9$ \\
\hline 7 & THF & $"$ & trace & 81 & 15 & $86: 14$ \\
\hline 8 & $\mathrm{CH}_{3} \mathrm{CN}$ & $"$ & 42 & 42 & 10 & $74: 26$ \\
\hline 9 & acetone & $"$ & 0 & 83 & 16 & $86: 14$ \\
\hline 10 & DCM & $"$ & 7 & 78 & 8 & $88: 12$ \\
\hline $11^{a)}$ & $\mathrm{PhCl}$ & $"$ & 43 & 53 & trace & $92: 8$ \\
\hline $12^{b)}$ & " & $"$ & 18 & 63 & 18 & $91: 9$ \\
\hline $13^{c)}$ & $"$ & $"$ & 0 & 90 & trace & $91: 9$ \\
\hline
\end{tabular}

a) Reaction was conducted at $-30^{\circ} \mathrm{C}$

b) MS4A (1 g/mmol) was added

c) MS3A (1 g/mmol) was added 
Unsuccessful examples and intermolecular reactions.
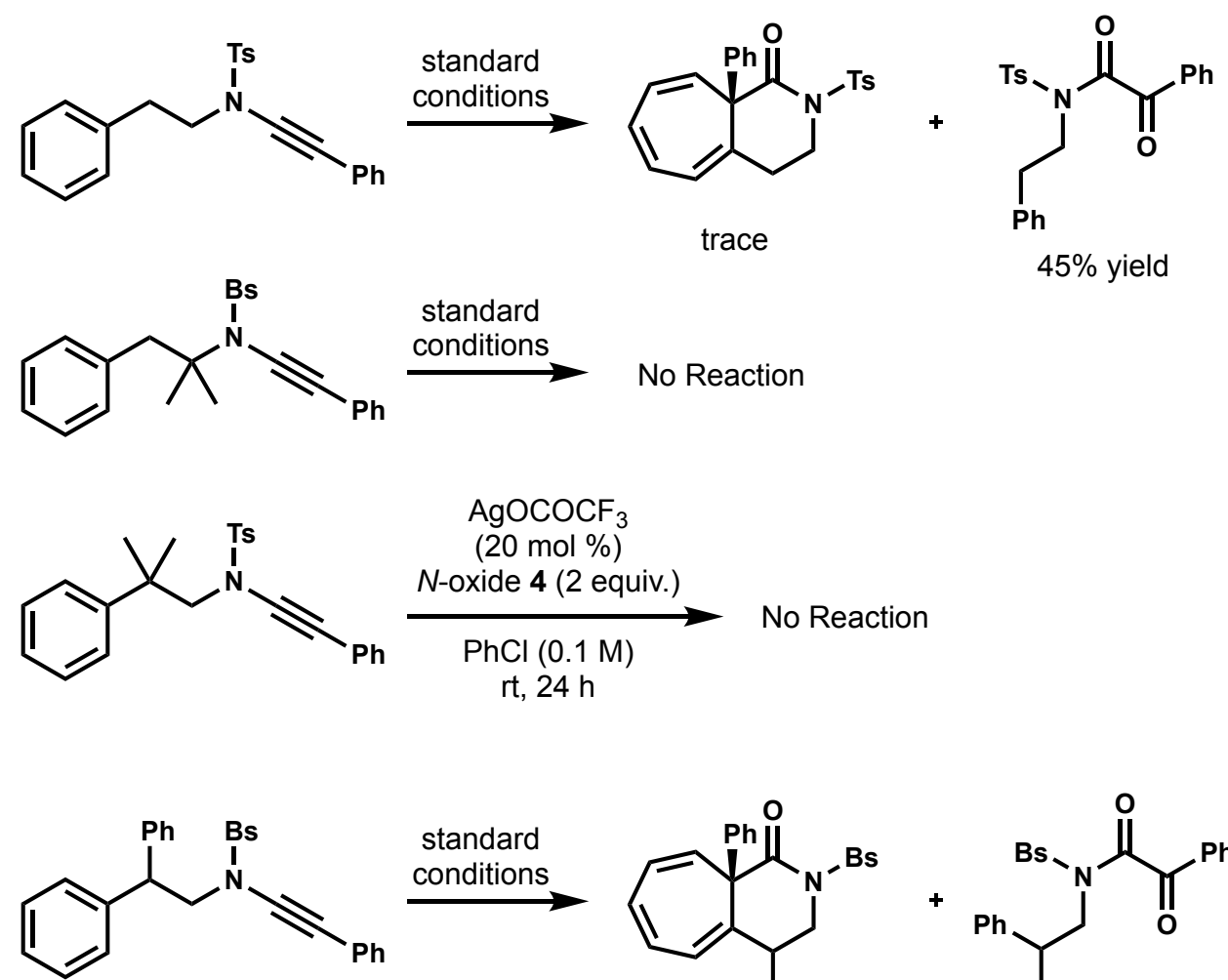<smiles>[2H][C@]12C=CC=CC=C1C(c1ccccc1)CN([13CH3])C2=O</smiles>

trace<smiles>O=C(C(=O)N(CC(c1ccccc1)c1ccccc1)c1ccccc1)c1ccccc1</smiles>

$23 \%$ yield<smiles>CN(C#Cc1ccccc1)c1ccccc1</smiles>

$\mathrm{AgOCOCF}_{3}$

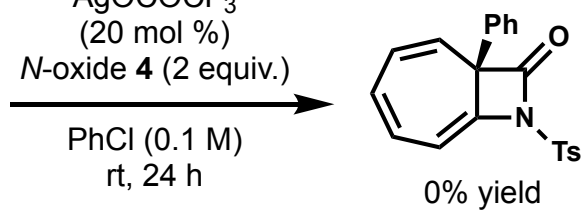<smiles>CN(C)C#Cc1ccccc1</smiles><smiles>c1ccccc1</smiles>

standard

conditions

No TM

(0.1 M)<smiles>CN(C#C[P+]([O-])c1ccco1)[13C](=O)c1ccccc1</smiles>

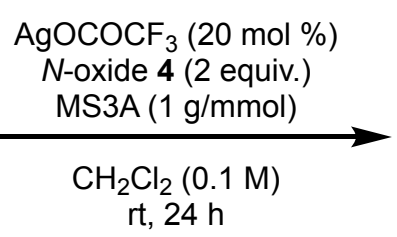

$\mathrm{AgOCOCF}_{3}(20 \mathrm{~mol} \%)$

$1 \mathrm{t}$
$+17$

$(0.1 \mathrm{M})$
$\mathrm{N}$-oxide 4 (2 equiv.) MS3A $(1 \mathrm{~g} / \mathrm{mmol})$

rt, $24 \mathrm{~h}$<smiles>C/C=C(/C(=O)N(C)Cc1ccccc1)c1ccccc1</smiles>

$17 \%$ yield 


\section{Kinetic Studies}

\section{General Procedure for Kinetic Experiments}

A pre-dried $10 \mathrm{~mL}$ round-bottom flask equipped with a magnetic stir bar was covered with aluminum foil to avoid light exposure. The flask was charged with ynamide compound 1a $(0.0375-0.15 \mathrm{mmol})$ and triphenylmethane (as an internal standard, 1 equiv.), $\mathrm{N}$-oxide 4 (0.1125-0.3 mmol), [(S)-TRIPAg]2 (1.25-5.0 mol \%), and MS3A (1 $\mathrm{g} / \mathrm{mmol})$, which were subsequently dissolved partially in dry $\mathrm{PhCl}(0.75 \mathrm{~mL})$ under argon gas atmosphere. The reaction mixture was stirred at the room temperature. Aliquots were taken at recorded times according to the following procedure. The reaction mixture $(0.1 \mathrm{~mL})$ was withdrawn with a syringe, which was immediately passed through a short pad of $\mathrm{NH}$ silica gel. After the solvent was evaporated in vacuo, the crude residue was analyzed by ${ }^{1} \mathrm{H}$ NMR. Yields of the products were measured by comparison with internal standard. 
Initial Rate Kinetics for $[(S)-T R I P A g]_{2}$

$\left[(S)-T_{R I P A g}\right]_{2}=1.25-7.50 \mathrm{mM},[1 \mathrm{a}]=0.1 \mathrm{M},[N$-oxide 4$]=0.2 \mathrm{M}$.

Figure S-1. Initial Rate Kinetics ([(S)-TRIPAg] $]_{2}$

\begin{tabular}{cccc}
\hline $\begin{array}{c}\left.[(\mathbf{S})-\mathrm{TRIPAg}]_{2}\right] \\
(\mathrm{M})\end{array}$ & $\begin{array}{c}\text { log } \\
{\left[[(\mathrm{S})-\mathrm{TRIPAg}]_{2}\right]}\end{array}$ & $\begin{array}{c}\text { Initial rate } \\
(\mathrm{M} / \mathrm{min})\end{array}$ & $\begin{array}{c}\text { log } \\
\text { (Initial rate) }\end{array}$ \\
\hline $1.25 \times 10^{-3}$ & -2.903 & $2.88 \times 10^{-3}$ & -2.541 \\
$1.88 \times 10^{-3}$ & -2.727 & $3.69 \times 10^{-3}$ & -2.433 \\
$2.50 \times 10^{-3}$ & -2.602 & $4.90 \times 10^{-3}$ & -2.310 \\
$3.75 \times 10^{-3}$ & -2.426 & $5.41 \times 10^{-3}$ & -2.267 \\
$7.50 \times 10^{-3}$ & -2.301 & $6.63 \times 10^{-3}$ & -2.178 \\
\hline
\end{tabular}

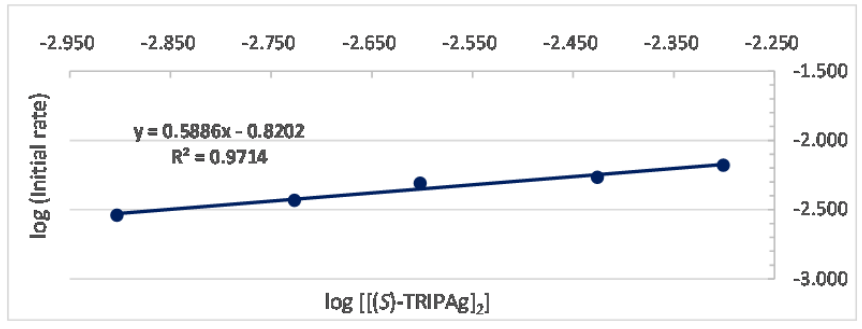

The order dependency of the reaction with regard to the $[(S)-\text { TRIPAg }]_{2}$ was determined

to be 0.589 . Detailed kinetics data are summarized in Figure S-2.

Figure S-2. Kinetics Data for [(S)-TRIPAg]2

\begin{tabular}{cc}
$\begin{array}{c}\left.\text { [I(S)-TRIPAg }]_{2}\right]= \\
\mathbf{1 . 2 5} \times \mathbf{1 0}^{-3}\end{array}$ \\
\hline $\begin{array}{c}\text { Time } \\
(\mathbf{m i n})\end{array}$ & $\begin{array}{c}\text { Yield } \\
(\%)\end{array}$ \\
\hline 10 & 3.6 \\
17 & 5.6 \\
24 & 7.3 \\
38 & 11.7 \\
\hline
\end{tabular}

\begin{tabular}{cc}
$\begin{array}{c}\left.[\text { (S) }- \text { TRIPAg }]_{2}\right]= \\
\mathbf{1 . 8 8} \times \mathbf{1 0}^{-3}\end{array}$ \\
\hline $\begin{array}{c}\text { Time } \\
(\mathbf{m i n})\end{array}$ & $\begin{array}{c}\text { Yield } \\
(\%)\end{array}$ \\
\hline 7 & 2.2 \\
15 & 5.4 \\
20 & 7.8 \\
25 & 9.1 \\
\hline
\end{tabular}

\begin{tabular}{cc}
$\begin{array}{c}\left.\text { [I(S)-TRIPAg }]_{2}\right]= \\
\mathbf{2 . 5 0} \times \mathbf{1 0}^{-3}\end{array}$ \\
\hline $\begin{array}{c}\text { Time } \\
(\mathbf{m i n})\end{array}$ & $\begin{array}{c}\text { Yield } \\
(\%)\end{array}$ \\
\hline 10 & 5.1 \\
15 & 7.3 \\
20 & 9.3 \\
30 & 14.9 \\
\hline
\end{tabular}

\begin{tabular}{cc}
$\begin{array}{c}{\left[(S)-\text { TRIPAg }_{2}\right]=} \\
\mathbf{3 . 7 5} \times \mathbf{1 0}^{-3}\end{array}$ \\
\hline $\begin{array}{cc}\text { Time } \\
(\text { min) }\end{array}$ & $\begin{array}{c}\text { Yield } \\
(\%)\end{array}$ \\
\hline 8 & 5.3 \\
12 & 7.1 \\
16 & 9.9 \\
24 & 13.8 \\
\hline
\end{tabular}

\begin{tabular}{cc}
$\begin{array}{c}\left.[(\mathbf{S})-\mathrm{TRIPAg}]_{2}\right] \\
\mathbf{7 . 5 0} \times \mathbf{1 0}^{-3}\end{array}$ \\
\hline $\begin{array}{c}\text { Time } \\
(\mathbf{m i n})\end{array}$ & $\begin{array}{c}\text { Yield } \\
(\%)\end{array}$ \\
\hline 6 & 4.5 \\
9 & 6.2 \\
12 & 8.7 \\
15 & 10.3 \\
\hline
\end{tabular}

\begin{tabular}{cc}
$\begin{array}{c}{\left[\mathbf{( S )}-\mathrm{TRIPAg}_{2}\right]} \\
(\mathrm{M})\end{array}$ & $\begin{array}{c}\text { Initial rate } \\
(\mathrm{M} / \mathrm{min})\end{array}$ \\
\hline $1.25 \times 10^{-3}$ & $2.88 \times 10^{-3}$ \\
$1.88 \times 10^{-3}$ & $3.69 \times 10^{-3}$ \\
$2.50 \times 10^{-3}$ & $4.90 \times 10^{-3}$ \\
$3.75 \times 10^{-3}$ & $5.41 \times 10^{-3}$ \\
$7.50 \times 10^{-3}$ & $6.63 \times 10^{-3}$ \\
\hline
\end{tabular}

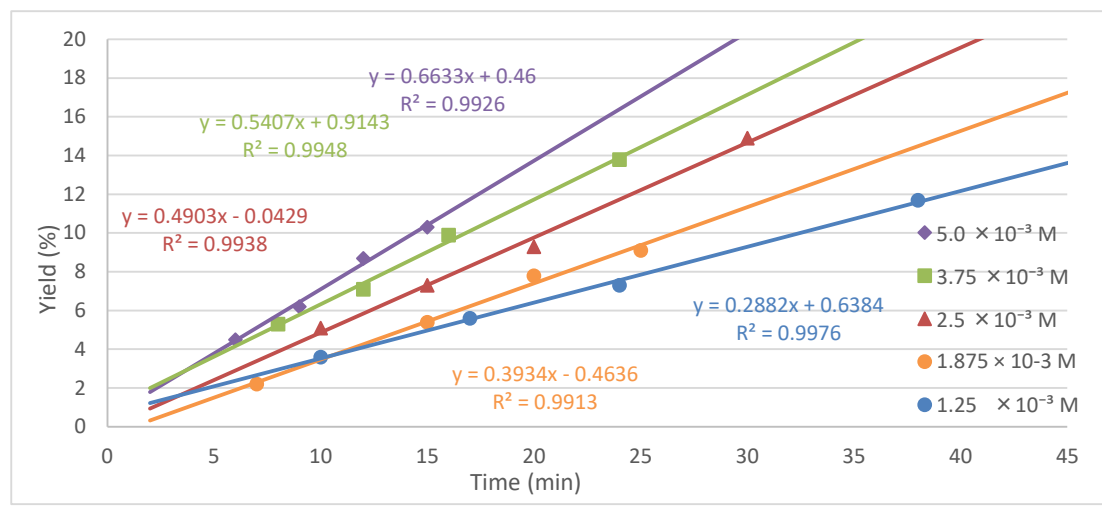


Initial Rate Kinetics for [N-oxide 4]

$[(S)-T R I P A g]_{2}=2.5 \mathrm{mM},[1 \mathrm{a}]=0.1 \mathrm{M},[N$-oxide 4$]=0.15-0.3 \mathrm{M}$.

Figure S-3. Initial Rate Kinetics (N-oxide 4)

\begin{tabular}{cccc}
\hline $\begin{array}{c}\text { [N-oxide 4] } \\
\text { (M) }\end{array}$ & $\begin{array}{c}\text { log } \\
\text { [N-oxide 4] }\end{array}$ & $\begin{array}{c}\text { Initial rate } \\
(\mathrm{M} / \mathrm{min})\end{array}$ & $\begin{array}{c}\text { log } \\
\text { (Initial rate) }\end{array}$ \\
\hline 0.15 & -0.824 & $5.21 \times 10^{-3}$ & -2.283 \\
0.2 & -0.699 & $4.90 \times 10^{-3}$ & -2.310 \\
0.25 & -0.602 & $4.72 \times 10^{-3}$ & -2.326 \\
0.3 & -0.523 & $4.45 \times 10^{-3}$ & -2.352 \\
0.4 & -0.398 & $4.33 \times 10^{-3}$ & -2.364 \\
\hline
\end{tabular}

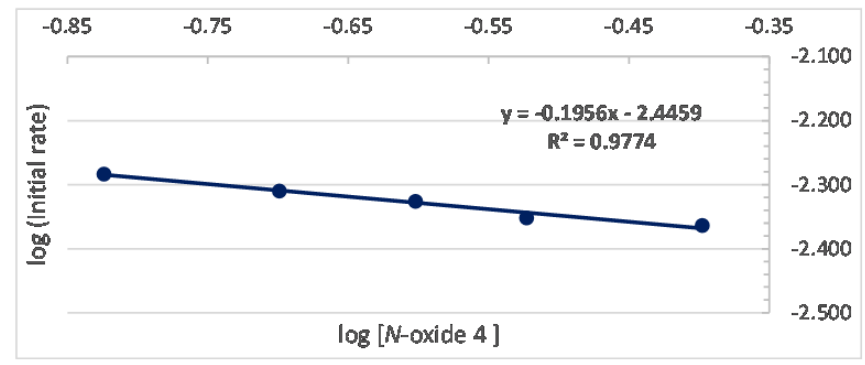

The order dependency of the reaction with regard to the $\mathrm{N}$-oxide 4 was determined to be -0.196 . Detailed kinetics data are summarized in Figure S-4.

Figure S-4. Kinetics Data for $\mathrm{N}$-oxide 4

\begin{tabular}{cc}
\multicolumn{2}{c}{$\begin{array}{c}\text { [N-oxide } \\
\mathbf{0 . 1 5}\end{array}$} \\
\hline $\begin{array}{c}\text { Time } \\
\text { (min) }\end{array}$ & $\begin{array}{c}\text { Yield } \\
(\%)\end{array}$ \\
\hline 10 & 4.6 \\
17 & 9.3 \\
38 & 19.8 \\
52 & 26.8 \\
\hline
\end{tabular}

\begin{tabular}{cc}
\multicolumn{2}{c}{$\begin{array}{c}\text { [N-oxide 4] }= \\
\mathbf{0 . 1 5}\end{array}$} \\
\hline $\begin{array}{c}\text { Time } \\
\text { (min) }\end{array}$ & $\begin{array}{c}\text { Yield } \\
(\%)\end{array}$ \\
\hline 10 & 5.1 \\
15 & 7.3 \\
20 & 9.3 \\
30 & 14.9 \\
\hline
\end{tabular}
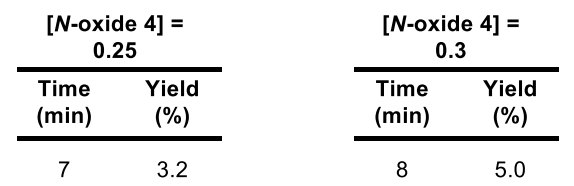

$10 \quad 5.1$

\begin{tabular}{cc}
15 & 6.9 \\
25 & 11.9 \\
\hline
\end{tabular}
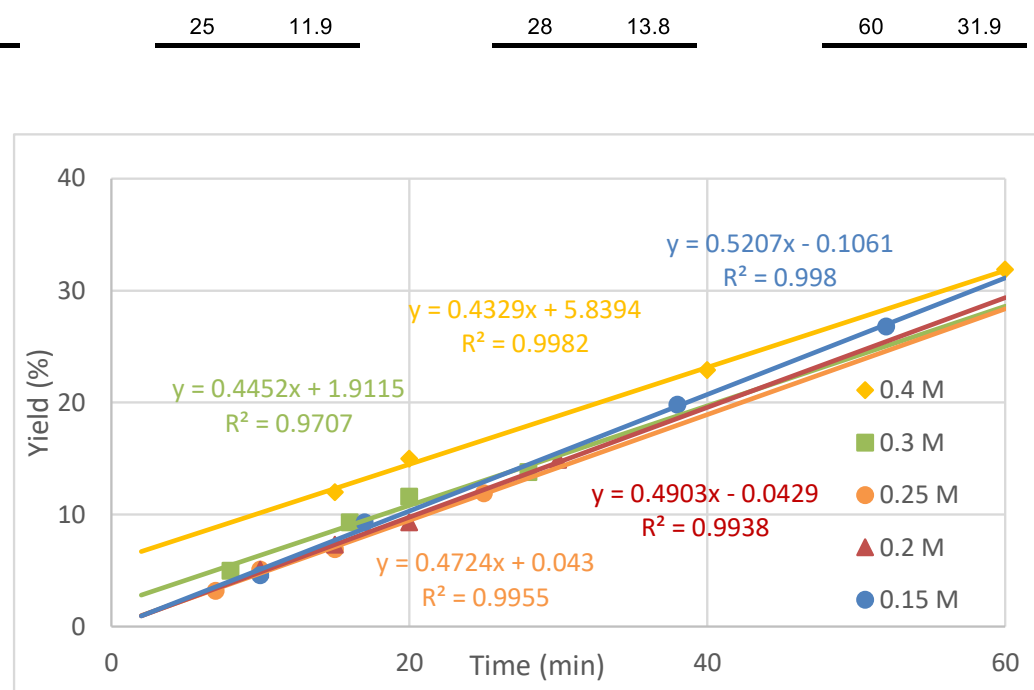
Initial Rate Kinetics for [1a]

$\left[(S)-T_{R I P A g}\right]_{2}=2.5 \mathrm{mM}, \quad[1 \mathrm{a}]=0.05-1.5 \mathrm{M},[N$-oxide 4$]=0.2 \mathrm{M}$.

Figure S-5. Initial Rate Kinetics (1a)

\begin{tabular}{cccc}
\hline $\begin{array}{c}\text { [1a] } \\
\text { (M) }\end{array}$ & $\begin{array}{c}\text { log } \\
\text { [1a] }\end{array}$ & $\begin{array}{c}\text { Initial rate } \\
\text { (M/min) }\end{array}$ & $\begin{array}{c}\text { log } \\
\text { (Initial rate) }\end{array}$ \\
\hline 0.05 & -1.301 & $4.09 \times 10^{-3}$ & -3.388 \\
0.075 & -1.125 & $4.65 \times 10^{-3}$ & -3.333 \\
0.1 & -1.000 & $4.74 \times 10^{-3}$ & -3.324 \\
0.12 & -0.921 & $5.05 \times 10^{-3}$ & -3.297 \\
0.15 & -0.824 & $5.25 \times 10^{-3}$ & -3.280 \\
\hline
\end{tabular}

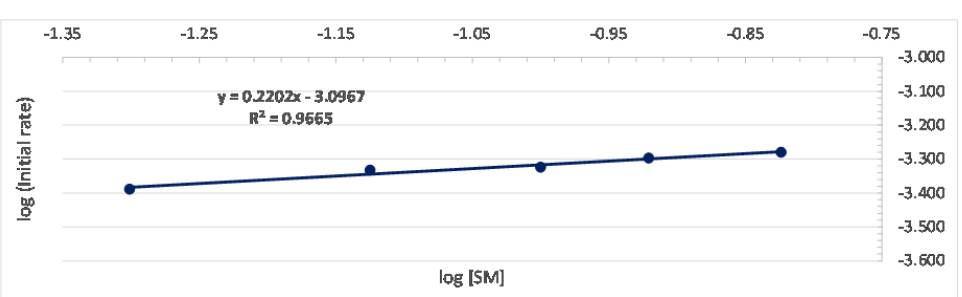

The order dependency of the reaction with regard to the 1a was determined to be

0.220. Detailed kinetics data are summarized in Figure S-6.

Figure S-6. Kinetics Data for [1a]

\begin{tabular}{cc}
\multicolumn{2}{c}{$[\mathbf{1 a}]=\mathbf{0 . 0 5}$} \\
\hline $\begin{array}{c}\text { Time } \\
(\mathbf{m i n})\end{array}$ & $\begin{array}{c}{[\mathbf{2 a}]} \\
\left(\mathbf{1 0}^{-3} \mathbf{M}\right)\end{array}$ \\
\hline 5 & 3.2 \\
14 & 7.4 \\
22 & 10.1 \\
33 & 15.7 \\
50 & 21.5 \\
\hline
\end{tabular}

\begin{tabular}{cc}
\multicolumn{2}{c}{$[1 \mathrm{a}]=\mathbf{0 . 1}$} \\
\hline $\begin{array}{c}\text { Time } \\
(\mathbf{m i n})\end{array}$ & $\begin{array}{c}{[\mathbf{2 a}]} \\
\left(\mathbf{1 0 ^ { - 3 }} \mathrm{M}\right)\end{array}$ \\
\hline 5 & 2.55 \\
12.5 & 6.2 \\
17.5 & 8.3 \\
25 & 12.1 \\
\hline
\end{tabular}

\begin{tabular}{cc}
\multicolumn{2}{c}{$[1 \mathrm{a}]=\mathbf{0 . 1 2}$} \\
\hline $\begin{array}{c}\text { Time } \\
(\mathbf{m i n})\end{array}$ & $\begin{array}{c}{[\mathbf{2 a}]} \\
\left(10^{-3} \mathrm{M}\right)\end{array}$ \\
\hline 6 & 5.0 \\
14 & 10.2 \\
18 & 11.9 \\
24 & 14.9 \\
32 & 18.9
\end{tabular}

\begin{tabular}{cc}
\multicolumn{2}{c}{$[1 \mathrm{a}]=\mathbf{0 . 1 5}$} \\
\hline $\begin{array}{c}\text { Time } \\
(\mathrm{min})\end{array}$ & $\begin{array}{c}{[2 \mathrm{a}]} \\
\left(10^{-3} \mathrm{M}\right)\end{array}$ \\
\hline 3.5 & $\mathbf{2 . 1}$ \\
8.5 & $\mathbf{4 . 8}$ \\
12.5 & $\mathbf{6 . 6}$ \\
17.5 & $\mathbf{9 . 4}$ \\
22.5 & $\mathbf{1 1 . 7}$ \\
\hline
\end{tabular}

\begin{tabular}{cc}
\hline $\begin{array}{c}{[1 \mathrm{a}]} \\
\text { (M) }\end{array}$ & $\begin{array}{c}\text { Initial rate } \\
(\mathrm{M} / \mathrm{min})\end{array}$ \\
\hline 0.05 & $4.09 \times 10^{-3}$ \\
0.075 & $4.65 \times 10^{-3}$ \\
0.1 & $4.74 \times 10^{-3}$ \\
0.12 & $5.05 \times 10^{-3}$ \\
0.15 & $5.25 \times 10^{-3}$ \\
\hline
\end{tabular}

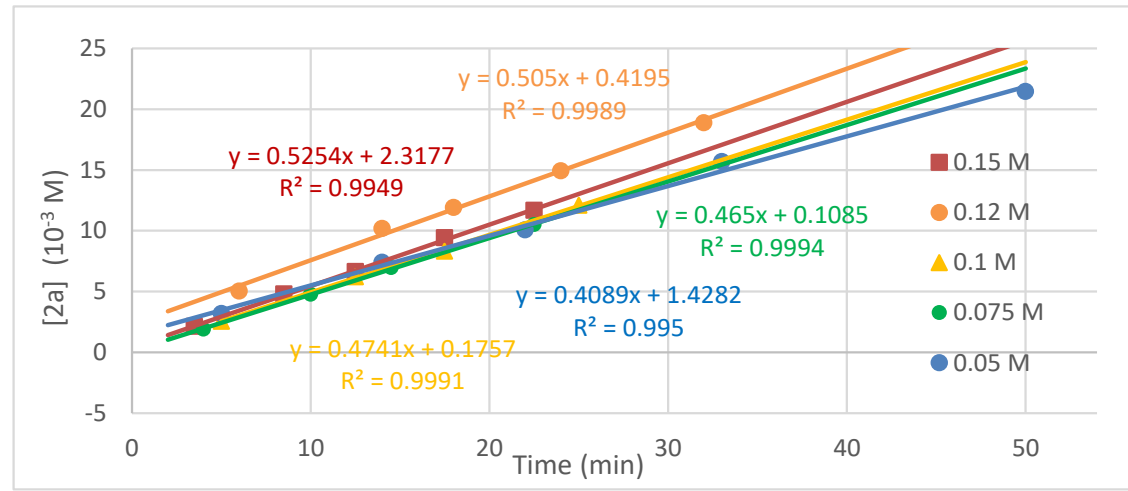


Supporting information

A control experiment in the presence of the product ( 0.5 equiv.) was conducted and exhibited a slight decrease in the reaction rate.

\begin{tabular}{|c|c|c|c|}
\hline \multicolumn{2}{|c|}{ Standard } & \multicolumn{2}{|c|}{ added pro. } \\
\hline $\begin{array}{l}\text { Time } \\
\text { (min) }\end{array}$ & $\begin{array}{c}\text { Yield } \\
(\%)\end{array}$ & $\begin{array}{l}\text { Time } \\
(\mathrm{min})\end{array}$ & $\begin{array}{c}\text { Yield } \\
(\%)\end{array}$ \\
\hline 0 & 0 & 0 & 0 \\
\hline 10 & 5.5 & 30 & 8.6 \\
\hline 20 & 10.6 & 60 & 18.7 \\
\hline 30 & 15.2 & 120 & 34.2 \\
\hline 50 & 22.8 & 240 & 45.3 \\
\hline 80 & 32.7 & & \\
\hline 150 & 45.9 & & \\
\hline 260 & 57.8 & & \\
\hline
\end{tabular}

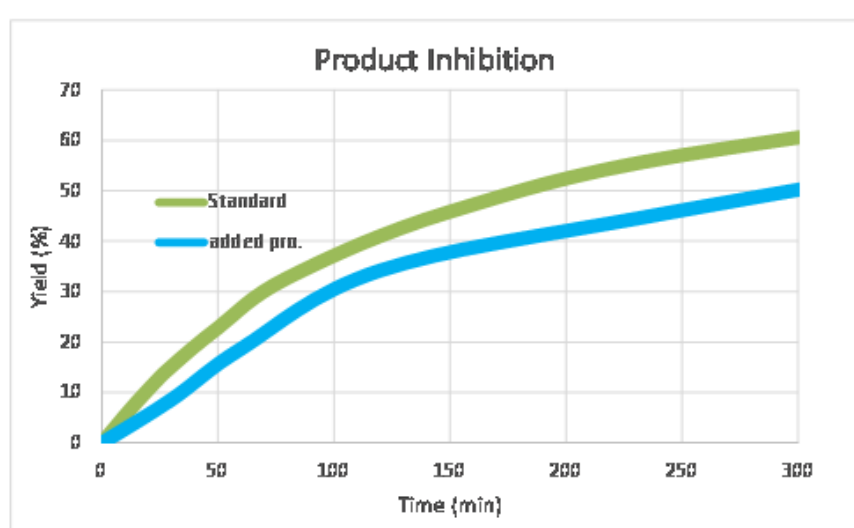




\section{Chiral Amplification}

\section{General Procedure for Chiral Amplification Studies (40\% ee of the Catalyst)}

A pre-dried $10 \mathrm{~mL}$ round-bottom flask equipped with a magnetic stir bar was covered with aluminum foil to avoid light exposure. The flask was charged with ynamide compound 1a (101 mg, 0.2 mmol, 1 equiv.), $N$-oxide 4 (63.8 mg, 0.4 mmol, 2 equiv.), [(S)-TRIPAg]2 (8.6 mg, $0.005 \mathrm{mmol}, 2.5 \mathrm{~mol} \%$ ), and MS3A (200 mg, $1 \mathrm{~g} / \mathrm{mmol})$, which were subsequently dissolved partially in dry $\mathrm{PhCl}(2 \mathrm{~mL}, 0.1 \mathrm{M})$ under argon gas atmosphere. The reaction mixture was stirred for 48 hours at the room temperature, and then passed through a celite to remove MS3A. After the solvent was evaporated in vacuo, the resulting residue was purified by flash chromatography ( $n$-hexane/EtOAc, $15 / 1$ to $8 / 1, v / v)$ to afford product $2 \mathbf{a}$.

\begin{tabular}{cc}
\hline $\begin{array}{c}\mathrm{ee}_{\text {Cat. }} \\
(\% \text { ee })\end{array}$ & $\begin{array}{c}\mathrm{ee}_{2 \mathrm{a}} \\
(\% \text { ee })\end{array}$ \\
\hline 20 & 20 \\
40 & 43.2 \\
60 & 52.8 \\
80 & 64 \\
100 & 94 \\
\hline
\end{tabular}

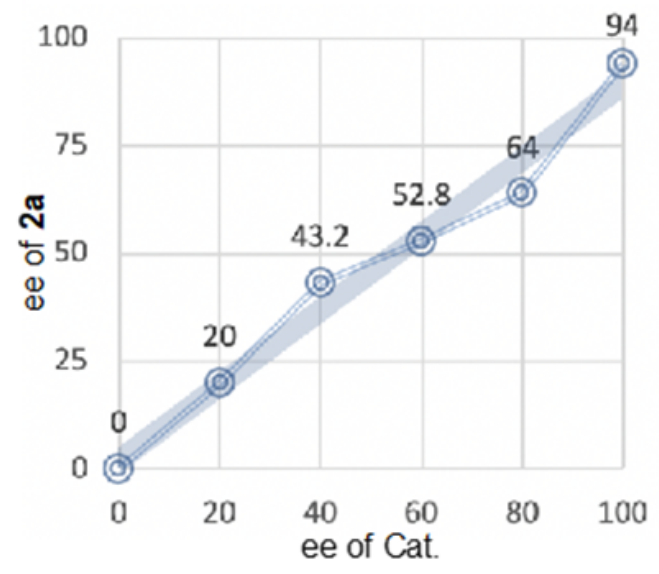




\section{Synthesis and Characterization of Cycloheptatriene 2 and 3}<smiles>[R]c1ccc(C#CN([R])Cc2ccccc2)cc1</smiles>

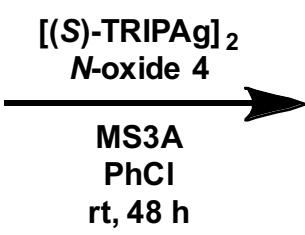

rt, $48 \mathrm{~h}$

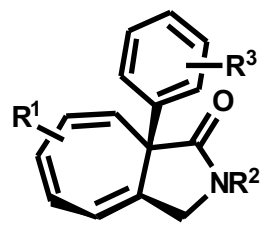

2

\section{General Procedure A for the Ag-catalyzed Asymmetric Büchner Reaction}

A pre-dried $10 \mathrm{~mL}$ test tube equipped with a magnetic stir bar was covered with aluminum foil to avoid light exposure. The test tube was charged with ynamide compounds 1 (0.2 mmol, 1 equiv.), N-oxide 4 (63.8 mg, 0.4 mmol, 2 equiv.), [(S)TRIPAg]2 (8.6 mg, $0.005 \mathrm{mmol}, 2.5 \mathrm{~mol} \%$ ), and MS3A (200 mg, $1 \mathrm{~g} / \mathrm{mmol}$ ), which were subsequently dissolved partially in dry $\mathrm{PhCl}(2 \mathrm{~mL}, 0.1 \mathrm{M})$ under argon gas atmosphere. The reaction mixture was stirred for 48 hours at the room temperature, and then passed through a celite to remove MS3A. After the solvent was evaporated in vacuo, the resulting residue was purified by flash chromatography ( $n$-hexane/EtOAc $=15 / 1$ to $8 / 1, \mathrm{v} / \mathrm{v})$ to afford desired products 2 .

\section{General Protocol for accessing racemic products 2}

A pre-dried $10 \mathrm{~mL}$ test tube equipped with a magnetic stir bar was covered with aluminum foil to avoid light exposure. The test tube was charged with ynamide

compounds 1 (0.2 mmol, 1 equiv.), $N$-oxide 4 (63.8 mg, $0.4 \mathrm{mmol}, 2$ equiv.), and 
$\mathrm{CF}_{3} \mathrm{CO}_{2} \mathrm{Ag}(8.8 \mathrm{mg}, 0.04 \mathrm{mmol}, 20 \mathrm{~mol} \%$ ), which were subsequently dissolved partially in dry $\mathrm{PhCl}(2 \mathrm{~mL}, 0.1 \mathrm{M})$ under argon gas atmosphere. The reaction mixture was stirred for 24 hours at the room temperature. After the solvent was evaporated in vacuo, the resulting residue was purified by flash chromatography $(n$-hexane/EtOAc $=$ $15 / 1$ to $8 / 1, v / v$ ) to afford racemic products 2 .

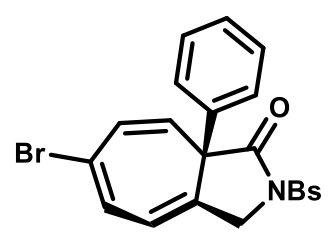

(R)-6-Bromo-2-((4-bromophenyl)sulfonyl)-8a-phenyl-3,8a-

\section{dihydrocyclohepta[c]pyrrol-1(2H)-one (2a)}

White solid (94.4 mg, $91 \%$ yield, $97: 3$ er): m.p. $164-165{ }^{\circ} \mathrm{C}$; TLC $R_{f}=0.2(n-$ hexane/EtOAc, 5/1, v/v); ${ }^{1} \mathrm{H} \mathrm{NMR}\left(400 \mathrm{MHz}, \mathrm{CDCl}_{3}\right) \delta 7.87(\mathrm{~d}, J=8.4 \mathrm{~Hz}, 2 \mathrm{H}), 7.63$ (d, $J=8.4 \mathrm{~Hz}, 2 \mathrm{H}), 7.19-7.13(\mathrm{~m}, 3 \mathrm{H}), 7.05-7.3(\mathrm{~m}, 2 \mathrm{H}), 6.65(\mathrm{~d}, J=7.2 \mathrm{~Hz}, 1 \mathrm{H}), 6.48$ $(\mathrm{d}, J=10.0 \mathrm{~Hz}, 1 \mathrm{H}), 6.27(\mathrm{~d}, J=7.2 \mathrm{~Hz}, 1 \mathrm{H}), 5.50(\mathrm{~d}, J=10.0 \mathrm{~Hz}, 1 \mathrm{H}), 4.82(\mathrm{~d}, J=$ $15.2 \mathrm{~Hz}, 1 \mathrm{H}), 4.67(\mathrm{~d}, J=15.2 \mathrm{~Hz}, 1 \mathrm{H}) ;{ }^{13} \mathrm{C} \mathrm{NMR}\left(100 \mathrm{MHz}, \mathrm{CDCl}_{3}\right) \delta 173.1,136.2$, 136.1, $132.4(2 \mathrm{C}), 130.90,130.86,129.9,129.5(2 \mathrm{C}), 128.2(2 \mathrm{C}), 128.1,127.1,126.3$ (2C), 124.1, 122.9, 121.3, 56.4, 50.4; IR (ATR) 2954, 1739, 1368, 1217, 1011, 818, $743,644,623,611 \mathrm{~cm}^{-1} ;$ HRMS (ESI-TOF) $[\mathrm{M}+\mathrm{Na}]^{+}$calcd for $\mathrm{C}_{21} \mathrm{H}_{15} \mathrm{Br}_{2} \mathrm{NNaO}_{3} \mathrm{~S}^{+}$ $\mathrm{m} / \mathrm{z} 541.9032$, found $541.9044 ;[\alpha]^{25} \mathrm{D}+99.2\left(\mathrm{c} 1.0, \mathrm{CHCl}_{3}\right)$. The enantiomeric ratio was 
determined by analytical chiral HPLC. Retention time: 20.4 min (minor isomer), 24.4 min (major isomer), AD-H column, 80/20 n-hexane/i-PrOH, $1 \mathrm{~mL} / \mathrm{min}, 254 \mathrm{~nm}$.

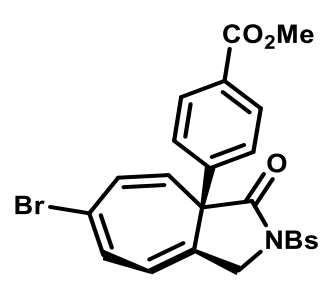

\section{Methyl (R)-4-(6-bromo-2-((4-bromophenyl)sulfonyl)-3-oxo-2,3-}

\section{dihydrocyclohepta[c]pyrrol-3a(1H)-yl)benzoate (2b)}

White solid (100.9 mg, $87 \%$ yield, 95 : 5 er): m.p. $167-169{ }^{\circ} \mathrm{C}$; TLC $R_{f}=0.5(n-$ hexane/EtOAc, 2/1, v/v); ${ }^{1} \mathrm{H}$ NMR (400 MHz, $\left.\mathrm{CDCl}_{3}\right) \delta 7.88(\mathrm{~d}, \mathrm{~J}=8.8 \mathrm{~Hz}, 2 \mathrm{H}), 7.83$ $(\mathrm{d}, J=8.4 \mathrm{~Hz}, 2 \mathrm{H}), 7.64(\mathrm{~d}, J=8.8 \mathrm{~Hz}, 2 \mathrm{H}), 7.15(\mathrm{~d}, J=8.4 \mathrm{~Hz}, 2 \mathrm{H}), 6.67(\mathrm{~d}, J=7.2$ $\mathrm{Hz}, 1 \mathrm{H}), 6.50(\mathrm{~d}, J=10.0 \mathrm{~Hz}, 1 \mathrm{H}), 6.33(\mathrm{~d}, J=7.2 \mathrm{~Hz}, 1 \mathrm{H}), 5.51(\mathrm{~d}, J=10.0 \mathrm{~Hz}, 1 \mathrm{H})$, $4.87(\mathrm{~d}, J=15.2 \mathrm{~Hz}, 1 \mathrm{H}), 4.70(\mathrm{~d}, J=15.2 \mathrm{~Hz}, 1 \mathrm{H}), 3.86(\mathrm{~s}, 3 \mathrm{H}) ;{ }^{13} \mathrm{C} \mathrm{NMR}(100 \mathrm{MHz}$, $\left.\mathrm{CDCl}_{3}\right) \delta 172.4,166.3,141.1,135.9,132.5(2 \mathrm{C}), 131.11,131.07,130.0,129.9,129.5$ (2C), 129.3 (2C), 126.5 (2C), 126.4, 124.2, 122.4, 121.6, 56.4, 52.1, 50.3; IR (ATR) 2951, 1716, 1607, 1573, 1469, 1435, 1407, 1390, 1366, $1335 \mathrm{~cm}^{-1}$; HRMS (ESI-TOF) $[\mathrm{M}+\mathrm{Na}]^{+}$calcd for $\mathrm{C}_{23} \mathrm{H}_{17} \mathrm{Br}_{2} \mathrm{NNaO}_{5} \mathrm{~S}^{+} \mathrm{m} / \mathrm{z} 599.9086$, found $599.9070 ;[\mathrm{\alpha}]^{25}{ }_{\mathrm{D}}+66.9$ (c 1.0, $\mathrm{CHCl}_{3}$ ). The enantiomeric ratio was determined by analytical chiral HPLC. Retention time: 26.0 min (minor isomer), $29.1 \mathrm{~min}$ (major isomer), OX-3 column, 60/40 n-hexane/i-PrOH, $1 \mathrm{~mL} / \mathrm{min}, 254 \mathrm{~nm}$. 


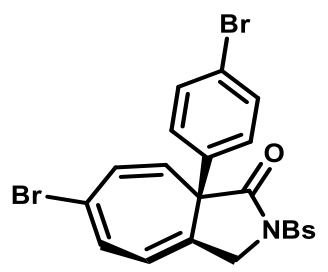

(R)-6-Bromo-8a-(4-bromophenyl)-2-((4-bromophenyl)sulfonyl)-3,8a-

dihydrocyclohepta[c]pyrrol-1(2H)-one (2c)

White solid (105.6 mg, 88\% yield, $94: 6$ er): m.p. $180{ }^{\circ} \mathrm{C}$; TLC $R_{f}=0.2(n-$ hexane/EtOAc, 5/1, v/v); ${ }^{1} \mathrm{H}$ NMR (400 MHz, $\left.\mathrm{CDCl}_{3}\right) \delta 7.88(\mathrm{~d}, \mathrm{~J}=8.8 \mathrm{~Hz}, 2 \mathrm{H}), 7.66$ (d, J = 8.8 Hz, 2H), $7.29(\mathrm{~d}, J=8.8 \mathrm{~Hz}, 2 \mathrm{H}), 6.94(\mathrm{~d}, J=8.8 \mathrm{~Hz}, 2 \mathrm{H}), 6.69(\mathrm{~d}, J=7.2$ $\mathrm{Hz}, 1 \mathrm{H}), 6.48$ (dd, $J=10.0,1.2 \mathrm{~Hz}, 1 \mathrm{H}), 6.31(\mathrm{~d}, J=7.2 \mathrm{~Hz}, 1 \mathrm{H}), 5.47(\mathrm{~d}, J=10.0 \mathrm{~Hz}$, $1 \mathrm{H}), 4.81(\mathrm{~d}, J=15.6 \mathrm{~Hz}, 1 \mathrm{H}), 4.66(\mathrm{~d}, J=15.6 \mathrm{~Hz}, 1 \mathrm{H}) ;{ }^{13} \mathrm{C} \mathrm{NMR}\left(100 \mathrm{MHz}, \mathrm{CDCl}_{3}\right)$ ठ 172.6, 136.0, 135.2, 132.5 (2C), $131.3(2 \mathrm{C}), 131.1(2 \mathrm{C}), 130.0,129.6(2 \mathrm{C}), 128.1$ (2C), 126.1, 124.2, 122.3, 122.2, 121.5, 55.9, 50.3; IR (ATR) 3092, 1739, 1574, 1485, 1469, 1391, 1368, 1335, 1279, $1222 \mathrm{~cm}^{-1}$; HRMS (ESI-TOF) [M + Na] ${ }^{+}$calcd for $\mathrm{C}_{21} \mathrm{H}_{14} \mathrm{Br}_{3} \mathrm{NNaO}_{3} \mathrm{~S}^{+} \mathrm{m} / \mathrm{z}$ 619.8137, found 619.8156; $[\alpha]^{25} \mathrm{D}+77.2$ (c 1.0, $\mathrm{CHCl}_{3}$ ). The enantiomeric ratio was determined by analytical chiral HPLC. Retention time: 15.9 min (minor isomer), 24.2 min (major isomer), AD-H column, 60/40 $n$-hexane/i-PrOH, 1 $\mathrm{mL} / \mathrm{min}, 254 \mathrm{~nm}$. 


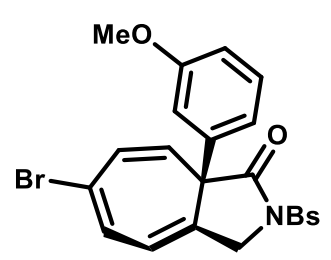

(R)-6-Bromo-2-((4-bromophenyl)sulfonyl)-8a-(3-methoxyphenyl)-3,8adihydrocyclohepta[c]pyrrol-1(2H)-one (2d)

White solid (79.4 mg, $65 \%$ yield, $96: 4$ er): m.p. $144-146{ }^{\circ} \mathrm{C} ; R_{f}=0.4$ ( $n$-hexane/EtOAc, 2/1, v/v); ${ }^{1} \mathrm{H} \mathrm{NMR}\left(400 \mathrm{MHz}, \mathrm{CDCl}_{3}\right) \delta 7.88(\mathrm{~d}, J=8.8 \mathrm{~Hz}, 2 \mathrm{H}), 7.64(\mathrm{~d}, J=8.8 \mathrm{~Hz}, 2 \mathrm{H})$, $7.07(\mathrm{t}, J=8.4 \mathrm{~Hz}, 1 \mathrm{H}), 6.71(\mathrm{dd}, J=8.4 \mathrm{~Hz}, 2.4 \mathrm{~Hz}, 1 \mathrm{H}), 6.76(\mathrm{~d}, J=6.8 \mathrm{~Hz}, 1 \mathrm{H})$, 6.60-6.58 (m, 2H), 6.49 (d, $J=10.0 \mathrm{~Hz}, 1 \mathrm{H}), 6.25(\mathrm{~d}, J=6.8 \mathrm{~Hz}, 1 \mathrm{H}), 5.51(\mathrm{~d}, J=10.0$ $\mathrm{Hz}, 1 \mathrm{H}), 4.80(\mathrm{~d}, J=16.0 \mathrm{~Hz}, 1 \mathrm{H}), 4.67(\mathrm{~d}, J=16.0 \mathrm{~Hz}, 1 \mathrm{H}), 3.71(\mathrm{~s}, 3 \mathrm{H}) ;{ }^{13} \mathrm{C} \mathrm{NMR}$ $\left(100 \mathrm{MHz}, \mathrm{CDCl}_{3}\right) \delta 173.0,159.2,137.8,136.1,132.4(2 \mathrm{C}), 131.1,130.9,129.8,129.5$ (2C), 129.2, 128.2, 124.2, 123.4, 121.2, 118.5, 113.0, 112.7, 56.7, 55.1, 50.4; IR (ATR) 2943, 1741, 1599, 1574, 1486, 1469, 1433, 1390, 1369, $1335 \mathrm{~cm}^{-1}$; HRMS (ESI-TOF) $[\mathrm{M}+\mathrm{Na}]^{+}$calcd for $\mathrm{C}_{22} \mathrm{H}_{17} \mathrm{Br}_{2} \mathrm{NNaO}_{4} \mathrm{~S}^{+} \mathrm{m} / \mathrm{z}$ 571.9137, found $571.9154 ;[\alpha]^{25} \mathrm{D}+855.3$ (c 1.2, $\mathrm{CHCl}_{3}$ ). The enantiomeric ratio was determined by analytical chiral HPLC. Retention time: $27.1 \mathrm{~min}$ (major isomer), $31.9 \mathrm{~min}$ (minor isomer), OX-3 column, 80/20 n-hexane/i-PrOH, $1 \mathrm{~mL} / \mathrm{min}, 254 \mathrm{~nm}$.

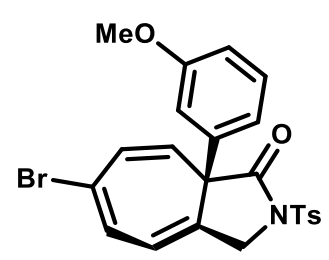


(R)-6-Bromo-8a-(3-methoxyphenyl)-2-tosyl-3,8a-dihydrocyclohepta[c]pyrrol-

$1(2 H)$-one $(2 e)$

White solid $\left(77.2 \mathrm{mg}, 82 \%\right.$ yield, $95: 5$ er): m.p. $156-157{ }^{\circ} \mathrm{C}$; TLC $R_{f}=0.4(n-$ hexane/EtOAc, 2/1, v/v); ${ }^{1} \mathrm{H}$ NMR (400 MHz, $\left.\mathrm{CDCl}_{3}\right) \delta 7.90(\mathrm{~d}, \mathrm{~J}=8.4 \mathrm{~Hz}, 2 \mathrm{H}), 7.30$ $(\mathrm{d}, J=8.0 \mathrm{~Hz}, 2 \mathrm{H}), 7.05(\mathrm{t}, J=8.0 \mathrm{~Hz}, 1 \mathrm{H}), 6.70(\mathrm{ddd}, J=8.4,6.8,0.8 \mathrm{~Hz}, 1 \mathrm{H}), 6.66$ $(\mathrm{d}, J=6.8 \mathrm{~Hz}, 1 \mathrm{H}), 6.60-6.58(\mathrm{~m}, 2 \mathrm{H}), 6.48(\mathrm{~d}, J=10.4 \mathrm{~Hz}, 1 \mathrm{H}), 6.25(\mathrm{dt}, J=6.8,1.6$ $\mathrm{Hz}, 1 \mathrm{H}), 5.51(\mathrm{~d}, J=10.4 \mathrm{~Hz}, 1 \mathrm{H}), 4.81(\mathrm{~d}, J=15.6 \mathrm{~Hz}, 1 \mathrm{H}), 4.67(\mathrm{~d}, J=15.6 \mathrm{~Hz}, 1 \mathrm{H})$, 3.70 (s, 3H), 2.41 (s, 3H); ${ }^{13} \mathrm{C}$ NMR (100 MHz, $\left.\mathrm{CDCl}_{3}\right) \delta$ 172.9, 159.1, 145.6, 138.0, 134.3, 131.0, 130.9, 129.7 (2C), 129.1, 129.0, 128.0 (2C), 124.1, 124.0, 121.0, 118.6, 113.1, 112.5, 56.9, 55.1, 50.4, 21.7; IR (ATR) 2938, 2257, 1738, 1596, 1583, 1485, $1465,1433,1398,1364 \mathrm{~cm}^{-1}$; HRMS (ESI-TOF) $[\mathrm{M}+\mathrm{Na}]^{+}$calcd for $\mathrm{C}_{23} \mathrm{H}_{20} \mathrm{BrNNaO}_{4} \mathrm{~S}^{+}$ $\mathrm{m} / \mathrm{z}$ 508.0189, found 508.0185; $[\alpha]^{25} \mathrm{D}+93.1\left(c 1.0, \mathrm{CHCl}_{3}\right)$. The enantiomeric ratio was determined by analytical chiral HPLC. Retention time: 20.1 min (major isomer), 26.7 min (minor isomer), OX-3 column, 60/40 n-hexane/i-PrOH, $1 \mathrm{~mL} / \mathrm{min}, 254 \mathrm{~nm}$.

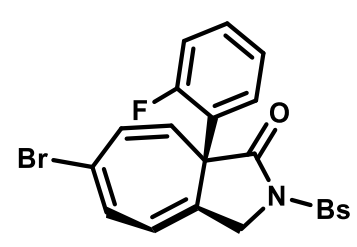

(S)-6-Bromo-2-((4-bromophenyl)sulfonyl)-8a-(2-fluorophenyl)-3,8adihydrocyclohepta[c]pyrrol-1(2H)-one (2f) 
White solid $\left(91.7 \mathrm{mg}, 85 \%\right.$ yield, $93: 7$ er): m.p. $68-69{ }^{\circ} \mathrm{C}$; TLC $R_{f}=0.2(n-$ hexane/EtOAc, 2/1); ${ }^{1} \mathrm{H}$ NMR $\left(400 \mathrm{MHz}, \mathrm{CDCl}_{3}\right) \delta 7.93(\mathrm{~d}, J=8.8 \mathrm{~Hz}, 2 \mathrm{H}), 7.67(\mathrm{~d}, J$ $=8.8 \mathrm{~Hz}, 2 \mathrm{H}), 7.12-7.19(\mathrm{~m}, 1 \mathrm{H}), 6.94-7.04(\mathrm{~m}, 2 \mathrm{H}), 6.77-6.82(\mathrm{~m}, 1 \mathrm{H}), 6.64(\mathrm{~d}, J=8.8$ $\mathrm{Hz}, 1 \mathrm{H}), 6.48(\mathrm{~d}, J=7.2 \mathrm{~Hz}, 1 \mathrm{H}), 6.09(\mathrm{~d}, J=7.2 \mathrm{~Hz}, 1 \mathrm{H}), 4.77(\mathrm{~d}, J=8.8 \mathrm{~Hz}, 1 \mathrm{H})$, $4.70(\mathrm{~d}, J=13.6 \mathrm{~Hz}, 1 \mathrm{H}), 4.59(\mathrm{~d}, J=13.6 \mathrm{~Hz}, 1 \mathrm{H}) ;{ }^{13} \mathrm{C} \mathrm{NMR}\left(100 \mathrm{MHz}, \mathrm{CDCl}_{3}\right) \delta$ 172.7, $160.6(\mathrm{~d}, J=245.0 \mathrm{~Hz}), 136.1,132.3(2 \mathrm{C}), 130.7$ (d, $J=2.8 \mathrm{~Hz}), 130.5,130.1$ (d, $J=8.6 \mathrm{~Hz}), 129.9,129.7(3 \mathrm{C}), 123.5(\mathrm{~d}, J=2.9 \mathrm{~Hz}), 122.7,120.9,120.4(\mathrm{~d}, J=$ $12.4 \mathrm{~Hz}), 115.7(\mathrm{~d}, J=21.9 \mathrm{~Hz}), 107.1,101.9,50.5(\mathrm{~d}, J=5.8 \mathrm{~Hz}), 47.3 ;{ }^{19} \mathrm{~F}$ NMR (376 MHz, $\left.\mathrm{CDCl}_{3}\right) \delta-115.9$; IR (ATR) 3026, 1736, 1612, 1574, 1483, 1470, 1455, 1390, 1365, $1335 \mathrm{~cm}^{-1}$; HRMS (ESI-TOF) $[\mathrm{M}+\mathrm{Na}]^{+}$calcd for $\mathrm{C}_{21} \mathrm{H}_{14} \mathrm{Br}_{2} \mathrm{FNNaO}_{3} \mathrm{~S}^{+} \mathrm{m} / \mathrm{z}$ 559.8937, found 559.8958; $[\alpha]^{25} \mathrm{D}+182.3\left(\mathrm{c} 1.0, \mathrm{CHCl}_{3}\right)$. The enantiomeric ratio was determined by analytical chiral HPLC. Retention time: 34.1 min (major isomer), 43.8 min (minor isomer), AD-H column, 85/15 n-hexane/i-PrOH, $1 \mathrm{~mL} / \mathrm{min}, 254 \mathrm{~nm}$.

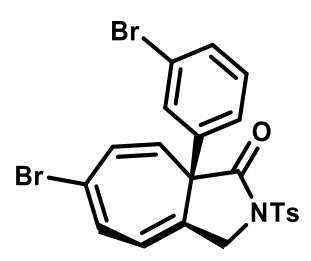

(R)-6-Bromo-8a-(3-bromophenyl)-2-tosyl-3,8a-dihydrocyclohepta[c]pyrrol$1(2 H)$-one $(2 \mathrm{~g})$ 
White solid (84.7 mg, $79 \%$ yield, $96: 4$ er): m.p. $160-162{ }^{\circ} \mathrm{C}$; TLC $R_{f}=0.2(n-$ hexane/EtOAc, 5/1, v/v); ${ }^{1} \mathrm{H}$ NMR $\left(400 \mathrm{MHz}, \mathrm{CDCl}_{3}\right) \delta 7.88(\mathrm{~d}, J=8.4 \mathrm{~Hz}, 2 \mathrm{H}), 7.30-$ $7.25(\mathrm{~m}, 3 \mathrm{H}), 7.07(\mathrm{~s}, 1 \mathrm{H}), 7.01-7.00(\mathrm{~m}, 2 \mathrm{H}), 6.68(\mathrm{~d}, J=6.8 \mathrm{~Hz}, 1 \mathrm{H}), 6.48(\mathrm{~d}, J=$ $10.0 \mathrm{~Hz}, 1 \mathrm{H}), 6.29(\mathrm{~d}, J=6.8 \mathrm{~Hz}, 1 \mathrm{H}), 5.48(\mathrm{~d}, J=10.0 \mathrm{~Hz}, 1 \mathrm{H}), 4.81(\mathrm{~d}, J=15.2 \mathrm{~Hz}$, 1H), $4.66(\mathrm{~d}, J=15.2 \mathrm{~Hz}, 1 \mathrm{H}), 2.40(\mathrm{~s}, 3 \mathrm{H}) ;{ }^{13} \mathrm{C} \mathrm{NMR}\left(100 \mathrm{MHz}, \mathrm{CDCl}_{3}\right) \delta$ 172.4, 145.8, $138.7,134.0,131.2,131.13,131.07,129.8$ (2C), 129.6, 129.2, $128.0(2 \mathrm{C}), 127.7$ 125.2, 124.2, 123.3, 122.2, 121.4, 56.4, 50.3, 21.7; IR (ATR) 2923, 1738, 1595, 1566, 1469, 1416, 1365, 1335, 1225, $1187 \mathrm{~cm}^{-1}$; HRMS (ESI-TOF) $[\mathrm{M}+\mathrm{Na}]^{+}$calcd for $\mathrm{C}_{22} \mathrm{H}_{17} \mathrm{Br}_{2} \mathrm{NNaO}_{3} \mathrm{~S}^{+} \mathrm{m} / \mathrm{z}$ 555.9188, found 555.9198; $[\alpha]^{25} \mathrm{D}+98.8$ (c 1.0, $\left.\mathrm{CHCl}_{3}\right)$. The enantiomeric ratio was determined by analytical chiral HPLC. Retention time: $23.3 \mathrm{~min}$ (minor isomer), 26.4 min (major isomer), AD-H column, 80/20 $n$-hexane/i-PrOH, 1 $\mathrm{mL} / \mathrm{min}, 254 \mathrm{~nm}$.

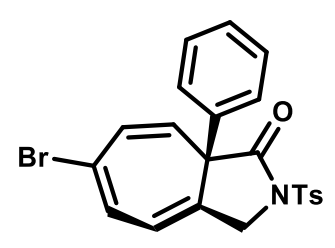

(R)-6-Bromo-8a-phenyl-2-tosyl-3,8a-dihydrocyclohepta[c]pyrrol-1(2H)-one (2h)

White solid $\left(72.9 \mathrm{mg}, 80 \%\right.$ yield, $96: 4$ er): m.p. $175-177^{\circ} \mathrm{C} ;$ TLC $R_{f}=0.3(n-$ hexane/EtOAc, 5/1, v/v); ${ }^{1} \mathrm{H}$ NMR $\left(400 \mathrm{MHz}, \mathrm{CDCl}_{3}\right) \delta 7.90(\mathrm{~d}, \mathrm{~J}=8.0 \mathrm{~Hz}, 2 \mathrm{H}), 7.29$ (d, $J=8.0 \mathrm{~Hz}, 2 \mathrm{H}), 7.16-7.14(\mathrm{~m}, 3 \mathrm{H}), 7.04(\mathrm{dd}, J=8.0,2.0 \mathrm{~Hz}, 2 \mathrm{H}), 6.65(\mathrm{~d}, J=6.8$ 
$\mathrm{Hz}, 1 \mathrm{H}), 6.47(\mathrm{~d}, J=10.0 \mathrm{~Hz}, 1 \mathrm{H}), 6.27(\mathrm{~d}, J=6.8 \mathrm{~Hz}, 1 \mathrm{H}), 5.51(\mathrm{~d}, J=10.0 \mathrm{~Hz}, 1 \mathrm{H})$, $4.83(\mathrm{~d}, J=15.2 \mathrm{~Hz}, 1 \mathrm{H}), 4.67(\mathrm{~d}, J=15.2 \mathrm{~Hz}, 1 \mathrm{H}), 2.41(\mathrm{~s}, 3 \mathrm{H}) ;{ }^{13} \mathrm{C} \mathrm{NMR}(100 \mathrm{MHz}$, $\left.\mathrm{CDCl}_{3}\right) \delta$ 173.0, 145.6, 136.4, 134.2, 130.9, 130.8, $129.7(2 \mathrm{C}), 128.1(3 \mathrm{C}), 128.0(2 \mathrm{C})$ 127.9, 126.3 (2C), 124.1, 123.5, 121.1, 56.6, 50.4, 21.7; IR (ATR) 3058, 1737, 1596, 1493, 1467, 1447, 1365, 1335, 1224, $1170 \mathrm{~cm}^{-1}$; HRMS (ESI-TOF) [M + Na] $]^{+}$calcd for $\mathrm{C}_{22} \mathrm{H}_{18} \mathrm{BrNNaO}_{3} \mathrm{~S}^{+} \mathrm{m} / \mathrm{z}$ 478.0083, found 478.0094; [a] ${ }^{25} \mathrm{D}+122.59$ (c 0.85, $\mathrm{CHCl}_{3}$ ). The enantiomeric ratio was determined by analytical chiral HPLC. Retention time: $20.9 \mathrm{~min}$ (minor isomer), $25.7 \mathrm{~min}$ (major isomer), AD-H column, 80/20 $n$ hexane/i-PrOH, $1 \mathrm{~mL} / \mathrm{min}, 254 \mathrm{~nm}$.

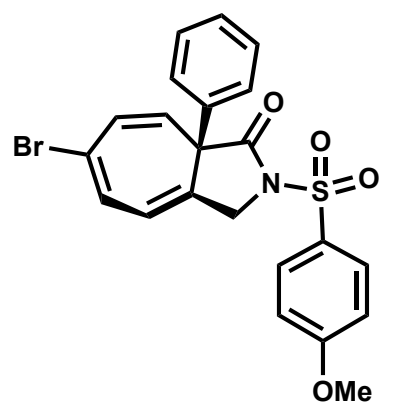

(R)-6-Bromo-2-((4-methoxyphenyl)sulfonyl)-8a-phenyl-3,8a-

\section{dihydrocyclohepta[c]pyrrol-1(2H)-one (2i)}

White solid $\left(79.3 \mathrm{mg}, 84 \%\right.$ yield, $95: 5$ er): m.p. $138-139{ }^{\circ} \mathrm{C}$; TLC $R_{f}=0.3(n-$ hexane/EtOAc, 2/1, v/v); ${ }^{1} \mathrm{H}$ NMR $\left(400 \mathrm{MHz}, \mathrm{CDCl}_{3}\right) \delta 7.95(\mathrm{~d}, J=8.4 \mathrm{~Hz}, 2 \mathrm{H}), 7.18-$ $7.13(\mathrm{~m}, 3 \mathrm{H}), 7.06-7.03(\mathrm{~m}, 2 \mathrm{H}), 6.94(\mathrm{~d}, J=8.4 \mathrm{~Hz}, 2 \mathrm{H}), 6.64(\mathrm{~d}, J=6.8 \mathrm{~Hz}, 1 \mathrm{H}), 6.47$ (d, $J=10.0 \mathrm{~Hz}, 1 \mathrm{H}), 6.27(\mathrm{~d}, J=6.8 \mathrm{~Hz}, 1 \mathrm{H}), 5.52(\mathrm{~d}, J=10.0 \mathrm{~Hz}, 1 \mathrm{H}), 4.83(\mathrm{~d}, J=$ 
$15.6 \mathrm{~Hz}, 1 \mathrm{H}), 4.68(\mathrm{~d}, J=15.6 \mathrm{~Hz}, 1 \mathrm{H}), 3.82(\mathrm{~s}, 3 \mathrm{H}) ;{ }^{13} \mathrm{C} \mathrm{NMR}\left(100 \mathrm{MHz}, \mathrm{CDCl}_{3}\right) \delta$ $172.9,164.2,136.4,130.9,130.8,130.4(2 \mathrm{C}), 128.5,128.3,128.1(2 \mathrm{C}), 127.9,126.3$ (2C), 124.0, 123.6, 121.0, 114.2 (2C), 56.7, 55.6, 50.4; IR (ATR) 2945, 1736, 1593, 1577, 1496, 1464, 1446, 1416, 1364, $1335 \mathrm{~cm}^{-1}$; HRMS (ESI-TOF) $[\mathrm{M}+\mathrm{Na}]^{+}$calcd for $\mathrm{C}_{22} \mathrm{H}_{18} \mathrm{BrNNaO}_{4} \mathrm{~S}^{+} \mathrm{m} / \mathrm{z} 494.0032$, found 494.0045; $[\alpha]^{25} \mathrm{D}+89.1\left(c 1.0, \mathrm{CHCl}_{3}\right)$. The enantiomeric ratio was determined by analytical chiral HPLC. Retention time: $20.7 \mathrm{~min}$ (major isomer), $28.0 \mathrm{~min}$ (minor isomer), OX-3 column, 60/40 $n$-hexane/i-PrOH, 1 $\mathrm{mL} / \mathrm{min}, 254 \mathrm{~nm}$.

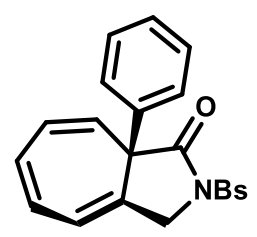

(R)-2-((4-Bromophenyl)sulfonyl)-8a-phenyl-3,8a-dihydrocyclohepta[c]pyrrol-

\section{$1(2 H)$-one (2j)}

White solid $\left(78.3 \mathrm{mg}, 89 \%\right.$ yield, $94: 6$ er): m.p. $154-155{ }^{\circ} \mathrm{C}$; TLC $R_{f}=0.3(n-$ hexane/EtOAc, 5/1, v/v); ${ }^{1} \mathrm{H}$ NMR $\left(400 \mathrm{MHz}, \mathrm{CDCl}_{3}\right) \delta 7.88(\mathrm{~d}, \mathrm{~J}=8.8 \mathrm{~Hz}, 2 \mathrm{H}), 7.62$ (d, $J=8.8 \mathrm{~Hz}, 2 \mathrm{H}), 7.14-7.08(\mathrm{~m}, 5 \mathrm{H}), 6.45(\mathrm{~d}, J=6.4 \mathrm{~Hz}, 1 \mathrm{H}), 6.34-6.20(\mathrm{~m}, 3 \mathrm{H}), 5.51$ (d, $J=9.2 \mathrm{~Hz}, 1 \mathrm{H}), 4.85(\mathrm{~d}, J=14.4 \mathrm{~Hz}, 1 \mathrm{H}), 4.70(\mathrm{~d}, J=14.4 \mathrm{~Hz}, 1 \mathrm{H}) ;{ }^{13} \mathrm{C}$ NMR $(100$ $\left.\mathrm{MHz}, \mathrm{CDCl}_{3}\right) \delta 173.9,136.6,136.3,132.3(2 \mathrm{C}), 129.8,129.6,129.5(2 \mathrm{C}), 128.5,127.8$ (2C), 127.7, 127.1, 126.7 (2C), 123.6, 122.1, 119.9, 55.7, 50.5; IR (ATR) 3023, 2255, 
1736, 1650, 1573, 1492,1469, 1447, 1389, $1360 \mathrm{~cm}^{-1} ;$ HRMS (ESI-TOF) $[\mathrm{M}+\mathrm{Na}]^{+}$ calcd for $\mathrm{C}_{21} \mathrm{H}_{16} \mathrm{BrNNaO}_{3} \mathrm{~S}^{+} \mathrm{m} / \mathrm{z}$ 463.9926, found 463.9912; [ $\alpha{ }^{25} \mathrm{D}+68.2\left(c 1.0, \mathrm{CHCl}_{3}\right)$. The enantiomeric ratio was determined by analytical chiral HPLC. Retention time: 14.7 min (minor isomer), 18.8 min (major isomer), AD-H column, 80/20 $n$-hexane/i-PrOH, 1 $\mathrm{mL} / \mathrm{min}, 254 \mathrm{~nm}$.

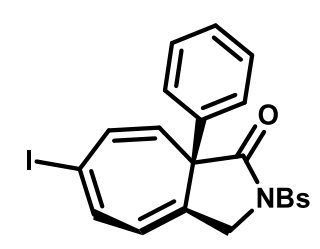

(R)-2-((4-Bromophenyl)sulfonyl)-6-iodo-8a-phenyl-3,8a-

\section{dihydrocyclohepta[c]pyrrol-1(2H)-one (2k)}

White solid (100.6 mg, 89\% yield, $93: 7$ er): m.p. $169{ }^{\circ} \mathrm{C}$; TLC $R_{f}=0.2(n-$ hexane/EtOAc, 5/1, v/v); ${ }^{1} \mathrm{H}$ NMR $\left(400 \mathrm{MHz}, \mathrm{CDCl}_{3}\right) \delta 7.88(\mathrm{~d}, J=8.8 \mathrm{~Hz}, 2 \mathrm{H}), 7.65$ $(\mathrm{d}, J=8.8 \mathrm{~Hz}, 2 \mathrm{H}), 7.22-7.14(\mathrm{~m}, 3 \mathrm{H}), 7.03-7.01(\mathrm{~m}, 2 \mathrm{H}), 6.90(\mathrm{~d}, J=6.8 \mathrm{~Hz}, 1 \mathrm{H}), 6.63$ (d, $J=10.0 \mathrm{~Hz}, 1 \mathrm{H}), 6.15(\mathrm{~d}, J=6.8 \mathrm{~Hz}, 1 \mathrm{H}), 5.28(\mathrm{~d}, J=10.0 \mathrm{~Hz}, 1 \mathrm{H}), 4.79(\mathrm{~d}, J=$ $14.8 \mathrm{~Hz}, 1 \mathrm{H}), 4.66(\mathrm{~d}, J=14.8 \mathrm{~Hz}, 1 \mathrm{H}) ;{ }^{13} \mathrm{C}$ NMR $\left(100 \mathrm{MHz}, \mathrm{CDCl}_{3}\right) \delta 173.1,138.1$, 136.1, 136.0, 135.3, 132.4 (2C), 129.8, 129.5 (2C), 128.14 (2C), 128.05, 127.6, 126.3 (2C), 122.1, 121.9, 97.9, 56.3, 50.4; IR (ATR) 3087, 2363, 2256, 1738, 1597, 1573, 1492, 1469, 1446, $1390 \mathrm{~cm}^{-1}$; HRMS (ESI-TOF) $\left[\mathrm{M}+\mathrm{Na}^{+}\right.$calcd for $\mathrm{C}_{21} \mathrm{H}_{15} \mathrm{BrINNaO}_{3} \mathrm{~S}^{+} \mathrm{m} / \mathrm{z}$ 589.8893, found 589.8888; $[\alpha]^{25} \mathrm{D}+35.6\left(c\right.$ 1.0, $\left.\mathrm{CHCl}_{3}\right)$. The 
enantiomeric ratio was determined by analytical chiral HPLC. Retention time: $22.7 \mathrm{~min}$ (minor isomer), $28.9 \mathrm{~min}$ (major isomer), AD-H column, 80/20 $n$-hexane/i-PrOH, 1 $\mathrm{mL} / \mathrm{min}, 254 \mathrm{~nm}$.

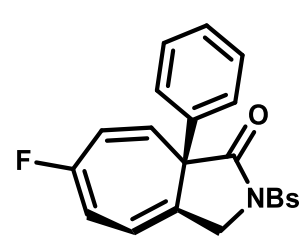

(R)-2-((4-Bromophenyl)sulfonyl)-6-fluoro-8a-phenyl-3,8a-

\section{dihydrocyclohepta[c]pyrrol-1(2H)-one (2I)}

White solid ( $80.1 \mathrm{mg}, 87 \%$ yield, $95: 5$ er): m.p. $140-141{ }^{\circ} \mathrm{C} ;$ TLC $R_{f}=0.2(n-$ hexane/EtOAc, 5/1, v/v); ${ }^{1} \mathrm{H} \mathrm{NMR}\left(400 \mathrm{MHz}, \mathrm{CDCl}_{3}\right) \delta 7.86(\mathrm{~d}, \mathrm{~J}=8.8 \mathrm{~Hz}, 2 \mathrm{H}), 7.62$ (d, $J=8.8 \mathrm{~Hz}, 2 \mathrm{H}), 7.19-7.09(\mathrm{~m}, 5 \mathrm{H}), 6.41-6.38(\mathrm{~m}, 1 \mathrm{H}), 6.31(\mathrm{dt}, J=8.4,1.2 \mathrm{~Hz}$, 1H), $6.05(\mathrm{dd}, J=12.0,7.2 \mathrm{~Hz}, 1 \mathrm{H}), 5.84(\mathrm{dd}, J=10.4,5.2 \mathrm{~Hz}, 1 \mathrm{H}), 4.85(\mathrm{~d}, J=14.8$ $\mathrm{Hz}, 1 \mathrm{H}), 4.67(\mathrm{~d}, J=14.8 \mathrm{~Hz}, 1 \mathrm{H}) ;{ }^{13} \mathrm{C} \mathrm{NMR}\left(100 \mathrm{MHz}, \mathrm{CDCl}_{3}\right) \delta 173.2,160.9(\mathrm{~d}, J=$ $246.0 \mathrm{~Hz}), 136.6,136.1,132.4(2 \mathrm{C}), 129.8,129.5(2 \mathrm{C}), 128.2(2 \mathrm{C}), 128.1,126.5(\mathrm{~d}$, $J=13.3 \mathrm{~Hz}), 126.2(2 \mathrm{C}), 124.9,121.7(\mathrm{~d}, J=36.2 \mathrm{~Hz}), 119.3(\mathrm{~d}, J=11.5 \mathrm{~Hz}), 110.0$ (d, $J=27.6 \mathrm{~Hz}), 56.9,50.4 ;{ }^{19} \mathrm{~F} \mathrm{NMR}\left(376 \mathrm{MHz}, \mathrm{CDCl}_{3}\right) \delta-98.1$; IR (ATR) 3085, 2237, 1746, 1604, 1574, 1509, 1469, 1442, 1390, $1365 \mathrm{~cm}^{-1}$; HRMS (ESI-TOF) [M + $\mathrm{Na}]^{+}$calcd for $\mathrm{C}_{21} \mathrm{H}_{15} \mathrm{BrFNNaO}_{3} \mathrm{~S}^{+} \mathrm{m} / \mathrm{z}$ 481.9832, found 481.9829; [ $\left.\mathrm{\alpha}\right]^{25} \mathrm{D}-3.58$ ( $\mathrm{c}$ 1.0, $\mathrm{CHCl}_{3}$ ). The enantiomeric ratio was determined by analytical chiral HPLC. 
Retention time: 19.4 min (minor isomer), 24.8 min (major isomer), AD-H column, 80/20 n-hexane/i-PrOH, $1 \mathrm{~mL} / \mathrm{min}, 254 \mathrm{~nm}$.

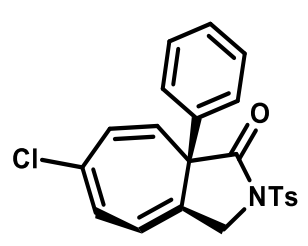

(R)-6-Chloro-8a-phenyl-2-tosyl-3,8a-dihydrocyclohepta[c]pyrrol-1(2H)-one (2m) White solid $\left(69.5 \mathrm{mg}, 84 \%\right.$ yield, $93: 7$ er): m.p. $116-118{ }^{\circ} \mathrm{C} ;$ TLC $R_{f}=0.5(n-$ hexane/EtOAc, 5/1, v/v); ${ }^{1} \mathrm{H}$ NMR (400 MHz, $\left.\mathrm{CDCl}_{3}\right) \delta 7.90(\mathrm{~d}, J=7.6 \mathrm{~Hz}, 2 \mathrm{H}), 7.29$ $(\mathrm{d}, J=8.4 \mathrm{~Hz}, 2 \mathrm{H}), 7.16-7.11(\mathrm{~m}, 3 \mathrm{H}), 7.06-7.04(\mathrm{~m}, 2 \mathrm{H}), 6.43(\mathrm{~d}, J=7.2 \mathrm{~Hz}, 1 \mathrm{H})$, 6.44-6.32 (m, 2H), $5.61(\mathrm{~d}, J=10.8 \mathrm{~Hz}, 1 \mathrm{H}), 4.86(\mathrm{~d}, J=14.8 \mathrm{~Hz}, 1 \mathrm{H}), 4.69(\mathrm{~d}, J=$ $14.8 \mathrm{~Hz}, 1 \mathrm{H}), 2.40$ (s, 3H); ${ }^{13} \mathrm{C}$ NMR (100 MHz, $\left.\mathrm{CDCl}_{3}\right) \delta 173.0,145.6,136.5,134.6$, 134.3, 129.7 (2C), 128.8, $128.1(2 \mathrm{C}), 128.0(2 \mathrm{C}), 127.9,127.3,127.1,126.3(2 \mathrm{C})$, 123.4, 120.4, 56.4, 50.4, 21.6; IR (ATR) 3032, 1738, 1596, 1493, 1468, 1447, 1399, 1364, 1335, $1225 \mathrm{~cm}^{-1}$; HRMS (ESI-TOF) $[\mathrm{M}+\mathrm{Na}]^{+}$calcd for $\mathrm{C}_{22} \mathrm{H}_{18} \mathrm{CINNaO}_{3} \mathrm{~S}^{+} \mathrm{m} / \mathrm{z}$ 434.0588, found 434.0600; $[\alpha]^{25} \mathrm{D}+70.5\left(c\right.$ 1.0, $\left.\mathrm{CHCl}_{3}\right)$. The enantiomeric ratio was determined by analytical chiral HPLC. Retention time: 20.7 min (minor isomer), 24.6 min (major isomer), AD-H column, 80/20 n-hexane/i-PrOH, $1 \mathrm{~mL} / \mathrm{min}, 254 \mathrm{~nm}$. 


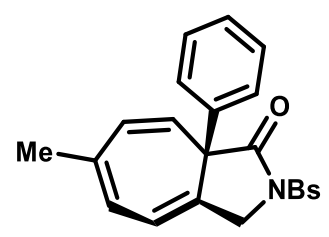

(R)-2-((4-Bromophenyl)sulfonyl)-6-methyl-8a-phenyl-3,8a-

\section{dihydrocyclohepta[c]pyrrol-1(2H)-one (2n)}

White solid $\left(79.6 \mathrm{mg}, 87 \%\right.$ yield, $94: 6$ er): m.p. $158-160{ }^{\circ} \mathrm{C}$; TLC $R_{f}=0.3(n-$ hexane/EtOAc, 5/1, v/v); ${ }^{1} \mathrm{H}$ NMR $\left(400 \mathrm{MHz}, \mathrm{CDCl}_{3}\right) \delta$ 7.89-7.87 (m, 2H), 7.63-7.61 (m, 2H), 7.14-7.11 (m, 3H), 7.07-7.05 (m, 2H), $6.30(\mathrm{~d}, J=6.8 \mathrm{~Hz}, 1 \mathrm{H}), 6.13(\mathrm{~d}, J=$ $10.0 \mathrm{~Hz}, 1 \mathrm{H}), 6.04(\mathrm{~d}, J=6.8 \mathrm{~Hz}, 1 \mathrm{H}), 5.31(\mathrm{~d}, J=10.0 \mathrm{~Hz}, 1 \mathrm{H}), 4.79(\mathrm{~d}, J=14.4 \mathrm{~Hz}$, 1H), $4.64(\mathrm{~d}, J=14.4 \mathrm{~Hz}, 1 \mathrm{H}), 1.77(\mathrm{~s}, 3 \mathrm{H}) ;{ }^{13} \mathrm{C} \mathrm{NMR}\left(100 \mathrm{MHz}, \mathrm{CDCl}_{3}\right) \delta$ 174.1, 138.6, 136.6, 136.4, $132.3(2 \mathrm{C}), 129.5,129.5(2 \mathrm{C}), 129.2,127.8(2 \mathrm{C}), 127.6,126.9(2 \mathrm{C})$, 126.2, 121.8, 116.5, 114.2, 53.8, 50.5, 23.8; IR (ATR) 3020, 1739, 1574, 1492, 1470, 1447, 1390, 1370, 1280, $1226 \mathrm{~cm}^{-1}$; HRMS (ESI-TOF) $[\mathrm{M}+\mathrm{Na}]^{+}$calcd for $\mathrm{C}_{22} \mathrm{H}_{18} \mathrm{BrNNaO}_{3} \mathrm{~S}^{+} \mathrm{m} / \mathrm{z}$ 478.0083, found 478.0080; $[\alpha]^{25} \mathrm{D}+85.6$ (c 1.0, $\mathrm{CHCl}_{3}$ ). The enantiomeric ratio was determined by analytical chiral HPLC. Retention time: $14.7 \mathrm{~min}$ (minor isomer), $16.5 \mathrm{~min}$ (major isomer), AD-H column, 80/20 $n$-hexane/i-PrOH, 1 $\mathrm{mL} / \mathrm{min}, 254 \mathrm{~nm}$.

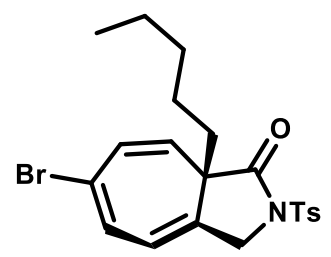


(S)-6-bromo-8a-pentyl-2-tosyl-3,8a-dihydrocyclohepta[c]pyrrol-1(2H)-one (20)

Yellow oil $(28.0 \mathrm{mg}, 31 \%$ yield, $87: 13 \mathrm{er})$; TLC $R_{f}=0.5(n$-hexane/EtOAc, 5/1, v/v); ${ }^{1} \mathrm{H}$ NMR $\left(400 \mathrm{MHz}, \mathrm{CDCl}_{3}\right) \delta 7.96(\mathrm{~d}, J=8.0 \mathrm{~Hz}, 2 \mathrm{H}), 7.35(\mathrm{~d}, J=8.0 \mathrm{~Hz}, 2 \mathrm{H}), 6.78$ (d, $J=7.2 \mathrm{~Hz}, 1 \mathrm{H}), 6.26(\mathrm{~d}, J=10.8 \mathrm{~Hz}, 1 \mathrm{H}), 6.10(\mathrm{~d}, J=7.2 \mathrm{~Hz}, 1 \mathrm{H}), 5.21(\mathrm{~d}, J=10.8$ $\mathrm{Hz}, 1 \mathrm{H}), 4.59(\mathrm{~d}, J=15.6 \mathrm{~Hz}, 1 \mathrm{H}), 4.47(\mathrm{~d}, J=15.6 \mathrm{~Hz}, 1 \mathrm{H}), 2.44(\mathrm{~s}, 3 \mathrm{H}), 1.48-1.47$ (m, 2H), 1.06-0.92 (m, 4H), 0.90-0.81 (m, 1H), $0.71(\mathrm{t}, J=7.2 \mathrm{~Hz}, 3 \mathrm{H}), 0.53-0.44(\mathrm{~m}$, 1H); $\left.{ }^{13} \mathrm{C} \mathrm{NMR} \mathrm{(100} \mathrm{MHz,} \mathrm{CDCl}_{3}\right) \delta 175.1,145.7,134.7,130.1,129.74(2 \mathrm{C}), 129.68$, 128.9, $128.2(2 \mathrm{C}), 125.6,123.6,119.8,53.5,50.5,31.3,30.8,22.9,22.2,21.7,13.7$; IR (ATR) 2925, 2856, 2360, 1740, 1597, 1466, 1370, 1337, 1216, $1173 \mathrm{~cm}^{-1}$; HRMS (ESI-TOF) $[\mathrm{M}+\mathrm{Na}]^{+}$calcd for $\mathrm{C}_{21} \mathrm{H}_{24} \mathrm{BrNNaO}_{3} \mathrm{~S}^{+} \mathrm{m} / \mathrm{z} 472.0552$, found $472.0561 ;[\alpha]^{25} \mathrm{D}$ +99.9 ( $\left.\mathrm{c} 0.72, \mathrm{CHCl}_{3}\right)$. The enantiomeric ratio was determined by analytical chiral HPLC. Retention time: 12.2 min (minor isomer), 19.4 min (major isomer), AD-H column, 90/10 n-hexane/i-PrOH, $1 \mathrm{~mL} / \mathrm{min}, 254 \mathrm{~nm}$.

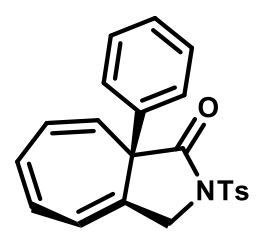

\section{(R)-8a-phenyl-2-tosyl-3,8a-dihydrocyclohepta[c]pyrrol-1(2H)-one (2p)}

White solid $\left(73.0 \mathrm{mg}, 96 \%\right.$ yield, 90:10 er): m.p. $56-58{ }^{\circ} \mathrm{C}$; TLC $R_{f}=0.3(n-$ hexane/EtOAc, 4/1); ${ }^{1} \mathrm{H} \mathrm{NMR}\left(400 \mathrm{MHz}, \mathrm{CDCl}_{3}\right) \delta 7.89$ (d, $\left.J=8.8 \mathrm{~Hz}, 2 \mathrm{H}\right), 7.26$ (dd, $J=8.8,8.4 \mathrm{~Hz}, 2 \mathrm{H}), 7.12-7.10(\mathrm{~m}, 4 \mathrm{H}), 7.25-7.20(\mathrm{~m}, 2 \mathrm{H}), 6.44(\mathrm{~d}, J=6.0 \mathrm{~Hz}, 1 \mathrm{H})$, 
6.32-6.19 (m, 3H), $5.31(\mathrm{~d}, J=9.6 \mathrm{~Hz}, 1 \mathrm{H}), 4.86(\mathrm{~d}, J=16.0 \mathrm{~Hz}, 1 \mathrm{H}), 4.69(\mathrm{~d}, J=16.0$ $\mathrm{Hz}, 1 \mathrm{H}), 2.39(\mathrm{~s}, 3 \mathrm{H}) ;{ }^{13} \mathrm{C} \mathrm{NMR}\left(100 \mathrm{MHz}, \mathrm{CDCl}_{3}\right) \delta 173.7,145.4,136.9,134.5,129.7$, $129.6(2 \mathrm{C}), 128.6,128.0(2 \mathrm{C}), 127.7$ (2C), 127.5, 127.0, 126.7 (2C), 124.9, 121.9, 120.9, 56.0, 50.5, 21.6; IR (ATR) 3022, 1735, 1595, 1492, 1467, 1447, 1358, 1327 , $1243,1206 \mathrm{~cm}^{-1} ;[\mathrm{M}+\mathrm{Na}]^{+}$calcd for $\mathrm{C}_{22} \mathrm{H}_{19} \mathrm{NNaO}_{3} \mathrm{~S}^{+} \mathrm{m} / \mathrm{z} 400.0978$, found 400.0981 ; $[\alpha]^{25} \mathrm{D}+72.6\left(\mathrm{c} 1.0, \mathrm{CHCl}_{3}\right)$. The enantiomeric ratio was determined by analytical chiral HPLC. Retention time: 14.9 min (major isomer), 19.0 min (minor isomer), OX-3 column, 60/40 n-hexane/i-PrOH, $1 \mathrm{~mL} / \mathrm{min}, 254 \mathrm{~nm}$.

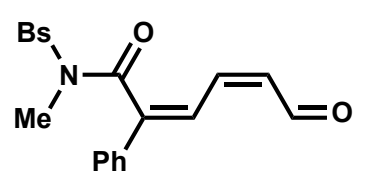

\section{$N$-((4-bromophenyl)sulfonyl)- $N$-methyl-6-oxo-2-phenylhexa-2,4-dienamide (2t)}

Yellow oil; TLC $R_{f}=0.3\left(n\right.$-hexane/EtOAc, 2/1); ${ }^{1} \mathrm{H} \mathrm{NMR}\left(400 \mathrm{MHz}, \mathrm{CDCl}_{3}\right) \delta 10.15$ (d, $J=7.2 \mathrm{~Hz}, 1 \mathrm{H}), 7.65(\mathrm{~s}, 4 \mathrm{H}), 7.46-7.43(\mathrm{~m}, 4 \mathrm{H}), 7.32-7.27(\mathrm{~m}, 2 \mathrm{H}), 7.09(\mathrm{t}, J=12.0$ $\mathrm{Hz}, 1 \mathrm{H}), 6.08(\mathrm{dd}, J=10.8,7.2 \mathrm{~Hz}, 1 \mathrm{H}), 3.11(\mathrm{~s}, 3 \mathrm{H}) ;{ }^{13} \mathrm{C} \mathrm{NMR}\left(100 \mathrm{MHz}, \mathrm{CDCl}_{3}\right) \delta$ $189.9,169,8,144.5,140.1,136.9,132.3(2 \mathrm{C}), 132.1,131.3,129.7$ (2C), $129.5(2 \mathrm{C})$, 129.3, 128.9 (2C), 126.1 (2C), 34.1; IR (ATR) 3088, 2854, 2257, 1669, 1613, 1573, 1492, 1470, 1444, $1390 \mathrm{~cm}^{-1}$; HRMS (ESI-TOF) $[\mathrm{M}+\mathrm{H}]^{+}$calcd for $\mathrm{C}_{19} \mathrm{H}_{17} \mathrm{BrNO}_{4} \mathrm{~S}^{+}$ $\mathrm{m} / \mathrm{z} 434.0056$, found 434.0050 . 
<smiles>[13CH3]N(Cc1ccc(Br)cc1)C(=O)C(=O)c1ccccc1</smiles>

N-(4-Bromobenzyl)-N-((4-bromophenyl)sulfonyl)-2-oxo-2-phenylacetamide (3a)

White solid: m.p. $121-123{ }^{\circ} \mathrm{C} ; R_{f}=0.6$ (n-hexane/EtOAc, $\left.2 / 1, \mathrm{v} / \mathrm{v}\right) ;{ }^{1} \mathrm{H}$ NMR $(400 \mathrm{MHz}$, $\left.\mathrm{CDCl}_{3}\right) \delta 7.86(\mathrm{dd}, J=8.8,2.4 \mathrm{~Hz}, 2 \mathrm{H}), 7.70(\mathrm{dd}, J=8.8 .2 .4 \mathrm{~Hz}, 2 \mathrm{H}), 7.67-7.58(\mathrm{~m}$, 3H), 7.51 (t, $J=8.8 \mathrm{~Hz}, 2 \mathrm{H}), 7.32(\mathrm{dt}, J=8.8,2.4 \mathrm{~Hz}, 2 \mathrm{H}), 7.12(\mathrm{dd}, J=8.8,2.4 \mathrm{~Hz}$, 2H), 4.94 (s, 2H); ${ }^{13} \mathrm{C}$ NMR (100 MHz, $\left.\mathrm{CDCl}_{3}\right) \delta 187.6,167.2,136.4,134.7,133.3$, $132.5(2 \mathrm{C}), 132.4,131.7(2 \mathrm{C}), 130.0,129.9(2 \mathrm{C}), 129.8(2 \mathrm{C}), 129.7(2 \mathrm{C}), 128.9(2 \mathrm{C})$ 122.2, 47.7; IR (ATR) 2925, 1680, 1596, 1573, 1488, 1473, 1450, 1392, 1371, 1321 $\mathrm{cm}^{-1}$; HRMS (ESI-TOF) $[\mathrm{M}+\mathrm{Na}]^{+}$calcd for $\mathrm{C}_{21} \mathrm{H}_{15} \mathrm{Br}_{2} \mathrm{NNaO}_{4} \mathrm{~S}^{+} \mathrm{m} / \mathrm{z} 557.8981$, found 557.8969. 


\section{Synthesis and Characterization of Compounds 6}

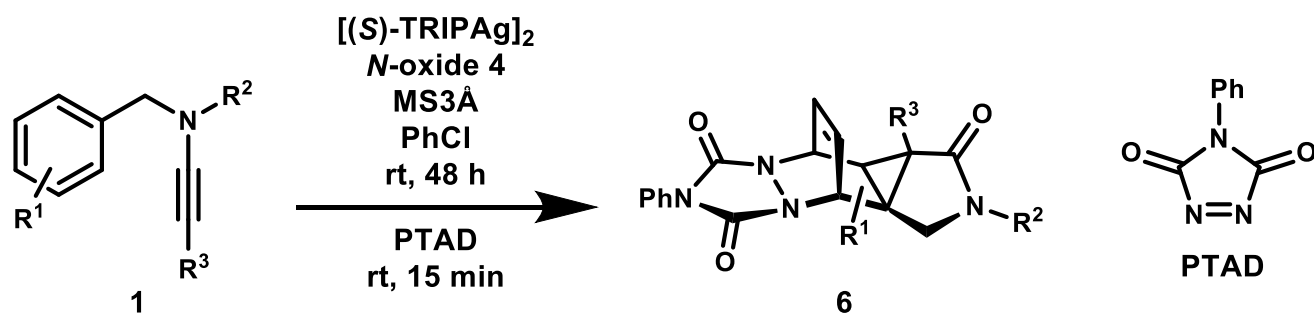

\section{General Procedure B for the One-pot Asymmetric Cyclization Reactions}

A pre-dried $10 \mathrm{~mL}$ test tube equipped with a magnetic stir bar was covered with aluminum foil to avoid light exposure. The test tube was charged with ynamide compounds 1 (0.2 mmol, 1 equiv.), $N$-oxide 4 (63.8 mg, 0.4 mmol, 2 equiv.), [(S)-

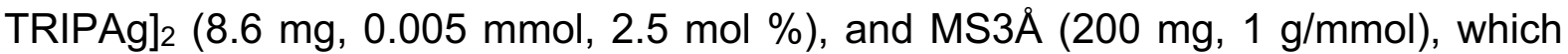
were subsequently dissolved partially in dry $\mathrm{PhCl}(2 \mathrm{~mL}, 0.1 \mathrm{M})$ under argon gas atmosphere. The reaction mixture was stirred for 48 hours at the room temperature, and then the aluminum foil was removed. PTAD (35.1 mg, $0.2 \mathrm{mmol}, 1$ equiv.) was added to the reaction mixture, and the reaction mixture was stirred another $15 \mathrm{~min}$ at the room temperature. Then, the reaction mixture was passed through a celite to remove MS3A. After the solvent was evaporated in vacuo, the resulting residue was purified by flash chromatography ( $n$-hexane/EtOAc $=3 / 1$ to $2 / 1, v / v)$ to afford desired products 6 .

General Protocol for accessing racemic products 6 
A pre-dried $10 \mathrm{~mL}$ test tube equipped with a magnetic stir bar was covered with aluminum foil to avoid light exposure. The test tube was charged with ynamide compounds 1 (0.2 mmol, 1 equiv.), $N$-oxide 4 (63.8 mg, $0.4 \mathrm{mmol}, 2$ equiv.), and $\mathrm{CF}_{3} \mathrm{CO}_{2} \mathrm{Ag}(8.8 \mathrm{mg}, 0.04 \mathrm{mmol}, 20 \mathrm{~mol} \%$ ), which were subsequently dissolved partially in dry $\mathrm{PhCl}(2 \mathrm{~mL}, 0.1 \mathrm{M})$ under argon gas atmosphere. The reaction mixture was stirred for 24 hours at the room temperature, and then the aluminum foil was removed. PTAD (35.1 mg, $0.2 \mathrm{mmol}, 1$ equiv.) was added to the reaction mixture, and the reaction mixture was stirred another $15 \mathrm{~min}$ at the room temperature. After the solvent was evaporated in vacuo, the resulting residue was purified by flash chromatography ( $n$-hexane/EtOAc $=3 / 1$ to $2 / 1, \mathrm{v} / \mathrm{v}$ ) to afford racemic products 6 .

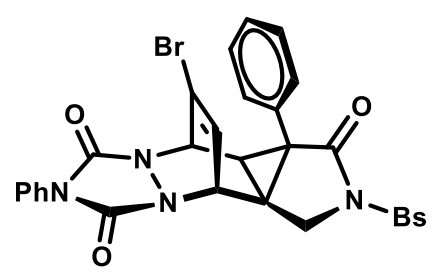

(3bS,4S,10S,10aR)-12-Bromo-2-((4-bromophenyl)sulfonyl)-3a,7-diphenyl-

\section{1,2,3b,4-tetrahydro-6H,10H-4,10-ethenopyrrolo[3',4':1,3]cyclopropa[1,2-}

d] $[1,2,4]$ triazolo[1,2-a]pyridazine-3,6,8(3aH,7H)-trione (6a)

White solid (103.9 mg, $75 \%$ yield, $97: 3$ er): m.p. $139{ }^{\circ} \mathrm{C}$ (decomp.); TLC $R_{f}=0.3(n-$ hexane/EtOAc, 2/1, v/v); ${ }^{1} \mathrm{H}$ NMR (400 MHz, $\left.\mathrm{CDCl}_{3}\right) \delta 7.88(\mathrm{~d}, \mathrm{~J}=8.4 \mathrm{~Hz}, 2 \mathrm{H}), 7.67$ (d, J = 8.4 Hz, 2H), 7.48-7.44 (m, 2H), 7.41-7.33 (m, 4H), 7.29-7.21 (m, 3H), 6.74 (d, 
$J=7.6 \mathrm{~Hz}, 1 \mathrm{H}), 5.96(\mathrm{dd}, J=6.8,2.0 \mathrm{~Hz}, 1 \mathrm{H}), 5.40(\mathrm{dd}, J=4.8,2.0 \mathrm{~Hz}, 1 \mathrm{H}), 5.36(\mathrm{~d}$, $J=7.6 \mathrm{~Hz}, 1 \mathrm{H}), 4.37(\mathrm{~d}, J=10.4 \mathrm{~Hz}, 1 \mathrm{H}), 4.25(\mathrm{~d}, J=10.4 \mathrm{~Hz}, 1 \mathrm{H}), 1.94(\mathrm{~d}, J=4.8$, 1H); ${ }^{13} \mathrm{C}$ NMR $\left(100 \mathrm{MHz}, \mathrm{CDCl}_{3}\right) \delta 169.2,156.9,155.9,136.1,132.6$ (2C), 131.6, 130.6, 130.3, 130.0, 129.7 (2C), 129.5, 129.2 (2C), 128.9, 128.8, 128.6, 128.4, 127.9, 125.3 (2C), 116.7, 60.5, 56.2, 48.8, 45.6, 28.9, 25.2; IR (ATR) 2954, 2925, 2327, 1721 , 1574, 1457, 1402, 1365, 1229, $1217 \mathrm{~cm}^{-1}$; HRMS (ESI-TOF) $[\mathrm{M}+\mathrm{Na}]^{+}$calcd for $\mathrm{C}_{29} \mathrm{H}_{20}{ }^{79} \mathrm{Br}^{81} \mathrm{BrN}_{4} \mathrm{NaO}_{5} \mathrm{~S}^{+} \mathrm{m} / \mathrm{z}$ 718.9393, found 718.9390; [a $]^{25} \mathrm{D}+10.6$ (c 1.1, $\mathrm{CHCl}_{3}$ ). The enantiomeric ratio was determined by analytical chiral HPLC. Retention time: 12.7 min (minor isomer), 13.9 min (major isomer), IA-3 column, 50/50 n-hexane/DCM 1 $\mathrm{mL} / \mathrm{min}, 254 \mathrm{~nm}$.

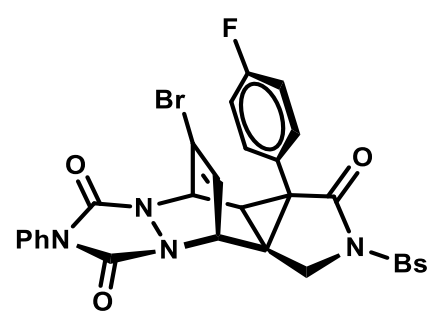

(3bR,4R,10R,10aS)-12-Bromo-2-((4-bromophenyl)sulfonyl)-3a-(4-fluorophenyl)7-phenyl-1,2,3b,4-tetrahydro-6H,10H-4,10ethenopyrrolo[3',4':1,3]cyclopropa[1,2-d][1,2,4]triazolo[1,2-a]pyridazine3,6,8(3aH,7H)-trione (6b) 
White solid (106.6 mg, $75 \%$ yield, $95: 5$ er): m.p. $155^{\circ} \mathrm{C}$ (decomp.); TLC $R_{f}=0.2(n-$ hexane/EtOAc, 2/1, v/v); ${ }^{1} \mathrm{H} \mathrm{NMR}\left(400 \mathrm{MHz}, \mathrm{CDCl}_{3}\right) \delta 7.87(\mathrm{~d}, \mathrm{~J}=8.8 \mathrm{~Hz}, 2 \mathrm{H}), 7.67$ $(\mathrm{d}, J=8.8 \mathrm{~Hz}, 2 \mathrm{H}), 7.48-7.37(\mathrm{~m}, 5 \mathrm{H}), 7.23(\mathrm{ddd}, J=8.4,5.2,2.4 \mathrm{~Hz}, 1 \mathrm{H}), 7.02(\mathrm{dt}, J$ $=8.4,2.4 \mathrm{~Hz}, 1 \mathrm{H}), 6.90(\mathrm{dt}, J=8.4,2.4 \mathrm{~Hz}, 1 \mathrm{H}), 6.72(\mathrm{ddd}, J=8.4,5.2,2.4 \mathrm{~Hz}, 1 \mathrm{H})$, $5.96(\mathrm{dd}, J=6.4,2.4 \mathrm{~Hz}, 1 \mathrm{H}), 5.38(\mathrm{dd}, J=4.8,2.4 \mathrm{~Hz}, 1 \mathrm{H}), 5.36(\mathrm{~d}, J=6.4 \mathrm{~Hz}, 1 \mathrm{H})$, $4.34(\mathrm{~d}, J=10.8 \mathrm{~Hz}, 1 \mathrm{H}), 4.25(\mathrm{~d}, J=10.8 \mathrm{~Hz}, 1 \mathrm{H}), 1.92(\mathrm{~d}, J=4.8 \mathrm{~Hz}, 1 \mathrm{H}) ;{ }^{13} \mathrm{C} N M R$ $\left(100 \mathrm{MHz}, \mathrm{CDCl}_{3}\right) \delta 169.1,162.3(\mathrm{~d}, J=246.0 \mathrm{~Hz}), 156.8,155.9,136.1,133.3(\mathrm{~d}, J=$ 8.6 Hz), $132.6(2 \mathrm{C}), 132.1$ (d, J = 8.6 Hz), 130.5, 130.0, $129.6(2 \mathrm{C}), 129.3(2 \mathrm{C}), 128.8$, $127.8,125.3(2 \mathrm{C}), 124.8(\mathrm{~d}, J=2.9 \mathrm{~Hz}), 116.8,116.3(\mathrm{~d}, J=21.9 \mathrm{~Hz}), 115.9(\mathrm{~d}, J=$ $21.9 \mathrm{~Hz}), 60.4,56.1,48.8,45.0,29.0,25.2 ;{ }^{19} \mathrm{~F} \mathrm{NMR}\left(376 \mathrm{MHz}, \mathrm{CDCl}_{3}\right) \delta-112.2$; IR (ATR) $3021,1714,1605,1574,1514,1500,1392,1365,1219,1171 \mathrm{~cm}^{-1}$; HRMS (ESI-TOF) $[\mathrm{M}+\mathrm{Na}]^{+}$calcd for $\mathrm{C}_{29} \mathrm{H}_{19} \mathrm{Br}_{2} \mathrm{FN}_{4} \mathrm{NaO}_{5} \mathrm{~S}^{+} \mathrm{m} / \mathrm{z} 734.9319$, found 734.9302; $[\alpha]^{25} \mathrm{D}+10.6\left(c 1.0, \mathrm{CHCl}_{3}\right)$. The enantiomeric ratio was determined by analytical chiral HPLC. Retention time: 12.7 min (major isomer),13.8 min (minor isomer), IG-3 column, 1/99 AcOEt/DCM, $0.3 \mathrm{~mL} / \mathrm{min}, 254 \mathrm{~nm}$.

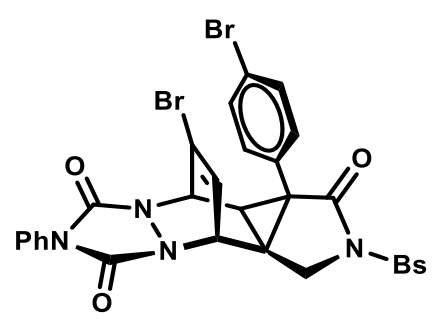


(3bR,4R,10R,10aS)-12-Bromo-3a-(4-bromophenyl)-2-((4-bromophenyl)sulfonyl)-

7-phenyl-1,2,3b,4-tetrahydro-6H,10H-4,10-

ethenopyrrolo[3',4':1,3]cyclopropa[1,2-d][1,2,4]triazolo[1,2-a]pyridazine-

\section{3,6,8(3aH,7H)-trione (6c)}

White solid (134.9 mg, $87 \%$ yield, $95: 5$ er): m.p. $153^{\circ} \mathrm{C}$ (decomp.); TLC $R_{f}=0.3(n-$ hexane/EtOAc, 2/1, v/v); ${ }^{1} \mathrm{H}$ NMR $\left(400 \mathrm{MHz}, \mathrm{CDCl}_{3}\right) \delta 7.86(\mathrm{~d}, J=8.8 \mathrm{~Hz}, 2 \mathrm{H}), 7.66$ (d, $J=8.8 \mathrm{~Hz}, 2 \mathrm{H}), 7.49-7.37(\mathrm{~m}, 6 \mathrm{H}), 7.31(\mathrm{dd}, J=8.0,1.6 \mathrm{~Hz}, 1 \mathrm{H}), 7.12(\mathrm{dd}, J=8.0$, $1.6 \mathrm{~Hz}, 1 \mathrm{H}), 6.63(\mathrm{dd}, J=8.4,1.6 \mathrm{~Hz}, 1 \mathrm{H}), 5.95(\mathrm{dd}, J=6.4,1.6 \mathrm{~Hz}, 1 \mathrm{H}), 5.36-5.34$ (m, 2H), $4.33(\mathrm{~d}, J=10.0 \mathrm{~Hz}, 1 \mathrm{H}), 4.24(\mathrm{~d}, J=10.0 \mathrm{~Hz}, 1 \mathrm{H}), 1.92(\mathrm{~d}, J=4.4 \mathrm{~Hz}, 1 \mathrm{H})$; ${ }^{13} \mathrm{C}$ NMR $\left(100 \mathrm{MHz}, \mathrm{CDCl}_{3}\right) \delta 168.8,156.7,155.8,136.0,133.1,132.6$ (2C), 132.4, $131.9,131.7,130.5,130.0,129.6(2 \mathrm{C}), 129.2(2 \mathrm{C}), 128.8,128.1,128.0,125.3(2 \mathrm{C})$ 122.5, 116.8, 60.3, 56.0, 48.7, 44.9, 29.0, 25.0; IR (ATR) 3087, 1779, 1714, 1605, 1574, 1492, 1392, 1365, 1308, $1278 \mathrm{~cm}^{-1}$; HRMS (ESI-TOF) $[\mathrm{M}+\mathrm{H}]^{+}$calcd for $\mathrm{C}_{29} \mathrm{H}_{20} \mathrm{Br}_{3} \mathrm{~N}_{4} \mathrm{O}_{5} \mathrm{~S}^{+} \mathrm{m} / \mathrm{z}$ 772.8699, found $772.8713 ;[\alpha]^{25} \mathrm{D}+2.5$ (c 1.2, $\mathrm{CHCl}_{3}$ ). The enantiomeric ratio was determined by analytical chiral HPLC. Retention time: $13.1 \mathrm{~min}$ (major isomer), $13.8 \mathrm{~min}$ (minor isomer), IG-3 column, 1/99 EtOAc/DCM, $0.3 \mathrm{~mL} / \mathrm{min}$, $254 \mathrm{~nm}$. 


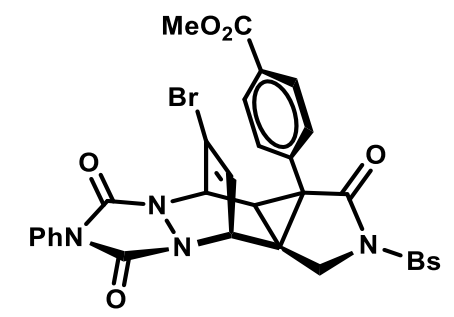

Methyl 4-((3bR,4R,10R,10aS)-12-bromo-2-((4-bromophenyl)sulfonyl)-3,6,8-

trioxo-7-phenyl-2,3,3b,4,7,8-hexahydro-6H,10H-4,10-

ethenopyrrolo[3',4':1,3]cyclopropa[1,2-d][1,2,4]triazolo[1,2-a]pyridazin-3a(1H)-

yl)benzoate (6d)

White solid (119.2 mg, $79 \%$ yield, $93: 7$ er): m.p. $154{ }^{\circ} \mathrm{C}$ (decomp.); TLC $R_{f}=0.2(n-$ hexane/EtOAc, 2/1, v/v); ${ }^{1} \mathrm{H} \mathrm{NMR}\left(400 \mathrm{MHz}, \mathrm{CDCl}_{3}\right) \delta 8.03(\mathrm{dd}, J=8.4,2.0 \mathrm{~Hz}, 1 \mathrm{H})$, 7.91-7.87 (m, 3H), 7.68 (d, J = 8.4 Hz, 2H), 7.48-7.33 (m, 6H), $6.85(\mathrm{dd}, J=7.6,1.2$ $\mathrm{Hz}, 1 \mathrm{H}), 5.96(\mathrm{dd}, J=6.4,2.4 \mathrm{~Hz}, 1 \mathrm{H}), 5.41(\mathrm{dd}, J=4.4,2.4 \mathrm{~Hz}, 1 \mathrm{H}), 5.38(\mathrm{~d}, J=6.4$ $\mathrm{Hz}, 1 \mathrm{H}), 4.38(\mathrm{~d}, J=10.8 \mathrm{~Hz}, 1 \mathrm{H}), 4.28(\mathrm{~d}, J=10.8 \mathrm{~Hz}, 1 \mathrm{H}), 3.90(\mathrm{~s}, 3 \mathrm{H}), 1.98(\mathrm{~d}, J=$ 4.4 Hz, 1H); $\left.{ }^{13} \mathrm{C} \mathrm{NMR} \mathrm{(100} \mathrm{MHz,} \mathrm{CDCl}_{3}\right) \delta 168.6,166.3,156.8,155.8,136.0,134.2$, 132.6 (2C), 131.7, 130.6, 130.51, 130.46, 130.11, 130.05, 129.7 (2C), 129.6, 129.3 (2C), 128.8, 128.4, 125.3 (2C), 116.9, 60.3, 56.0, 52.2, 48.7, 45.3, 29.3, 25.2; IR (ATR) 3021, 1714, 1608, 1574, 1500, 1436, 1401, 1365, 1276, $1172 \mathrm{~cm}^{-1}$; HRMS (ESI-TOF) $[\mathrm{M}+\mathrm{Na}]^{+}$calcd for $\mathrm{C}_{31} \mathrm{H}_{22} \mathrm{Br}_{2} \mathrm{~N}_{4} \mathrm{NaO}_{7} \mathrm{~S}^{+} \mathrm{m} / \mathrm{z}$ 774.9468, found 774.9450; $[\alpha]^{25} \mathrm{D}-1.9$ (c 1.0, $\mathrm{CHCl}_{3}$ ). The enantiomeric ratio was determined by analytical chiral HPLC. 
Retention time: 10.0 min (minor isomer), 11.6 min (major isomer), $\mathrm{IH}-3$ column, 50/50 n-hexane/DCM, $1 \mathrm{~mL} / \mathrm{min}, 254 \mathrm{~nm}$.

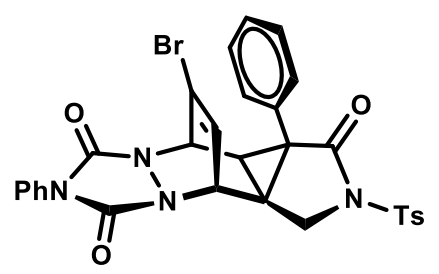

(3bS,4S,10S,10aR)-12-Bromo-3a,7-diphenyl-2-tosyl-1,2,3b,4-tetrahydro-6H,10H4,10-ethenopyrrolo[ $\left.3^{\prime}, 4^{\prime}: 1,3\right]$ cyclopropa[1,2-d][1,2,4]triazolo[1,2-a]pyridazine-

\section{3,6,8(3aH,7H)-trione (6e)}

White solid (104.0 mg, $82 \%$ yield, $96: 4$ er): m.p. $150^{\circ} \mathrm{C}$ (decomp.); TLC $R_{f}=0.3(n-$ hexane/EtOAc, 2/1, v/v); ${ }^{1} \mathrm{H}$ NMR $\left(400 \mathrm{MHz}, \mathrm{CDCl}_{3}\right) \delta 7.89(\mathrm{~d}, J=8.0 \mathrm{~Hz}, 2 \mathrm{H}), 7.47-$ $7.30(\mathrm{~m}, 5 \mathrm{H}), 7.34-7.30(\mathrm{~m}, 3 \mathrm{H}), 7.25-7.17(\mathrm{~m}, 2 \mathrm{H}), 7.18(\mathrm{~d}, J=7.6 \mathrm{~Hz}, 1 \mathrm{H}), 6.74(\mathrm{~d}$, $J=7.6 \mathrm{~Hz}, 1 \mathrm{H}), 5.93(\mathrm{dd}, J=6.4,2.0 \mathrm{~Hz}, 1 \mathrm{H}), 5.38-5.36(\mathrm{~m}, 2 \mathrm{H}), 4.34(\mathrm{~d}, J=10.0 \mathrm{~Hz}$, 1H), $4.27(\mathrm{~d}, J=10.0 \mathrm{~Hz}, 1 \mathrm{H}), 2.41(\mathrm{~s}, 3 \mathrm{H}), 1.87(\mathrm{~d}, J=4.8,1 \mathrm{H}) ;{ }^{13} \mathrm{C} \mathrm{NMR}(100 \mathrm{MHz}$, $\left.\mathrm{CDCl}_{3}\right) \delta 169.1,156.8,155.9,145.6,134.2,131.5,130.6,130.3,129.8(2 \mathrm{C}), 129.3$ $129.2(2 \mathrm{C}), 129.1,128.7,128.4,128.12,128.08(2 \mathrm{C}), 128.0,125.3(2 \mathrm{C}), 116.5,60.5$ 56.2, 48.7, 45.6, 28.7, 25.0, 21.6; IR (ATR) 3031, 1778, 1714, 1597, 1499, 1448, 1399, 1362, 1307, $1278 \mathrm{~cm}^{-1}$; HRMS (ESI-TOF) $[\mathrm{M}+\mathrm{Na}]^{+}$calcd for $\mathrm{C}_{30} \mathrm{H}_{23} \mathrm{BrN}_{4} \mathrm{NaO}_{5} \mathrm{~S}^{+} \mathrm{m} / \mathrm{z}$ 653.0465, found 653.0494; $[\mathrm{\alpha}]^{25} \mathrm{D}+11.3\left(\mathrm{c} 1.0, \mathrm{CHCl}_{3}\right)$. The enantiomeric ratio was 
determined by analytical chiral HPLC. Retention time: 26.6 min (minor isomer), 33.2 min (major isomer), IH-3 column, 70/30 n-hexane/DCM, 1 mL/min, 254 nm.

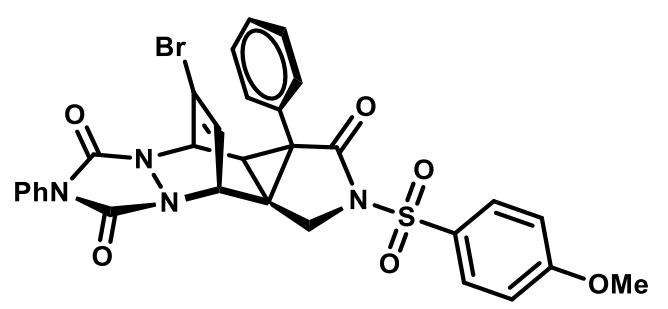

(3bR,4R,10R,10aS)-12-Bromo-2-((4-methoxyphenyl)sulfonyl)-3a,7-diphenyl-

\section{1,2,3b,4-tetrahydro-6H,10H-4,10-ethenopyrrolo[3',4':1,3]cyclopropa[1,2-}

\section{d][1,2,4]triazolo[1,2-a]pyridazine-3,6,8(3aH,7H)-trione (6f)}

White solid (96.9 mg, $75 \%$ yield, $92: 8$ er): m.p. $152{ }^{\circ} \mathrm{C}$ (decomp.); TLC $R_{f}=0.2(n-$ hexane/EtOAc, 1/2, v/v); ${ }^{1} \mathrm{H}$ NMR $\left(400 \mathrm{MHz} \mathrm{CDCl}_{3}\right) \delta 7.95(\mathrm{~d}, J=8.8 \mathrm{~Hz}, 2 \mathrm{H}), 7.47-$ $7.32(\mathrm{~m}, 6 \mathrm{H}), 7.27-7.21(\mathrm{~m}, 3 \mathrm{H}), 6.97(\mathrm{~d}, J=8.8 \mathrm{~Hz}, 2 \mathrm{H}), 6.74(\mathrm{~d}, J=7.2 \mathrm{~Hz}, 1 \mathrm{H}), 5.94$ (dd, $J=6.4,2.0 \mathrm{~Hz}, 1 \mathrm{H}), 5.39-5.36(\mathrm{~m}, 2 \mathrm{H}), 4.35(\mathrm{~d}, J=10.8 \mathrm{~Hz}, 1 \mathrm{H}), 4.27(\mathrm{~d}, J=10.8$ $\mathrm{Hz}, 1 \mathrm{H}), 3.85$ (s, 3H), 1.89 (d, $J=4.8 \mathrm{~Hz}, 1 \mathrm{H}) ;{ }^{13} \mathrm{C}$ NMR $\left(100 \mathrm{MHz}, \mathrm{CDCl}_{3}\right) \delta$ 169.1, $164.3,156.9,156.0,131.6,130.6,130.5(2 C), 130.3,129.4,129.2(3 C), 128.7,128.6$, $128.5,128.2,128.0,125.4(2 \mathrm{C}), 116.6,114.4(2 \mathrm{C}), 60.6,56.3,55.7,48.8,45.7,28.7$, 25.1; IR (ATR) 2947, 1778, 1713, 1594, 1497, 1458, 1448, 1399, 1362, $1311 \mathrm{~cm}^{-1}$; HRMS (ESI-TOF) $[\mathrm{M}+\mathrm{H}]^{+}$calcd for $\mathrm{C}_{30} \mathrm{H}_{24} \mathrm{BrN}_{4} \mathrm{O}_{5} \mathrm{~S}^{+} \mathrm{m} / \mathrm{z}$ 647.0594, found 647.0588; $[\alpha]^{25} \mathrm{D}+13.4\left(c 1.0, \mathrm{CHCl}_{3}\right)$. The enantiomeric ratio was determined by analytical chiral 
HPLC. Retention time: 12.1 min (major isomer), 13.4 min (minor isomer), IA-3 column, 50/50 n-hexane/DCM, $1 \mathrm{~mL} / \mathrm{min}, 254 \mathrm{~nm}$.

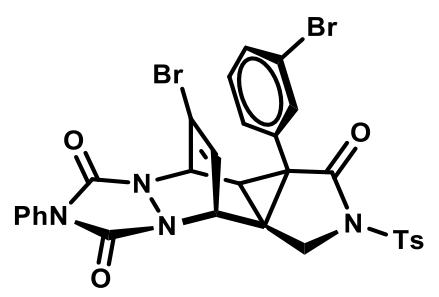

(3bR,4R,10R,10aS)-12-Bromo-3a-(3-bromophenyl)-7-phenyl-2-tosyl-1,2,3b,4tetrahydro-6H,10H-4,10-ethenopyrrolo[3',4':1,3]cyclopropa[1,2d] $[1,2,4]$ triazolo[1,2-a]pyridazine-3,6,8(3aH,7H)-trione $(6 \mathrm{~g})$ White solid (122.8 mg, 86\% yield, $93: 7$ er): m.p. $155^{\circ} \mathrm{C}$ (decomp.); TLC $R_{f}=0.3(n-$ hexane/EtOAc, 2/1, v/v); ${ }^{1} \mathrm{H}$ NMR (400 MHz, $\left.\mathrm{CDCl}_{3}\right) \delta 7.89(\mathrm{~d}, \mathrm{~J}=6.8 \mathrm{~Hz}, 2 \mathrm{H}), 7.46$ (dd, J = 8.4, 6.8 Hz, 2H), $7.38(\mathrm{~d}, J=8.4 \mathrm{~Hz}, 4 \mathrm{H}), 7.34(\mathrm{~d}, J=8.0 \mathrm{~Hz}, 2.5 \mathrm{H}), 7.24-7.17$ $(\mathrm{m}, 1 \mathrm{H}) 7.01(\mathrm{t}, J=8.0 \mathrm{~Hz}, 0.5 \mathrm{H}), 6.93(\mathrm{~s}, 0.5 \mathrm{H}), 6.73(\mathrm{~d}, J=7.6 \mathrm{~Hz}, 0.5 \mathrm{H}), 6.00$ (dd, $J=6.4,2.4 \mathrm{~Hz}, 0.5 \mathrm{H}), 5.92(\mathrm{dd}, J=6.4,2.4 \mathrm{~Hz}, 0.5 \mathrm{H}), 5.40-5.34(\mathrm{~m}, 2 \mathrm{H}), 4.36-4.24$ $(\mathrm{m}, 2 \mathrm{H}), 2.44(\mathrm{~s}, 3 \mathrm{H}), 1.89(\mathrm{~d}, J=4.4 \mathrm{~Hz}, 0.5 \mathrm{H}), 1.84(\mathrm{~d}, J=5.2 \mathrm{~Hz}, 0.5 \mathrm{H}) ;{ }^{13} \mathrm{C}$ NMR (100 MHz, $\left.\mathrm{CDCl}_{3}\right) \delta 168.6(0.5 \mathrm{C}), 168.5(0.5 \mathrm{C}), 156.82(0.5 \mathrm{C}), 156.78(0.5 \mathrm{C}), 155.95$ (0.5C). 155.88 (0.5C), 145.9, $134.7(0.5 C), 134.2(0.5 C), 133.1(0.5 C), 131.6(0.5 C)$ $131.5,130.9(0.5 \mathrm{C}), 130.6(0.5 \mathrm{C}), 130.3(0.5 \mathrm{C}), 130.0,129.9(2 \mathrm{C}), 129.3(2 \mathrm{C}), 128.8$ $128.7,128.25(0.5 \mathrm{C}), 128.19(2 \mathrm{C}), 127.9,125.4(2 \mathrm{C}), 123.6(0.5 \mathrm{C}), 122.4(0.5 \mathrm{C})$, 116.8 (0.5C), $116.6(0.5 C), 60.44$ (0.5C), 60.37 (0.5C), 56.1 (0.5 C), 56.0 (0.5C), 48.8 
(0.5C), 48.7 (0.5C), $45.2(0.5 \mathrm{C}), 45.1$ (0.5C), 29.0, 25.1 (0.5C), 25.0 (0.5C), 21.7 (NMR data of mixture of rotamers); IR (ATR) 2958, 2326, 1778, 1714, 1596, 1567, 1499, 1399, 1362, $1306 \mathrm{~cm}^{-1}$; HRMS (ESI-TOF) $\left[\mathrm{M}+\mathrm{Na}^{+}\right.$calcd for $\mathrm{C}_{30} \mathrm{H}_{22} \mathrm{Br}_{2} \mathrm{~N}_{4} \mathrm{NaO}_{5} \mathrm{~S}^{+} \mathrm{m} / \mathrm{z}$ 730.9570, found 730.9555; $[\alpha]^{25} \mathrm{D}-4.4\left(c\right.$ 1.0, $\left.\mathrm{CHCl}_{3}\right)$. The enantiomeric ratio was determined by analytical chiral HPLC. Retention time: 12.5 min (major isomer), 14.0 min (minor isomer), IG-3 column, 1/99 EtOAc/DCM, $0.3 \mathrm{~mL} / \mathrm{min}, 254 \mathrm{~nm}$.

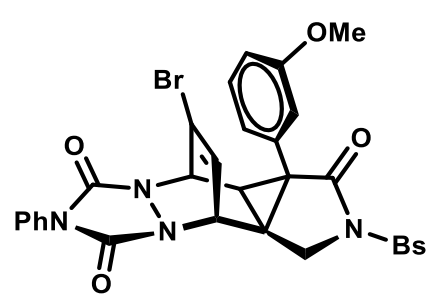

(3bR,4R,10R,10aR)-12-Bromo-2-((4-bromophenyl)sulfonyl)-3a-(3methoxyphenyl)-7-phenyl-1,2,3b,4-tetrahydro-6H,10H-4,10ethenopyrrolo[3',4':1,3]cyclopropa[1,2-d][1,2,4]triazolo[1,2-a]pyridazine-

\section{3,6,8(3aH,7H)-trione (6h)}

White solid (130.0 mg, $89 \%$ yield, $96: 4$ er): m.p. $148{ }^{\circ} \mathrm{C}$ (decomp.); $R_{f}=0.2(n-$ hexane/EtOAc, 2/1, v/v); ${ }^{1} \mathrm{H}$ NMR $\left(400 \mathrm{MHz}, \mathrm{CDCl}_{3}\right) \delta$ 7.88-7.85 (m, 2H), $7.64(\mathrm{~d}, J=$ $8.0 \mathrm{~Hz}, 2 \mathrm{H}), 7.48-7.37(\mathrm{~m}, 5 \mathrm{H}), 7.23(\mathrm{~d}, J=7.6 \mathrm{~Hz}, 0.4 \mathrm{H}), 7.10(\mathrm{t}, J=7.6 \mathrm{~Hz}, 0.6 \mathrm{H})$, 6.83-6.74 (m, 2H), 6.32 (d, J =7.6 Hz, 0.6H), 6.28 (s, 0.4H), 5.95 (dd, J = 7.6 Hz, 2.4 $\mathrm{Hz}, 0.4 \mathrm{H}), 5.91(\mathrm{dd}, J=7.6 \mathrm{~Hz}, 2.0 \mathrm{~Hz}, 0.6 \mathrm{H}), 5.42(\mathrm{dd}, J=4.0 \mathrm{~Hz}, 1.6 \mathrm{~Hz}, 0.6 \mathrm{H}), 5.39$ 
(dd, $J=4.0 \mathrm{~Hz}, 1.6 \mathrm{~Hz}, 0.4 \mathrm{H}), 5.34(\mathrm{t}, J=6.8 \mathrm{~Hz}, 1 \mathrm{H}), 4.32(\mathrm{t}, J=10.0 \mathrm{~Hz}, 1 \mathrm{H}), 4.23$ (d, J = 10.0 Hz, 1H), $3.75(\mathrm{~s}, 1.8 \mathrm{H}), 3.68(\mathrm{~s}, 1.2 \mathrm{H}), 1.95(\mathrm{~d}, J=4.4 \mathrm{~Hz}, 0.6 \mathrm{H}), 1.91(\mathrm{~d}$, $J=4.4 \mathrm{~Hz}, 0.4 \mathrm{H}) ;{ }^{13} \mathrm{C} \mathrm{NMR}\left(100 \mathrm{MHz}, \mathrm{CDCl}_{3}\right) \delta$ 169.2, 159.7 (0.6C), $159.5(0.4 \mathrm{C})$, 156.7, 155.9, 136.1, 132.5 (2C), 130.6 (0.6C), 130.5, 130.4 (0.4C), $129.78(0.6 \mathrm{C})$, $129.76(0.4 \mathrm{C}), 129.6(2 \mathrm{C}), 129.2(2 \mathrm{C}), 128.7,127.9(0.6 \mathrm{C}), 127.8(0.4 \mathrm{C}), 125.3(2 \mathrm{C})$ 124.0 (0.4 C), 122.2 (0.6 C), 117.2, 116.2 (0.6C), 116.1 (0.4C), 115.9, 114.0 (0.6C), $113.3(0.4 \mathrm{C}), 60.5(0.4 \mathrm{C}), 60.4(0.6 \mathrm{C}), 56.12$ (0.4C), 56.07 (0.6C), $55.12(0.4 \mathrm{C}), 55.07$ (0.6C), 48.7, 45.4, $29.1(0.6 \mathrm{C}), 28.9(0.4 \mathrm{C}), 25.1$ (NMR data of mixture of rotamers); IR (ATR) 3019, 1777, 1717, 1601, 1574, 1494, 1401, 1366, 1315, $1285 \mathrm{~cm}^{-1}$; HRMS (ESI-TOF) $[\mathrm{M}+\mathrm{Na}]^{+}$calcd for $\mathrm{C}_{30} \mathrm{H}_{22} \mathrm{Br}_{2} \mathrm{~N}_{4} \mathrm{NaO}_{6} \mathrm{~S}^{+} \mathrm{m} / \mathrm{z}$ 746.9519, found 746.9514; $[\alpha]^{25} \mathrm{D}+11.2\left(c 1.1, \mathrm{CHCl}_{3}\right)$. The enantiomeric ratio was determined by analytical chiral HPLC. Retention time: 15.5 min (minor isomer), 18.4 min (major isomer), IA-3 column, 50/50 n-hexane/DCM, $1 \mathrm{~mL} / \mathrm{min}, 254 \mathrm{~nm}$.

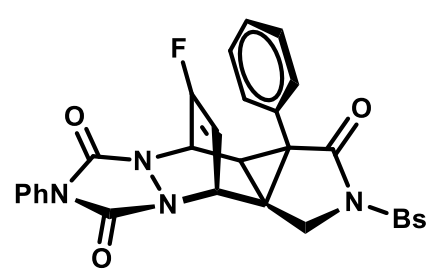


(3bR,4R,10R,10aS)-2-((4-Bromophenyl)sulfonyl)-12-fluoro-3a,7-diphenyl-

1,2,3b,4-tetrahydro-6H,10H-4,10-ethenopyrrolo[3',4':1,3]cyclopropa[1,2d] $[1,2,4]$ triazolo[1,2-a]pyridazine-3,6,8(3aH,7H)-trione (6i)

White solid (109.6 mg, 86\% yield, $95: 5$ er): m.p. $143^{\circ} \mathrm{C}$ (decomp.); TLC $R_{f}=0.4(n-$ hexane/EtOAc, 1/2, v/v); ${ }^{1} \mathrm{H}$ NMR $\left(400 \mathrm{MHz}, \mathrm{CDCl}_{3}\right) \delta 7.88(\mathrm{~d}, J=8.0 \mathrm{~Hz}, 2 \mathrm{H}), 7.66$ $(\mathrm{d}, J=8.0 \mathrm{~Hz}, 2 \mathrm{H}), 7.47-7.20(\mathrm{~m}, 8 \mathrm{H}), 7.11(\mathrm{~d}, J=7.6 \mathrm{~Hz}, 1 \mathrm{H}), 6.81(\mathrm{~d}, J=7.6 \mathrm{~Hz}$, 1H), $5.42(\mathrm{dd}, J=6.4,4.8 \mathrm{~Hz}, 1 \mathrm{H}), 5.36-5.32(\mathrm{~m}, 1 \mathrm{H}), 4.98-4.95(\mathrm{~m}, 1 \mathrm{H}), 4.37(\mathrm{~d}, J=$ $10.8 \mathrm{~Hz}, 1 \mathrm{H}), 4.28(\mathrm{~d}, J=10.8 \mathrm{~Hz}, 1 \mathrm{H}), 1.91-1.90(\mathrm{~m}, 1 \mathrm{H}) ;{ }^{13} \mathrm{C} \mathrm{NMR}\left(100 \mathrm{MHz}, \mathrm{CDCl}_{3}\right)$ ठ 169.4, 156.7, 156.2, 154.5 (d, J = 295.6 Hz), 136.1, $132.6(2 \mathrm{C}), 130.6,130.4,130.2$, $129.9,129.7(2 \mathrm{C}), 129.3,129.2(2 \mathrm{C}), 128.82,128.76,128.72,128.56,125.3(2 \mathrm{C})$, $101.6(\mathrm{~d}, J=4.7 \mathrm{~Hz}), 55.0(\mathrm{~d}, J=6.7 \mathrm{~Hz}), 54.1(\mathrm{~d}, J=26.7 \mathrm{~Hz}), 48.8,45.6,30.2,23.2$; ${ }^{19} \mathrm{~F}$ NMR (376 MHz, CDCl $)$ ) -101.1 ; IR (ATR) 3093, 2253, 1780, 1714, 1673, 1598, 1574, 1500, 1472, $1448 \mathrm{~cm}^{-1}$; HRMS (ESI-TOF) $\left[\mathrm{M}+\mathrm{Na}^{+}\right.$calcd for $\mathrm{C}_{29} \mathrm{H}_{20} \mathrm{BrFN}_{4} \mathrm{NaO}_{5} \mathrm{~S}^{+} \mathrm{m} / \mathrm{z} 657.0214$., found $657.0223 ;[\alpha]^{25} \mathrm{D}+62.0\left(c 1.0, \mathrm{CHCl}_{3}\right)$. The enantiomeric ratio was determined by analytical chiral HPLC. Retention time: 28.4 min (minor isomer), $30.8 \mathrm{~min}$ (major isomer), IH-3 column, 70/30 $n$-hexane/DCM, $1 \mathrm{~mL} / \mathrm{min}$, $254 \mathrm{~nm}$. 


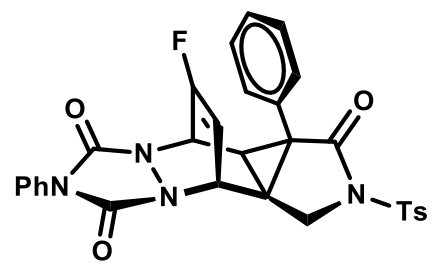

(3bR,4R,10R,10aS)-12-Fluoro-3a,7-diphenyl-2-tosyl-1,2,3b,4-tetrahydro-6H,10H-

\section{4,10-ethenopyrrolo[3',4':1,3]cyclopropa[1,2-d][1,2,4]triazolo[1,2-a]pyridazine-}

\section{3,6,8(3aH,7H)-trione $(6 \mathrm{j})$}

White solid (89.9 mg, 78\% yield, $97: 3$ er): m.p. $150{ }^{\circ} \mathrm{C}$ (decomp.); TLC $R_{f}=0.3(n-$ hexane/EtOAc, 2/1, v/v); ${ }^{1} \mathrm{H}$ NMR $\left(400 \mathrm{MHz} \mathrm{CDCl}_{3}\right) \delta 7.90(\mathrm{~d}, J=8.0 \mathrm{~Hz}, 2 \mathrm{H}), 7.47-$ $7.18(\mathrm{~m}, 10 \mathrm{H}), 7.10(\mathrm{~d}, J=7.6 \mathrm{~Hz}, 1 \mathrm{H}), 6.81(\mathrm{~d}, J=7.6 \mathrm{~Hz}, 1 \mathrm{H}), 5.43(\mathrm{dd}, J=6.8,5.2$ $\mathrm{Hz}, 1 \mathrm{H}), 5.34-5.32(\mathrm{~m}, 1 \mathrm{H}), 4.98-4.96(\mathrm{~m}, 1 \mathrm{H}), 4.36(\mathrm{~d}, J=10.8 \mathrm{~Hz}, 1 \mathrm{H}), 4.30(\mathrm{~d}, J=$ $10.8 \mathrm{~Hz}, 1 \mathrm{H}), 2.42$ (s, 3H), 1.85-1.83 (m, 1H); ${ }^{13} \mathrm{C}$ NMR (100 MHz, $\left.\mathrm{CDCl}_{3}\right) \delta 169.2$, 156.7, 156.2, 154.4 (d, J = 295.6 Hz), 145.7, 134.3, 130.6, 130.4, 130.2, 129.9 (2C), $129.2(3 \mathrm{C}), 128.9,128.8,128.7,128.4,128.1(2 \mathrm{C}), 125.3(2 \mathrm{C}), 101.6(\mathrm{~d}, J=4.8 \mathrm{~Hz})$, $55.0(\mathrm{~d}, J=6.6 \mathrm{~Hz}), 54.1$ (d, $J=26.6 \mathrm{~Hz}), 48.8,45.7,30.1,23.0$ (d, $J=2.9 \mathrm{~Hz}), 21.7$; ${ }^{19} \mathrm{~F}$ NMR (376 MHz, $\mathrm{CDCl}_{3}$ ) $\delta$-101.3; IR (ATR) 3069, 1715, 1598, 1514, 1500, 1457, 1399, 1363, 1308, $1224 \mathrm{~cm}^{-1}$; HRMS (ESI-TOF) $[\mathrm{M}+\mathrm{Na}]^{+}$calcd for $\mathrm{C}_{30} \mathrm{H}_{23} \mathrm{FN}_{4} \mathrm{NaO}_{5} \mathrm{~S}^{+}$ $\mathrm{m} / \mathrm{z}$ 593.1265, found 593.1268; $[\alpha]^{25} \mathrm{D}+6.9\left(\mathrm{c}\right.$ 1.0, $\left.\mathrm{CHCl}_{3}\right)$. The enantiomeric ratio was determined by analytical chiral HPLC. Retention time: 27.8 min (minor isomer), 31.6 min (major isomer), IH-3 column, 70/30 n-hexane/DCM, 1 mL/min, 254 nm. 


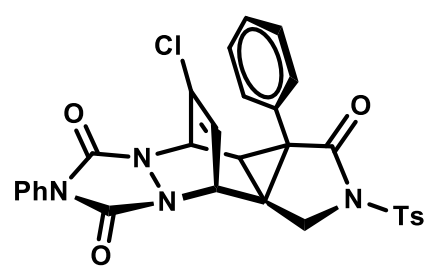

(3bR,4R,10R,10aS)-12-Chloro-3a,7-diphenyl-2-tosyl-1,2,3b,4-tetrahydro-6 $\mathrm{H}, 10 \mathrm{H}$ 4,10-ethenopyrrolo[3',4':1,3]cyclopropa[1,2-d][1,2,4]triazolo[1,2-a]pyridazine-

\section{3,6,8(3aH,7H)-trione $(6 \mathrm{k})$}

White solid (108.1 mg, 91\% yield, 95 : 5 er): m.p. $147{ }^{\circ} \mathrm{C} ;$ TLC $R_{f}=0.3(n-$ hexane/EtOAc, 2/1, v/v); ${ }^{1} \mathrm{H}$ NMR $\left(400 \mathrm{MHz} \mathrm{CDCl}_{3}\right) \delta 7.89(\mathrm{~d}, J=8.4 \mathrm{~Hz}, 2 \mathrm{H}), 7.47-$ $7.35(\mathrm{~m}, 5 \mathrm{H}), 7.32-7.30(\mathrm{~m}, 3 \mathrm{H}), 7.25-7.17(\mathrm{~m}, 3 \mathrm{H}), 6.75(\mathrm{~d}, J=7.2 \mathrm{~Hz}, 1 \mathrm{H}), 5.68$ (dd, $J=6.8,2.4 \mathrm{~Hz}, 1 \mathrm{H}), 5.40(\mathrm{~d}, J=6.8 \mathrm{~Hz}, 1 \mathrm{H}), 5.29(\mathrm{dd}, J=4.8,2.4 \mathrm{~Hz}, 1 \mathrm{H}), 4.35(\mathrm{~d}, J$ $=10.8 \mathrm{~Hz}, 1 \mathrm{H}), 4.28(\mathrm{~d}, J=10.8 \mathrm{~Hz}, 1 \mathrm{H}), 2.41(\mathrm{~s}, 3 \mathrm{H}), 1.87(\mathrm{~d}, J=4.8 \mathrm{~Hz}, 1 \mathrm{H}) ;{ }^{13} \mathrm{C}$ NMR $\left(100 \mathrm{MHz}, \mathrm{CDCl}_{3}\right) \delta 169.1,156.7,156.0,145.7,134.3,131.2,130.6,130.3$ 129.9 (2C), 129.3, 129.2 (3C), 129.0, 128.7, 128.4, 128.2, $128.1(2 \mathrm{C}), 125.3$ (2C), 123.5, 59.2, 55.6, 48.7, 45.6, 29.1, 24.3, 21.7; IR (ATR) 3030, 2255, 1778, 1714, 1615, 1597, 1500, 1448, 1399, $1363 \mathrm{~cm}^{-1}$; HRMS (ESI-TOF) $[\mathrm{M}+\mathrm{Na}]^{+}$calcd for $\mathrm{C}_{30} \mathrm{H}_{23} \mathrm{CIN}_{4} \mathrm{NaO}_{5} \mathrm{~S}^{+} \mathrm{m} / \mathrm{z}$ 609.0970, found 609.0951; $[\alpha]^{25} \mathrm{D}+30.8$ (c 1.0, $\mathrm{CHCl}_{3}$ ). The enantiomeric ratio was determined by analytical chiral HPLC. Retention time: 10.3 min (major isomer), $11.3 \mathrm{~min}$ (minor isomer), IA-3 column, 50/50 $n$-hexane/DCM, $1 \mathrm{~mL} / \mathrm{min}$, $254 \mathrm{~nm}$. 


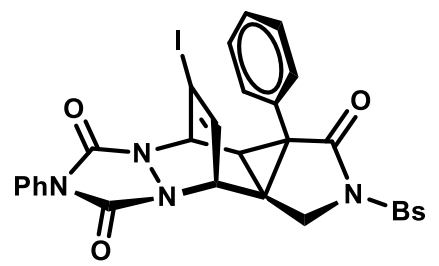

(3bR,4R,10R,10aS)-2-((4-Bromophenyl)sulfonyl)-12-iodo-3a,7-diphenyl-1,2,3b,4tetrahydro-6H,10H-4,10-ethenopyrrolo[3',4':1,3]cyclopropa[1,2-

d] $[1,2,4]$ triazolo[1,2-a]pyridazine-3,6,8(3aH,7H)-trione (6I)

White solid (127.3 mg, 86\% yield, $97: 3$ er): m.p. $154{ }^{\circ} \mathrm{C}$ (decomp.); TLC $R_{f}=0.3(n-$ hexane/EtOAc, 1/2, v/v); ${ }^{1} \mathrm{H}$ NMR (400 MHz, $\left.\mathrm{CDCl}_{3}\right) \delta 7.86(\mathrm{~d}, \mathrm{~J}=8.8 \mathrm{~Hz}, 2 \mathrm{H}), 7.64$ (d, $J=8.8 \mathrm{~Hz}, 2 \mathrm{H}), 7.48-7.34(\mathrm{~m}, 5 \mathrm{H}), 7.30-7.19(\mathrm{~m}, 4 \mathrm{H}), 6.71(\mathrm{~d}, J=7.6 \mathrm{~Hz}, 1 \mathrm{H}), 6.27$ (dd, $J=6.4,2.4 \mathrm{~Hz}, 1 \mathrm{H}), 5.44(\mathrm{dd}, J=4.8,2.4 \mathrm{~Hz}, 1 \mathrm{H}), 5.24(\mathrm{~d}, J=6.4 \mathrm{~Hz}, 1 \mathrm{H}), 4.33$ $(\mathrm{d}, J=10.4 \mathrm{~Hz}, 1 \mathrm{H}), 4.23(\mathrm{~d}, J=10.4 \mathrm{~Hz}, 1 \mathrm{H}), 1.90(\mathrm{~d}, J=4.8 \mathrm{~Hz}, 1 \mathrm{H}) ;{ }^{13} \mathrm{C}$ NMR $(100$ $\left.\mathrm{MHz}, \mathrm{CDCl}_{3}\right) \delta 169.2,156.8,155.8,136.3,136.1,132.5(2 \mathrm{C}), 132.1,130.6,130.4$ 129.8, 129.6 (3C), 129.3, $129.2(2 \mathrm{C}), 128.7,128.6,128.2,125.3(2 \mathrm{C}), 86.2,63.0,56.6$, 48.8, 45.5, 28.4, 26.0; IR (ATR) 3024, 1776, 1713, 1593, 1574, 1499, 1472, 1458, 1448, $1391 \mathrm{~cm}^{-1} ; \mathrm{HRMS}$ (ESI-TOF) $[\mathrm{M}+\mathrm{Na}]^{+}$calcd for $\mathrm{C}_{29} \mathrm{H}_{20} \mathrm{BrIN}_{4} \mathrm{NaO}_{5} \mathrm{~S}^{+} \mathrm{m} / \mathrm{z}$ 764.9275, found 764.9260; $[\alpha]^{25} \mathrm{D}-13.8\left(\mathrm{c}\right.$ 1.0, $\left.\mathrm{CHCl}_{3}\right)$. The enantiomeric ratio was determined by analytical chiral HPLC. Retention time: $13.1 \mathrm{~min}$ (minor isomer), 15.6 min (major isomer), IA-3 column, 50/50 n-hexane/DCM, 1 mL/min, 254 nm. 


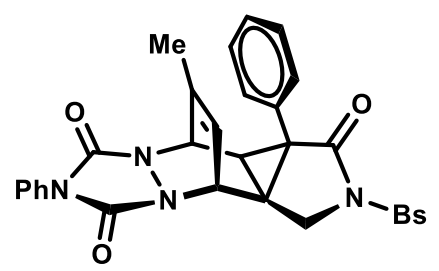

(3bR,4R,10R,10aS)-2-((4-Bromophenyl)sulfonyl)-12-methyl-3a,7-diphenyl-

\section{1,2,3b,4-tetrahydro-6H,10H-4,10-ethenopyrrolo[3',4':1,3]cyclopropa[1,2- \\ d] $[1,2,4]$ triazolo[1,2-a]pyridazine-3,6,8(3aH,7H)-trione $(6 \mathrm{~m})$}

White solid (108.5 mg, 86\% yield, $96: 4$ er): m.p. $145{ }^{\circ} \mathrm{C}$ (decomp.); TLC $R_{f}=0.3(n-$ hexane/EtOAc, 1/2, v/v); ${ }^{1} \mathrm{H}$ NMR (400 MHz, $\left.\mathrm{CDCl}_{3}\right) \delta 7.88(\mathrm{~d}, \mathrm{~J}=8.8 \mathrm{~Hz}, 2 \mathrm{H}), 7.65$ $(\mathrm{d}, J=8.8 \mathrm{~Hz}, 2 \mathrm{H}), 7.46-7.34(\mathrm{~m}, 5 \mathrm{H}), 7.30-7.27(\mathrm{~m}, 1 \mathrm{H}), 7.23-7.16(\mathrm{~m}, 3 \mathrm{H}), 6.75(\mathrm{~d}$, $J=6.8 \mathrm{~Hz}, 1 \mathrm{H}), 5.41-5.39(\mathrm{~m}, 1 \mathrm{H}), 5.27(\mathrm{~d}, J=6.0 \mathrm{~Hz}, 1 \mathrm{H}), 5.08-5.07(\mathrm{~m}, 1 \mathrm{H}), 4.38$ (d, J = 10.4 Hz, 1H), 4.26 (d, J=10.4 Hz, 1H), 1.87 (d, J = 4.8 Hz, 1H), $1.17(\mathrm{~s}, 3 \mathrm{H})$; ${ }^{13} \mathrm{C}$ NMR $\left(100 \mathrm{MHz} \mathrm{CDCl}_{3}\right) \delta 169.8,157.1,156.6,137.7,136.2,132.5(2 \mathrm{C}), 131.0$ $130.9,130.7,130.0,129.7,129.6(2 \mathrm{C}), 129.1(2 \mathrm{C}), 128.7,128.4,128.2,128.0,125.2$ (2C), 121.2, 56.8, 55.3, 49.1, 45.5, 29.6, 24.8, 19.2; IR (ATR) 3063, 2356, 1771, 1709, 1599, 1574, 1500, 1472, 1447, $1400 \mathrm{~cm}^{-1}$; HRMS (ESI-TOF) $[\mathrm{M}+\mathrm{H}]^{+}$calcd for $\mathrm{C}_{30} \mathrm{H}_{24} \mathrm{BrN}_{4} \mathrm{O}_{5} \mathrm{~S}^{+} \mathrm{m} / \mathrm{z}$ 631.0645, found 631.0661; $[\alpha]^{25} \mathrm{D}+37.4$ (c 1.0, $\mathrm{CHCl}_{3}$ ). The enantiomeric ratio was determined by analytical chiral HPLC. Retention time: 20.0 min (minor isomer), $26.3 \mathrm{~min}$ (major isomer), IH-3 column, 70/30 n-hexane/DCM, $1 \mathrm{~mL} / \mathrm{min}$, $254 \mathrm{~nm}$. 


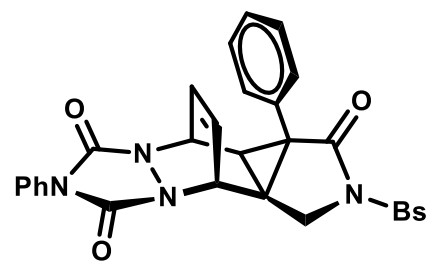

(3bR,4S,10R,10aS)-2-((4-Bromophenyl)sulfonyl)-3a,7-diphenyl-1,2,3b,4-

\section{tetrahydro-6H,10H-4,10-ethenopyrrolo[3',4':1,3]cyclopropa[1,2-}

\section{d] $[1,2,4]$ triazolo[1,2-a]pyridazine-3,6,8(3aH,7H)-trione (6n)}

White solid (106.6 mg, 86\% yield, $94: 6$ er): m.p. $152{ }^{\circ} \mathrm{C}$ (decomp.); TLC $R_{f}=0.3(n-$ hexane/EtOAc, 2/1, v/v); ${ }^{1} \mathrm{H}$ NMR (400 MHz, $\left.\mathrm{CDCl}_{3}\right) \delta 7.89(\mathrm{~d}, \mathrm{~J}=8.8 \mathrm{~Hz}, 2 \mathrm{H}), 7.68$ $(\mathrm{d}, J=8.8 \mathrm{~Hz}, 2 \mathrm{H}), 7.46-7.34(\mathrm{~m}, 5 \mathrm{H}), 7.32-7.20(\mathrm{~m}, 3 \mathrm{H}), 7.02(\mathrm{~d}, J=7.2 \mathrm{~Hz}, 1 \mathrm{H}), 6.77$ $(\mathrm{d}, J=6.8 \mathrm{~Hz}, 1 \mathrm{H}), 5.70(\mathrm{ddd}, J=8.0,6.4,2.0 \mathrm{~Hz}, 1 \mathrm{H}), 5.54-5.51(\mathrm{~m}, 1 \mathrm{H}), 5.36(\mathrm{t}, J=$ $4.4 \mathrm{~Hz}, 1 \mathrm{H}), 5.29(\mathrm{dd}, J=6.4,2.0 \mathrm{~Hz}, 1 \mathrm{H}), 4.42(\mathrm{~d}, J=10.8 \mathrm{~Hz}, 1 \mathrm{H}), 4.28(\mathrm{~d}, J=10.8$ $\mathrm{Hz}, 1 \mathrm{H}), 1.89$ (d, J = 4.4 Hz, 1H); ${ }^{13} \mathrm{C}$ NMR (100 MHz, $\left.\mathrm{CDCl}_{3}\right) \delta$ 169.9, 156.8, 156.4, 136.3, 132.5 (2C), 130.9, 130.8, 130.33, 130.26, 129.8, 129.7 (2C), $129.1(2 \mathrm{C}), 129.0$, $128.9,128.5,128.2,127.2,126.1,125.4$ (2C), 54.3, 52.4, 49.2, 45.6, 28.9, 24.7; IR (ATR) 2957, 1707, 1574, 1499, 1391, 1362, 1315, 1263, 1171, $1134 \mathrm{~cm}^{-1}$; HRMS (ESI-TOF) $[\mathrm{M}+\mathrm{Na}]^{+}$calcd for $\mathrm{C}_{29} \mathrm{H}_{21} \mathrm{BrN}_{4} \mathrm{NaO}_{5} \mathrm{~S}^{+} \mathrm{m} / \mathrm{z}$ 639.0308, found 639.03285; $[\alpha]^{25} \mathrm{D}+56.4\left(c 1.0, \mathrm{CHCl}_{3}\right)$. The enantiomeric ratio was determined by analytical chiral HPLC. Retention time: 8.7 min (major isomer), 12.1 min (minor isomer), IG-3 column, 30/70 n-hexane/DCM, 1 mL/min, 254 nm. 


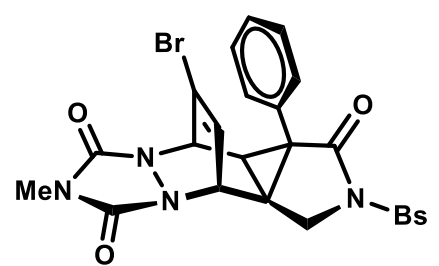

(3bR,4R,10R,10aS)-12-Bromo-2-((4-bromophenyl)sulfonyl)-7-methyl-3a-phenyl-

\section{1,2,3b,4-tetrahydro-6H,10H-4,10-ethenopyrrolo[3',4':1,3]cyclopropa[1,2- \\ d] $[1,2,4]$ triazolo[1,2-a]pyridazine-3,6,8(3aH,7H)-trione (6o)}

This compound was synthesized according to the general procedure A. MTAD (22.6 mg, 0.2 mmol, 1 equiv.) was used, instead of PTAD.

White solid (113.9 mg, $90 \%$ yield, $95: 5$ er): m.p. $143{ }^{\circ} \mathrm{C}$ (decomp.); TLC $R_{f}=0.15$ ( $n$ hexane/EtOAc, 2/1, v/v); ${ }^{1} \mathrm{H}$ NMR (400 MHz, $\left.\mathrm{CDCl}_{3}\right) \delta 7.86(\mathrm{~d}, \mathrm{~J}=8.8 \mathrm{~Hz}, 2 \mathrm{H}), 7.66$ $(\mathrm{d}, J=8.8 \mathrm{~Hz}, 2 \mathrm{H}), 7.34(\mathrm{t}, J=6.0 \mathrm{~Hz}, 1 \mathrm{H}), 7.27-7.19(\mathrm{~m}, 3 \mathrm{H}), 6.74(\mathrm{~d}, J=7.2 \mathrm{~Hz}, 1 \mathrm{H})$, $5.87(\mathrm{dd}, J=6.4,2.4 \mathrm{~Hz}, 1 \mathrm{H}), 5.30(\mathrm{dd}, J=4.0,2.4 \mathrm{~Hz}, 1 \mathrm{H}), 5.28(\mathrm{~d}, J=6.4 \mathrm{~Hz}, 1 \mathrm{H})$, $4.31(\mathrm{~d}, J=10.4 \mathrm{~Hz}, 1 \mathrm{H}), 4.24(\mathrm{~d}, J=10.4 \mathrm{~Hz}, 1 \mathrm{H}), 3.01(\mathrm{~s}, 3 \mathrm{H}), 1.90$ (d, J = 4.0 Hz, $1 \mathrm{H}) ;{ }^{13} \mathrm{C}$ NMR $\left(100 \mathrm{MHz}, \mathrm{CDCl}_{3}\right) \delta 169.2,158.3,157.4,136.2,132.6$ (2C), 131.6, 130.3, $130.0,129.7$ (2C), 129.4, 129.0, 128.5, 128.3, 127.6, 116.7, 60.2, 55.9, 48.8, 45.6, 28.9, 25.8, 25.2; IR (ATR) 3027, 2363, 2352, 1780, 1606, 1574, 1367, 1276, 1088, $1068 \mathrm{~cm}^{-1}$; HRMS (ESI-TOF) $[\mathrm{M}+\mathrm{H}]^{+}$calcd for $\mathrm{C}_{24} \mathrm{H}_{19} \mathrm{Br}_{2} \mathrm{~N}_{4} \mathrm{O}_{5} \mathrm{~S}^{+} \mathrm{m} / \mathrm{z} 632.9437$, found 632.9435; $[\mathrm{\alpha}]^{25} \mathrm{D}+28.7\left(\mathrm{c} 1.0, \mathrm{CHCl}_{3}\right.$ ). The enantiomeric ratio was determined by analytical chiral HPLC. Retention time: 10.5 min (major isomer),18.2 min (minor isomer), IA-3 column, 3/97 AcOEt/DCM, $0.3 \mathrm{~mL} / \mathrm{min}, 254 \mathrm{~nm}$. 


\section{X-ray Crystallographic Data}

ORTEP of 2a, CCDC No. 2005346

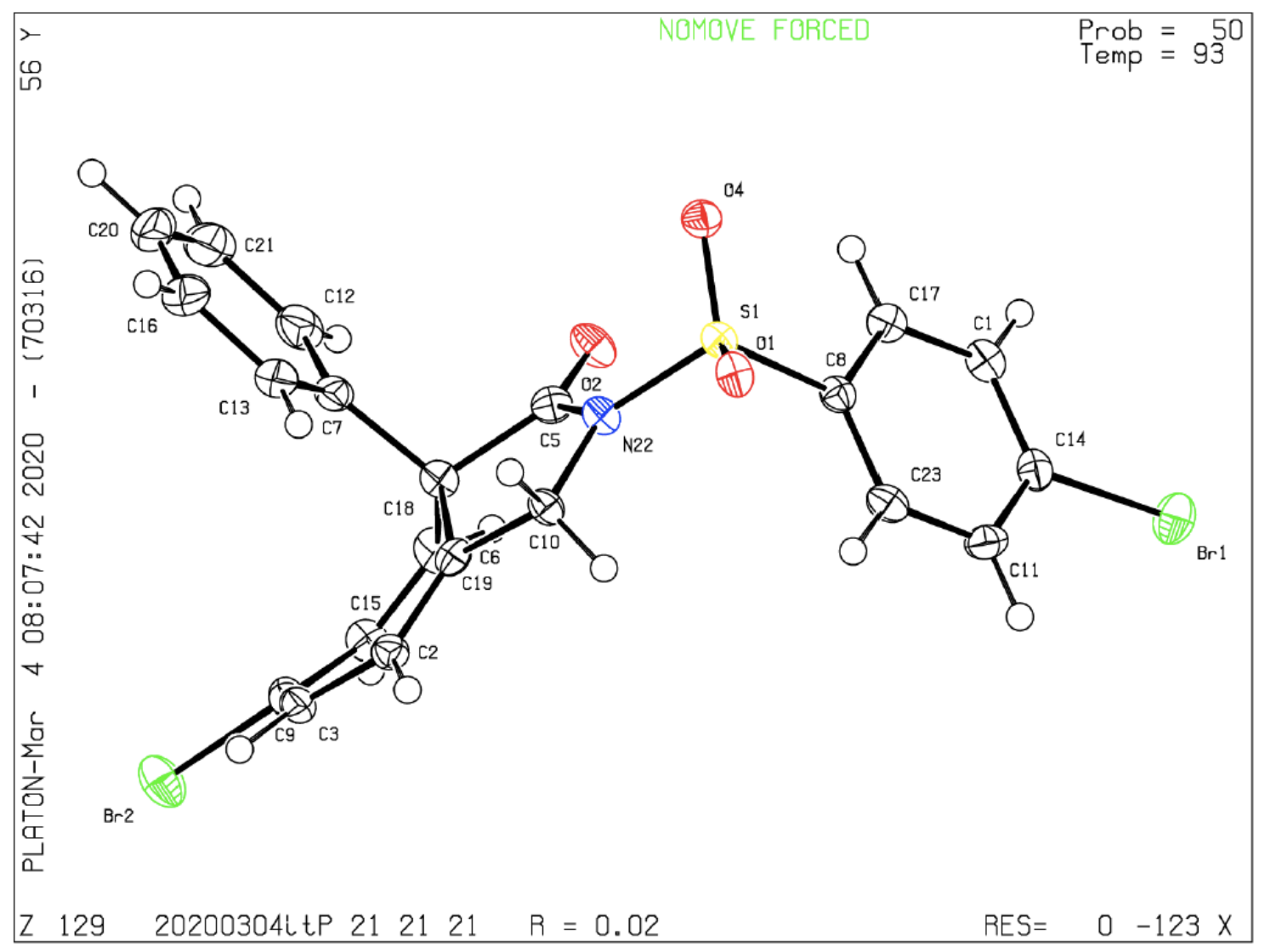

The ellipsoid contour probability level in the ORTEP is $50 \%$.

\section{EXPERIMENTAL DETAILS}

The sample for X-ray crystal structure analysis was prepared by vapor diffusion method. A pre-dried $1 \mathrm{~mL}$ sample tube was charged with $\mathbf{2 a}$, which was dissolved in DCM. The sample tube was left to stand in a $5 \mathrm{~mL}$ sample tube charged with $n$-hexane until a single crystal forms.

A. Crystal Data 
Empirical Formula

Formula Weight

Crystal Color, Habit

Crystal Dimensions

Crystal System

Lattice Type

Lattice Parameters

Space Group

$Z$ value

D calc

F000

$\mathrm{m}$ (CuKa)
$\mathrm{C}_{21} \mathrm{H}_{15} \mathrm{Br}_{2} \mathrm{NO}_{3} \mathrm{~S}$

521.22

colorless, block

$0.200 \times 0.200 \times 0.100 \mathrm{~mm}$

orthorhombic

Primitive

$$
\begin{aligned}
& a=6.75499(15) \AA \\
& b=10.0036(2) \AA \\
& c=28.9000(7) \AA \\
& V=1952.89(8) \AA^{3}
\end{aligned}
$$

$\mathrm{P} 2{ }_{1} 2{ }_{1}{ }_{1}(\# 19)$

4

$1.773 \mathrm{~g} / \mathrm{cm}^{3}$

1032.00

$64.665 \mathrm{~cm}^{-1}$ 
B. Intensity Measurements

Diffractometer

Radiation

Voltage, Current

Temperature

Detector Aperture

Data Images

w oscillation Range $(c=54.0, f=0.0)$

Exposure Rate

w oscillation Range $(c=54.0, f=90.0)$

Exposure Rate

w oscillation Range $(c=54.0, f=180.0)$

Exposure Rate

w oscillation Range $(c=54.0, f=270.0)$

Exposure Rate

w oscillation Range $(c=0.0, f=0.0)$

Exposure Rate

Detector Position
R-AXIS RAPID

CuKa $(I=1.54187 \AA)$

multi-layer mirror monochromated

$40 \mathrm{kV}, 30 \mathrm{~mA}$

$-180.0^{\circ} \mathrm{C}$

$460.0 \times 256.0 \mathrm{~mm}$

45 exposures

$80.0-260.0^{0}$

$3.0 \mathrm{sec} . / \mathrm{O}$

$80.0-260.0^{0}$

$3.0 \mathrm{sec} . / 0$

$80.0-260.0^{0}$

$3.0 \mathrm{sec} . / 0$

$80.0-260.0^{0}$

$3.0 \mathrm{sec} . / 0$

$80.0-260.0^{0}$

$3.0 \mathrm{sec} . / 0$

$127.40 \mathrm{~mm}$ 
Pixel Size

$2 q \max$

No. of Reflections Measured

Corrections
$0.100 \mathrm{~mm}$

$136.5^{0}$

Total: 22758

Unique: $3571\left(R_{\text {int }}=0.0475\right)$

Friedel pairs: 1483

Lorentz-polarization

Absorption

(trans. factors: $0.381-0.524$ ) 
C. Structure Solution and Refinement

Structure Solution

Refinement

Function Minimized

Least Squares Weights

2qmax cutoff

Anomalous Dispersion

No. Observations (All reflections)

No. Variables

Reflection/Parameter Ratio

Residuals: R1 (I>2.00s(I))

Residuals: R (All reflections)

Residuals: wR2 (All reflections)

Goodness of Fit Indicator

Flack Parameter $($ Friedel pairs $=1483)$

Max Shift/Error in Final Cycle

Maximum peak in Final Diff. Map
Direct Methods

Full-matrix least-squares on $\mathrm{F}^{2}$

$S w\left(F o^{2}-F c^{2}\right)^{2}$

$$
\begin{aligned}
& w=1 /\left[\mathrm{s}^{2}\left(\mathrm{Fo}^{2}\right)+(0.0281 \cdot \mathrm{P})^{2}\right. \\
& +0.6204 \cdot \mathrm{P}] \\
& \text { where } \mathrm{P}=\left(\operatorname{Max}\left(\mathrm{Fo}^{2}, 0\right)+2 \mathrm{Fc}^{2}\right) / 3
\end{aligned}
$$

$136.4^{\circ}$

All non-hydrogen atoms

3571

253

14.11

0.0237

0.0246

0.0579

1.065

$-0.014(16)$

0.001

$0.75 \mathrm{e}^{-/ \AA^{3}}$ 
Supporting information

Minimum peak in Final Diff. Map

$-0.24 e^{-/ / A^{3}}$ 
ORTEP of 6 n. $\mathrm{CH}_{2} \mathrm{Cl}_{2}, \mathrm{CCDC}$ No. 2034985

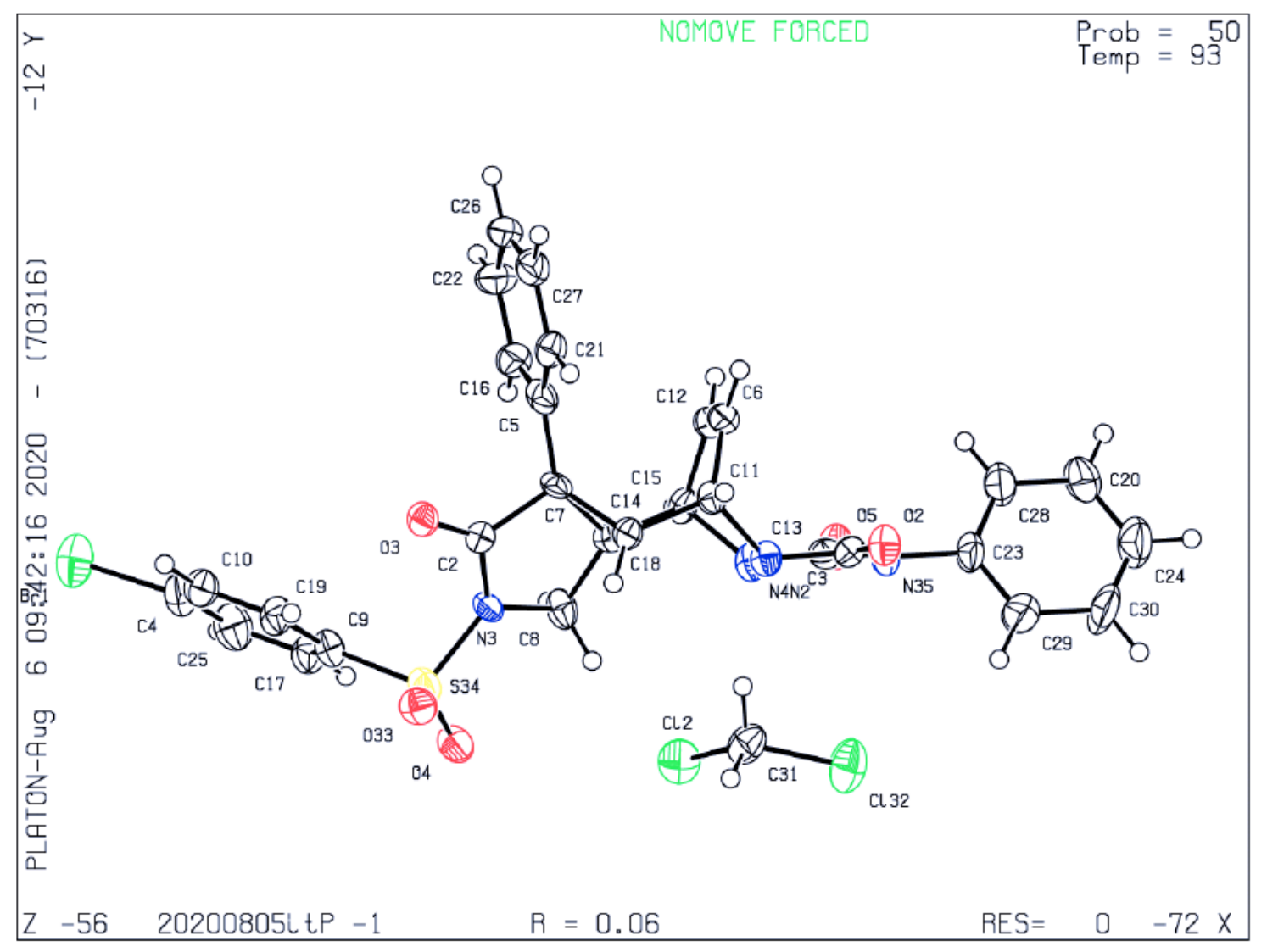

The ellipsoid contour probability level in the ORTEP is $50 \%$.

\section{EXPERIMENTAL DETAILS}

The sample for X-ray crystal structure analysis was prepared by vapor diffusion method. A pre-dried $1 \mathrm{~mL}$ sample tube was charged with $6 \mathbf{n}$, which was dissolved in DCM. The sample tube was left to stand in a $5 \mathrm{~mL}$ sample tube charged with $n$-hexane until a single crystal forms.

\section{A. Crystal Data}


Formula Weight

Crystal Color, Habit

Crystal Dimensions

Crystal System

Lattice Type

Lattice Parameters

Space Group

$Z$ value

Dcalc

F000

$\mathrm{m}(\mathrm{CuKa})$
702.41

colorless, block

$0.200 \times 0.200 \times 0.100 \mathrm{~mm}$

triclinic

Primitive

$$
\begin{aligned}
& a=10.1732(5) \AA \\
& b=10.8571(5) \AA \\
& c=14.6473(7) \AA \\
& a=71.482(5) 0 \\
& b=78.632(6) 0 \\
& g=79.222(6) 0 \\
& V=1490.43(13) \AA^{3}
\end{aligned}
$$

$\mathrm{P}-1$ (\#2)

2

$1.565 \mathrm{~g} / \mathrm{cm}^{3}$

712.00

$45.876 \mathrm{~cm}^{-1}$ 
B. Intensity Measurements

Diffractometer

Radiation

Voltage, Current

Temperature

Detector Aperture

Data Images

w oscillation Range $(c=54.0, f=0.0)$

Exposure Rate

w oscillation Range $(c=54.0, f=90.0)$

Exposure Rate

w oscillation Range $(c=54.0, f=180.0)$

Exposure Rate

w oscillation Range $(c=54.0, f=270.0)$

Exposure Rate

w oscillation Range $(c=0.0, f=0.0)$

Exposure Rate

Detector Position
R-AXIS RAPID

CuKa $(I=1.54187 \AA)$

multi-layer mirror monochromated

$40 \mathrm{kV}, 30 \mathrm{~mA}$

$-180.0^{\circ} \mathrm{C}$

$460.0 \times 256.0 \mathrm{~mm}$

41 exposures

$80.0-260.0^{0}$

$4.0 \mathrm{sec} . / \mathrm{O}$

$80.0-260.0^{0}$

$4.0 \mathrm{sec} . / \mathrm{O}$

$80.0-260.0^{0}$

$4.0 \mathrm{sec} . / 0$

$80.0-260.0^{0}$

$4.0 \mathrm{sec} . / 0$

$80.0-260.0^{0}$

$4.0 \mathrm{sec} . / 0$

$127.40 \mathrm{~mm}$ 
Pixel Size

$2 q \max$

No. of Reflections Measured

Corrections
$0.100 \mathrm{~mm}$

136.40

Total: 15515

Unique: $5287\left(R_{\text {int }}=0.0942\right)$

Lorentz-polarization

Absorption

(trans. factors: $0.366-0.632$ ) 
C. Structure Solution and Refinement

Structure Solution

Refinement

Function Minimized

Least Squares Weights

2qmax cutoff

Anomalous Dispersion

No. Observations (All reflections)

No. Variables

Reflection/Parameter Ratio

Residuals: R1 (I>2.00s(I))

Residuals: R (All reflections)

Residuals: wR2 (All reflections)

Goodness of Fit Indicator

Max Shift/Error in Final Cycle

Maximum peak in Final Diff. Map

Minimum peak in Final Diff. Map
Direct Methods

Full-matrix least-squares on $\mathrm{F}^{2}$

$S w\left(F o^{2}-F c^{2}\right)^{2}$

$$
\begin{aligned}
& w=1 /\left[\mathrm{s}^{2}\left(\mathrm{Fo}^{2}\right)+(0.0395 \cdot \mathrm{P})^{2}\right. \\
& +0.0000 \cdot \mathrm{P}] \\
& \text { where } \mathrm{P}=\left(\operatorname{Max}\left(\mathrm{Fo}^{2}, 0\right)+2 \mathrm{Fc}^{2}\right) / 3
\end{aligned}
$$

$136.4^{\circ}$

All non-hydrogen atoms

5287

388

13.63

0.0647

0.1436

0.1286

0.896

0.000

$0.77 e^{-/ \AA^{3}}$

$-0.58 e^{-/ / \AA^{3}}$ 


\section{Synthesis and Characterization of Substrates}

\section{General Procedure C for Synthesis Stating Materials}
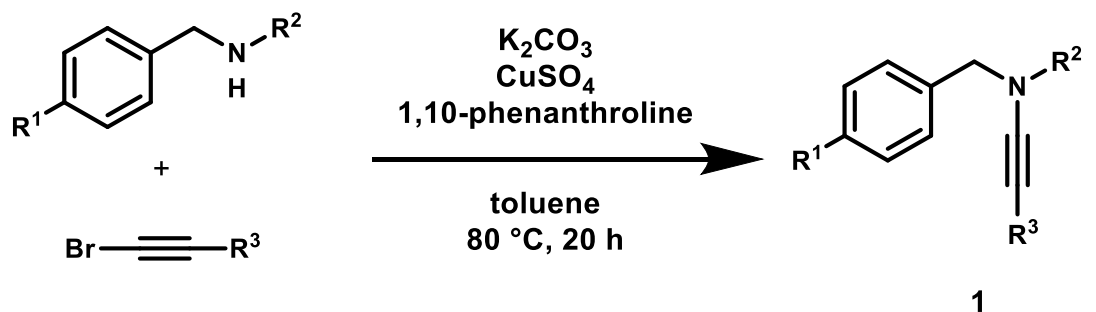

N-Alkyl substituted amides (1 equiv.), $\mathrm{CuSO}_{4}$ (10 mol \%), 1,10-phenanthroline (20 mol \%), and $\mathrm{K}_{2} \mathrm{CO}_{3}$ (2 equiv.) were suspended in toluene $(0.5 \mathrm{M})$ under argon gas atmosphere. Then alkynyl bromide (1.2 equiv.) was introduced to the reaction mixture, and the whole was stirred at $80{ }^{\circ} \mathrm{C}$ for 20 hours. After that, the reaction mixture was filtered through a short pad of celite and washed with EtOAc. Removal of the solvent and purification by flash chromatography yielded the $\mathrm{N}$-alkyl substituted ynamides 1a$1 q$.<smiles>[18O]=C(N[123I])c1ccccc1</smiles>

\section{4-Bromo-N-(4-bromobenzyl)-N-(phenylethynyl)benzenesulfonamide (1a)}

Prepared according to the general procedure $\mathrm{C}$ (1.5 mmol scale) using 4-bromo- $\mathrm{N}$-(4bromobenzyl)benzenesulfonamide and (bromoethynyl)benzene, and isolated as white 
solid in $73 \%$ yield (553.1 mg, $1.1 \mathrm{mmol}$ ) (column condition; $n$-hexane/EtOAc, 15/1, v/v).

${ }^{1} \mathrm{H}$ and ${ }^{13} \mathrm{C}$ NMR, IR, and MS data were identical to those reported ${ }^{1)}$.<smiles>CC(=O)c1ccc(C#CN([18CH2])Cc2ccc(Br)cc2)cc1</smiles>

\section{Methyl 4-((4-bromo-N-(4-bromobenzyl)phenyl)sulfonamido)ethynyl)benzoate}

Prepared according to the general procedure C $(0.8 \mathrm{mmol}$ scale $)$ using $\mathrm{N}-(4-$ bromobenzyl)-4-methylbenzenesulfonamide and methyl 4-(bromoethynyl)benzoate, and isolated as white solid in 79\% yield ( $353.4 \mathrm{mg}, 0.63 \mathrm{mmol}$ ) (column condition; $n$ hexane/EtOAc, $15 / 1$, v/v); m.p. $90-92{ }^{\circ} \mathrm{C} ;$ TLC $R_{f}=0.4(n$-hexane/EtOAc, $5 / 1, \mathrm{v} / \mathrm{v}) ;{ }^{1} \mathrm{H}$ $\operatorname{NMR}\left(400 \mathrm{MHz}, \mathrm{CDCl}_{3}\right) \delta 7.94(\mathrm{~d}, J=8.4 \mathrm{~Hz}, 2 \mathrm{H}), 7.74(\mathrm{~d}, J=8.8 \mathrm{~Hz}, 2 \mathrm{H}), 7.68(\mathrm{~d}, J$ $=8.8 \mathrm{~Hz}, 2 \mathrm{H}), 7.46(\mathrm{~d}, J=8.4 \mathrm{~Hz}, 2 \mathrm{H}), 7.28(\mathrm{~d}, J=8.0 \mathrm{~Hz}, 2 \mathrm{H}), 7.20(\mathrm{~d}, J=8.0 \mathrm{~Hz}$, 2H), 4.57 (s, 2H), 3.90 (s, 3H); ${ }^{13} \mathrm{C}$ NMR (100 MHz, $\left.\mathrm{CDCl}_{3}\right) \delta$ 166.4, 136.3, 132.9, $132.5(2 \mathrm{C}), 131.8(2 \mathrm{C}), 130.6(2 \mathrm{C}), 130.4(2 \mathrm{C}), 129.4(2 \mathrm{C}), 129.2,129.04,128.98(2 \mathrm{C})$, 127.0, 122.8, 84.8, 71.6, 55.2, 52.2; IR (ATR) 2950, 2232, 1719, 1605, 1574, 1489, 1471, 1435, 1406, $1390 \mathrm{~cm}^{-1}$; HRMS (ESI-TOF) $\left[\mathrm{M}+\mathrm{Na}^{+}\right.$calcd for $\mathrm{C}_{23} \mathrm{H}_{17} \mathrm{Br}_{2} \mathrm{NNaO}_{4} \mathrm{~S}^{+} \mathrm{m} / \mathrm{z}$ 583.9137, found 583.9151. 
<smiles>[18N]C(C#Cc1ccc(Br)cc1)Cc1ccc(Br)cc1</smiles>

\section{4-Bromo-N-(4-bromobenzyl)-N-((4-bromophenyl)ethynyl)benzenesulfonamide}

(1c)

Prepared according to the general procedure $\mathrm{C}(0.6 \mathrm{mmol}$ scale) using 4-bromo- $\mathrm{N}-(4-$ bromobenzyl)benzenesulfonamide and 1-bromo-4-(bromoethynyl)benzene, and isolated as white solid in $83 \%$ yield $(292.2 \mathrm{mg}, 0.5 \mathrm{mmol})$ (column condition; $n$ hexane/EtOAc, $15 / 1$, v/v); m.p. $83-84{ }^{\circ} \mathrm{C} ;$ TLC $R_{f}=0.5(n$-hexane/EtOAc, $5 / 1, \mathrm{v} / \mathrm{v}) ;{ }^{1} \mathrm{H}$ NMR $\left(400 \mathrm{MHz}, \mathrm{CDCl}_{3}\right) \delta 7.72(\mathrm{~d}, J=8.0 \mathrm{~Hz}, 2 \mathrm{H}), 7.67(\mathrm{~d}, J=8.4 \mathrm{~Hz}, 2 \mathrm{H}), 7.45(\mathrm{~d}, J$ $=8.4 \mathrm{~Hz}, 2 \mathrm{H}), 7.40(\mathrm{~d}, J=8.0 \mathrm{~Hz}, 2 \mathrm{H}), 7.19(\mathrm{~d}, J=8.4 \mathrm{~Hz}, 2 \mathrm{H}), 7.11(\mathrm{~d}, J=8.4 \mathrm{~Hz}$, 2H), $4.54(\mathrm{~s}, 2 \mathrm{H}) ;{ }^{13} \mathrm{C} \mathrm{NMR}\left(100 \mathrm{MHz}, \mathrm{CDCl}_{3}\right) \delta 136.3,133.0,132.7(2 \mathrm{C}), 132.5(2 \mathrm{C})$, $131.8(2 \mathrm{C}), 131.5(2 \mathrm{C}), 130.4(2 \mathrm{C}), 129.1,129.0(2 \mathrm{C}), 122.8,122.3,121.1,82.8,70.9$ 55.2; IR (ATR) 3087, 2236, 1906, 1747, 1592, 1573, 1487, 1470, 1407, $1390 \mathrm{~cm}^{-1}$; HRMS (ESI-TOF) $[\mathrm{M}+\mathrm{Na}]^{+}$calcd for $\mathrm{C}_{21} \mathrm{H}_{14}{ }^{79} \mathrm{Br}^{81} \mathrm{BrNNaO}_{2} \mathrm{~S}^{+} \mathrm{m} / \mathrm{z}$ 605.8168, found 605.8168. 
<smiles>COc1cccc(C#CN([18Br])Cc2ccc(Br)cc2)c1</smiles>

\section{4-Bromo-N-(4-bromobenzyl)-N-((3-}

\section{methoxyphenyl)ethynyl)benzenesulfonamide (1d)}

Prepared according to the general procedure $\mathrm{C}$ (1 mmol scale) using 4-bromo- $\mathrm{N}-(4-$ bromobenzyl)benzenesulfonamide and 1-(bromoethynyl)-3-methoxybenzene, and isolated as yellow oil in $95 \%$ yield $(510.0 \mathrm{mg}, 0.95 \mathrm{mmol})$ (column condition; $n$ hexane/EtOAc, 15/1, v/v); $R_{f}=0.6(n$-hexane/EtOAc, $2 / 1, \mathrm{v} / \mathrm{v}) ;{ }^{1} \mathrm{H}$ NMR $(400 \mathrm{MHz}$, $\left.\mathrm{CDCl}_{3}\right) \delta 7.73(\mathrm{~d}, J=8.8 \mathrm{~Hz}, 2 \mathrm{H}), 7.65(\mathrm{~d}, J=8.4 \mathrm{~Hz}, 2 \mathrm{H}), 7.43(\mathrm{~d}, J=8.4 \mathrm{~Hz}, 2 \mathrm{H})$, $7.21(\mathrm{~d}, J=8.8 \mathrm{~Hz}, 2 \mathrm{H}), 7.15(\mathrm{~d}, J=7.6 \mathrm{~Hz}, 1 \mathrm{H}), 6.86-6.76(\mathrm{~m}, 3 \mathrm{H}), 4.50(\mathrm{~s}, 2 \mathrm{H}), 3.75$ (s, 3H); ${ }^{13} \mathrm{C}$ NMR (100 MHz, $\left.\mathrm{CDCl}_{3}\right) \delta$ 159.2, 136.3, 133.1, 132.4 (2C), 131.7 (2C), $130.4(2 \mathrm{C}), 129.3(2 \mathrm{C}), 129.0(2 \mathrm{C}), 123.7,123.1,122.6,116.2,114.4,81.6,71.7,55.2$ 55.1; IR (ATR) 2936, 2235, 1596, 1573, 1488, 1467, 1422, 1389, 1364, $1282 \mathrm{~cm}^{-1}$; HRMS (ESI-TOF) $[\mathrm{M}+\mathrm{Na}]^{+}$calcd for $\mathrm{C}_{22} \mathrm{H}_{17} \mathrm{Br}_{2} \mathrm{NNaO}_{3} \mathrm{~S}^{+} \mathrm{m} / \mathrm{z}$ 555.9188, found 555.9184. 
<smiles>COc1cccc(C#CN(C)Cc2ccc(Br)cc2)c1</smiles>

\section{$N$-(4-Bromobenzyl)-N-((3-methoxyphenyl)ethynyl)-4-}

\section{methylbenzenesulfonamide (1e)}

Prepared according to the general procedure $\mathrm{C}(0.49 \mathrm{mmol}$ scale $)$ using $\mathrm{N}$-(4bromobenzyl)-4-methylbenzenesulfonamide and 1-(bromoethynyl)-3methoxybenzene, and isolated as yellow oil in $73 \%$ yield $(170.6 \mathrm{mg}, 0.36 \mathrm{mmol})$ (column condition; $n$-hexane/EtOAc, 10/1, v/v); TLC $R_{f}=0.3$ ( $n$-hexane/EtOAc, 5/1, v/v); ${ }^{1} \mathrm{H}$ NMR (400 MHz, $\left.\mathrm{CDCl}_{3}\right) \delta 7.78(\mathrm{~d}, J=8.4 \mathrm{~Hz}, 2 \mathrm{H}), 7.39(\mathrm{~d}, J=8.0 \mathrm{~Hz}, 2 \mathrm{H})$, $7.29(\mathrm{~d}, J=8.4 \mathrm{~Hz}, 2 \mathrm{H}), 7.18(\mathrm{~d}, J=8.8 \mathrm{~Hz}, 2 \mathrm{H}), 7.13(\mathrm{t}, J=8.0 \mathrm{~Hz}, 1 \mathrm{H}), 6.83(\mathrm{~d}, J=$ $7.2 \mathrm{~Hz}, 1 \mathrm{H}), 6.79-6.75(\mathrm{~m}, 2 \mathrm{H}), 4.50(\mathrm{~s}, 2 \mathrm{H}), 3.71(\mathrm{~s}, 3 \mathrm{H}), 2.39(\mathrm{~s}, 3 \mathrm{H}) ;{ }^{13} \mathrm{C}$ NMR $(100$ $\left.\mathrm{MHz}, \mathrm{CDCl}_{3}\right) \delta 159.0,144.7,134.2,133.3,131.4(2 \mathrm{C}), 130.3(2 \mathrm{C}), 129.6(2 \mathrm{C}), 129.1$ 127.4 (2C), 123.4, 123.3, 122.2, 115.9, 114.0, 82.1, 71.3, 55.0, 54.8, 21.4; IR (ATR) 2938, 2234, 1596, 1574, 1489, 1451, 1423, 1407, 1362, $1284 \mathrm{~cm}^{-1}$; HRMS (ESI-TOF) $[\mathrm{M}+\mathrm{Na}]^{+}$calcd for $\mathrm{C}_{23} \mathrm{H}_{20} \mathrm{BrNNaO}_{3} \mathrm{~S}^{+} \mathrm{m} / \mathrm{z}$ 492.0239, found 492.0242 . 
<smiles>Fc1ccccc1C#CN([18Br])Cc1ccc(Br)cc1</smiles>

$N$-(4-Bromobenzyl)-N-((2-fluorophenyl)ethynyl)-4-methylbenzenesulfonamide

Prepared according to the general procedure $\mathrm{C}$ (1 mmol scale) using 4-bromo- $\mathrm{N}-(4-$ bromobenzyl)benzenesulfonamide and 1-(bromoethynyl)-2-fluorobenzene, and isolated as white solid in $34 \%$ yield $(177.5 \mathrm{mg}, 0.34 \mathrm{mmol})$ (column condition; $n$ hexane/EtOAc, $15 / 1)$; m.p. $59-60{ }^{\circ} \mathrm{C}$; TLC $R_{f}=0.5$ (n-hexane/EtOAc, $\left.5 / 1, \mathrm{v} / \mathrm{v}\right) ;{ }^{1} \mathrm{H}$ $\operatorname{NMR}\left(400 \mathrm{MHz}, \mathrm{CDCl}_{3}\right) \delta 7.76(\mathrm{~d}, J=8.8 \mathrm{~Hz}, 2 \mathrm{H}), 7.66(\mathrm{~d}, J=8.8 \mathrm{~Hz}, 2 \mathrm{H}), 7.44(\mathrm{~d}, J$ $=8.4 \mathrm{~Hz}, 2 \mathrm{H}), 7.27-7.21(\mathrm{~m}, 4 \mathrm{H}), 7.04(\mathrm{~d}, J=7.2 \mathrm{~Hz}, 1 \mathrm{H}), 7.01(\mathrm{~d}, J=7.2 \mathrm{~Hz}, 1 \mathrm{H})$, 4.57 (s, 2H); ${ }^{13} \mathrm{C}$ NMR (100 MHz, $\left.\mathrm{CDCl}_{3}\right) \delta 162.3$ (d, J = 249.8 Hz), 136.2, 132.9 (d, J = 7.6 Hz), 132.8, $132.4(2 \mathrm{C}), 131.7(2 \mathrm{C}), 130.4(2 \mathrm{C}), 129.7$ (d, J = 7.7 Hz), 129.0 (3C), $123.9(\mathrm{~d}, J=3.8 \mathrm{~Hz}), 122.6,115.3(\mathrm{~d}, J=20.9 \mathrm{~Hz}), 110.7$ (d, J = $16.2 \mathrm{~Hz}), 86.5$ (d, J $=2.9 \mathrm{~Hz}), 65.4,55.2 ;{ }^{19} \mathrm{~F} \mathrm{NMR}\left(376 \mathrm{MHz}, \mathrm{CDCl}_{3}\right) \delta-117.0 ; \mathrm{IR}$ (ATR) 3023, 2241, 1704, 1613, 1574, 1489, 1471, 1455, 1407, $1390 \mathrm{~cm}^{-1}$; HRMS (ESI-TOF) [M + Na] ${ }^{+}$calcd for $\mathrm{C}_{21} \mathrm{H}_{14} \mathrm{Br}_{2} \mathrm{FNNaO}_{2} \mathrm{~S}^{+} \mathrm{m} / \mathrm{z}$ 543.8988, found 543.8977. 
<smiles>CN(C#Cc1cccc(Br)c1)Cc1ccc(Br)cc1</smiles>

N-(4-Bromobenzyl)-N-((3-bromophenyl)ethynyl)-4-methylbenzenesulfonamide (1g)

Prepared according to the general procedure $\mathrm{C}(2.1 \mathrm{mmol}$ scale $)$ using $\mathrm{N}$-(4bromobenzyl)-4-methylbenzenesulfonamide and 1-bromo-3-(bromoethynyl)benzene, and isolated as white solid in $76 \%$ yield $(645 \mathrm{mg}, 1.63 \mathrm{mmol}$ ) (column condition; $n$ hexane/EtOAc, $15 / 1$, v/v); m.p. $75-77^{\circ} \mathrm{C}$; TLC $R_{f}=0.5$ ( $n$-hexane/EtOAc, 4/1, v/v); ${ }^{1} \mathrm{H}$ $\operatorname{NMR}\left(400 \mathrm{MHz} \mathrm{CDCl}_{3}\right) \delta 7.77(\mathrm{~d}, J=8.4 \mathrm{~Hz}, 2 \mathrm{H}), 7.44(\mathrm{~d}, J=8.0 \mathrm{~Hz}, 2 \mathrm{H}), 7.38-7.32$ (m, 4H), 7.19-7.13 (m, 3H), $7.10(\mathrm{~d}, J=8.4 \mathrm{~Hz}, 1 \mathrm{H}), 4.52(\mathrm{~s}, 2 \mathrm{H}), 2.45(\mathrm{~s}, 3 \mathrm{H}) ;{ }^{13} \mathrm{C}$ NMR (100 MHz, $\left.\mathrm{CDCl}_{3}\right) \delta 145.0,134.3,133.6,133.3,131.7$ (2C), 130.8, 130.3 (2C), $129.8(2 \mathrm{C}), 129.6,129.5,127.6(2 \mathrm{C}), 124.5,122.5,122.0,83.6,70.2,54.9,21.7$; IR (ATR) 3063, 2232, 1593, 1553, 1488, 1443, 1406, 1362, 1292, $1212 \mathrm{~cm}^{-1}$; HRMS (ESI-TOF) $[\mathrm{M}+\mathrm{Na}]^{+}$calcd for $\mathrm{C}_{22} \mathrm{H}_{17} \mathrm{Br}_{2} \mathrm{NNaO}_{2} \mathrm{~S}^{+} \mathrm{m} / \mathrm{z} 539.9239$, found 539.9231.<smiles>CN(C#Cc1ccccc1)Cc1ccc(Br)cc1</smiles>

N-(4-Bromobenzyl)-4-methyl-N-(phenylethynyl)benzenesulfonamide (1h) 
Prepared according to the general procedure C (1.5 mmol scale) using $\mathrm{N}-(4-$ bromobenzyl)-4-methylbenzenesulfonamide and (bromoethynyl)benzene, and isolated as white solid in $83 \%$ yield $(548.2 \mathrm{mg}, 1.2 \mathrm{mmol}$ ) (column condition; $n$ hexane/EtOAc, 15/1, v/v).

${ }^{1} \mathrm{H}$ and ${ }^{13} \mathrm{C} N M R, I R$, and MS data were identical to those reported ${ }^{2}$.<smiles>COc1ccc(S(=O)(=O)N(C#Cc2ccccc2)Cc2ccc(Br)cc2)cc1</smiles>

\section{$N$-(4-Bromobenzyl)-4-methoxy- $N$-(phenylethynyl)benzenesulfonamide (1i)}

Prepared according to the general procedure C (2.0 mmol scale) using $\mathrm{N}-(4-$ bromobenzyl)-4-methoxybenzenesulfonamide and (bromoethynyl)benzene, and isolated as yellow solid in $34 \%$ yield ( $309.1 \mathrm{mg}, 0.67 \mathrm{mmol}$ ) (column condition; $n$ hexane/EtOAc, 15/1, v/v); m.p. $120-122^{\circ} \mathrm{C} ;$ TLC $R_{f}=0.3(n$-hexane/EtOAc, 5/1, v/v); ${ }^{1} \mathrm{H}$ NMR $\left(400 \mathrm{MHz}, \mathrm{CDCl}_{3}\right) \delta 7.72(\mathrm{~d}, J=8.4 \mathrm{~Hz}, 2 \mathrm{H}), 7.37(\mathrm{~d}, J=7.2 \mathrm{~Hz}, 2 \mathrm{H}), 7.23-$ $7.17(\mathrm{~m}, 7 \mathrm{H}), 6.92(\mathrm{~d}, J=7.2 \mathrm{~Hz}, 2 \mathrm{H}), 4.49(\mathrm{~s}, 2 \mathrm{H}), 3.76(\mathrm{~s}, 3 \mathrm{H}) ;{ }^{13} \mathrm{C} \mathrm{NMR}(100 \mathrm{MHz}$, $\left.\mathrm{CDCl}_{3}\right) \delta$ 163.5, 133.4, $131.3(2 \mathrm{C}), 130.8(2 \mathrm{C}), 130.2(2 \mathrm{C}), 129.5(2 \mathrm{C}), 128.4,128.0$ (2C), 127.6, 122.2, 122.0, 114.1 (2C), 82.4, 71.1, 55.4, 54.7; IR (ATR) 2945, 2236, 1595, 1577, 1496, 1461, 1442, 1408, 1362, $1309 \mathrm{~cm}^{-1}$; HRMS (ESI-TOF) [M + Na] $]^{+}$ 
calcd for $\mathrm{C}_{22} \mathrm{H}_{18} \mathrm{BrNNaO}_{3} \mathrm{~S}^{+} \mathrm{m} / \mathrm{z} 478.0083$, found 478.0075 .<smiles>[18N]C(=[18O])C#Cc1ccccc1</smiles>

\section{N-Benzyl-4-bromo-N-(phenylethynyl)benzenesulfonamide (1j)}

Prepared according to the general procedure $\mathrm{C}(1.8 \mathrm{mmol}$ scale) using $\mathrm{N}$-benzyl-4bromobenzenesulfonamide and (bromoethynyl)benzene, and isolated as white solid in $31 \%$ yield (240.2 mg, $0.56 \mathrm{mmol}$ ) (column condition; $n$-hexane/EtOAc, 15/1, v/v). ${ }^{1} \mathrm{H}$ and ${ }^{13} \mathrm{C}$ NMR, IR, and MS data were identical to those reported ${ }^{1}$.<smiles>[13CH2]N(C#Cc1ccccc1)Cc1ccc(I)cc1</smiles>

\section{4-Bromo-N-(4-iodobenzyl)-N-(phenylethynyl)benzenesulfonamide (1k)}

Prepared according to the general procedure $\mathrm{C}$ (1.3 mmol scale) using 4-bromo- $\mathrm{N}-(4-$ iodobenzyl)benzenesulfonamide and (bromoethynyl)benzene, and isolated as white solid in $62 \%$ yield $(445.8 \mathrm{mg}, 0.81 \mathrm{mmol}$ ) (column condition; $n$-hexane/EtOAc, 15/1, v/v); m.p. $85-87^{\circ} \mathrm{C}$; TLC $R_{f}=0.5\left(n\right.$-hexane/EtOAc, 5/1, v/v); ${ }^{1} \mathrm{H}$ NMR $(400 \mathrm{MHz}$, $\left.\mathrm{CDCl}_{3}\right) \delta 7.72(\mathrm{~d}, J=8.4 \mathrm{~Hz}, 2 \mathrm{H}), 7.66-7.63(\mathrm{~m}, 4 \mathrm{H}), 7.27(\mathrm{~s}, 5 \mathrm{H}), 7.07(\mathrm{~d}, J=8.4 \mathrm{~Hz}$ 
2H), $4.55(\mathrm{~s}, 2 \mathrm{H}) ;{ }^{13} \mathrm{C} \mathrm{NMR}\left(100 \mathrm{MHz}, \mathrm{CDCl}_{3}\right) \delta 137.7$ (2C), 136.4, 133.8, 132.4 (2C),

$131.3(2 \mathrm{C}), 130.6(2 \mathrm{C}), 129.02(2 \mathrm{C}), 128.96,128.3(2 \mathrm{C}), 128.1,122.1,94.3,81.8,71.7$, 55.3; IR (ATR) 3083, 2235, 1589, 1573, 1484, 1469, 1442, $1389 \mathrm{~cm}^{-1}$; HRMS (ESITOF) $[\mathrm{M}+\mathrm{Na}]^{+}$calcd for $\mathrm{C}_{21} \mathrm{H}_{15} \mathrm{BrINNaO}_{2} \mathrm{~S}^{+} \mathrm{m} / \mathrm{z} 573.8944$, found 573.8968 .<smiles>[18O]Cc1ccc(F)cc1</smiles>

\section{4-Bromo-N-(4-fluorobenzyl)-N-(phenylethynyl)benzenesulfonamide (1I)}

Prepared according to the general procedure $\mathrm{C}$ (1 mmol scale) using 4-bromo- $\mathrm{N}-(4-$ fluorobenzyl)benzenesulfonamide and (bromoethynyl)benzene, and isolated as white solid in $49 \%$ yield (217.2 mg, $0.49 \mathrm{mmol}$ ) (column condition; $n$-hexane/EtOAc, 15/1, v/v); m.p. $104-106{ }^{\circ} \mathrm{C} ;$ TLC $R_{f}=0.5$ (n-hexane/EtOAc, 5/1, v/v); ${ }^{1} \mathrm{H}$ NMR $(400 \mathrm{MHz}$, $\left.\mathrm{CDCl}_{3}\right) \delta 7.74(\mathrm{~d}, J=8.8 \mathrm{~Hz}, 2 \mathrm{H}), 7.71(\mathrm{~d}, J=8.8 \mathrm{~Hz}, 2 \mathrm{H}), 7.33-7.26(\mathrm{~m}, 7 \mathrm{H}), 7.01(\mathrm{t}$, $J=8.4 \mathrm{~Hz}, 2 \mathrm{H}), 4.57(\mathrm{~s}, 2 \mathrm{H}) ;{ }^{13} \mathrm{C} \mathrm{NMR}\left(100 \mathrm{MHz}, \mathrm{CDCl}_{3}\right) \delta 162.8(\mathrm{~d}, J=246 \mathrm{~Hz})$, 136.4, 132.4 (2C), 131.3 (2C), 130.7 (d, $J=8.6 \mathrm{~Hz}, 2 \mathrm{C}), 129.9,129.1$ (2C), 128.9, $128.3(2 \mathrm{C}), 128.1,122.2,115.5(\mathrm{~d}, J=21.0 \mathrm{~Hz}, 2 \mathrm{C}), 81.8,71.7,55.1 ;{ }^{19} \mathrm{~F}$ NMR $(376$ $\left.\mathrm{MHz}, \mathrm{CDCl}_{3}\right) \delta-112.9$; IR (ATR) 3085, 2237, 1746, 1604, 1574, 1509, 1469, 1442, 1390, $1365 \mathrm{~cm}^{-1}$; HRMS (ESI-TOF) $\left[\mathrm{M}+\mathrm{Na}^{+}\right.$calcd for $\mathrm{C}_{21} \mathrm{H}_{15} \mathrm{BrFNNaO}_{2} \mathrm{~S}^{+} \mathrm{m} / \mathrm{z}$ 465.9883, found 465.9893 . 
<smiles></smiles>

\section{$N$-(4-Chlorobenzyl)-4-methyl- $N$-(phenylethynyl)benzenesulfonamide (1m)}

Prepared according to the general procedure C (1.2 mmol scale) using $\mathrm{N}-(4-$ chlorobenzyl)-4-methylbenzenesulfonamide and (bromoethynyl)benzene, and isolated as white solid in $25 \%$ yield $(118.5 \mathrm{mg}, 0.3 \mathrm{mmol}$ ) (column condition; $n$ hexane/EtOAc, $15 / 1$, v/v); m.p. $81-82{ }^{\circ} \mathrm{C} ; \mathrm{TLC} R_{f}=0.5(n$-hexane/EtOAc, $5 / 1, \mathrm{v} / \mathrm{v}) ;{ }^{1} \mathrm{H}$ NMR $\left(400 \mathrm{MHz}, \mathrm{CDCl}_{3}\right) \delta 7.79(\mathrm{~d}, J=8.0 \mathrm{~Hz}, 2 \mathrm{H}), 7.32(\mathrm{~d}, J=8.0 \mathrm{~Hz}, 2 \mathrm{H}), 7.27(\mathrm{~s}$, 4H), $7.25(\mathrm{~s}, 5 \mathrm{H}), 4.54(\mathrm{~s}, 2 \mathrm{H}), 2.44(\mathrm{~s}, 3 \mathrm{H}) ;{ }^{13} \mathrm{C} \mathrm{NMR}\left(100 \mathrm{MHz}, \mathrm{CDCl}_{3}\right) \delta$ 144.8, 134.5, 134.3, 133.0, $131.2(2 \mathrm{C}), 130.1(2 \mathrm{C}), 129.7$ (2C), $128.7(2 \mathrm{C}), 128.2(2 \mathrm{C}), 127.8,127.7$ (2C), 122.5, 82.3, 71.5, 54.9, 21.6; IR (ATR) 3054, 2234, 1597, 1492, 1442, 1409, 1362, $1306 \mathrm{~cm}^{-1}$; HRMS (ESI-TOF) $[\mathrm{M}+\mathrm{Na}]^{+}$calcd for $\mathrm{C}_{22} \mathrm{H}_{18} \mathrm{CINNaO}_{2} \mathrm{~S}^{+} \mathrm{m} / \mathrm{z}$ 418.0639, found $\mathrm{m} / \mathrm{z} 418.0647$.<smiles>Cc1ccc(CN([18OH])C#Cc2ccccc2)cc1</smiles>

4-Bromo-N-(4-methylbenzyl)-N-(phenylethynyl)benzenesulfonamide (1n) 
Prepared according to the general procedure $\mathrm{C}$ (5.1 mmol scale) using 4-bromo- $\mathrm{N}-(4-$ methylbenzyl)benzenesulfonamide and (bromoethynyl)benzene, and isolated as white solid in $87 \%$ yield $(1.9 \mathrm{~g}, 4.4 \mathrm{mmol}$ ) (column condition; $n$-hexane/EtOAc, 15/1, v/v); m.p. $60-61{ }^{\circ} \mathrm{C} ;$ TLC $R_{f}=0.5(n$-hexane/EtOAc, $4 / 1, \mathrm{v} / \mathrm{v}) ;{ }^{1} \mathrm{H}$ NMR $\left(400 \mathrm{MHz}, \mathrm{CDCl}_{3}\right)$ $\delta 7.71(\mathrm{~d}, J=8.8 \mathrm{~Hz}, 2 \mathrm{H}), 7.62(\mathrm{~d}, J=8.8 \mathrm{~Hz}, 2 \mathrm{H}), 7.26(\mathrm{~s}, 5 \mathrm{H}), 7.21(\mathrm{~d}, J=8.0 \mathrm{~Hz}$, 2H), $7.11(\mathrm{~d}, J=8.0 \mathrm{~Hz}, 2 \mathrm{H}), 4.56(\mathrm{~s}, 2 \mathrm{H}), 2.33(\mathrm{~s}, 3 \mathrm{H}) ;{ }^{13} \mathrm{C} \mathrm{NMR}\left(100 \mathrm{MHz}, \mathrm{CDCl}_{3}\right) \delta$ 138.3, 136.6, $132.3(2 \mathrm{C}), 131.2(2 \mathrm{C}), 131.0,129.2(2 \mathrm{C}), 129.1(2 \mathrm{C}), 128.8(2 \mathrm{C}), 128.7$ 128.2 (2C), 127.9, 122.4, 82.2, 71.5, 55.8, 21.2; IR (ATR) 3055, 2922, 2237, 1598, 1573, 1516, 1492, 1469, 1442, $1389 \mathrm{~cm}^{-1}$; HRMS (ESI-TOF) $[\mathrm{M}+\mathrm{Na}]^{+}$calcd for $\mathrm{C}_{22} \mathrm{H}_{18} \mathrm{BrNNaO}_{2} \mathrm{~S}^{+} \mathrm{m} / \mathrm{z}$ 462.0134, found 462.0132 .<smiles>CCCCCC#CN(C)Cc1ccc(Br)cc1</smiles>

$N$-(4-Bromobenzyl)-N-(hept-1-yn-1-yl)-4-methylbenzenesulfonamide (10)

Prepared according to the general procedure $C$ (1.5 mmol scale) using $\mathrm{N}-(4-$ bromobenzyl)-4-methylbenzenesulfonamide and 1-bromohept-1-yne, and isolated as colerless oil in $43 \%$ yield $(282.0 \mathrm{mg}, 0.65 \mathrm{mmol}$ ) (column condition; $n$-hexane/EtOAc, 15/1, v/v); $R_{f}=0.6(n$-hexane/EtOAc, $1 / 5) ;{ }^{1} \mathrm{H}$ NMR $\left(400 \mathrm{MHz}, \mathrm{CDCl}_{3}\right) \delta 7.74(\mathrm{~d}, J=$ $8.4 \mathrm{~Hz}, 2 \mathrm{H}), 7.40(\mathrm{~d}, J=8.4 \mathrm{~Hz}, 2 \mathrm{H}), 7.31(\mathrm{~d}, J=8.0 \mathrm{~Hz}, 2 \mathrm{H}), 7.15(\mathrm{~d}, J=8.0 \mathrm{~Hz}, 2 \mathrm{H})$, 
$4.39(\mathrm{~s}, 2 \mathrm{H}), 2.44(\mathrm{~s}, 3 \mathrm{H}), 2.16(\mathrm{t}, J=6.8 \mathrm{~Hz}, 2 \mathrm{H}$ ), 1.37 (quin, $J=7.2 \mathrm{~Hz}, 2 \mathrm{H}$ ), $1.27-$

$1.16(\mathrm{~m}, 4 \mathrm{H}), 0.85(\mathrm{t}, J=7.2 \mathrm{~Hz}, 3 \mathrm{H}) ;{ }^{13} \mathrm{C} \mathrm{NMR}\left(150 \mathrm{MHz}, \mathrm{CDCl}_{3}\right) \delta$ 144.4, 134.6, 133.9, 131.5 (2C), $130.4(2 \mathrm{C}), 129.6$ (2C), 127.7 (2C), 122.2, 73.1, 71.2, 54.9, 30.8, 28.4, 22.1, 21.6, 18.3, 14.0; IR (ATR) 2929, 2857, 2253, 1596, 1489, 1455, 1407, 1361 $\mathrm{cm}^{-1}$; HRMS (ESI-TOF) $[\mathrm{M}+\mathrm{Na}]^{+}$calcd for $\mathrm{C}_{21} \mathrm{H}_{24} \mathrm{BrNNaO}_{2} \mathrm{~S}^{+} \mathrm{m} / \mathrm{z} 456.0603$, found $\mathrm{m} / \mathrm{z} 456.0621$.<smiles>CN(Cc1ccccc1)Cc1ccccc1</smiles>

\section{$N$-benzyl-4-methyl-N-(phenylethynyl)benzenesulfonamide (1p)}

Prepared according to the general procedure C (1.85 mmol scale) using $\mathrm{N}$-benzyl-4methylbenzenesulfonamide and (bromoethynyl)benzene, and isolated as white solid in $85 \%$ yield (570.2 mg, $1.58 \mathrm{mmol}$ ) (column condition; $n$-hexane/EtOAc, 15/1, v/v). ${ }^{1} \mathrm{H}$ and ${ }^{13} \mathrm{C} N M R$, IR, and MS data were identical to those reported. ${ }^{3)}$

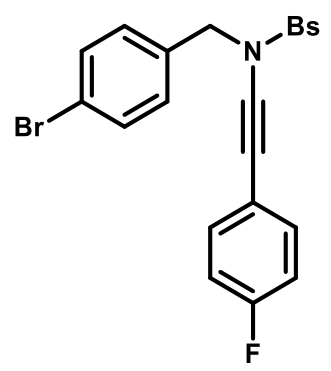




\section{4-Bromo-N-(4-bromobenzyl)-N-((4-fluorophenyl)ethynyl)benzenesulfonamide}

(1u)

Prepared according to the general procedure C (1.2 mmol scale) using 4-bromo- $\mathrm{N}-(4-$ bromobenzyl)benzenesulfonamide and 1-(bromoethynyl)-4-fluorobenzene, and isolated as white solid in $76 \%$ yield $(474.2 \mathrm{mg}, 0.91 \mathrm{mmol})$ (column condition; $n$ hexane/EtOAc, $15 / 1)$; m.p. $88-90{ }^{\circ} \mathrm{C}$; TLC $R_{f}=0.6$ (n-hexane/EtOAc, $2 / 1$, v/v); ${ }^{1} \mathrm{H}$ $\operatorname{NMR}\left(400 \mathrm{MHz}_{\mathrm{CDCl}}\right) \delta 7.73(\mathrm{~d}, J=7.2 \mathrm{~Hz}, 2 \mathrm{H}), 7.65(\mathrm{dd}, J=8.8,0.8 \mathrm{~Hz}, 2 \mathrm{H}), 7.43$ (dd, $J=8.8,0.8 \mathrm{~Hz}, 2 \mathrm{H}), 7.25-7.18(\mathrm{~m}, 4 \mathrm{H}), 6.95(\mathrm{t}, J=8.4 \mathrm{~Hz}, 2 \mathrm{H}), 4.53(\mathrm{~s}, 2 \mathrm{H}) ;{ }^{13} \mathrm{C}$ $\operatorname{NMR}\left(100 \mathrm{MHz}, \mathrm{CDCl}_{3}\right) \delta 162.3$ (d, J = 248.9 Hz), 136.2, 133.4 (d, J = 8.6 Hz, 2C), 133.1, 132.4 (2C), 131.7 (2C), 130.3 (2C), 128.9 (3C), 122.6, 118.0 (d, J = 2.8 Hz), $115.5(\mathrm{~d}, J=21.9 \mathrm{~Hz}, 2 \mathrm{C}), 81.3,70.6,55.1 ;{ }^{19} \mathrm{~F} \mathrm{NMR}\left(376 \mathrm{MHz}, \mathrm{CDCl}_{3}\right) \delta-110.6$; IR (ATR) 3088, 2238, 1909, 1714, 1599, 1573, 1508, 1488, 1471, $1407 \mathrm{~cm}^{-1}$; HRMS (ESI-TOF) $[\mathrm{M}+\mathrm{Na}]^{+}$calcd for $\mathrm{C}_{21} \mathrm{H}_{14} \mathrm{Br}_{2} \mathrm{FNNaO}_{2} \mathrm{~S}^{+} \mathrm{m} / \mathrm{z}$ 543.8988, found 543.8989.<smiles>CN(C#Cc1ccccc1)Cc1ccc(F)cc1</smiles>

$N$-(4-Fluorobenzyl)-4-methyl-N-(phenylethynyl)benzenesulfonamide (1v)

Prepared according to the general procedure $\mathrm{C}(1.4 \mathrm{mmol}$ scale) using $\mathrm{N}$-(4fluorobenzyl)-4-methylbenzenesulfonamide and (bromoethynyl)benzene, and isolated 
as white solid in $80 \%$ yield ( $400.7 \mathrm{mg}, 1.1 \mathrm{mmol}$ ) (column condition; $n$-hexane/EtOAc, 15/1, v/v).

${ }^{1} \mathrm{H}$ and ${ }^{13} \mathrm{C}$ NMR, IR, and MS data were identical to those reported. ${ }^{3)}$ 


\section{$9 \underline{\text { Transformation of Product }}$}

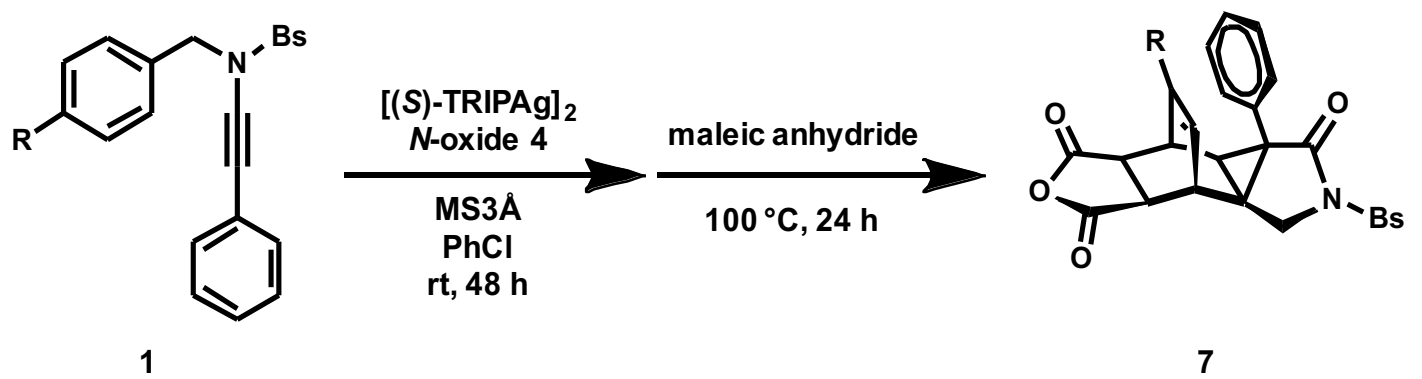

A pre-dried $10 \mathrm{~mL}$ test tube equipped with a magnetic stir bar was covered with aluminum foil to avoid light exposure. The test tube was charged with ynamide compounds 1 (0.2 mmol, 1 equiv.), $N$-oxide 4 (63.8 mg, $0.4 \mathrm{mmol}, 2$ equiv.), [(S)TRIPAg]2 (8.6 mg, $0.005 \mathrm{mmol}, 2.5 \mathrm{~mol} \%$ ), and MS3Å (200 mg, $1 \mathrm{~g} / \mathrm{mmol})$, which were subsequently dissolved partially in dry $\mathrm{PhCl}(2 \mathrm{~mL}, 0.1 \mathrm{M})$ under argon gas atmosphere. The reaction mixture was stirred for 48 hours at the room temperature, and then the aluminum foil was removed. Maleic anhydride $(392 \mathrm{mg}, 4.0 \mathrm{mmol}, 20$ equiv.) was added to the reaction mixture, and the whole was stirred another 24 hours at $100^{\circ} \mathrm{C}$. Then, the reaction mixture was passed through a celite to remove MS3A. After the solvent was evaporated in vacuo, the resulting residue was purified by flash chromatography ( $n$-hexane/EtOAc $=3 / 1$ to $2 / 1, \mathrm{v} / \mathrm{v})$ to afford desired product 7 . 


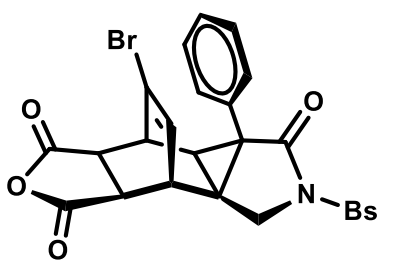

(3bR,4S,7aR,8R,8aR)-10-Bromo-2-((4-bromophenyl)sulfonyl)-3a-phenyl-

1,2,3a,3b,4,4a,7a,8-octahydro-7H-4,8-

ethenoisobenzofuro[5',6':1,3]cyclopropa[1,2-c]pyrrole-3,5,7-trione (7a)

White solid (97.1 mg, 78\% yield): m.p. $152{ }^{\circ} \mathrm{C}$ (decomp.); TLC $R_{f}=0.3$ ( $n$ hexane/EtOAc, 2/1, v/v); ${ }^{1} \mathrm{H}$ NMR (400 MHz, $\left.\mathrm{CDCl}_{3}\right) \delta 7.85$ (d, J = $\left.8.8 \mathrm{~Hz}, 2 \mathrm{H}\right), 7.66$ $(\mathrm{d}, J=8.8 \mathrm{~Hz}, 2 \mathrm{H}), 7.36(\mathrm{t}, J=6.8 \mathrm{~Hz}, 1 \mathrm{H}), 7.29-7.19(\mathrm{~m}, 4 \mathrm{H}), 6.71(\mathrm{~d}, J=7.2 \mathrm{~Hz}, 1 \mathrm{H})$, $5.74(\mathrm{dd}, J=7.2,2.0 \mathrm{~Hz}, 1 \mathrm{H}), 4.12(\mathrm{~d}, J=10.4 \mathrm{~Hz}, 1 \mathrm{H}), 4.05(\mathrm{~d}, J=10.4 \mathrm{~Hz}, 1 \mathrm{H}), 3.83$ $(\mathrm{d}, J=2.8 \mathrm{~Hz}, 1 \mathrm{H}), 3.72(\mathrm{dd}, J=2.8,7.2 \mathrm{~Hz}, 1 \mathrm{H}), 3.58-3.51(\mathrm{~m}, 2 \mathrm{H}), 1.63(\mathrm{~d}, J=3.6$ $\mathrm{Hz}, 1 \mathrm{H}) ;{ }^{13} \mathrm{C}$ NMR $\left(100 \mathrm{MHz}, \mathrm{CDCl}_{3}\right) \delta 170.8,170.5,169.3,136.0,132.7$ (2C), 131.8, $131.4,130.5,130.0(2 \mathrm{C}), 129.6(2 \mathrm{C}), 129.3,128.5,128.0,120.3,49.1,45.9,44.6,43.6$, 43.2, 37.3, 32.6, 29.1; IR (ATR) 2924, 2351, 1777, 1730, 1573, 1471, 1345, 1282 , 1225, $1168 \mathrm{~cm}^{-1}$; HRMS (ESI-TOF) $[\mathrm{M}+\mathrm{H}]^{+}$calcd for $\mathrm{C}_{25} \mathrm{H}_{18} \mathrm{Br}_{2} \mathrm{NO}_{6} \mathrm{~S}^{+} \mathrm{m} / \mathrm{z} 617.9216$, found $617.9222 ;[\alpha]^{25} \mathrm{D}+63.0\left(c\right.$ 1.0, $\left.\mathrm{CHCl}_{3}\right)$. The enantiomeric ratio was determined after conversion into $\mathbf{8 a}$. 


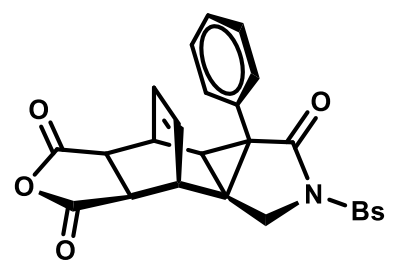

(3bR,4S,7aR,8S,8aR)-2-((4-bromophenyl)sulfonyl)-3a-phenyl-

\section{1,2,3a,3b,4,4a,7a,8-octahydro-7H-4,8-}

ethenoisobenzofuro[ $\left.5^{\prime}, 6^{\prime}: 1,3\right]$ cyclopropa[1,2-c]pyrrole-3,5,7-trione (7j)

White solid (86.2 mg, $79 \%$ yield, $94: 6$ er): m.p. $>200{ }^{\circ} \mathrm{C}$ (decomp.); $R_{f}=0.3(n-$ hexane/EtOAc, 2/1, v/v); ${ }^{1} \mathrm{H} \mathrm{NMR}\left(400 \mathrm{MHz}, \mathrm{CDCl}_{3}\right) \delta 7.87$ (dd, $\left.J=8.8,2.0 \mathrm{~Hz}, 2 \mathrm{H}\right)$, $7.67(\mathrm{~d}, J=8.8,2.0 \mathrm{~Hz}, 2 \mathrm{H}), 7.30-7.26(\mathrm{~m}, 1 \mathrm{H}), 7.23-7.15(\mathrm{~m}, 2 \mathrm{H}), 7.04(\mathrm{~d}, J=7.6 \mathrm{~Hz}$, 1H), $6.7(\mathrm{~d}, J=7.2 \mathrm{~Hz}, 1 \mathrm{H}), 5.49(\mathrm{dd}, J=6.4,5.6 \mathrm{~Hz}, 1 \mathrm{H}), 5.30(\mathrm{t}, J=7.2 \mathrm{~Hz}, 1 \mathrm{H})$, $4.14(\mathrm{~d}, J=10.0 \mathrm{~Hz}, 1 \mathrm{H}), 4.05(\mathrm{~d}, J=10.0 \mathrm{~Hz}, 1 \mathrm{H}), 3.76-3.75(\mathrm{~m}, 1 \mathrm{H}), 3.66-3.63(\mathrm{~m}$, 1H), $3.52(\mathrm{dd}, J=8.8,3.6 \mathrm{~Hz}, 1 \mathrm{H}), 3.41(\mathrm{dd}, J=8.8,3.6 \mathrm{~Hz}, 1 \mathrm{H}), 1.62(\mathrm{~d}, J=3.6 \mathrm{~Hz}$, $1 \mathrm{H}) ;{ }^{13} \mathrm{C} \mathrm{NMR}\left(100 \mathrm{MHz}, \mathrm{CDCl}_{3}\right) \delta 171.2,171.1,170.9,136.5,136.2,132.6(2 \mathrm{C})$, $132.1,130.8,130.5,130.0,129.8,129.6(2 \mathrm{C}), 128.9,128.7,127.9,49.5,45.2,44.7$, 43.7, 34.6, 33.3, 32.8, 29.2; IR (ATR) 3117, 2922, 2358, 1848, 1771, 1730, 1572, 1471, 1390, $1350 \mathrm{~cm}^{-1}$; HRMS (ESI-TOF) [M + H] $]^{+}$calcd for $\mathrm{C}_{25} \mathrm{H}_{19} \mathrm{BrNO}_{6} \mathrm{~S}^{+} \mathrm{m} / \mathrm{z} 540.0111$, found $540.0118 ;[\alpha]^{25} \mathrm{D}+61.9\left(c 1.0, \mathrm{CHCl}_{3}\right)$; The enantiomeric ratio was determined by analytical chiral HPLC. Retention time: 16.0 min (minor isomer), 17.1 min (major isomer), IH-3 column, 50/50 n-hexane/DCM $1 \mathrm{~mL} / \mathrm{min}, 254 \mathrm{~nm}$. 


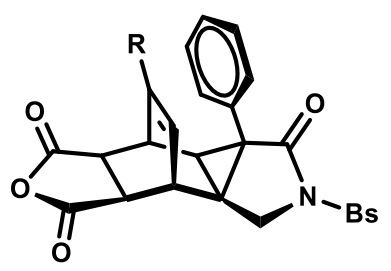

7

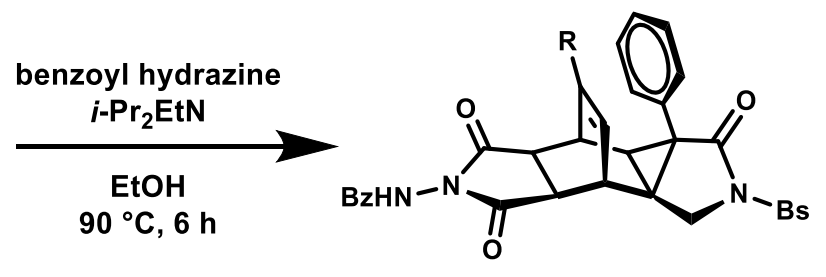

8

\section{General Procedure D for Condensation with benzoyl hydrazine}

A pre-dried $10 \mathrm{~mL}$ sealed tube equipped with a magnetic stir bar was charged with compound 7 ( 1 equiv.), benzoyl hydrazine (1 equiv.), which were subsequently dissolved in dry $\mathrm{EtOH}(0.25 \mathrm{M})$ under argon gas atmosphere. $N, N$ Diisopropylethylamine (15 mol \%) was added to the reaction mixture, and the whole was stirred for 6 hours at $90{ }^{\circ} \mathrm{C}$. After the solvent was evaporated in vacuo, the resulting residue was purified by flash chromatography ( $n$-hexane/EtOAc, $3 / 1$ to 2/1, v/v) to afford desired products 8 .

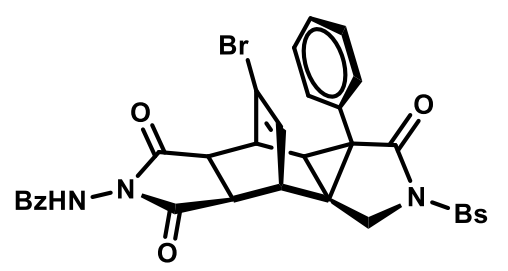

$N-((3 \mathrm{~b} R, 4 S, 7 \mathrm{a} R, 8 R, 8 \mathrm{a} R)-10-\mathrm{Bromo}-2-((4-\mathrm{b} r o m o p h e n y l) s u l f o n y l)-3,5,7-t r i o x o-3 a-$ phenyl-1,2,3,3a,3b,4,4a,5,7a,8-decahydro-4,8-

\section{ethenopyrrolo[3',4':1,3]cyclopropa[1,2-f]isoindol-6(7H)-yl)benzamide (8a)}

Prepared according to the general procedure $\mathrm{D}(0.075 \mathrm{mmol}$ scale $)$ using compound 7a, and isolated as white solid in $80 \%$ yield $(44.5 \mathrm{mg}, 0.06 \mathrm{mmol}, 95: 5$ er): m.p. > 
$200{ }^{\circ} \mathrm{C} ;$ TLC $R_{f}=0.3(n$-hexane/EtOAc, $1 / 1, \mathrm{v} / \mathrm{v}) ;{ }^{1} \mathrm{H}$ NMR $\left(400 \mathrm{MHz}, \mathrm{CDCl}_{3}\right) \delta 8.52$ (br s, $1 \mathrm{H}), 7.85(\mathrm{~d}, J=8.4 \mathrm{~Hz}, 2 \mathrm{H}), 7.76(\mathrm{~d}, J=7.6 \mathrm{~Hz}, 2 \mathrm{H}), 7.64(\mathrm{~d}, J=8.8 \mathrm{~Hz}, 2 \mathrm{H})$, $7.52(\mathrm{t}, J=7.2 \mathrm{~Hz}, 1 \mathrm{H}), 7.37(\mathrm{dd}, J=8.0,7.6 \mathrm{~Hz}, 2 \mathrm{H}), 7.32(\mathrm{t}, J=7.2 \mathrm{~Hz}, 1 \mathrm{H}), 7.25-$ $7.17(\mathrm{~m}, 3 \mathrm{H}), 6.71(\mathrm{~d}, J=7.2 \mathrm{~Hz}, 1 \mathrm{H}), 5.70(\mathrm{~d}, J=6.4 \mathrm{~Hz}, 1 \mathrm{H}), 4.19(\mathrm{~d}, J=10.4 \mathrm{~Hz}$, $1 \mathrm{H}), 4.04(\mathrm{~d}, J=10.0 \mathrm{~Hz}, 1 \mathrm{H}), 3.80(\mathrm{~s}, 1 \mathrm{H}), 3.71(\mathrm{~d}, J=6.8 \mathrm{~Hz}, 1 \mathrm{H}), 3.38(\mathrm{~s}, 2 \mathrm{H}), 1.65$ $(\mathrm{d}, J=4.0 \mathrm{~Hz}, 1 \mathrm{H}) ;{ }^{13} \mathrm{C} \mathrm{NMR}\left(100 \mathrm{MHz}, \mathrm{CDCl}_{3}\right) \delta 172.8,172.0,170.7,165.0,136.2$, 133.0, $132.6(2 \mathrm{C}), 131.5,131.4,130.8,130.3,130.1,129.9,129.8(2 \mathrm{C}), 129.2,128.8$ (2C), 128.4, 127.9, 127.7 (2C), 119.7, 49.3, 43.9, 43.1 (2C), 42.4, 37.3, 32.7, 29.6; IR (ATR) 2955, 1725, 1573, 1472, 1390, 1362, 1276, 1169, 1136, $1087 \mathrm{~cm}^{-1}$; HRMS (ESI-TOF) $[\mathrm{M}+\mathrm{Na}]^{+}$calcd for $\mathrm{C}_{32} \mathrm{H}_{23} \mathrm{Br}_{2} \mathrm{~N}_{3} \mathrm{NaO}_{6} \mathrm{~S}^{+} \mathrm{m} / \mathrm{z}$ 757.9567, found 757.9546; $[\alpha]^{25} \mathrm{D}+28.3\left(c 1.0, \mathrm{CHCl}_{3}\right)$. The enantiomeric ratio was determined by analytical chiral HPLC. Retention time: 11.3 min (minor isomer), 13.1 min (major isomer), IG-3 column, 1/99 EtOAc/DCM, $0.5 \mathrm{~mL} / \mathrm{min}, 254 \mathrm{~nm}$.

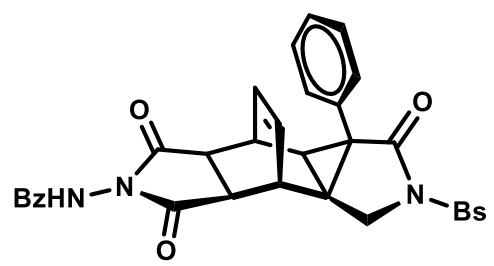

$N-((3 \mathrm{~b} R, 4 S, 7 \mathrm{a} R, 8 S, 8 \mathrm{a} R)-2-((4-\mathrm{bromophenyl})$ sulfonyl)-3,5,7-trioxo-3a-phenyl1,2,3,3a,3b,4,4a,5,7a,8-decahydro-4,8-ethenopyrrolo[3',4':1,3]cyclopropa[1,2f]isoindol-6(7H)-yl)benzamide (8j) 
Prepared according to the general procedure $\mathrm{D}(0.13 \mathrm{mmol}$ scale $)$ using compound $7 \mathbf{j}$, and isolated as white solid in $80 \%$ yield $(70.8 \mathrm{mg}, 0.11 \mathrm{mmol}):$ m.p. $188-190{ }^{\circ} \mathrm{C} ; R_{f}=$ $0.4(n$-hexane/EtOAc, $1 / 1, \mathrm{v} / \mathrm{v}) ;{ }^{1} \mathrm{H}$ NMR $\left(400 \mathrm{MHz}, \mathrm{CDCl}_{3}\right) \delta 8.37$ (br s, $\left.1 \mathrm{H}\right), 7.86(\mathrm{dd}$, $J=8.8,2.0 \mathrm{~Hz}, 2 \mathrm{H}), 7.74(\mathrm{~d}, J=7.6 \mathrm{~Hz}, 2 \mathrm{H}), 7.65(\mathrm{dd}, J=8.8,2.0 \mathrm{~Hz}, 2 \mathrm{H}), 7.52(\mathrm{t}, J$ $=7.6 \mathrm{~Hz}, 1 \mathrm{H}), 7.38(\mathrm{t}, J=7.6 \mathrm{~Hz}, 2 \mathrm{H}), 7.24(\mathrm{dt}, J=7.2,1.6 \mathrm{~Hz}, 1 \mathrm{H}), 7.19-7.12(\mathrm{~m}, 2 \mathrm{H})$, $7.01(\mathrm{~d}, J=7.2 \mathrm{~Hz}, 1 \mathrm{H}), 6.72(\mathrm{~d}, J=7.6 \mathrm{~Hz}, 1 \mathrm{H}), 5.38(\mathrm{t}, J=6.8 \mathrm{~Hz}, 1 \mathrm{H}), 5.17(\mathrm{t}, J=$ $6.8 \mathrm{~Hz}, 1 \mathrm{H}), 4.20(\mathrm{~d}, J=10.0 \mathrm{~Hz}, 1 \mathrm{H}), 4.04(\mathrm{~d}, J=10.0 \mathrm{~Hz}, 1 \mathrm{H}), 3.65-3.64(\mathrm{~m}, 1 \mathrm{H})$, 3.59-3.57 (m, 1H), $3.34(\mathrm{~d}, 4.8 \mathrm{~Hz}, 1 \mathrm{H}), 3.17(\mathrm{~d}, J=4.8 \mathrm{~Hz}, 1 \mathrm{H}), 1.60(\mathrm{~d}, J=3.6 \mathrm{~Hz}$, 1H); $\left.{ }^{13} \mathrm{C} \mathrm{NMR} \mathrm{(100} \mathrm{MHz,} \mathrm{CDCl}_{3}\right) \delta 173.5,173.3,171.5,165.0,136.3,132.9,132.6$ (2C), 132.5, 130.5, 130.4, 130.1, 129.8, 129.7 (2C), 129.0, 128.8 (3C), 128.6, 128.1, 127.7, 127.6 (2C), 49.8, 43.2, 43.1, 42.5, 34.6, 33.2, 33.0, 29.6; IR (ATR) 3299, 2958, 1793, 1728, 1602, 1574, 1518, 1473, 1448, $1390 \mathrm{~cm}^{-1}$; HRMS (ESI-TOF) $[\mathrm{M}+\mathrm{Na}]^{+}$ calcd for $\mathrm{C}_{32} \mathrm{H}_{24} \mathrm{BrN}_{3} \mathrm{NaO}_{6} \mathrm{~S}^{+} \mathrm{m} / \mathrm{z}$ 680.0461, found 680.0465; [a] ${ }^{25} \mathrm{D}+53.6$ (c 1.0, $\left.\mathrm{CHCl}_{3}\right)$.

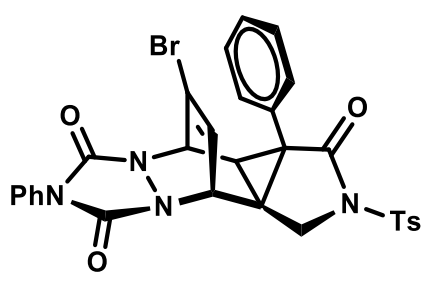

$6 e$

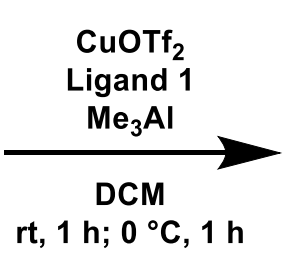

$\mathrm{rt}, 1 \mathrm{~h} ; 0^{\circ} \mathrm{C}, 1 \mathrm{~h}$

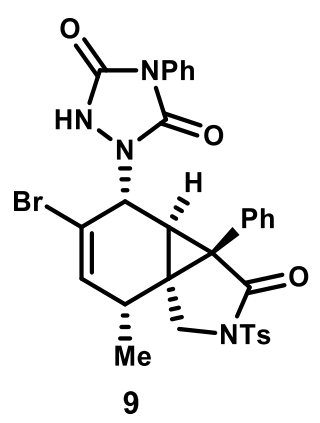

9

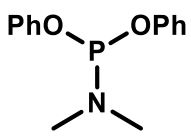

Ligand 1 
$\mathrm{CuOTf}_{2}(15.5 \mathrm{mg}, 0.043 \mathrm{mmol}, 10 \mathrm{~mol} \%)$ and Ligand $1(40 \mu \mathrm{L}, 0.086 \mathrm{mmol}, 20 \mathrm{~mol} \%)$ in dry DCM $(8.4 \mathrm{~mL}, 0.05 \mathrm{M})$ were placed in a flame-dried Schlenk tube, and the whole was stirred at room temperature for 1 hour. A solution of compound $6 \mathbf{e}(270 \mathrm{mg}, 0.43$ mmol, 1 equiv. $)$ in dry DCM $(8.4 \mathrm{~mL}, 0.05 \mathrm{M})$ was added to the mixture, and the whole was cooled to $0^{\circ} \mathrm{C}$. Trimethylaluminum $(1.4 \mathrm{M}$ in $n$-hexane, $1.1 \mathrm{~mL}, 0.48 \mathrm{mmol}, 3$ equiv.) was added, then the reaction was stirred at $0{ }^{\circ} \mathrm{C}$ for 1 hour. The reaction was quenched with the addition of $1 \mathrm{~N}$ aqueous $\mathrm{HCl}$, and the aqueous solution was extracted with DCM. The combined organic layers were washed with brine, dried over $\mathrm{Na}_{2} \mathrm{SO}_{4}$, and concentrated under reduced pressure. The crude was purified by flash chromatography ( $n$-hexane/EtOAc, 3/2, v/v) to afford desired product 9.

\section{1-((3aR,3bR,4R,7R,7a R)-5-Bromo-7-methyl-3-oxo-3a-phenyl-2-tosyl-}

\section{2,3,3a,3b,4,7-hexahydro-1H-benzo[1,3]cyclopropa[1,2-c]pyrrol-4-yl)-4-phenyl-}

\section{1,2,4-triazolidine-3,5-dione (9)}

White solid (256.4 mg, $93 \%$ yield): m.p. $162-164{ }^{\circ} \mathrm{C}$; TLC $R_{f}=0.2$ ( $n$-hexane/EtOAc, 1/1, v/v); ${ }^{1} \mathrm{H}$ NMR (400 MHz, $\left.\mathrm{CDCl}_{3}\right) \delta 8.97(\mathrm{br} \mathrm{s}, 1 \mathrm{H}), 7.87(\mathrm{~d}, J=8.4 \mathrm{~Hz}, 2 \mathrm{H}), 7.51$ $(\mathrm{d}, J=4.0 \mathrm{~Hz}, 4 \mathrm{H}), 7.42-7.38(\mathrm{~m}, 1 \mathrm{H}), 7.31-7.25(\mathrm{~m}, 5 \mathrm{H}), 7.17-7.16(\mathrm{~m}, 2 \mathrm{H}), 5.88(\mathrm{~d}$, $J=5.2 \mathrm{~Hz}, 1 \mathrm{H}), 5.00(\mathrm{~d}, J=1.6 \mathrm{~Hz}, 1 \mathrm{H}), 4.24(\mathrm{~d}, J=10.4 \mathrm{~Hz}, 1 \mathrm{H}), 3.80(\mathrm{~d}, J=10.4$ $\mathrm{Hz}, 1 \mathrm{H}), 2.97-2.93(\mathrm{~m}, 1 \mathrm{H}), 2.37(\mathrm{~s}, 3 \mathrm{H}), 1.72(\mathrm{~s}, 1 \mathrm{H}), 1.20(\mathrm{~d}, J=6.8 \mathrm{~Hz}, 3 \mathrm{H}) ;{ }^{13} \mathrm{C}$ 
$\operatorname{NMR}\left(100 \mathrm{MHz}, \mathrm{CDCl}_{3}\right) \delta 171.2,155.1,153.2,145.4,138.0,134.4,130.9,129.8(2 \mathrm{C})$, $129.6(2 \mathrm{C}), 129.2(2 \mathrm{C}), 128.9(2 \mathrm{C}), 128.7,128.5,128.4,128.1(2 \mathrm{C}), 125.5(2 \mathrm{C}), 115.4$ 55.2, 50.2, 37.9, 31.8, 30.4, 29.7, 21.6, 16.9; IR (ATR) 3029, 1777, 1702, 1597, 1501, 1428, 1358, 1291, 1230, $1168 \mathrm{~cm}^{-1}$; HRMS (ESI-TOF) $[\mathrm{M}+\mathrm{Na}]^{+}$calcd for $\mathrm{C}_{31} \mathrm{H}_{27} \mathrm{BrN}_{4} \mathrm{NaO}_{5} \mathrm{~S}^{+} \mathrm{m} / \mathrm{z} 669.0778$, found 669.0797; $[\alpha]^{25} \mathrm{D}+151.2\left(c 1.0, \mathrm{CHCl}_{3}\right)$.

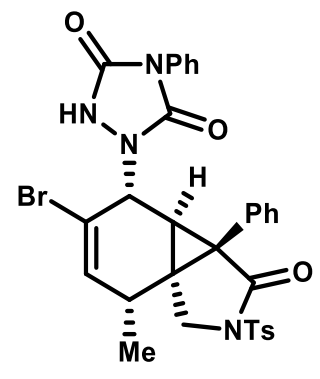

9

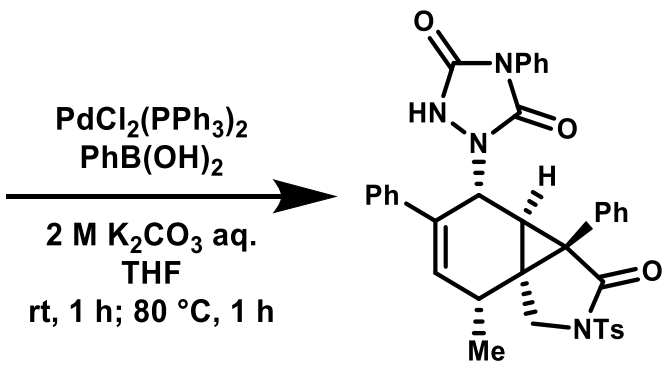

10

In a $50 \mathrm{~mL}$ flask, compound 9 (142.9 mg, 0.22 mmol, 1 equiv.), $\mathrm{PdCl}_{2}\left(\mathrm{PPh}_{3}\right)_{2}(31.2$ $\mathrm{mg}, 0.044 \mathrm{mmol}, 20 \mathrm{~mol} \%), \mathrm{PhB}(\mathrm{OH})_{2}(94.7 \mathrm{mg}, 0.78 \mathrm{mmol}, 3.5$ equiv.), and THF (4.5 $\mathrm{mL}, 0.05 \mathrm{M}$ ) were added in sequence under argon gas atmosphere. The mixture was stirred at room temperature for 1 hour. Then, $2 \mathrm{M}$ aqueous $\mathrm{K}_{2} \mathrm{CO}_{3}(0.9 \mathrm{~mL}, 0.25 \mathrm{M})$ was added to the mixture, and the reaction mixture was stirred at $80^{\circ} \mathrm{C}$ for 1 hour. The reaction was quenched with the addition of $\mathrm{H}_{2} \mathrm{O}$, and the aqueous solution was extracted with DCM. The combined organic layers were washed with brine, dried over $\mathrm{Na}_{2} \mathrm{SO}_{4}$, and concentrated under reduced pressure. The crude was purified by flash chromatography ( $n$-hexane/EtOAc, 3/2, v/v) to afford desired product 10. 
1-((3aR,3bR,4R,7R,7aR)-7-Methyl-3-oxo-3a,5-diphenyl-2-tosyl-2,3,3a,3b,4,7-

hexahydro-1H-benzo[1,3]cyclopropa[1,2-c]pyrrol-4-yl)-4-phenyl-1,2,4-

triazolidine-3,5-dione (10)

Pale yellow solid (110.7 mg, 77\% yield): m.p. $159{ }^{\circ} \mathrm{C}$ (decomp.); TLC $R_{f}=0.2(n-$ hexane/EtOAc, 1/1, v/v); ${ }^{1} \mathrm{H}$ NMR $\left(400 \mathrm{MHz}, \mathrm{CDCl}_{3}\right) \delta 8.63(\mathrm{br} \mathrm{s}, 1 \mathrm{H}), 7.92(\mathrm{~d}, \mathrm{~J}=8.4$ $\mathrm{Hz}, 2 \mathrm{H}), 7.45-7.21(\mathrm{~m}, 12 \mathrm{H}), 7.15-7.05(\mathrm{~m}, 3 \mathrm{H}), 6.75(\mathrm{~d}, J=6.8 \mathrm{~Hz}, 2 \mathrm{H}) 5.68(\mathrm{~d}, J=$ $5.6 \mathrm{~Hz}, 1 \mathrm{H}), 5.39(\mathrm{~d}, J=1.6 \mathrm{~Hz}, 1 \mathrm{H}), 4.29(\mathrm{~d}, J=10.0 \mathrm{~Hz}, 1 \mathrm{H}), 3.92(\mathrm{~d}, J=10.0 \mathrm{~Hz}$, 1H), 3.13-3.10 (m, $1 \mathrm{H}), 2.38(\mathrm{~s}, 3 \mathrm{H}), 1.74(\mathrm{~s}, 1 \mathrm{H}), 1.41(\mathrm{~d}, J=6.8 \mathrm{~Hz}, 3 \mathrm{H}) ;{ }^{13} \mathrm{C}$ NMR $\left(100 \mathrm{MHz} \mathrm{CDCl}_{3}\right) \delta 171.5,154.9,152.8,145.4,138.3,134.7,134.6,131.5,130.9$, $130.1(2 \mathrm{C}), 129.8(2 \mathrm{C}), 129.2,129.0(2 \mathrm{C}), 128.6(2 \mathrm{C}), 128.5,128.3(2 \mathrm{C}), 128.2(3 \mathrm{C})$ 127.9, 126.5 (2C), 125.3 (2C), 51.4, 50.5, 38.3, 30.9, 29.6, 29.4, 21.7, 17.7; IR (ATR) $3060,1775,1704,1598,1502,1428,1360,1293,1170,1121 \mathrm{~cm}^{-1}$; HRMS (ESI-TOF) $[\mathrm{M}+\mathrm{Na}]^{+}$calcd for $\mathrm{C}_{37} \mathrm{H}_{32} \mathrm{~N}_{4} \mathrm{NaO}_{5} \mathrm{~S}^{+} \mathrm{m} / \mathrm{z} 667.1986$, found $667.1975 ;[\alpha]^{25} \mathrm{D}+153.2(c$ 1.0, $\mathrm{CHCl}_{3}$ ).

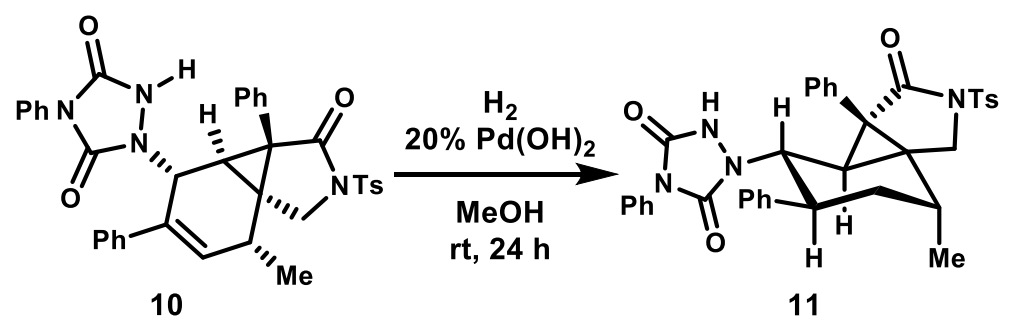


In a $2 \mathrm{~mL}$ test tube, compound 10 (20 mg, $0.03 \mathrm{mmol}, 1$ equiv.) and $\mathrm{MeOH}(400 \mu \mathrm{L}$, $0.075 \mathrm{M}$ ) were added in sequence under argon gas atmosphere. $20 \% \mathrm{Pd}(\mathrm{OH})_{2}$ (wetted with ca. $50 \%$ water, $4.0 \mathrm{mg}, 20 \mathrm{wt} \%$ ) was added slowly to the test tube. The reaction mixture was stirred under $\mathrm{H}_{2}$ gas at a pressure of $8.1 \mathrm{MPa}$ at the room temperature for 24 hours. Then, reaction mixture was filtered through a short pad of celite and washed with DCM. Removal of the solvent and purification by flash chromatography (n-hexane/EtOAc, 3/2, v/v) afforded desired product 11.

\section{1-((3aR,3b $R, 4 S, 5 S, 7 R, 7 \mathrm{a} R)-7-M e t h y l-3-0 x 0-3 \mathrm{a}, 5$-diphenyl-2-tosyloctahydro-1H-}

\section{benzo[1,3]cyclopropa[1,2-c]pyrrol-4-yl)-4-phenyl-1,2,4-triazolidine-3,5-dione}

White solid (18.6 mg, 93\% yield): m.p. $164{ }^{\circ} \mathrm{C}$ (decomp.); TLC $R_{f}=0.2(n-$ hexane/EtOAc, 1/1, v/v); ${ }^{1} \mathrm{H} \mathrm{NMR}\left(400 \mathrm{MHz}, \mathrm{CDCl}_{3}\right) \delta 9.65(\mathrm{br} \mathrm{s}, 1 \mathrm{H}), 7.92(\mathrm{~d}, J=8.4$ $\mathrm{Hz}, 2 \mathrm{H}), 7.47-7.34(\mathrm{~m}, 8 \mathrm{H}), 7.32-7.28(\mathrm{~m}, 4 \mathrm{H}), 7.12-7.10(\mathrm{~m}, 3 \mathrm{H}), 6.74-6.72(\mathrm{~m}, 2 \mathrm{H})$, $4.52(\mathrm{dd}, J=12.0,2.0 \mathrm{~Hz}, 1 \mathrm{H}), 3.82(\mathrm{~d}, J=10.0 \mathrm{~Hz}, 1 \mathrm{H}), 3.07(\mathrm{dt}, J=12.0,2.8 \mathrm{~Hz}$, $1 \mathrm{H}), 2.56-2.53(\mathrm{~m}, 1 \mathrm{H}), 3.13-3.10(\mathrm{~m}, 1 \mathrm{H}), 2.38(\mathrm{~s}, 3 \mathrm{H}), 1.71(\mathrm{~d}, J=2.8 \mathrm{H}, 1 \mathrm{H}), 1.29-$ $1.24(\mathrm{~m}, 4 \mathrm{H}), 1.14(\mathrm{dt}, J=10.0,4.8 \mathrm{~Hz}, 1 \mathrm{H}) ;{ }^{13} \mathrm{C} \mathrm{NMR}\left(100 \mathrm{MHz}, \mathrm{CDCl}_{3}\right) \delta 172.1$, 154.4, 151.9, 145.3, 141.4, 134.7, 131.7, 130.9, 129.8 (2C), 129.6, 129.3 (2C), 129.0 (2C), 128.9, $128.7(2 \mathrm{C}), 128.3(2 \mathrm{C}), 128.2(2 \mathrm{C}), 127.3,127.2(2 \mathrm{C}), 125.5(2 \mathrm{C}), 54.5$, 
51.1, 41.0, 37.5, 35.3, 31.8, 30.5, 26.9, 21.7, 17.5; IR (ATR) 3029, 2965, 1772, 1714, 1599, 1503, 1430, 1362, 1292, $1218 \mathrm{~cm}^{-1}$; HRMS (ESI-TOF) $[\mathrm{M}+\mathrm{Na}]^{+}$calcd for $\mathrm{C}_{37} \mathrm{H}_{34} \mathrm{~N}_{4} \mathrm{NaO}_{5} \mathrm{~S}^{+} \mathrm{m} / \mathrm{z}$ 669.2142, found 669.2152; [a $]^{25} \mathrm{D}+63.0$ (c 0.83, $\left.\mathrm{CHCl}_{3}\right)$.

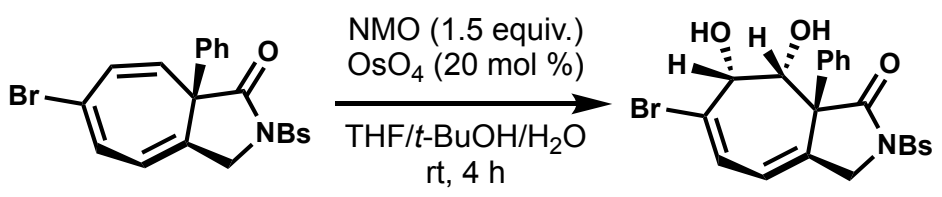

In a $10 \mathrm{~mL}$ flask, compound $2 \mathrm{a}$ ( $26.1 \mathrm{mg}, 0.05 \mathrm{mmol}, 1$ equiv.), $\mathrm{N}$-methylmorpholine$\mathrm{N}$-oxide (8.8 mg, $0.075 \mathrm{mmol}, 1.5$ equiv.), $\mathrm{OsO}_{4}(2.54 \mathrm{mg}, 0.01 \mathrm{mmol}, 20 \mathrm{~mol} \%$ ), and $\mathrm{THF} / t-\mathrm{BuOH} / \mathrm{H}_{2} \mathrm{O}(0.75 \mathrm{~mL}, 6 / 8 / 1, \mathrm{v} / \mathrm{v}$ respectively $)$ were added in sequence under argon gas atmosphere. The mixture was stirred at room temperature for 4 hours. The reaction was quenched with the addition of $\mathrm{Na}_{2} \mathrm{~S}_{2} \mathrm{O}_{4}$ aq, and the aqueous solution was extracted with EtOAc. The combined organic layers were dried over $\mathrm{Na}_{2} \mathrm{SO}_{4}$, and concentrated under reduced pressure. The crude was purified by flash chromatography ( $n$-hexane/EtOAc, 5/1 to 3/1, v/v) to afford desired product 12.

$(7 R, 8 R, 8 \mathrm{a} R)-6$-Bromo-2-((4-bromophenyl)sulfonyl)-7,8-dihydroxy-8a-phenyl-

\section{3,7,8,8a-tetrahydrocyclohepta[c]pyrrol-1(2H)-one (12)}

White solid (24.9 mg, 90\% yield): m.p. $>200{ }^{\circ} \mathrm{C}$; TLC $R_{f}=0.7$ ( $n$-hexane/EtOAc, $1 / 1$, v/v); ${ }^{1} \mathrm{H}$ NMR $\left(400 \mathrm{MHz}, \mathrm{CDCl}_{3}\right) \delta 7.79(\mathrm{~d}, J=8.4 \mathrm{~Hz}, 2 \mathrm{H}), 7.60(\mathrm{~d}, J=8.4 \mathrm{~Hz}, 2 \mathrm{H})$, 7.35-7.30 (m, 5H), $6.53(\mathrm{~d}, J=8.4 \mathrm{~Hz}, 1 \mathrm{H}), 6.15(\mathrm{~d}, J=8.4 \mathrm{~Hz}, 1 \mathrm{H}), 7.80(\mathrm{dd}, J=4.4$, 
$3.2 \mathrm{~Hz}, 1 \mathrm{H}), 4.45(\mathrm{~s}, 2 \mathrm{H}), 3.68(\mathrm{dd}, J=4.4,2.4 \mathrm{~Hz}, 1 \mathrm{H}), 2.58(\mathrm{~d}, J=3.2 \mathrm{~Hz}, 1 \mathrm{H}), 2.57$

$(\mathrm{d}, J=2.4 \mathrm{~Hz}, 1 \mathrm{H}) ;{ }^{13} \mathrm{C} \mathrm{NMR}\left(100 \mathrm{MHz}\right.$, acetone- $\left.\mathrm{d}_{6}\right) \delta 172.9,137.9,134.7,133.8$, 133.7, 133.1 (2C), $130.4(2 \mathrm{C}), 130.3(2 \mathrm{C}), 129.6,129.4,127.2(2 \mathrm{C}), 127.0,123.0,74.6$, 72.9, 61.6, 51.0; IR (ATR) v 3445, 2115, 1737, 1695, 1574, 1695, 1574, 1470, 1365, $1248 \mathrm{~cm}^{-1}$; HRMS (ESI-TOF) [M + Na] $]^{+}$calcd for $\mathrm{C}_{21} \mathrm{H}_{17} \mathrm{Br}_{2} \mathrm{NNaO}_{5} \mathrm{~S}^{+} \mathrm{m} / \mathrm{z} 575.9086$, found $575.9100 ;[\alpha]^{25} \mathrm{D}+37.7\left(c 0.5, \mathrm{CHCl}_{3}\right)$.

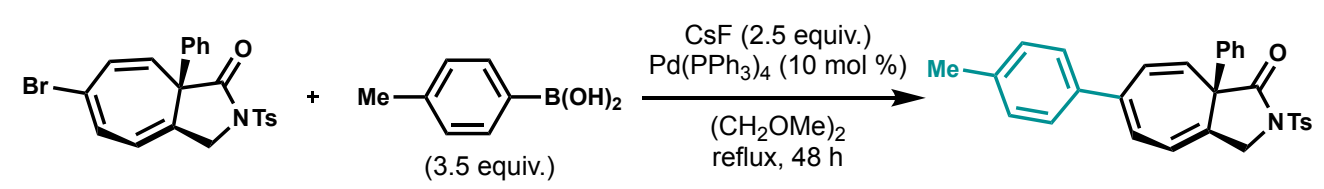

In a $10 \mathrm{~mL}$ test tube, compound $2 \mathrm{~h}\left(22.8 \mathrm{mg}, 0.05 \mathrm{mmol}, 1\right.$ equiv.), $\mathrm{Pd}\left(\mathrm{PPh}_{3}\right)_{4}(5.8 \mathrm{mg}$, $0.005 \mathrm{mmol}, 10 \mathrm{~mol} \%$ ), $p$-TolB(OH)2 (23.8 mg, $0.175 \mathrm{mmol}, 3.5$ equiv.), CsF (19.0 mg, $0.125 \mathrm{mmol}, 2.5$ equiv. $)$, and $\mathrm{DME}(0.5 \mathrm{~mL}, 0.1 \mathrm{M})$ were added in sequence under argon gas atmosphere. The mixture was stirred at reflux for 48 hours. The reaction was quenched with the addition of $\mathrm{H}_{2} \mathrm{O}$, and the aqueous solution was extracted with EtOAc. The combined organic layers were washed with $\mathrm{NaOH}$ aq., dried over $\mathrm{Na}_{2} \mathrm{SO}_{4}$, and concentrated under reduced pressure. The crude was purified by flash chromatography ( $n$-hexane/EtOAc, 3/1, v/v) to afford desired product 13. 
White solid (17.2 mg, 74\% yield): m.p. 166-168; TLC $R_{f}=0.4$ ( $n$-hexane/EtOAc, 3/1, v/v); ${ }^{1} \mathrm{H}$ NMR $\left(400 \mathrm{MHz}, \mathrm{CDCl}_{3}\right) \delta 7.93(\mathrm{~d}, \mathrm{~J}=8.0 \mathrm{~Hz}, 2 \mathrm{H}), 7.30(\mathrm{~d}, \mathrm{~J}=8.0 \mathrm{~Hz}, 2 \mathrm{H})$, 7.09-7.03 (m, 9H), 6.52-6.47 (m, 3H), $5.53(\mathrm{~d}, \mathrm{~J}=9.6 \mathrm{~Hz}, 1 \mathrm{H}), 4.87(\mathrm{~d}, \mathrm{~J}=14.8 \mathrm{~Hz}$, 1H), $4.71(\mathrm{~d}, \mathrm{~J}=14.8 \mathrm{~Hz}, 1 \mathrm{H}), 2.42(\mathrm{~s}, 3 \mathrm{H}), 2,29(\mathrm{~s}, 3 \mathrm{H}) ;{ }^{13} \mathrm{C} \mathrm{NMR}\left(100 \mathrm{MHz}, \mathrm{CDCl}_{3}\right)$ $\delta$ 173.8, 145.4, 141.9, 138.4, 137.5, 136.5, 134.6, $129.7(2 \mathrm{C}), 129.1$ (2C) $128.1(2 \mathrm{C})$ $127.8(2 \mathrm{C}), 127.8,127.6,127.1(2 \mathrm{C}), 126.4(3 \mathrm{C}), 126.0,121.8,114.5,53.9,50.7,21.7$, 21.0; IR (ATR) 3680, 2973, 2866, 2844, 1739, 1596, 1510, 1368, 1223, $1173 \mathrm{~cm}^{-1}$; HRMS (ESI-TOF) $[\mathrm{M}+\mathrm{Na}]^{+}$calcd for $\mathrm{C}_{29} \mathrm{H}_{25} \mathrm{NNaO}_{3} \mathrm{~S}^{+} \mathrm{m} / \mathrm{z} 490.1447$, found 490.1444; $[\alpha]^{25} \mathrm{D}+28.0\left(\mathrm{c} 0.55, \mathrm{CHCl}_{3}\right)$
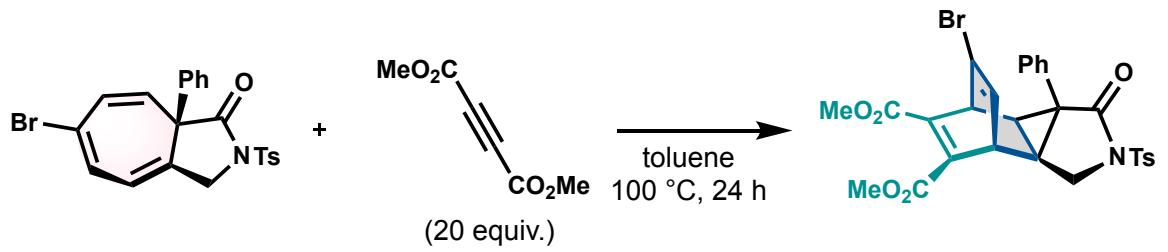

In a $10 \mathrm{~mL}$ test tube, compound $\mathbf{2 h}(22.8 \mathrm{mg}, 0.05 \mathrm{mmol}, 1$ equiv.), Dimethyl acetylenedicarboxylate $(0.12 \mathrm{~mL}, 1.0 \mathrm{mmol}, 20$ equiv. $)$, and toluene $(0.5 \mathrm{~mL}, 0.1 \mathrm{M})$ were added in sequence under argon gas atmosphere. The mixture was stirred at 100 ${ }^{\circ} \mathrm{C}$ for 24 hours. After the solvent was evaporated in vacuo, the resulting residue was purified by flash chromatography $(n$-hexane/EtOAc $=3 / 1, v / v)$ to afford desired product

14. 


\section{Dimethyl}

(3bR,4S,7R,7aS)-9-bromo-3-oxo-3a-phenyl-2-tosyl-2,3,3a,3b,4,7hexahydro-1H-4,7-ethenobenzo[1,3]cyclopropa[1,2-c]pyrrole-5,6-dicarboxylate

White solid (24.5 mg, $0.041 \mathrm{mmol}, 82 \%$ yield): m.p. $154-156{ }^{\circ} \mathrm{C} ;$ TLC $R_{f}=0.1(n-$ hexane/EtOAc, 3/1, v/v); ${ }^{1} \mathrm{H}$ NMR $\left(400 \mathrm{MHz}, \mathrm{CDCl}_{3}\right) \delta 7.87(\mathrm{~d}, J=8.0 \mathrm{~Hz}, 2 \mathrm{H}), 7.33-$ $7.28(\mathrm{~m}, 2 \mathrm{H}), 7.29(\mathrm{~d}, J=8.0 \mathrm{~Hz}, 2 \mathrm{H}), 7.22-7.20(\mathrm{~m}, 2 \mathrm{H}), 6.72(\mathrm{dt}, J=6.8,1.6 \mathrm{~Hz}, 1 \mathrm{H})$, $5.91(\mathrm{dd}, J=6.4,2.0 \mathrm{~Hz}, 1 \mathrm{H}), 4.37(\mathrm{dd}, J=3.6,2.0 \mathrm{~Hz}, 1 \mathrm{H}), 4.28(\mathrm{~d}, J=6.4 \mathrm{~Hz}, 1 \mathrm{H})$, $4.13(\mathrm{~d}, J=10.4 \mathrm{~Hz}, 1 \mathrm{H}), 4.01(\mathrm{~d}, J=10.4 \mathrm{~Hz}, 1 \mathrm{H}), 3.87(\mathrm{~s}, 3 \mathrm{H}), 3.85(\mathrm{~s}, 3 \mathrm{H}), 2.42(\mathrm{~s}$, 3H), $1.67(\mathrm{~d}, J=3.6 \mathrm{~Hz}, 1 \mathrm{H}) ;{ }^{13} \mathrm{C} \mathrm{NMR}\left(100 \mathrm{MHz}, \mathrm{CDCl}_{3}\right) \delta$ 169.9, 165.7, 164.4, 147.3, 146.2, 145.4, 134.5 (2C), 131.6, 130.7, 130.0, 129.7 (2C), 128.9, 128.3 (2C), 127.9, 127.6, 123.3, 56.4, 52.9, 52.7, 50.4, 49.8, 44.4, 41.8, 34.9, 21.7; IR (ATR) 3680, 2952, $2844,1717,1635,1597,1435,1358,1269,1238 \mathrm{~cm}^{-1} ;$ HRMS (ESI-TOF) [M + Na] ${ }^{+}$ calcd for $\mathrm{C}_{28} \mathrm{H}_{24} \mathrm{BrNNaO}_{7} \mathrm{~S}^{+} \mathrm{m} / \mathrm{z}$ 620.0355, found 620.0355; [a $]^{25} \mathrm{D}+29.9$ (c 1.0, $\left.\mathrm{CHCl}_{3}\right)$ 


\section{Computational Details}

All DFT calculations were performed with Gaussian 16 program. ${ }^{4)}$ The molecular structure optimizations in Figure 1 were carried out using the hybrid density functional method based on Becke's three-parameter exchange function and the Lee-Yang-Parr nonlocal correlation functional $(B 3 L Y P)^{5}$ and the LANL2DZ basis set for $\mathrm{Ag}$, and the $6-31 \mathrm{G}^{*}$ basis set for $\mathrm{H}, \mathrm{C}, \mathrm{N}, \mathrm{O}, \mathrm{P}$, and $\mathrm{S}$. The vibrational frequencies were computed at the same level to check whether each optimized structure is at an energy minimum (no imaginary frequency) or a transition state (one imaginary frequency) and to evaluate its zero-point vibrational energy (ZPVE) and thermal corrections at $298.15 \mathrm{~K}$. The intrinsic reaction coordinate (IRC) method was used to track minimum energy paths from transition structures to the corresponding local minima. ${ }^{6}$ Single point energies were calculated at the RM06 level using the SDD basis set for $\mathrm{Ag}$ and the $6-311++\mathrm{G}^{* *}$ basis set for $\mathrm{H}, \mathrm{C}, \mathrm{N}, \mathrm{O}$, $\mathrm{P}$, and $\mathrm{S}$ in chlorobenzene solvent. 


\begin{tabular}{|c|c|c|}
\hline & $E(r B 3 L Y P / L A N L 2 D Z / 6-$ & $\begin{array}{c}E(r m 06 / S D D / 6- \\
\left.311++G^{* *}\right)(A . U .)\end{array}$ \\
\hline CP1 & -2320.975243 & -2321.688641 \\
TS1 & -2320.97429 & -2321.687955 \\
CP2 & -2320.980972 & -2321.693918 \\
TS2 & -2837.374554 & -2837.871615 \\
CP3 & -2837.457768 & -2837.882772 \\
TS3 & -2837.370023 & -2837.869364 \\
CP4 & -2396.203593 & -2396.920052 \\
TS4 & -2396.184172 & -2396.912253 \\
CP5 & -2396.226498 & -2396.945700 \\
TS5 & -2396.213957 & -2396.928156 \\
CP6 & -2150.821066 & -2150.143832 \\
TS6 & -2150.80701 & -2150.133113 \\
TS7 & -2912.608271 & -2913.103760 \\
PRO6 & -1603.560258 & -1603.073856 \\
PRO2 & -2396.222884 & -2396.944281 \\
PRO3 & -2150.850757 & -2150.185527 \\
Silver Cat. & -867.917101 & -869.093292 \\
PTAD & -622.522401 & -622.306229 \\
8-methylquinoline & -441.249748 & -441.024193 \\
N-oxide 4 & -516.406518 & -516.174122 \\
\hline & & \\
\hline & & \\
\hline
\end{tabular}


CP1

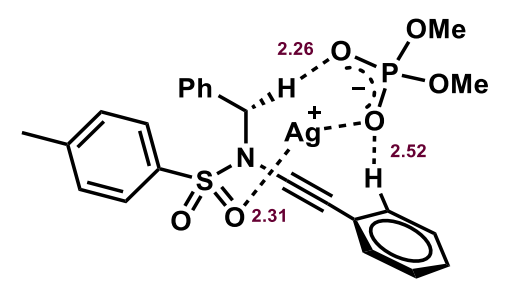

Zero-point correction=

Thermal correction to Energy=

Thermal correction to Enthalpy=

Thermal correction to Gibbs Free Energy=

Sum of electronic and zero-point Energies=

Sum of electronic and thermal Energies=

Sum of electronic and thermal Enthalpies=

Sum of electronic and thermal Free Energies=

C

C

$\mathrm{H}$

C

C

C

C

$\mathrm{H}$

$\mathrm{H}$

C

$\mathrm{H}$

$\mathrm{H}$

$\mathrm{N}$

$S$

O

O

C

C

C

C

C

C

$\mathrm{H}$
$-0.94789600$

0.34701900

$-2.18071500$

$-1.17405200$

1.41006200

1.18381400

$-0.11052600$

2.41853600

2.00299400

$-0.32951700$

0.62825600

$-0.89229600$

$-1.17405900$

$-1.92770300$

$-2.15331600$

$-1.14286700$

$-0.75069800$

$-0.39047000$

$-0.02981700$

$-0.82809100$

1.11628700

$-0.48986500$

$-1.70740900$
4.49414000

4.59172000

3.71676000

3.79040900

3.98048500

3.26730700

3.17193700

4.05664600

2.78486100

2.43021800

2.13307300

3.03108900

1.19336800

0.50300800

1.59706300

$-0.68805100$

0.27521000

$-0.57335700$

$-1.55106100$

$-1.72398800$

$-2.35551200$

$-2.67999500$

$-1.10064100$
0.452578 (Hartree/Particle)

0.488836

0.489780

0.374675

$-2320.522665$

$-2320.486408$

$-2320.485464$

$-2320.600569$

$-2.48807400$

$-3.00676800$

$-0.90097400$

$-1.30637400$

$-2.34073000$

$-1.16043500$

$-0.63508200$

$-2.73866500$

$-0.63287900$

0.66077700

1.09633300

1.37714800

0.42470000

1.82068600

2.76133700

2.25647900

$-0.47628600$

$-1.27192800$

$-2.25308900$

$-3.40183000$

$-2.08463700$

$-4.35618500$

$-3.53256300$ 


$\begin{array}{lrrr}\mathrm{C} & 1.44426400 & -3.31040100 & -3.04568200 \\ \mathrm{H} & 1.74608900 & -2.21809500 & -1.20904400 \\ \mathrm{C} & 0.64532100 & -3.47610100 & -4.17998000 \\ \mathrm{H} & -1.11138600 & -2.80407600 & -5.23881900 \\ \mathrm{H} & 2.32860800 & -3.92624600 & -2.90721900 \\ \mathrm{H} & 0.90691300 & -4.22205100 & -4.92566800 \\ \mathrm{Ag} & 0.99384200 & -1.08406200 & 1.48103200 \\ \mathrm{H} & 0.52409300 & 5.14513300 & -3.92537600 \\ \mathrm{H} & -1.77802000 & 4.97241800 & -3.00152100 \\ \mathrm{O} & 2.61160000 & 1.04918100 & 1.10503800 \\ \mathrm{O} & 3.07111100 & -1.46687900 & 0.79498400 \\ \mathrm{O} & 4.62265800 & 0.23279300 & -0.37037500 \\ \mathrm{C} & 4.08905400 & 0.10419200 & -1.68763300 \\ \mathrm{H} & 4.86978300 & 0.43449800 & -2.37753700 \\ \mathrm{H} & 3.82684500 & -0.93674800 & -1.90544300 \\ \mathrm{H} & 3.20184600 & 0.73477700 & -1.82004200 \\ \mathrm{P} & 3.64363300 & -0.03344700 & 0.90798300 \\ \mathrm{O} & 4.73856000 & 0.04450500 & 2.09619200 \\ \mathrm{C} & 5.75435900 & -0.96033900 & 2.18125600 \\ \mathrm{H} & 6.30434000 & -0.77074600 & 3.10599200 \\ \mathrm{H} & 5.30894500 & -1.96012500 & 2.21369000 \\ \mathrm{H} & 6.43804000 & -0.89061000 & 1.32824000 \\ \mathrm{C} & -3.48429100 & -0.10493100 & 1.20520100 \\ \mathrm{C} & -3.58869100 & -1.41097900 & 0.72291300 \\ \mathrm{C} & -4.58419500 & 0.75733100 & 1.22164400 \\ \mathrm{C} & -4.82104700 & -1.85024800 & 0.24280000 \\ \mathrm{H} & -2.72821000 & -2.06964200 & 0.73106700 \\ \mathrm{C} & -5.80511800 & 0.29522600 & 0.73978200 \\ \mathrm{H} & -4.48379900 & 1.76249900 & 1.61672500 \\ \mathrm{C} & -5.94412000 & -1.01088700 & 0.24394700 \\ \mathrm{H} & -4.91082400 & -2.86490700 & -0.13593000 \\ \mathrm{H} & -6.66640300 & 0.95809000 & 0.75195600 \\ \mathrm{C} & -7.28206200 & -1.50789400 & -0.24759200 \\ \mathrm{H} & -7.84943600 & -0.71068200 & -0.73903300 \\ \mathrm{H} & -7.16778200 & -2.33410800 & -0.95608700 \\ \mathrm{H} & -7.89274400 & -1.87401500 & 0.58823200\end{array}$




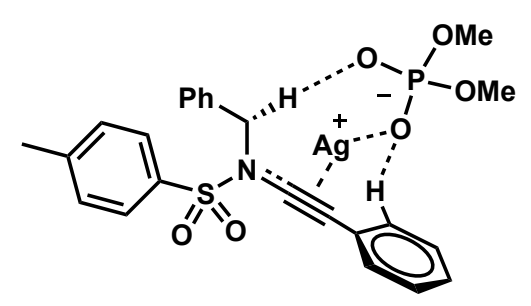

Zero-point correction=

0.451964 (Hartree/Particle)

Thermal correction to Energy= 0.487528

Thermal correction to Enthalpy=

0.488472

Thermal correction to Gibbs Free Energy=

Sum of electronic and zero-point Energies=

0.375146

Sum of electronic and thermal Energies=

$-2320.522326$

$-2320.486762$

Sum of electronic and thermal Enthalpies=

$-2320.485817$

Sum of electronic and thermal Free Energies=

$-2320.599143$

C

C

$\mathrm{H}$

C

C

C

C

$\mathrm{H}$

$\mathrm{H}$

C

$\mathrm{H}$

$\mathrm{H}$

$\mathrm{N}$

$S$

O

O

C

C

C

C

C

C

$\mathrm{H}$

C
0.87005900

$-0.38847700$

2.07339700

1.09617700

$-1.41471900$

$-1.18907900$

0.06934100

$-2.39530800$

$-1.98038500$

0.29382000

$-0.65871400$

0.81826200

1.19575000

1.85701800

2.02516100

1.02770100

0.81956000

0.44900300

0.26114400

1.22212500

$-0.87216100$

1.05239700

2.09287000

$-1.03428200$
1.14287600

0.64474900

2.00553900

1.61335500

0.61808700

1.08331600

1.58611500

0.23611000

1.06891700

2.10881400

2.21124300

3.06538400

1.16552500

1.81671800

3.24914400

1.32616100

$-0.12205500$

$-1.28241500$

$-2.70503800$

$-3.51365400$

$-3.31284900$

$-4.89423900$

$-3.04542200$

$-4.69522400$
5.03833000

5.38956500

3.47278300

3.74599000

4.44419200

3.14598600

2.79211300

4.71562400

2.39974700

1.39541400

0.86665100

1.39979200

0.61663400

$-0.87076700$

$-0.64077900$

$-1.99482100$

0.50102500

0.35692300

0.40797700

1.04811900

$-0.16679000$

1.10578400

1.49649900

$-0.10293100$ 


$\begin{array}{lrrc}\mathrm{H} & -1.62339600 & -2.69484600 & -0.65228600 \\ \mathrm{C} & -0.07440100 & -5.48834800 & 0.53029900 \\ \mathrm{H} & 1.79908800 & -5.50796900 & 1.60201800 \\ \mathrm{H} & -1.91247800 & -5.15298600 & -0.54921700 \\ \mathrm{H} & -0.20407200 & -6.56611500 & 0.57677900 \\ \mathrm{Ag} & -1.04015800 & -0.00929800 & -1.42952900 \\ \mathrm{H} & -0.56687700 & 0.28308200 & 6.39897800 \\ \mathrm{H} & 1.67014100 & 1.17072600 & 5.77354100 \\ \mathrm{O} & -2.71556400 & 1.30403000 & 0.09961500 \\ \mathrm{O} & -3.24489400 & -0.35531000 & -1.79667400 \\ \mathrm{O} & -4.99019800 & -0.00362800 & 0.06747800 \\ \mathrm{C} & -4.75526200 & -1.26764800 & 0.68394100 \\ \mathrm{H} & -5.67093600 & -1.53561700 & 1.21735100 \\ \mathrm{H} & -4.53006300 & -2.03297600 & -0.06731000 \\ \mathrm{H} & -3.92750900 & -1.20906400 & 1.40210600 \\ \mathrm{P} & -3.77307300 & 0.67984700 & -0.78410600 \\ \mathrm{O} & -4.59851100 & 1.85541400 & -1.52834600 \\ \mathrm{C} & -5.57136700 & 1.50610600 & -2.51821100 \\ \mathrm{H} & -5.92715900 & 2.44414700 & -2.95086100 \\ \mathrm{H} & -5.12285600 & 0.88418200 & -3.29977800 \\ \mathrm{H} & -6.41198400 & 0.97212500 & -2.06159700 \\ \mathrm{C} & 3.45011500 & 1.02599200 & -0.97931900 \\ \mathrm{C} & 3.60470600 & -0.12637700 & -1.75108300 \\ \mathrm{C} & 4.52572700 & 1.59121100 & -0.28805300 \\ \mathrm{C} & 4.86277600 & -0.72306500 & -1.81975700 \\ \mathrm{H} & 2.76141700 & -0.53801900 & -2.29372900 \\ \mathrm{C} & 5.77217300 & 0.97914000 & -0.37217000 \\ \mathrm{H} & 4.38690500 & 2.49743200 & 0.29175600 \\ \mathrm{C} & 5.96152100 & -0.18389700 & -1.13648900 \\ \mathrm{H} & 4.99197600 & -1.62081700 & -2.41840800 \\ \mathrm{H} & 6.61457100 & 1.41278500 & 0.16052800 \\ \mathrm{C} & 7.32704700 & -0.81877900 & -1.24171000 \\ \mathrm{H} & 7.86874000 & -0.76269400 & -0.29156100 \\ \mathrm{H} & 7.25936900 & -1.87058700 & -1.53592000 \\ \mathrm{H} & & & \\ & & -0.30494900 & -1.99484700\end{array}$

\section{CP2}


<smiles>[3H][N+](Cc1ccccc1)=C([14CH3])[14CH3]</smiles>

Zero-point correction=

Thermal correction to Energy=

Thermal correction to Enthalpy=

Thermal correction to Gibbs Free Energy=

Sum of electronic and zero-point Energies=

Sum of electronic and thermal Energies=

Sum of electronic and thermal Enthalpies=

Sum of electronic and thermal Free Energies=

$\begin{array}{lrrr}\text { C } & -1.29483000 & 0.86846300 & 4.62033100 \\ \mathrm{C} & -0.18952600 & 1.69072900 & 4.86124400 \\ \mathrm{H} & -2.07301400 & -0.78402600 & 3.47832900 \\ \mathrm{C} & -1.21553600 & -0.13913100 & 3.66048600 \\ \mathrm{C} & 0.98925100 & 1.50049000 & 4.13905600 \\ \mathrm{C} & 1.07018900 & 0.49493400 & 3.17149700 \\ \mathrm{C} & -0.03455200 & -0.33143400 & 2.92909300 \\ \mathrm{H} & 1.85137300 & 2.13464900 & 4.32796500 \\ \mathrm{H} & 1.98273100 & 0.33419100 & 2.60217900 \\ \mathrm{C} & 0.06765100 & -1.42444400 & 1.89435900 \\ \mathrm{H} & 1.10303200 & -1.55853000 & 1.56586300 \\ \mathrm{H} & -0.33232200 & -2.37264400 & 2.25634300 \\ \mathrm{~N} & -0.75571300 & -1.08783700 & 0.66702700 \\ \mathrm{~S} & -1.44264500 & -2.43757300 & -0.29556200 \\ \mathrm{O} & -1.40379300 & -3.58350000 & 0.60936600 \\ \mathrm{O} & -0.76785100 & -2.43127400 & -1.59019500 \\ \mathrm{C} & -0.64733400 & 0.06060800 & 0.05852900 \\ \mathrm{C} & -0.48267500 & 1.12916900 & -0.58817900 \\ \mathrm{C} & -1.09465400 & 2.42588000 & -0.83168700 \\ \mathrm{C} & -2.20829600 & 2.83974800 & -0.07330100 \\ \mathrm{C} & -0.59192700 & 3.28761000 & -1.82198500 \\ \mathrm{C} & -2.79712500 & 4.07928800 & -0.30433900 \\ \mathrm{H} & -2.59357200 & 2.18511400 & 0.70261500 \\ \mathrm{C} & -1.18857300 & 4.52566800 & -2.05439400 \\ \mathrm{H} & 0.26810800 & 2.97525900 & -2.40799900 \\ \mathrm{C} & -2.29107400 & 4.92494200 & -1.29652000 \\ & & & \\ \mathrm{H} & & & \\ \mathrm{C} & & & \end{array}$

0.452352 (Hartree/Particle)

0.488578

0.489523

0.372866

$-2320.528621$

$-2320.492394$

$-2320.491450$

$-2320.608107$

4.62033100

4.86124400

32796500

1.89435900

.56586300

25634300

29556200

$-1.59019500$

0.05852900

0.58817900 
$\mathrm{H}$

$\mathrm{H}$

$\mathrm{H}$

$\mathrm{Ag}$

$\mathrm{H}$

$\mathrm{H}$

$\mathrm{O}$

O

O

C

$\mathrm{H}$

$\mathrm{H}$

$\mathrm{H}$

$P$

$\mathrm{O}$

C

$\mathrm{H}$

$\mathrm{H}$

$\mathrm{H}$

C

C

C

C

$\mathrm{H}$

C

$\mathrm{H}$

C

$\mathrm{H}$

$\mathrm{H}$

C

$\mathrm{H}$

$\mathrm{H}$

$\mathrm{H}$
$-3.65112600$

$-0.78904200$

$-2.75260100$

1.58484900

$-0.24869100$

$-2.21221300$

3.10197300

3.64776200

5.55559200

5.68085400

6.72112500

5.43756200

5.02643500

4.09403100

4.51442800

5.33954500

5.41572000

4.88852700

6.33870800

$-3.13111600$

$-3.47638900$

$-4.08070400$

$-4.79912700$

$-2.72645600$

$-5.39653900$

$-3.79392300$

$-5.77647400$

$-5.07668100$

$-6.14313500$

$-7.21313900$

$-7.70936600$

$-7.28661200$

$-7.78290000$
4.38859000

0.29199800

5.17944200

5.89233200

0.57185900

2.47319800

1.00797200

$-0.71095000$

0.05522100

$-0.10541800$

1.30257500

1.49362400

1.84642400

1.64605700

$-0.69608200$

$-2.21353800$

$-2.45555900$

$-3.54031800$

$-2.02606700$

$-2.03136200$

$-1.90791300$

$-1.18676400$

$-2.23391300$

$-0.77812400$

$-0.96271700$

$-1.81790000$

$-2.81409400$

$-1.08554700$

$-0.21481300$

$-2.07008700$

$-0.66771300$

$-0.46522100$

0.22981000

$-1.46119300$
$-2.82442600$

$-1.47440900$

$-1.20615400$

5.61337400

5.18642800

0.85536400

$-1.55498200$

0.15131700

0.34539100

0.62091700

$-0.57419800$

1.15641400

$-0.27715200$

$-0.64505900$

$-1.78921900$

$-1.89485700$

$-2.68946700$

$-1.64103500$

$-0.52026900$

$-1.66507600$

0.45159800

$-1.82507800$

$-2.41556300$

0.26858800

1.32225600

$-0.86739400$

$-2.71206000$

1.01723500

$-1.06841200$

$-0.11370300$

$-1.69050500$

$-1.56977500$ 
TS2

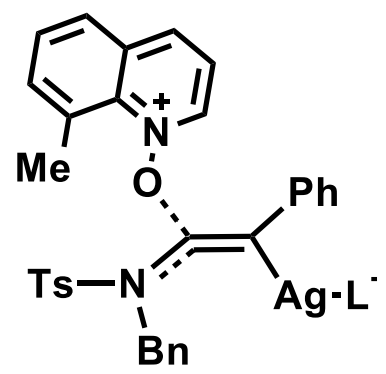

Zero-point correction=

Thermal correction to Energy=

Thermal correction to Enthalpy=

Thermal correction to Gibbs Free Energy=

Sum of electronic and zero-point Energies=

Sum of electronic and thermal Energies=

Sum of electronic and thermal Enthalpies=

Sum of electronic and thermal Free Energies=

0.622441 (Hartree/Particle)
0.668090
0.669034
0.534442
-2836.752113
-2836.706464
-2836.705520
-2836.840112

$\begin{array}{lrrr}\mathrm{C} & -1.03334000 & -1.38934100 & 4.44835700 \\ \mathrm{C} & -2.36348700 & -1.51781900 & 4.04710400 \\ \mathrm{H} & 0.84935100 & -0.39932100 & 4.09714300 \\ \mathrm{C} & -0.18805100 & -0.49717700 & 3.78353500 \\ \mathrm{C} & -2.84714100 & -0.75038200 & 2.98293400 \\ \mathrm{C} & -2.00415600 & 0.14663500 & 2.32501400 \\ \mathrm{C} & -0.66440500 & 0.27821300 & 2.72116000 \\ \mathrm{H} & -3.87642500 & -0.84622300 & 2.64970100 \\ \mathrm{H} & -2.40151700 & 0.72743900 & 1.49668100 \\ \mathrm{C} & 0.25541600 & 1.26192700 & 2.03600100 \\ \mathrm{H} & -0.17547000 & 2.26762200 & 2.06610300 \\ \mathrm{H} & 1.22711200 & 1.30331900 & 2.53898800 \\ \mathrm{~N} & 0.45895200 & 0.94960400 & 0.58824900 \\ \mathrm{~S} & 0.90462600 & 2.31400900 & -0.40648700 \\ \mathrm{O} & 1.96693900 & 3.07191000 & 0.27330700 \\ \mathrm{O} & 1.15685800 & 1.74312100 & -1.73411500 \\ \mathrm{C} & 0.95722400 & -0.25238900 & 0.20930300 \\ \mathrm{C} & 0.54340700 & -1.45826100 & -0.10137500 \\ \mathrm{C} & 1.37782100 & -2.66713200 & -0.21790500 \\ \mathrm{C} & 1.23639500 & -3.51549100 & -1.33378700 \\ \mathrm{C} & 2.29538100 & -3.03906200 & 0.78477900\end{array}$


C

$\mathrm{H}$

C

$\mathrm{H}$

C

$\mathrm{H}$

$\mathrm{H}$

$\mathrm{H}$

Ag

$\mathrm{O}$

$\mathrm{H}$

$\mathrm{H}$

C

C

C

C

C

$\mathrm{H}$

C

C

C

$\mathrm{H}$

C

$\mathrm{H}$

$\mathrm{H}$

$\mathrm{H}$

$\mathrm{H}$

C

$\mathrm{H}$

$\mathrm{H}$

$\mathrm{H}$

$\mathrm{N}$

O

O

O

C

$\mathrm{H}$

$\mathrm{H}$

$\begin{array}{rrr}2.01058000 & -4.66746800 & -1.46375700 \\ 0.50422600 & -3.26288300 & -2.09662600 \\ 3.04681900 & -4.20735000 & 0.66573600 \\ 2.40150500 & -2.41070500 & 1.66193500 \\ 2.91717500 & -5.02169800 & -0.46198000 \\ 1.88939900 & -5.30010100 & -2.33927300 \\ 3.73794800 & -4.48276400 & 1.45850000 \\ 3.50613400 & -5.93047600 & -0.55217500 \\ -1.56331100 & -1.65720700 & -0.46429600 \\ 2.72108600 & -0.09233100 & 0.51740500 \\ -0.65054700 & -1.98277800 & 5.27465500 \\ -3.02205900 & -2.21378300 & 4.56028500 \\ 4.88529700 & 0.41123100 & -0.20939700 \\ 3.22768900 & -0.42994700 & -1.72796200 \\ 5.26476800 & 0.91271200 & 1.07610700 \\ 5.84033400 & 0.34900000 & -1.27651700 \\ 4.14041100 & -0.45380300 & -2.78901500 \\ 2.19367000 & -0.71130100 & -1.83666000 \\ 6.59627400 & 1.27617600 & 1.23279100 \\ 7.18052700 & 0.74165800 & -1.03695100 \\ 5.44190500 & -0.08828400 & -2.56499600 \\ 3.78841500 & -0.77736100 & -3.76156300 \\ 7.55548300 & 1.18848100 & 0.20520300 \\ 6.90514000 & 1.66129100 & 2.20023400 \\ 7.89172300 & 0.68345000 & -1.85565300 \\ 6.17971200 & -0.11870700 & -3.36091700 \\ 8.58049300 & 1.48978300 & 0.39922900 \\ 4.33598800 & 1.13379900 & 2.24956500 \\ 3.52276000 & 1.81096100 & 1.97575600 \\ 3.88616700 & 0.20334300 & 2.60263300 \\ 4.90151500 & 1.58284100 & 3.07205600 \\ 3.59063000 & -0.03951400 & -0.50261000 \\ -3.93598000 & 0.55635800 & -0.56392000 \\ -3.62671000 & -2.00957400 & -0.91562200 \\ -5.54450600 & -1.07712000 & 0.54525100 \\ -6.20470400 & -2.34222200 & 0.64091700 \\ -6.74468700 & -2.34483500 & 1.59150900 \\ -6.91616800 & -2.46942600 & -0.18222400\end{array}$




$\begin{array}{lrrr}\mathrm{H} & -5.47892100 & -3.16220900 & 0.62470700 \\ \mathrm{P} & -4.55975500 & -0.79752600 & -0.72219900 \\ \mathrm{O} & -5.66981200 & -0.85692800 & -1.92501500 \\ \mathrm{C} & -5.19138600 & -0.88410700 & -3.26819100 \\ \mathrm{H} & -6.06924900 & -0.92652900 & -3.91860000 \\ \mathrm{H} & -4.61786500 & 0.02248700 & -3.49935700 \\ \mathrm{H} & -4.56259500 & -1.76468100 & -3.44185200 \\ \mathrm{C} & -0.57921600 & 3.30036200 & -0.39123800 \\ \mathrm{C} & -0.46482900 & 4.66328000 & -0.10373000 \\ \mathrm{C} & -1.81299700 & 2.71855600 & -0.69493500 \\ \mathrm{C} & -1.61472900 & 5.45080500 & -0.12999600 \\ \mathrm{H} & 0.50204900 & 5.08799500 & 0.14265500 \\ \mathrm{C} & -2.95054500 & 3.52041400 & -0.69262800 \\ \mathrm{H} & -1.92735100 & 1.65864200 & -0.89242900 \\ \mathrm{C} & -2.86919500 & 4.89524100 & -0.42144200 \\ \mathrm{H} & -1.53637800 & 6.51215800 & 0.09225300 \\ \mathrm{H} & -3.90445800 & 3.03756000 & -0.88131100 \\ \mathrm{C} & -4.11224900 & 5.75195700 & -0.44473700 \\ \mathrm{H} & -4.46581600 & 5.90108400 & -1.47315600 \\ \mathrm{H} & -4.92985100 & 5.27689700 & 0.10872900 \\ \mathrm{H} & -3.93093300 & 6.73948900 & -0.00884500\end{array}$

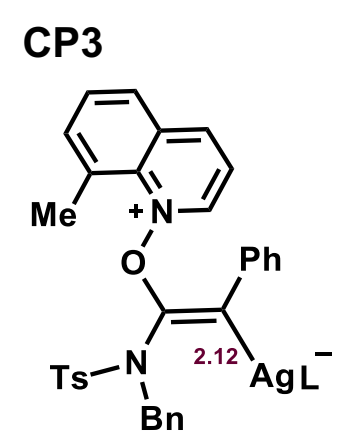

Zero-point correction=

Thermal correction to Energy=

0.622218 (Hartree/Particle)

Thermal correction to Enthalpy=

0.669373

Thermal correction to Gibbs Free Energy=

0.670317

Sum of electronic and zero-point Energies=

0.528986

Sum of electronic and thermal Energies=

$-2836.835550$

Sum of electronic and thermal Enthalpies= $-2836.788395$

Sum of electronic and thermal Free Energies=

$-2836.787451$

$-2836.928782$ 
Supporting information

\begin{tabular}{|c|c|c|c|}
\hline C & -0.07376900 & -1.43635700 & 4.65883400 \\
\hline C & -1.43084200 & -1.71057100 & 4.48709800 \\
\hline $\mathrm{H}$ & 1.61723700 & -0.29795000 & 3.96446400 \\
\hline C & 0.56034800 & -0.51404800 & 3.82219400 \\
\hline C & -2.14672200 & -1.05637200 & 3.48040500 \\
\hline C & -1.51590800 & -0.13266900 & 2.64692700 \\
\hline C & -0.15060300 & 0.14408800 & 2.81244900 \\
\hline $\mathrm{H}$ & -3.20440500 & -1.25687300 & 3.33546000 \\
\hline $\mathrm{H}$ & -2.10175800 & 0.35314600 & 1.87120200 \\
\hline C & 0.54456600 & 1.16547900 & 1.93839600 \\
\hline $\mathrm{H}$ & 0.02662000 & 2.12731800 & 2.00700300 \\
\hline $\mathrm{H}$ & 1.57405900 & 1.33042300 & 2.27860400 \\
\hline $\mathrm{N}$ & 0.52741700 & 0.78367700 & 0.49853800 \\
\hline$S$ & 0.76293800 & 2.10551900 & -0.58562800 \\
\hline 0 & 0.72108800 & 1.52471200 & -1.93685700 \\
\hline 0 & 1.96429600 & 2.86867600 & -0.19312500 \\
\hline C & 1.12436000 & -0.43803100 & 0.12954100 \\
\hline C & 0.52951000 & -1.56150900 & -0.29269800 \\
\hline C & 1.32015300 & -2.78885600 & -0.53795800 \\
\hline C & 1.29491300 & -3.40904600 & -1.80223300 \\
\hline C & 2.07434800 & -3.40135600 & 0.48257300 \\
\hline C & 2.02987500 & -4.56838100 & -2.04947600 \\
\hline $\mathrm{H}$ & 0.67796900 & -2.97889700 & -2.58786200 \\
\hline C & 2.78319400 & -4.57756800 & 0.24107500 \\
\hline $\mathrm{H}$ & 2.07594300 & -2.95829400 & 1.47411800 \\
\hline C & 2.77599400 & -5.16127800 & -1.02831700 \\
\hline $\mathrm{H}$ & 2.00087800 & -5.02162500 & -3.03725500 \\
\hline $\mathrm{H}$ & 3.34461900 & -5.04071300 & 1.04902400 \\
\hline $\mathrm{H}$ & 3.33115400 & -6.07665000 & -1.21472900 \\
\hline $\mathrm{Ag}$ & -1.55994800 & -1.62184000 & -0.63854600 \\
\hline 0 & 2.58790300 & -0.45898100 & 0.41181100 \\
\hline $\mathrm{H}$ & -1.92862200 & -2.42783800 & 5.13468100 \\
\hline $\mathrm{H}$ & 0.49218000 & -1.93672800 & 5.44058700 \\
\hline C & 6.33487000 & 1.18112500 & 1.34718500 \\
\hline C & 5.11577300 & 0.55189500 & 1.12648200 \\
\hline C & 4.63786800 & 0.54634800 & -0.22001600 \\
\hline C & 5.43481900 & 1.09655600 & -1.27778800 \\
\hline C & 6.66734000 & 1.72231100 & -0.97069300 \\
\hline
\end{tabular}


C

$\mathrm{H}$

C

$\mathrm{H}$

$\mathrm{H}$

C

C

$\mathrm{H}$

$\mathrm{H}$

$\mathrm{H}$

$\mathrm{N}$

C

$\mathrm{H}$

$\mathrm{H}$

$\mathrm{H}$

$\mathrm{O}$

O

O

C

$\mathrm{H}$

$\mathrm{H}$

$\mathrm{H}$

$P$

O

C

$\mathrm{H}$

$\mathrm{H}$

$\mathrm{H}$

C

C

C

C

$\mathrm{H}$

C

$\mathrm{H}$

C

$\mathrm{H}$

$\mathrm{H}$

$\begin{array}{rrr}7.10416400 & 1.77700700 & 0.32936500 \\ 6.71675300 & 1.19916700 & 2.36376800 \\ 4.99507800 & 0.99944400 & -2.62219500 \\ 7.25148500 & 2.14508400 & -1.78245500 \\ 8.04616400 & 2.25583500 & 0.57791400 \\ 3.81438300 & 0.37140500 & -2.92281700 \\ 3.02722000 & -0.12386700 & -1.87664100 \\ 5.61458900 & 1.42590400 & -3.40544800 \\ 3.44728700 & 0.27173000 & -3.93690300 \\ 2.05816800 & -0.57448600 & -2.02793800 \\ 3.41963100 & -0.00331700 & -0.61199200 \\ 4.44135900 & -0.10546700 & 2.31005800 \\ 3.51322200 & 0.40098100 & 2.58358200 \\ 4.18988100 & -1.14919000 & 2.10878000 \\ 5.11602300 & -0.07081900 & 3.17039100 \\ -3.97234200 & 0.31280500 & 0.44626900 \\ -3.64520800 & -1.77310500 & -1.08969200 \\ -5.95252000 & -1.31523500 & -0.09822600 \\ -5.89562000 & -2.36698700 & 0.86132600 \\ -6.91937300 & -2.72289000 & 1.00681400 \\ -5.26760500 & -3.19098600 & 0.50420900 \\ -5.50577000 & -2.00302200 & 1.82084800 \\ -4.54063700 & -0.63007700 & -0.57209800 \\ -5.09215400 & 0.23822700 & -1.83250400 \\ -5.59018200 & -0.44749800 & -2.98263800 \\ -5.81762700 & 0.31577400 & -3.73174900 \\ -4.84025500 & -1.14073400 & -3.37801600 \\ -6.50378100 & -1.00121000 & -2.73803300 \\ -0.67304400 & 3.12047900 & -0.29185900 \\ -0.48535900 & 4.48966400 & -0.08760500 \\ -1.95092900 & 2.55614800 & -0.32438400 \\ -1.60709200 & 5.30086900 & 0.07976800 \\ 0.51730700 & 4.90170800 & -0.05490600 \\ -3.05497500 & 3.38158400 & -0.13621900 \\ -2.11213200 & 1.49191800 & -0.44987600 \\ -2.90226200 & 4.76340300 & 0.05822700 \\ -1.47192600 & 6.36792600 & 0.23887000 \\ -4.03630300 & 2.91673800 & -0.12717200\end{array}$




$\begin{array}{lrrr}\mathrm{C} & -4.11399800 & 5.64546800 & 0.24181300 \\ \mathrm{H} & -4.70046800 & 5.70351700 & -0.68387100 \\ \mathrm{H} & -4.77921000 & 5.24637800 & 1.01610300 \\ \mathrm{H} & -3.83300500 & 6.66488300 & 0.52467900\end{array}$

\section{TS3}

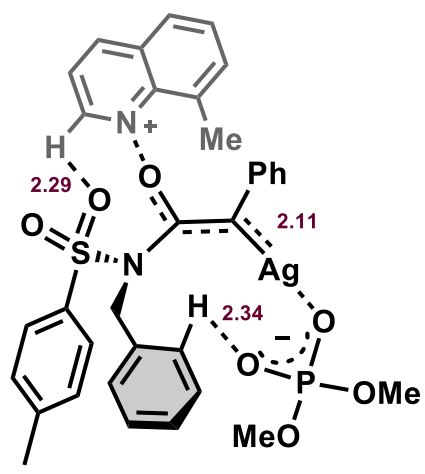

Zero-point correction=

Thermal correction to Energy=

0.621948 (Hartree/Particle)

Thermal correction to Enthalpy=

0.667444

Thermal correction to Gibbs Free Energy=

0.668388

Sum of electronic and zero-point Energies=

0.534051

Sum of electronic and thermal Energies=

$-2836.748075$

Sum of electronic and thermal Enthalpies= $-2836.702579$

Sum of electronic and thermal Free Energies=

$-2836.701635$

$-2836.835972$

$\begin{array}{lrcc}\mathrm{C} & 0.63499000 & 0.00243900 & -5.02018300 \\ \mathrm{C} & -0.63207400 & -0.58153800 & -5.04519300 \\ \mathrm{H} & 2.04708500 & 1.15109600 & -3.86846500 \\ \mathrm{C} & 1.06365600 & 0.68463300 & -3.87879900 \\ \mathrm{C} & -1.46572700 & -0.47032200 & -3.92902500 \\ \mathrm{C} & -1.04077000 & 0.21486800 & -2.79020200 \\ \mathrm{C} & 0.23578000 & 0.79419300 & -2.75493200 \\ \mathrm{H} & -2.45972700 & -0.90841900 & -3.93431000 \\ \mathrm{H} & -1.71972600 & 0.28905500 & -1.94604700 \\ \mathrm{C} & 0.70612000 & 1.59298600 & -1.55521900 \\ \mathrm{H} & 0.14999000 & 2.53508000 & -1.50269200 \\ \mathrm{H} & 1.76599000 & 1.85425200 & -1.65872800 \\ \mathrm{~N} & 0.45764100 & 0.88853500 & -0.27312500 \\ \mathrm{~S} & 0.35034300 & 1.94948600 & 1.08423300 \\ \mathrm{O} & 0.14893200 & 1.08702900 & 2.25942800\end{array}$


Supporting information

O

C

C

C

C

C

C

$\mathrm{H}$

C

$\mathrm{H}$

C

$\mathrm{H}$

$\mathrm{H}$

$\mathrm{H}$

Ag

$\mathrm{O}$

$\mathrm{H}$

$\mathrm{H}$

C

C

C

C

C

C

$\mathrm{H}$

C

$\mathrm{H}$

$\mathrm{H}$

C

C

$\mathrm{H}$

$\mathrm{H}$

$\mathrm{H}$

$\mathrm{N}$

C

$\mathrm{H}$

$\mathrm{H}$

$\mathrm{H}$
1.50428500

1.13901500

0.53711000

1.35076800

1.25392400

2.18237700

2.00193500

0.58249100

2.89377800

2.23440900

2.82206600

1.92361200

3.50856400

3.38335900

$-1.54536200$

2.43019000

$-0.96945100$

1.29046900

6.49760200

5.24424200

4.76750900

5.60443800

6.87321300

7.30444700

6.87976500

5.15406600

7.48977400

8.27392900

3.93060100

3.11560200

5.79931300

3.55490600

2.09944700

3.53248300

4.51620400

3.59735700

4.22960500

5.17113200
2.87125300

$-0.35359800$

$-1.48501500$

$-2.68633900$

$-3.31151300$

$-3.28295000$

$-4.44870600$

$-2.89114200$

$-4.44776900$

$-2.84694800$

$-5.02747200$

$-4.89936100$

$-4.90344800$

$-5.93083200$

$-1.63042400$

$-0.39108700$

$-1.11568500$

$-0.07186400$

0.81669000

0.30627700

0.54006900

1.19403200

1.68636000

1.51459800

0.65107700

1.31813600

2.18871400

1.88979500

0.82411800

0.24851100

1.81177700

0.90051400

$-0.08843100$

0.14843400

$-0.50384400$

$-0.02183200$

$-1.48484700$

$-0.65148400$
1.09414300

$-0.06493000$

0.43236500

0.70474900

1.96503000

$-0.26552500$

2.26225300

2.70927900

0.02143800

$-1.25738500$

1.28977700

3.24831900

$-0.75099700$

1.51269500

0.71673800

$-0.49009500$

$-5.92982100$

$-5.88436800$

$-1.50231800$

$-1.19350500$

0.13398700

1.10442000

0.71212300

$-0.58031900$

$-2.50560300$

2.44435000

1.45170300

$-0.89341800$

2.82270300

1.83323200

3.16543700

3.83594400

2.03379000

0.59457300

$-2.24003600$

$-2.57969400$

$-1.85171300$

$-3.10356900$ 


$\begin{array}{lrrr}\text { O } & -3.78479400 & -0.17151100 & -0.95587000 \\ \mathrm{O} & -3.65334300 & -1.90204900 & 0.98507000 \\ \mathrm{O} & -5.66421000 & -1.96429300 & -0.59911900 \\ \mathrm{C} & -5.24575900 & -3.12332800 & -1.31680100 \\ \mathrm{H} & -6.15189600 & -3.63272700 & -1.65580100 \\ \mathrm{H} & -4.66638500 & -3.79465700 & -0.67276400 \\ \mathrm{H} & -4.64177200 & -2.84778000 & -2.19058000 \\ \mathrm{P} & -4.50483300 & -1.01143300 & 0.05692500 \\ \mathrm{O} & -5.45147300 & -0.04314900 & 0.95913500 \\ \mathrm{C} & -6.15111800 & -0.59964600 & 2.07478500 \\ \mathrm{H} & -6.64316500 & 0.23219000 & 2.58590200 \\ \mathrm{H} & -5.45686000 & -1.09594000 & 2.76066100 \\ \mathrm{H} & -6.90681500 & -1.31800900 & 1.73765600 \\ \mathrm{C} & -1.11877200 & 2.90096700 & 0.73745500 \\ \mathrm{C} & -1.04790100 & 4.29071400 & 0.86011700 \\ \mathrm{C} & -2.31920100 & 2.25762100 & 0.42684100 \\ \mathrm{C} & -2.20868100 & 5.03963500 & 0.66937300 \\ \mathrm{H} & -0.10261800 & 4.76766900 & 1.09451600 \\ \mathrm{C} & -3.46289200 & 3.02511700 & 0.23079300 \\ \mathrm{H} & -2.38471300 & 1.18381400 & 0.29569700 \\ \mathrm{C} & -3.42819400 & 4.42294200 & 0.35542200 \\ \mathrm{H} & -2.16353300 & 6.12197100 & 0.76227500 \\ \mathrm{H} & -4.38372800 & 2.50895600 & -0.02470300 \\ \mathrm{C} & -4.68283200 & 5.23776200 & 0.15026700 \\ \mathrm{H} & -5.43993400 & 4.98976500 & 0.90444600 \\ \mathrm{H} & -5.13031300 & 5.03340500 & -0.82961500 \\ \mathrm{H} & -4.48224500 & 6.31181500 & 0.21505400\end{array}$

\section{CP4}

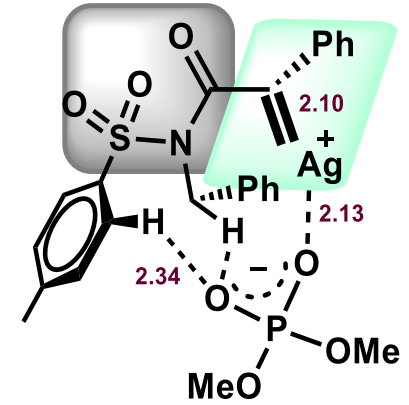

Zero-point correction= 0.457498 (Hartree/Particle)

Thermal correction to Energy= 0.494098 
Thermal correction to Enthalpy=

Thermal correction to Gibbs Free Energy=

Sum of electronic and zero-point Energies=

Sum of electronic and thermal Energies=

Sum of electronic and thermal Enthalpies=

Sum of electronic and thermal Free Energies=

C

C

$\mathrm{H}$

C

C

C

C

$\mathrm{H}$

$\mathrm{H}$

C

$\mathrm{H}$

$\mathrm{H}$

$\mathrm{N}$

$S$

O

O

C

C

C

C

C

C

$\mathrm{H}$

C

$\mathrm{H}$

C

$\mathrm{H}$

$\mathrm{H}$

$\mathrm{H}$

$\mathrm{Ag}$

$\mathrm{O}$
3.85857700

4.92472200

1.71665500

2.54972500

4.67704100

3.37133300

2.29390200

5.50367600

3.18097200

0.86537300

0.15375000

0.60106100

0.65597500

$-0.23422400$

0.51979900

$-0.47109300$

0.92204000

1.45099100

2.74305200

3.17435100

3.62951100

4.42895300

2.49505000

4.87646900

3.30903900

5.27723000

4.74831100

5.54338500

6.25732300

$-0.01571900$

0.63756800
1.87695100

0.98643500

2.08612600

1.39342600

$-0.39035600$

$-0.87099700$

0.01686900

$-1.08759000$

$-1.94070100$

$-0.48424100$

0.33201100

$-0.95044900$

$-1.54019700$

$-3.00152500$

$-4.14977100$

$-2.83136500$

$-1.34454000$

$-0.07372200$

$-0.05877300$

1.11304000

$-1.17263900$

1.17604600

1.95912600

$-1.10878100$

$-2.08291700$

0.06398000

2.07724400

$-1.96463600$

0.10834900

1.42495700

$-2.13088500$
0.495042

0.381021

$-2395.746095$

$-2395.709495$

$-2395.708551$

$-2395.822572$

1.97661300

2.10031900

1.80277200

1.89273000

2.15035600

2.07444800

1.93732400

2.25881600

2.11918800

1.89642800

1.72813200

2.84777800

0.87315200

1.31850000

0.82394700

2.75272100

$-0.45346700$

$-0.98941500$

$-1.59180600$

$-2.27002500$

$-1.53818400$

$-2.85826600$

$-2.31712600$

$-2.13825300$

$-1.04382200$

$-2.79427000$

$-3.37238400$

$-2.10081100$

$-3.26183800$

$-0.98714100$

$-1.36256000$ 
Supporting information

$\begin{array}{lrrr}H & 5.94349700 & 1.35914000 & 2.16414300 \\ \mathrm{H} & 4.04147600 & 2.94773700 & 1.94504500 \\ \mathrm{O} & -1.80983600 & 1.43605100 & 1.36143200 \\ \mathrm{O} & -1.58182400 & 2.85530100 & -0.79364200 \\ \mathrm{O} & -2.79714700 & 3.86405600 & 1.22405200 \\ \mathrm{C} & -1.73462700 & 4.70400800 & 1.67527200 \\ \mathrm{H} & -2.19723400 & 5.56625300 & 2.16156300 \\ \mathrm{H} & -1.12161100 & 5.04350300 & 0.83256200 \\ \mathrm{H} & -1.10345800 & 4.17755900 & 2.40152200 \\ \mathrm{P} & -2.41122300 & 2.47622700 & 0.45954800 \\ \mathrm{O} & -3.89621500 & 2.00618900 & 0.00416200 \\ \mathrm{C} & -4.62056100 & 2.80358000 & -0.94050100 \\ \mathrm{H} & -5.54557100 & 2.26533300 & -1.16074900 \\ \mathrm{H} & -4.04081800 & 2.93657900 & -1.85952400 \\ \mathrm{H} & -4.86086900 & 3.78248100 & -0.51283400 \\ \mathrm{C} & -1.76670700 & -2.83706300 & 0.42389200 \\ \mathrm{C} & -2.55206400 & -1.69418400 & 0.60531400 \\ \mathrm{C} & -2.16211300 & -3.87622900 & -0.41737200 \\ \mathrm{C} & -3.75814300 & -1.60289700 & -0.08472600 \\ \mathrm{H} & -2.23657000 & -0.86524500 & 1.23332100 \\ \mathrm{C} & -3.38385100 & -3.76973700 & -1.08007400 \\ \mathrm{H} & -1.51844400 & -4.73754900 & -0.55558200 \\ \mathrm{C} & -4.19680500 & -2.63840300 & -0.92561000 \\ \mathrm{H} & -4.35151200 & -0.69984300 & 0.02935200 \\ \mathrm{H} & -3.70301500 & -4.57418500 & -1.73788000 \\ \mathrm{C} & -5.52377200 & -2.53373700 & -1.63887800 \\ \mathrm{H} & -5.67754400 & -1.52885800 & -2.04732700 \\ \mathrm{H} & -6.35569100 & -2.73277100 & -0.95051900 \\ \mathrm{H} & -5.59675400 & -3.25213300 & -2.46146200\end{array}$

\section{TS4}

Zero-point correction=

0.457160 (Hartree/Particle)

Thermal correction to Energy= 0.492456

Thermal correction to Enthalpy=

0.493400

Thermal correction to Gibbs Free Energy=

0.384309

Sum of electronic and zero-point Energies=

$-2395.727012$

Sum of electronic and thermal Energies= $-2395.691717$

Sum of electronic and thermal Enthalpies= $-2395.690773$ 
$-2395.799863$

C

C

$3.28921500 \quad 1.59954900$

2.25471000

$\mathrm{H}$

4.46020600

0.79915600

2.09430800

C

1.14195900

1.66390100

2.09325900

2.05568600

1.07426700

2.00726900

C

4.37813000

$-0.55538600$

1.80148000

C

3.12205100

$-1.13533400$

1.62882000

C

1.91562200

$-0.31594900$

1.54455300

$\mathrm{H}$

5.27595000

$-1.16201400$

1.74021200

$\mathrm{H}$

3.02213600

$-2.20659800$

1.48997600

C

0.62834400

$-1.03831900$

1.98380400

$\mathrm{H}$

$-0.21691000$

$-0.33986700$

1.91698200

$\mathrm{H}$

0.70669800

$-1.42156600$

3.00155000

N

0.51828600

$-2.14798200$

1.02892500

$\mathrm{S}$

$-0.75887900$

$-3.29743400$

1.20146800

O

$-0.28339600$

$-4.56414800$

0.66173400

O

$-1.14876500$

$-3.15263100$

2.60568300

C

1.08457400

$-1.83484600$

$-0.22647400$

C

1.79577000

$-0.53200200$

$-0.11853400$

C

2.85663300

$-0.13777100$

$-1.08094200$

C

3.26948900

1.20915900

$-1.17562600$

C

3.45958300

$-1.07279100$

$-1.94965500$

C

4.23814400

1.60630800

$-2.09758500$

$\mathrm{H}$

2.81741900

1.95248600

$-0.52644500$

C

4.41491000

$-0.66848500$

$-2.87783000$

$\mathrm{H}$

3.14530700

$-2.10875200$

$-1.91427300$

C

4.81530600

0.66947500

$-2.95481900$

$\mathrm{H}$

4.53179700

2.65130600

$-2.15038800$

$\mathrm{H}$

4.85326300

$-1.40522300$

$-3.54584300$

$\mathrm{H}$

5.56447000

0.97686500

$-3.67917600$

$\mathrm{Ag}$

0.04896900

0.83480600

$-0.71475000$

0.92101800

$-2.50354000$

$-1.23783700$

$\mathrm{H}$

5.43479400

1.25553200

2.24796800

3.39217700

2.63507600

2.56484800

$\mathrm{O}$

$-0.84694800$

2.15599800

1.62670400

O

$-1.55904800$

2.30590600

$-0.85634900$

O

$-1.87682300$

4.36159000

0.64692400 


$\begin{array}{lrrr}\mathrm{C} & -0.83583700 & 5.10632100 & 0.01660100 \\ \mathrm{H} & -1.09689600 & 6.16281800 & 0.11636000 \\ \mathrm{H} & -0.75418900 & 4.84370000 & -1.04358700 \\ \mathrm{H} & 0.12769500 & 4.92958200 & 0.51208000 \\ \mathrm{P} & -1.79949400 & 2.73007100 & 0.60924900 \\ \mathrm{O} & -3.31906800 & 2.38247800 & 1.05326000 \\ \mathrm{C} & -4.40085500 & 2.81559400 & 0.22197900 \\ \mathrm{H} & -5.31762100 & 2.42909900 & 0.67443700 \\ \mathrm{H} & -4.29259800 & 2.42014200 & -0.79373400 \\ \mathrm{H} & -4.44589300 & 3.90892400 & 0.18558100 \\ \mathrm{C} & -2.06169800 & -2.65312900 & 0.16810700 \\ \mathrm{C} & -2.93442600 & -1.69548400 & 0.69634000 \\ \mathrm{C} & -2.17132900 & -3.08643000 & -1.15492900 \\ \mathrm{C} & -3.92477700 & -1.16303800 & -0.12439400 \\ \mathrm{H} & -2.84913700 & -1.38591200 & 1.73223900 \\ \mathrm{C} & -3.17695700 & -2.54550500 & -1.95460200 \\ \mathrm{H} & -1.48069800 & -3.82649000 & -1.54063400 \\ \mathrm{C} & -4.05937400 & -1.57490800 & -1.45935700 \\ \mathrm{H} & -4.59894300 & -0.41398200 & 0.28098400 \\ \mathrm{H} & -3.27487900 & -2.88207000 & -2.98352100 \\ \mathrm{C} & -5.11818900 & -0.96450500 & -2.34593500 \\ \mathrm{H} & -4.80574500 & 0.02809000 & -2.69598300 \\ \mathrm{H} & -6.06475600 & -0.83636600 & -1.80931800 \\ \mathrm{H} & -5.30755800 & -1.58167200 & -3.22969200\end{array}$

\section{CP5}

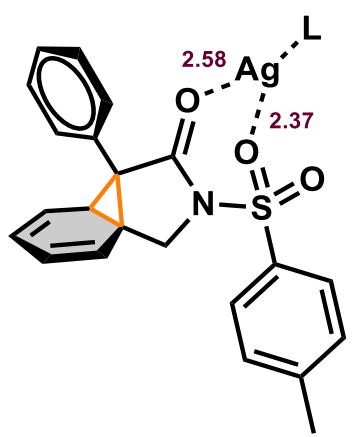

Zero-point correction=

0.458676 (Hartree/Particle)

Thermal correction to Energy= 0.494488

Thermal correction to Enthalpy=

0.495433

Thermal correction to Gibbs Free Energy=

0.383247

Sum of electronic and zero-point Energies= 
Sum of electronic and thermal Energies=

Sum of electronic and thermal Enthalpies=

Sum of electronic and thermal Free Energies=

$-2395.732010$

$-2395.731066$

$-2395.843251$

C

C

$\mathrm{H}$

C

C

C

C

$\mathrm{H}$

$\mathrm{H}$

C

$\mathrm{H}$

$\mathrm{H}$

$\mathrm{N}$

$S$

O

O

C

C

C

C

C

C

$\mathrm{H}$

C

$\mathrm{H}$

C

$\mathrm{H}$

$\mathrm{H}$

$\mathrm{H}$

$\mathrm{O}$

$\mathrm{H}$

$\mathrm{H}$

$\mathrm{O}$

O
2.27082200

2.17628100

1.20010700

1.07888100

0.88869600

$-0.26719700$

$-0.27522200$

0.86856800

$-1.21669400$

$-1.42042000$

$-1.25913700$

$-2.38269300$

$-1.41172600$

$-2.33318000$

$-2.71602100$

$-1.54221400$

$-0.62276100$

0.17674800

0.60978300

1.70931800

$-0.09228100$

2.09907900

2.24091700

0.30513000

$-0.94602300$

1.40115300

2.94733700

$-0.24335500$

1.70918200

$-0.62204300$

3.22809400

3.07519700

2.59561400

2.78360000
2.36409700

3.67315800

0.47908500

1.53135100

4.29530200

3.60298100

2.21497300

5.32663300

4.04785200

1.28790600

0.82299200

1.81648100

0.26464500

$-1.16417000$

$-1.26831300$

$-2.23696800$

0.60749300

1.81345500

2.69409700

2.30011100

3.85597300

3.06590400

1.38888100

4.62549500

4.16306400

4.23155100

2.75074300

5.52914600

4.82893900

$-0.01430800$

1.85916900

4.26520900

$-2.81588400$

$-0.68938400$
$-1.83008200$

$-2.15331900$

$-1.85278500$

$-1.61430600$

$-2.40751900$

$-2.29705600$

$-1.80932300$

$-2.74977700$

$-2.58723300$

$-2.21264000$

$-3.18732300$

$-2.22736900$

$-1.14943700$

$-1.25696200$

$-2.66188000$

$-0.62186300$

$-0.03889800$

$-0.38958600$

0.73606800

1.51291600

1.07431700

2.61097500

1.25148100

2.16980300

0.47679000

2.93905400

3.21261300

2.42282000

3.79331100

1.01680500

$-1.74690100$

$-2.30152000$

1.00065500

$-0.46506000$ 
Supporting information

$\begin{array}{lrrr}\mathrm{O} & 4.35285000 & -1.09881800 & 1.58631900 \\ \mathrm{P} & 3.48751000 & -1.73620100 & 0.37139800 \\ \mathrm{O} & 4.70128600 & -2.44219400 & -0.46780500 \\ \mathrm{C} & 4.38173500 & -3.04105600 & -1.72277200 \\ \mathrm{H} & 3.70596400 & -3.89411500 & -1.58564200 \\ \mathrm{H} & 5.32112300 & -3.39460600 & -2.15555800 \\ \mathrm{H} & 3.91770000 & -2.31269600 & -2.39798300 \\ \mathrm{Ag} & 0.55559500 & -2.24095800 & 0.47552000 \\ \mathrm{C} & 5.32048600 & -0.09022300 & 1.29058600 \\ \mathrm{H} & 5.70620100 & 0.26649200 & 2.24896500 \\ \mathrm{H} & 4.86458600 & 0.74449100 & 0.74562700 \\ \mathrm{H} & 6.14322100 & -0.50484800 & 0.69808400 \\ \mathrm{C} & -3.79415000 & -0.88939200 & -0.27251200 \\ \mathrm{C} & -3.71461200 & -0.90591600 & 1.12492100 \\ \mathrm{C} & -5.00493000 & -0.67238400 & -0.93286500 \\ \mathrm{C} & -4.87698300 & -0.69395600 & 1.85900300 \\ \mathrm{H} & -2.76262700 & -1.06112500 & 1.61894100 \\ \mathrm{C} & -6.15613600 & -0.46737300 & -0.17330800 \\ \mathrm{H} & -5.04224700 & -0.67934800 & -2.01662100 \\ \mathrm{C} & -6.11276700 & -0.47569500 & 1.22717800 \\ \mathrm{H} & -4.82452600 & -0.70017000 & 2.94469200 \\ \mathrm{H} & -7.10357000 & -0.30235500 & -0.67941600 \\ \mathrm{C} & -7.36506300 & -0.27518100 & 2.04573100 \\ \mathrm{H} & -7.19274900 & 0.42203500 & 2.87318200 \\ \mathrm{H} & -7.69935200 & -1.22306000 & 2.48678900 \\ \mathrm{H} & -8.18597200 & 0.11427600 & 1.43632100\end{array}$

TS5

Zero-point correction=

0.456934 (Hartree/Particle)

Thermal correction to Energy=

0.492621

Thermal correction to Enthalpy=

0.493566

Thermal correction to Gibbs Free Energy=

0.381014

Sum of electronic and zero-point Energies=

$-2395.757023$

Sum of electronic and thermal Energies= $-2395.721335$

Sum of electronic and thermal Enthalpies= $-2395.720391$

Sum of electronic and thermal Free Energies= $-2395.832943$

C

1.72529700

3.24142000

$-1.34091200$ 
Supporting information

C

$\mathrm{H}$

C

C

C

C

$\mathrm{H}$

$\mathrm{H}$

C

$\mathrm{H}$

$\mathrm{H}$

$\mathrm{N}$

$S$

O

O

C

C

C

C

C

C

$\mathrm{H}$

C

$\mathrm{H}$

C

$\mathrm{H}$

$\mathrm{H}$

$\mathrm{H}$

$\mathrm{O}$

$\mathrm{H}$

$\mathrm{H}$

O

0

O

$\mathrm{P}$

O

C

$\mathrm{H}$
1.08978300

1.68643800

1.09029600

$-0.17995100$

$-0.90288600$

$-0.66258900$

$-0.51092500$

$-1.63939900$

$-1.16591100$

$-0.55572000$

$-2.20100100$

$-1.08576400$

$-1.63517100$

$-1.83247400$

$-0.70367900$

$-0.59235600$

$-0.26964400$

$-0.70143000$

0.15149500

$-1.97856500$

$-0.26583400$

1.14155000

$-2.39572500$

$-2.64320400$

$-1.53958700$

0.40404700

$-3.38785900$

$-1.86253900$

$-0.45851200$

2.81307200

1.67018100

3.36246600

3.03403100

4.67953500

3.98121200

5.31668300

5.13563000

4.67712300
4.40295400

1.09588900

2.00809300

4.39712100

3.23581900

2.03014700

5.30918400

3.21765100

0.68982000

0.26967000

0.76062900

$-0.16644400$

$-1.78766200$

$-2.09184900$

$-2.59113800$

0.48080700

1.92332200

2.90724600

3.18295100

3.47711100

4.02205000

2.73773300

4.31989400

3.27250700

4.59479900

4.22759900

4.76257600

5.25236200

$-0.03864600$

3.23402100

5.32102100

$-2.07393600$

$-0.42761100$

0.08588100

$-1.02680600$

$-1.62659000$

$-2.57423300$

$-3.49476400$
$-1.74947200$

$-1.12187200$

$-1.09108900$

$-2.36421500$

$-2.58092400$

$-1.89377700$

$-2.85494000$

$-3.38337400$

$-2.40260000$

$-3.20587500$

$-2.76027000$

$-1.20410600$

$-1.23296500$

$-2.64628300$

$-0.41924500$

$-0.07133300$

$-0.45468200$

0.60751500

1.68250100

0.58347800

2.71531500

1.70692400

1.61510100

$-0.25111300$

2.68283600

3.54580800

1.58250400

3.48564600

1.02509400

$-1.29865700$

$-1.80082400$

1.40281500

$-0.55343800$

1.41874200

0.46903800

$-0.25576300$

$-1.30823100$

$-0.92690800$ 


$\begin{array}{lrrr}\mathrm{H} & 6.12872400 & -2.80501400 & -1.70199300 \\ \mathrm{H} & 4.51050700 & -2.15757600 & -2.10611400 \\ \mathrm{Ag} & 1.25847600 & -2.03147300 & 0.73455500 \\ \mathrm{C} & 5.33960000 & 1.19665300 & 0.81026100 \\ \mathrm{H} & 5.63341600 & 1.87044700 & 1.61894800 \\ \mathrm{H} & 4.66724200 & 1.72119300 & 0.12147100 \\ \mathrm{H} & 6.23177900 & 0.86754800 & 0.26638400 \\ \mathrm{C} & -3.21221300 & -1.76362600 & -0.40441900 \\ \mathrm{C} & -3.26990800 & -1.68085400 & 0.99164100 \\ \mathrm{C} & -4.37033700 & -1.83771600 & -1.18122600 \\ \mathrm{C} & -4.51854200 & -1.66516700 & 1.60493900 \\ \mathrm{H} & -2.36054900 & -1.61515400 & 1.57743500 \\ \mathrm{C} & -5.60857900 & -1.82604600 & -0.54116100 \\ \mathrm{H} & -4.29802200 & -1.91860700 & -2.26029600 \\ \mathrm{C} & -5.70368000 & -1.74074800 & 0.85464600 \\ \mathrm{H} & -4.57408300 & -1.59579700 & 2.68816400 \\ \mathrm{H} & -6.51496000 & -1.88840600 & -1.13745000 \\ \mathrm{C} & -7.04636700 & -1.75383700 & 1.54397900 \\ \mathrm{H} & -7.07915300 & -1.03198700 & 2.36718600 \\ \mathrm{H} & -7.25482000 & -2.74280500 & 1.97242200 \\ \mathrm{H} & -7.85768900 & -1.51767500 & 0.84894100\end{array}$

\section{CP6}

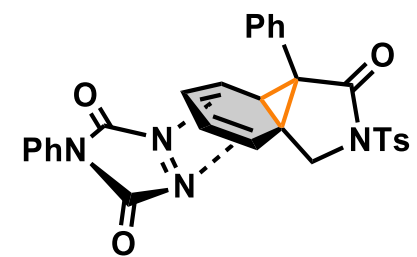

Zero-point correction=

0.487732 (Hartree/Particle)

Thermal correction to Energy= 0.522631

Thermal correction to Enthalpy=

0.523575

Thermal correction to Gibbs Free Energy=

0.414120

Sum of electronic and zero-point Energies=

$-2150.333334$

Sum of electronic and thermal Energies=

$-2150.298435$

Sum of electronic and thermal Enthalpies=

$-2150.297491$

Sum of electronic and thermal Free Energies=

$-2150.406946$

C

$0.99709200 \quad 0.83128200$

1.49202800 
Supporting information

\begin{tabular}{lrrr}
$\mathrm{C}$ & & & \\
$\mathrm{C}$ & 2.09229000 & 1.39948800 & 0.92044100 \\
$\mathrm{C}$ & -0.21285200 & 0.51735200 & 0.72314900 \\
$\mathrm{C}$ & 2.20597500 & 1.51881600 & -0.51175100 \\
$\mathrm{C}$ & 1.21017000 & 1.09171000 & -1.33331000 \\
$\mathrm{H}$ & -0.10187300 & 0.69616300 & -0.81572400 \\
$\mathrm{H}$ & 3.14532300 & 1.86114900 & -0.93707700 \\
$\mathrm{C}$ & 1.36994200 & 1.03103200 & -2.40688700 \\
$\mathrm{H}$ & -0.99679500 & -0.21067000 & -1.65346400 \\
$\mathrm{H}$ & -0.75189400 & -1.26640600 & -1.52027300 \\
$\mathrm{~N}$ & -0.91632700 & 0.02861800 & -2.72033600 \\
$\mathrm{~S}$ & -2.34717500 & 0.09053600 & -1.15167100 \\
$\mathrm{O}$ & -3.73408400 & -0.69854200 & -1.80877100 \\
$\mathrm{O}$ & -3.15506200 & -1.76893900 & -2.62393700 \\
$\mathrm{C}$ & -4.61112300 & 0.31171300 & -2.38966100 \\
$\mathrm{C}$ & -2.41495500 & 1.18819100 & -0.29066800 \\
$\mathrm{C}$ & -0.99279200 & 1.62541000 & 0.00640800 \\
$\mathrm{C}$ & -0.77799700 & 3.08194000 & 0.26298900 \\
$\mathrm{C}$ & -0.45898600 & 3.95722100 & -0.78172000 \\
$\mathrm{C}$ & -0.97430000 & 3.60175000 & 1.54852200 \\
$\mathrm{H}$ & -0.32872800 & 5.32612400 & -0.54496900 \\
$\mathrm{C}$ & -0.31364400 & 3.56567300 & -1.78466600 \\
$\mathrm{H}$ & -0.84664500 & 4.96941500 & 1.78679600 \\
$\mathrm{C}$ & -1.23658400 & 2.92984200 & 2.36079800 \\
$\mathrm{H}$ & -0.52039700 & 5.83472800 & 0.74041900 \\
$\mathrm{H}$ & -0.08106500 & 5.99423500 & -1.36545400 \\
$\mathrm{H}$ & -1.00566500 & 5.36000200 & 2.78835000 \\
$\mathrm{O}$ & -0.41959200 & 6.90083800 & 0.92564800 \\
$\mathrm{H}$ & -3.43029200 & 1.68170300 & 0.15426300 \\
$\mathrm{H}$ & -0.80569700 & -0.31812100 & 1.08993200 \\
$\mathrm{H}$ & 0.99992500 & 0.57846500 & 2.54869100 \\
$\mathrm{C}$ & 2.94609000 & 1.66812100 & 1.53665900 \\
$\mathrm{C}$ & 3.41026100 & -1.45509100 & -1.12309700 \\
$\mathrm{O}$ & 3.27128600 & -1.67934800 & 1.11839000 \\
$\mathrm{~N}$ & 3.78278600 & -1.33737500 & -2.25909400 \\
& 3.50085200 & -1.78172800 & 2.29288800 \\
$\mathrm{~N}$ & & -1.69904300 & -0.72746800 \\
$\mathrm{~N}$ & -1.82434400 & 0.51437300 \\
\hline & -1.45077200 & 0.05913900
\end{tabular}




$\begin{array}{lrrr}\text { C } & 5.56305600 & -1.26061300 & 0.16720200 \\ \text { C } & 6.08560200 & -0.57789100 & 1.27044400 \\ \text { C } & 6.40778200 & -1.75869700 & -0.83001900 \\ \text { C } & 7.46456600 & -0.39971700 & 1.37208500 \\ \text { H } & 5.42523900 & -0.20741000 & 2.04475500 \\ \text { C } & 7.78350300 & -1.56147500 & -0.71858000 \\ \text { H } & 5.99430200 & -2.27925300 & -1.68468900 \\ \text { C } & 8.31694400 & -0.88597200 & 0.37986300 \\ \text { H } & 7.86998300 & 0.12572300 & 2.23196100 \\ \text { H } & 8.43863900 & -1.94464000 & -1.49559000 \\ \text { H } & 9.39009700 & -0.74026200 & 0.46264800 \\ \text { C } & -4.53629900 & -1.42338300 & -0.38669900 \\ \text { C } & -5.45132500 & -0.67070300 & 0.35298000 \\ \text { C } & -4.25149100 & -2.75073000 & -0.05924000 \\ \text { C } & -6.07812500 & -1.26675500 & 1.44447300 \\ \text { H } & -5.64931700 & 0.35938100 & 0.08323700 \\ \text { C } & -4.89215800 & -3.32582100 & 1.03574500 \\ \text { H } & -3.55200100 & -3.32024200 & -0.66164500 \\ \text { C } & -5.81318400 & -2.59700500 & 1.80181600 \\ \text { H } & -6.78804400 & -0.68704700 & 2.02911300 \\ \text { H } & -4.67680800 & -4.35905100 & 1.29639100 \\ \text { C } & -6.52713100 & -3.23832000 & 2.96750500 \\ \text { H } & -6.73632700 & -2.51071200 & 3.75853600 \\ \text { H } & -7.49005200 & -3.66152200 & 2.65212200 \\ \text { H } & -5.93843500 & -4.05376500 & 3.39972500\end{array}$

\section{TS6}

Zero-point correction=

Thermal correction to Energy=

0.488758 (Hartree/Particle)

Thermal correction to Enthalpy=

0.522046

Thermal correction to Gibbs Free Energy=

0.522990

Sum of electronic and zero-point Energies=

0.419208

Sum of electronic and thermal Energies=

$-2150.318252$

$-2150.284964$

Sum of electronic and thermal Enthalpies=

$-2150.284020$

Sum of electronic and thermal Free Energies=

$-2150.387802$

C

1.10745200

0.68955000

1.32226600

C

2.05935600

1.56926300

0.74318300 


\begin{tabular}{|c|c|c|c|}
\hline C & -0.18350900 & 0.44997900 & 0.63640400 \\
\hline C & 2.15082800 & 1.62414400 & -0.65121700 \\
\hline C & 1.21507400 & 0.95167400 & -1.41928300 \\
\hline C & -0.10488700 & 0.60492600 & -0.89132100 \\
\hline $\mathrm{H}$ & 3.02611400 & 2.05981100 & -1.12438600 \\
\hline $\mathrm{H}$ & 1.39435100 & 0.78820000 & -2.47829600 \\
\hline C & -1.02642400 & -0.29820100 & -1.69731600 \\
\hline $\mathrm{H}$ & -0.78641500 & -1.35323800 & -1.54972300 \\
\hline $\mathrm{H}$ & -0.96969900 & -0.07563600 & -2.76888100 \\
\hline$N$ & -2.36017600 & 0.02916700 & -1.16868600 \\
\hline s & -3.77565800 & -0.74911200 & -1.78958900 \\
\hline $\mathrm{O}$ & -3.22555500 & -1.81810100 & -2.62512300 \\
\hline 0 & -4.65651700 & 0.27456000 & -2.33915400 \\
\hline C & -2.39719700 & 1.12812400 & -0.31209900 \\
\hline C & -0.95955100 & 1.56285500 & -0.04792300 \\
\hline C & -0.74559500 & 3.02319500 & 0.18581000 \\
\hline C & -0.56687700 & 3.90007700 & -0.89106600 \\
\hline C & -0.80456100 & 3.54361400 & 1.48425900 \\
\hline C & -0.43719400 & 5.27141400 & -0.67306000 \\
\hline $\mathrm{H}$ & -0.52949000 & 3.50678100 & -1.90355000 \\
\hline C & -0.67681600 & 4.91496700 & 1.70378700 \\
\hline $\mathrm{H}$ & -0.96148700 & 2.87114000 & 2.32316900 \\
\hline C & -0.48980800 & 5.78167000 & 0.62551300 \\
\hline $\mathrm{H}$ & -0.29757800 & 5.94087900 & -1.51742200 \\
\hline $\mathrm{H}$ & -0.72724500 & 5.30617000 & 2.71624500 \\
\hline $\mathrm{H}$ & -0.38879700 & 6.85007300 & 0.79578400 \\
\hline $\mathrm{O}$ & -3.39324800 & 1.63348000 & 0.15995900 \\
\hline $\mathrm{H}$ & -0.75028100 & -0.39658400 & 1.01964000 \\
\hline $\mathrm{H}$ & 1.12016900 & 0.56397800 & 2.39948500 \\
\hline $\mathrm{H}$ & 2.83666000 & 1.99620700 & 1.36737600 \\
\hline C & 3.23228700 & -1.39987400 & -0.88352100 \\
\hline C & 3.35134000 & -0.85690800 & 1.32157800 \\
\hline $\mathrm{O}$ & 3.53557900 & -1.72895500 & -2.00699700 \\
\hline $\mathrm{O}$ & 3.70171100 & -0.61932000 & 2.45435700 \\
\hline $\mathrm{N}$ & 1.90056800 & -1.24902400 & -0.39406300 \\
\hline$N$ & 1.95763500 & -0.94946800 & 0.88026300 \\
\hline$N$ & 4.11169100 & -1.18104500 & 0.20714600 \\
\hline C & 5.53626900 & -1.28457500 & 0.17012200 \\
\hline
\end{tabular}




$\begin{array}{lrrr}\text { C } & 6.31603400 & -0.46345500 & 0.99341500 \\ \text { C } & 6.14445000 & -2.21118700 & -0.68508300 \\ \text { C } & 7.70502000 & -0.57761400 & 0.95871100 \\ \text { H } & 5.84017600 & 0.23907700 & 1.66566100 \\ \text { C } & 7.53511100 & -2.30432700 & -0.71536200 \\ \text { H } & 5.53631900 & -2.83439800 & -1.32753100 \\ \text { C } & 8.32074900 & -1.49320800 & 0.10454400 \\ \text { H } & 8.30563200 & 0.05761100 & 1.60382600 \\ \text { H } & 8.00308400 & -3.02166000 & -1.38375200 \\ \text { H } & 9.40373200 & -1.57476900 & 0.07896200 \\ \text { C } & -4.53995800 & -1.47270400 & -0.34743200 \\ \text { C } & -5.42724500 & -0.71549200 & 0.42097300 \\ \text { C } & -4.25390100 & -2.80293400 & -0.03203800 \\ \text { C } & -6.02458200 & -1.31009800 & 1.52961500 \\ \text { H } & -5.62759600 & 0.31622600 & 0.15911700 \\ \text { C } & -4.86501700 & -3.37611500 & 1.08052700 \\ \text { H } & -3.57716400 & -3.37603100 & -0.65665200 \\ \text { C } & -5.75764200 & -2.64293100 & 1.87581400 \\ \text { H } & -6.71294900 & -0.72736700 & 2.13649300 \\ \text { H } & -4.64898300 & -4.41140100 & 1.33206700 \\ \text { C } & -6.44022000 & -3.28272600 & 3.06080400 \\ \text { H } & -6.63449000 & -2.55288200 & 3.85345400 \\ \text { H } & -7.40791200 & -3.71227400 & 2.76963500 \\ \text { H } & -5.83732100 & -4.09345600 & 3.48213900\end{array}$

TS7

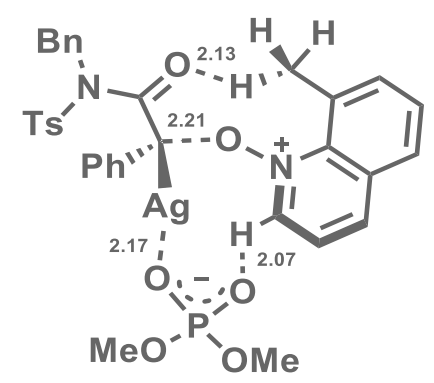

Zero-point correction= 0.627135 (Hartree/Particle)

Thermal correction to Energy= 0.673581

Thermal correction to Enthalpy= 0.674525

Thermal correction to Gibbs Free Energy= 0.538221

Sum of electronic and zero-point Energies= $-2911.981136$

Sum of electronic and thermal Energies= $-2911.934690$ 
Sum of electronic and thermal Enthalpies=

Sum of electronic and thermal Free Energies=
$-2911.933746$

$-2912.070050$
C

$\mathrm{C}$

$\mathrm{H}$

C

C

C

C

$\mathrm{H}$

$\mathrm{H}$

C

$\mathrm{H}$

$\mathrm{H}$

$\mathrm{N}$

$S$

O

$\mathrm{O}$

C

C

C

C

C

C

$\mathrm{H}$

C

$\mathrm{H}$

C

$\mathrm{H}$

$\mathrm{H}$

$\mathrm{H}$

$\mathrm{Ag}$

$\mathrm{O}$

O

$\mathrm{H}$

$\mathrm{H}$

C
$-5.43406300$

$-5.05404600$

$-3.76153600$

$-3.52828000$

$-3.97384400$

$-3.80680800$

$-3.34441700$

$-3.39483200$

$-3.62351400$

$-3.16766800$

$-3.25921600$

$-3.69204400$

$-4.11275300$

$-4.31367600$

$-2.37510300$

$-1.51585200$

$-1.05352200$

$-2.41527700$

$-2.06005900$

$-0.80511700$

$-0.89205400$

0.29141100

$-2.13479900$

0.23004700

1.25106400

$-2.18951600$

$-3.05006100$

$-1.00625900$

1.14590500

$-3.14859700$

$-1.05301200$

1.03869400

$-2.81748400$

$-0.68323500$

$-3.49240700$

$-3.90695500$

$-1.05054300$
$-1.47357200$

$-2.79609900$

0.56642500

$-0.46549500$

$-3.10708000$

$-2.10201400$

$-0.76839300$

$-4.13603600$

$-2.34100800$

0.33187300

1.23681300

0.02236100

0.70530500

2.01008200

2.91976500

2.49598000

0.09918200

0.50012800

1.53970700

2.15669800

1.97982700

3.18426700

1.82957700

3.00089300

1.50156700

3.60821200

3.65568800

3.33197900

4.41397000

$-0.15640800$

$-0.76923700$

$-1.21970300$

$-3.58156700$

$-1.22356100$

$-1.02398100$ 


$\begin{array}{lrrr}\mathrm{C} & 3.17478900 & 1.08320600 & -0.59241800 \\ \mathrm{C} & 4.12920200 & -2.35023800 & -1.61507500 \\ \mathrm{C} & 5.42283100 & -0.52561000 & -0.53286100 \\ \mathrm{C} & 4.39602100 & 1.63028400 & -0.18092600 \\ \mathrm{H} & 2.27413100 & 1.68736100 & -0.60497300 \\ \mathrm{C} & 5.29216600 & -3.10910600 & -1.55981400 \\ \mathrm{C} & 6.56807200 & -1.36031000 & -0.50965300 \\ \mathrm{C} & 5.50533400 & 0.82694000 & -0.10982000 \\ \mathrm{H} & 4.39877400 & 2.67641100 & 0.10321800 \\ \mathrm{C} & 6.49527300 & -2.64365300 & -0.99395600 \\ \mathrm{H} & 5.27214600 & -4.10132100 & -2.00203900 \\ \mathrm{H} & 7.49736200 & -0.95597700 & -0.11846700 \\ \mathrm{H} & 6.45800000 & 1.20857700 & 0.24592600 \\ \mathrm{H} & 7.36827600 & -3.28980600 & -0.97962900 \\ \mathrm{~N} & 3.05729700 & -0.20697600 & -0.95379500 \\ \mathrm{C} & 2.94410500 & -2.91014400 & -2.36402900 \\ \mathrm{H} & 2.08099800 & -3.08240600 & -1.72178100 \\ \mathrm{H} & 2.60667000 & -2.21397900 & -3.13840800 \\ \mathrm{H} & 3.23797600 & -3.84776000 & -2.84789200 \\ \mathrm{O} & 1.45560800 & 3.44598000 & 0.11780400 \\ \mathrm{O} & -0.93747300 & 3.02928500 & -0.81212800 \\ \mathrm{O} & -0.24984700 & 5.40826400 & -0.12920900 \\ \mathrm{C} & -0.72018900 & 5.47585500 & 1.21473000 \\ \mathrm{H} & -1.02029300 & 6.51110800 & 1.39691700 \\ \mathrm{H} & -1.58450700 & 4.81624900 & 1.36138700 \\ \mathrm{H} & 0.07193700 & 5.19679500 & 1.91942200 \\ \mathrm{P} & -2.28481000 & 3.96146000 & -0.67462600 \\ \mathrm{O} & 0.79283900 & 4.40817800 & -2.14922700 \\ \mathrm{C} & -3.16880500 & 4.80385200 & -3.13129800 \\ \mathrm{H} & -37945600 & 4.95702000 & -4.06414300 \\ \mathrm{H} & -0.92504800 & 4.02446000 & -3.27034900 \\ \mathrm{H} & -0.65667900 & 5.74042700 & -2.83860100 \\ \mathrm{C} & -2.98499600 & -0.07217400 & 1.28038100 \\ \mathrm{C} & -3.72423000 & -0.15959500 & 0.09860000 \\ \mathrm{C} & -2.91122600 & 1.12559400 & 1.99930500 \\ \mathrm{C} & -45247200 & 0.98570200 & -0.38665200 \\ \mathrm{H} & -1.09473200 & -0.44508000 \\ & 2.25137100 & 1.50364700\end{array}$




$\begin{array}{lrrr}\mathrm{H} & -2.33893500 & 1.16983000 & 2.91917300 \\ \mathrm{C} & -4.27216600 & 2.20803400 & 0.29525700 \\ \mathrm{H} & -4.90829200 & 0.92816100 & -1.31883900 \\ \mathrm{H} & -3.49957700 & 3.18640000 & 2.05395000 \\ \mathrm{C} & -4.88473100 & 3.45945600 & -0.28065100 \\ \mathrm{H} & -5.27826900 & 4.11457700 & 0.50408700 \\ \mathrm{H} & -4.12116900 & 4.02399000 & -0.83085500 \\ \mathrm{H} & -5.69782400 & 3.22914900 & -0.97644000\end{array}$

\section{PRO2}<smiles>[2H][C@@]12C=CC=CC=C1CN(S(=O)(=O)c1ccc(C)cc1)O[C@@H]2[C+]</smiles>

Zero-point correction=

0.458214 (Hartree/Particle)

Thermal correction to Energy=

0.494527

Thermal correction to Enthalpy=

0.495471

Thermal correction to Gibbs Free Energy=

0.380709

Sum of electronic and zero-point Energies= $-2395.764670$

Sum of electronic and thermal Energies= $-2395.728358$

Sum of electronic and thermal Enthalpies= $-2395.727413$

Sum of electronic and thermal Free Energies= $-2395.842175$

$\begin{array}{lrrr}\mathrm{C} & 0.94895600 & 1.64325100 & -2.85599300 \\ \mathrm{C} & 1.14969800 & 3.05527600 & -3.07322500 \\ \mathrm{H} & 0.13143700 & -0.02363800 & -1.86505900 \\ \mathrm{C} & 0.24876400 & 1.05701300 & -1.85895600 \\ \mathrm{C} & 0.30694000 & 4.06161100 & -2.69896100 \\ \mathrm{C} & -0.94956900 & 3.89083100 & -2.01548100 \\ \mathrm{C} & -1.27338100 & 2.86438200 & -1.19981100 \\ \mathrm{H} & 0.53259300 & 5.06567900 & -3.05235000 \\ \mathrm{H} & -1.71573100 & 4.63955200 & -2.22088900 \\ \mathrm{C} & -2.67162400 & 2.61016200 & -0.67291600 \\ \mathrm{H} & -3.40829700 & 2.43776800 & -1.46456000 \\ \mathrm{H} & -3.04584600 & 3.42455400 & -0.04263400\end{array}$


$-2.49863200$

$-3.82929600$

$-3.37948700$

$-4.94216500$

$-1.22696400$

$-0.30152700$

0.77886200

2.05976300

0.45271200

2.99286300

2.34855900

1.38955100

$-0.53331800$

2.66130400

3.97202500

1.12276000

3.38690100

$-0.90876100$

1.42488000

1.99926000

3.02811400

2.93137700

5.04492800

3.75996000

4.54168200

3.77018400

3.06132400

4.47269200

3.22271100

1.12932900

5.92573600

6.62668700

5.36713500

6.48010500

$-4.07388600$

$-4.93671800$

$-3.42390100$

$-5.13613400$
1.38733300

0.71078100

0.56948100

1.58313400

0.89899500

1.82271000

2.35764500

1.78814000

3.35016300

2.20045200

1.03826300

3.76944400

3.80748900

3.19101500

1.73039100

4.54443900

3.51180100

$-0.13839500$

0.97647000

3.32499000

$-2.01321400$

$-1.19167600$

$-0.83627600$

$-1.76325100$

$-3.12408500$

$-4.16530500$

$-4.58809600$

$-4.94367500$

$-3.79408000$

$-1.00256000$

$-0.44918100$

0.27599400

0.01058700

$-1.31604700$

$-0.89875300$

$-1.02487200$

$-2.00700700$

$-2.28675500$
0.12911800

1.04733500

2.42507000

0.67484800

0.14998100

$-0.67091900$

0.29298200

0.32214900

1.22690400

1.28059400

$-0.41010900$

2.17140500

1.21453900

2.20489900

1.29493800

2.88502000

2.94734900

0.74101000

$-3.57283900$

$-3.69762700$

1.24050000

$-1.21568200$

0.28075800

$-0.09057900$

$-0.54544800$

$-1.14363700$

$-0.42088900$

$-1.45250600$

$-2.01723000$

1.01845200

$-0.77829700$

$-0.35724900$

$-1.60102100$

$-1.15386900$

0.32569700

$-0.76617400$

0.87498700

$-1.32072800$ 


$\begin{array}{lrrr}\mathrm{H} & -5.45146700 & -0.15489900 & -1.15959700 \\ \mathrm{C} & -3.64045400 & -3.25860000 & 0.30446000 \\ \mathrm{H} & -2.76682300 & -1.88404000 & 1.72743100 \\ \mathrm{C} & -4.49425000 & -3.41953800 & -0.79784800 \\ \mathrm{H} & -5.80569900 & -2.39490900 & -2.16995300 \\ \mathrm{H} & -3.13871800 & -4.12655100 & 0.72433000 \\ \mathrm{C} & -4.73998200 & -4.78774900 & -1.38617500 \\ \mathrm{H} & -5.06267700 & -4.72420900 & -2.42999000 \\ \mathrm{H} & -5.52663300 & -5.31495100 & -0.83042600 \\ \mathrm{H} & -3.83973400 & -5.40935600 & -1.34262500\end{array}$

PRO3 + Silver cat.<smiles>O=C(C(=O)N([13F])Cc1ccccc1)c1ccccc1</smiles>

Zero-point correction=

Thermal correction to Energy=

Thermal correction to Enthalpy=

Thermal correction to Gibbs Free Energy=

Sum of electronic and zero-point Energies=

Sum of electronic and thermal Energies=

Sum of electronic and thermal Enthalpies=

Sum of electronic and thermal Free Energies=

0.365842 (Hartree/Particle)
0.390898
0.391842
0.305953
-1603.194415
-1603.169360
-1603.168415
-1603.254305

$\begin{array}{lrrr}\mathrm{C} & -4.19306300 & -2.24922100 & 0.16758000 \\ \mathrm{C} & -4.10922400 & -2.74910000 & -1.13192000 \\ \mathrm{H} & -3.09686200 & -1.61515000 & 1.91106000 \\ \mathrm{C} & -3.02832900 & -2.00453400 & 0.89793200 \\ \mathrm{C} & -2.85688900 & -3.00754600 & -1.69549700 \\ \mathrm{C} & -1.69463600 & -2.76802200 & -0.96391100 \\ \mathrm{C} & -1.77016100 & -2.25854000 & 0.34072400 \\ \mathrm{H} & -2.78555400 & -3.40399200 & -2.70492900 \\ \mathrm{H} & -0.72184500 & -2.98059800 & -1.39796200 \\ \mathrm{C} & -0.51305700 & -2.01319700 & 1.14886600 \\ \mathrm{H} & -0.75074400 & -1.81522200 & 2.19443000 \\ \mathrm{H} & 0.15018300 & -2.87991500 & 1.10384400 \\ \mathrm{~N} & 0.31071400 & -0.87036800 & 0.66349600 \\ \mathrm{~S} & 0.08517200 & 0.67418200 & 1.41736000\end{array}$




$\begin{array}{lrrr}\mathrm{O} & 1.29944900 & 1.43268700 & 1.11776100 \\ \mathrm{O} & -0.30172600 & 0.39549600 & 2.80101400 \\ \mathrm{C} & 1.37558000 & -1.15025400 & -0.18465100 \\ \mathrm{C} & 2.17258500 & -0.03095500 & -0.88138000 \\ \mathrm{C} & 3.62126600 & 0.08544200 & -0.59552400 \\ \mathrm{C} & 4.36522400 & 1.02048200 & -1.33215400 \\ \mathrm{C} & 4.25411000 & -0.68346600 & 0.39206100 \\ \mathrm{C} & 5.72380700 & 1.18288400 & -1.08439700 \\ \mathrm{H} & 3.85582000 & 1.60875600 & -2.08828600 \\ \mathrm{C} & 5.61546400 & -0.51813900 & 0.63771300 \\ \mathrm{H} & 3.68994900 & -1.41829700 & 0.95696500 \\ \mathrm{C} & 6.35037200 & 0.41367100 & -0.09855300 \\ \mathrm{H} & 6.29677800 & 1.90838500 & -1.65506100 \\ \mathrm{H} & 6.10314500 & -1.11642500 & 1.40194200 \\ \mathrm{H} & 7.41199500 & 0.54153200 & 0.09572600 \\ \mathrm{O} & 1.64415600 & -2.29577300 & -0.52032800 \\ \mathrm{O} & 1.60680100 & 0.59057700 & -1.76861500 \\ \mathrm{H} & -5.16323000 & -2.04960200 & 0.61498000 \\ \mathrm{H} & -5.01420200 & -2.94159300 & -1.70221100 \\ \mathrm{C} & -1.32494500 & 1.40021100 & 0.59041300 \\ \mathrm{C} & -1.29596800 & 1.63010900 & -0.78848400 \\ \mathrm{C} & -2.41568500 & 1.78418400 & 1.37054400 \\ \mathrm{C} & -2.39262000 & 2.24578100 & -1.38226100 \\ \mathrm{H} & -0.43850000 & 1.32794300 & -1.38203000 \\ \mathrm{C} & -3.49979700 & 2.40582300 & 0.75204300 \\ \mathrm{H} & -2.40938900 & 1.60072100 & 2.43907000 \\ \mathrm{C} & -3.50861800 & 2.64257100 & -0.62774200 \\ \mathrm{H} & -2.38081200 & 2.42475100 & -2.45458400 \\ \mathrm{H} & -4.35155200 & 2.71112600 & 1.35437200 \\ \mathrm{C} & -4.69331200 & 3.29441700 & -1.29888900 \\ \mathrm{H} & -5.37730900 & 3.73544900 & -0.56760800 \\ \mathrm{H} & -4.37568400 & 4.08509100 & -1.98795800 \\ \mathrm{H} & -5.26199600 & 2.56322100 & -1.88772600\end{array}$

\section{PRO6}

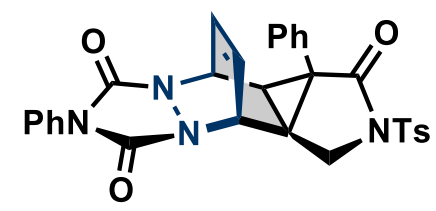


Zero-point correction=

Thermal correction to Energy=

Thermal correction to Enthalpy=

Thermal correction to Gibbs Free Energy=

Sum of electronic and zero-point Energies=

Sum of electronic and thermal Energies=

Sum of electronic and thermal Enthalpies=

Sum of electronic and thermal Free Energies=
0.413128 (Hartree/Particle)

0.440504

0.441448

0.353939

$-1919.385843$

$-1919.358466$

$-1919.357522$

$-1919.445032$

1.24883600

0.21053500

1.07539700

$-1.04812000$

$-1.14491000$

$-0.36086500$

$-0.99237100$

$-0.25264800$

$-1.93432900$

0.28305700

$-0.24269200$

$-2.16169600$

$-0.60620900$

$-0.19584800$

$-0.99300600$

$-1.62458000$

$-2.43555400$

$-1.67739800$

$-1.74772200$

$-1.80994800$

0.09117900

$-2.89271400$

$-1.53415900$

$-0.05313700$

$-4.02887700$

$-2.83034900$

$-0.32954300$

$-3.19310800$

$-3.99909900$

$-0.96979600$

$-3.24984100$

$-2.44100600$

0.47144100

$-2.05049600$

$-0.23805500$

0.22564900

$-2.42746200$

0.67904800

$-0.10713200$

$-2.75454200$

2.09049000

$-1.41977900$

$-2.55512500$

3.04093000

0.91104700

$-3.17548000$

3.74729200

$-1.71339300$

$-2.67697900$

1.71350900

$-2.21513400$

$-2.97626800$

4.33883800

0.61902300

$-2.32668700$

2.76072200

1.93590400

$-3.28395100$

4.69637000

$-0.69451900$

$-3.42190000$

4.01523600

$-2.73720400$

$-3.06845300$

5.06849200

1.41896300

$-3.61173700$

5.70675800

$-0.92287800$

$-4.31917800$

0.08815800

0.94318200 


$\begin{array}{lrrc}\mathrm{H} & -1.06601000 & -0.50108100 & 1.87503100 \\ \mathrm{H} & 0.61109900 & 1.32237000 & 2.27253900 \\ \mathrm{H} & 0.83473200 & 3.10233300 & 0.49349600 \\ \mathrm{C} & 2.65215300 & -0.60177400 & -0.93773500 \\ \mathrm{C} & 2.81839900 & 0.17093700 & 1.23620700 \\ \mathrm{O} & 2.92458200 & -0.89229200 & -2.08362800 \\ \mathrm{O} & 3.25498600 & 0.65374000 & 2.26088000 \\ \mathrm{~N} & 1.36558900 & -0.53991700 & -0.39273500 \\ \mathrm{~N} & 1.46963500 & -0.05811200 & 0.95287300 \\ \mathrm{~N} & 3.53145000 & -0.30773900 & 0.12119500 \\ \mathrm{C} & 4.95622200 & -0.39946700 & 0.03236300 \\ \mathrm{C} & 5.76494800 & 0.54411500 & 0.67495600 \\ \mathrm{C} & 5.52965600 & -1.44549700 & -0.69934100 \\ \mathrm{C} & 7.15196800 & 0.42819400 & 0.58634100 \\ \mathrm{H} & 5.31294500 & 1.34218700 & 1.24909800 \\ \mathrm{C} & 6.91716600 & -1.53886500 & -0.78939900 \\ \mathrm{H} & 4.89627800 & -2.16412600 & -1.20457500 \\ \mathrm{C} & 7.73346600 & -0.60731300 & -0.14580300 \\ \mathrm{H} & 7.77792500 & 1.15834000 & 1.09172900 \\ \mathrm{H} & 7.35867300 & -2.34904000 & -1.36288200 \\ \mathrm{H} & 8.81464000 & -0.68808300 & -0.21488400 \\ \mathrm{C} & -4.69518600 & -2.92807900 & 1.61266600 \\ \mathrm{H} & -5.11965100 & -1.95426300 & 1.85977400 \\ \mathrm{H} & -5.46753400 & -3.70047000 & 1.58585900 \\ \mathrm{H} & -3.89146400 & -3.20944800 & 2.29474800\end{array}$

\section{Silver cat.}

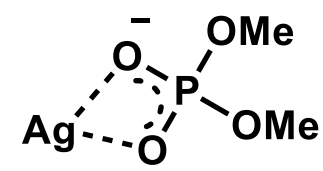

Zero-point correction=

Thermal correction to Energy=

0.095673 (Hartree/Particle)

Thermal correction to Enthalpy=

0.106281

Thermal correction to Gibbs Free Energy=

0.107225

Sum of electronic and zero-point Energies=

0.056945

Sum of electronic and thermal Energies=

$-867.821429$

$-867.810820$

Sum of electronic and thermal Enthalpies=

$-867.809876$

Sum of electronic and thermal Free Energies=

$-867.860157$ 


$\begin{array}{lrrr}\mathrm{O} & 0.05661300 & 0.16504100 & -1.37948000 \\ \mathrm{O} & -0.21832600 & -0.16325200 & 1.13913300 \\ \mathrm{O} & -1.86231100 & -1.29714300 & -0.55213300 \\ \mathrm{P} & -0.92337400 & -0.02963700 & -0.22147800 \\ \mathrm{O} & -2.04946300 & 1.14124600 & -0.15573000 \\ \mathrm{C} & -1.65320200 & 2.44105800 & 0.29050800 \\ \mathrm{H} & -0.92538900 & 2.88174400 & -0.40106900 \\ \mathrm{H} & -2.55509400 & 3.05704900 & 0.30373500 \\ \mathrm{H} & -1.22427000 & 2.39134900 & 1.29714200 \\ \mathrm{Ag} & 1.85260300 & -0.11219100 & 0.07209000 \\ \mathrm{C} & -2.80937300 & -1.73960500 & 0.42970400 \\ \mathrm{H} & -3.23697000 & -2.67018700 & 0.05060700 \\ \mathrm{H} & -2.31386100 & -1.92192900 & 1.38883600 \\ \mathrm{H} & -3.60280400 & -0.99635500 & 0.55910300\end{array}$

\section{PTAD}

Zero-point correction=

Thermal correction to Energy=

Thermal correction to Enthalpy=

Thermal correction to Gibbs Free Energy=

Sum of electronic and zero-point Energies=

Sum of electronic and thermal Energies=

Sum of electronic and thermal Enthalpies=

Sum of electronic and thermal Free Energies=

C

C

$\mathrm{O}$

$\mathrm{O}$

$\mathrm{N}$

$\mathrm{N}$

$\mathrm{N}$

C

C

C

C
1.57972700

1.57976600

1.28788400

1.28793500

2.99067000

2.99066700

0.76440000

$-0.66895700$

$-1.35769900$

$-1.35774300$

$-2.75182900$
$-1.10229800$

1.10228600

$-2.24084200$

2.24086900

$-0.60678900$

0.60672000

$-0.00000800$

0.00001700

1.09234500

$-1.09231200$

1.08703200
0.124941 (Hartree/Particle)

0.134687

0.135632

0.088653

$-622.397460$

$-622.387714$

$-622.386770$

$-622.433748$

0.23592100

$-0.23592500$

0.47100300

$-0.47085900$

0.13212600

$-0.13214000$

$-0.00009000$

$-0.00004700$

0.53473900

$-0.53482400$

0.52522800 


$\begin{array}{lrrc}\mathrm{H} & -0.81321600 & 1.93659900 & 0.94010200 \\ \mathrm{C} & -2.75186300 & -1.08702800 & -0.52520700 \\ \mathrm{H} & -0.81328500 & -1.93656100 & -0.94022500 \\ \mathrm{C} & -3.45207900 & 0.00000400 & 0.00003900 \\ \mathrm{H} & -3.28896000 & 1.93696500 & 0.93584300 \\ \mathrm{H} & -3.28901600 & -1.93696800 & -0.93577600 \\ \mathrm{H} & -4.53818700 & 0.00001500 & 0.00008700\end{array}$

\section{8-methylquinoline}

Zero-point correction=

0.163854 (Hartree/Particle)

Thermal correction to Energy=

0.172200

Thermal correction to Enthalpy=

0.173144

Thermal correction to Gibbs Free Energy=

0.130705

Sum of electronic and zero-point Energies=

Sum of electronic and thermal Energies=

Sum of electronic and thermal Enthalpies=

Sum of electronic and thermal Free Energies=

$-441.085894$

$-441.077549$

$-441.076604$

$-441.119043$

$\begin{array}{lrrr}\mathrm{C} & 2.18953300 & 1.18466000 & -0.00001200 \\ \mathrm{C} & 0.02316800 & 0.40506500 & -0.00000300 \\ \mathrm{C} & 2.73096900 & -0.12425400 & 0.00000200 \\ \mathrm{H} & 2.86089700 & 2.04381000 & -0.00002000 \\ \mathrm{C} & 0.46542000 & -0.95711800 & 0.00000100 \\ \mathrm{C} & -1.37869900 & 0.69737300 & -0.00001200 \\ \mathrm{C} & 1.86394400 & -1.19138700 & 0.00000800 \\ \mathrm{H} & 3.80746200 & -0.26635300 & -0.00000500 \\ \mathrm{C} & -0.49186100 & -2.00530900 & -0.00000500 \\ \mathrm{C} & -2.26903200 & -0.35859800 & -0.00000300 \\ \mathrm{H} & 2.23169800 & -2.21517300 & 0.00000900 \\ \mathrm{C} & -1.83353900 & -1.70665300 & 0.00000100 \\ \mathrm{H} & -0.14762700 & -3.03691600 & -0.00000100 \\ \mathrm{H} & -3.33649300 & -0.15094000 & 0.00000500 \\ \mathrm{H} & -2.57130200 & -2.50449600 & 0.00001000 \\ \mathrm{~N} & 0.89900300 & 1.45235800 & 0.00001300 \\ \mathrm{C} & -1.84439600 & 2.13000600 & 0.00000400 \\ \mathrm{H} & -1.46651300 & 2.66839800 & 0.87608400 \\ \mathrm{H} & -1.46652400 & 2.66840500 & -0.87607500\end{array}$




\section{$\mathrm{N}$-oxide 4}

Zero-point correction=

0.168373 (Hartree/Particle)

Thermal correction to Energy=

0.177425

Thermal correction to Enthalpy=

0.178369

Thermal correction to Gibbs Free Energy=

0.134264

Sum of electronic and zero-point Energies=

$-516.238145$

Sum of electronic and thermal Energies=

Sum of electronic and thermal Enthalpies=

Sum of electronic and thermal Free Energies=

$-516.229093$

$-516.228149$

$-516.272254$

$\mathrm{O}$

$0.95035800 \quad 2.31208200$

0.00009300

C

2.34902300

0.52368700

0.00001200

C

$-0.02396100$

0.17859300

$-0.00005100$

C

2.59394100

$-0.85074100$

0.00002100

$\mathrm{H}$

3.12022200

1.28186300

0.00007300

C

0.21638700

$-1.23348700$

$-0.00003000$

C

$-1.35718600$

0.69646400

$-0.00001100$

C

1.54274500

$-1.73562100$

$-0.00002300$

$\mathrm{H}$

3.62493800

$-1.18978500$

0.00007100

C

$-0.89327900$

$-2.11705800$

0.00000400

C

$-2.39066900$

$-0.23042700$

0.00003300

$\mathrm{H}$

1.70454500

$-2.80889600$

$-0.00000300$

C

$-2.17394400$

$-1.62264200$

0.00003700

$-0.70305900$

$-3.18685500$

0.00000900

$\mathrm{H}$

$-3.41173300$

0.14111400

0.00005500

$\mathrm{H}$

$-3.02503200$

$-2.29799700$

0.00007000

$\mathrm{N}$

1.09633600

1.04156400

$-0.00009200$

$-1.72049600$

2.16481100

$-0.00003500$

$\mathrm{H}$

$-1.31542100$

2.68341100

0.87165900

$\mathrm{H}$

$-1.31570800$

2.68330800

$-0.87192000$

$\mathrm{H}$

$-2.81133100$

2.26475400

0.00014600 
The molecular structure optimizations in Figure 2 were carried out using the hybrid density functional method based on B3LYP functional and the LANL2DZ basis set for $\mathrm{Ag}, \mathrm{Br}$, and the $6-31 \mathrm{G}^{*}$ basis set for $\mathrm{H}, \mathrm{C}, \mathrm{N}, \mathrm{O}, \mathrm{P}$, and $\mathrm{S}$. The vibrational frequencies were computed at the same level to check whether each optimized structure is at an energy minimum (no imaginary frequency) or a transition state (one imaginary frequency) and to evaluate its zero-point vibrational energy (ZPVE) and thermal corrections at $298.15 \mathrm{~K}$. Single point energies were calculated at the RM06 level using the LanL2TZ(f) basis set for Ag and the cc-pVTZ basis set for $\mathrm{H}$, $\mathrm{C}, \mathrm{N}, \mathrm{O}, \mathrm{P}, \mathrm{S}$ and $\mathrm{Br}$ in chlorobenzene solvent.

\begin{tabular}{|c|c|c|}
\hline & $\begin{array}{c}\text { E(rB3LYP/LANL2DZ(Ag)/6- } \\
\text { 31G*) (A.U.) }\end{array}$ & $\begin{array}{c}\mathrm{E}(\mathrm{rM06} / \mathrm{LanL2TZ} \text { (f)(Ag)/ cc- } \\
\text { pVTZ) (A.U.) }\end{array}$ \\
\hline $\mathrm{TS}_{\text {MAJOR }}$ & -3685.968348 & -6245.657178 \\
\hline $\mathrm{TS}_{\text {MINOR }}$ & -3685.961349 & -6245.652716 \\
\hline
\end{tabular}

$\mathrm{TS}_{\text {MAJOR }}$

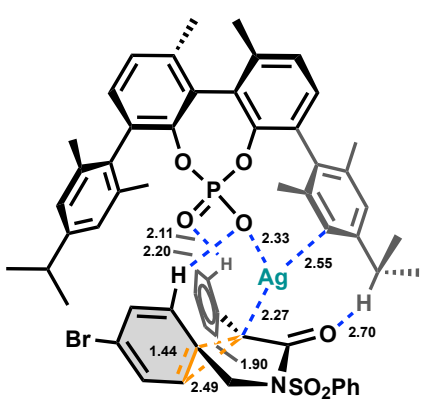

Zero-point correction= 1.004083 (Hartree/Particle) 
Thermal correction to Energy=

Thermal correction to Enthalpy=

Thermal correction to Gibbs Free Energy=

Sum of electronic and zero-point Energies=

Sum of electronic and thermal Energies=

Sum of electronic and thermal Enthalpies=

Sum of electronic and thermal Free Energies=
1.071039

1.071983

0.898430

$-3684.964266$

$-3684.897309$

$-3684.896365$

$-3685.069919$
$\mathrm{Ag}$

$\mathrm{O}$

O

$\mathrm{P}$

O

O

C

C

C

C

C

C

$\mathrm{H}$

C

C

C

C

C

C

$\mathrm{H}$

C

C

C

C

C

C

$\mathrm{H}$

$\mathrm{H}$

C

C
1.21775800

$-0.78517200$

$-1.88006000$

$-1.94224100$

$-2.28760600$

$-3.26287500$

$-4.50546600$

$-4.93036700$

$-5.27172500$

$-6.17492200$

$-6.53183600$

$-6.96359300$

$-7.15936900$

$-2.69899500$

$-4.02275500$

$-1.77752000$

$-4.41249500$

$-2.21375100$

$-3.50057300$

$-1.52523500$

$-4.73669000$

$-4.58650700$

$-4.35392200$

$-4.05628100$

$-3.83790300$

$-3.67279800$

$-3.94547200$

$-3.54029900$

$-0.37724100$

$-0.10177100$
$-1.13236800$

$-0.99201400$

0.35810100

$-0.75261400$

$-2.13632200$

$-0.69383400$

$-0.84718300$

$-2.14776200$

0.29728800

$-2.29421200$

0.11248300

$-1.15470900$

0.98032000

$-3.25088700$

$-3.29722100$

$-4.28580700$

$-4.41574300$

$-5.41292900$

$-5.46507800$

$-6.23823300$

1.65362000

2.06252800

2.51503000

3.32584500

3.77632500

4.19973600

3.62839200

4.43871300

$-4.12507300$

$-4.11854100$
0.10647300

$-1.07198800$

0.88316500

$-0.11459300$

0.73159600

$-1.09933100$

$-0.50327300$

$-0.16982900$

$-0.26933900$

0.47847500

0.31193500

0.68766800

0.49364600

0.02105300

$-0.45739200$

$-0.17372800$

$-1.22436400$

$-0.87796500$

$-1.40273600$

$-1.03532200$

$-0.58584000$

$-1.92581000$

0.46056500

$-2.19955800$

0.14240900

$-1.17796200$

$-3.23826000$

0.95164800

0.31310600

1.69521200 
C

C

C

C

$\mathrm{H}$

$\mathrm{H}$

$\mathrm{H}$

$\mathrm{H}$

C

$\mathrm{H}$

$\mathrm{H}$

$\mathrm{H}$

C

$\mathrm{H}$

$\mathrm{H}$

$\mathrm{H}$

C

$\mathrm{H}$

$\mathrm{H}$

$\mathrm{H}$

C

$\mathrm{H}$

$\mathrm{H}$

$\mathrm{H}$

C

C

C

C

C

C

$\mathrm{H}$

$\mathrm{H}$

C

$\mathrm{H}$

$\mathrm{H}$

N

$S$

O

$\begin{array}{rrr}0.65699300 & -3.87108700 & -0.61166900 \\ 1.19191900 & -3.82583000 & 2.14180600 \\ 1.94602700 & -3.56694100 & -0.12796900 \\ 2.23108900 & -3.52630600 & 1.25387500 \\ 1.38051100 & -3.81343400 & 3.21121600 \\ 2.75010700 & -3.38232300 & -0.83686200 \\ -3.80534900 & -6.33008300 & -1.98644800 \\ -7.92680900 & -1.26777500 & 1.17909200 \\ -5.75683300 & -4.50206600 & -1.90789900 \\ -6.43739200 & -5.18127000 & -1.38057500 \\ -6.24322000 & -3.52631100 & -1.97172100 \\ -5.63733100 & -4.89206700 & -2.92443400 \\ -6.66628100 & -3.62142600 & 1.00689600 \\ -7.12402800 & -3.48769200 & 1.99288500 \\ -7.43039700 & -4.06112700 & 0.35460500 \\ -5.85491400 & -4.34641900 & 1.10314400 \\ -1.19784300 & -4.36797900 & 2.70042400 \\ -1.84653300 & -3.48791400 & 2.77861200 \\ -1.82986200 & -5.21155600 & 2.40479700 \\ -0.78120500 & -4.57490900 & 3.69082800 \\ -4.41954000 & 2.07958300 & 1.90426400 \\ -5.42404900 & 1.76098900 & 2.19992000 \\ -3.73918600 & 1.23547900 & 2.05904600 \\ -4.11330900 & 2.89657800 & 2.56562400 \\ 1.80299400 & 4.03170600 & 0.44068000 \\ 0.42374400 & 3.87130100 & 0.61548600 \\ 2.47263800 & 3.09857200 & -0.32565100 \\ -0.32345700 & 2.87000900 & -0.03028900 \\ 0.35530000 & 1.94082000 & -0.78206700 \\ 1.79257200 & 1.93520600 & -0.82765400 \\ -1.39521800 & 2.79082600 & 0.10184700 \\ -0.18149600 & 1.11658600 & -1.24172100 \\ 2.50527300 & 1.12744300 & -1.90386000 \\ 2.72306100 & 1.72798000 & -2.78806500 \\ 1.87791100 & 0.27690000 & -2.19831500 \\ 3.76767600 & 0.69012900 & -1.28432200 \\ 4.70399800 & -0.41995400 & -2.20672800 \\ 4.41856800 & -0.04421400 & -3.59372100\end{array}$


Supporting information

\begin{tabular}{|c|c|c|c|}
\hline O & 4.43820700 & -1.78168500 & -1.73353800 \\
\hline C & 3.65863500 & 0.47619100 & 0.11778800 \\
\hline C & 2.31629100 & 0.79659100 & 0.60417700 \\
\hline C & 2.04805400 & 1.29503500 & 1.94780200 \\
\hline$C$ & 0.73655000 & 1.22703600 & 2.46320500 \\
\hline C & 3.06341000 & 1.86244900 & 2.74998600 \\
\hline C & 0.45261400 & 1.71226500 & 3.73945200 \\
\hline $\mathrm{H}$ & -0.06812800 & 0.80697700 & 1.86424000 \\
\hline C & 2.77754100 & 2.32118600 & 4.02828700 \\
\hline $\mathrm{H}$ & 4.07752700 & 1.90360200 & 2.36848000 \\
\hline C & 1.46727500 & 2.25863100 & 4.52318300 \\
\hline $\mathrm{H}$ & -0.56568200 & 1.65926400 & 4.11207200 \\
\hline $\mathrm{H}$ & 3.57104000 & 2.73591800 & 4.64357500 \\
\hline $\mathrm{H}$ & 1.24640800 & 2.63398500 & 5.51853000 \\
\hline 0 & 4.56401900 & -0.02365800 & 0.77753800 \\
\hline $\mathrm{H}$ & 3.54492000 & 3.17761600 & -0.47283200 \\
\hline $\mathrm{H}$ & 2.32614800 & 4.85390400 & 0.91281900 \\
\hline C & 6.38540000 & 0.02021400 & -1.82003400 \\
\hline C & 7.04462500 & -0.62425300 & -0.77340700 \\
\hline C & 7.00913200 & 0.98696200 & -2.61006100 \\
\hline C & 8.36850600 & -0.27728500 & -0.51054500 \\
\hline $\mathrm{H}$ & 6.52232800 & -1.36276400 & -0.18016000 \\
\hline C & 8.33252800 & 1.32456600 & -2.33149600 \\
\hline $\mathrm{H}$ & 6.46913000 & 1.44540700 & -3.43073900 \\
\hline C & 9.00837600 & 0.69464600 & -1.28370200 \\
\hline $\mathrm{H}$ & 8.90011000 & -0.76650300 & 0.29974700 \\
\hline $\mathrm{H}$ & 8.83668300 & 2.07212600 & -2.93623800 \\
\hline C & 0.39563700 & -3.84012500 & -2.09798800 \\
\hline $\mathrm{H}$ & 1.30570300 & -3.56803000 & -2.64113400 \\
\hline $\mathrm{H}$ & 0.05221900 & -4.81062600 & -2.47055700 \\
\hline $\mathrm{H}$ & -0.37811800 & -3.10048300 & -2.32505400 \\
\hline C & -4.97296300 & 1.14670200 & -3.06159700 \\
\hline $\mathrm{H}$ & -4.26622600 & 0.31309800 & -3.14239900 \\
\hline $\mathrm{H}$ & -5.96715800 & 0.71298100 & -2.90761200 \\
\hline $\mathrm{H}$ & -4.97680800 & 1.68506300 & -4.01448300 \\
\hline C & -3.10005000 & 5.57347000 & -1.48375300 \\
\hline $\mathrm{H}$ & -2.83083300 & 6.03385600 & -0.52413700 \\
\hline C & 3.64241000 & -3.21322500 & 1.72456700 \\
\hline
\end{tabular}




$\begin{array}{lrrr}\mathrm{H} & 4.14377800 & -2.68536200 & 0.90528100 \\ \mathrm{C} & -4.14477800 & 6.47717100 & -2.16061500 \\ \mathrm{H} & -3.74066500 & 7.48248400 & -2.33031900 \\ \mathrm{H} & -4.44616100 & 6.06740200 & -3.13172100 \\ \mathrm{H} & -5.04470600 & 6.56669900 & -1.54296000 \\ \mathrm{C} & -1.81946600 & 5.48633200 & -2.33100500 \\ \mathrm{H} & -2.02549700 & 5.04885000 & -3.31463800 \\ \mathrm{H} & -1.39237800 & 6.48346800 & -2.49143300 \\ \mathrm{H} & -1.06372100 & 4.86328700 & -1.84220100 \\ \mathrm{C} & 4.41166900 & -4.52061100 & 1.99026800 \\ \mathrm{H} & 4.43186000 & -5.15606300 & 1.09823700 \\ \mathrm{H} & 5.44642700 & -4.30676800 & 2.28310600 \\ \mathrm{H} & 3.94071400 & -5.09104300 & 2.80004000 \\ \mathrm{C} & 3.67743500 & -2.29274800 & 2.95243000 \\ \mathrm{H} & 3.12159400 & -1.36853700 & 2.77258500 \\ \mathrm{H} & 3.25851300 & -2.78315800 & 3.83905500 \\ \mathrm{H} & 4.71148500 & -2.01547100 & 3.18159600 \\ \mathrm{Br} & -0.50368500 & 5.12041100 & 1.77579800 \\ \mathrm{H} & 10.04028200 & 0.95917100 & -1.07170700\end{array}$

$\mathrm{TS}_{\text {MINOR }}$

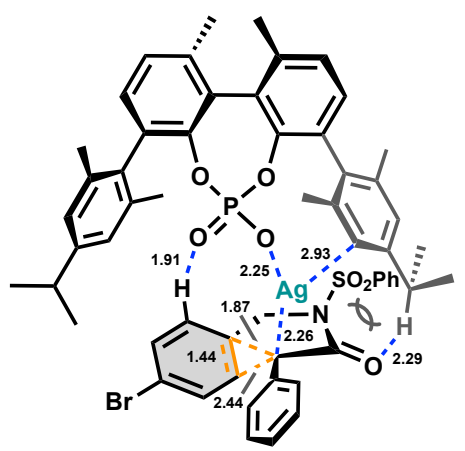

Zero-point correction=

Thermal correction to Energy=

1.004233 (Hartree/Particle)

Thermal correction to Enthalpy=

1.071110

Thermal correction to Gibbs Free Energy=

1.072054

Sum of electronic and zero-point Energies=

0.897583

Sum of electronic and thermal Energies=

$-3684.957115$

$-3684.890239$

Sum of electronic and thermal Enthalpies=

$-3684.889295$

Sum of electronic and thermal Free Energies=

$-3685.063765$ 


$\begin{array}{lrrr}\mathrm{C} & 3.79854700 & 2.64692200 & 0.80017000 \\ \mathrm{C} & 2.95121200 & 3.48778900 & 0.07656600 \\ \mathrm{C} & 3.26960000 & 1.46857000 & 1.29435400 \\ \mathrm{C} & 1.57179200 & 3.23744800 & -0.07556100 \\ \mathrm{C} & 1.05818900 & 2.05381300 & 0.39209900 \\ \mathrm{C} & 1.93066700 & 1.05272900 & 0.95928600 \\ \mathrm{H} & 0.93474400 & 3.96428900 & -0.56397600 \\ \mathrm{H} & -0.00188700 & 1.80940000 & 0.30209500 \\ \mathrm{C} & 1.34007300 & -0.07036400 & 1.81015500 \\ \mathrm{H} & 1.31327800 & 0.19578300 & 2.86753700 \\ \mathrm{H} & 0.32382400 & -0.30171000 & 1.47993600 \\ \mathrm{~N} & 2.25547700 & -1.20196300 & 1.60878500 \\ \mathrm{~S} & 2.60844900 & -2.32013500 & 2.84347400 \\ \mathrm{O} & 1.77746100 & -1.88665100 & 3.96703700 \\ \mathrm{O} & 2.53205700 & -3.67418900 & 2.30742500 \\ \mathrm{C} & 2.94288700 & -1.18629300 & 0.38621000 \\ \mathrm{C} & 2.47753500 & -0.05562000 & -0.44339900 \\ \mathrm{C} & 3.34552300 & 0.56689000 & -1.44882800 \\ \mathrm{C} & 2.77186100 & 1.40122700 & -2.42880100 \\ \mathrm{C} & 4.74254200 & 0.37076000 & -1.47804000 \\ \mathrm{C} & 3.55571000 & 2.01711400 & -3.40210400 \\ \mathrm{H} & 1.69702400 & 1.55715800 & -2.41962200 \\ \mathrm{C} & 5.52016600 & 0.96626200 & -2.46349200 \\ \mathrm{H} & 5.19820700 & -0.28193500 & -0.74323000 \\ \mathrm{C} & -2.93298300 & 1.80062700 & -3.42227800 \\ \mathrm{H} & 3.08990300 & 2.65881400 & -4.14369200 \\ \mathrm{H} & 6.59126400 & 0.78640600 & -2.48524200 \\ \mathrm{H} & 5.54828000 & 2.27361800 & -4.18206800 \\ \mathrm{Ag} & 0.56354800 & -0.79560800 & -1.39113100 \\ \mathrm{O} & 3.75767100 & -2.04127500 & 0.05743500 \\ \mathrm{O} & -1.60749000 & -0.27071500 & -1.67523800 \\ \mathrm{O} & -1.54329800 & 0.76256000 & 0.73138200 \\ \mathrm{P} & -2.21497900 & -0.10088000 & -0.29188700 \\ \mathrm{O} & -3.79113000 & 0.28368800 & -0.55376100 \\ \mathrm{O} & -.40711100 & -1.57193600 & 0.44030800 \\ \mathrm{C} & & -2.45927200 & -0.07478700 \\ \mathrm{C} & -2.24302400 & 0.20227900 \\ \mathrm{C} & -3.52702400 & -0.84651600\end{array}$


C

C

C

$\mathrm{H}$

C

C

C

C

C

C

$\mathrm{H}$

C

C

C

C

C

C

$\mathrm{H}$

$\mathrm{H}$

C

C

C

C

C

C

$\mathrm{H}$

$\mathrm{H}$

$\mathrm{H}$

$\mathrm{H}$

C

$\mathrm{H}$

$\mathrm{H}$

$\mathrm{H}$

C

$\mathrm{H}$

$\mathrm{H}$

$\mathrm{H}$

C
$-5.63891700$

$-3.81767900$

$-5.17191100$

$-3.48489200$

$-4.62072900$

$-5.09121400$

$-4.93122500$

$-5.87698700$

$-5.77578700$

$-6.22371600$

$-6.05190400$

$-1.43839700$

$-1.11531900$

$-0.41346800$

0.23126900

0.92189400

1.26922700

0.46709200

1.70755900

$-4.33021000$

$-3.33443900$

$-4.71746300$

$-2.73707500$

$-4.09648000$

$-3.09795500$

$-1.96184500$

$-4.39806100$

3.89892100

4.83886200

$-2.86684800$

$-3.67465700$

$-2.07148100$

$-2.47351800$

$-2.17763600$

$-2.92432600$

$-1.72661400$

$-2.69946600$

4.31362200
$-3.14600800$

$-4.44854700$

$-4.25553800$

$-5.29904800$

0.22622900

$-1.03316900$

1.42314500

$-1.08945000$

1.34088600

0.10933800

2.25404300

$-3.58464400$

$-3.26027200$

$-3.86316000$

$-3.23472200$

$-3.83243900$

$-3.53051900$

$-2.99264400$

$-4.04569200$

2.71166900

3.32981400

3.28427000

4.50265000

4.46262500

5.08263300

4.96466000

4.90133300

0.79235400

2.90620900

2.71926100

2.63625400

3.32729200

1.71504900

$-2.85529700$

$-3.64001400$

$-2.61766500$

$-1.96361800$

$-1.93399000$
$-0.33973700$

$-1.30519500$

$-1.05804000$

$-1.89339400$

0.56231600

0.98324300

1.21343400

2.15364700

2.32677300

2.79114600

2.84603300

$-1.26288300$

$-2.59682500$

$-0.33541400$

$-2.98482200$

$-0.75939400$

$-2.08573900$

$-4.01715800$

$-0.04056300$

0.76170400

1.54729500

$-0.46311500$

1.08104700

$-0.89031800$

$-0.13912100$

1.68845300

$-1.83948300$

1.86574900

0.95200700

2.84651500

3.58122800

3.29043500

2.66327400

$-3.58940000$

$-3.74419000$

$-4.55787700$

$-3.22794300$

3.20666600 
Supporting information

C

C

C

$\mathrm{H}$

C

$\mathrm{H}$

C

$\mathrm{H}$

$\mathrm{H}$

$\mathrm{H}$

$\mathrm{H}$

C

$\mathrm{H}$

$\mathrm{H}$

$\mathrm{H}$

C

$\mathrm{H}$

$\mathrm{H}$

$\mathrm{H}$

C

$\mathrm{H}$

$\mathrm{H}$

$\mathrm{H}$

C

$\mathrm{H}$

C

$\mathrm{H}$

$\mathrm{H}$

$\mathrm{H}$

C

$\mathrm{H}$

C

$\mathrm{H}$

$\mathrm{H}$

$\mathrm{H}$

C

$\mathrm{H}$

$\mathrm{H}$
$5.33148900 \quad-2.59607000$

$4.58117300 \quad-0.93142500$

$6.65344900-2.23155200$

$5.08144600-3.36279700$

$5.90768900-0.57750600$

$3.76379000-0.46066800$

$6.94024300-1.22451900$

$7.45944100-2.73638700$

6.13518200

$-5.89500400$

$-6.83822400$

$-7.13051500$

$-7.37670600$

$-7.57573200$

$-7.61755100$

$-6.30726100$

$-7.36948500$

$-6.16522500$

$-5.73347800$

$-5.77115100$

$-5.37357600$

$-6.64317700$

$-6.10837300$

$-2.41877800$

$-2.88336900$

$-0.73353900$

$-1.08167600$

$-1.53541600$

0.14849100

2.71660600

3.33958100

$-0.92043700$

$-0.44511000$

$-0.39942200$

$-0.77582200$

$-2.62839100$

$-2.17133700$

$-2.17562500$

0.19376600

$-4.95938800$

0.06680800

$-2.93487600$

$-1.90044200$

$-3.57815500$

$-3.18322300$

$-2.39398100$

$-2.60069000$

$-2.35212500$

$-3.23963500$

2.63543800

1.73432100

2.33034200

3.31943300

6.34534200

6.60662700

$-4.16378300$

$-3.25785500$

$-4.90564100$

$-4.53155000$

$-3.62998700$

$-3.50502300$

2.51968400

4.14256700

2.77603100

1.79825800

4.38509000

4.67795000

3.70017300

2.25238300

5.11474800

$-1.46259100$

3.68709700

$-0.22793900$

0.02177000

0.54046500

$-1.17695200$

2.78236800

2.60462500

3.86767600

2.39710400

$-1.32713800$

$-1.80770100$

$-0.73853800$

$-2.11225600$

$-0.64138500$

$-1.60149100$

1.10774100

1.61686500

1.19065000

1.63628500

$-2.53898700$

$-1.64907600$

$6.10946900-0.90166600$

$7.00621700-1.31678100$

$5.85629400 \quad 0.02952500$

$5.28462900-1.60928100$

$7.52941700 \quad 0.31704900$

$7.33120400 \quad 1.29348800$

$8.44341000 \quad-0.08587200$ 
Supporting information

$\begin{array}{lrrr}\mathrm{H} & -3.69493700 & 7.71596100 & 0.48049700 \\ \mathrm{C} & 3.12725400 & -2.54437800 & -3.54326200 \\ \mathrm{H} & 4.20103400 & -2.61063100 & -3.75107700 \\ \mathrm{H} & 2.60117600 & -2.65058700 & -4.49935800 \\ \mathrm{H} & 2.92599900 & -1.54222000 & -3.15342000 \\ \mathrm{C} & 2.97878700 & -5.03373200 & -3.11758200 \\ \mathrm{H} & 4.03255300 & -5.14656900 & -3.39962400 \\ \mathrm{H} & 2.73332500 & -5.81206800 & -2.38728800 \\ \mathrm{H} & 2.36682300 & -5.20661100 & -4.01134700 \\ \mathrm{Br} & 3.68757900 & 5.10181900 & -0.70897500 \\ \mathrm{H} & 7.97231500 & -0.94698900 & 3.89370200\end{array}$


11 Charts of $1 \mathrm{H}-$ and 13C-NMR Spectra

(R)-6-Bromo-2-((4-bromophenyl)sulfonyl)-8a-phenyl-3,8adihydrocyclohepta[c]pyrrol-1(2H)-one (2a)

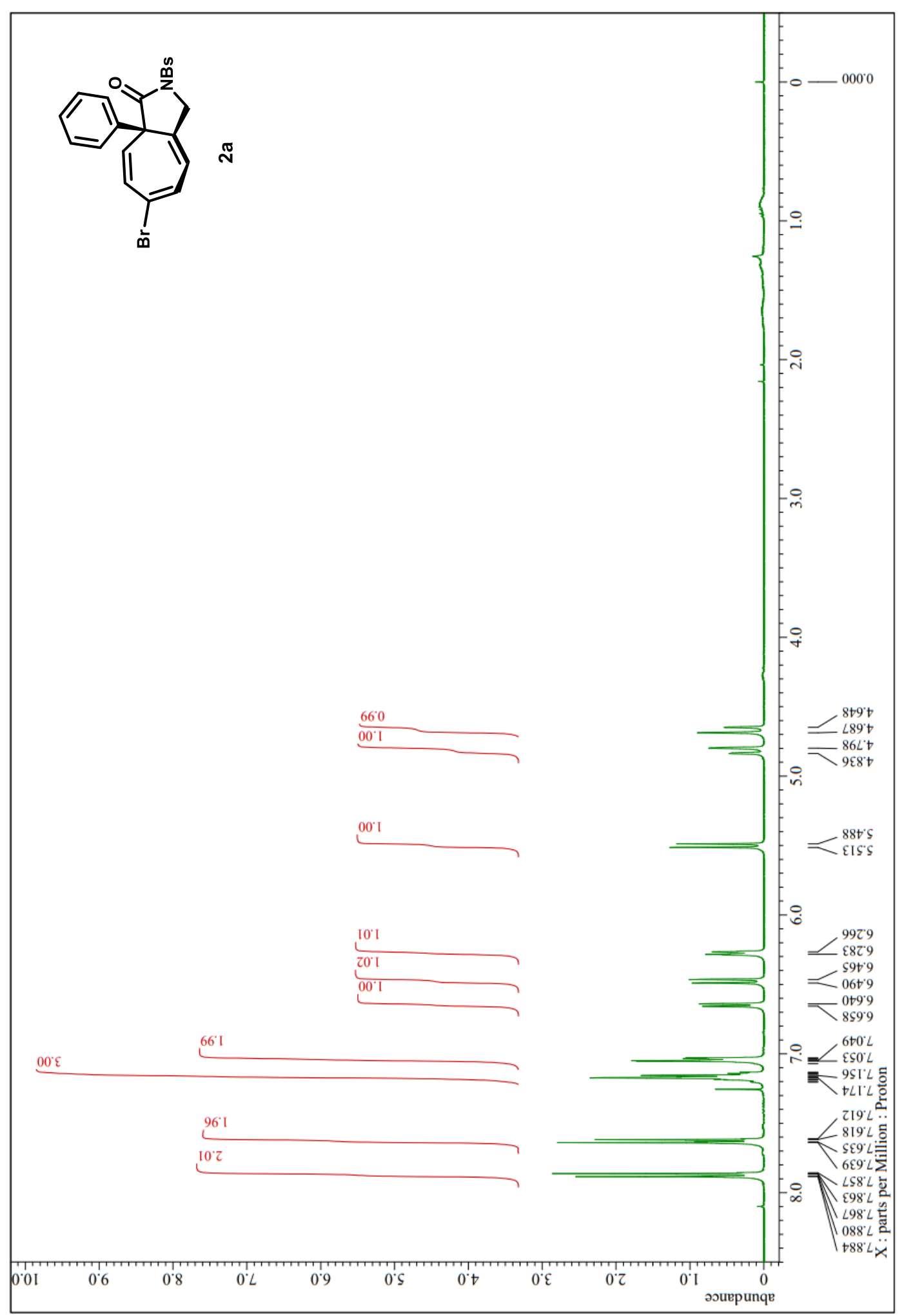


Supporting information

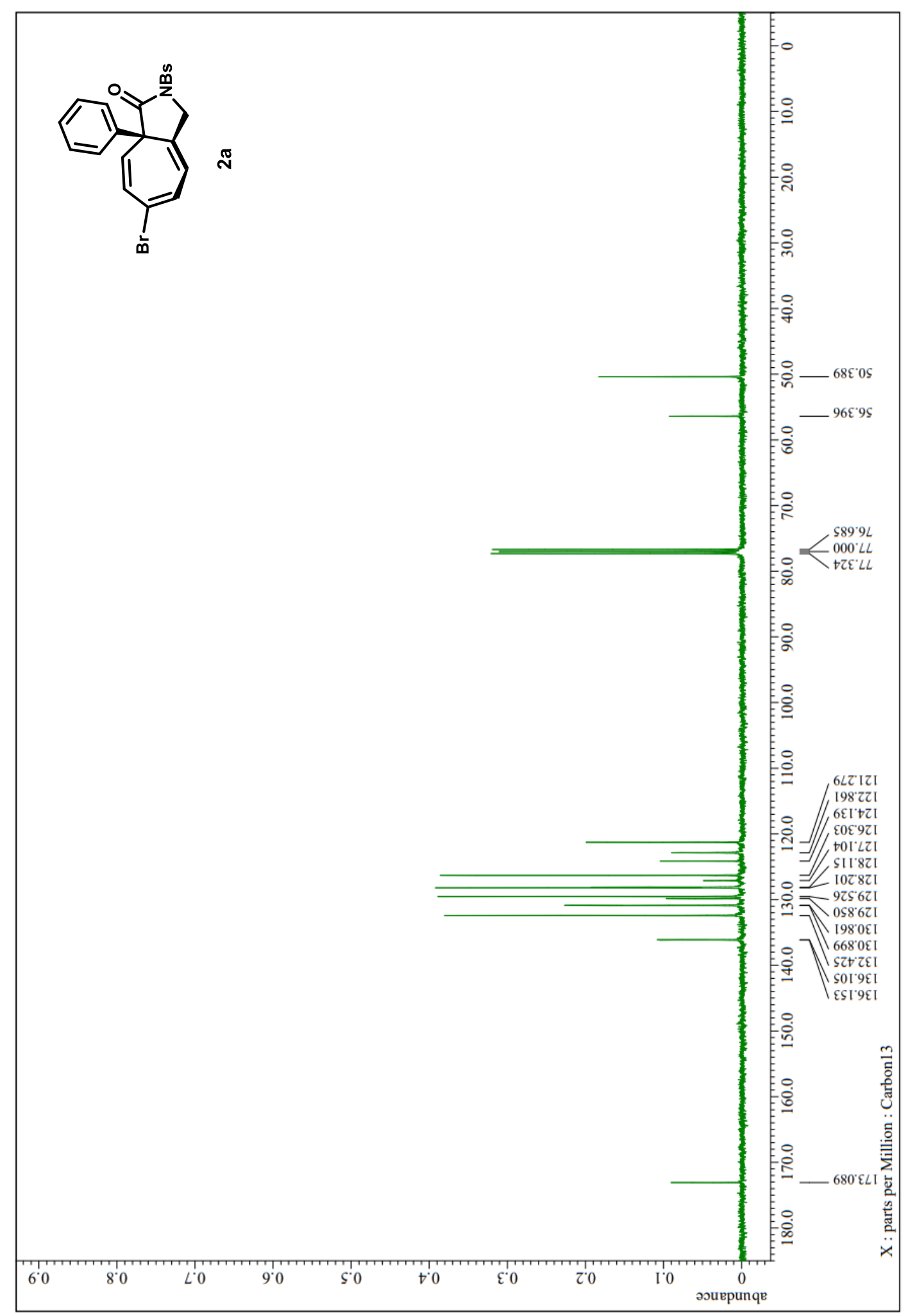


Supporting information

Methyl (R)-4-(6-bromo-2-((4-bromophenyl)sulfonyl)-3-oxo-2,3-

dihydrocyclohepta[c]pyrrol-3a(1H)-yl)benzoate (2b)

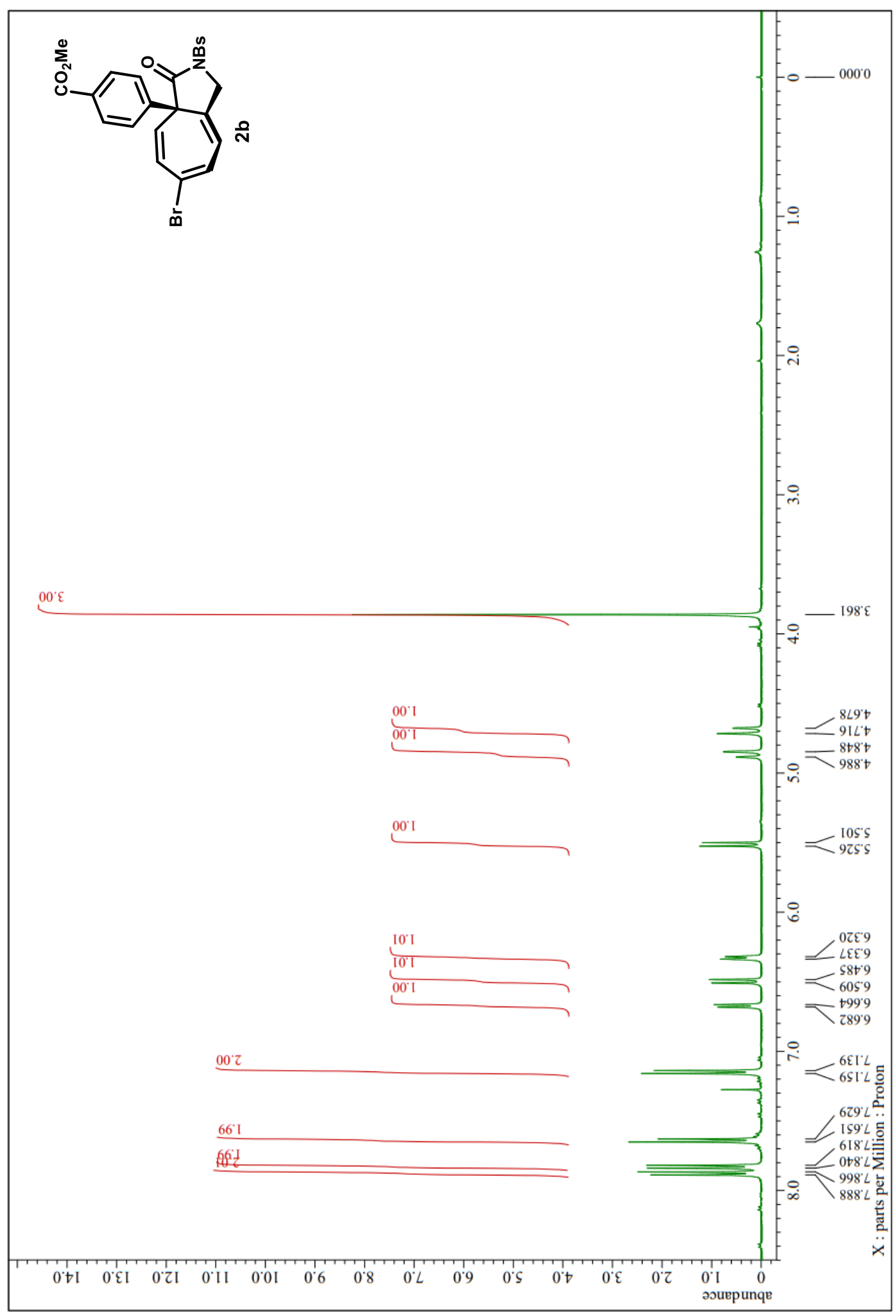


Supporting information

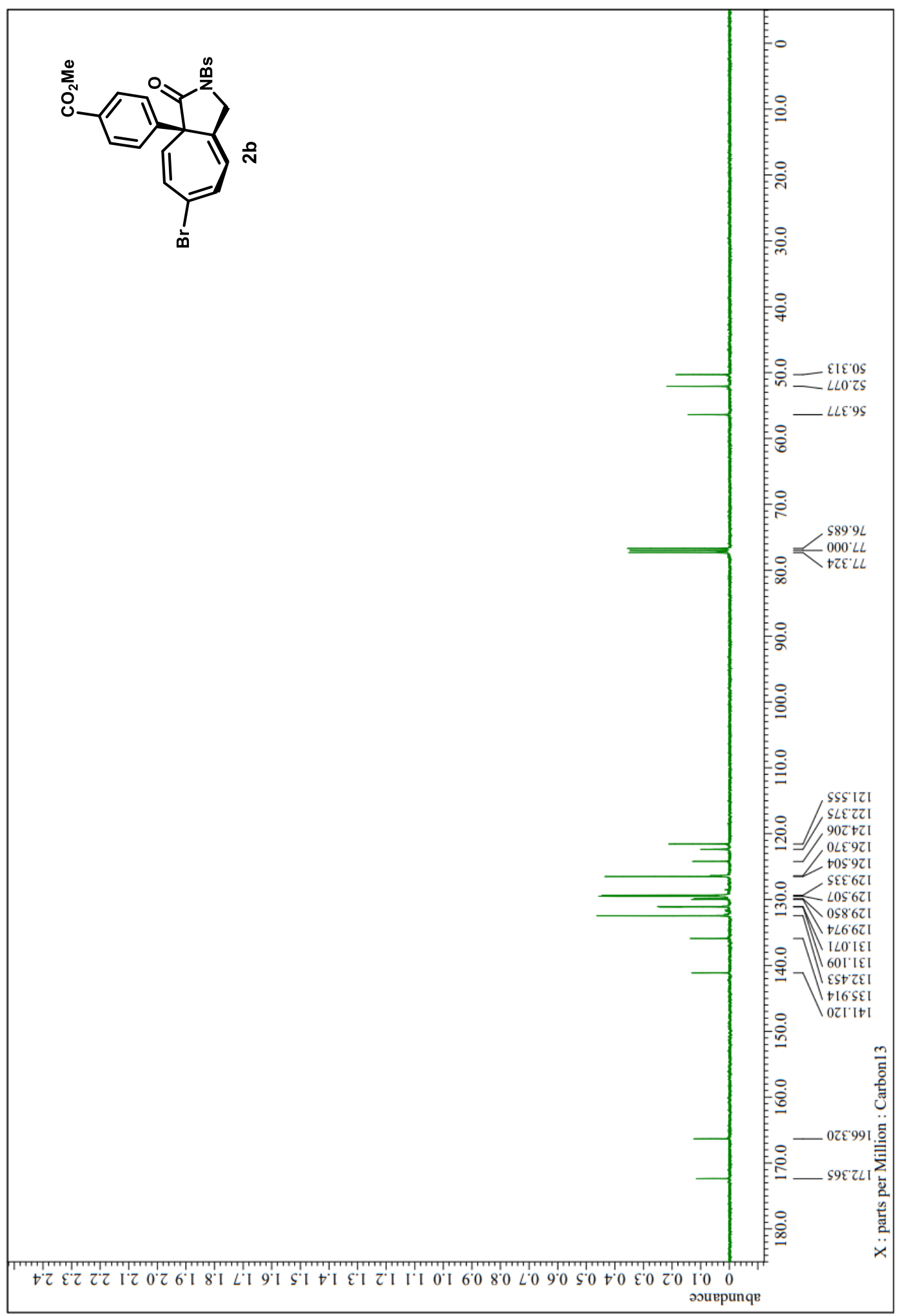


(R)-6-Bromo-8a-(4-bromophenyl)-2-((4-bromophenyl)sulfonyl)-3,8adihydrocyclohepta[c]pyrrol-1(2H)-one (2c)

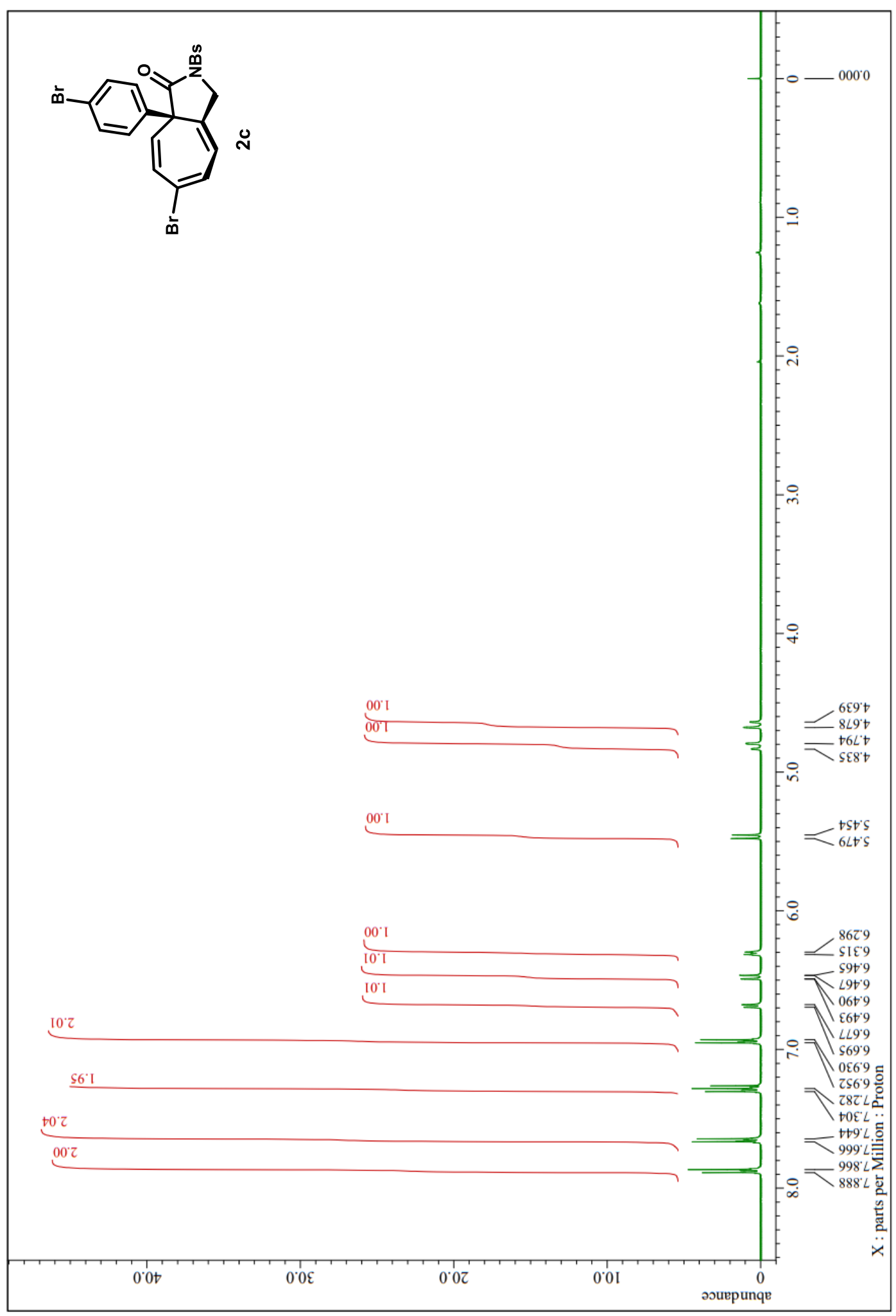


Supporting information

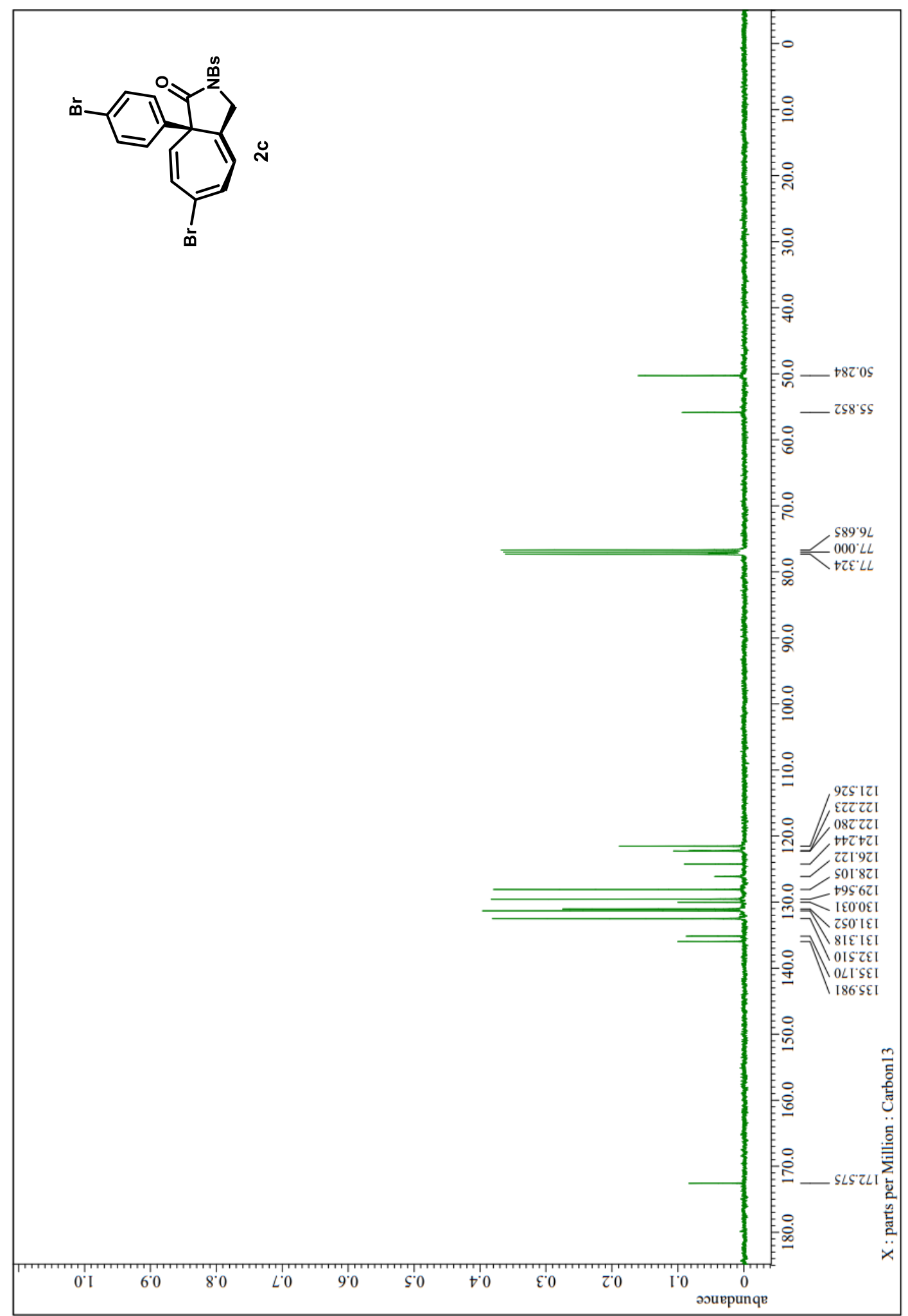


(R)-6-Bromo-2-((4-bromophenyl)sulfonyl)-8a-(3-methoxyphenyl)-3,8adihydrocyclohepta[c]pyrrol-1(2H)-one (2d)

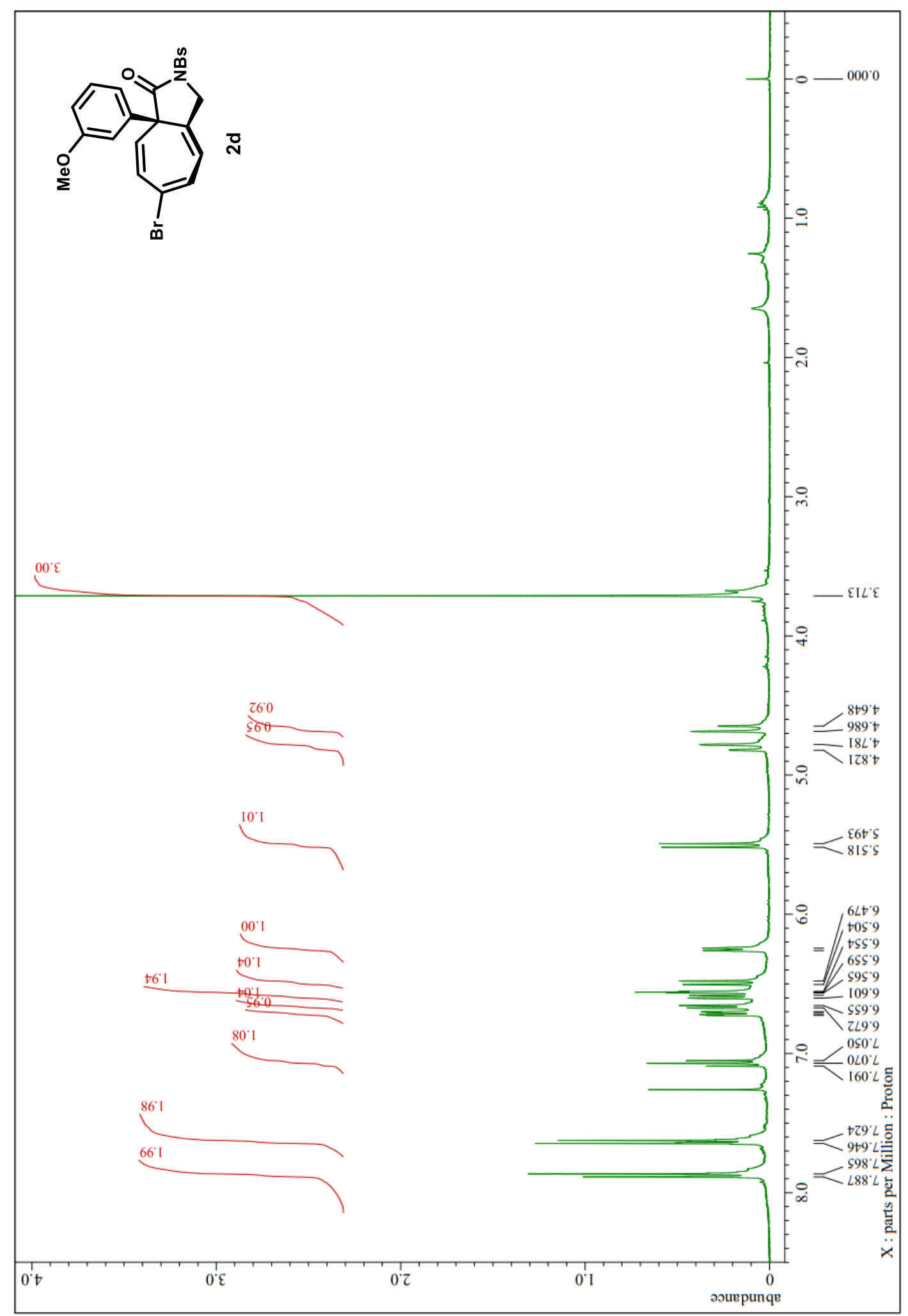


Supporting information

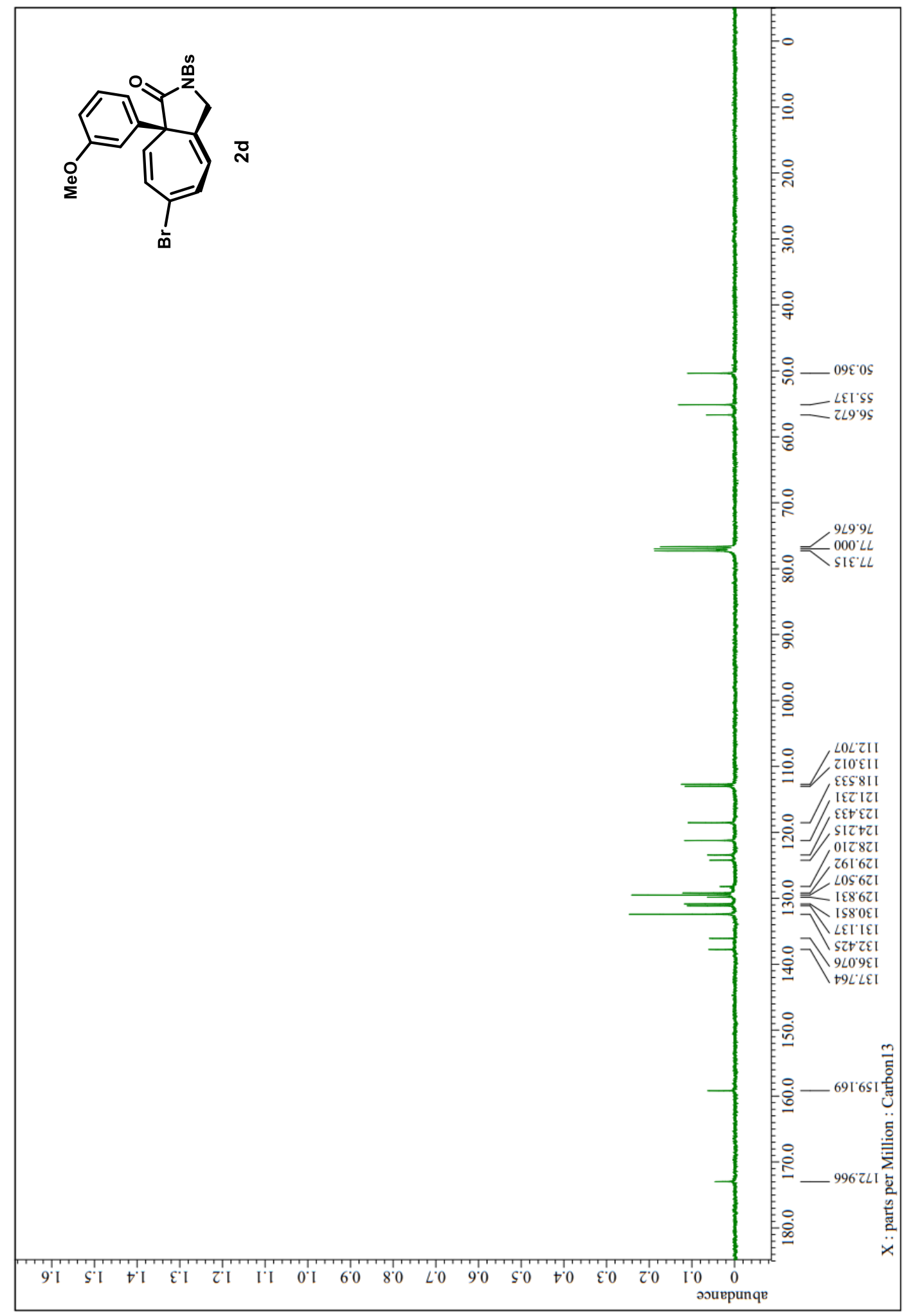


(R)-6-Bromo-8a-(3-methoxyphenyl)-2-tosyl-3,8a-dihydrocyclohepta[c]pyrrol$1(2 H)$-one (2e)

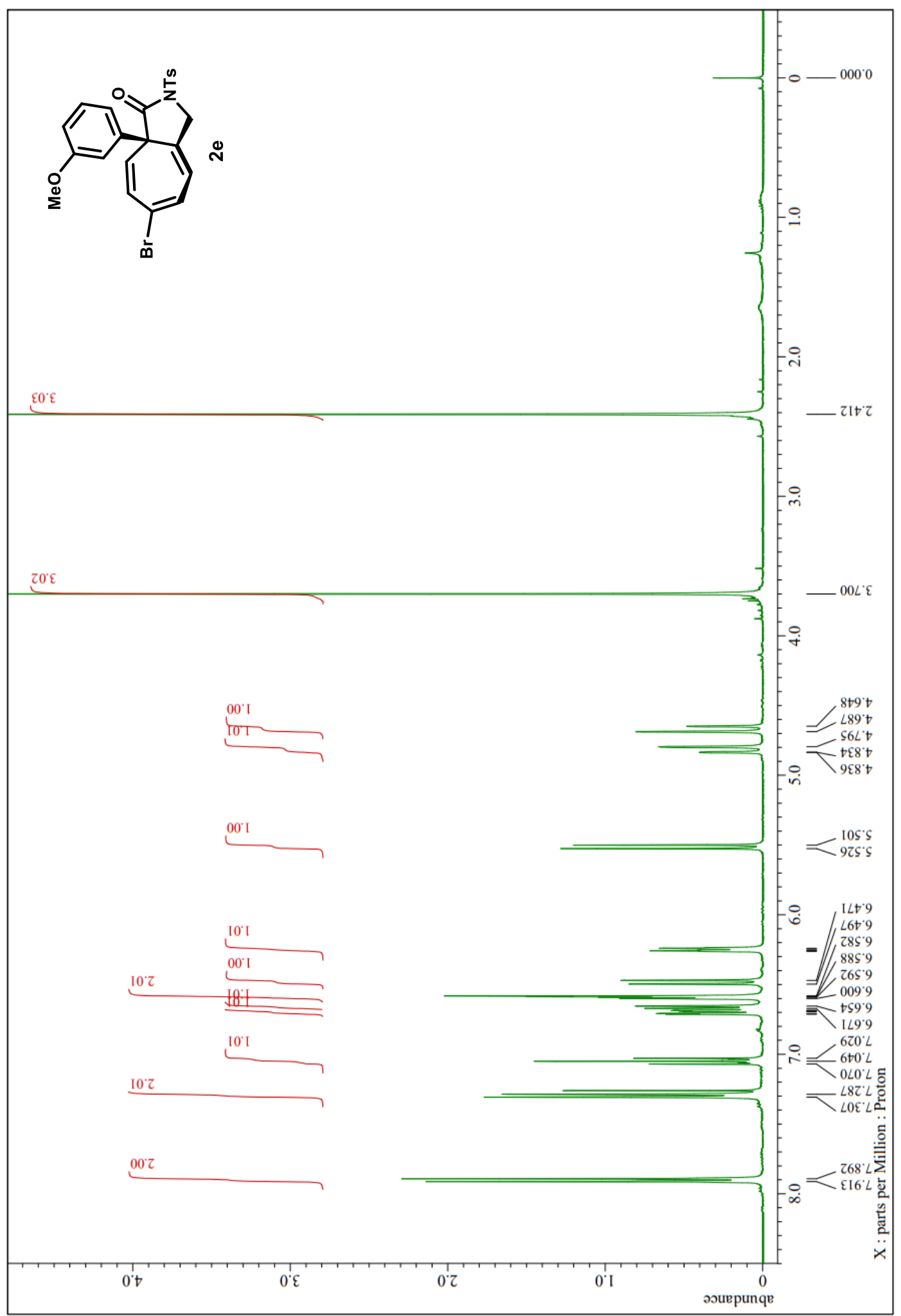


Supporting information

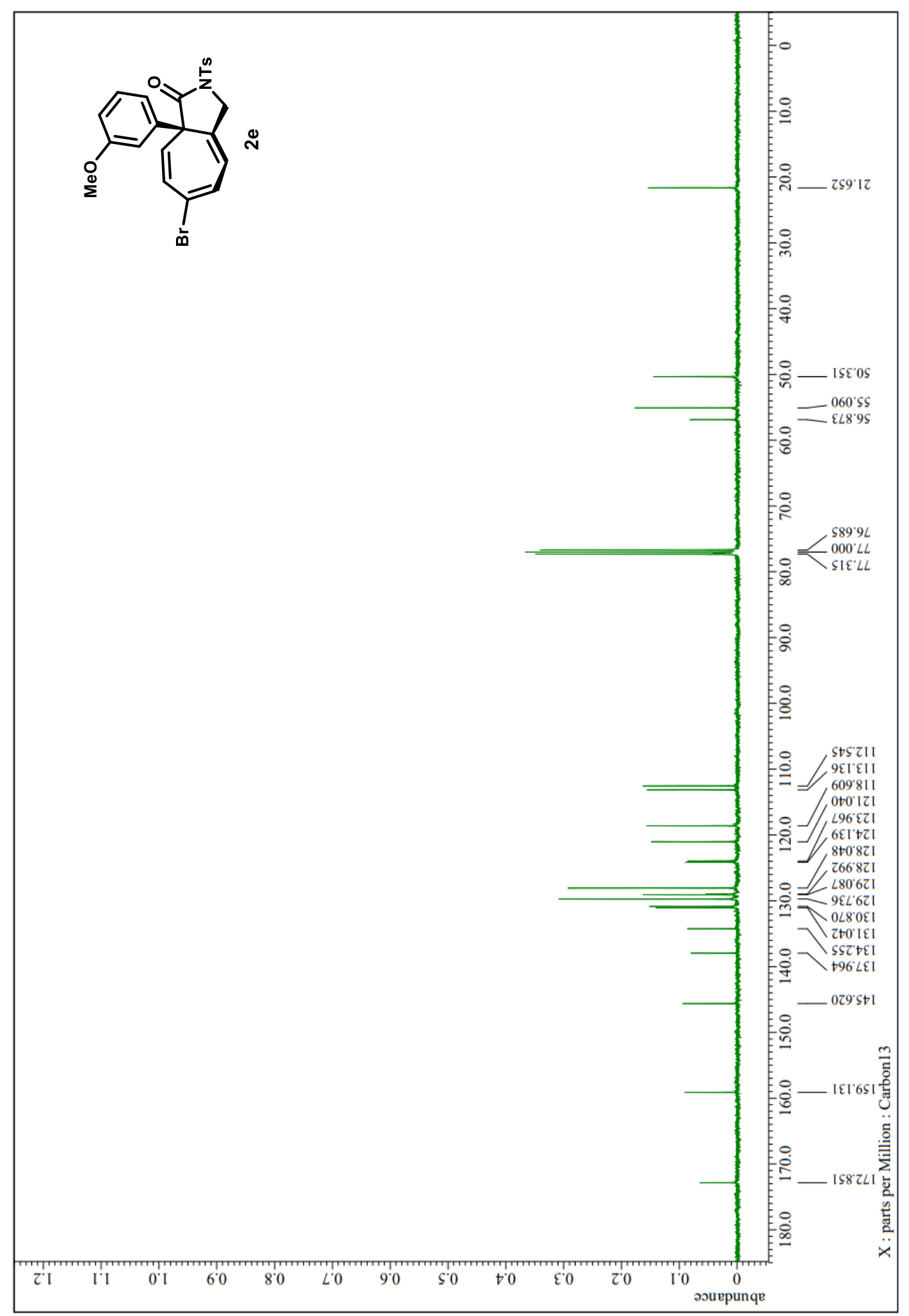


(S)-6-Bromo-2-((4-bromophenyl)sulfonyl)-8a-(2-fluorophenyl)-3,8adihydrocyclohepta[c]pyrrol-1(2H)-one (2f)

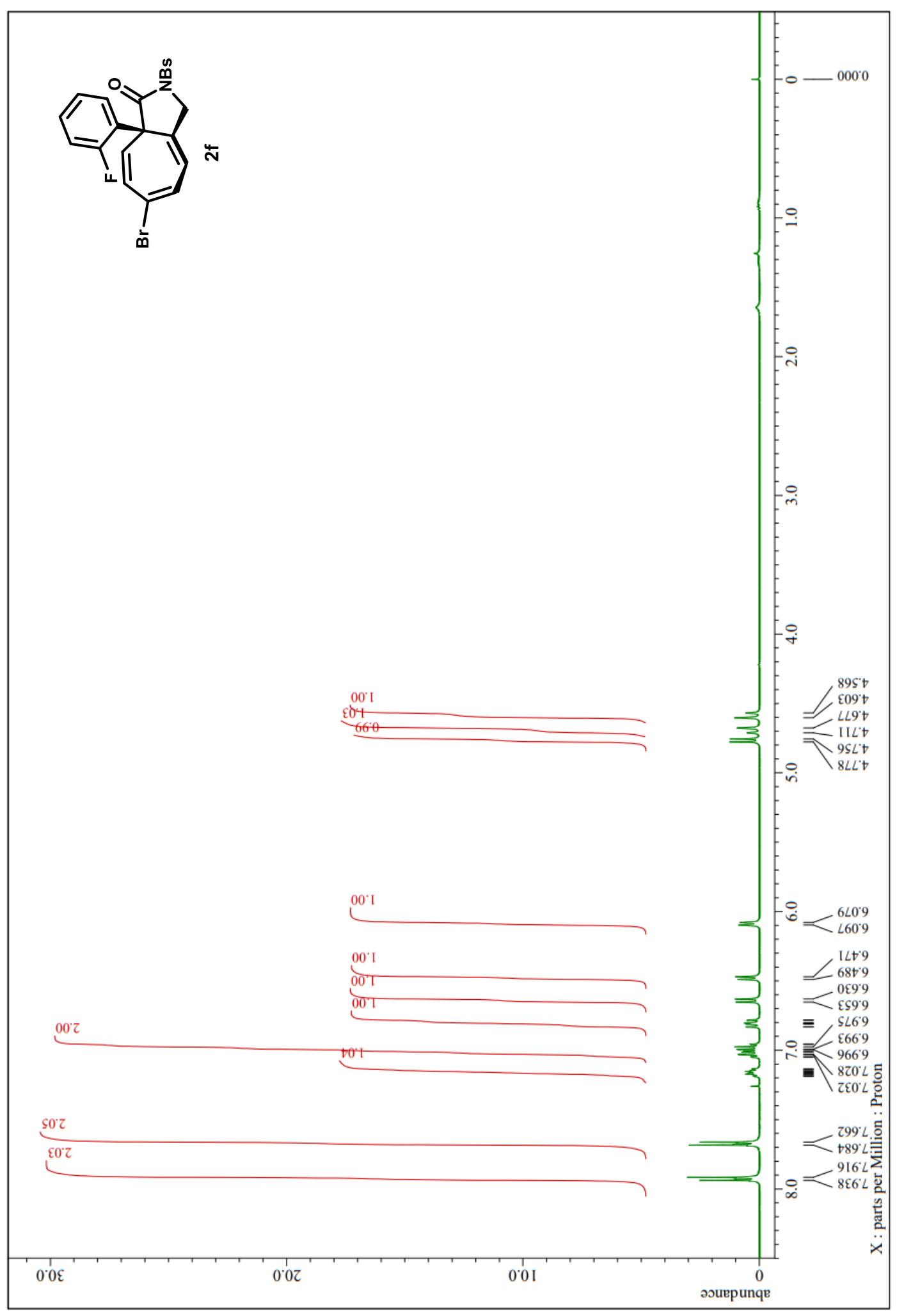


Supporting information

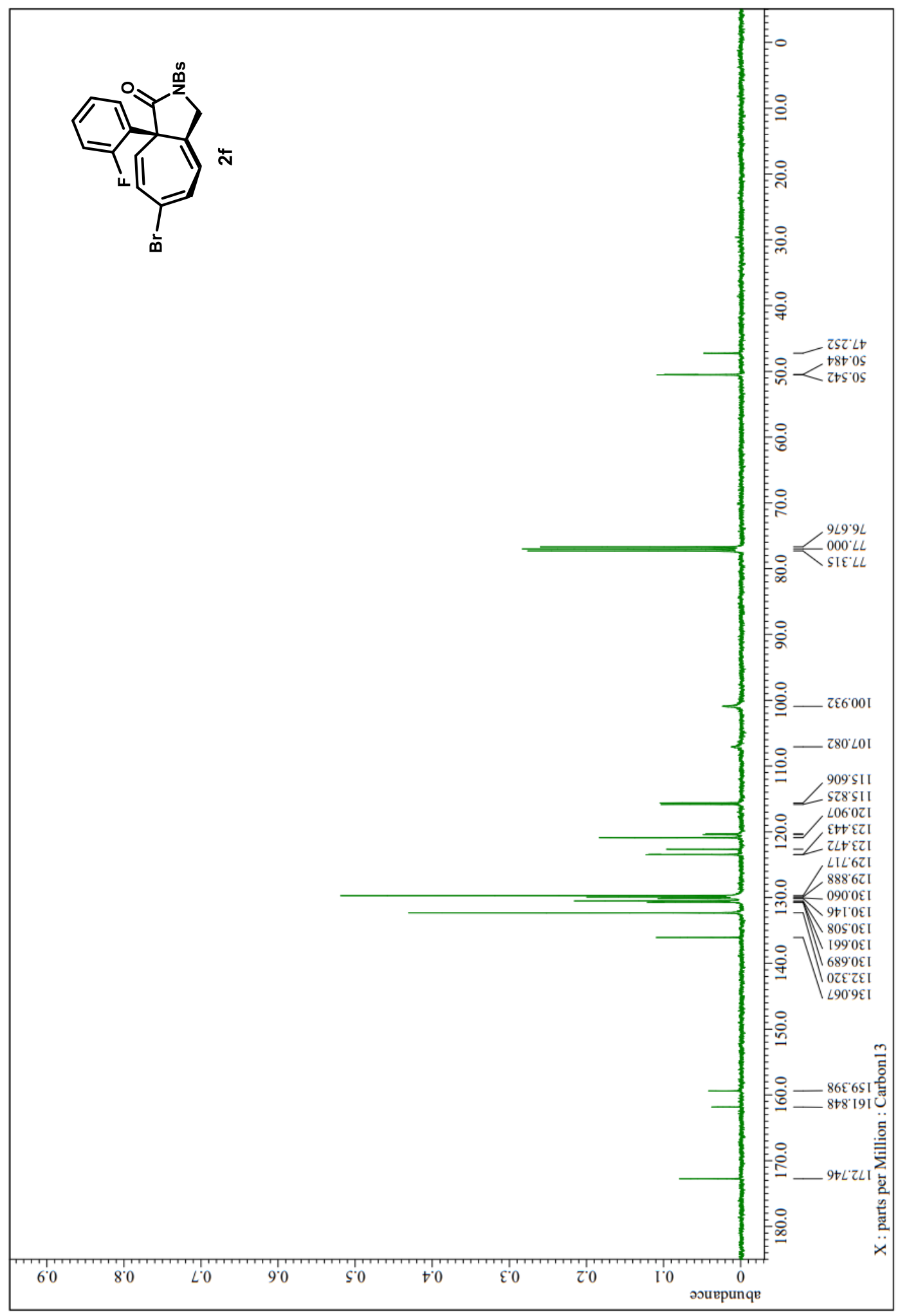


Supporting information

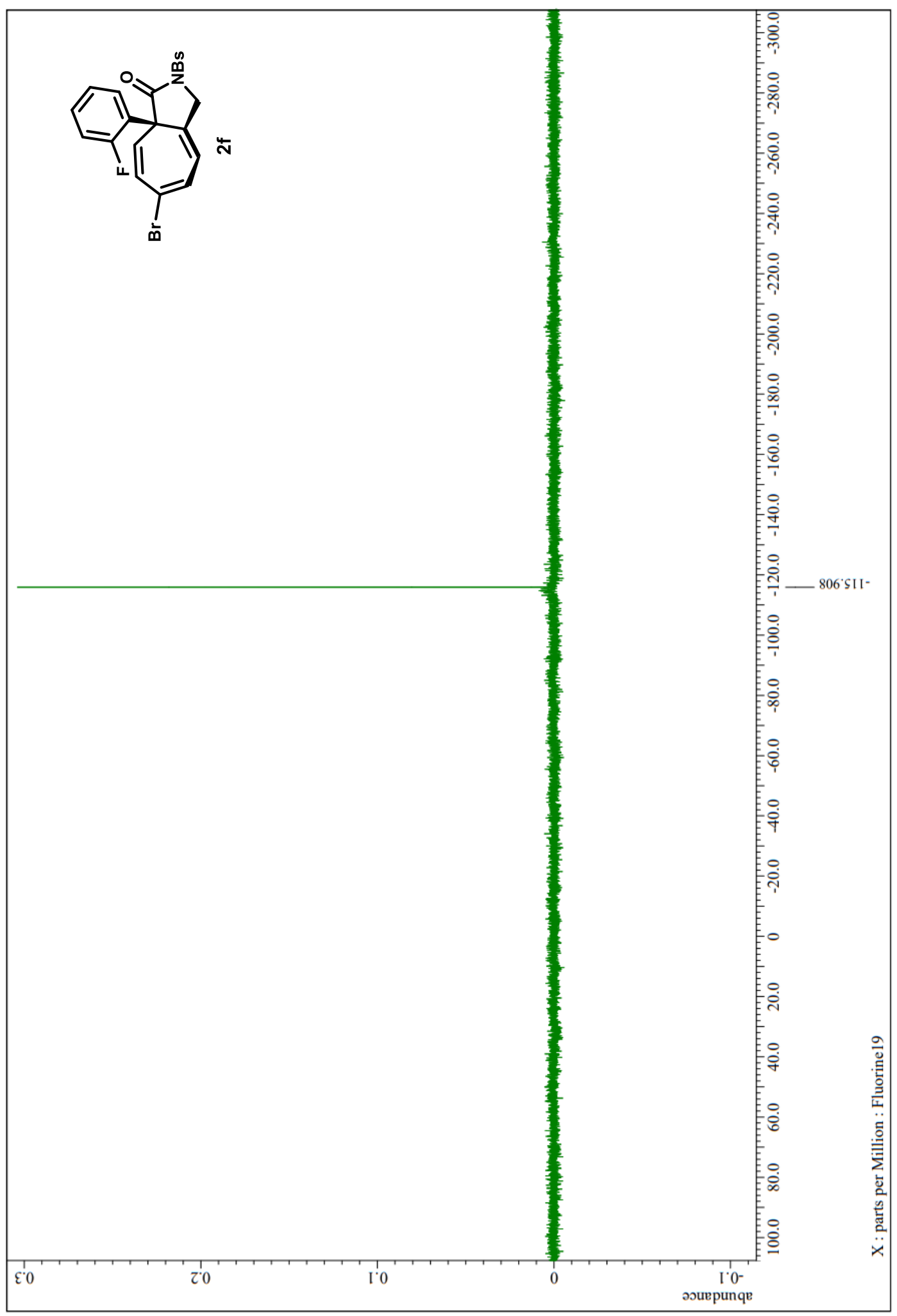


(R)-6-Bromo-8a-(3-bromophenyl)-2-tosyl-3,8a-dihydrocyclohepta[c]pyrrol$1(2 H)$-one $(2 \mathrm{~g})$

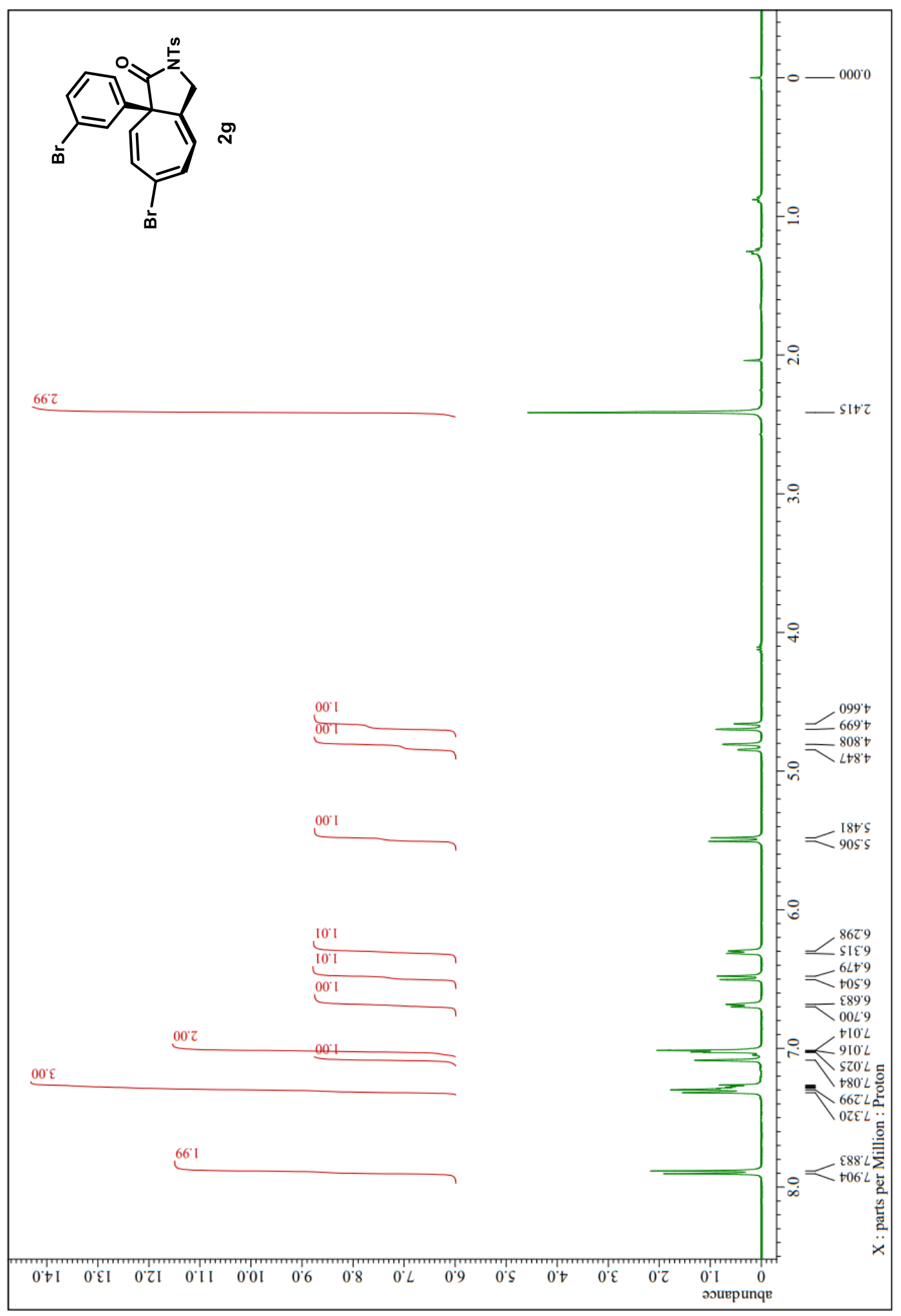


Supporting information

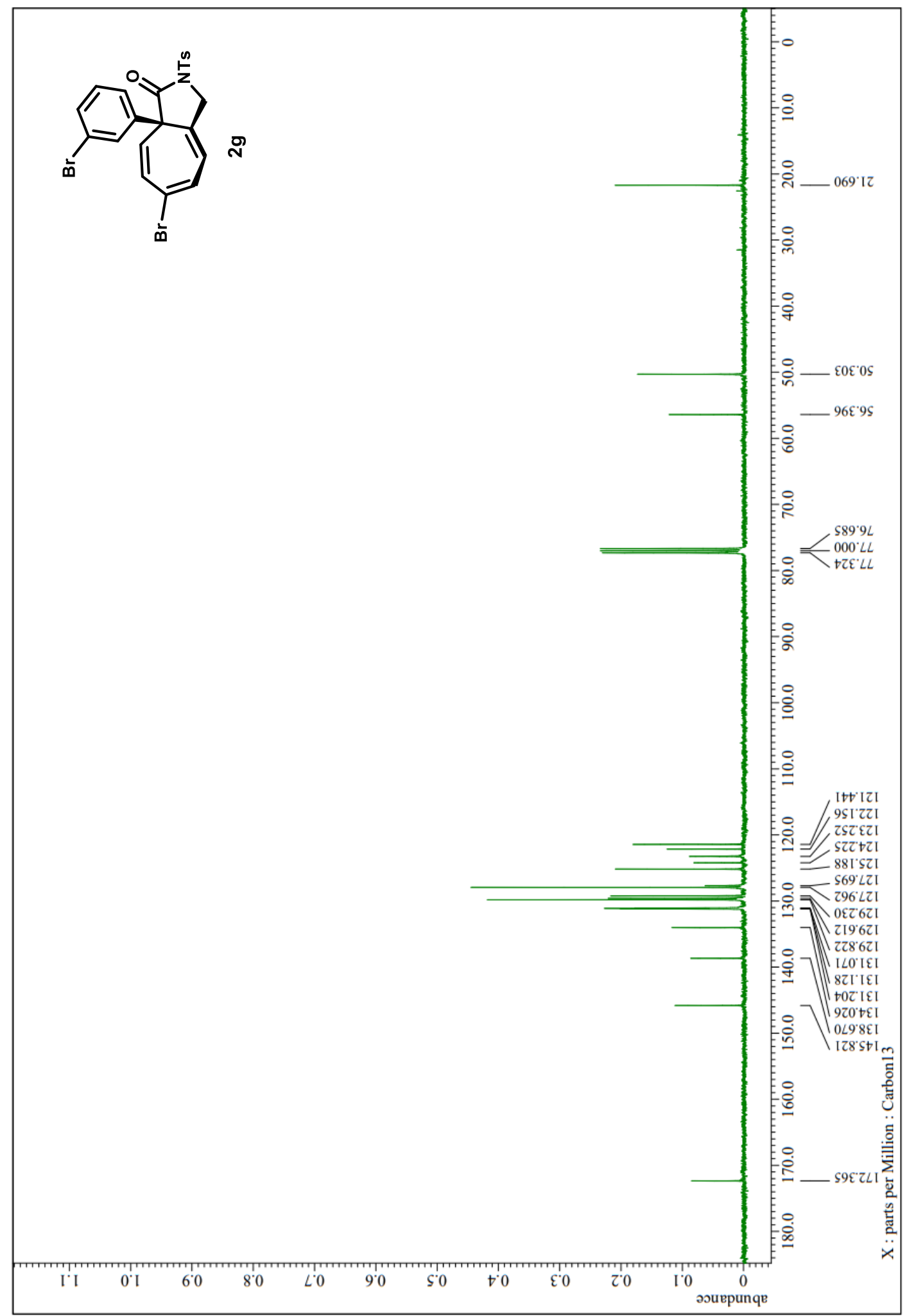




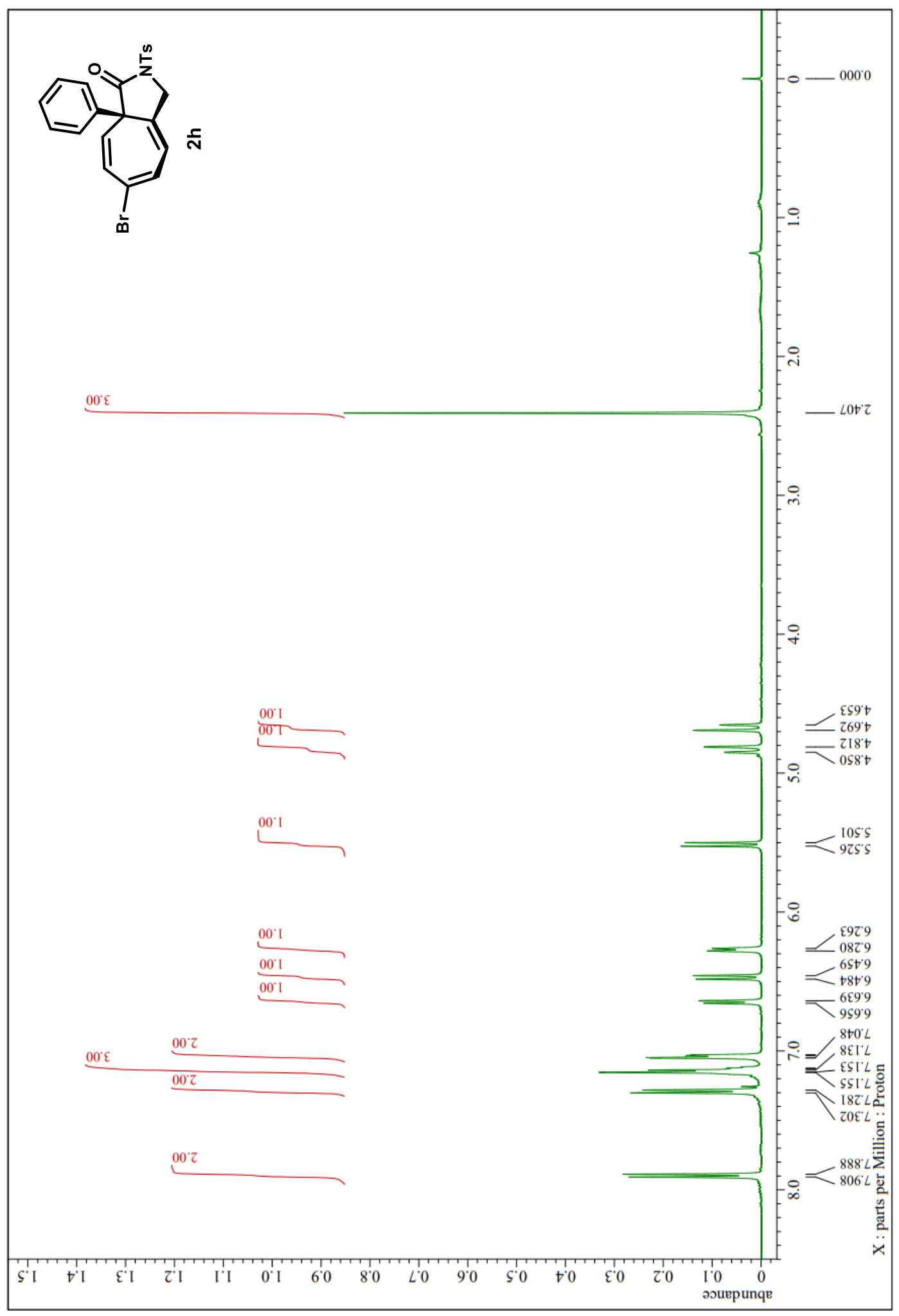


Supporting information

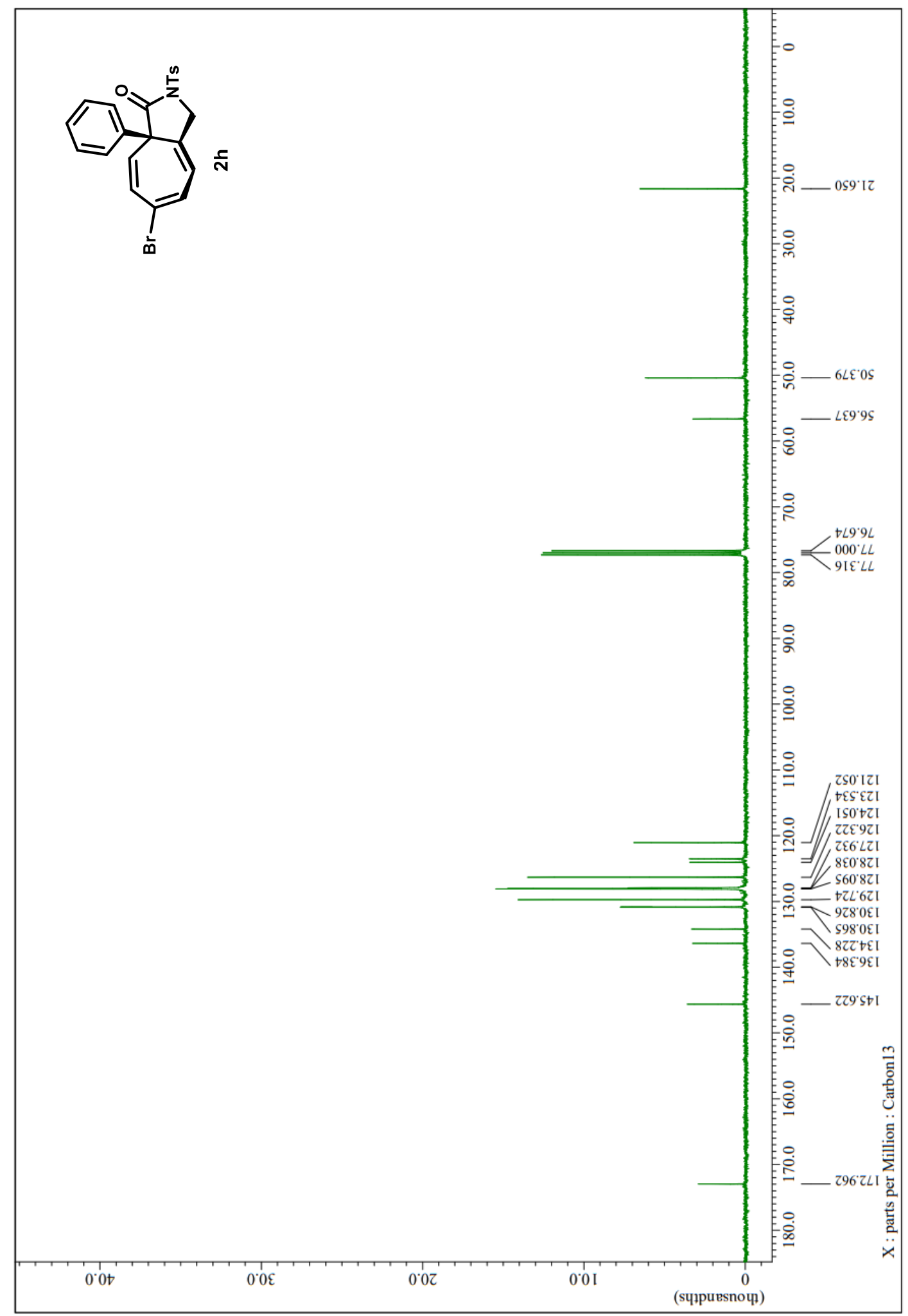


(R)-6-Bromo-2-((4-methoxyphenyl)sulfonyl)-8a-phenyl-3,8adihydrocyclohepta[c]pyrrol-1(2H)-one (2i)

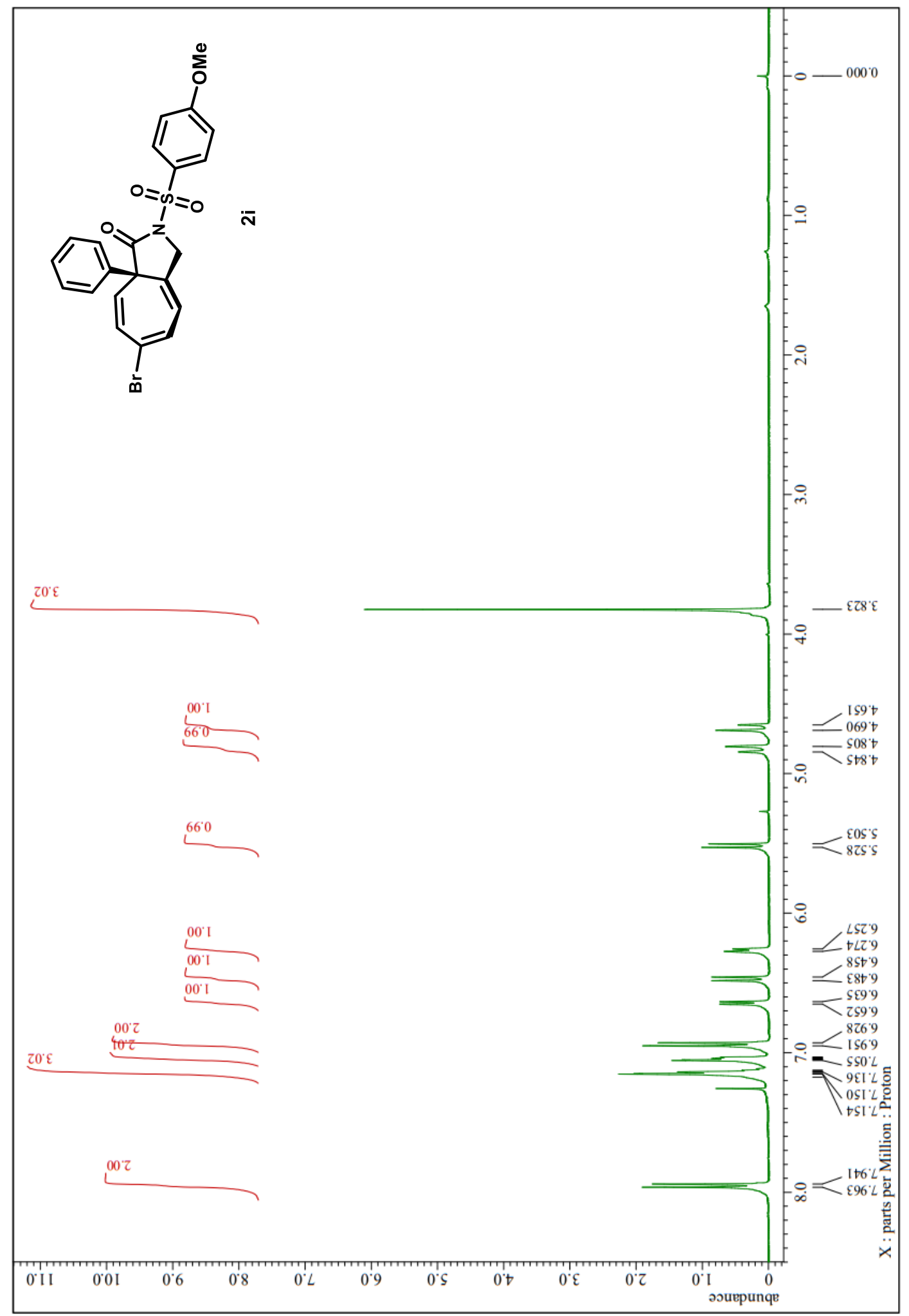


Supporting information

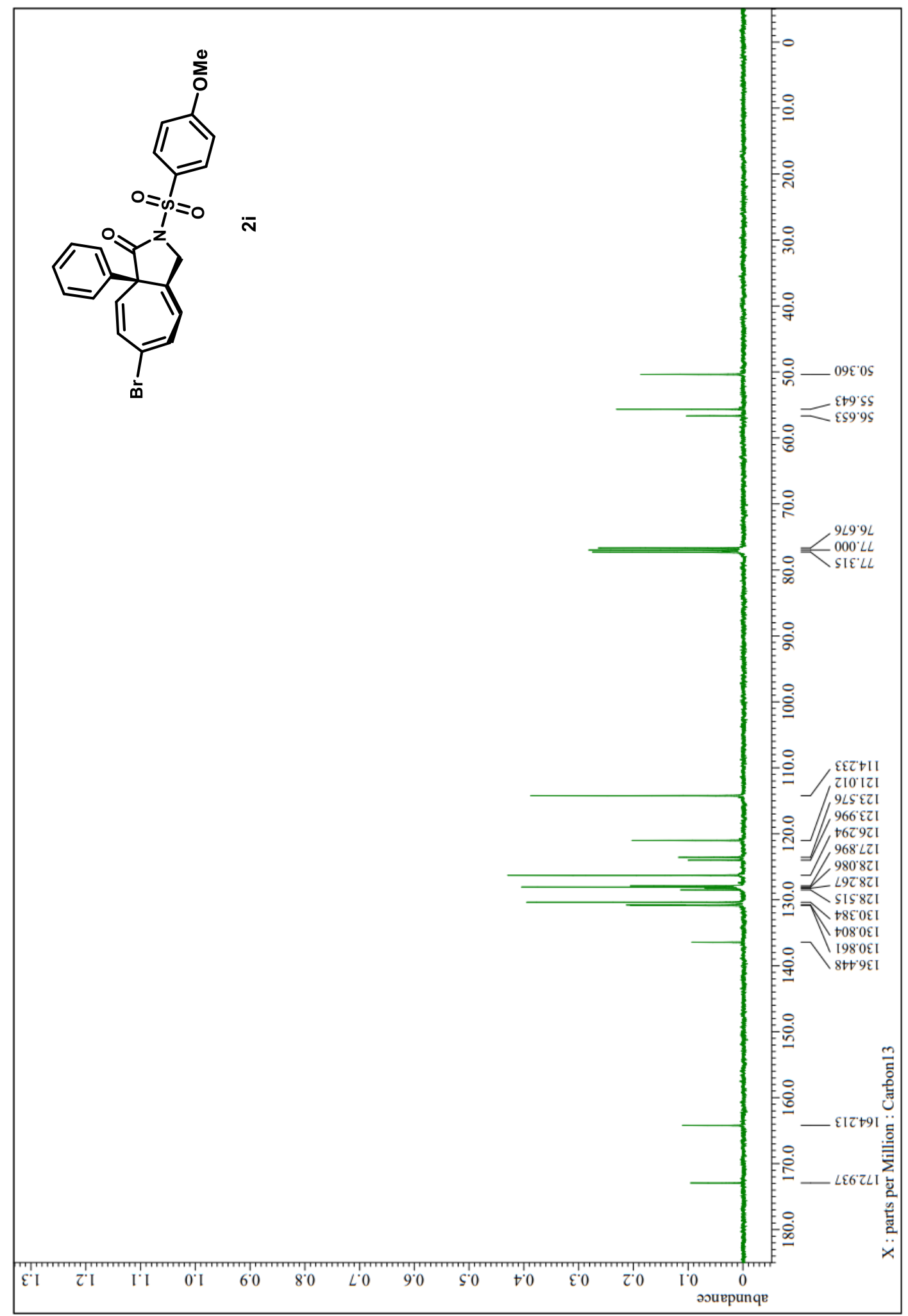


(R)-2-((4-Bromophenyl)sulfonyl)-8a-phenyl-3,8a-dihydrocyclohepta[c]pyrrol$1(2 H)$-one (2j)

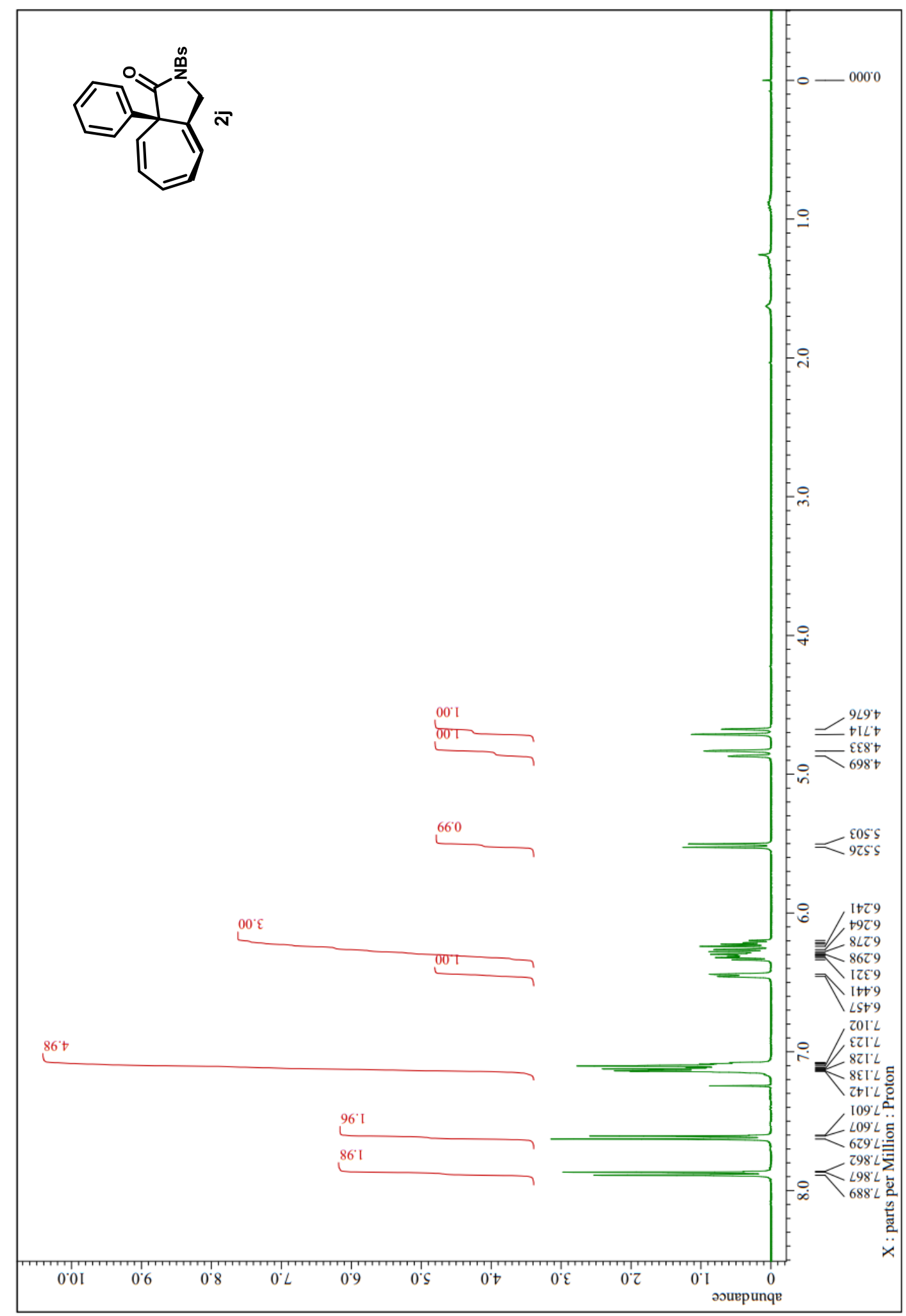


Supporting information

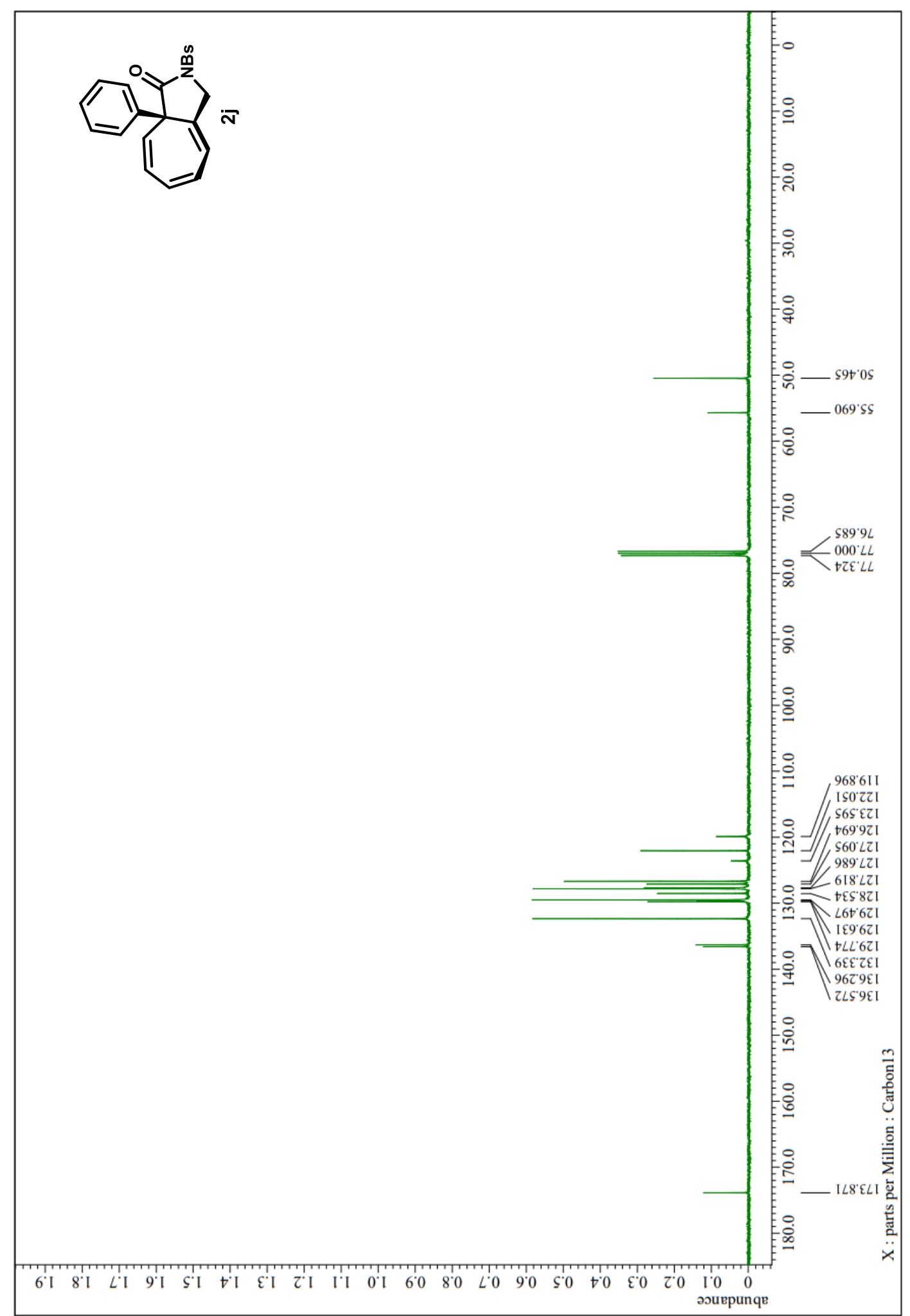


Supporting information

(R)-2-((4-Bromophenyl)sulfonyl)-6-iodo-8a-phenyl-3,8a-

dihydrocyclohepta[c]pyrrol-1(2H)-one (2k)

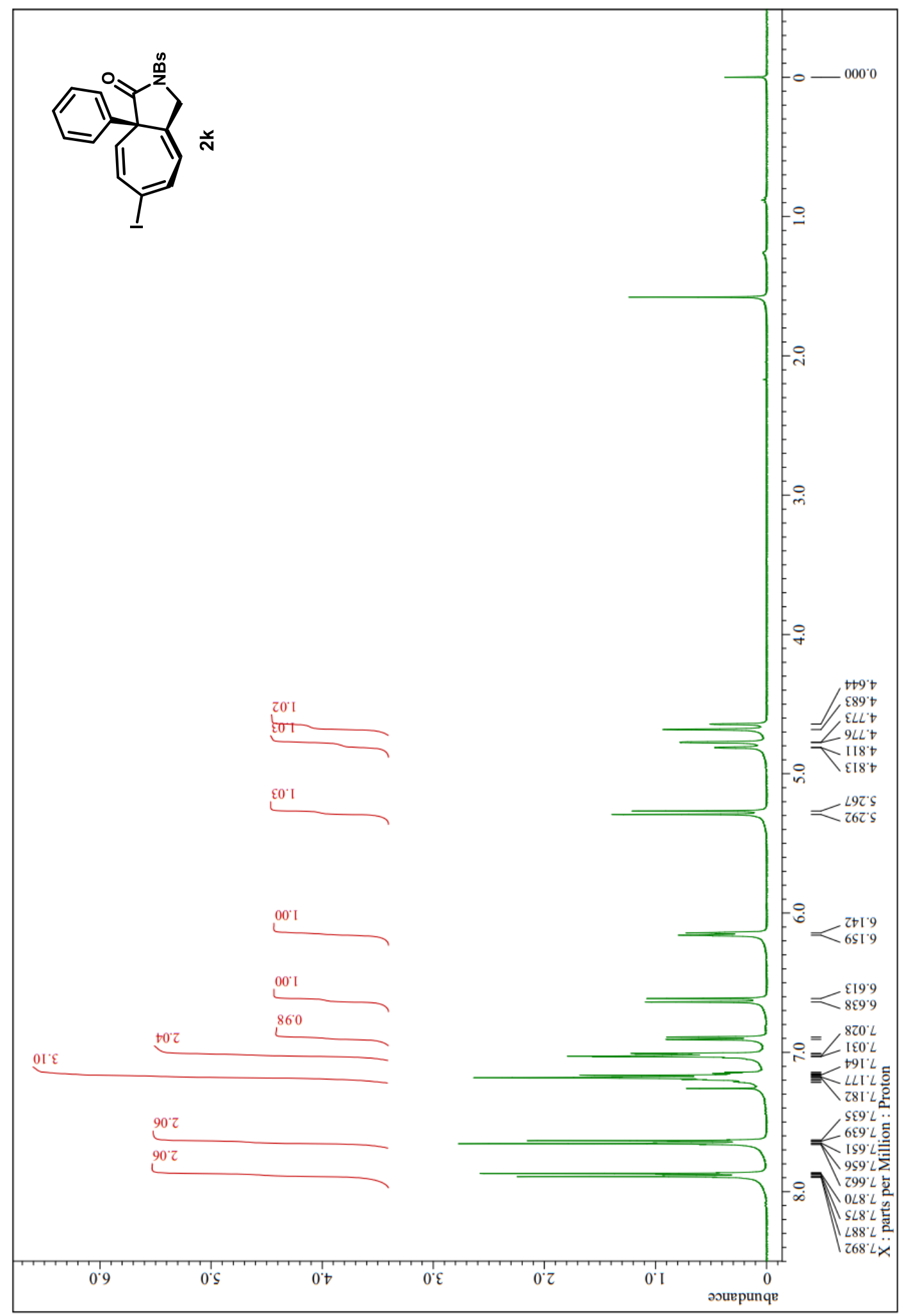


Supporting information

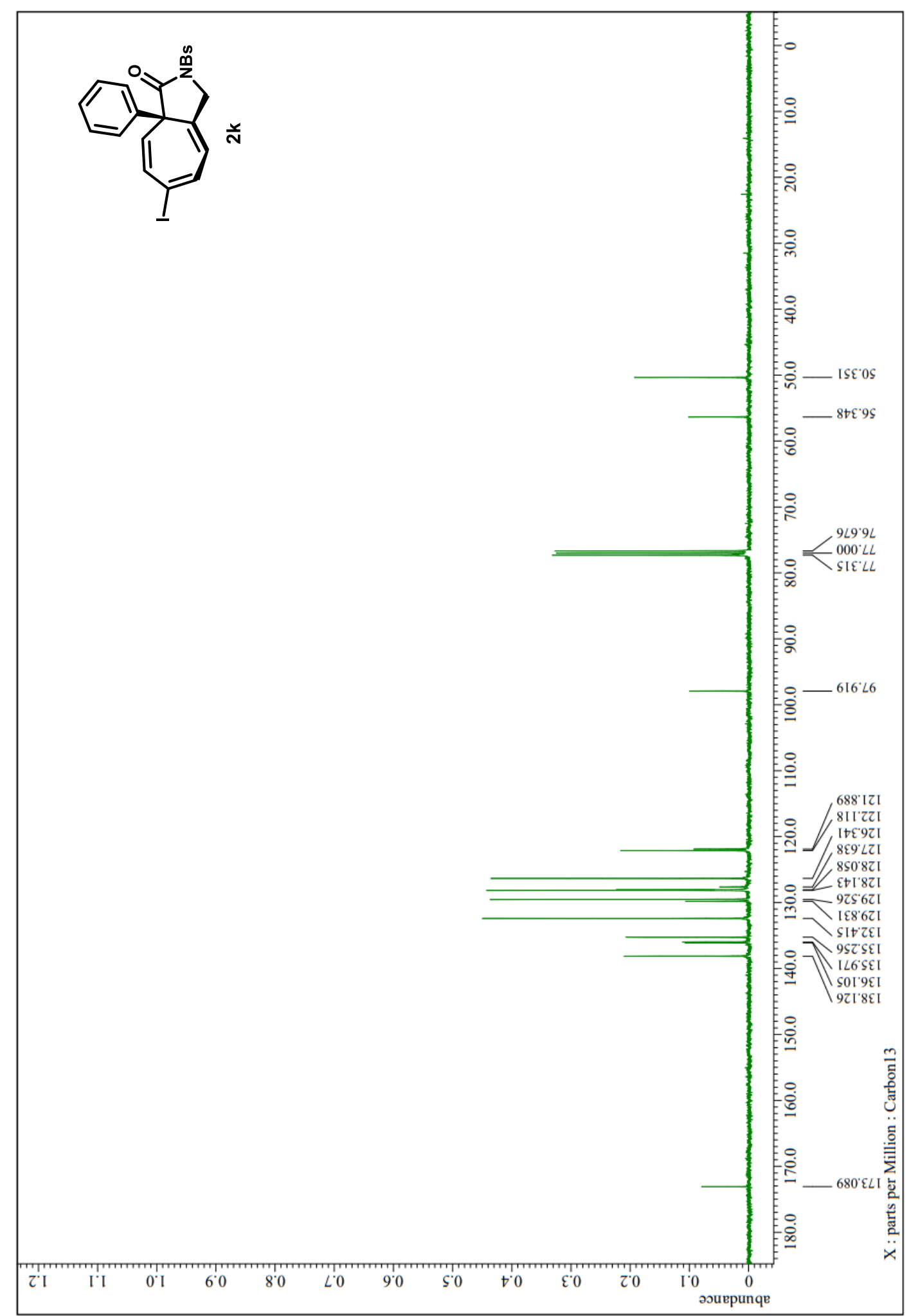


Supporting information

(R)-2-((4-Bromophenyl)sulfonyl)-6-fluoro-8a-phenyl-3,8adihydrocyclohepta[c]pyrrol-1(2H)-one (2I)

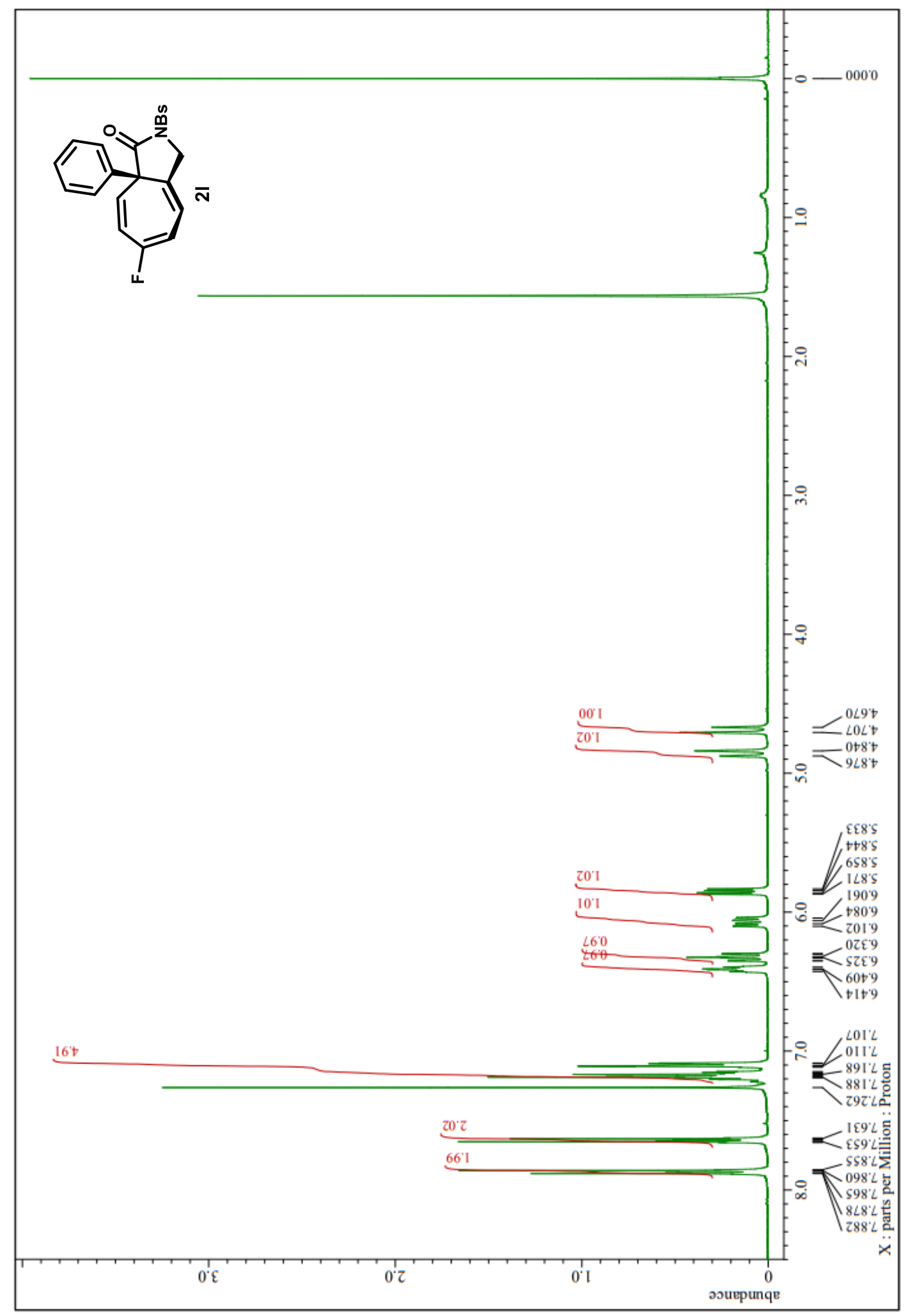


Supporting information

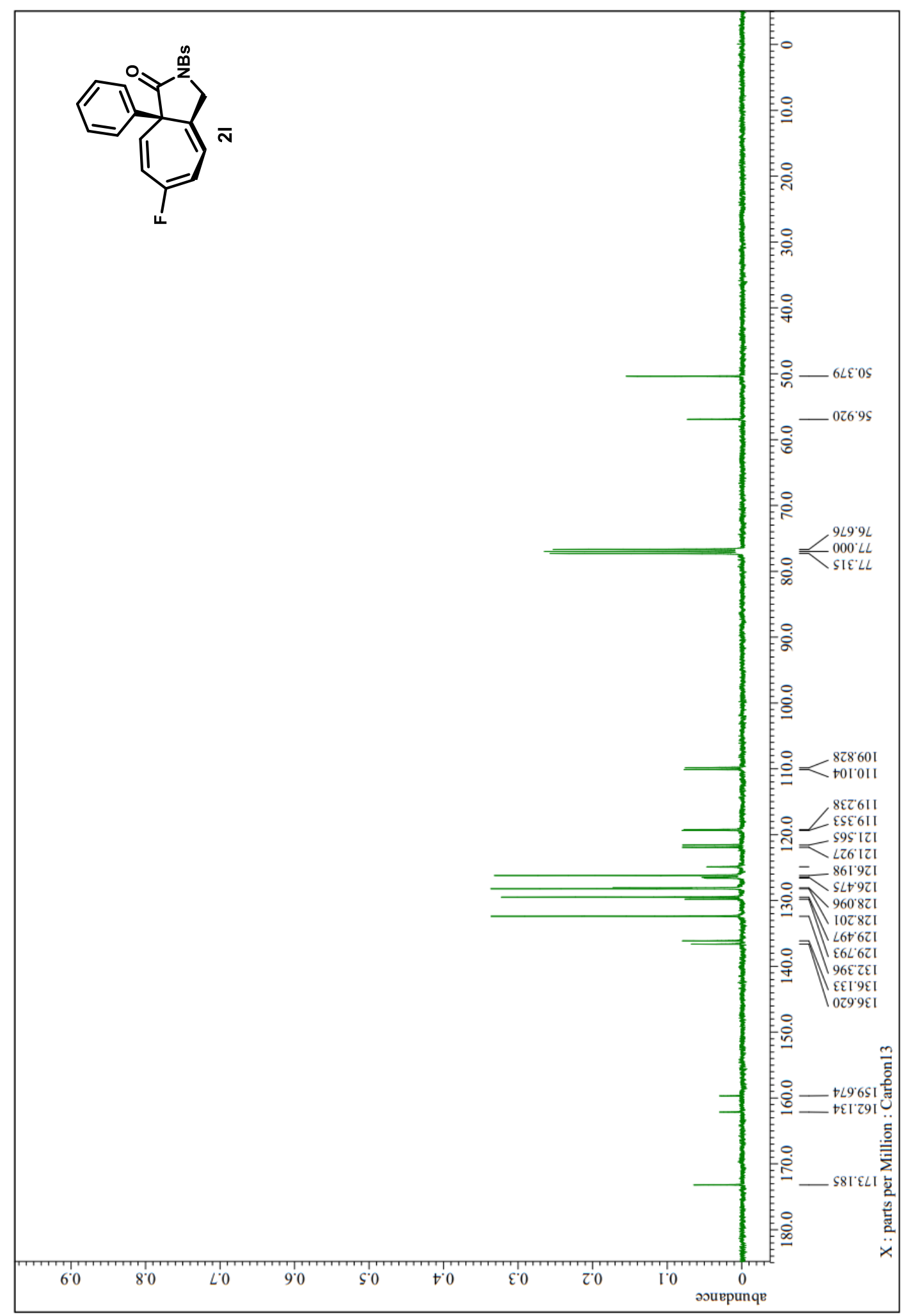


Supporting information

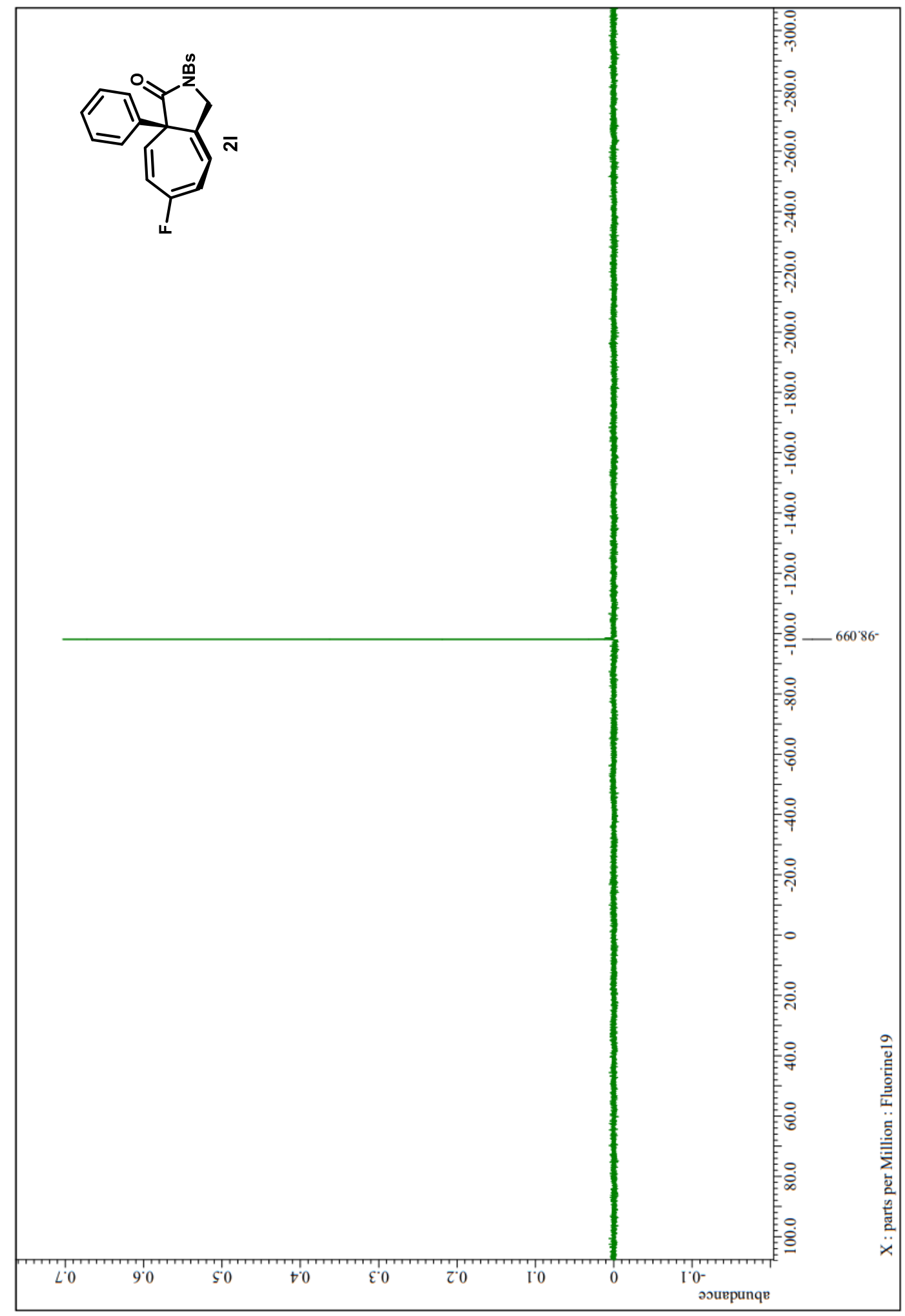


(R)-6-Chloro-8a-phenyl-2-tosyl-3,8a-dihydrocyclohepta[c]pyrrol-1(2H)-one (2m)

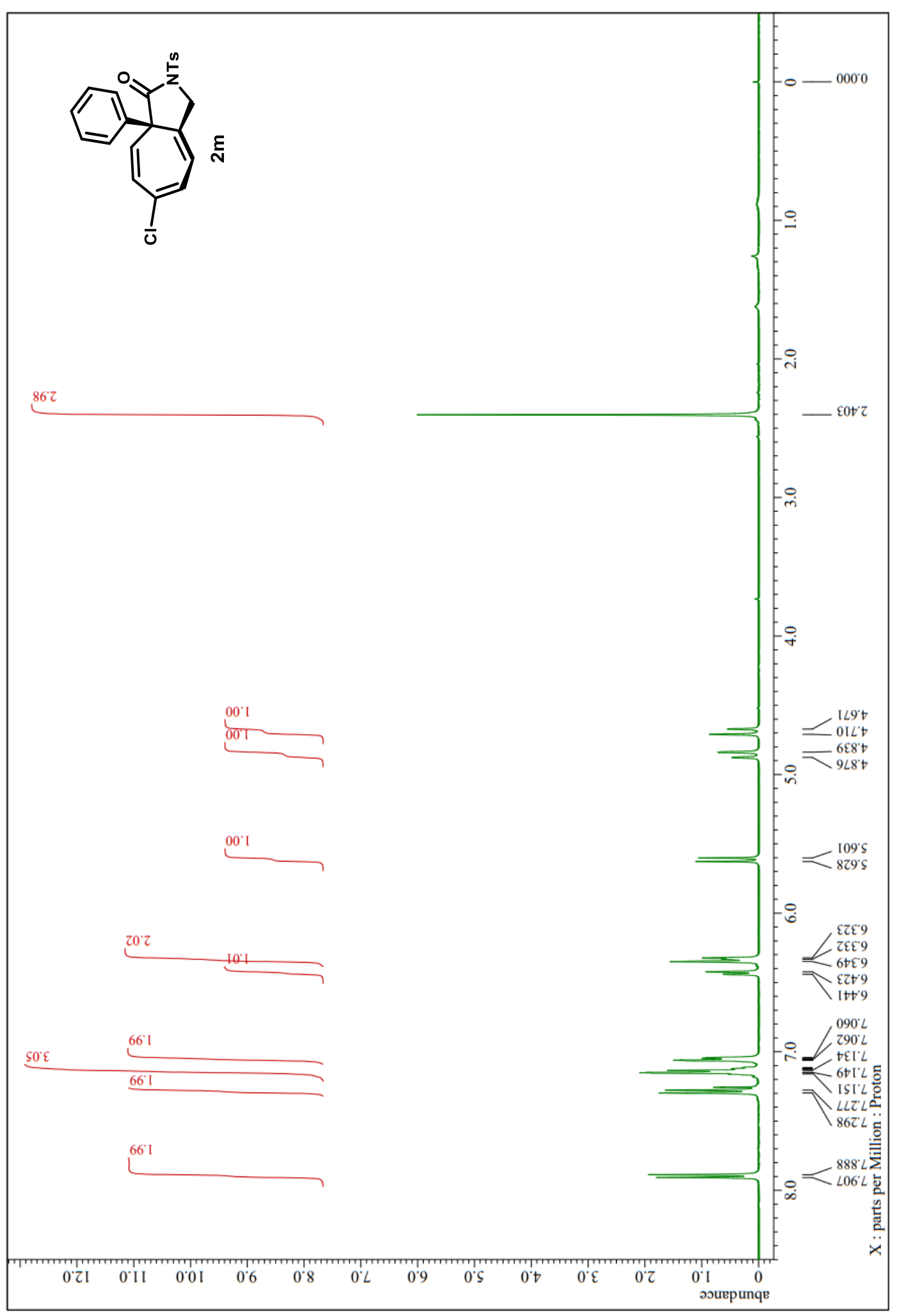


Supporting information

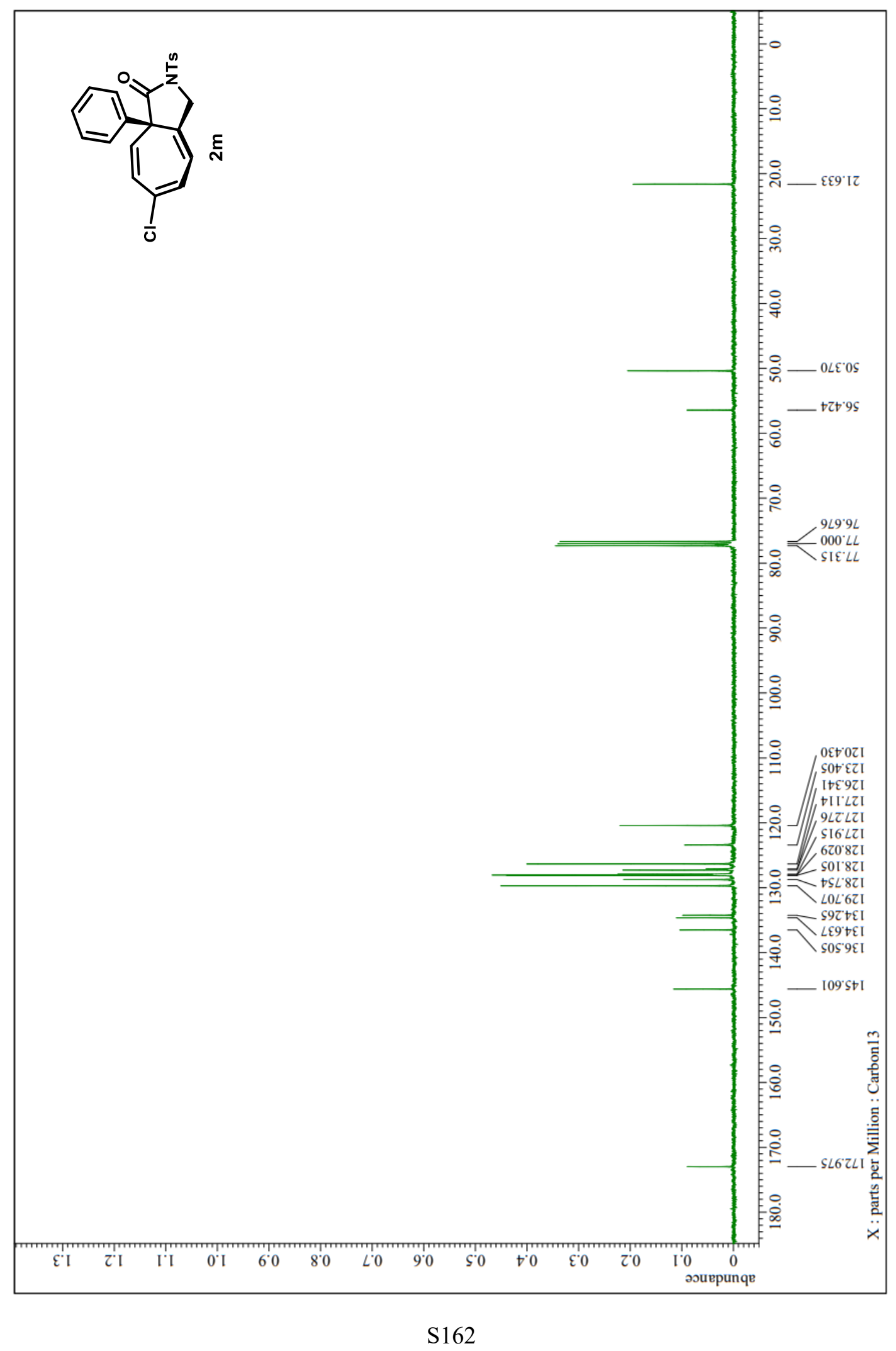


(R)-2-((4-Bromophenyl)sulfonyl)-6-methyl-8a-phenyl-3,8adihydrocyclohepta[c]pyrrol-1(2H)-one (2n)

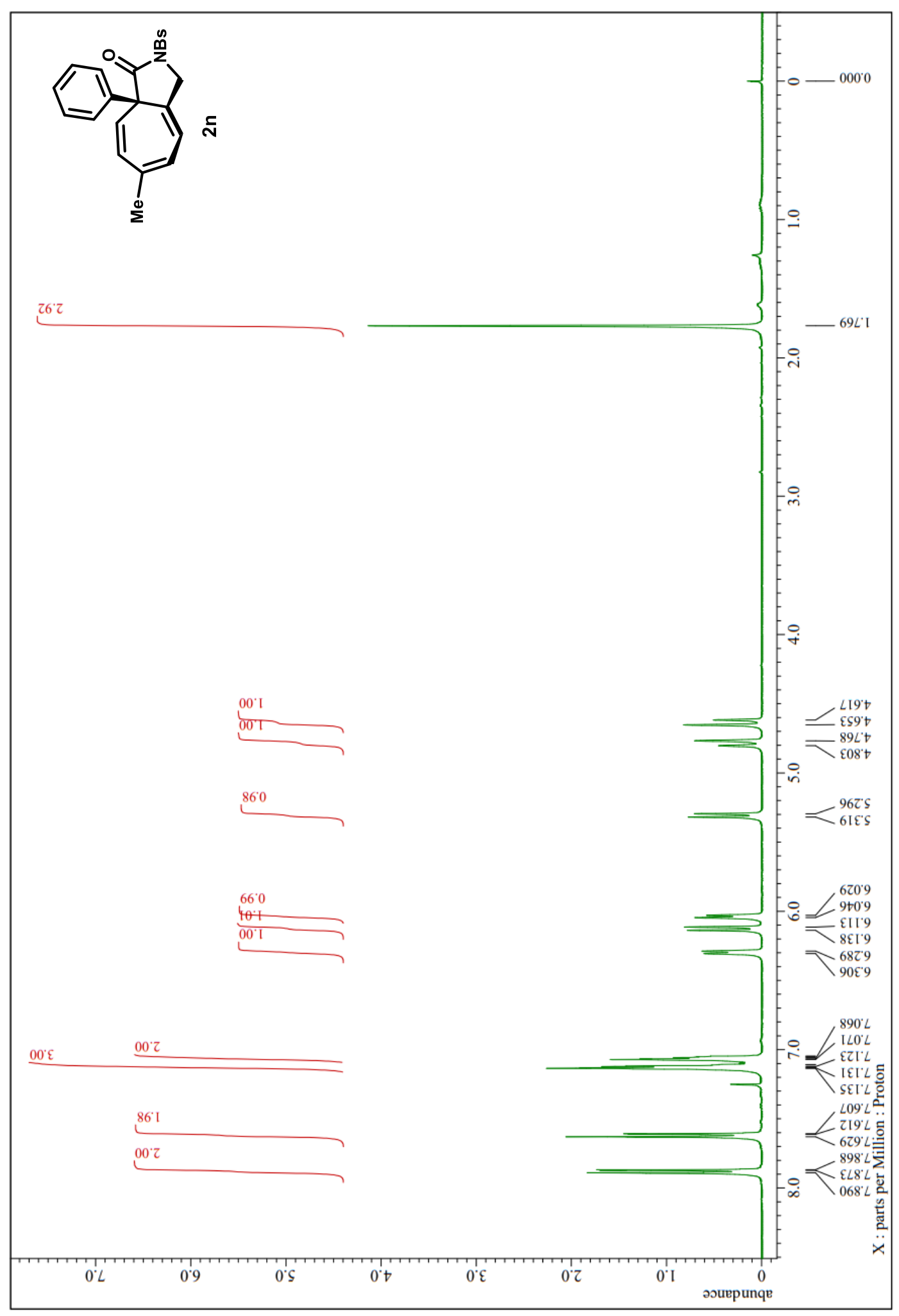


Supporting information

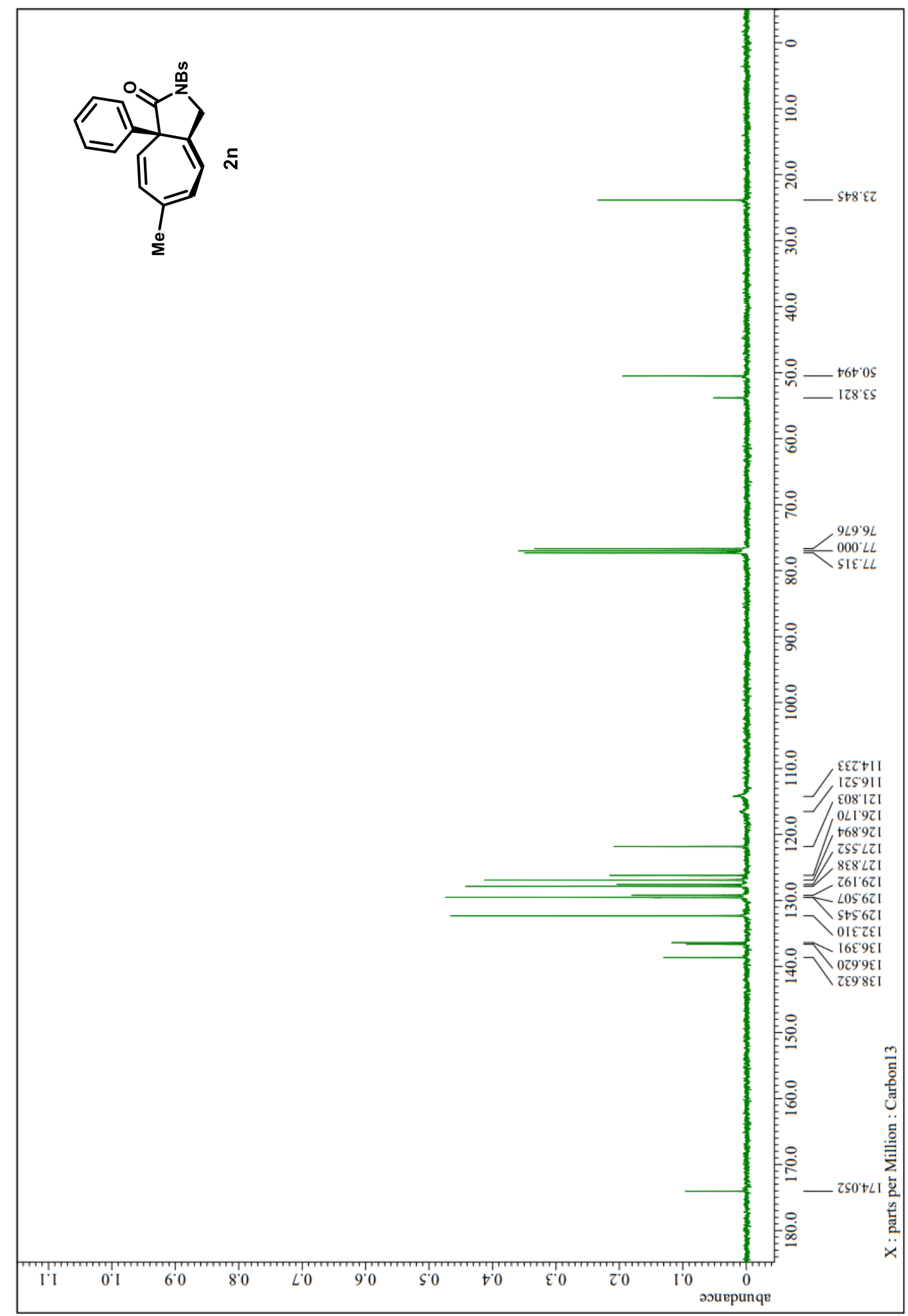


(S)-6-bromo-8a-pentyl-2-tosyl-3,8a-dihydrocyclohepta[c]pyrrol-1(2H)-one (20)

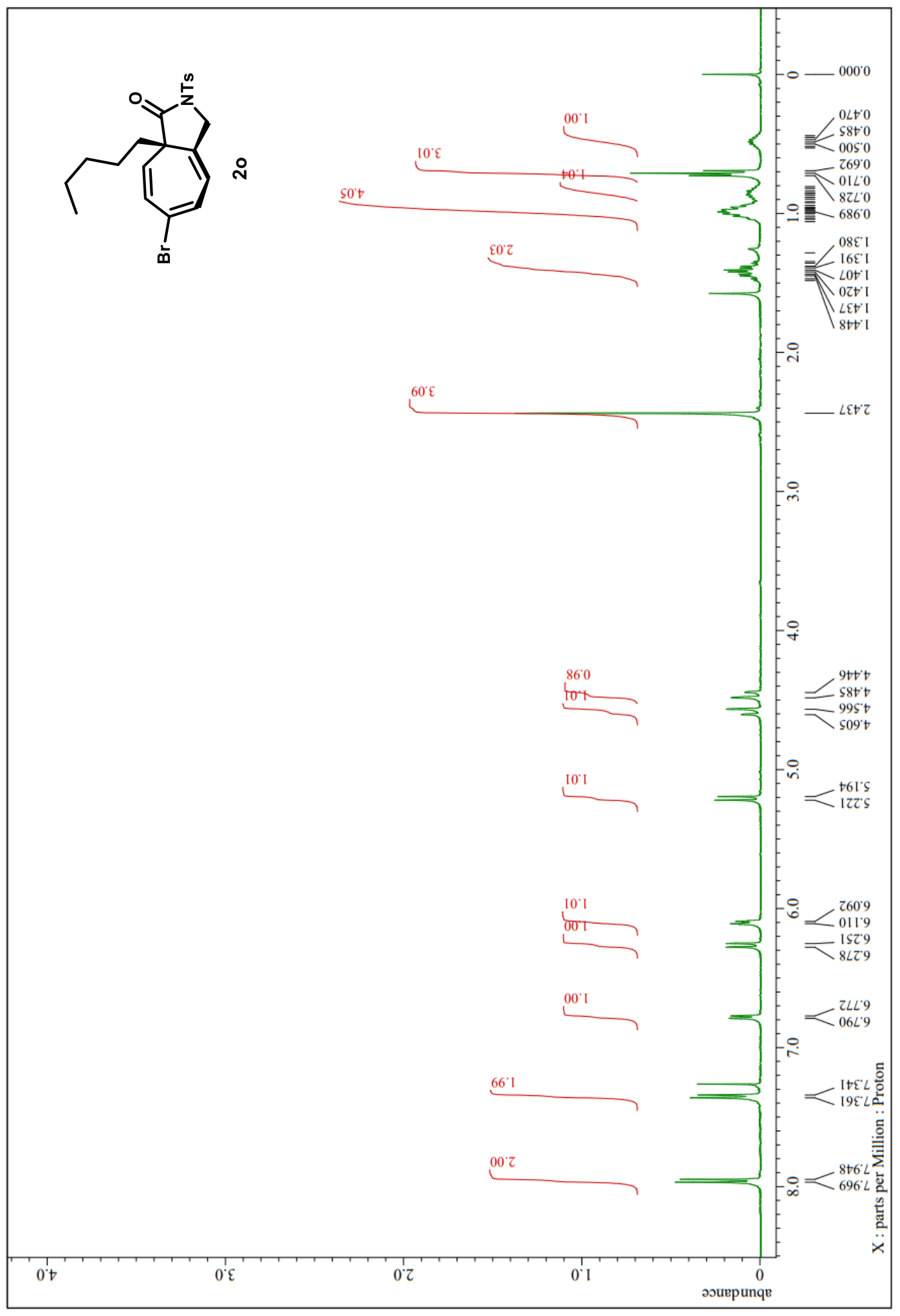


Supporting information

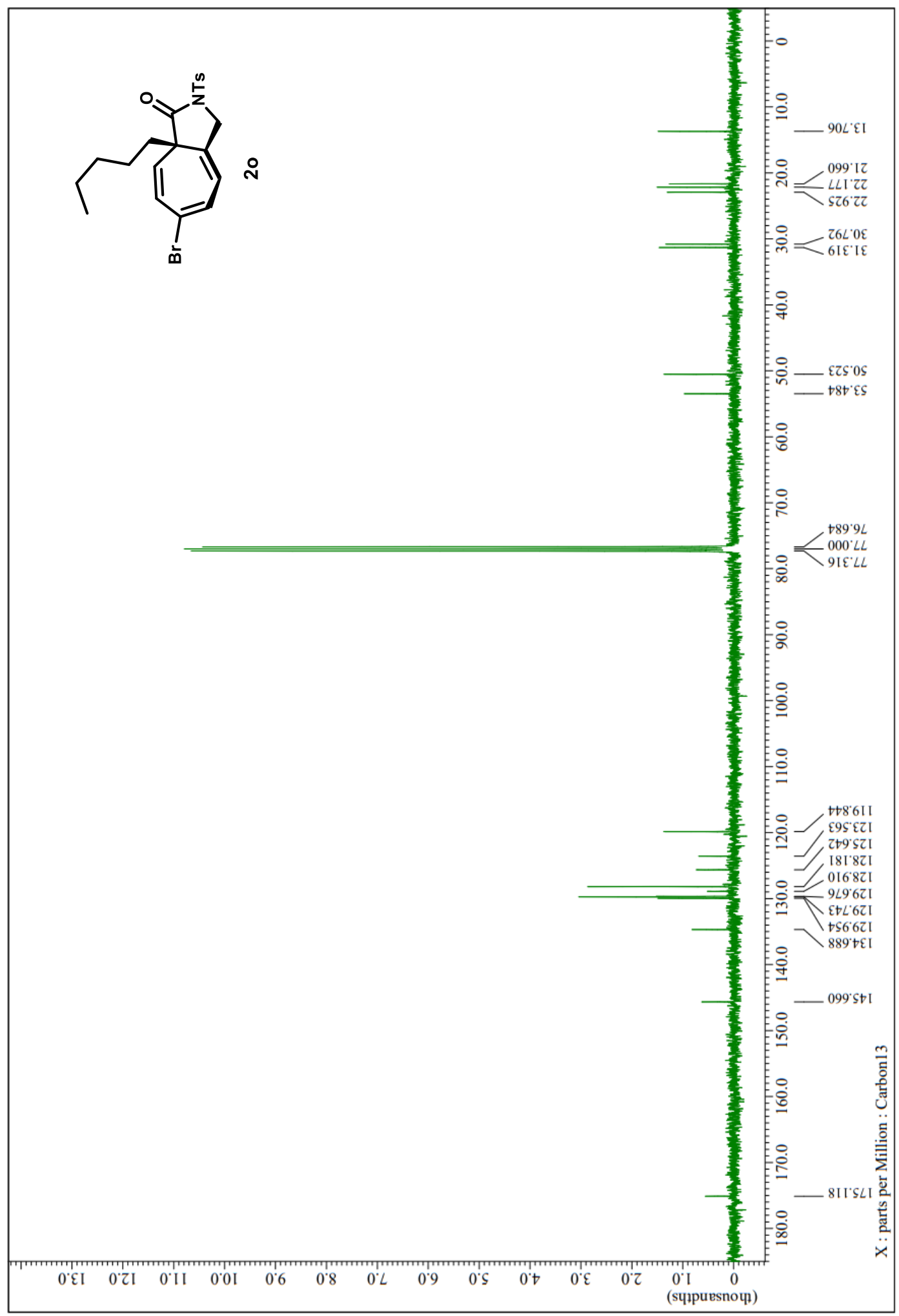


Supporting information

(R)-8a-phenyl-2-tosyl-3,8a-dihydrocyclohepta[c]pyrrol-1(2H)-one (2p)

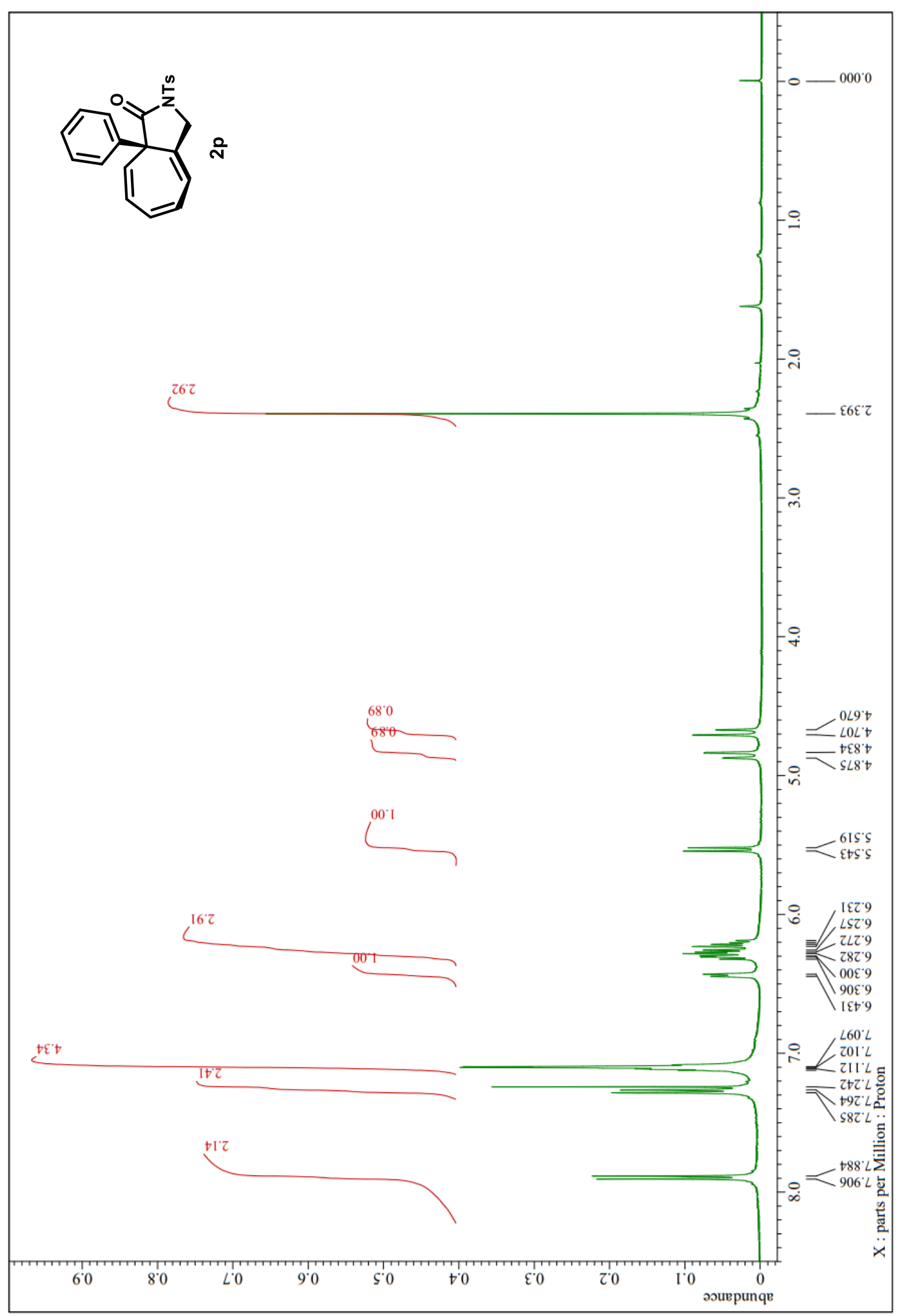


Supporting information

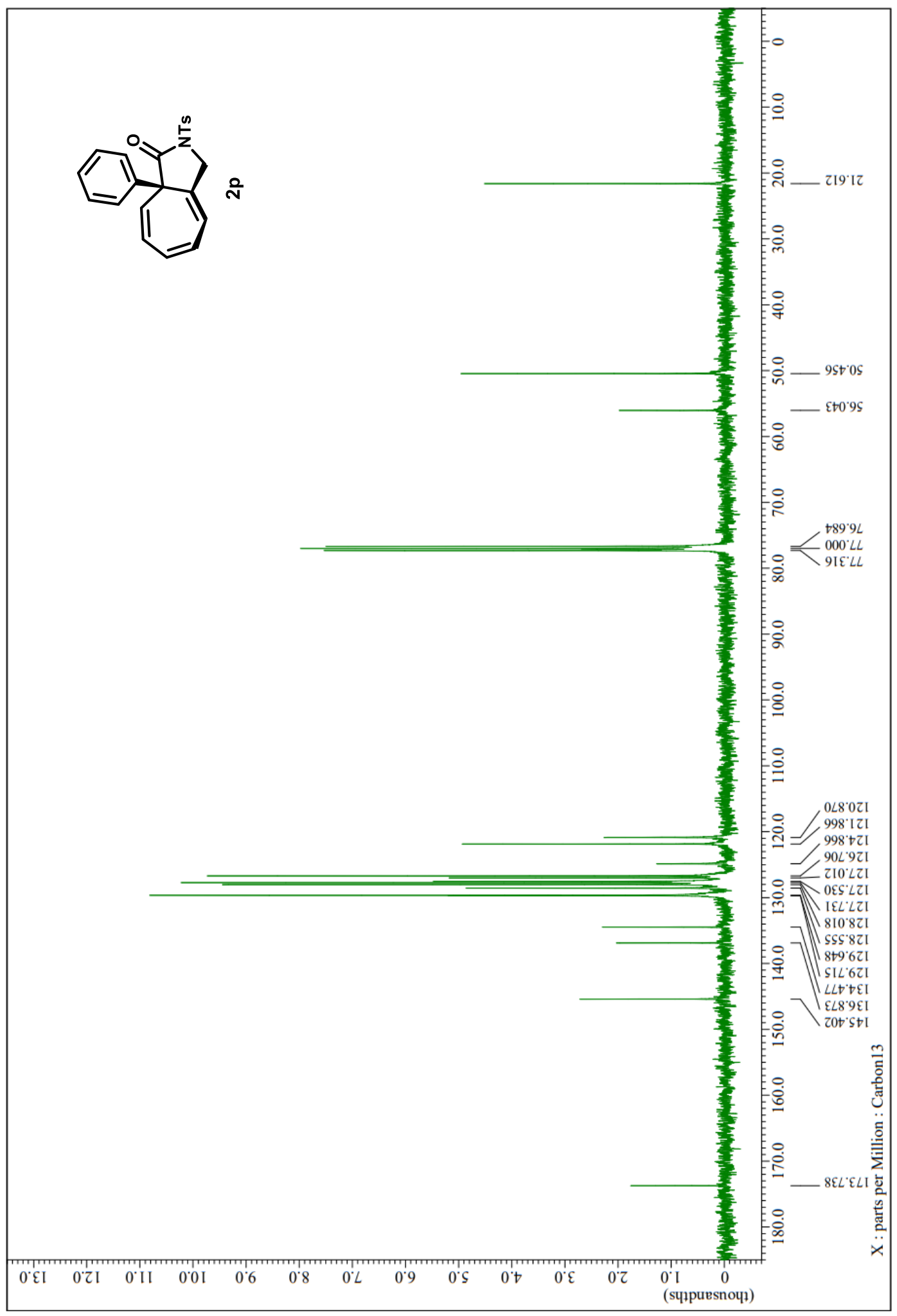




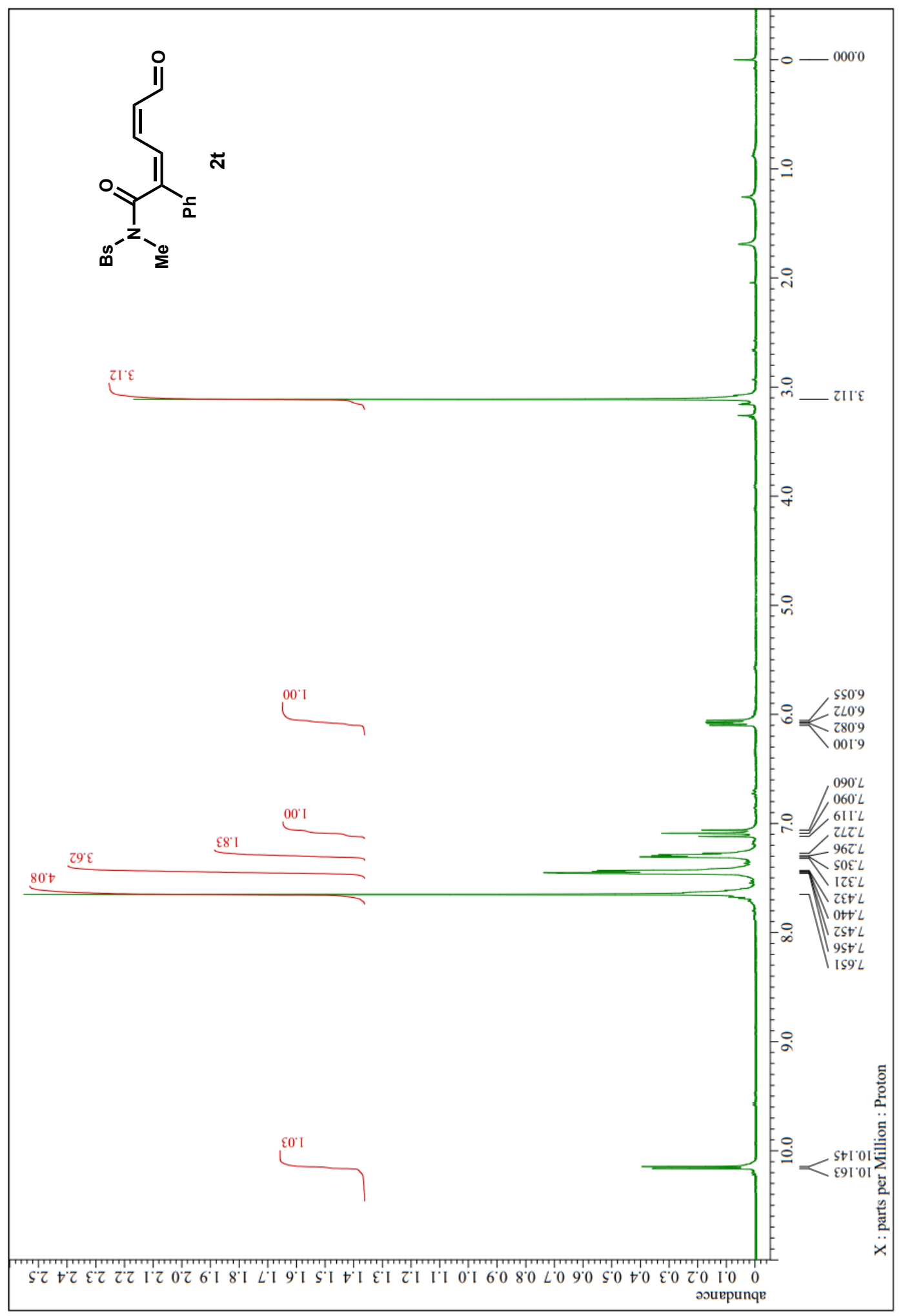


Supporting information

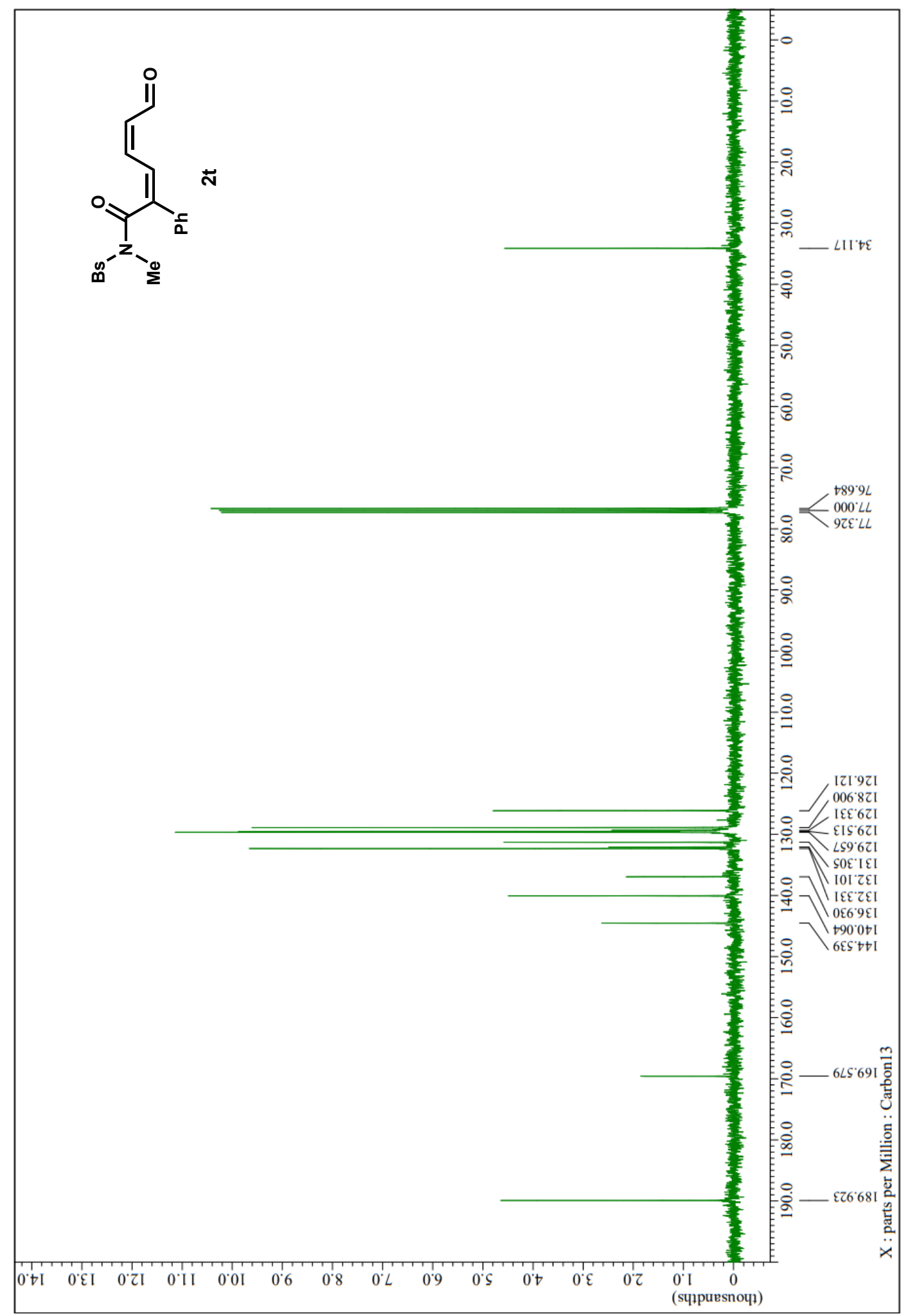


Supporting information

N-(4-Bromobenzyl)-N-((4-bromophenyl)sulfonyl)-2-oxo-2-phenylacetamide (3a)

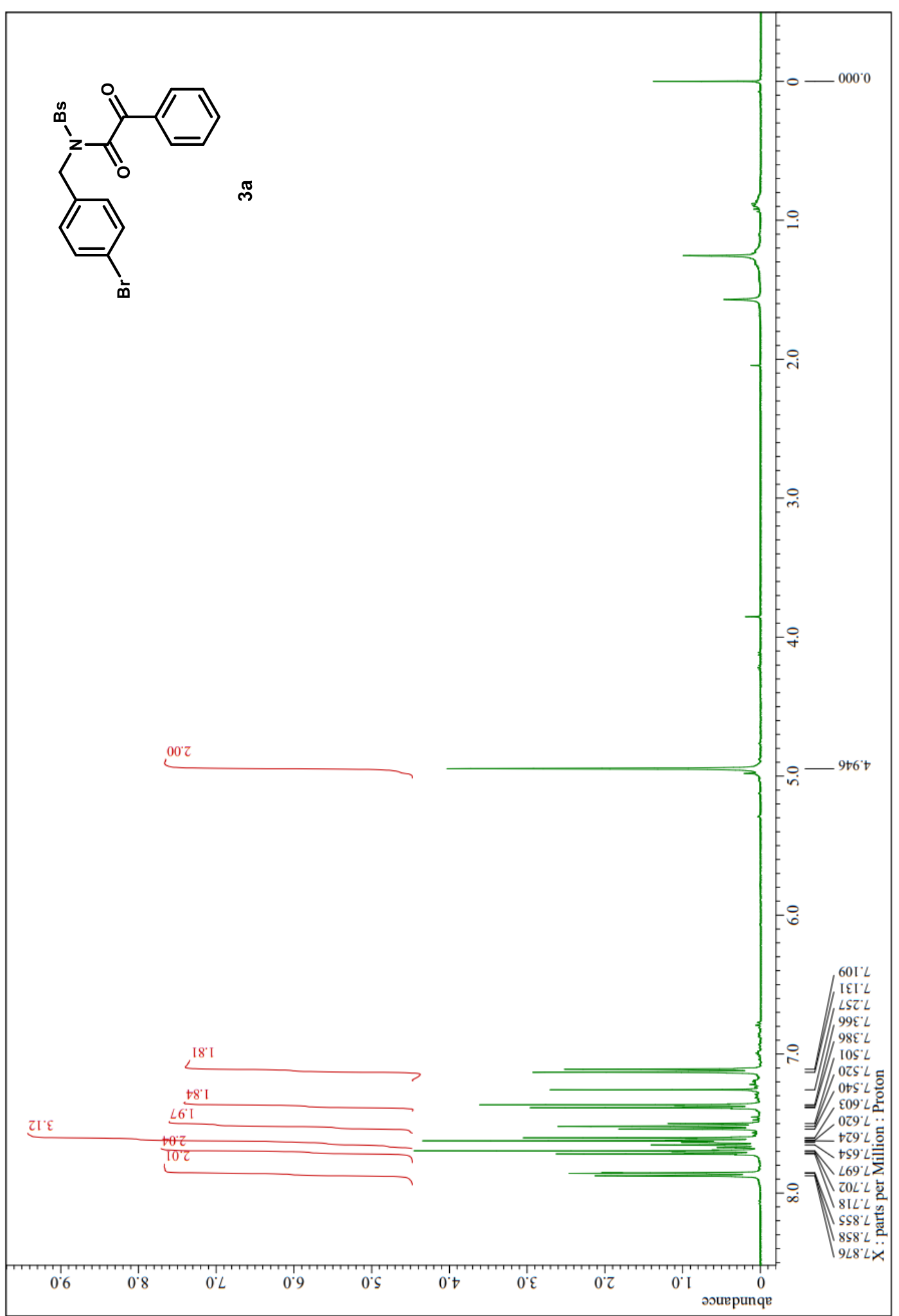


Supporting information

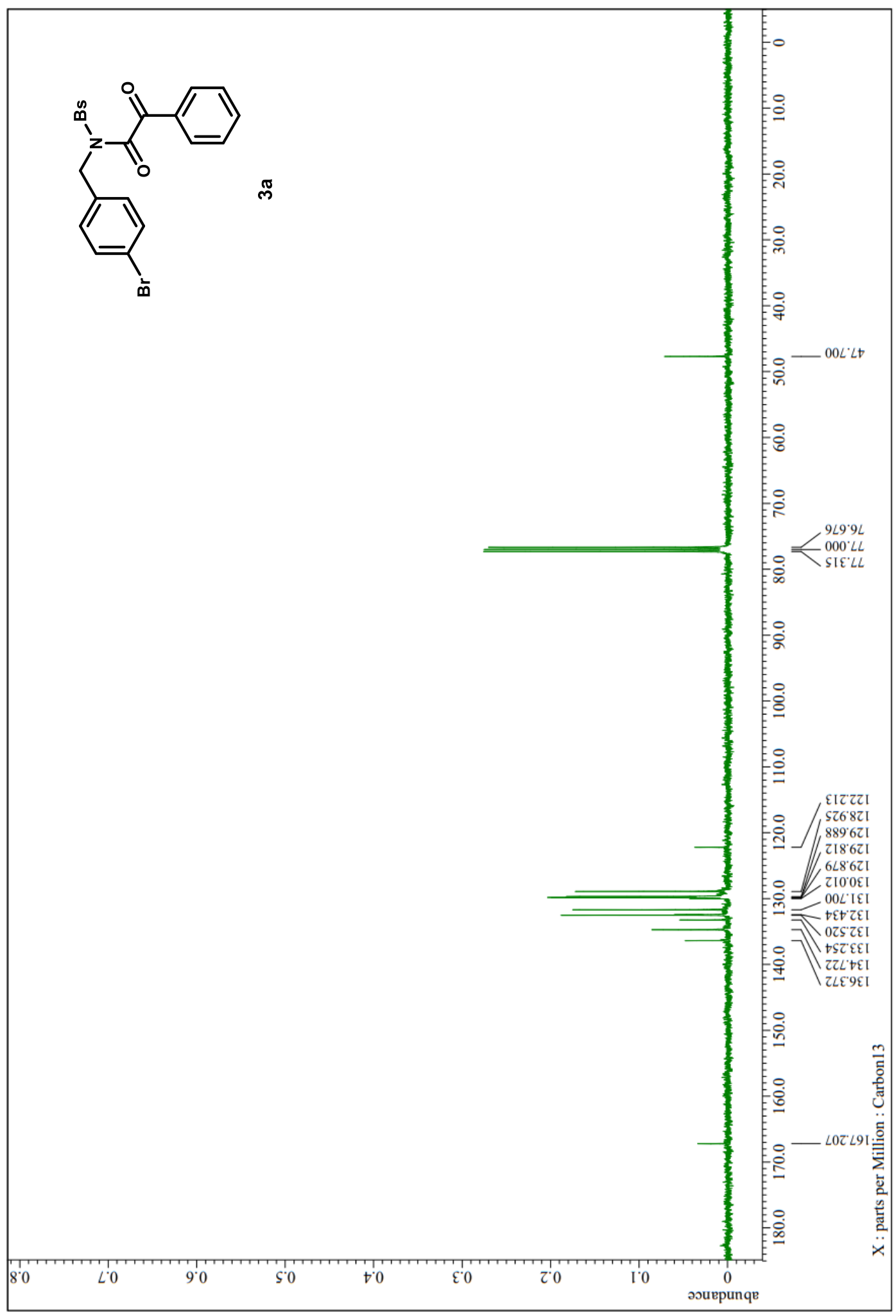


(3bS,4S,10S,10aR)-12-Bromo-2-((4-bromophenyl)sulfonyl)-3a,7-diphenyl1,2,3b,4-tetrahydro-6H,10H-4,10-ethenopyrrolo[3',4':1,3]cyclopropa[1,2d] $[1,2,4]$ triazolo[1,2-a]pyridazine-3,6,8(3aH,7H)-trione (6a)

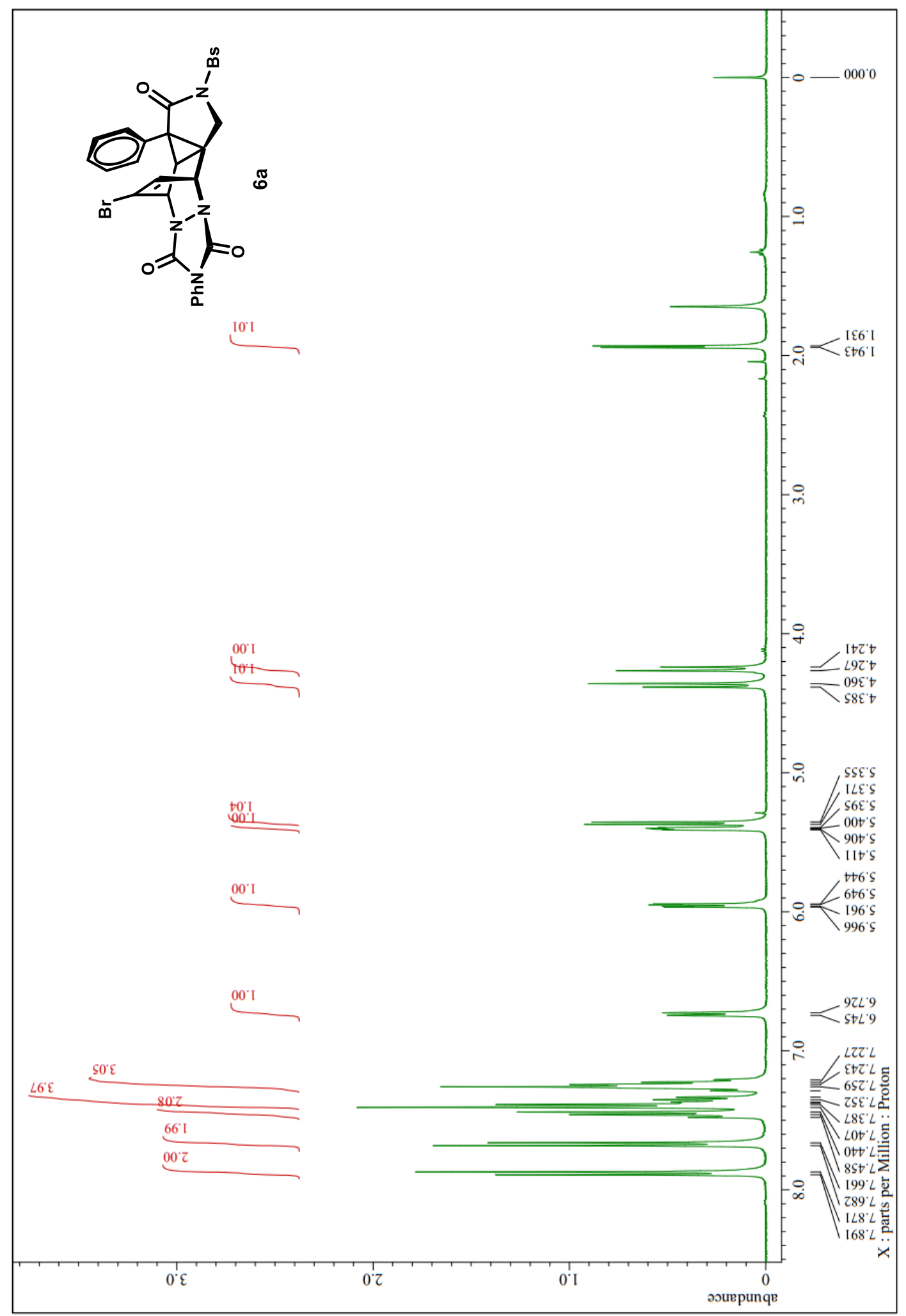


Supporting information

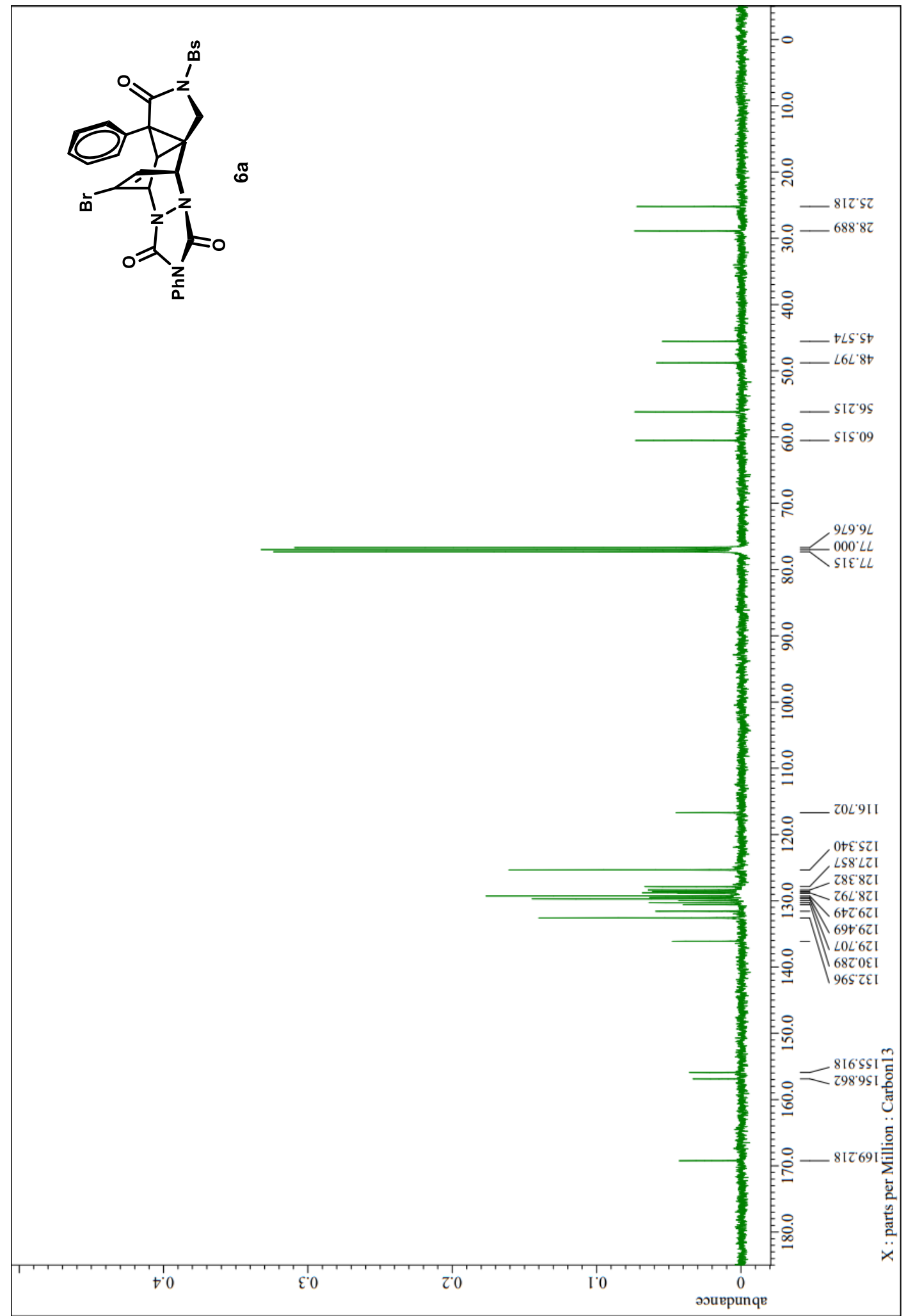


(3bR,4R,10R,10aS)-12-Bromo-2-((4-bromophenyl)sulfonyl)-3a-(4-fluorophenyl)7-phenyl-1,2,3b,4-tetrahydro-6H,10H-4,10ethenopyrrolo[3',4':1,3]cyclopropa[1,2-d][1,2,4]triazolo[1,2-a]pyridazine3,6,8(3aH,7H)-trione (6b)

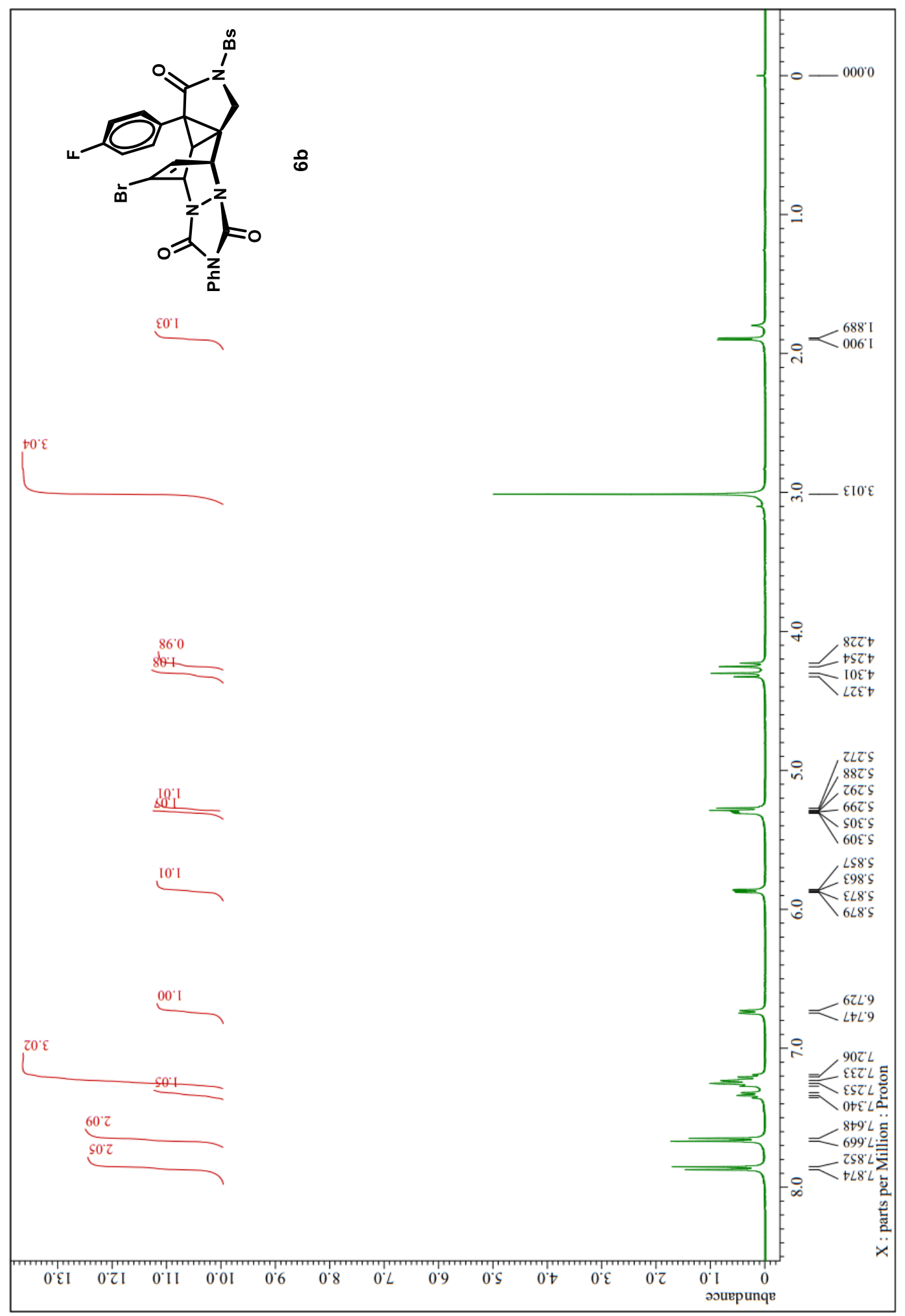


Supporting information

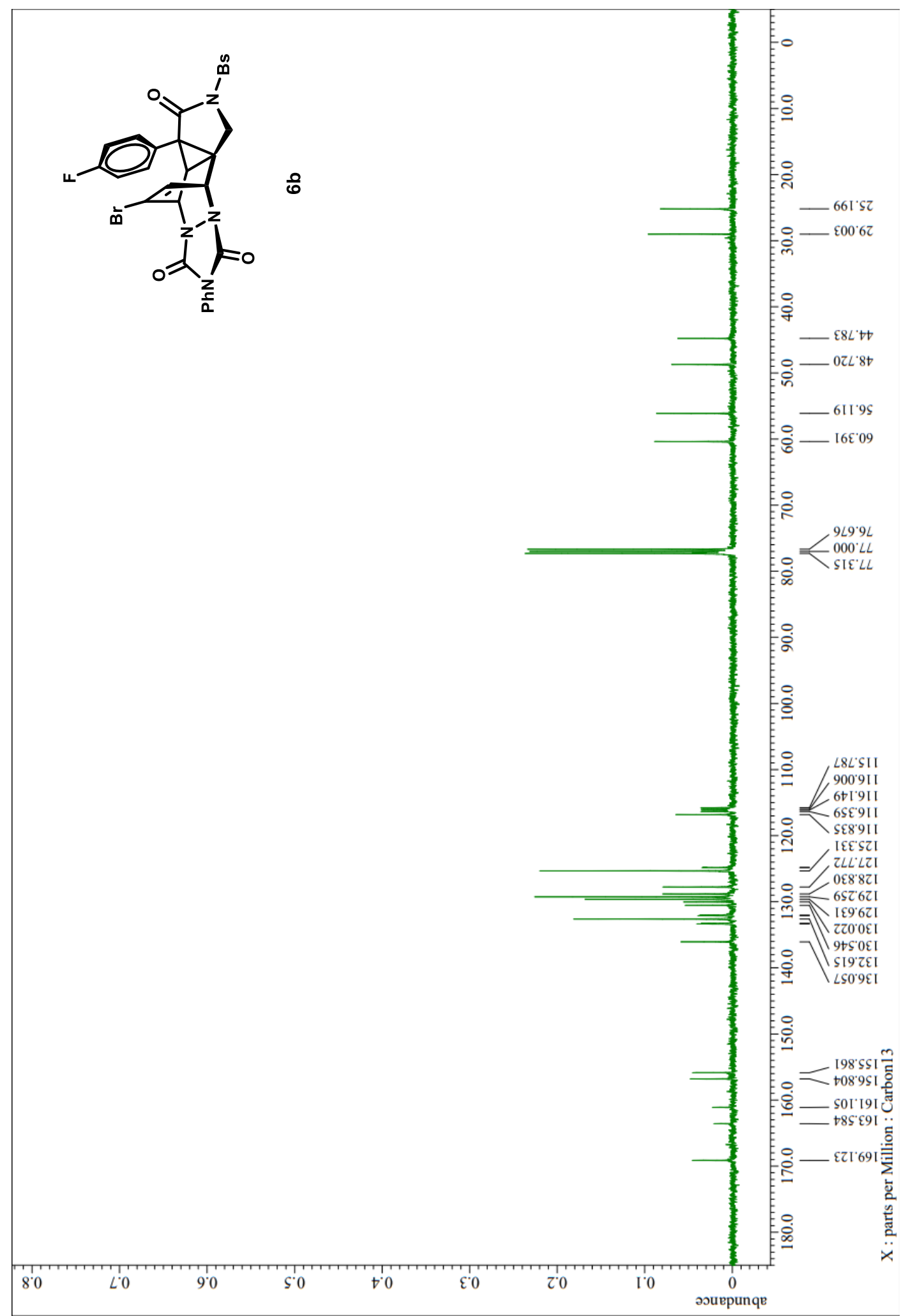


Supporting information

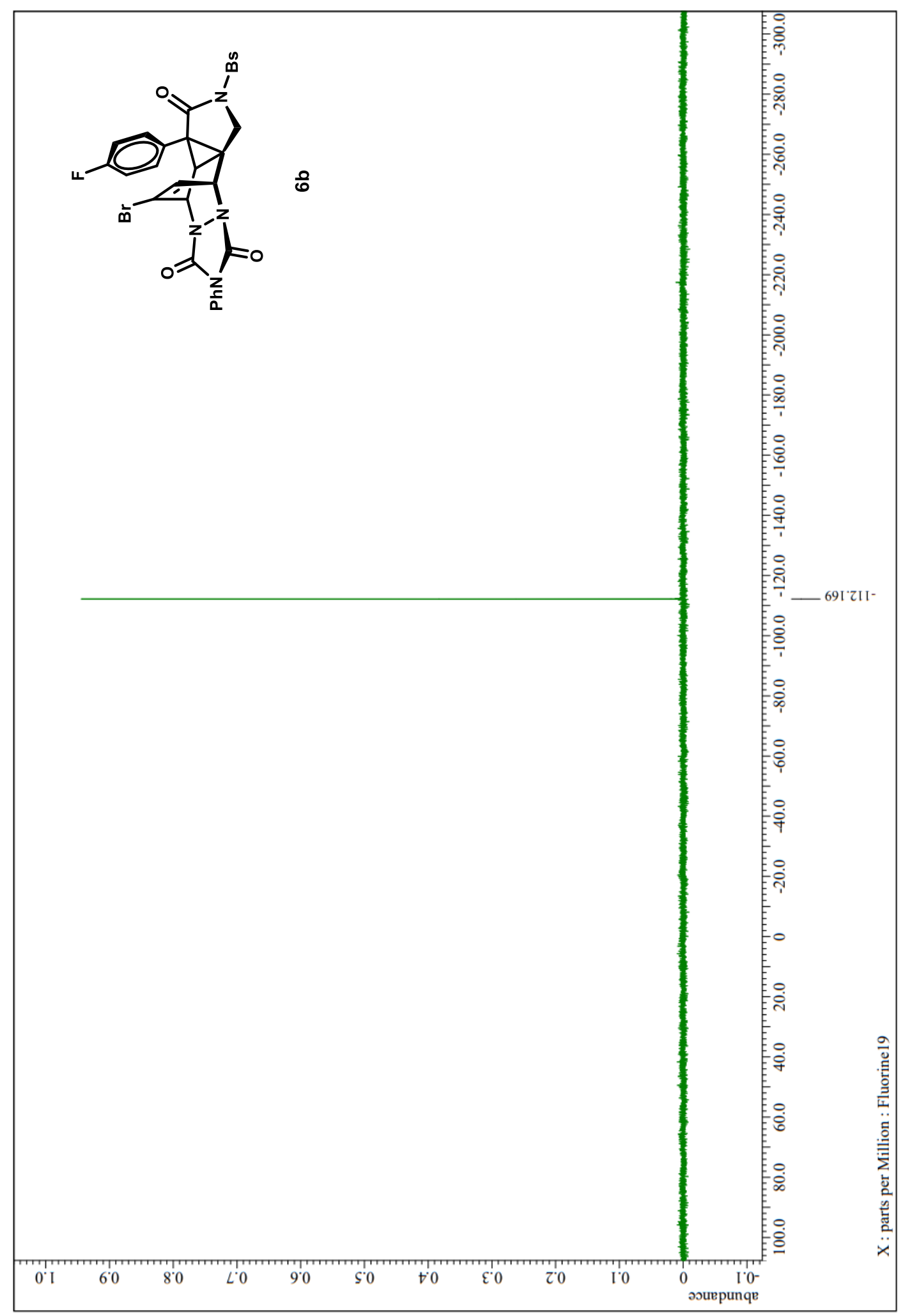


(3bR,4R,10R,10aS)-12-Bromo-3a-(4-bromophenyl)-2-((4-bromophenyl)sulfonyl)7-phenyl-1,2,3b,4-tetrahydro-6H,10H-4,10ethenopyrrolo[3',4':1,3]cyclopropa[1,2-d][1,2,4]triazolo[1,2-a]pyridazine3,6,8(3aH,7H)-trione (6c)

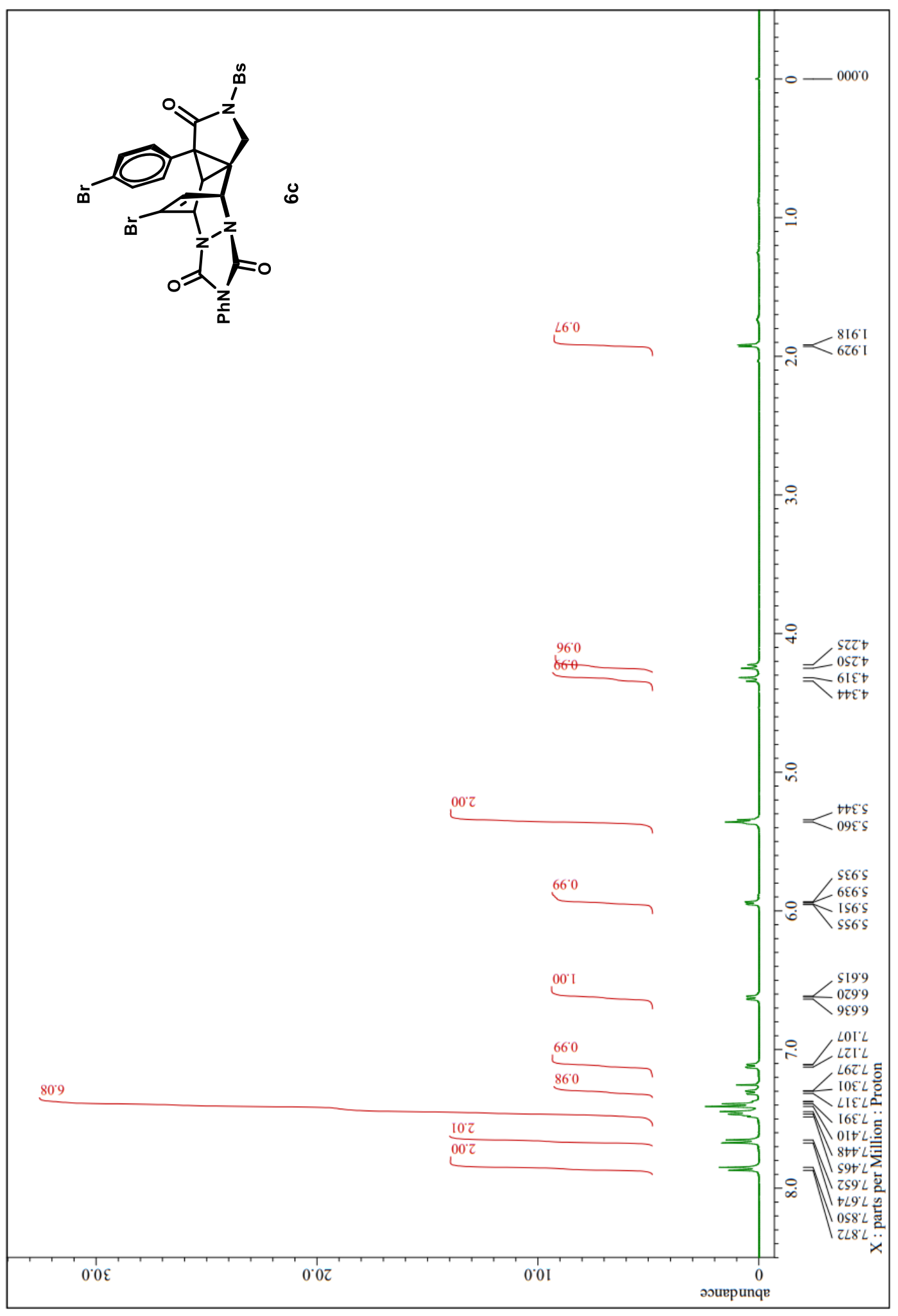


Supporting information

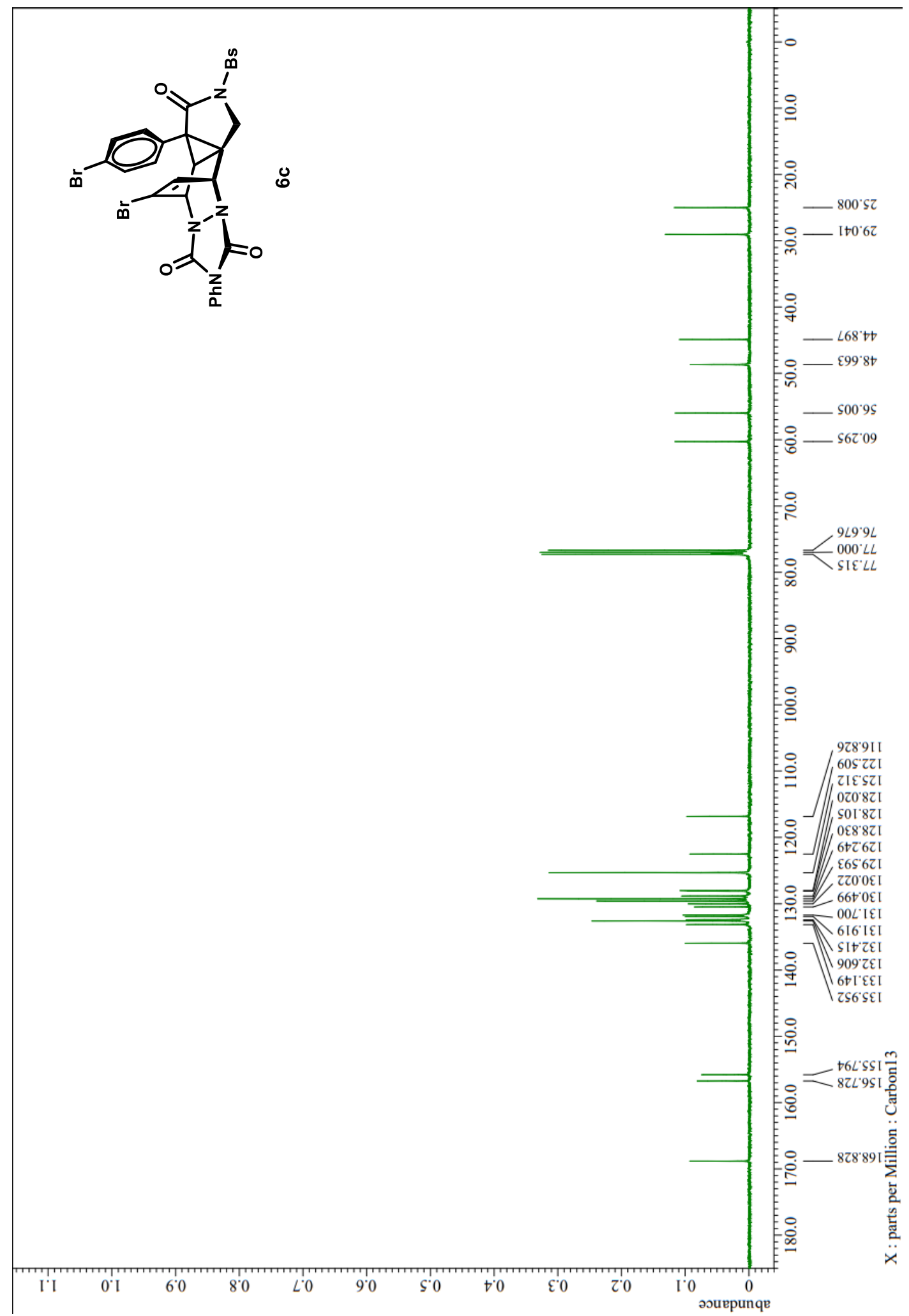


Methyl 4-((3bR,4R,10R,10aS)-12-Bromo-2-((4-bromophenyl)sulfonyl)-3,6,8trioxo-7-phenyl-2,3,3b,4,7,8-hexahydro-6H,10H-4,10ethenopyrrolo[3',4':1,3]cyclopropa[1,2-d][1,2,4]triazolo[1,2-a]pyridazin-3a(1H)yl)benzoate (6d)

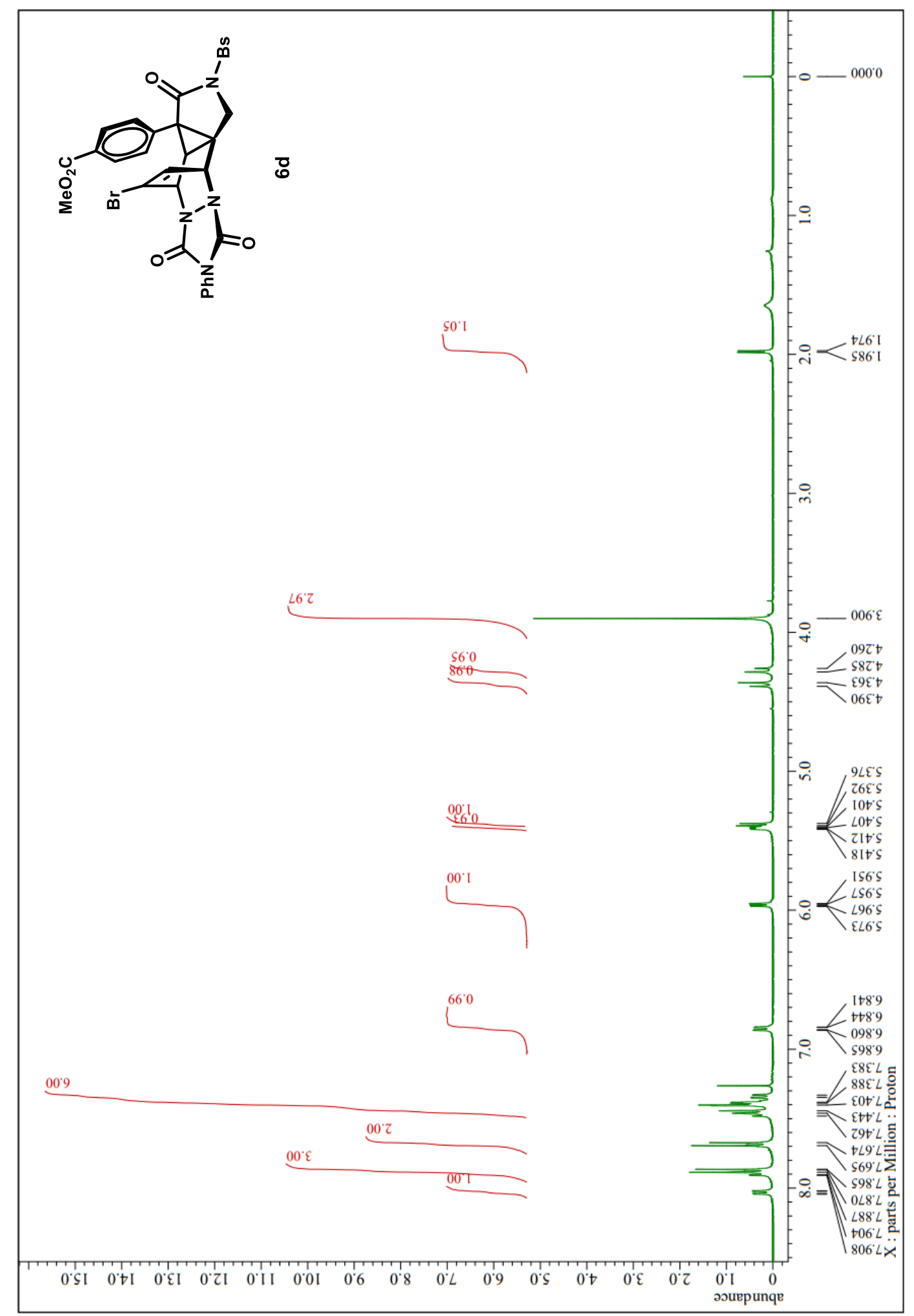


Supporting information

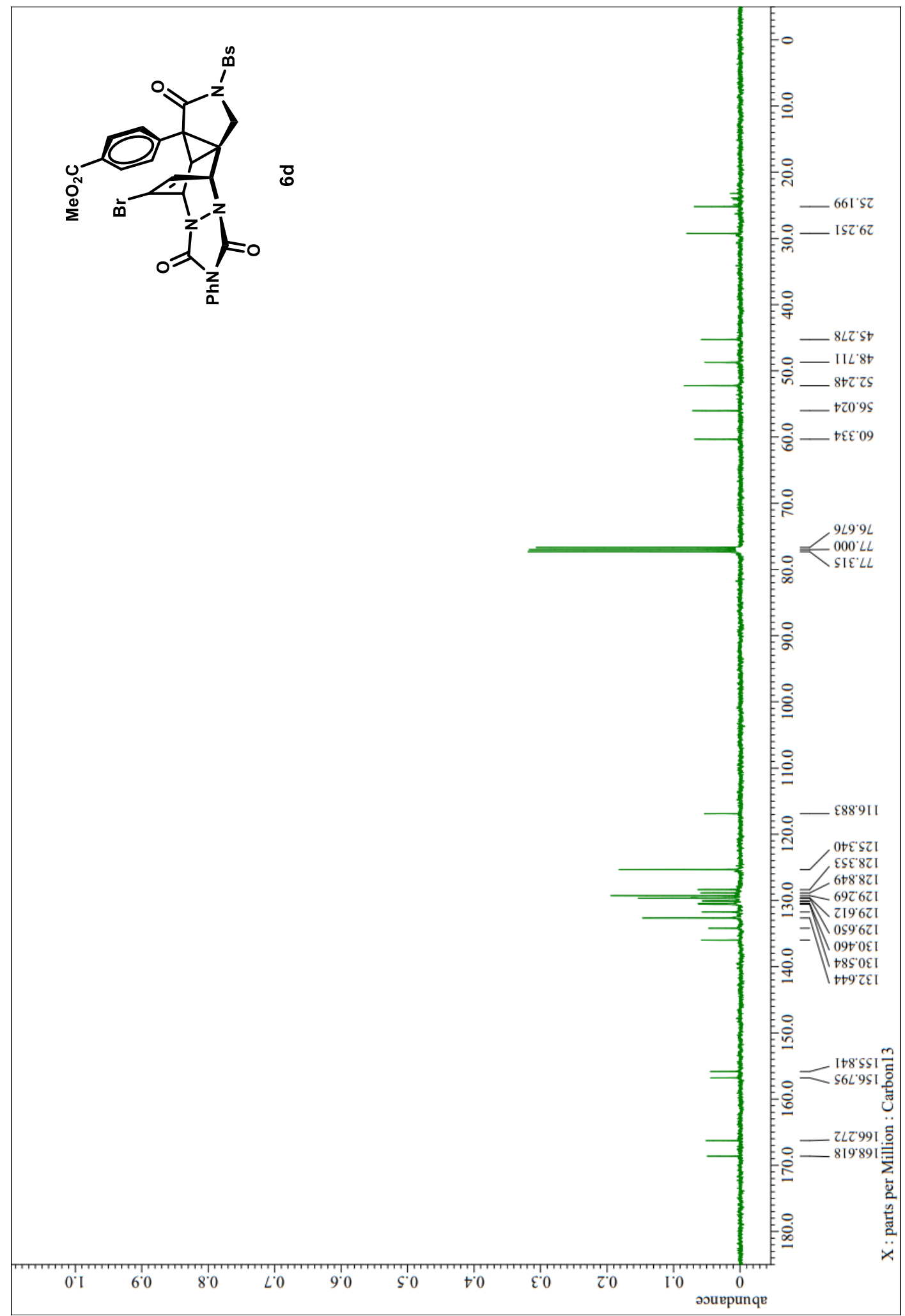


(3bS,4S,10S,10aR)-12-Bromo-3a,7-diphenyl-2-tosyl-1,2,3b,4-tetrahydro-6H,10H4,10-ethenopyrrolo[3',4':1,3]cyclopropa[1,2-d][1,2,4]triazolo[1,2-a]pyridazine$3,6,8(3 \mathrm{a} H, 7 H)$-trione $(6 \mathrm{e})$

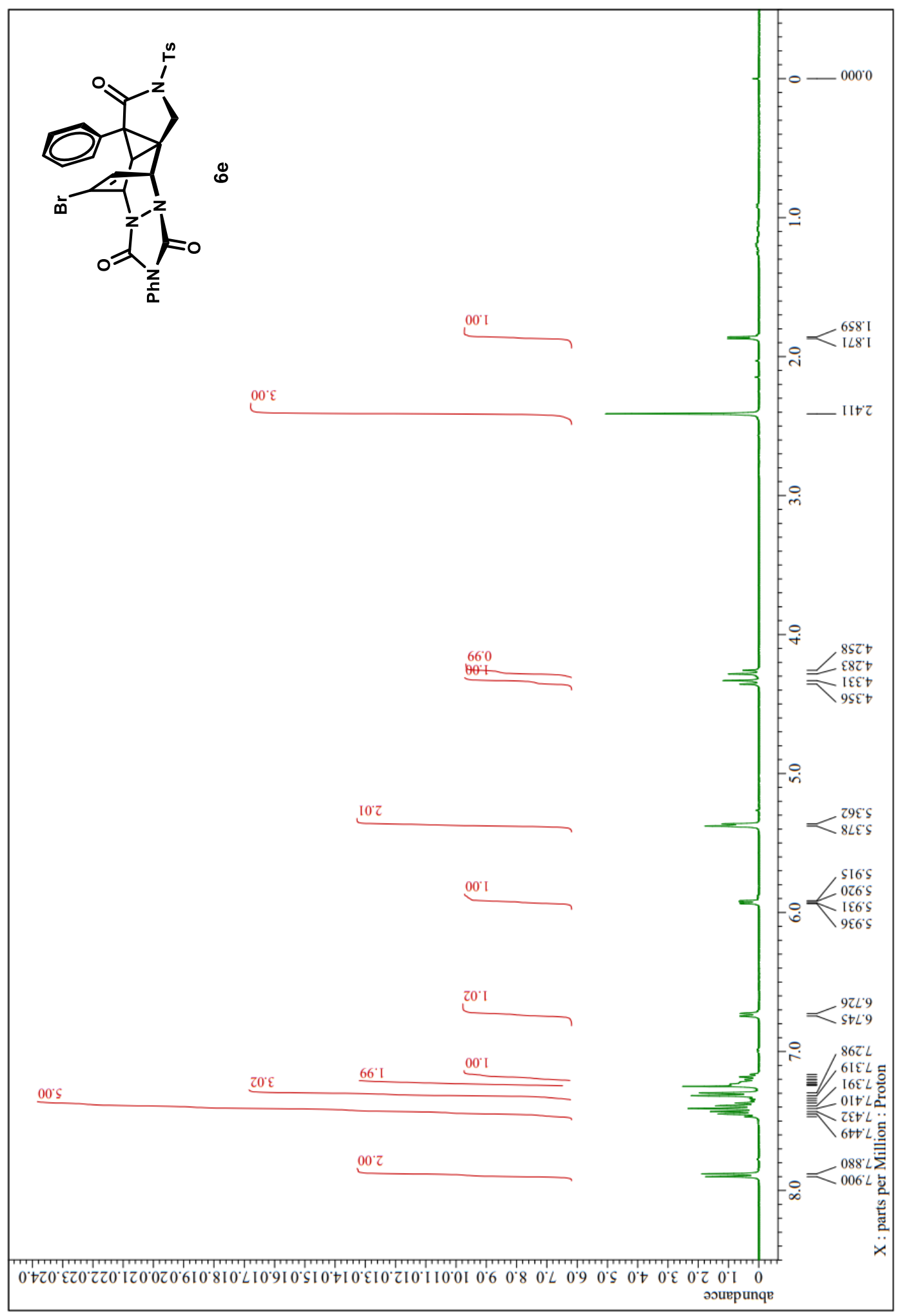


Supporting information

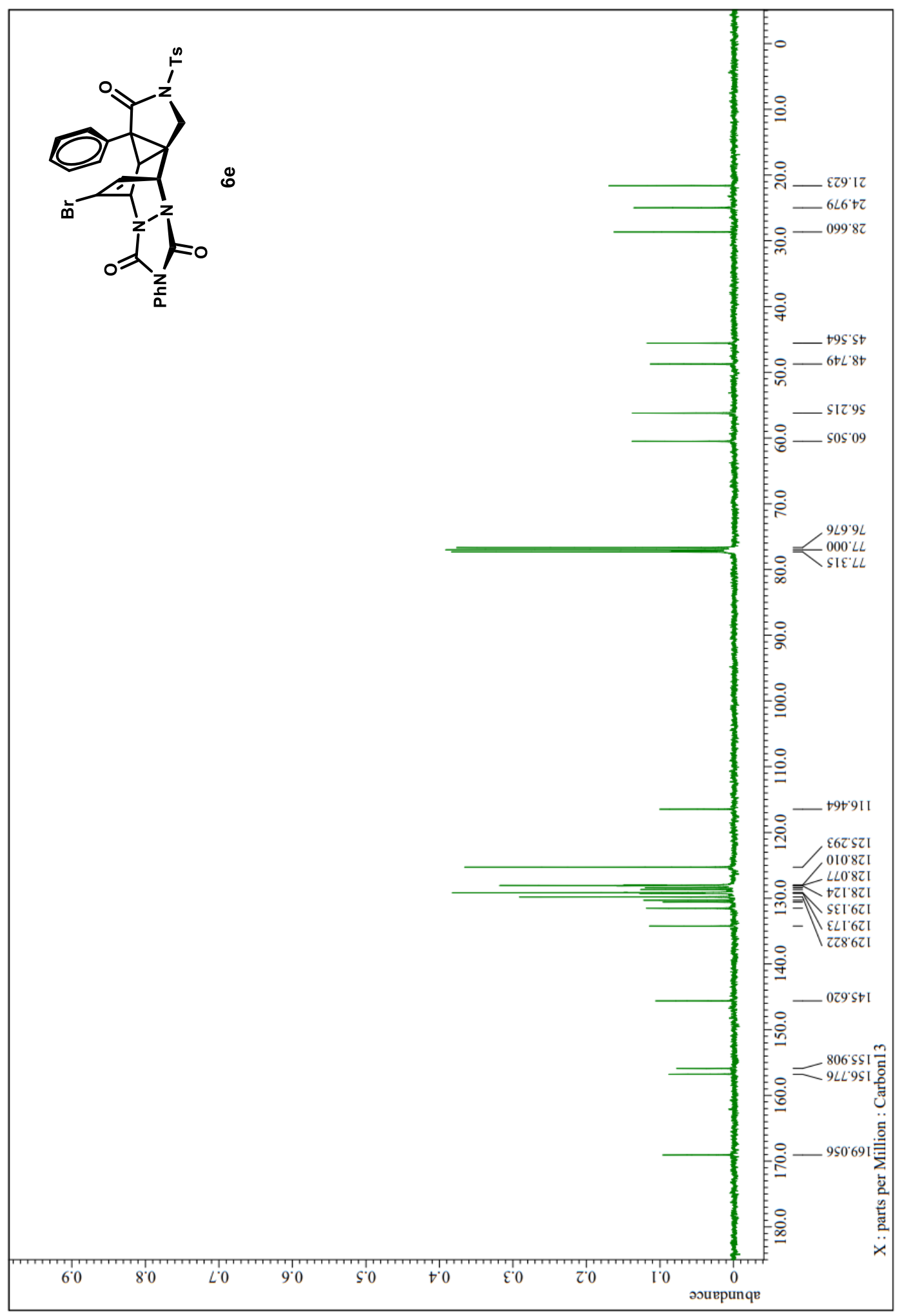


(3bR,4R,10R,10aS)-12-Bromo-2-((4-methoxyphenyl)sulfonyl)-3a,7-diphenyl1,2,3b,4-tetrahydro-6H,10H-4,10-ethenopyrrolo[3',4':1,3]cyclopropa[1,2d] $[1,2,4]$ triazolo[1,2-a]pyridazine-3,6,8(3aH,7H)-trione (6f)

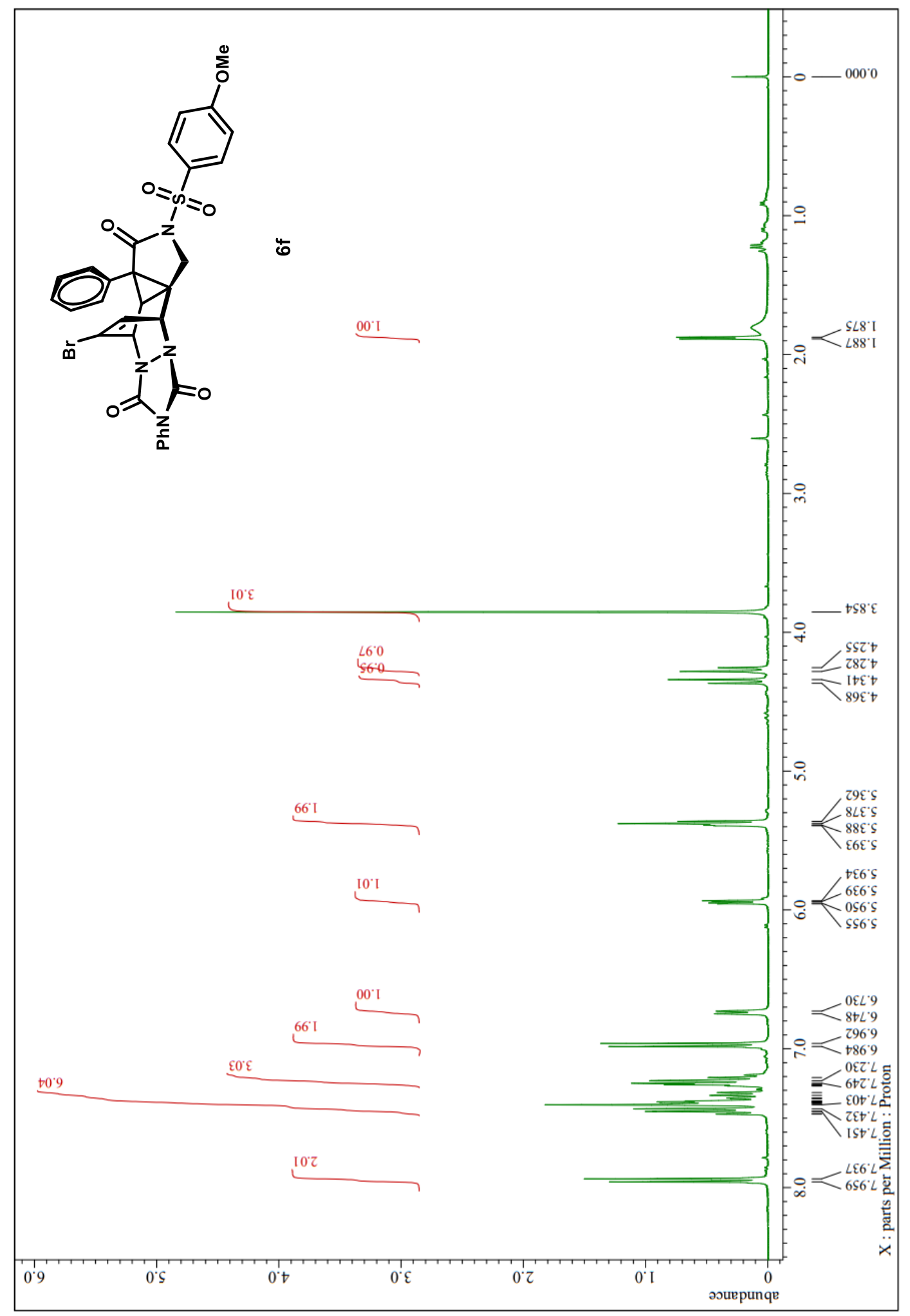


Supporting information

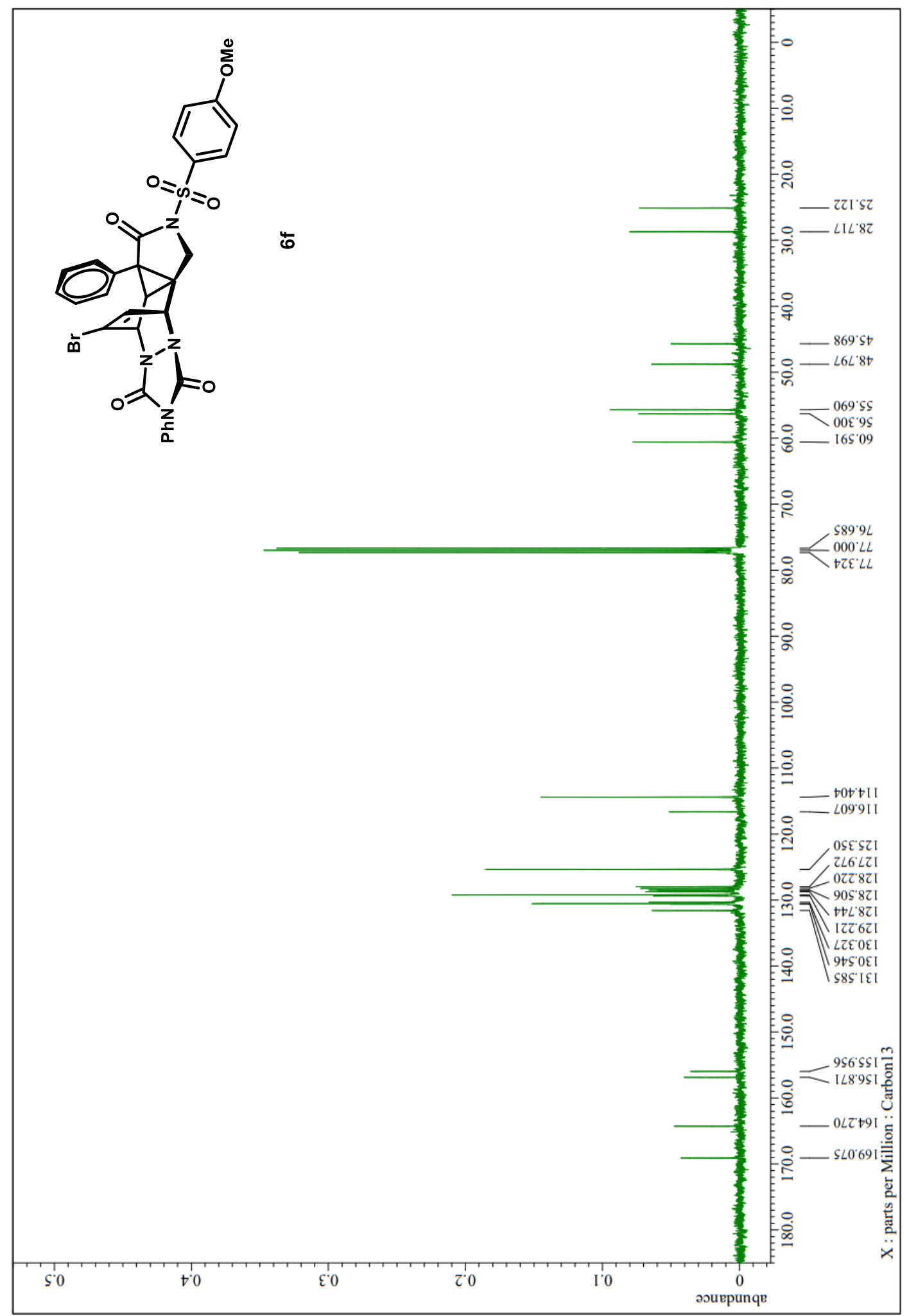


(3bR,4R,10R,10aS)-12-Bromo-3a-(3-bromophenyl)-7-phenyl-2-tosyl-1,2,3b,4tetrahydro-6H,10H-4,10-ethenopyrrolo[3',4':1,3]cyclopropa[1,2d] $[1,2,4]$ triazolo[1,2-a]pyridazine-3,6,8(3aH,7H)-trione $(6 \mathrm{~g})$

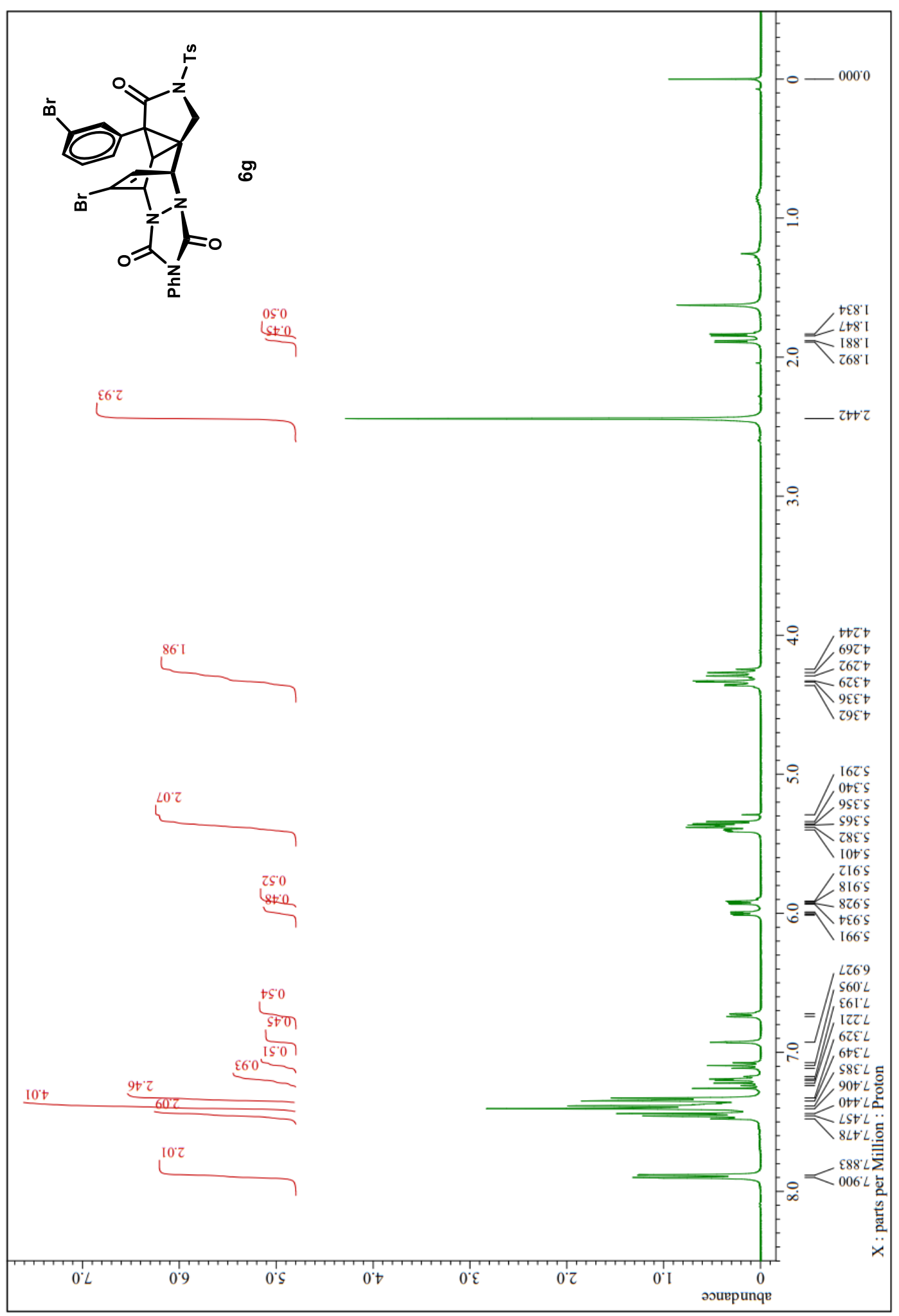


Supporting information

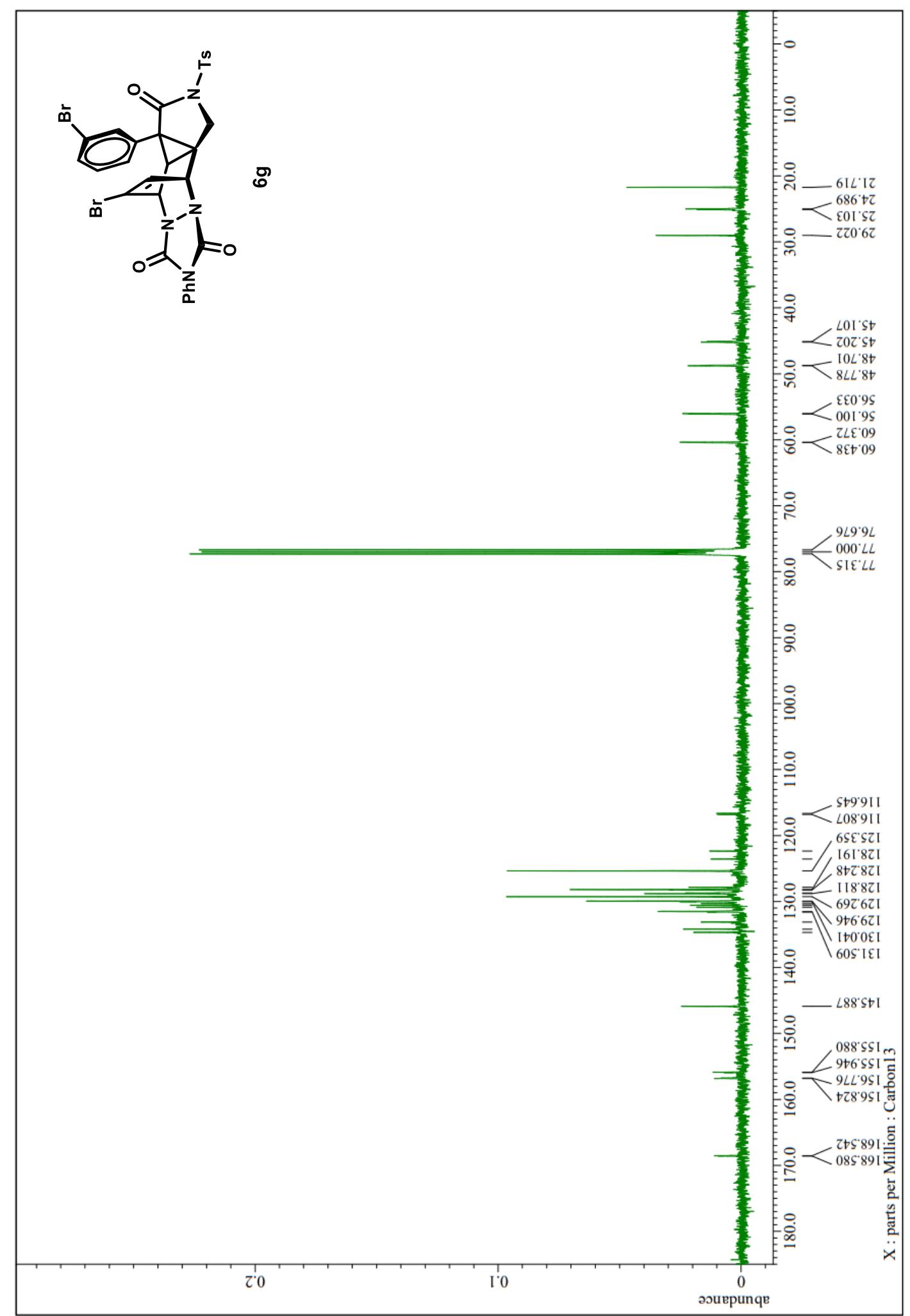


(3bR,4R,10R,10aR)-12-Bromo-2-((4-bromophenyl)sulfonyl)-3a-(3methoxyphenyl)-7-phenyl-1,2,3b,4-tetrahydro-6H,10H-4,10ethenopyrrolo[3',4':1,3]cyclopropa[1,2-ه][1,2,4]triazolo[1,2-a]pyridazine3,6,8(3aH,7H)-trione (6h)

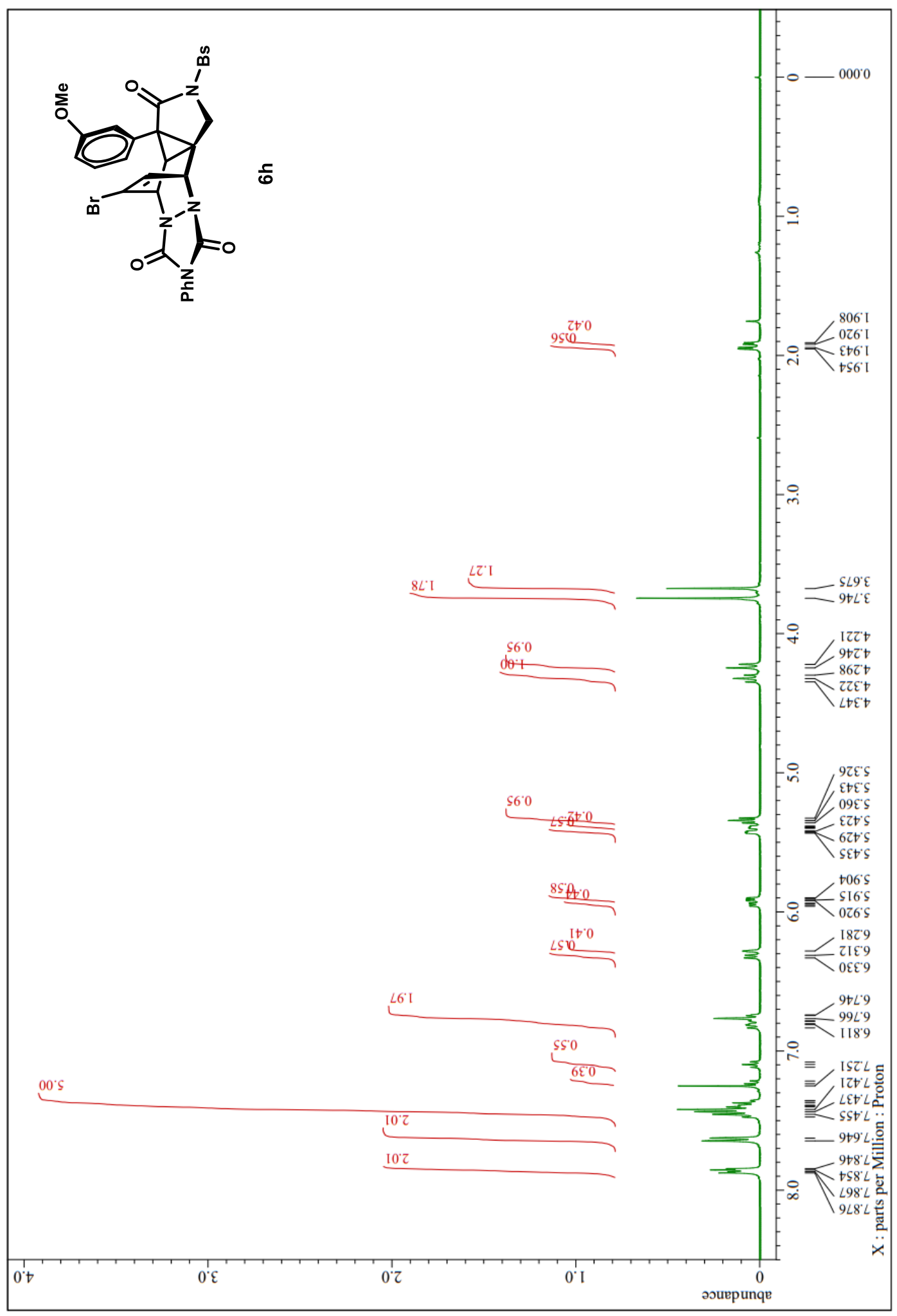


Supporting information

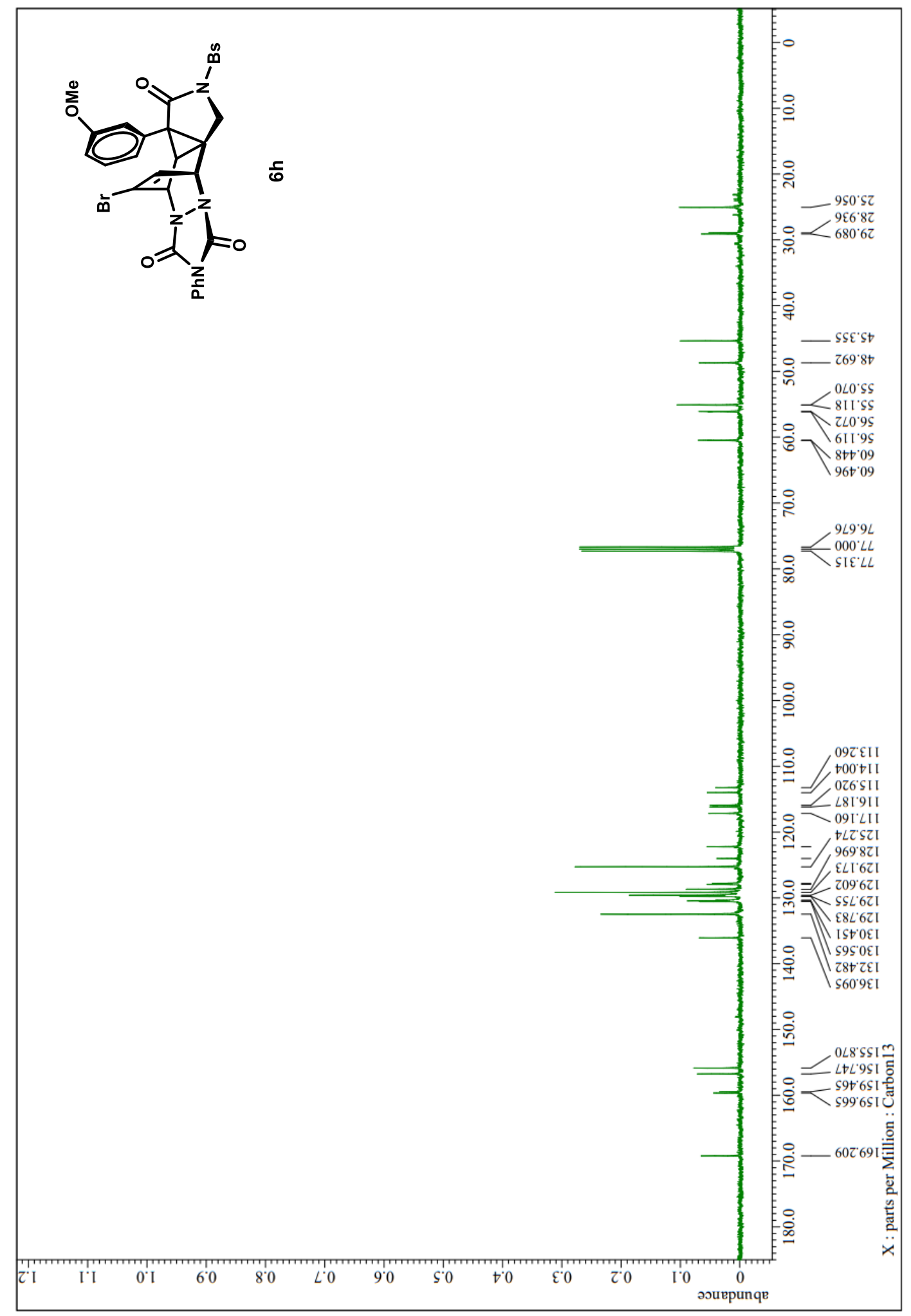


(3bR,4R,10R,10aS)-2-((4-Bromophenyl)sulfonyl)-12-fluoro-3a,7-diphenyl1,2,3b,4-tetrahydro-6H,10H-4,10-ethenopyrrolo[3',4':1,3]cyclopropa[1,2d] $[1,2,4]$ triazolo[1,2-a]pyridazine-3,6,8(3aH,7H)-trione (6i)

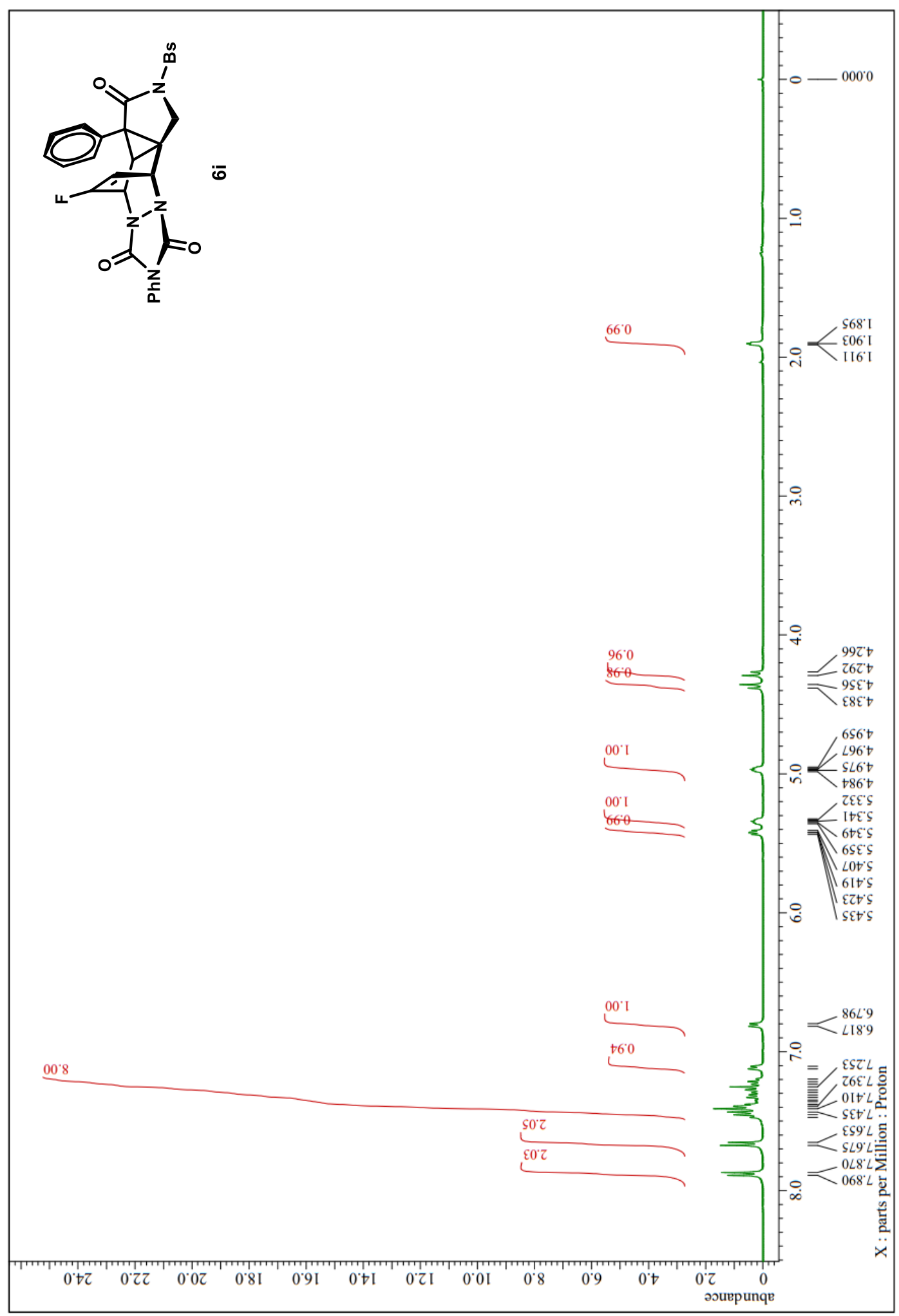


Supporting information

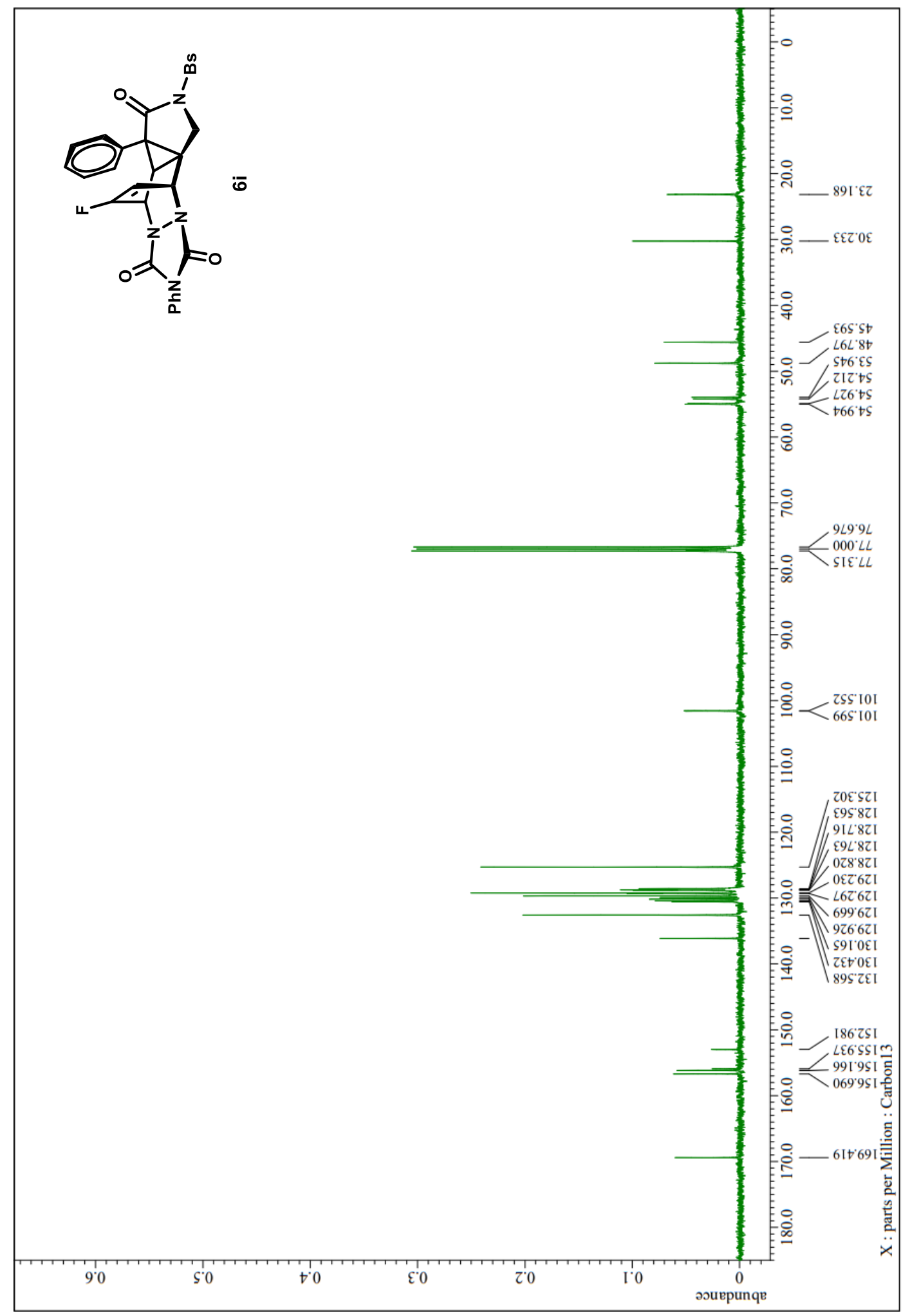


Supporting information

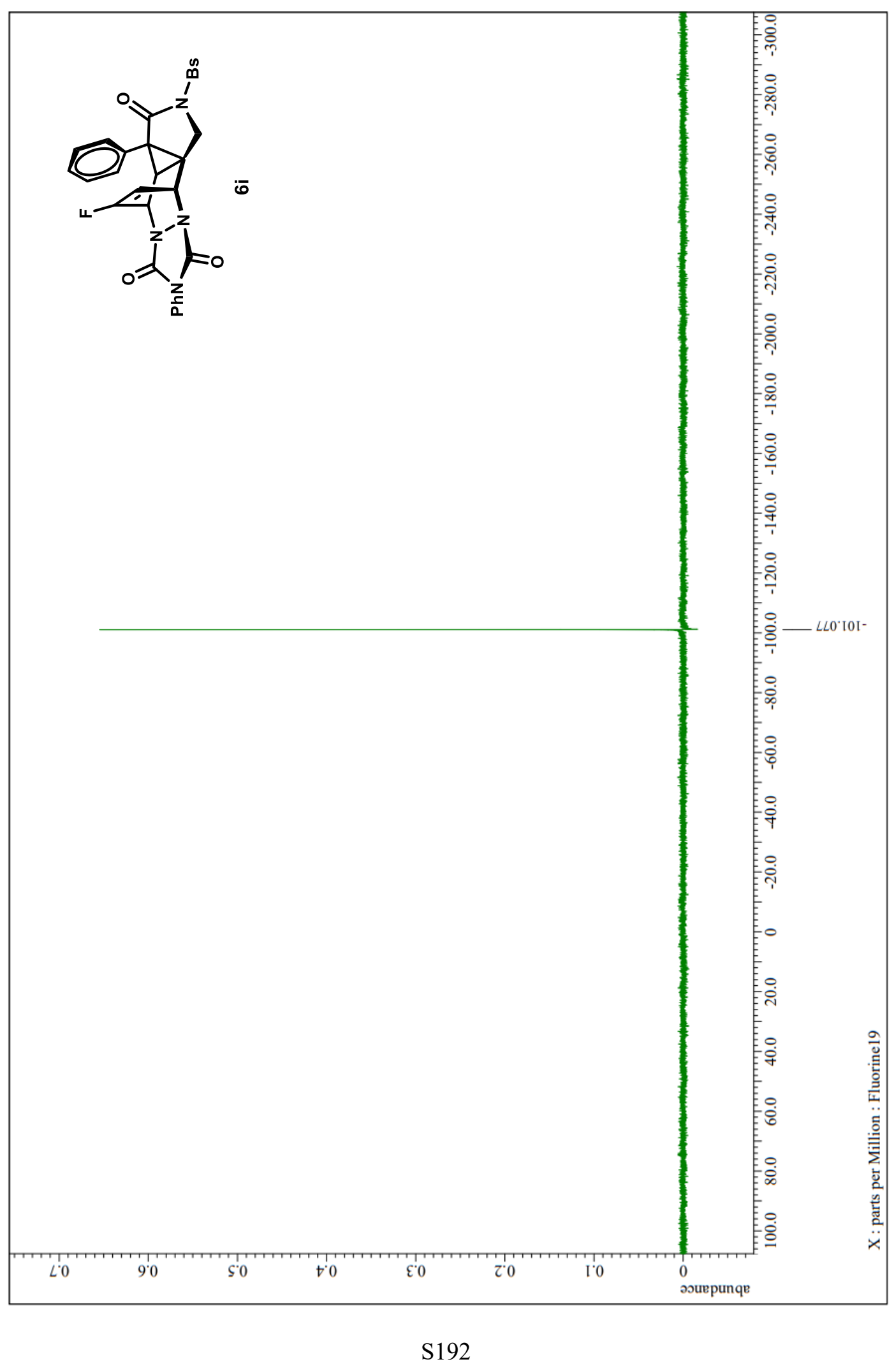


(3b $R, 4 R, 10 R, 10 a S)-12-F l u o r o-3 a, 7-d i p h e n y l-2-t o s y l-1,2,3 b, 4-t e t r a h y d r o-6 H, 10 H-$ 4,10-ethenopyrrolo[3',4':1,3]cyclopropa[1,2-o][1,2,4]triazolo[1,2-a]pyridazine3,6,8(3aH,7H)-trione (6j)

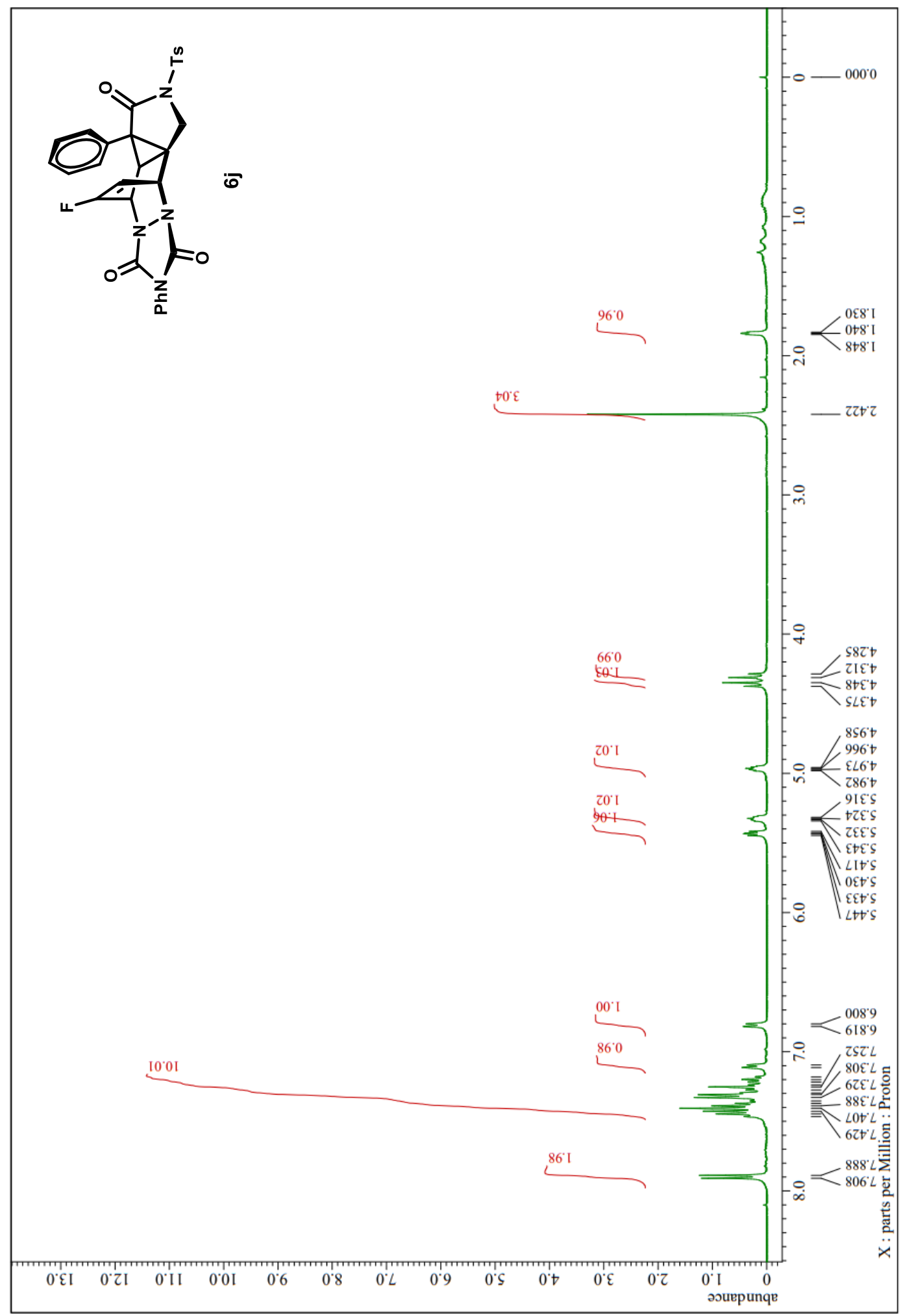


Supporting information

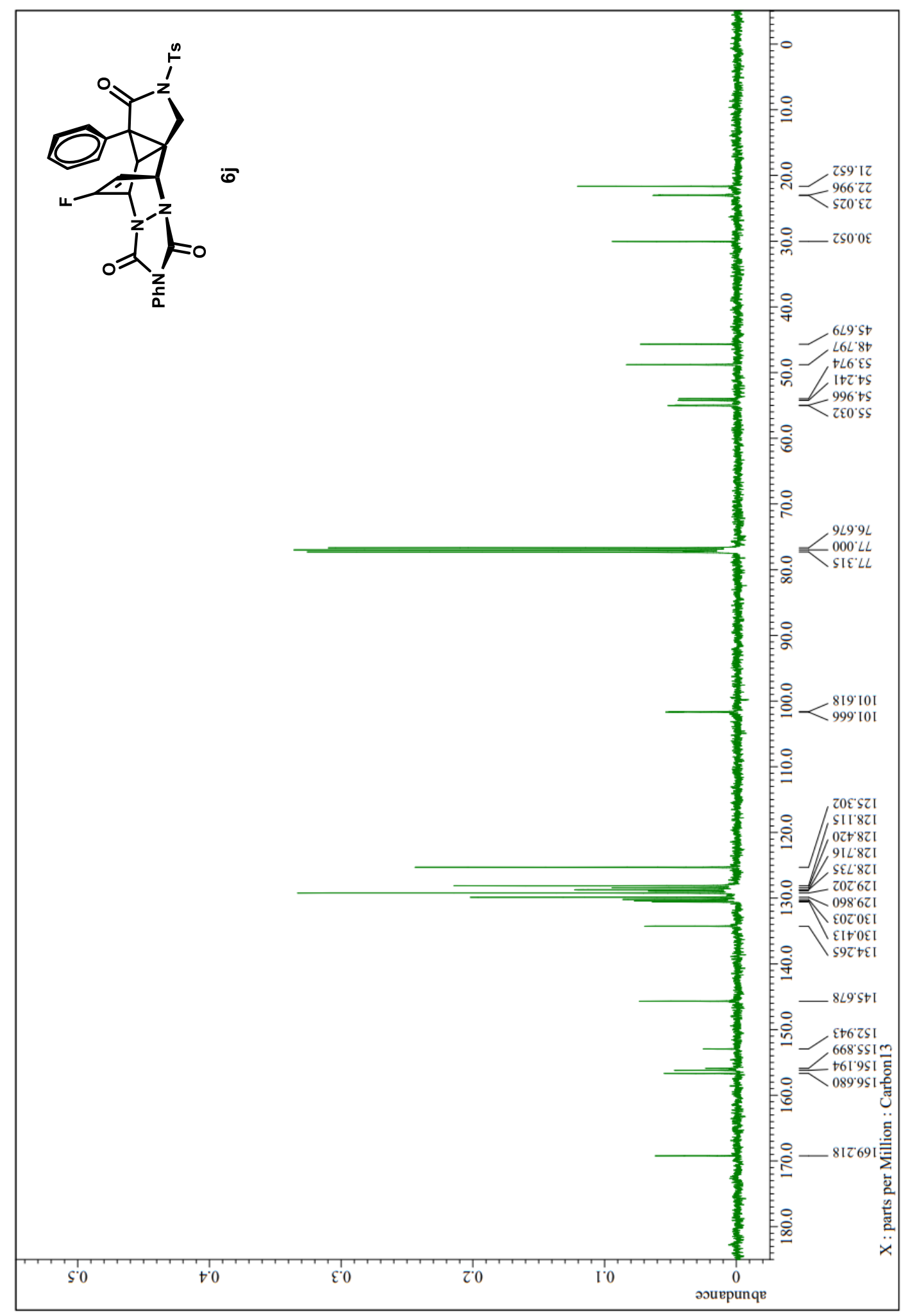


Supporting information

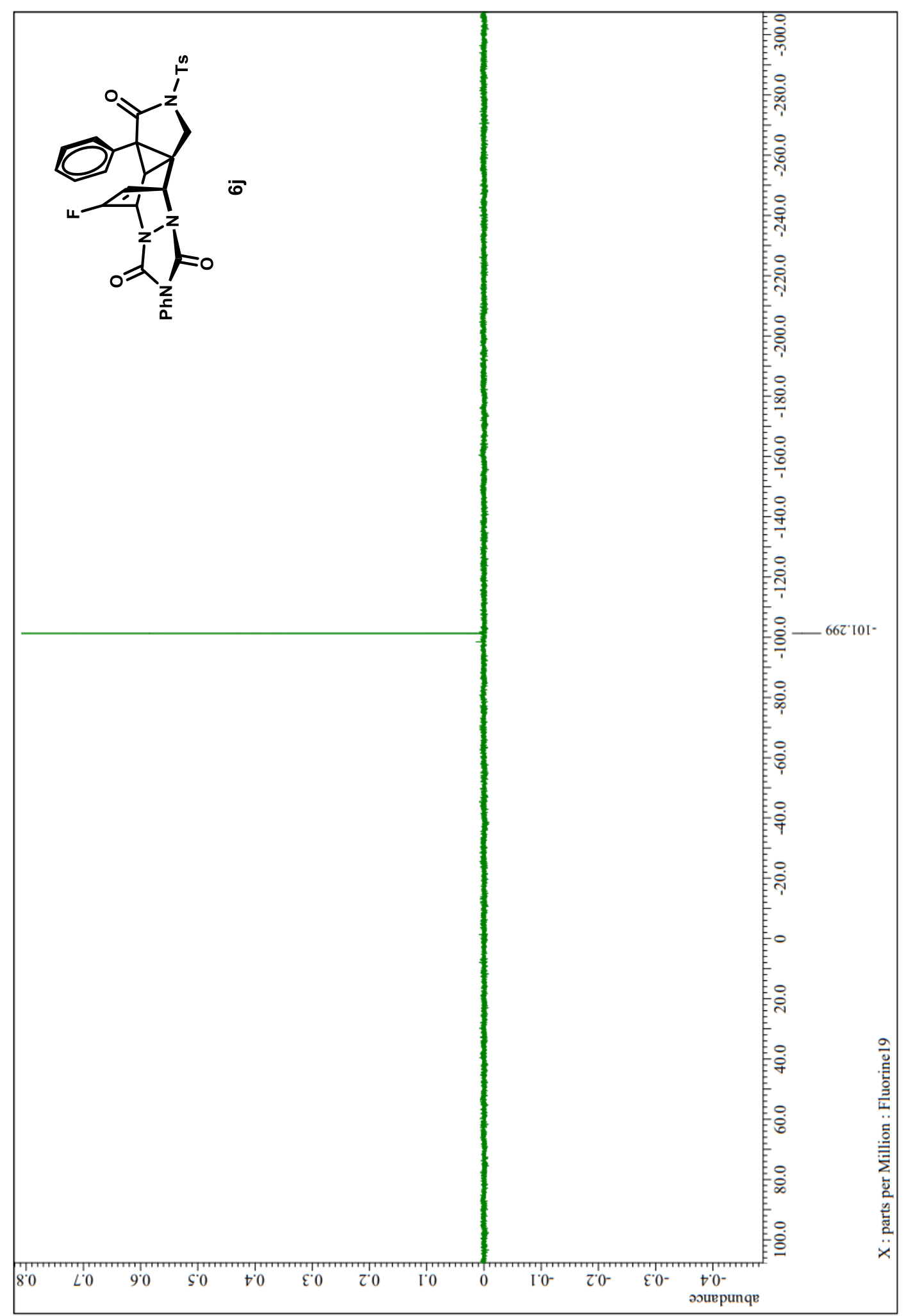


(3b $, 4 R, 10 R, 10 \mathrm{aS})$-12-Chloro-3a,7-diphenyl-2-tosyl-1,2,3b,4-tetrahydro-6H,10H4,10-ethenopyrrolo[3',4':1,3]cyclopropa[1,2-o][1,2,4]triazolo[1,2-a]pyridazine3,6,8(3aH,7H)-trione (6k)

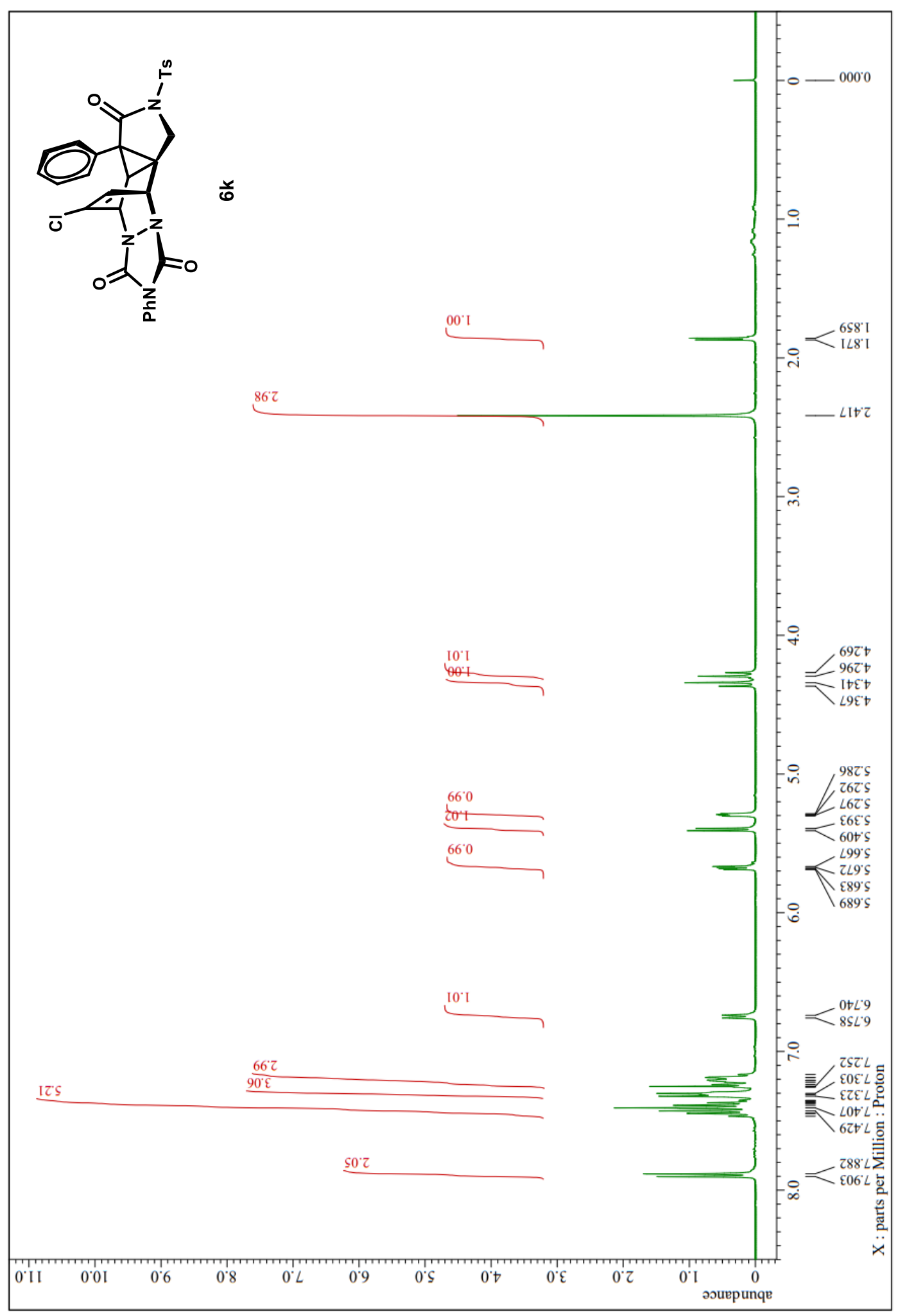


Supporting information

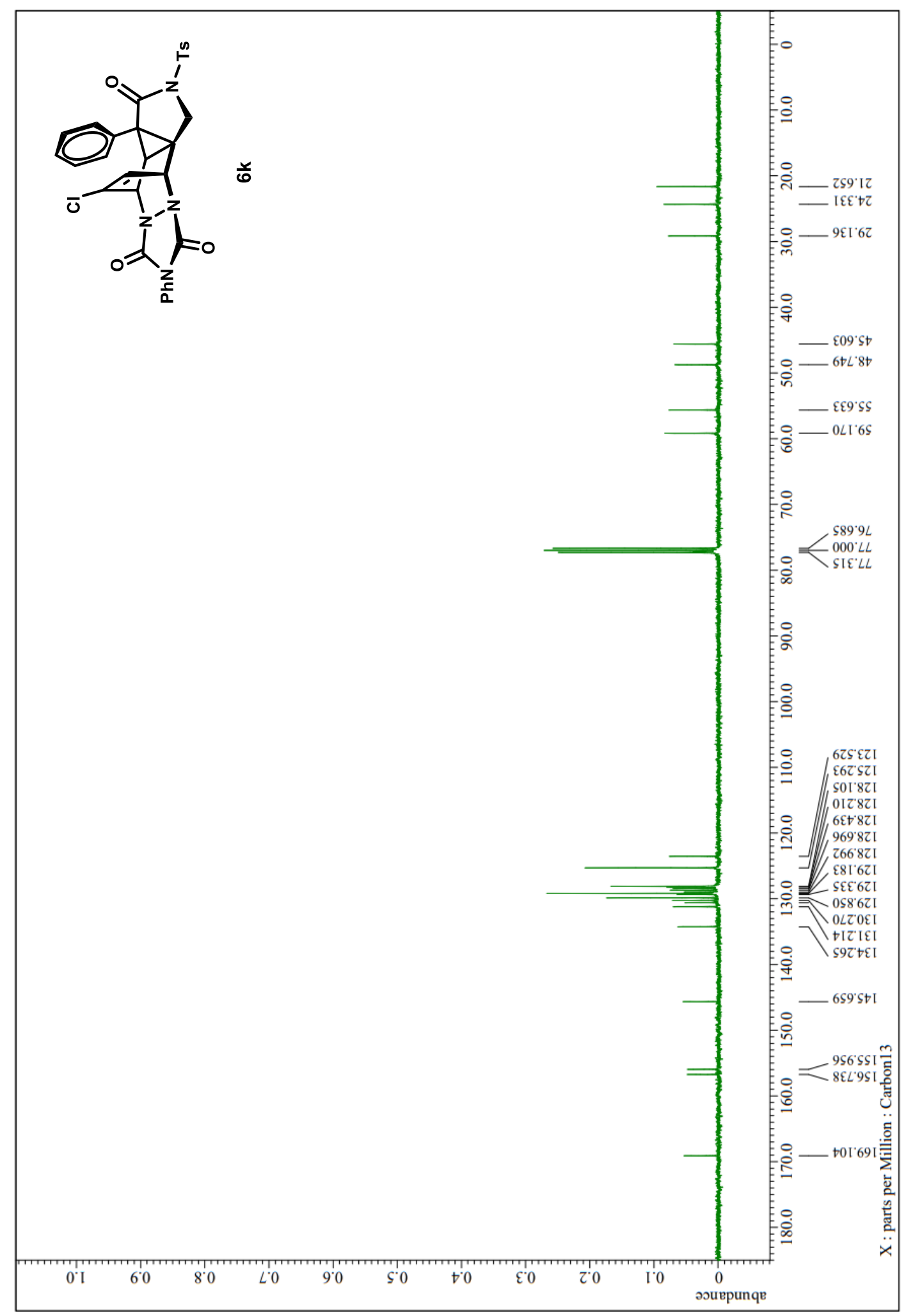


(3bR,4R,10R,10aS)-2-((4-Bromophenyl)sulfonyl)-12-iodo-3a,7-diphenyl-1,2,3b,4tetrahydro-6H,10H-4,10-ethenopyrrolo[3',4':1,3]cyclopropa[1,2d] $[1,2,4]$ triazolo[1,2-a]pyridazine-3,6,8(3aH,7H)-trione (6I)

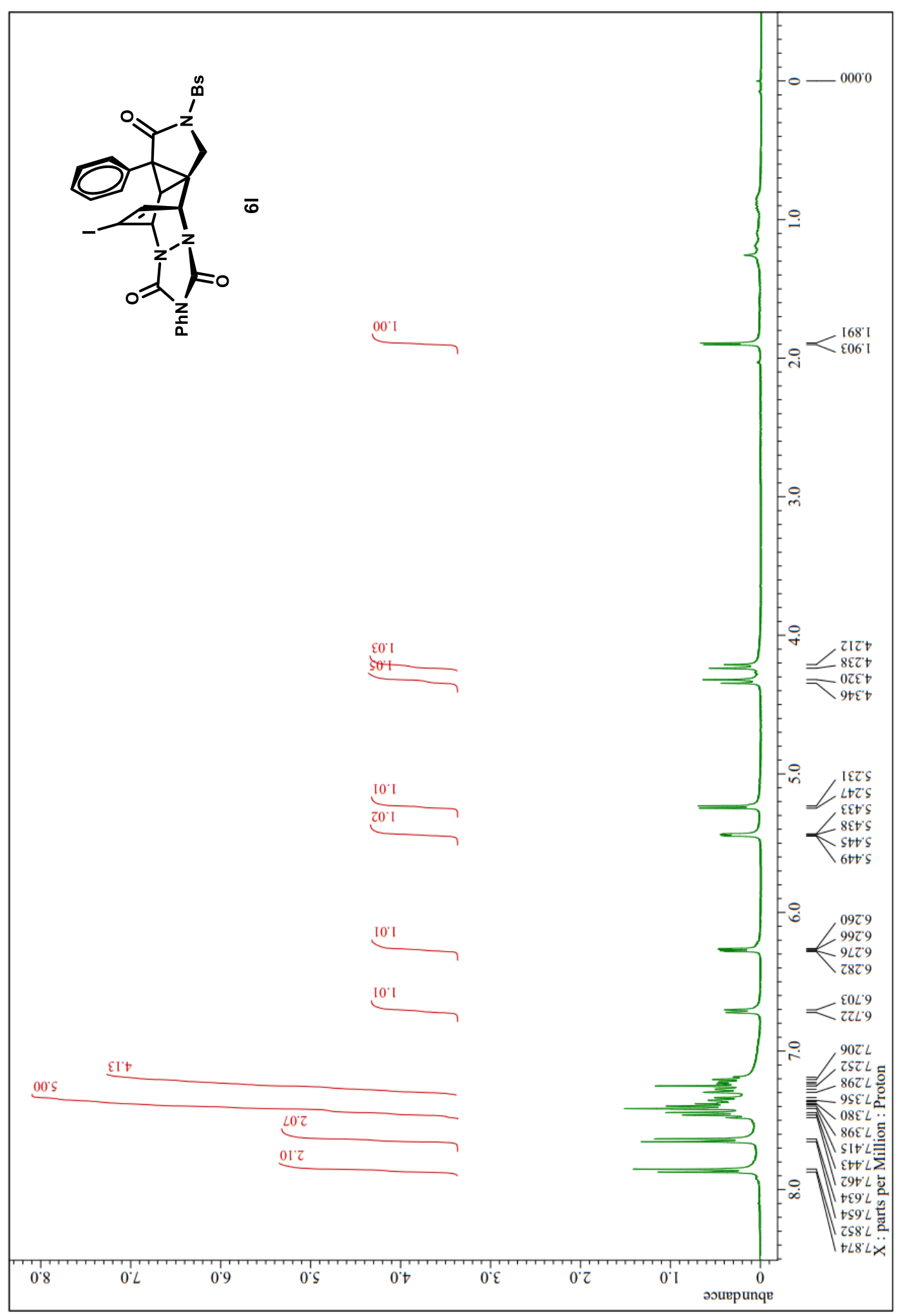


Supporting information

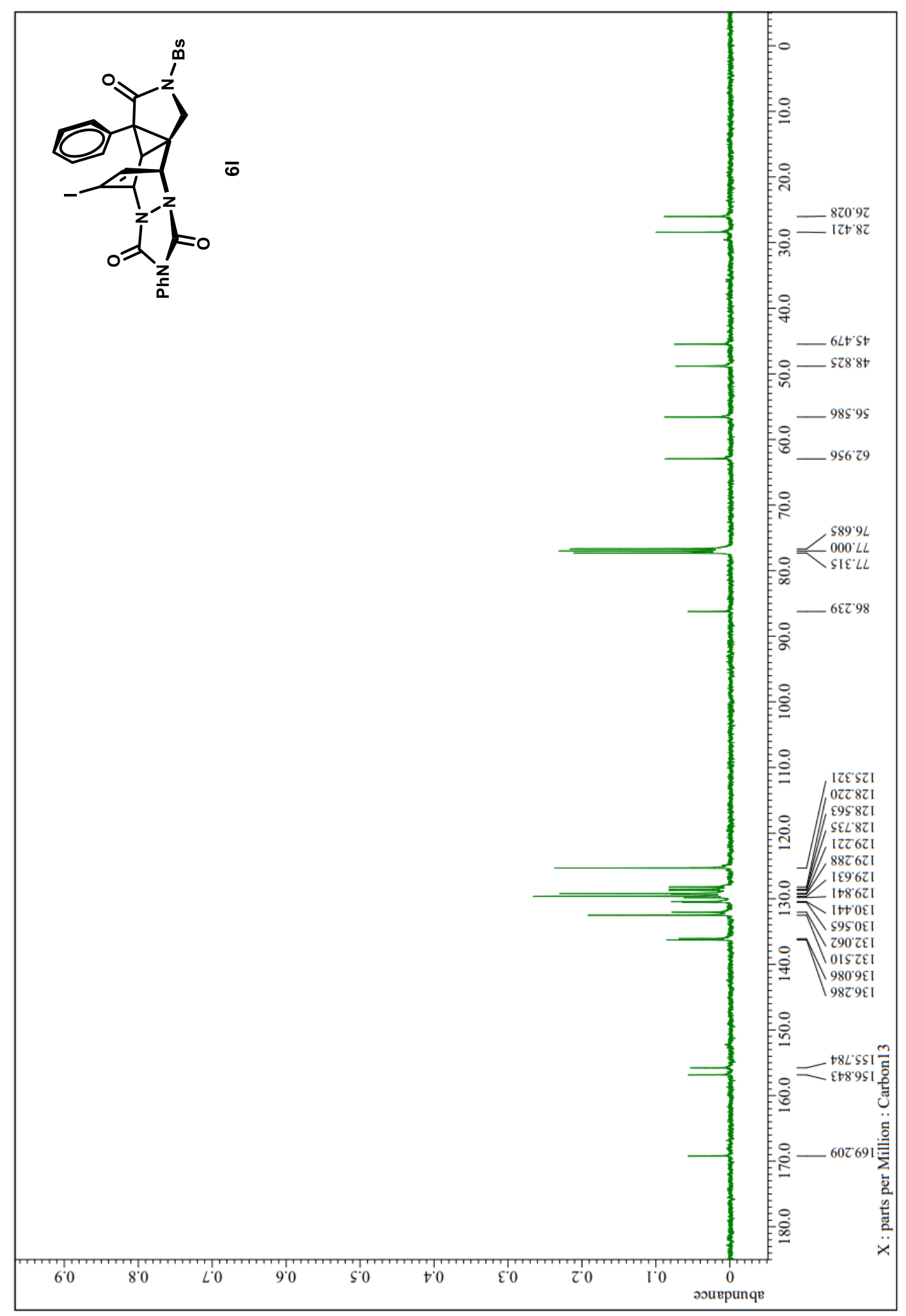


(3bR,4R,10R,10aS)-2-((4-Bromophenyl)sulfonyl)-12-methyl-3a,7-diphenyl1,2,3b,4-tetrahydro-6H,10H-4,10-ethenopyrrolo[3',4':1,3]cyclopropa[1,2d] $[1,2,4]$ triazolo[1,2-a]pyridazine-3,6,8(3aH,7H)-trione $(6 \mathrm{~m})$

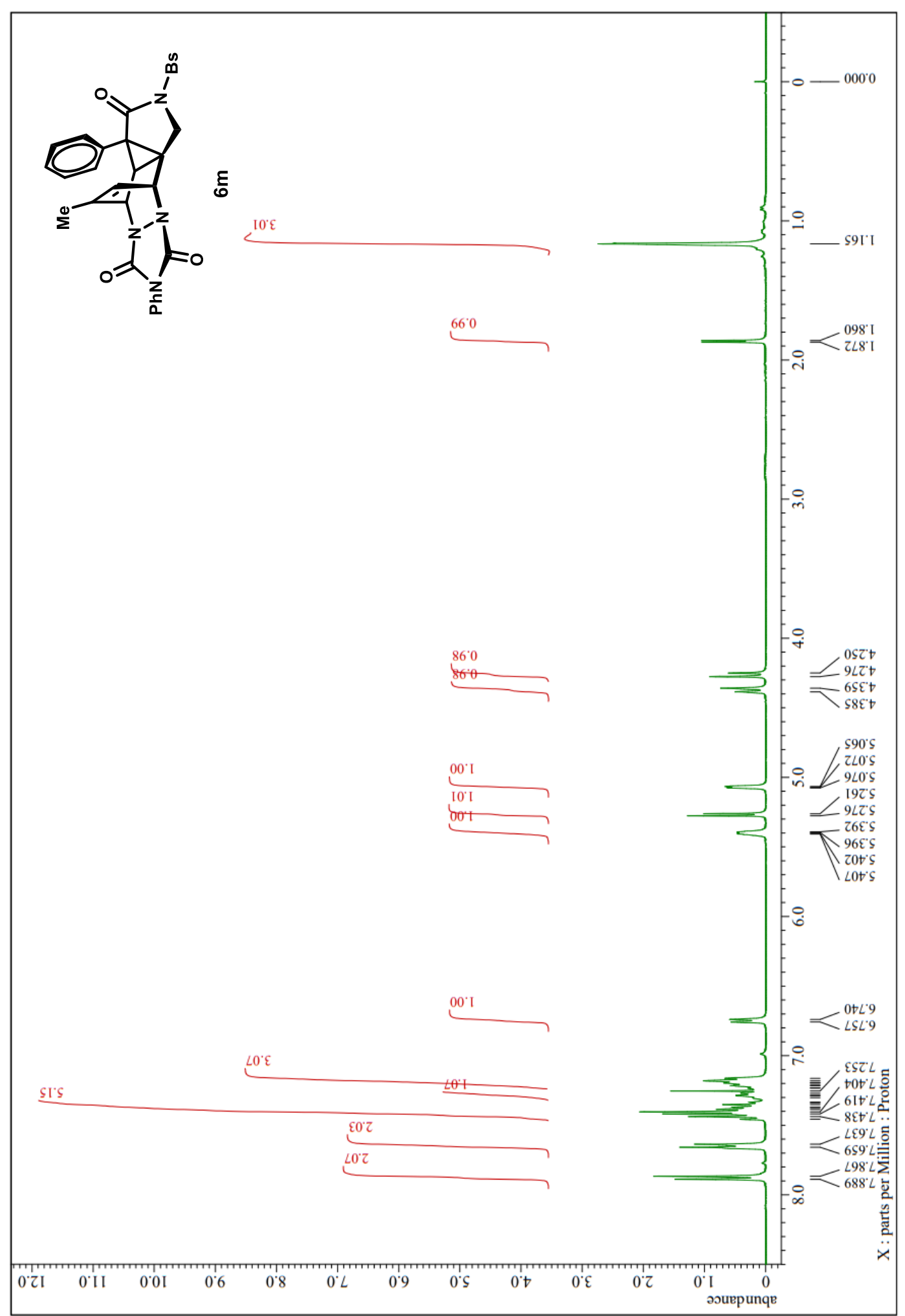


Supporting information

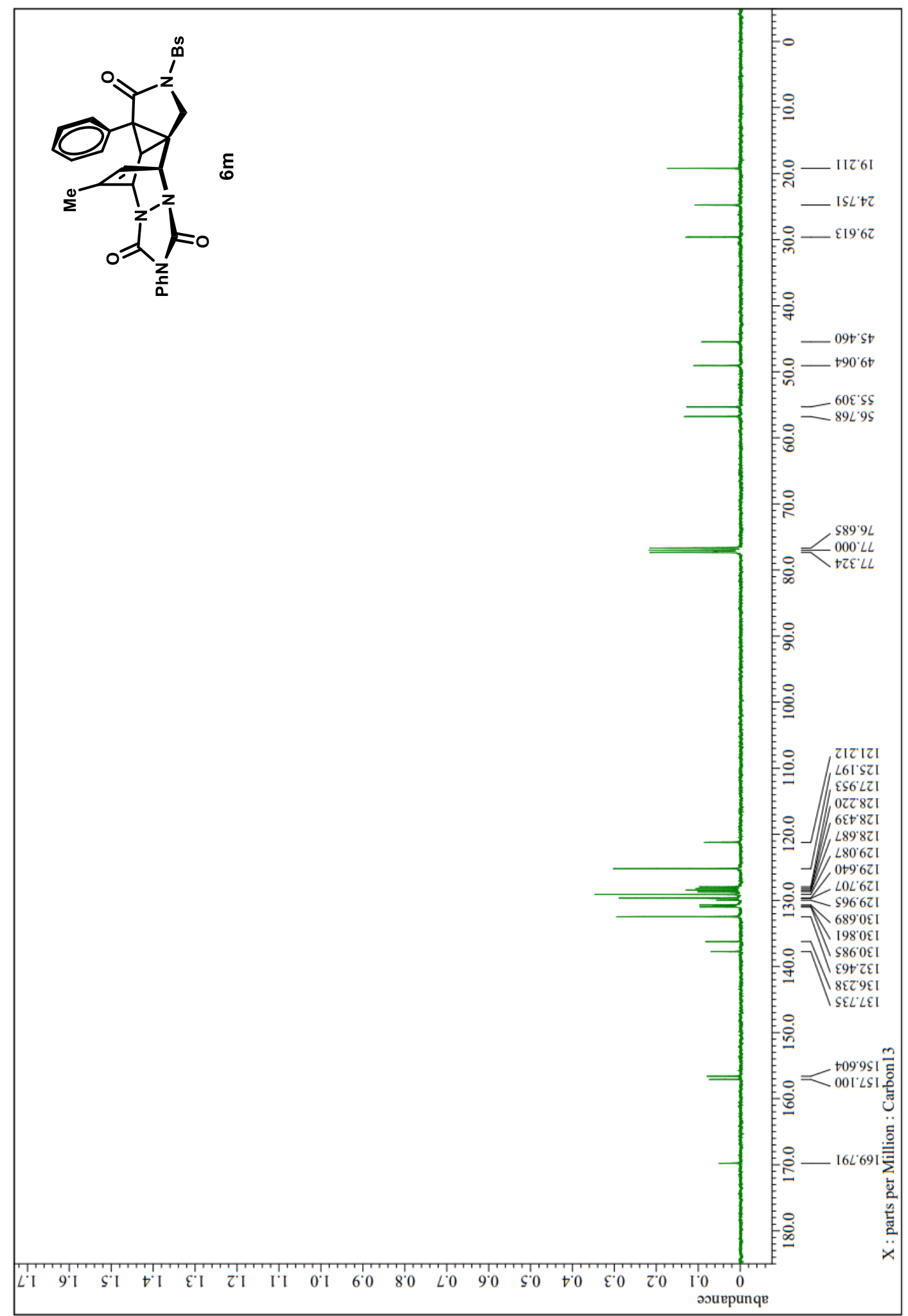


(3bR,4S,10R,10aS)-2-((4-Bromophenyl)sulfonyl)-3a,7-diphenyl-1,2,3b,4tetrahydro-6H,10H-4,10-ethenopyrrolo[3',4':1,3]cyclopropa[1,2d] $[1,2,4]$ triazolo[1,2-a]pyridazine-3,6,8(3aH,7H)-trione (6n)

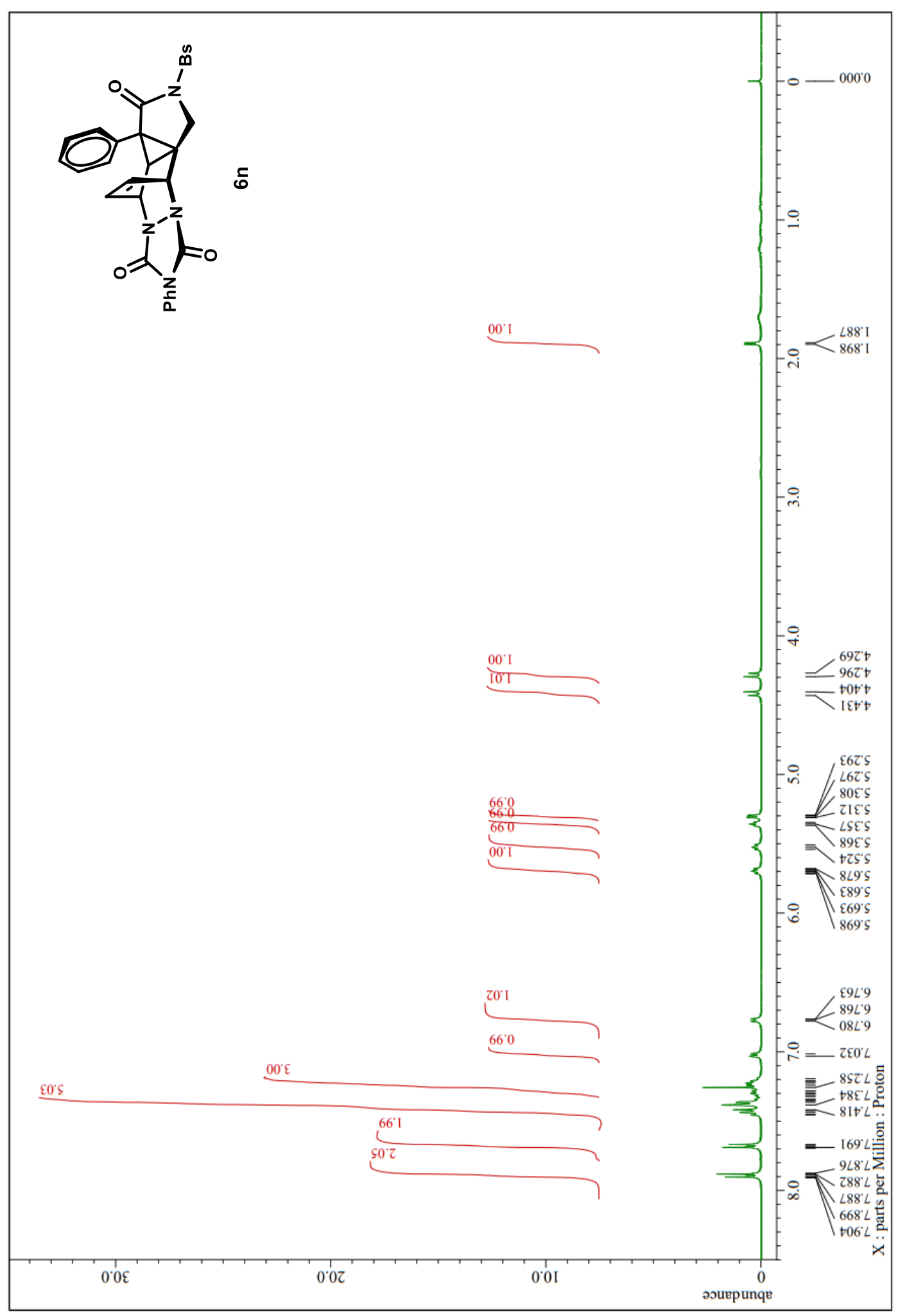


Supporting information

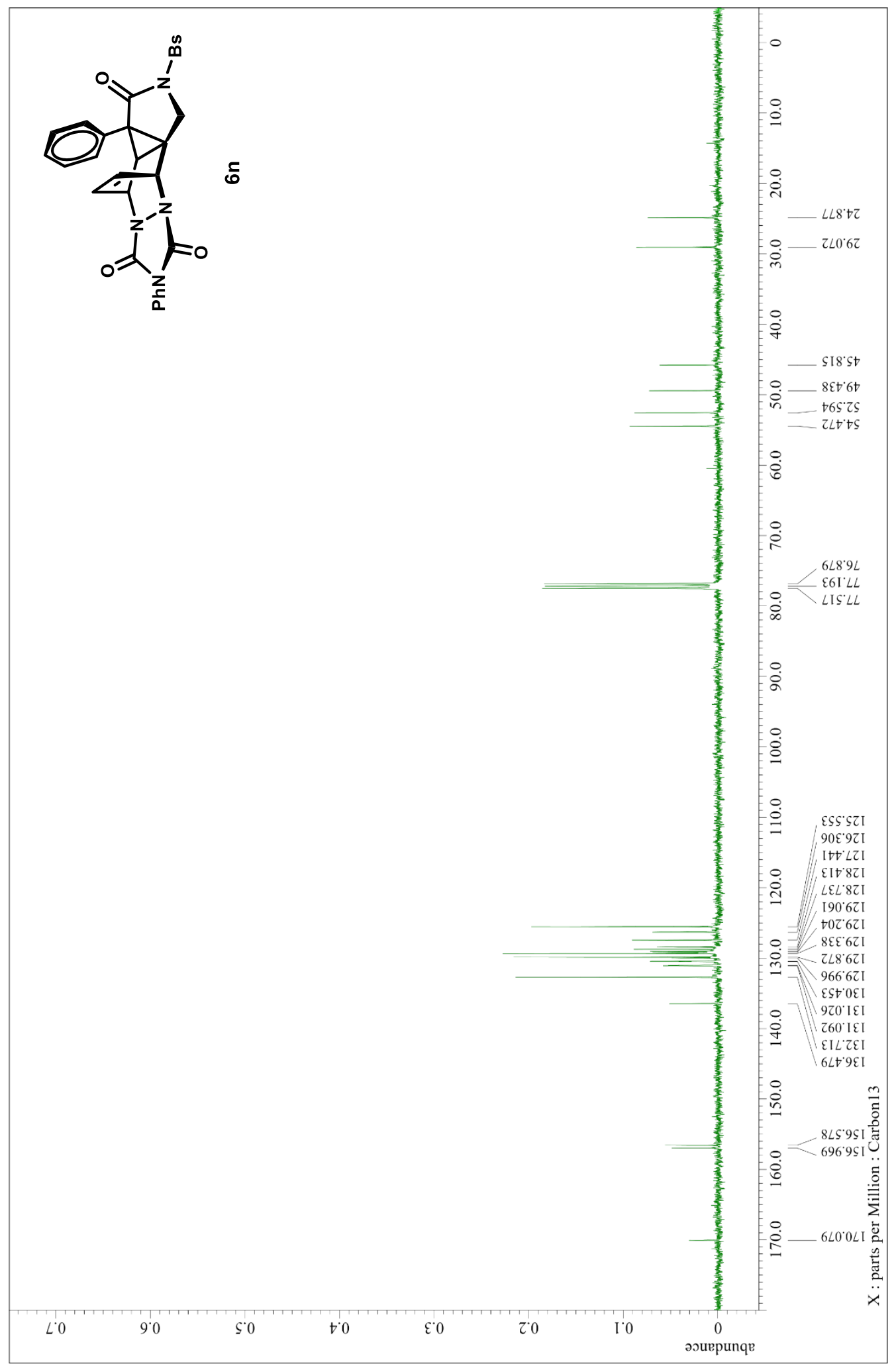


(3bR,4R,10R,10aS)-12-Bromo-2-((4-bromophenyl)sulfonyl)-7-methyl-3a-phenyl1,2,3b,4-tetrahydro-6H,10H-4,10-ethenopyrrolo[3',4':1,3]cyclopropa[1,2d] $[1,2,4]$ triazolo[1,2-a]pyridazine-3,6,8(3aH,7H)-trione (6o)

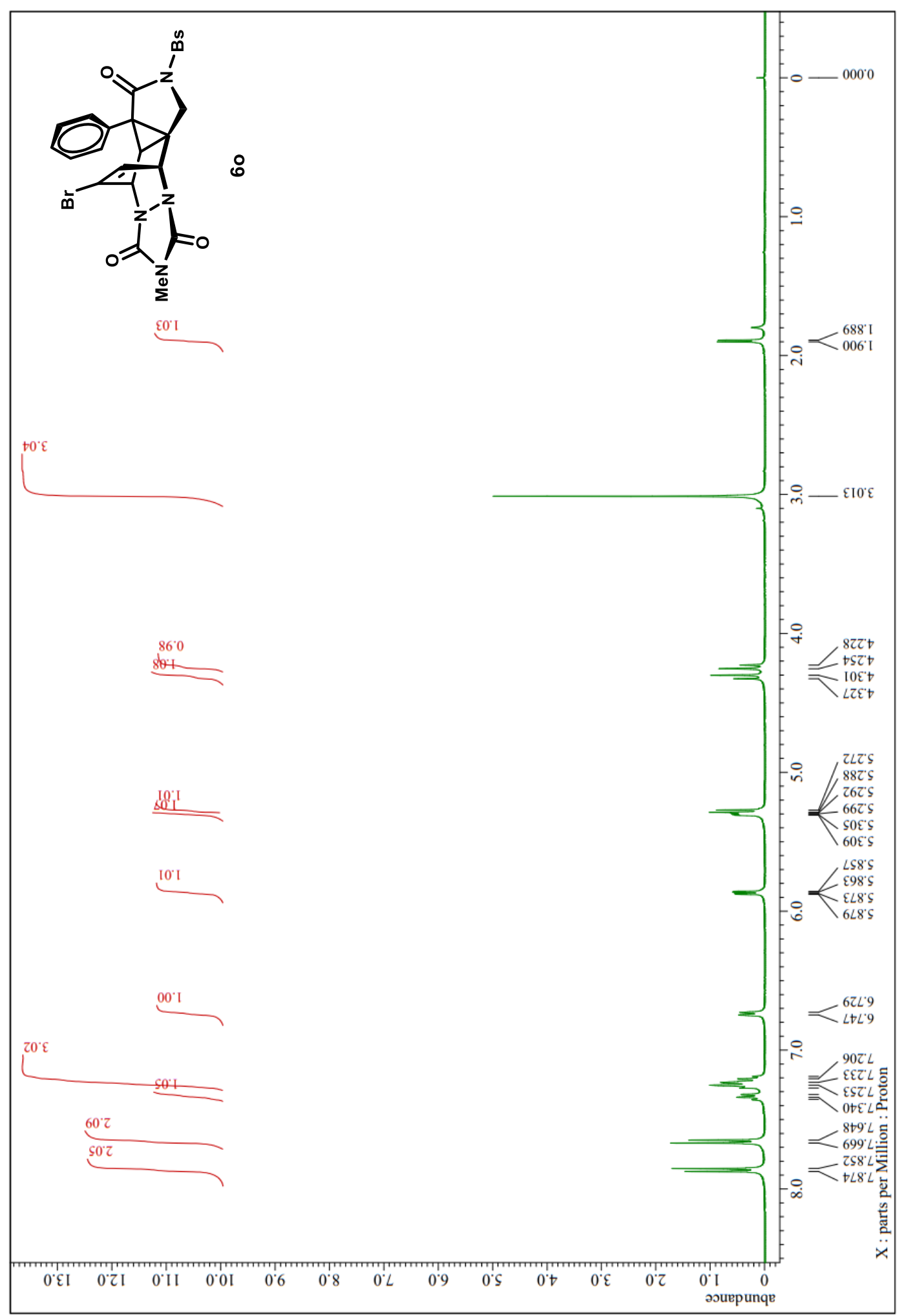


Supporting information

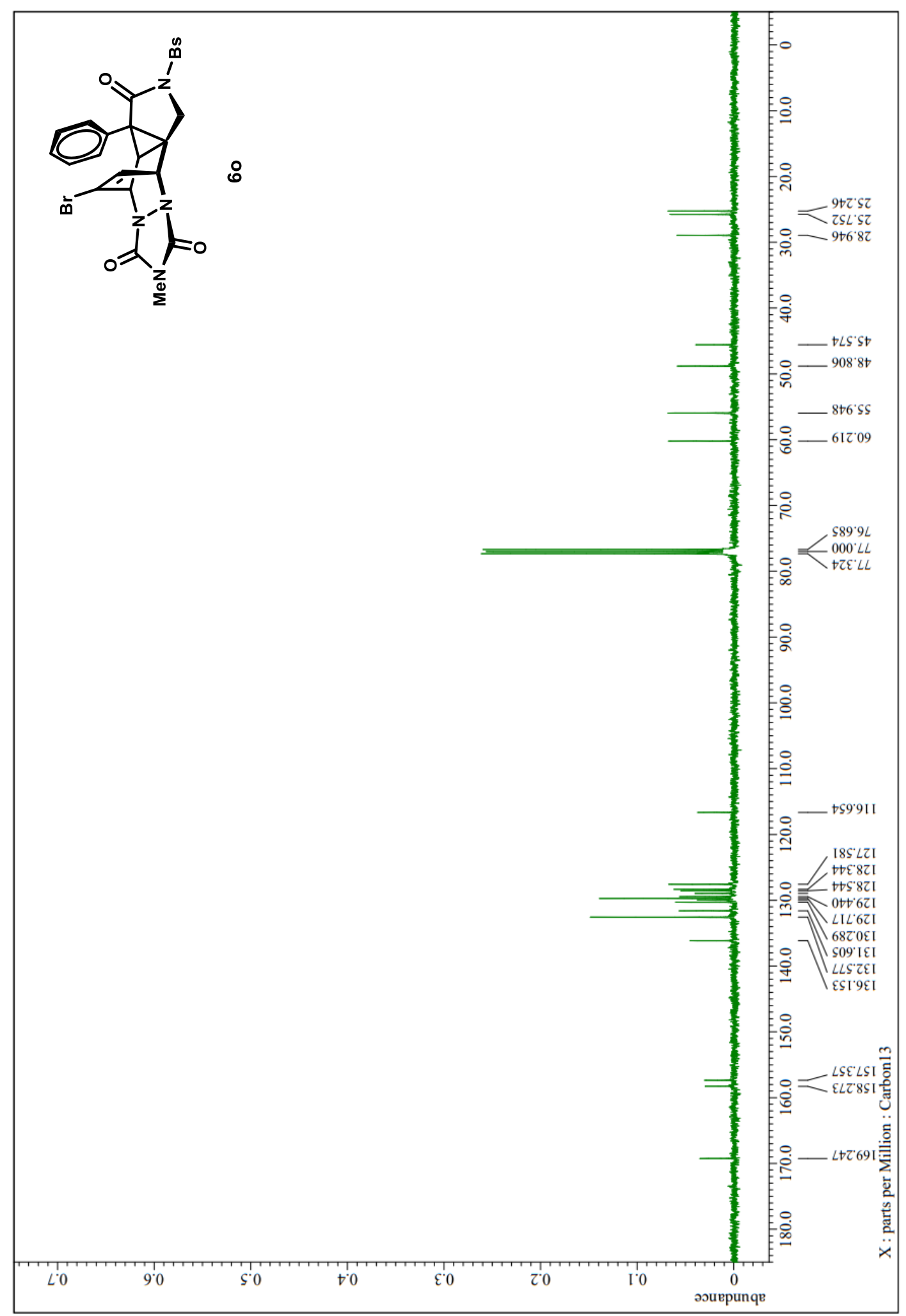


Methyl 4-((4-bromo-N-(4-bromobenzyl)phenyl)sulfonamido)ethynyl)-benzoate (1b)

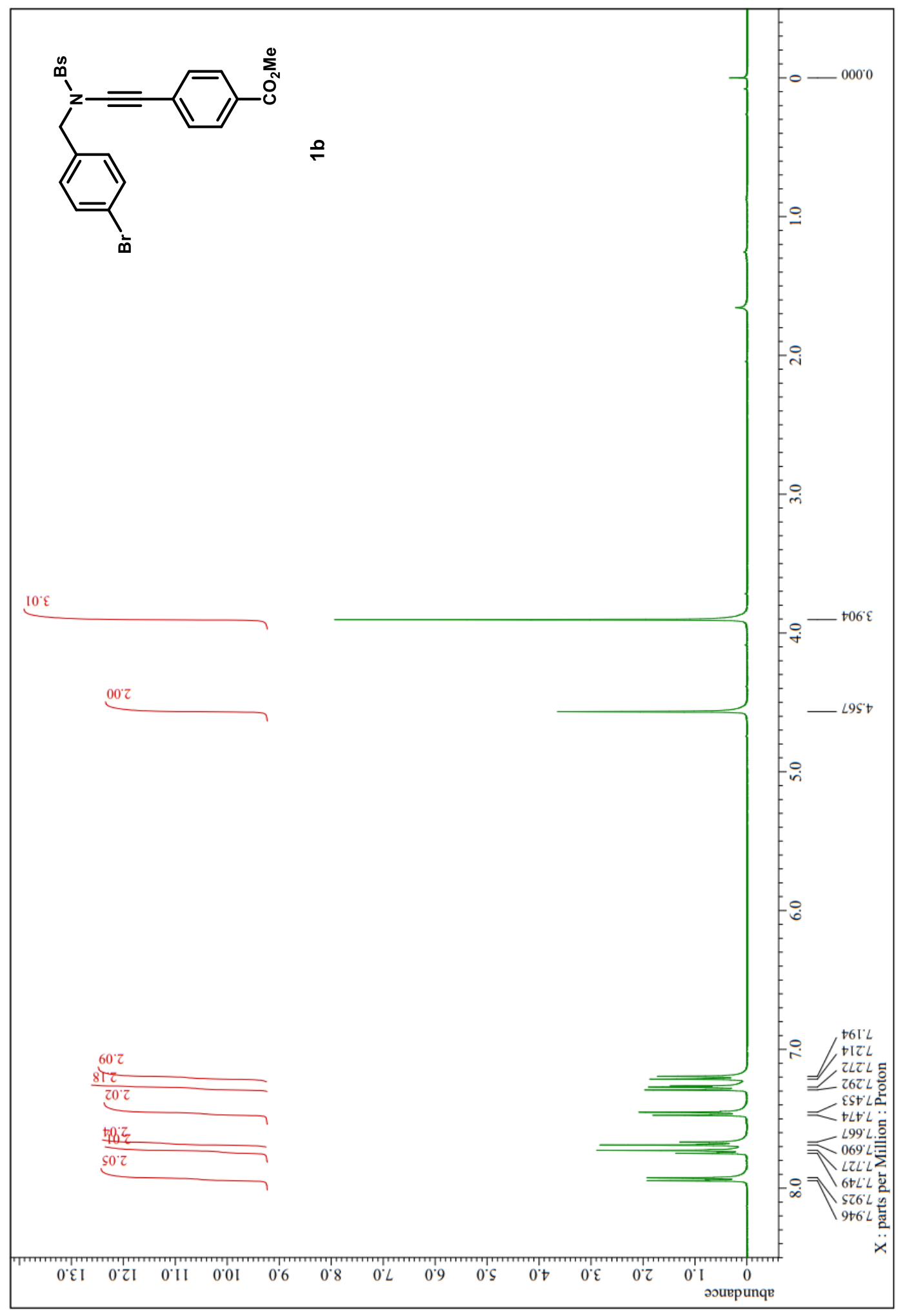


Supporting information

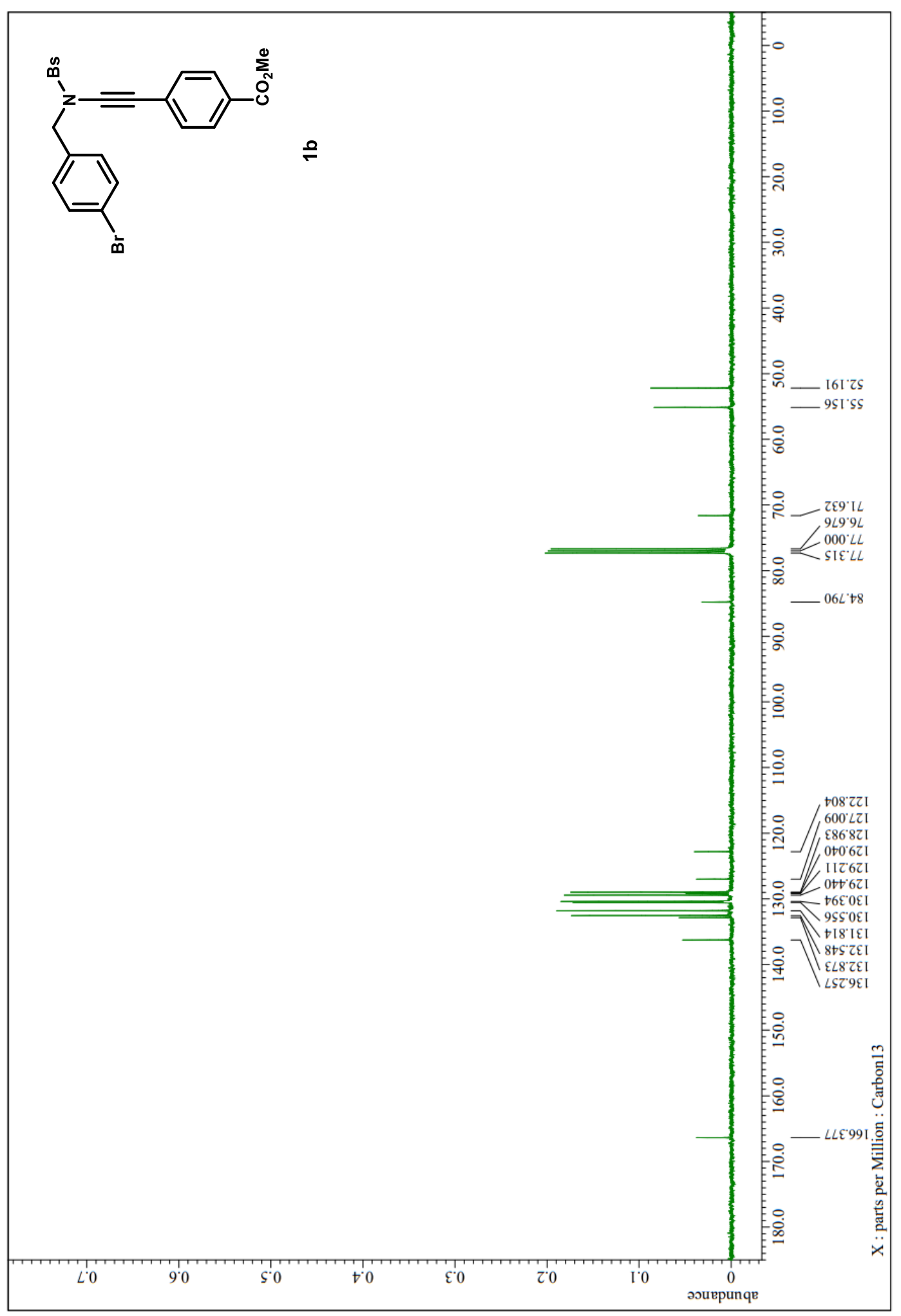


4-Bromo-N-(4-bromobenzyl)-N-((4-bromophenyl)ethynyl)benzenesulfonamide (1c)

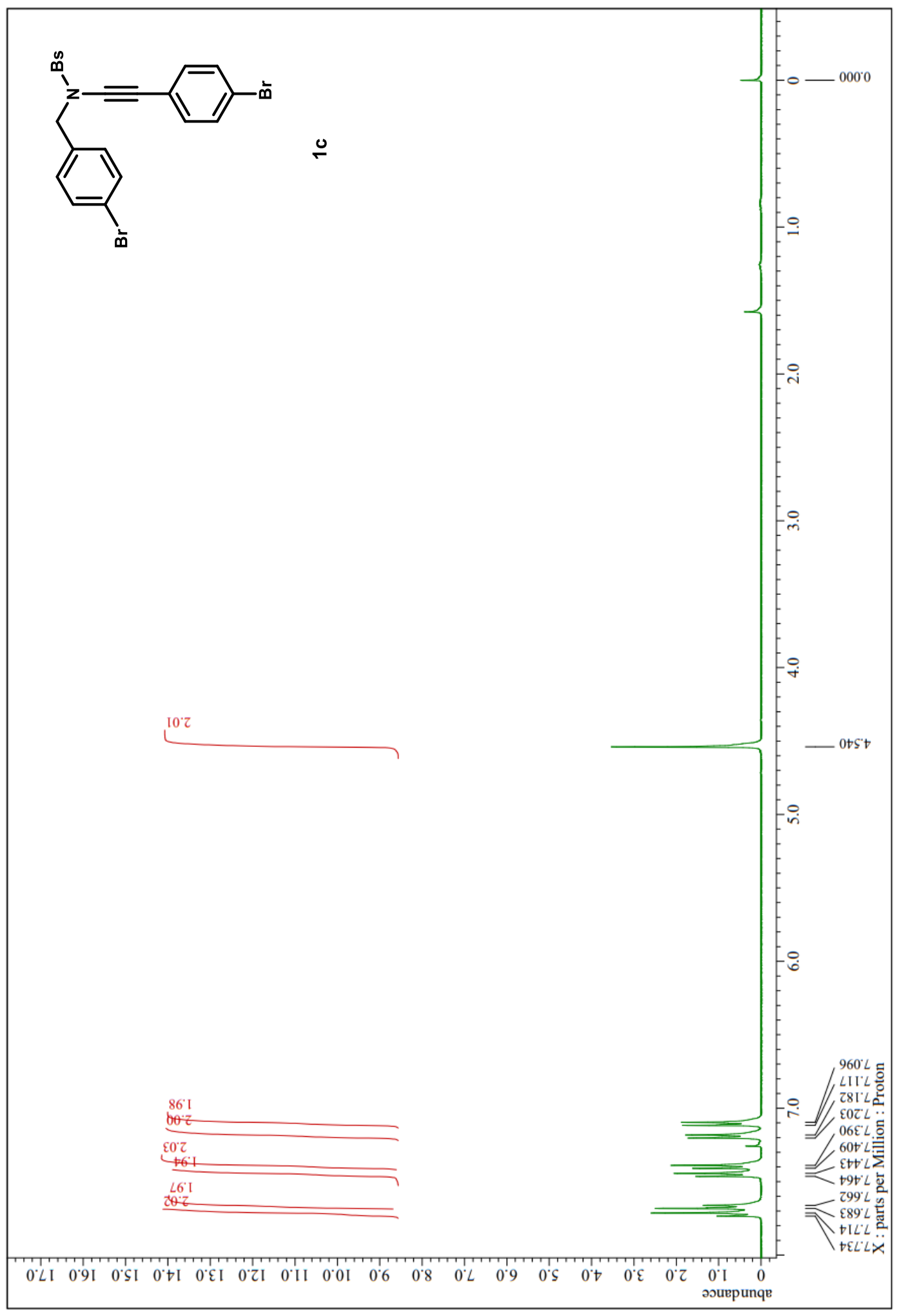


Supporting information

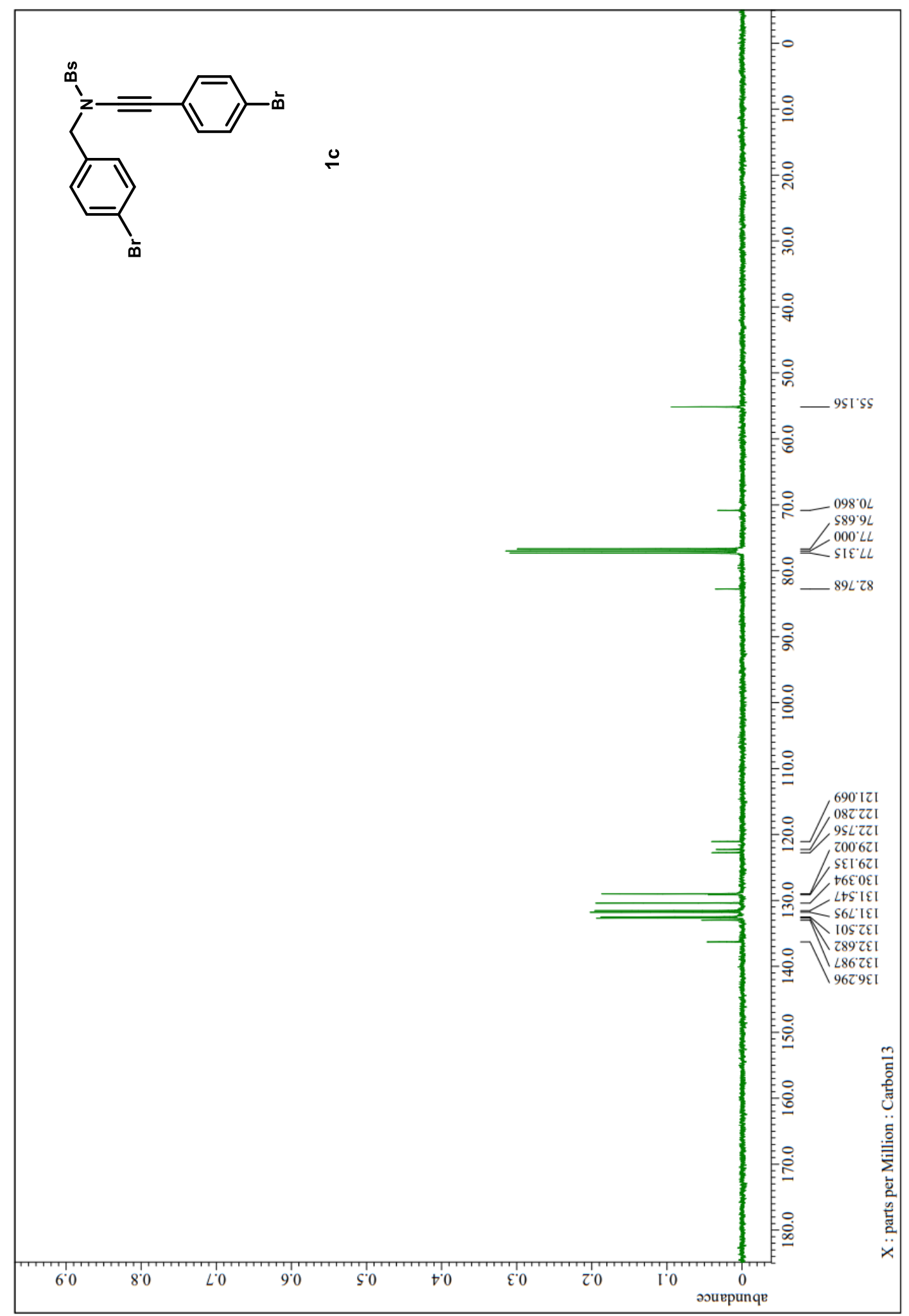


Supporting information

4-Bromo-N-(4-bromobenzyl)-N-((3-

methoxyphenyl)ethynyl)benzenesulfonamide (1d)

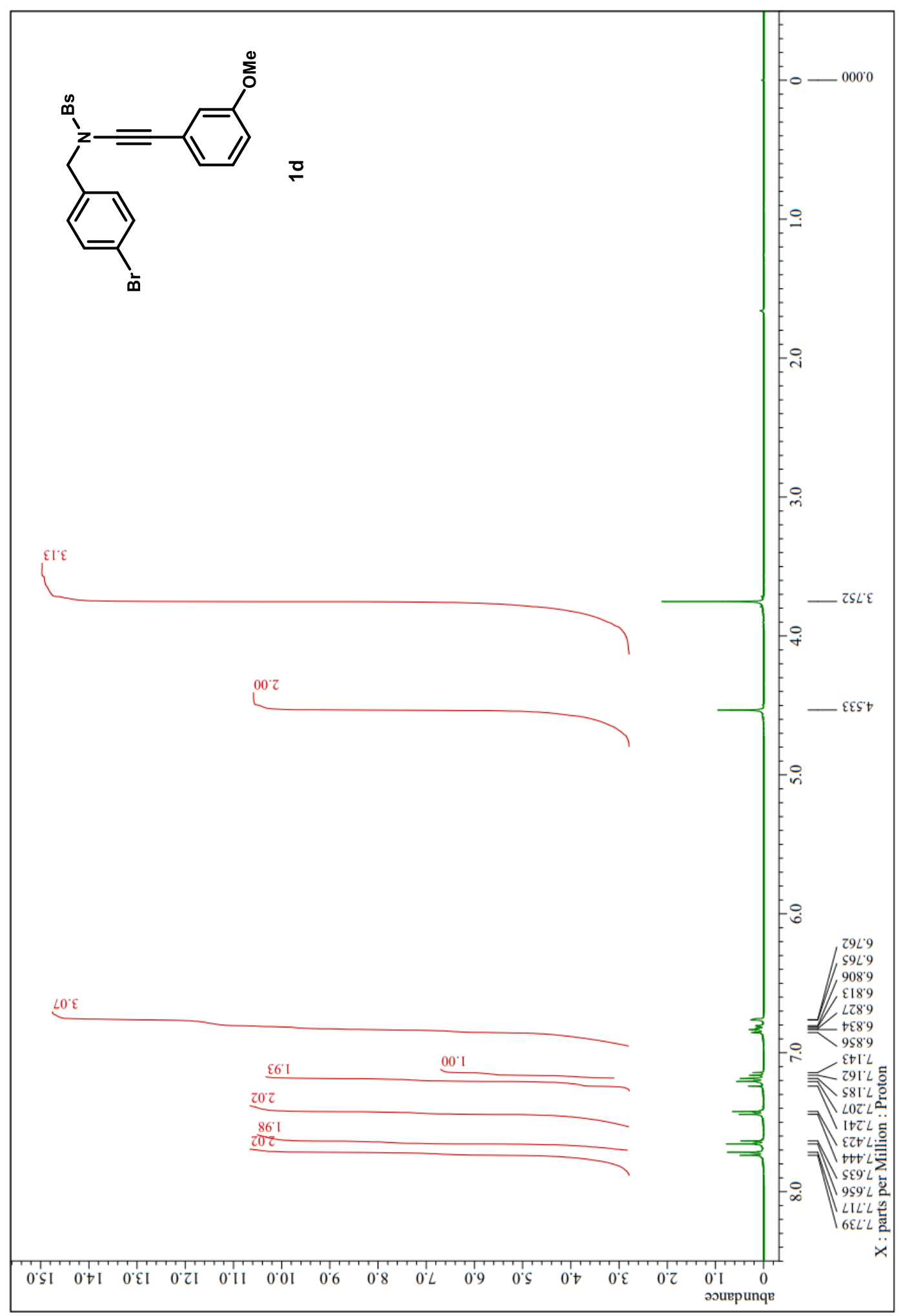


Supporting information

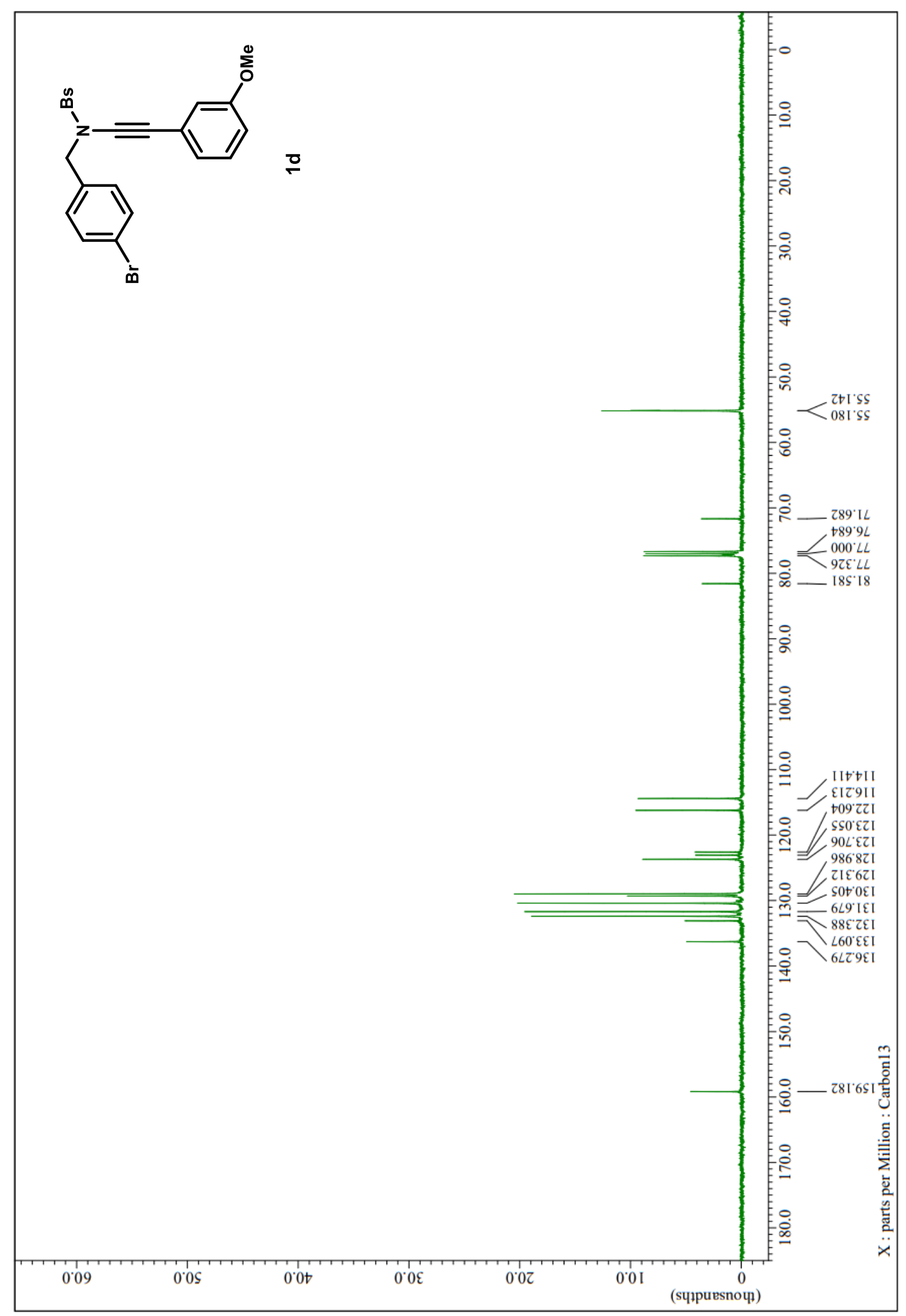


Supporting information

$N$-(4-Bromobenzyl)-N-((3-methoxyphenyl)ethynyl)-4-

methylbenzenesulfonamide (1e)

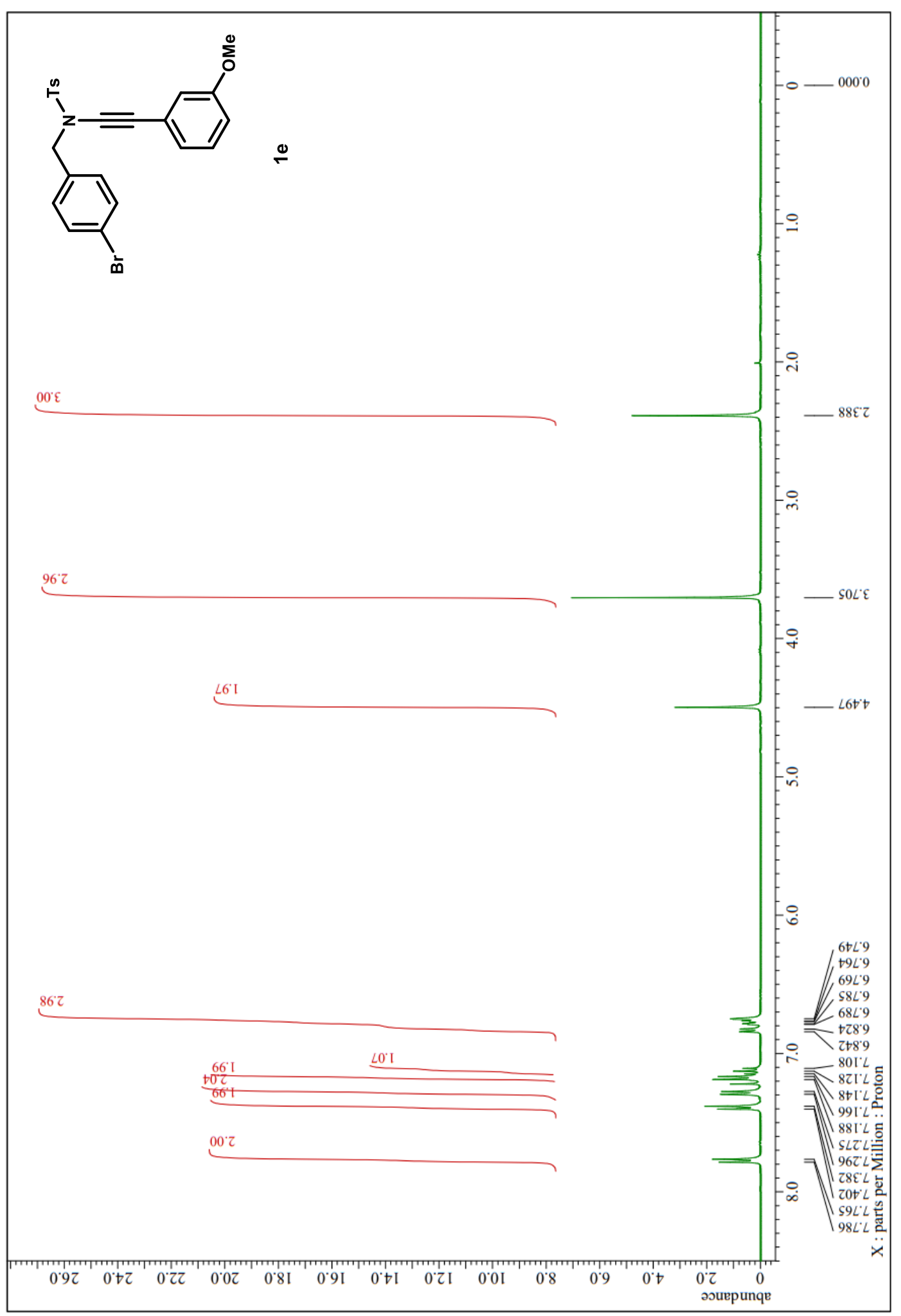


Supporting information

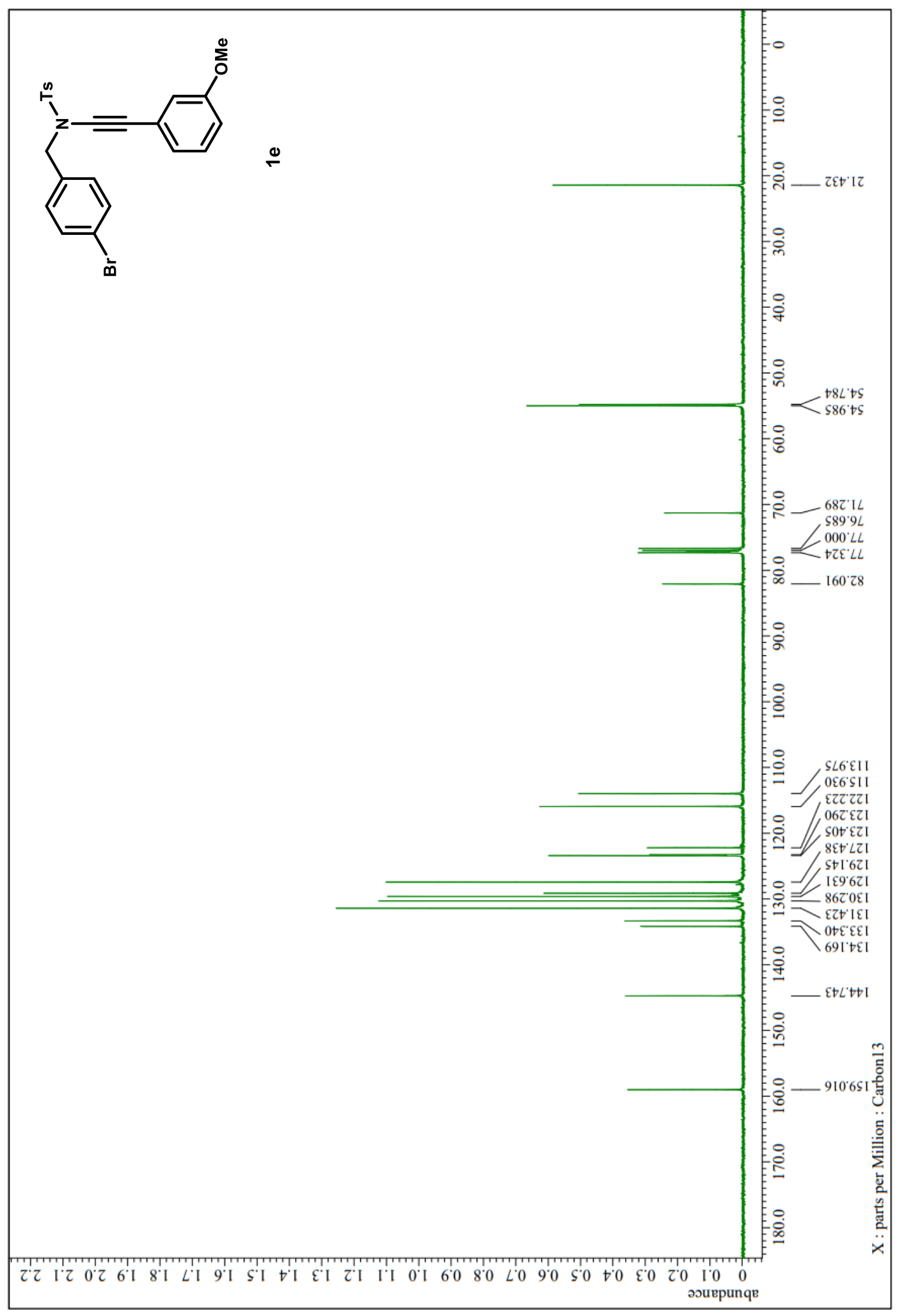


$N$-(4-Bromobenzyl)-N-((2-fluorophenyl)ethynyl)-4-methylbenzenesulfonamide (1f)

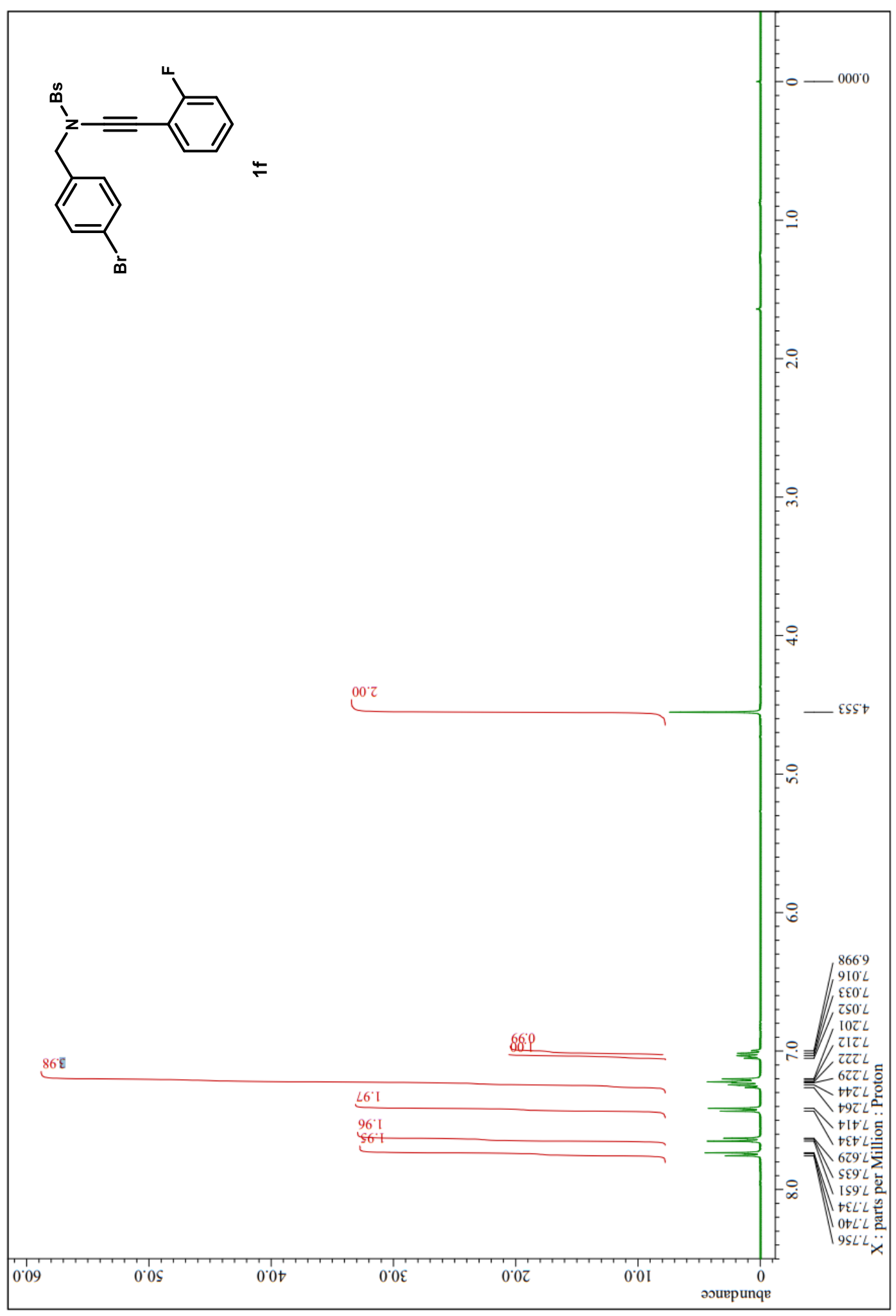


Supporting information

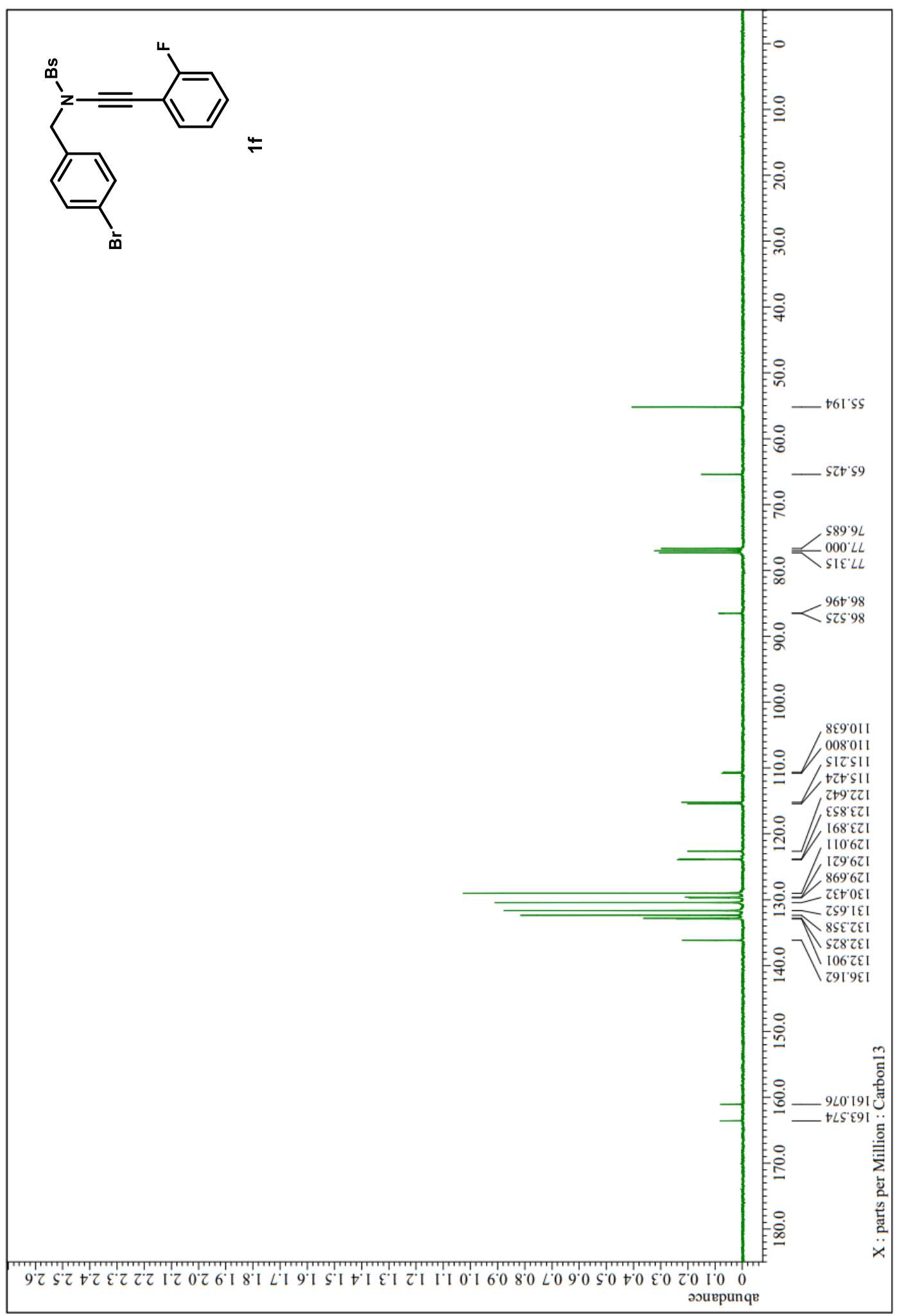


Supporting information

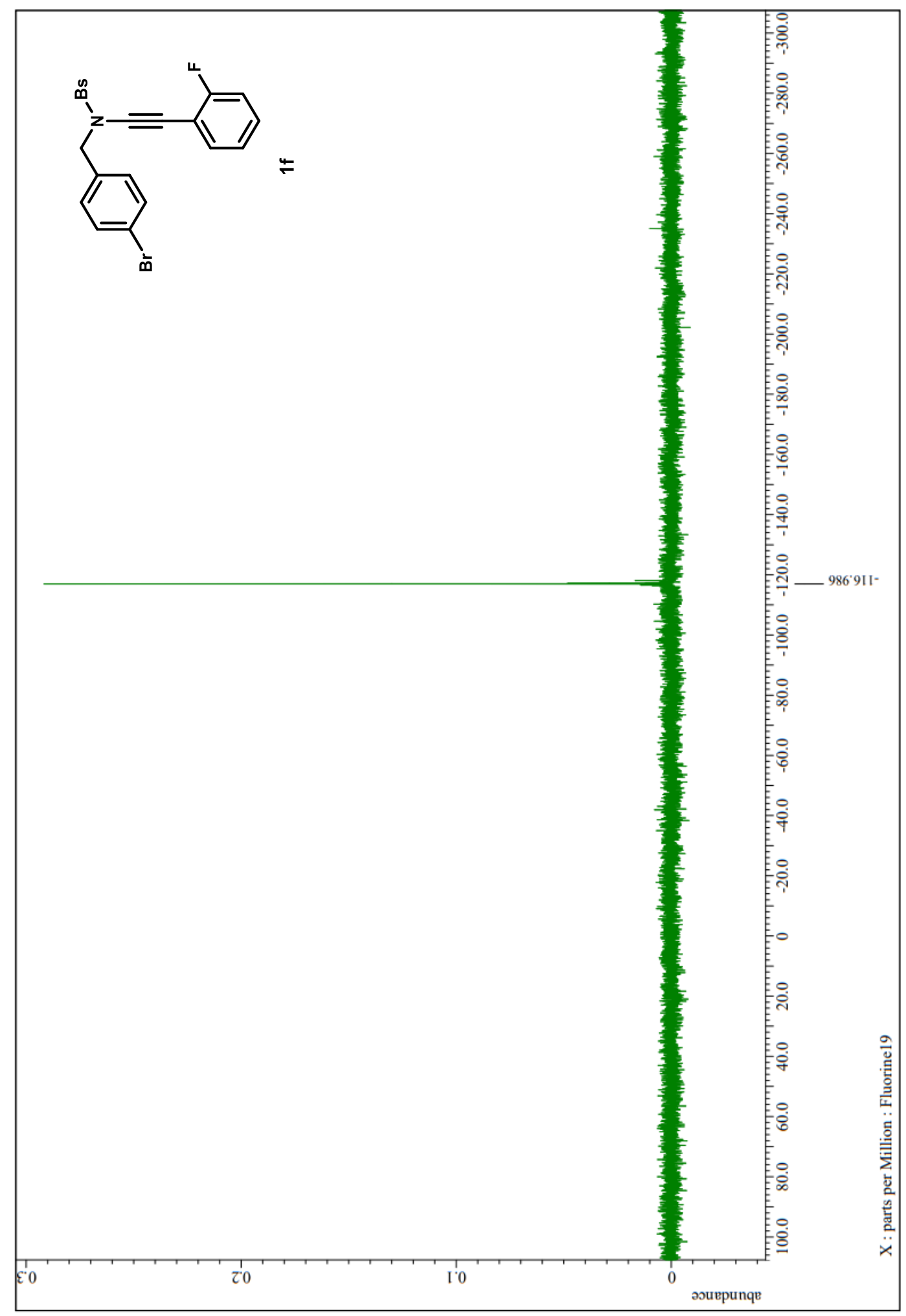


N-(4-Bromobenzyl)-N-((3-bromophenyl)ethynyl)-4-methylbenzenesulfonamide (1g)

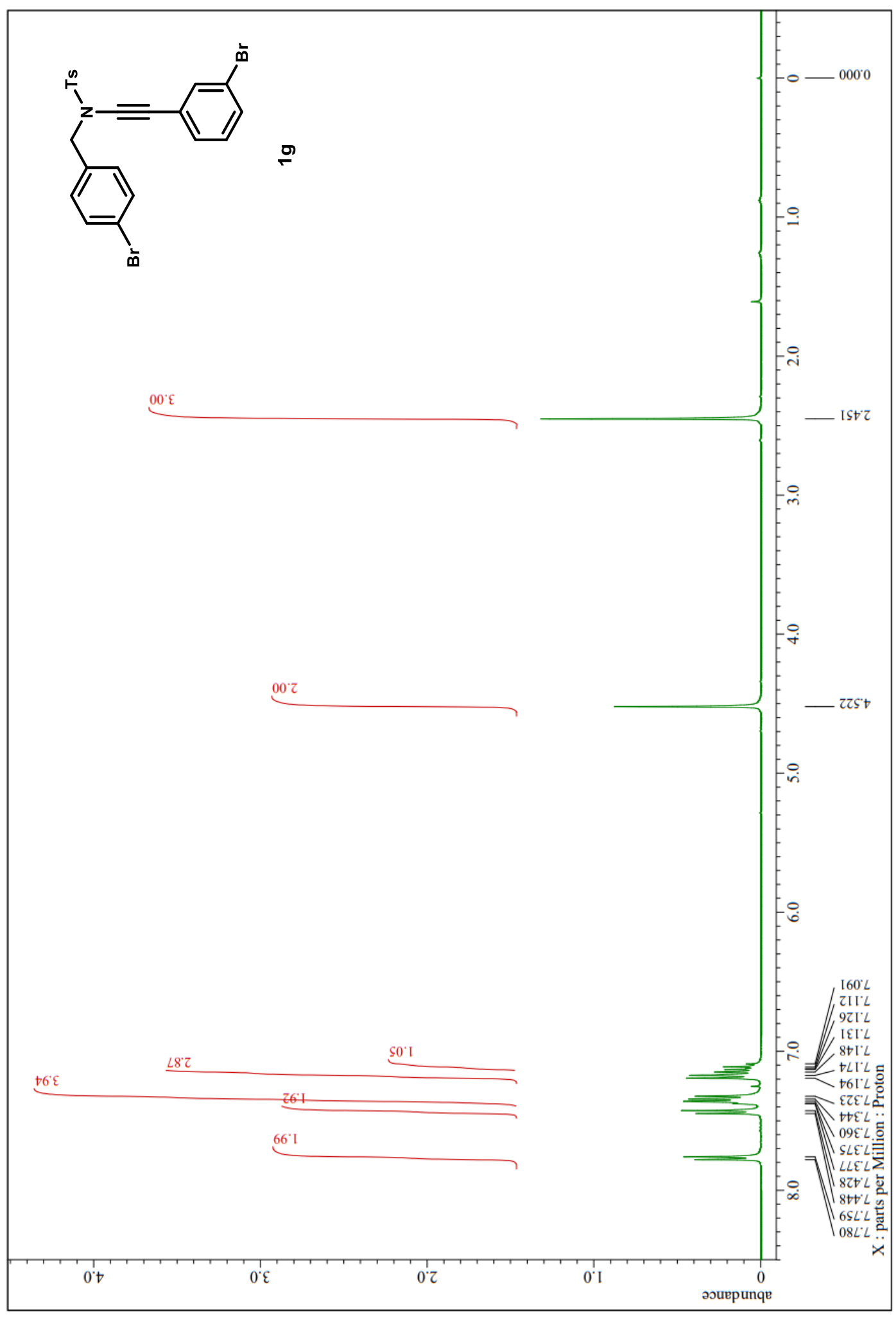


Supporting information

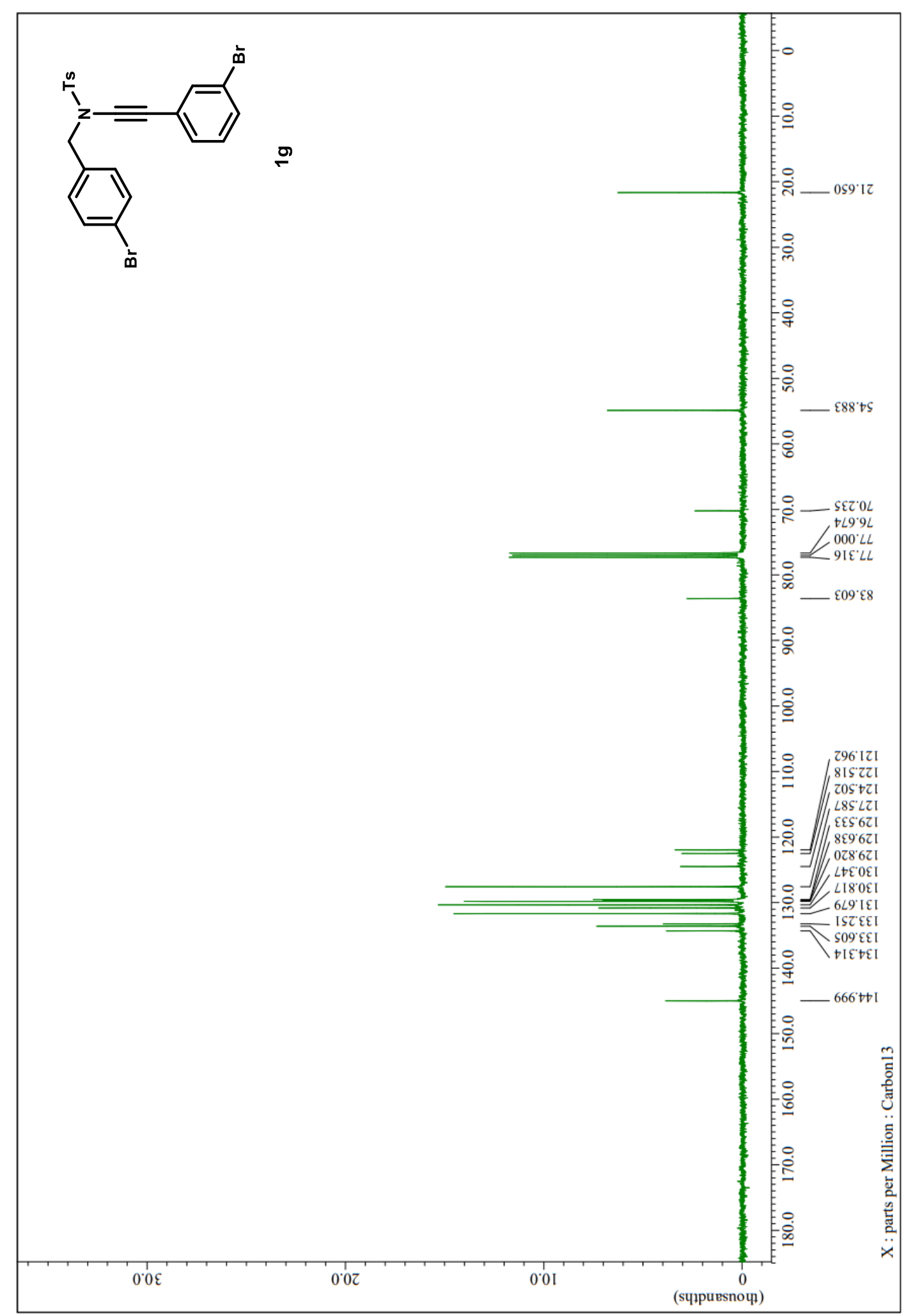




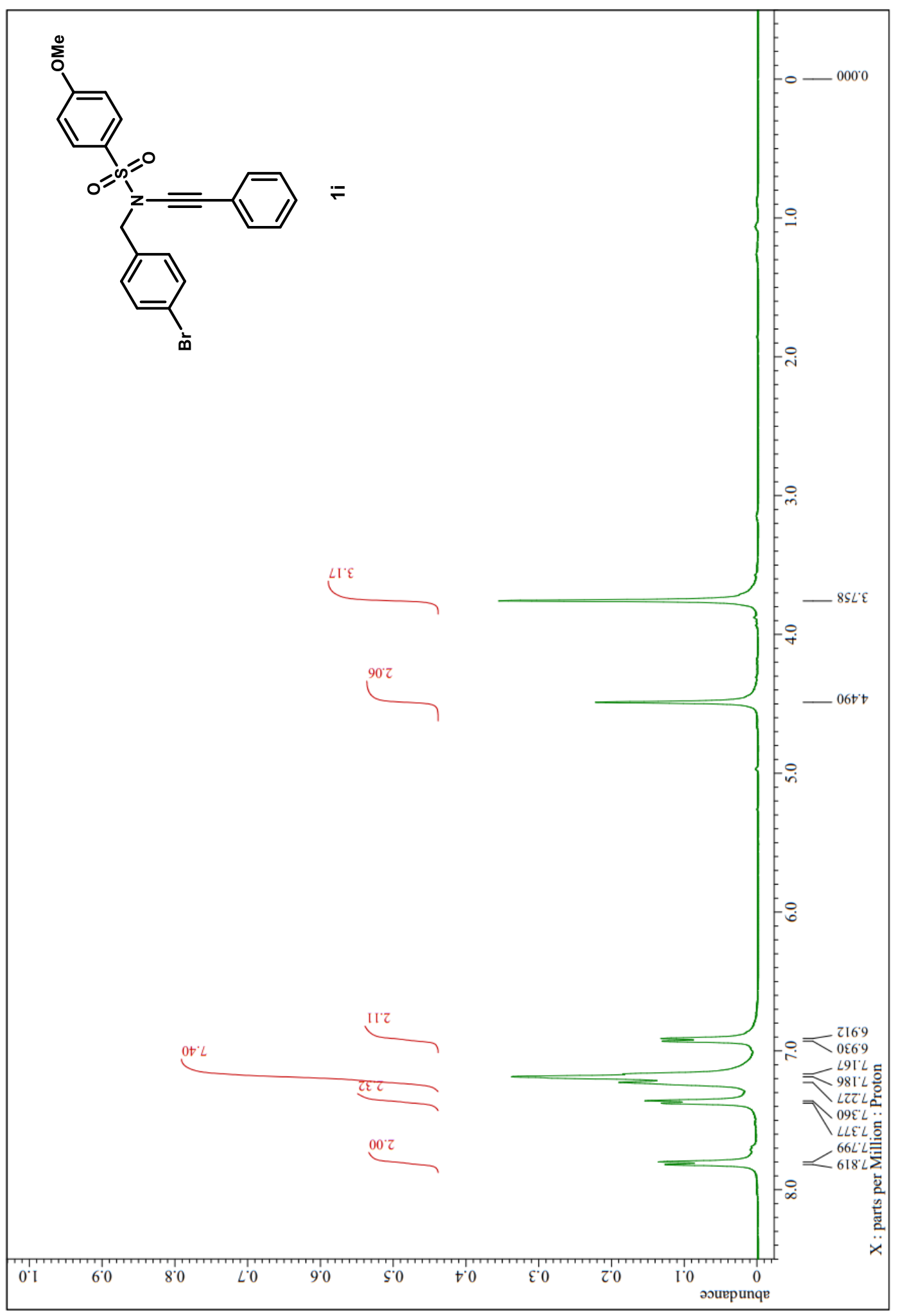


Supporting information

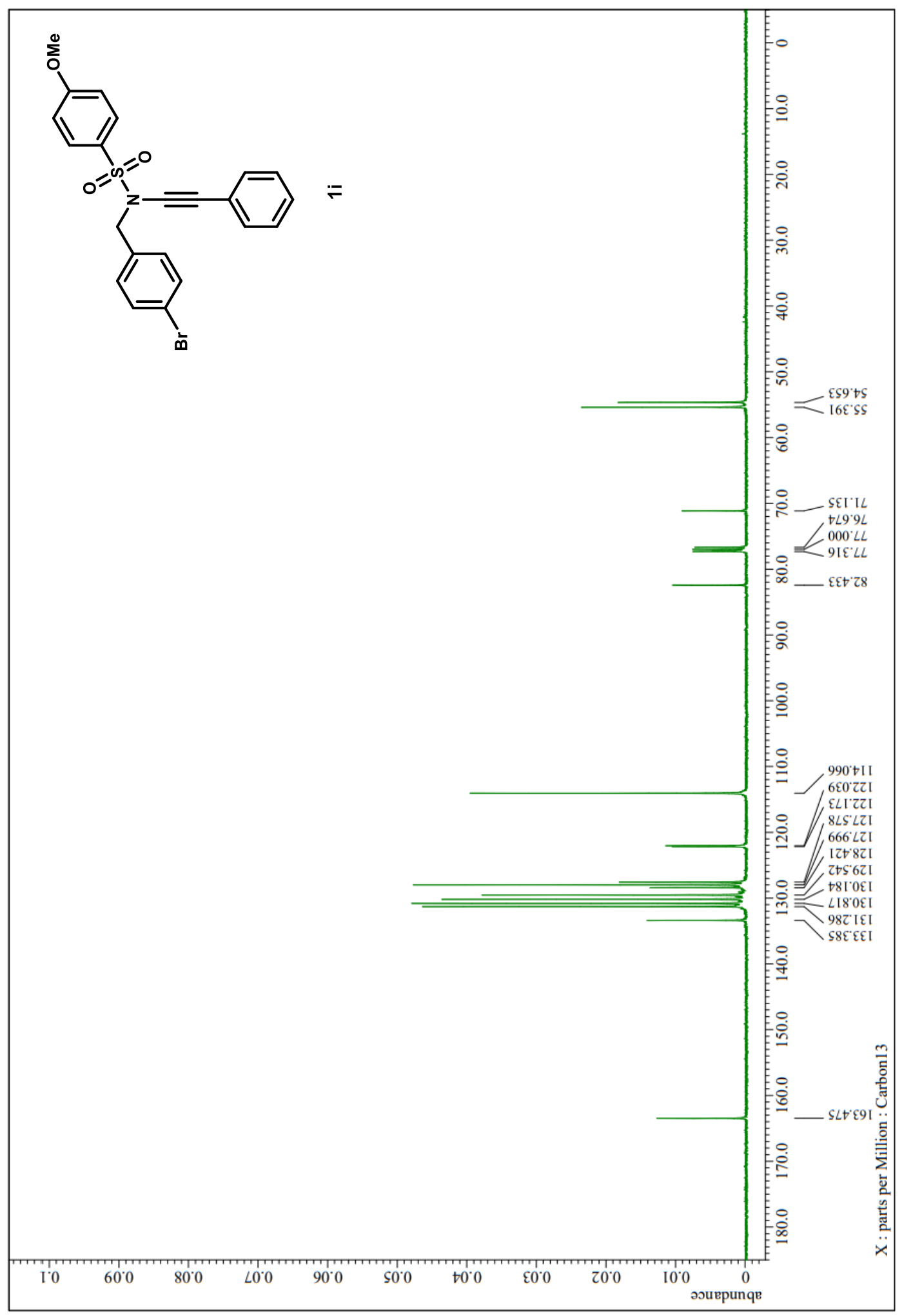


Supporting information

4-Bromo-N-(4-iodobenzyl)-N-(phenylethynyl)benzenesulfonamide (1k)

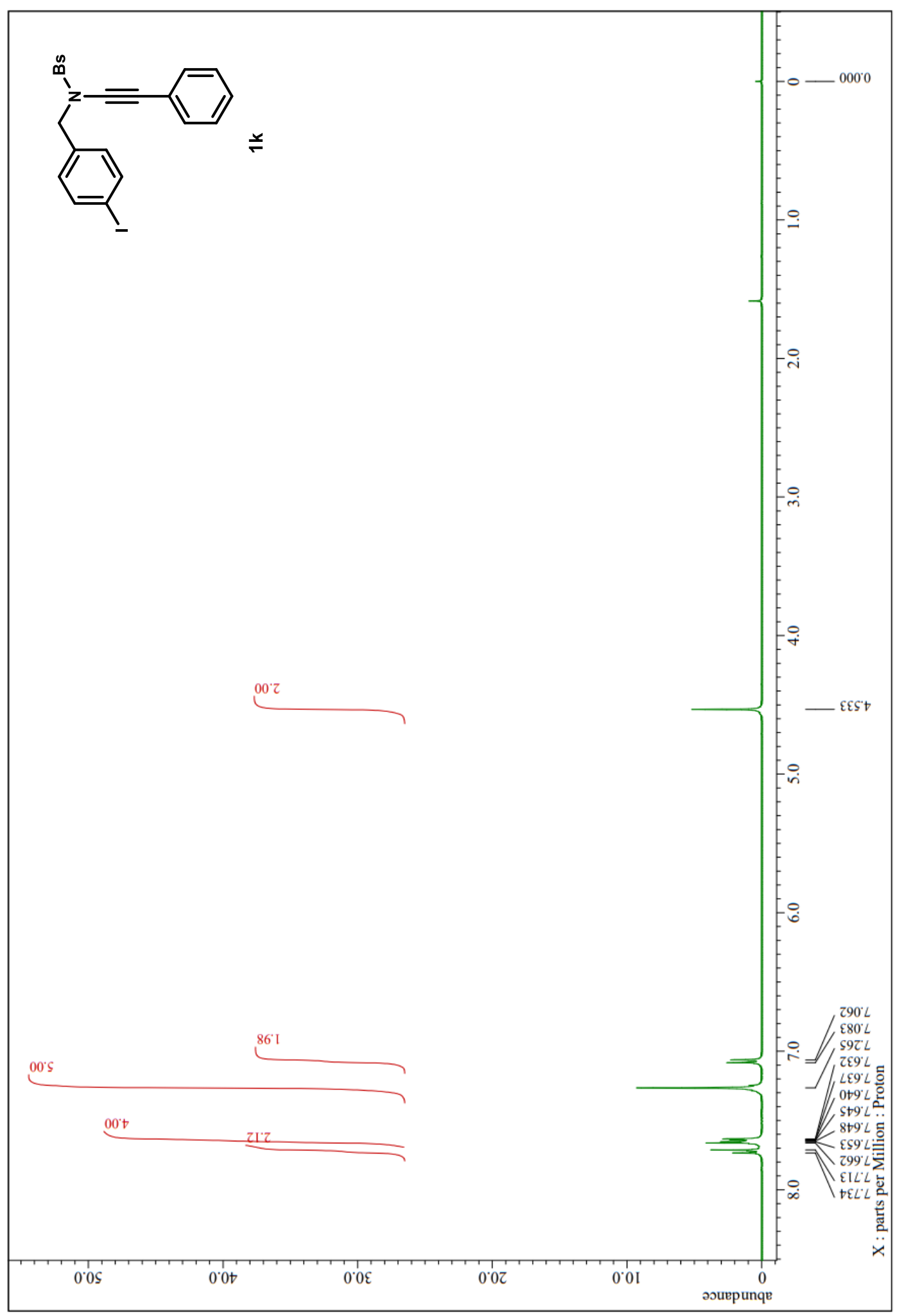


Supporting information

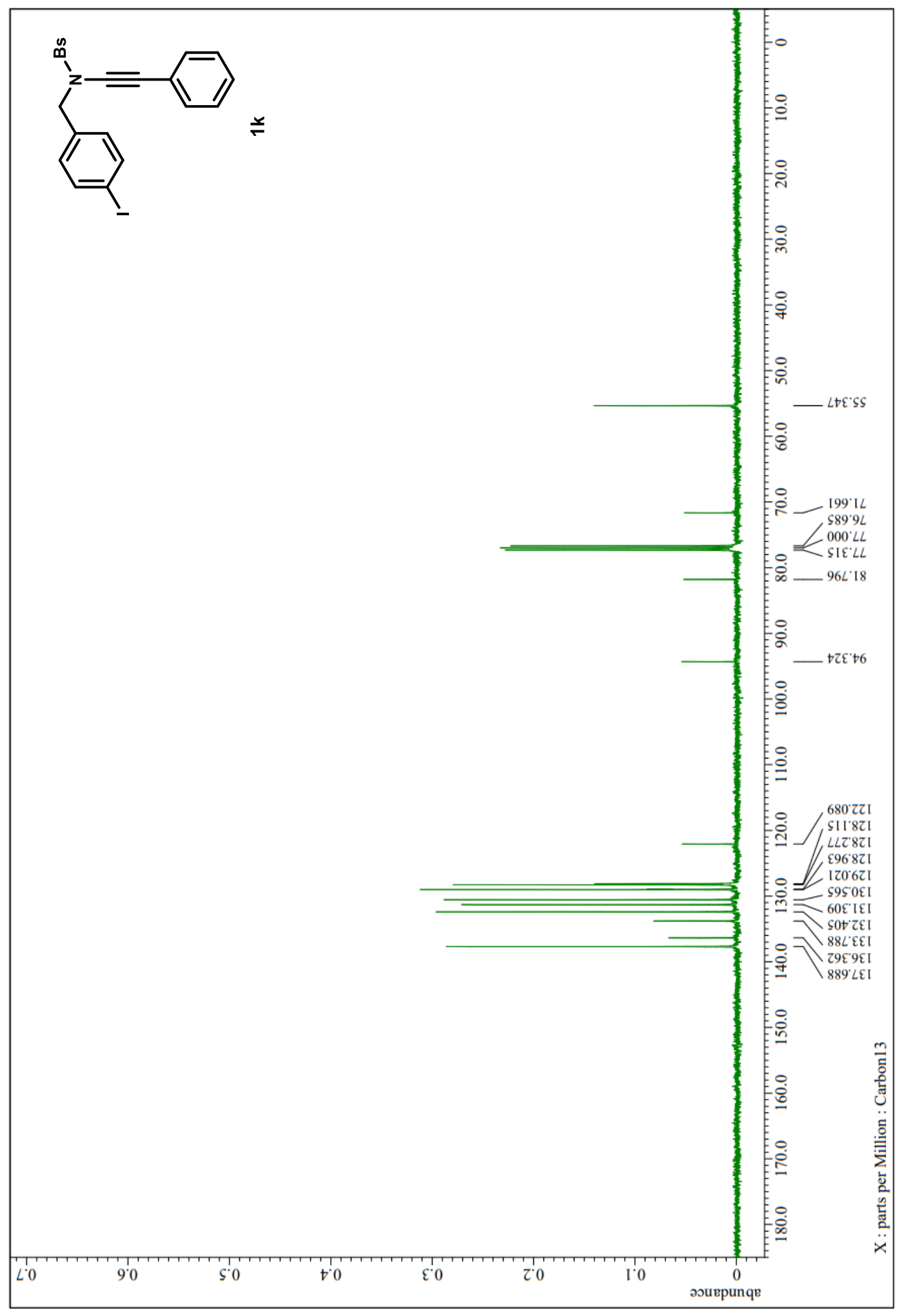


Supporting information

4-Bromo-N-(4-fluorobenzyl)-N-(phenylethynyl)benzenesulfonamide (1I)

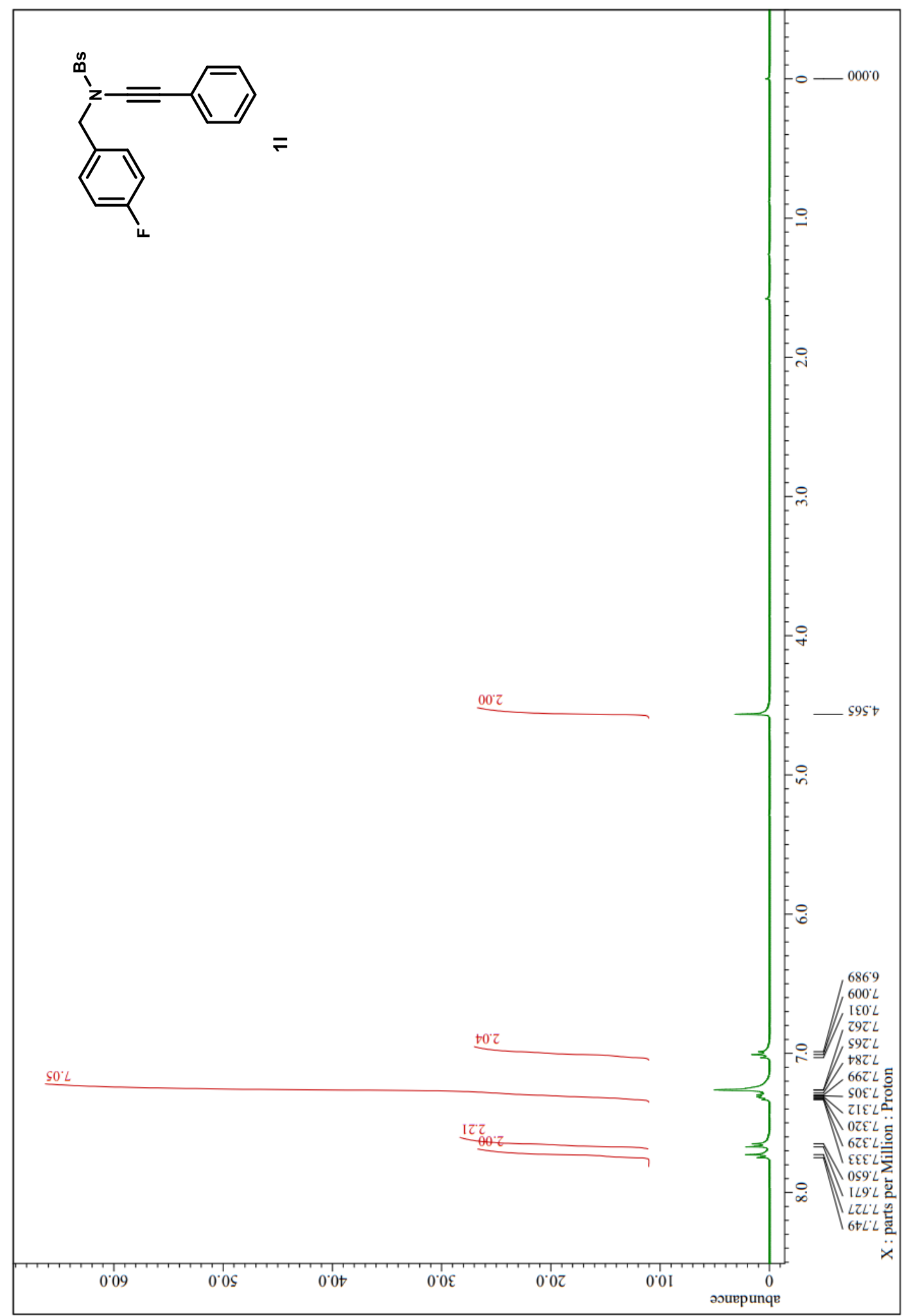


Supporting information

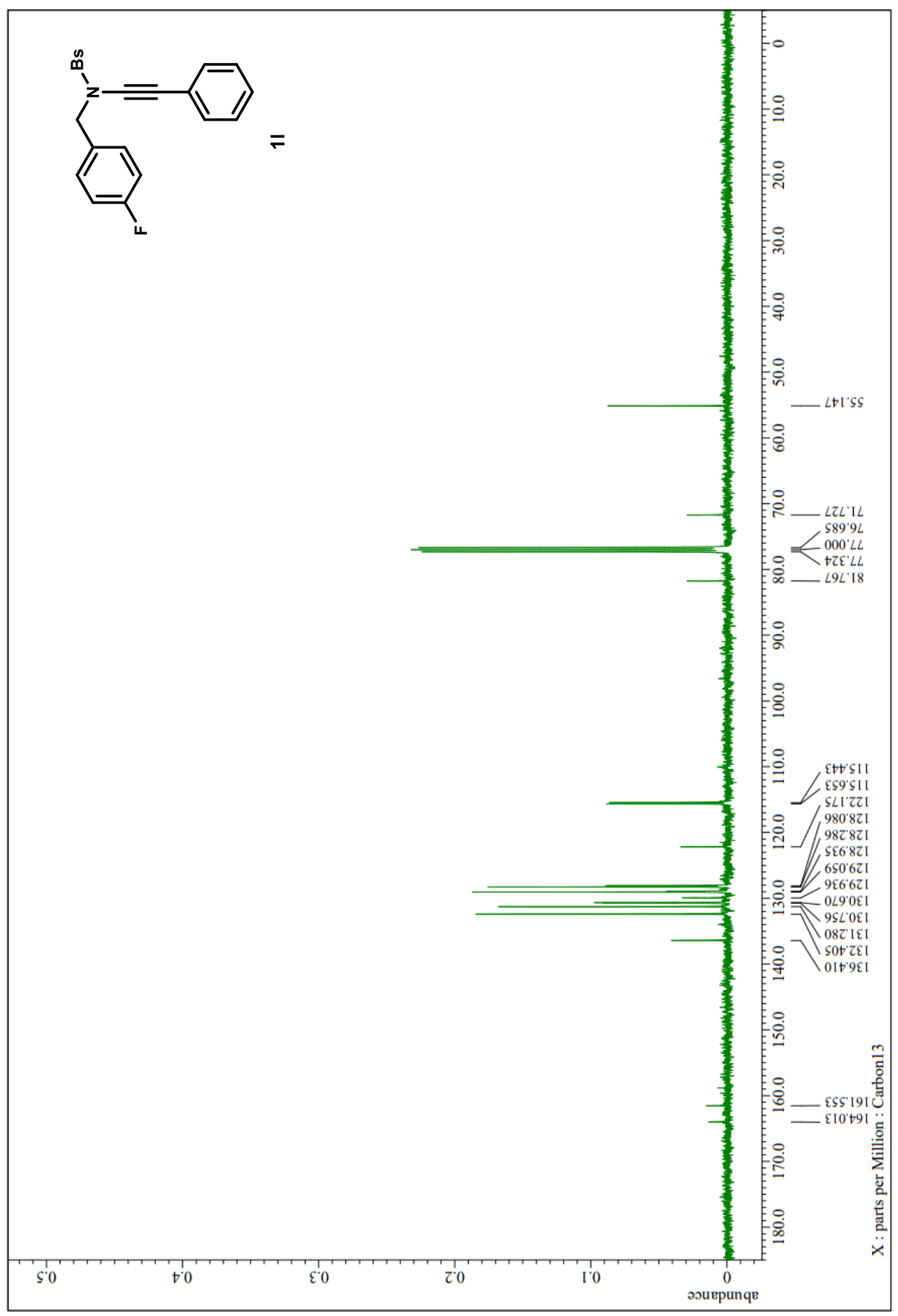


Supporting information

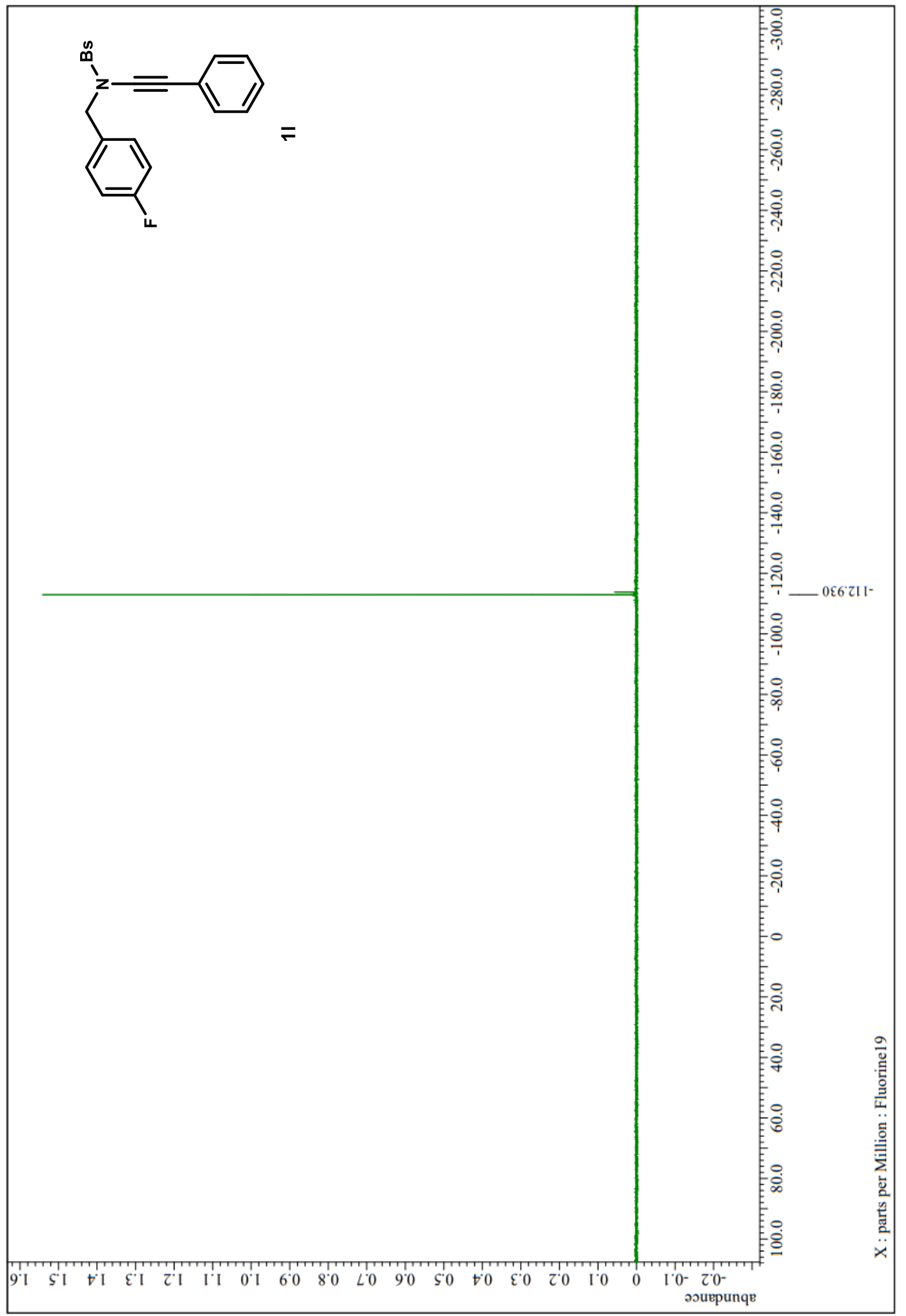


Supporting information

$N$-(4-Chlorobenzyl)-4-methyl- $N$-(phenylethynyl)benzenesulfonamide (1m)

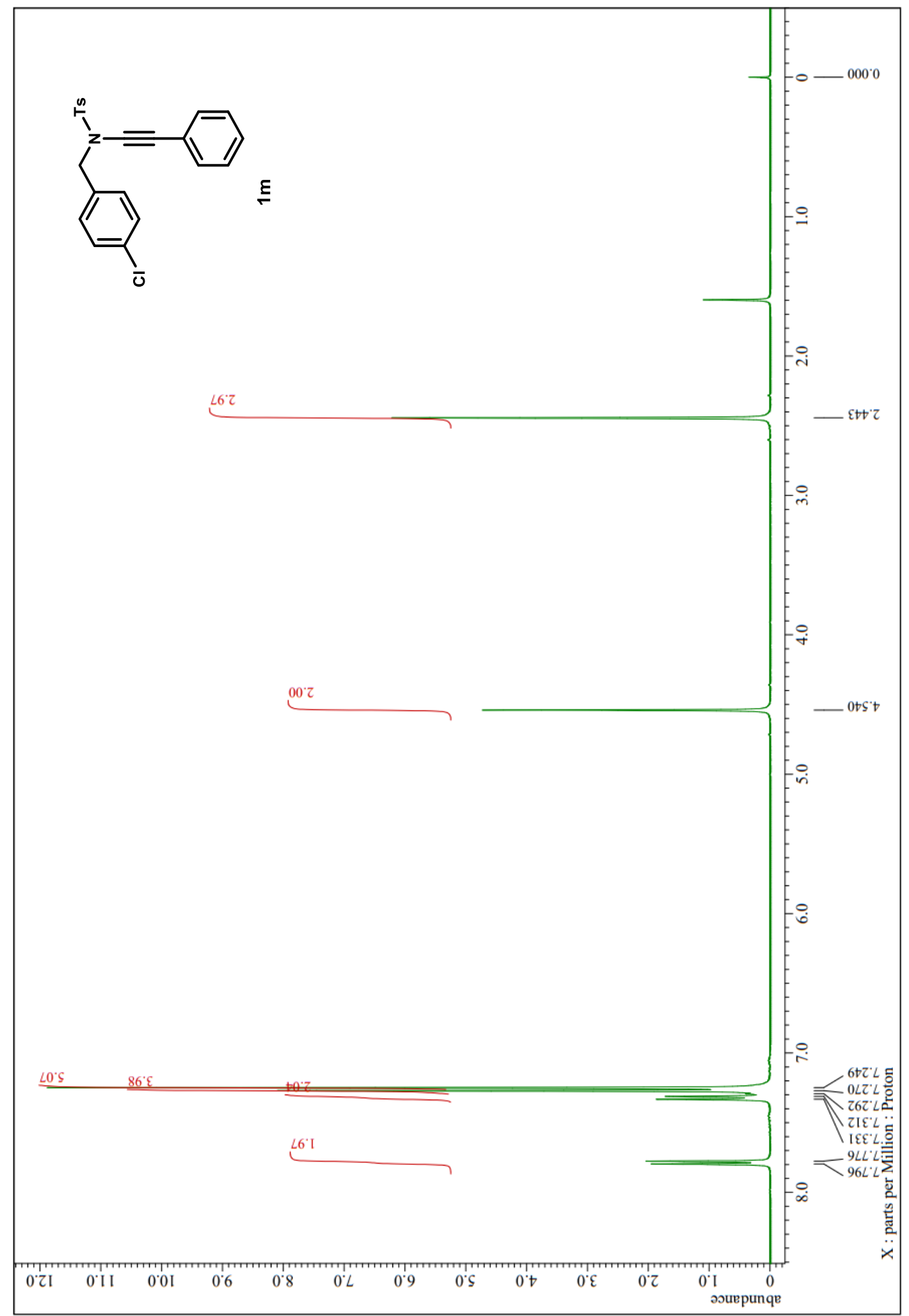


Supporting information

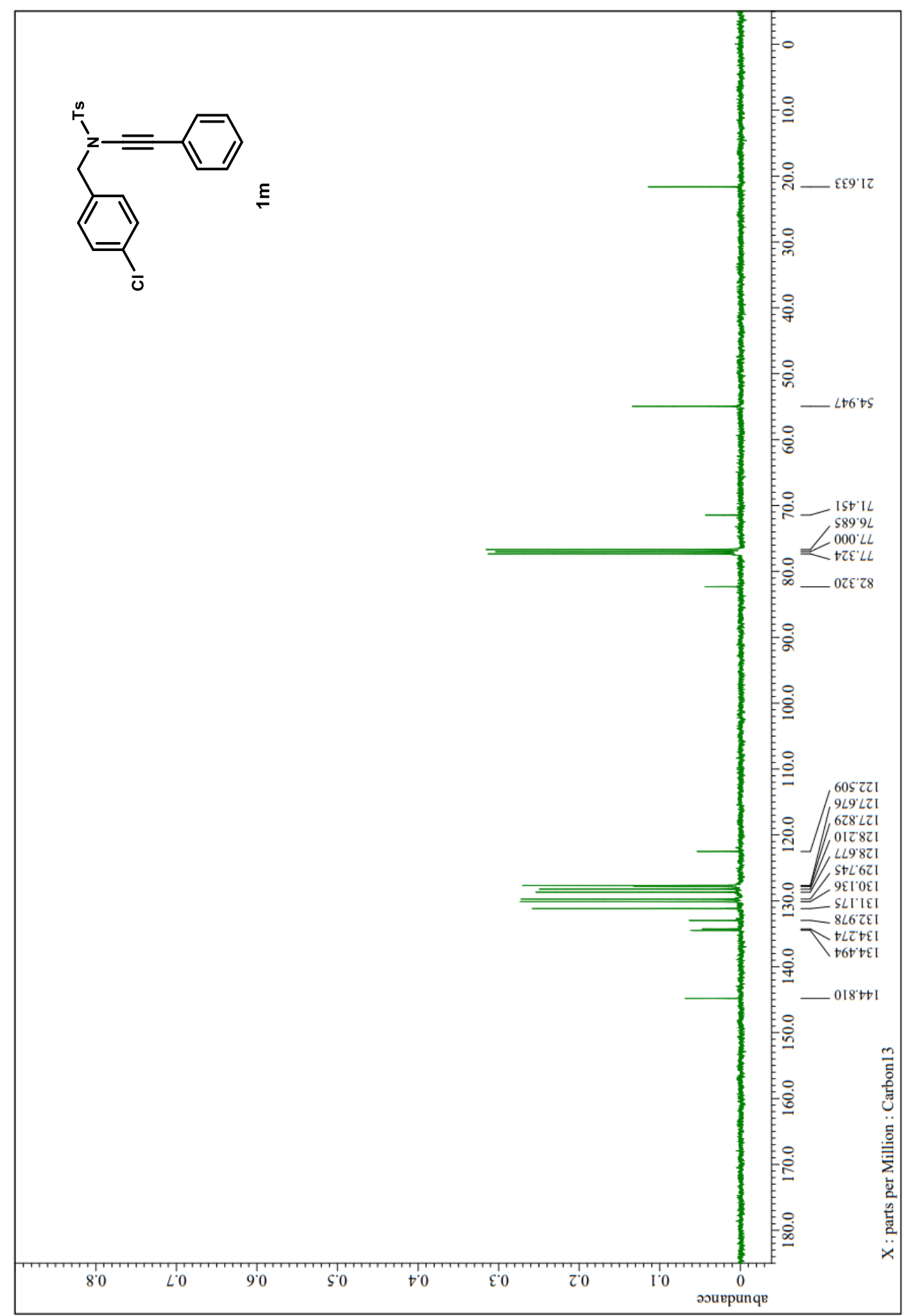


Supporting information

4-Bromo-N-(4-methylbenzyl)-N-(phenylethynyl)benzenesulfonamide (1n)

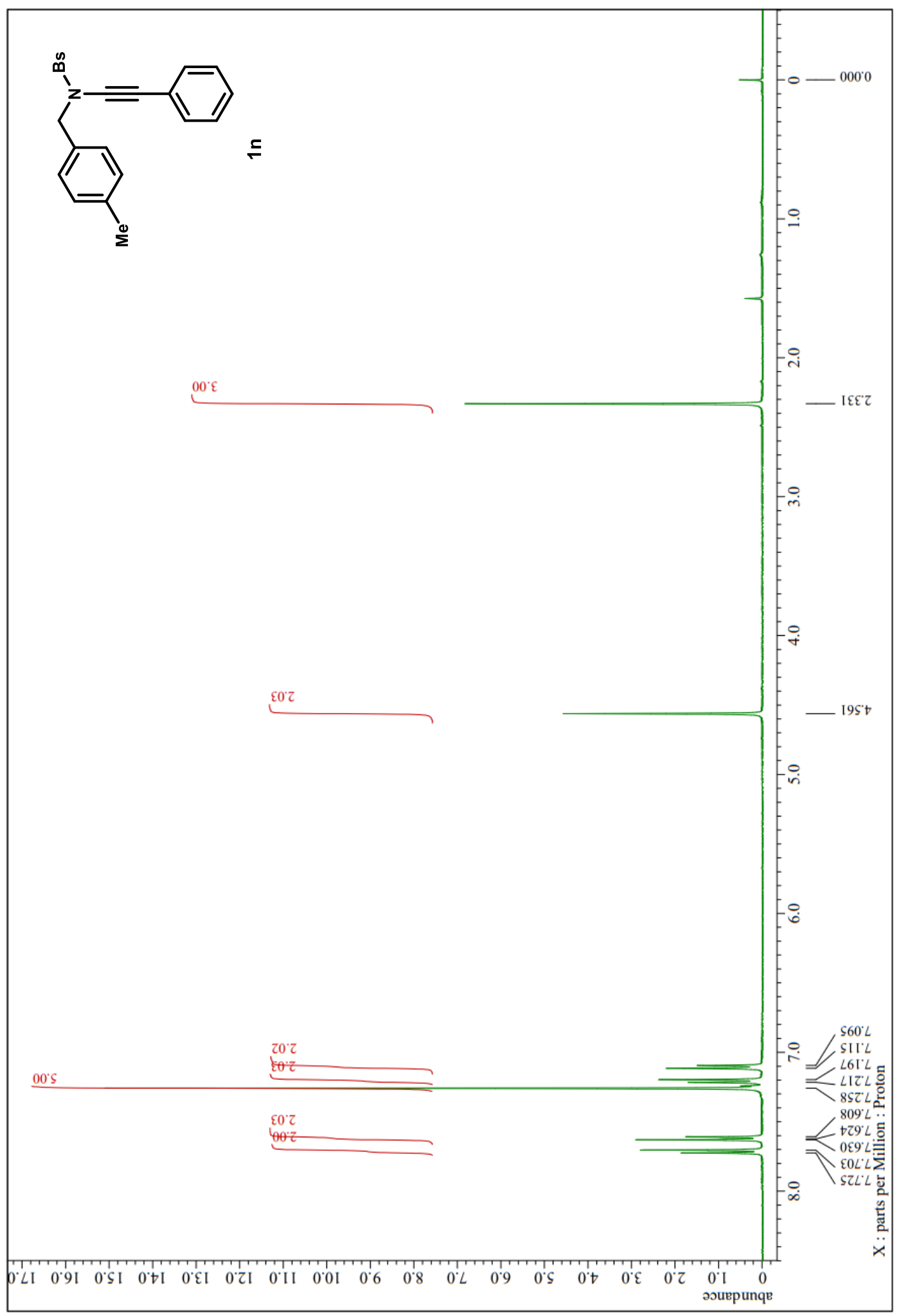


Supporting information

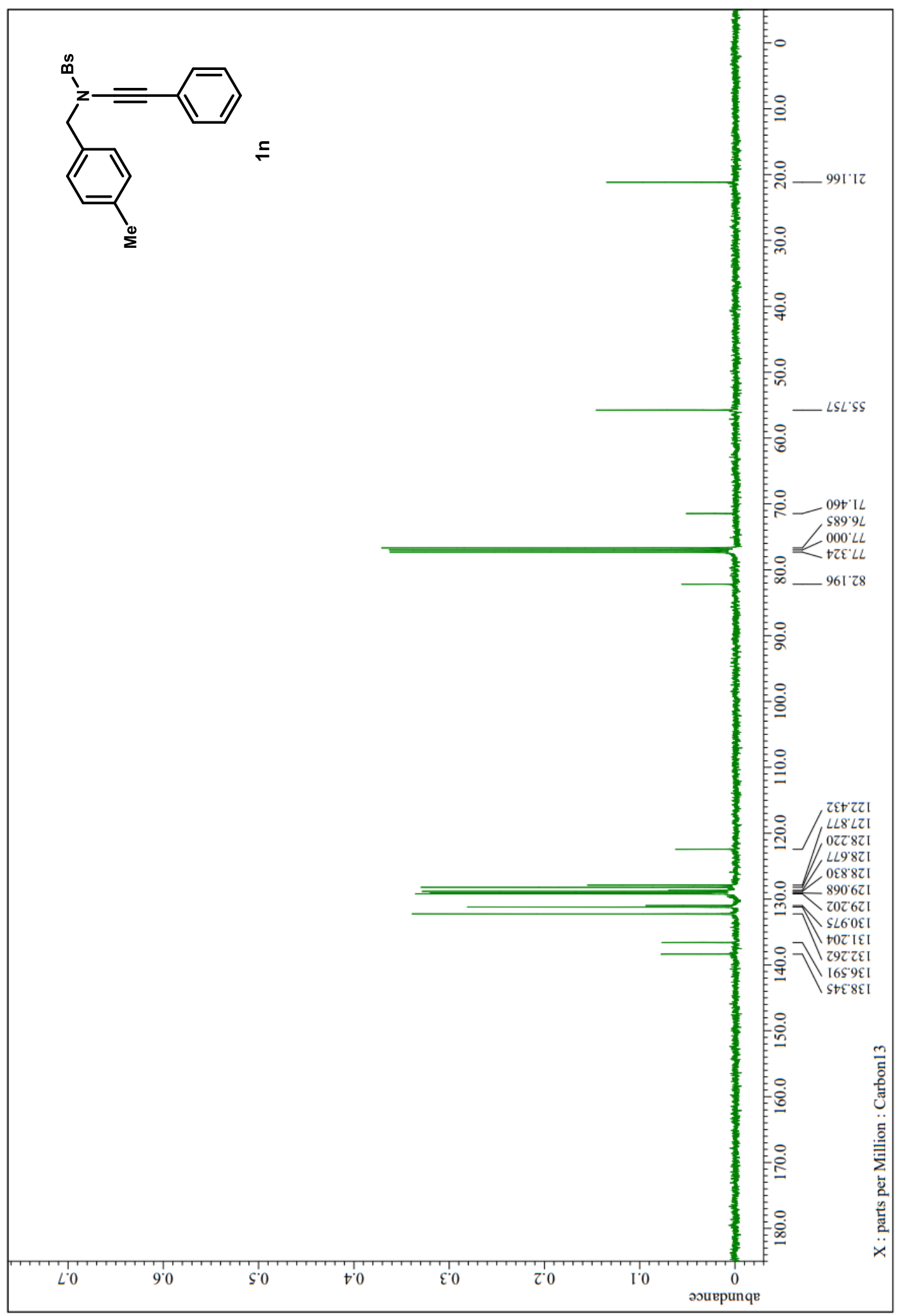




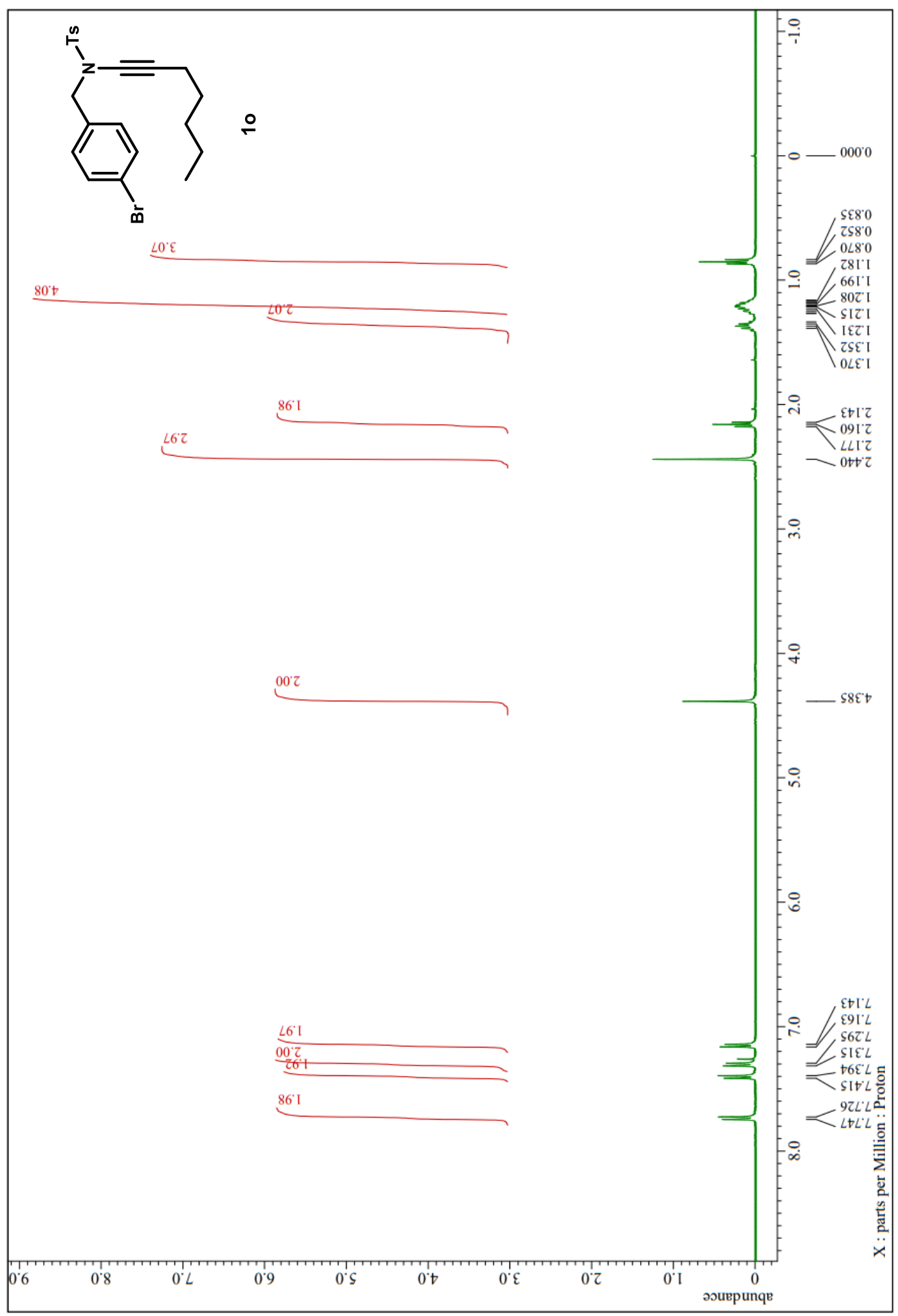


Supporting information

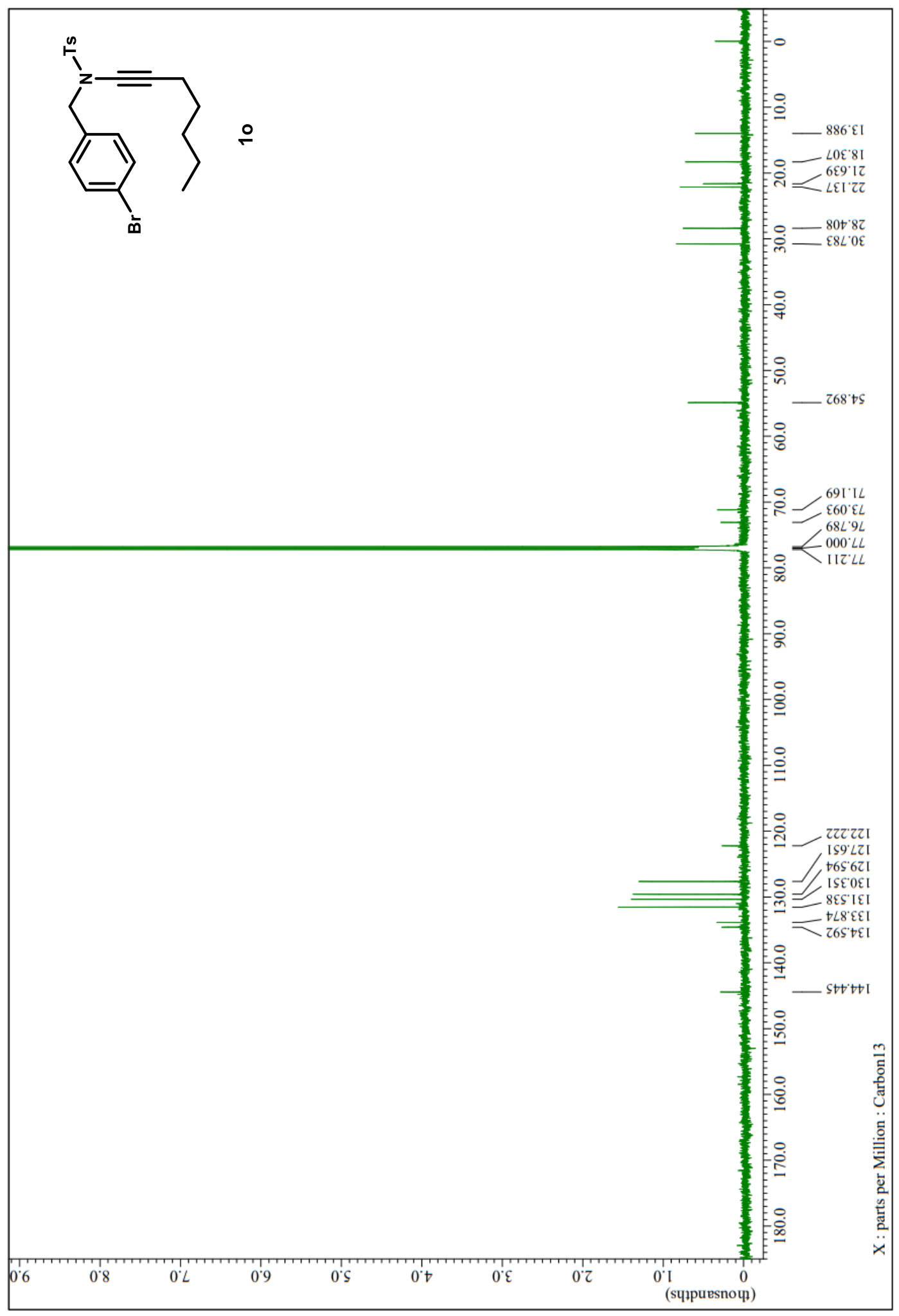


4-Bromo-N-(4-bromobenzyl)-N-((4-fluorophenyl)ethynyl)benzenesulfonamide (1u)

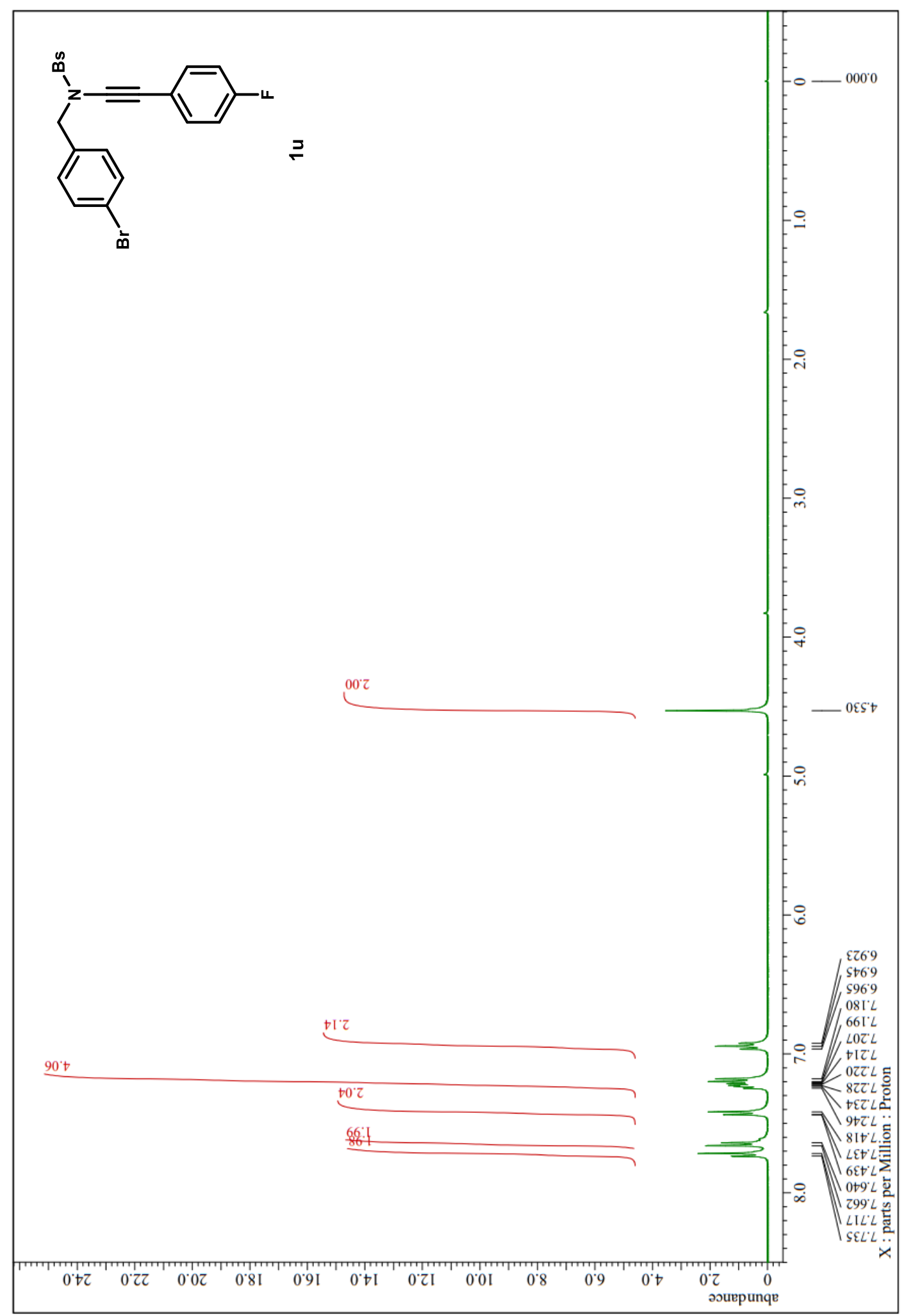


Supporting information

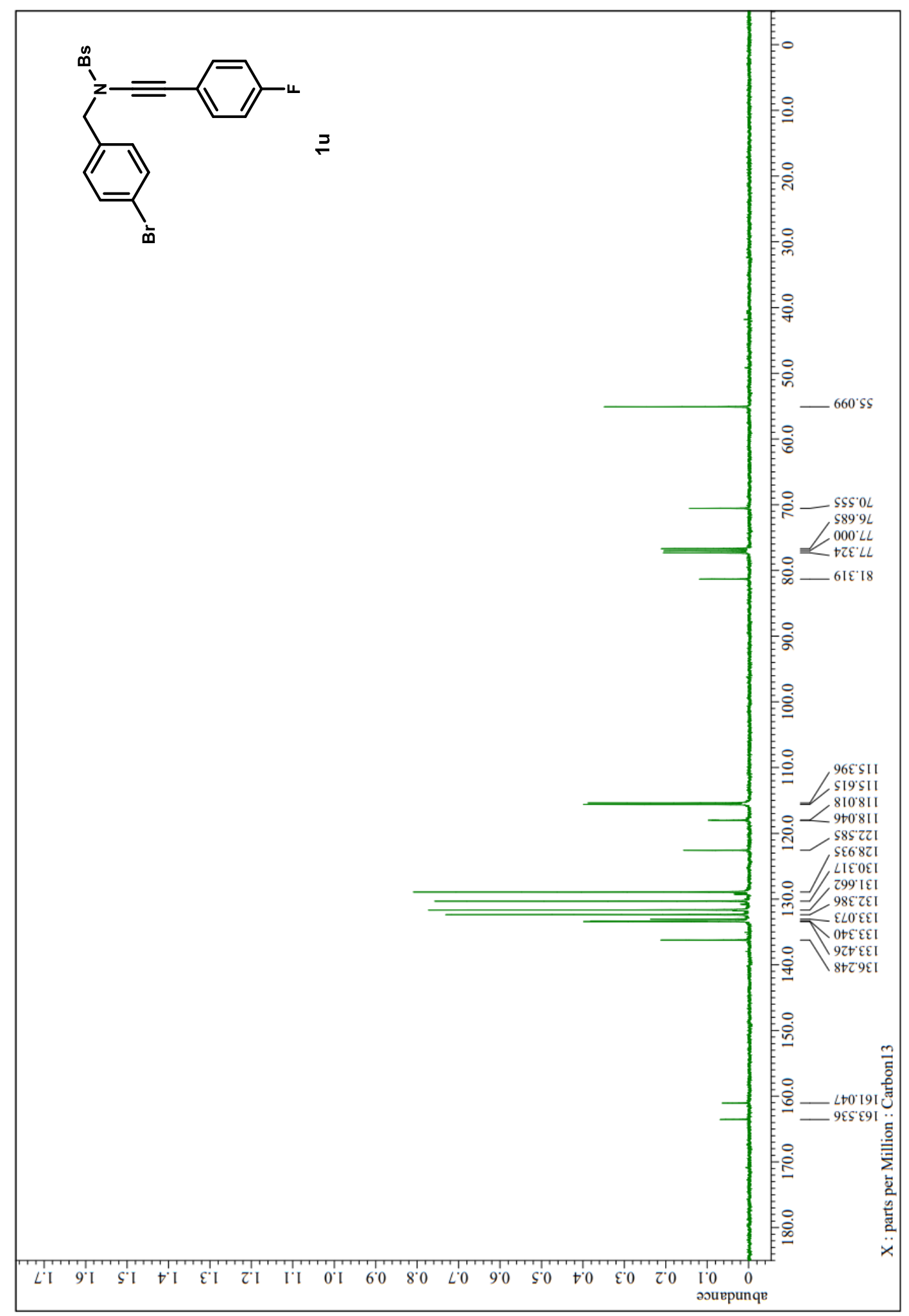


Supporting information

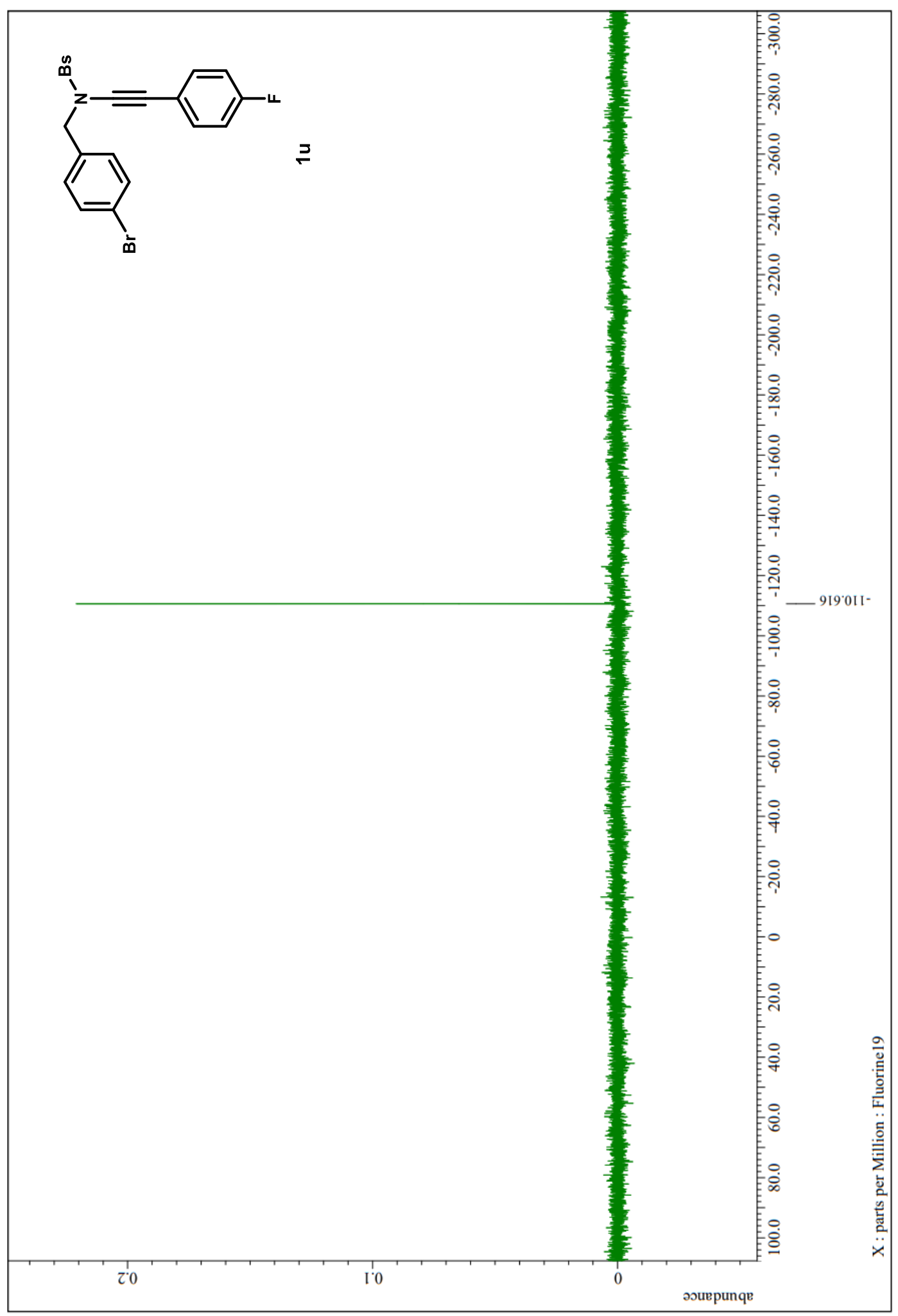


(3bR,4S,7a $R, 8 R, 8 \mathrm{a} R)$-10-Bromo-2-((4-bromophenyl)sulfonyl)-3a-phenyl1,2,3a,3b,4,4a,7a,8-octahydro-7H-4,8ethenoisobenzofuro[5',6':1,3]cyclopropa[1,2-c]pyrrole-3,5,7-trione (7a)

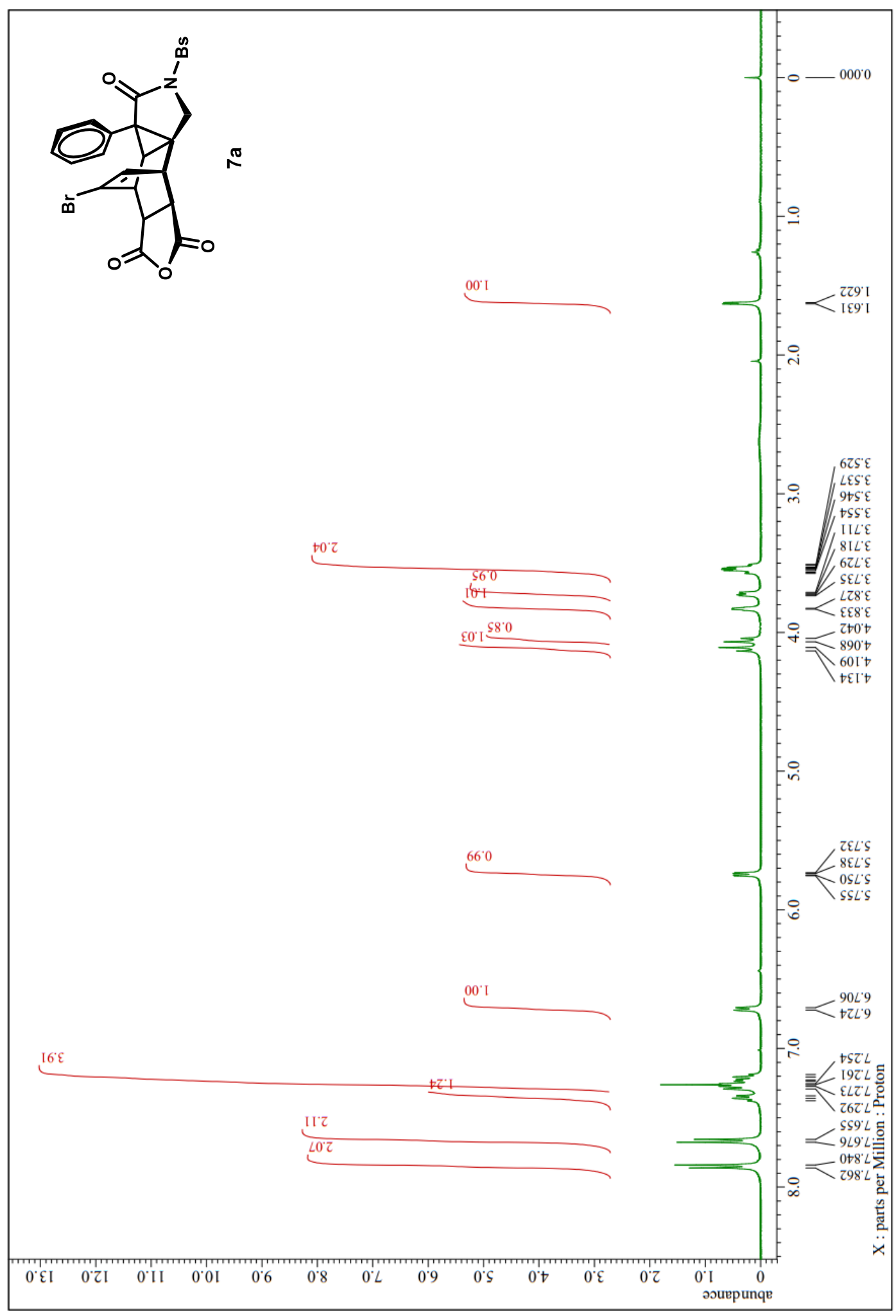


Supporting information

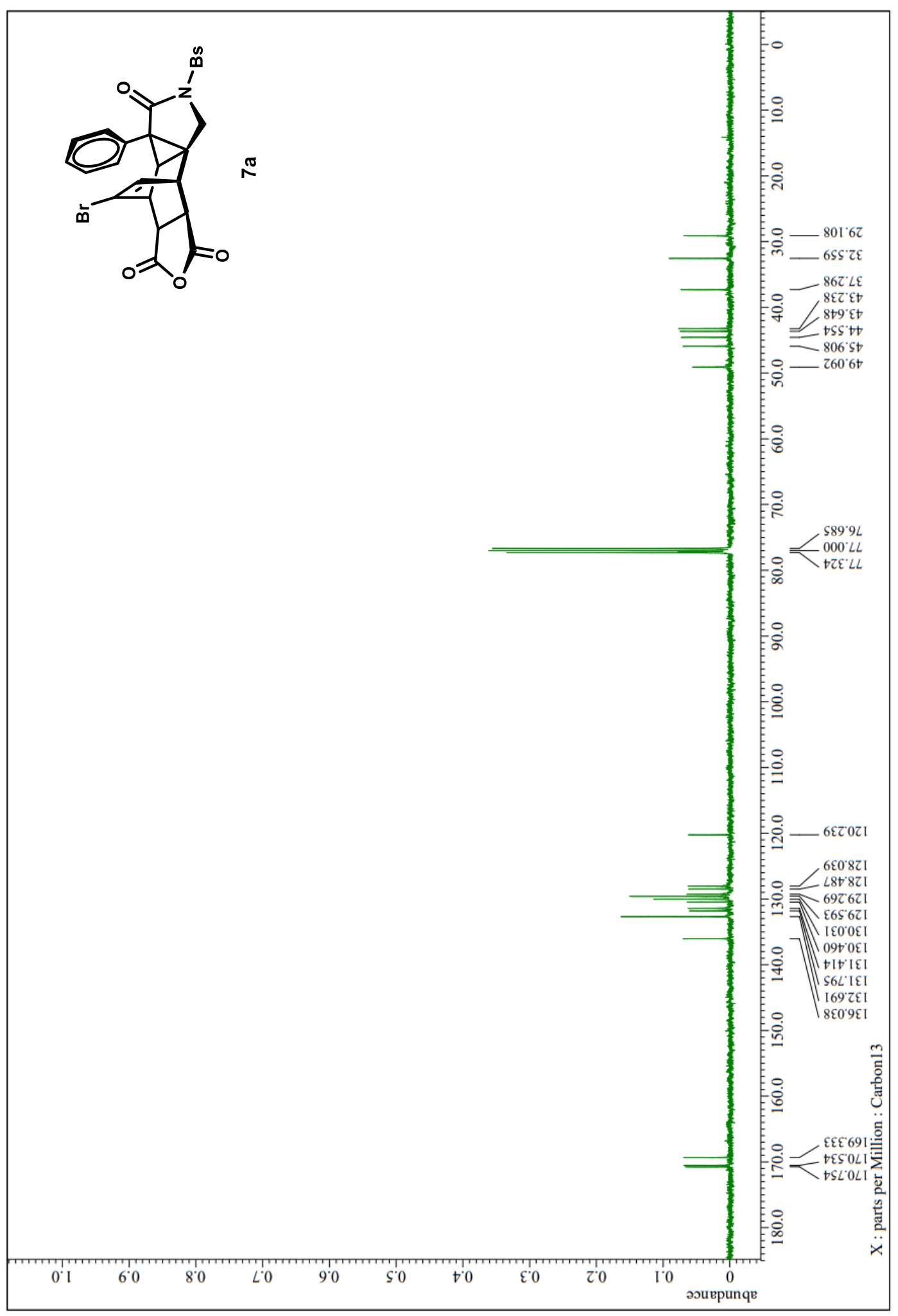




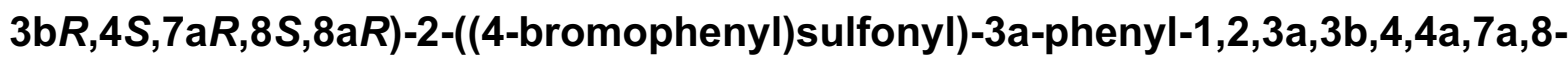
octahydro-7H-4,8-ethenoisobenzofuro[5',6':1,3]cyclopropa[1,2-c]pyrrole-3,5,7trione (7j)

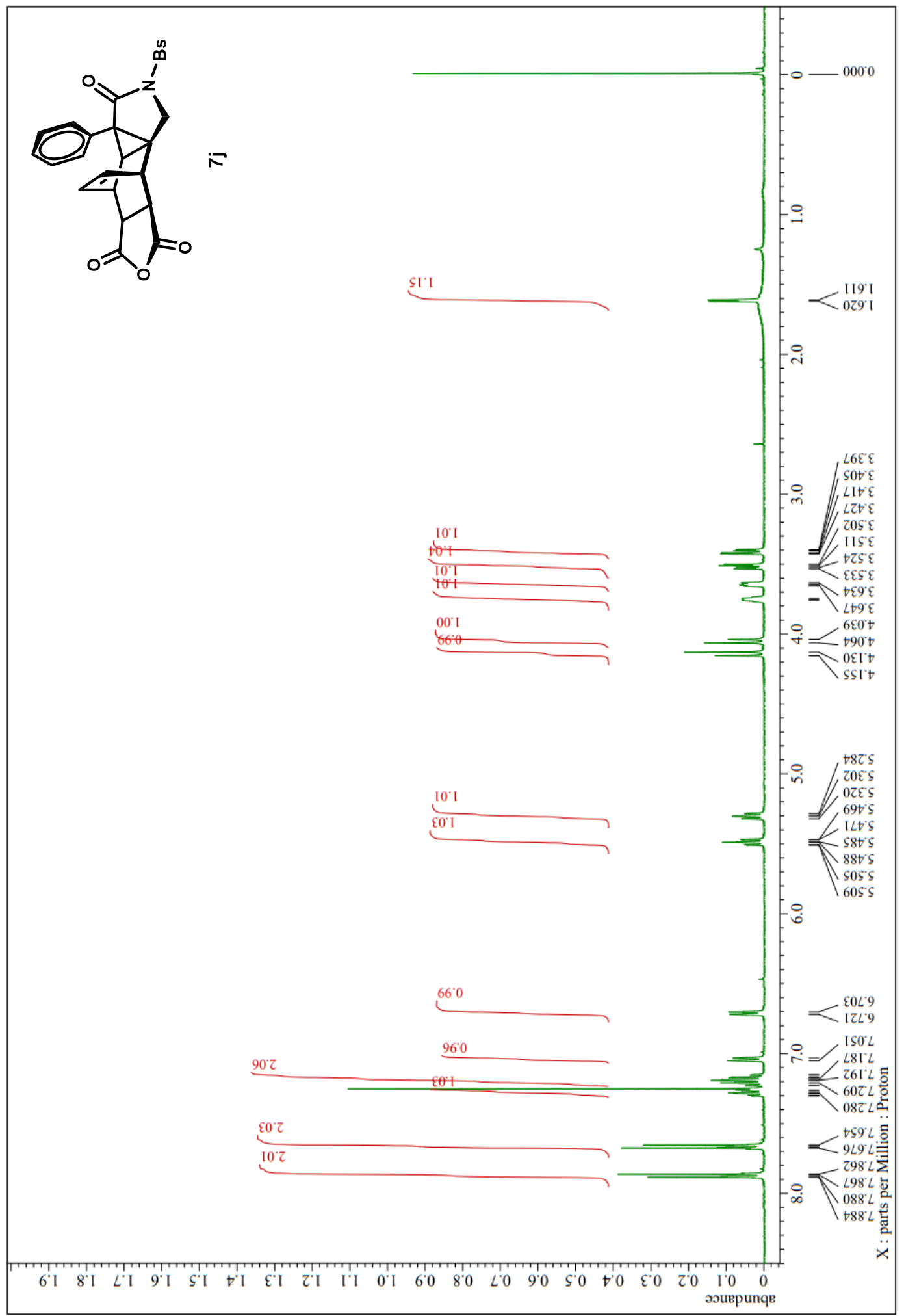


Supporting information

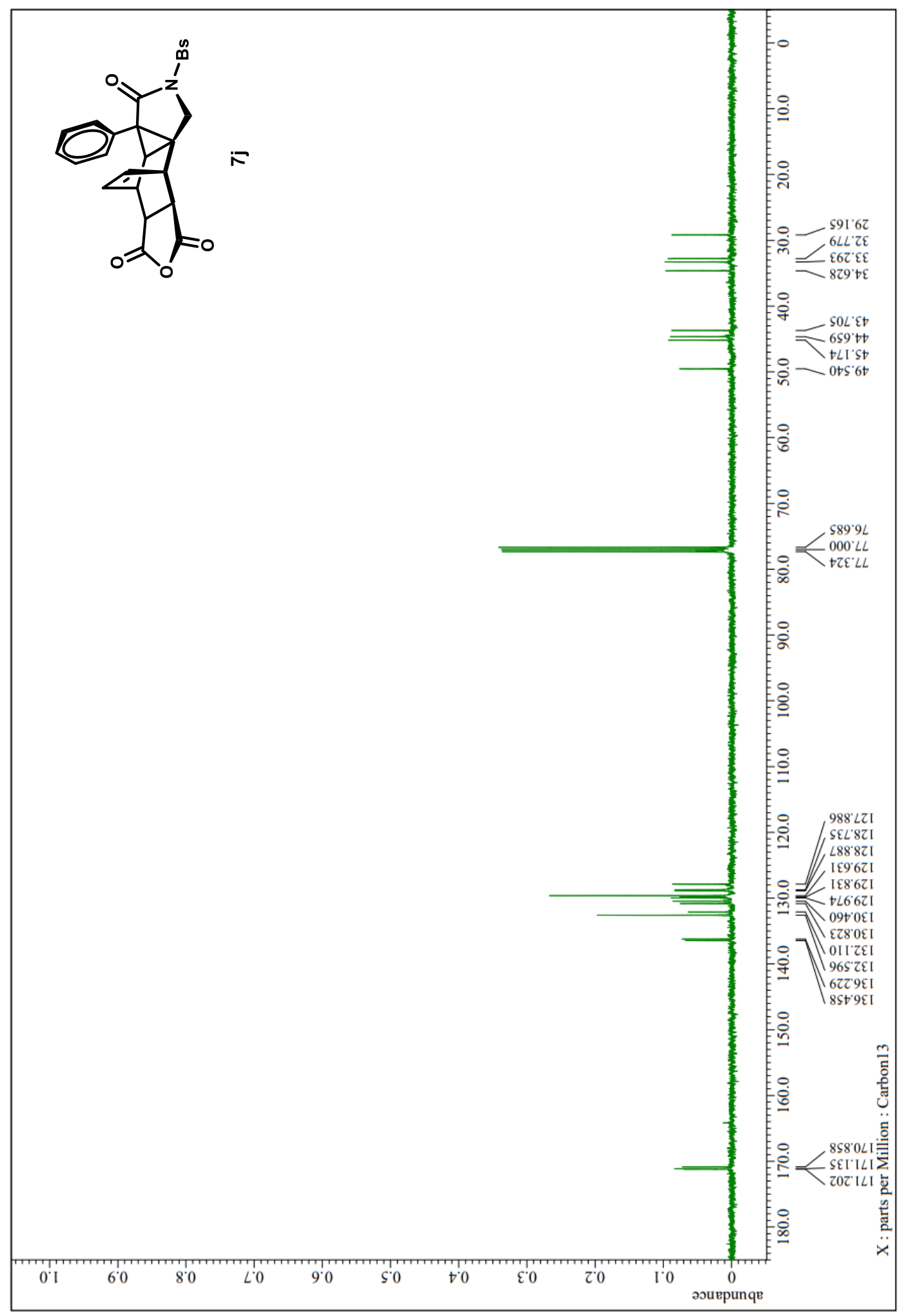


$N$-((3bR,4S,7aR,8R,8aR)-10-Bromo-2-((4-bromophenyl)sulfonyl)-3,5,7-trioxo-3aphenyl-1,2,3,3a,3b,4,4a,5,7a,8-decahydro-4,8-

ethenopyrrolo[3',4':1,3]cyclopropa[1,2-f]isoindol-6(7H)-yl)benzamide (8a)

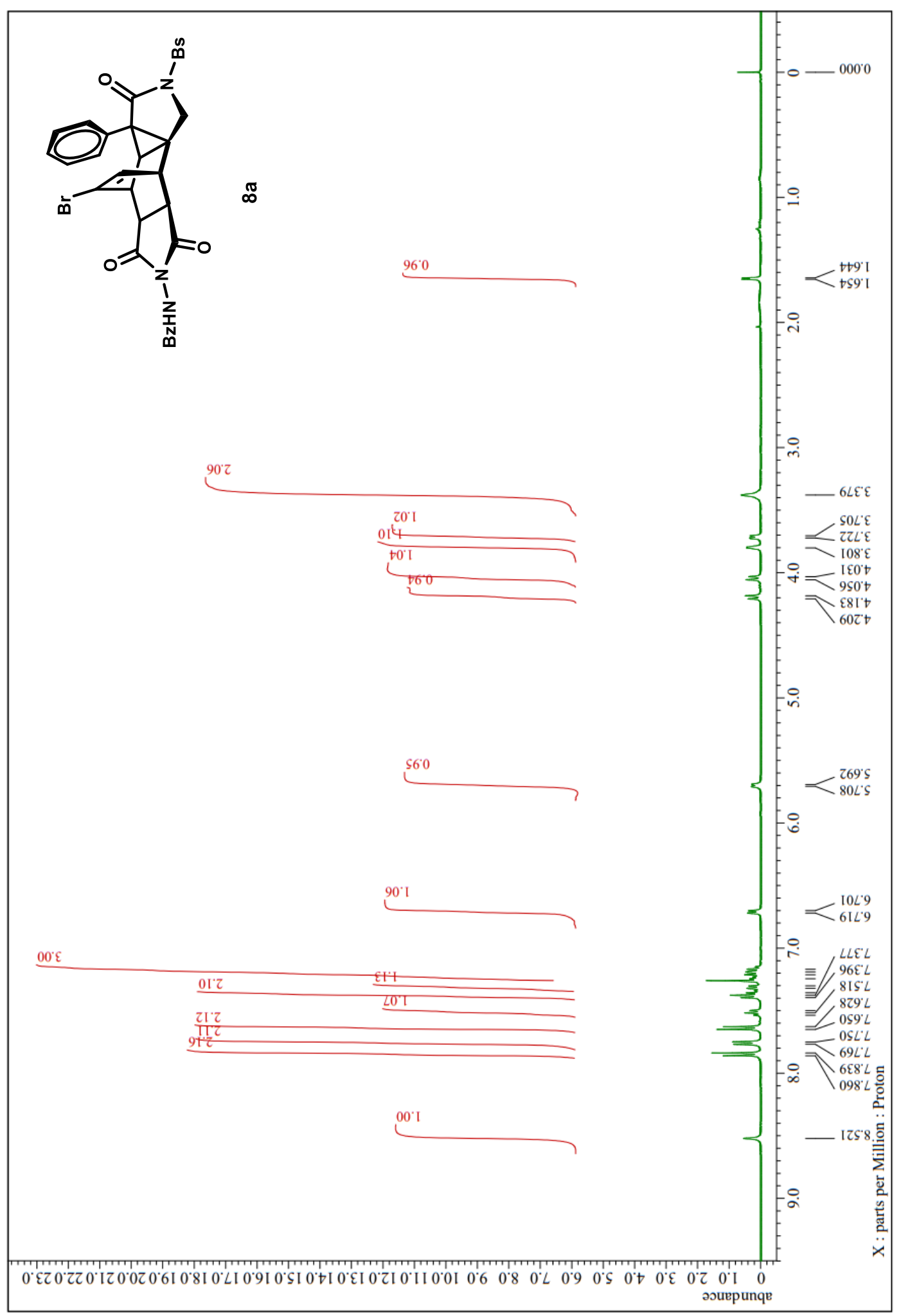


Supporting information

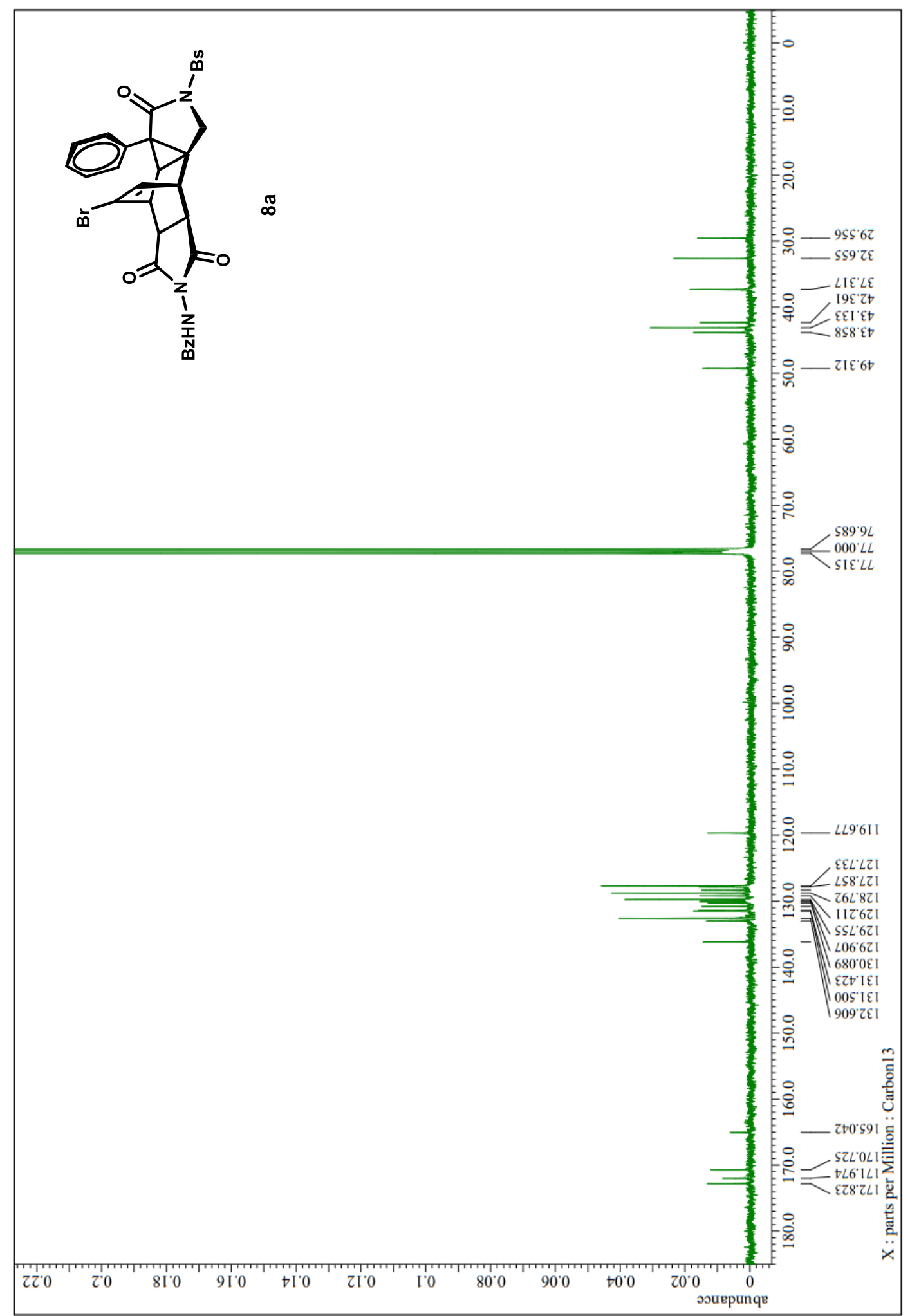


$N$-((3bR,4S,7aR,8S,8aR)-2-((4-bromophenyl)sulfonyl)-3,5,7-trioxo-3a-phenyl1,2,3,3a,3b,4,4a,5,7a,8-decahydro-4,8-ethenopyrrolo[3',4':1,3]cyclopropa[1,2f]isoindol-6(7H)-yl)benzamide (8j)

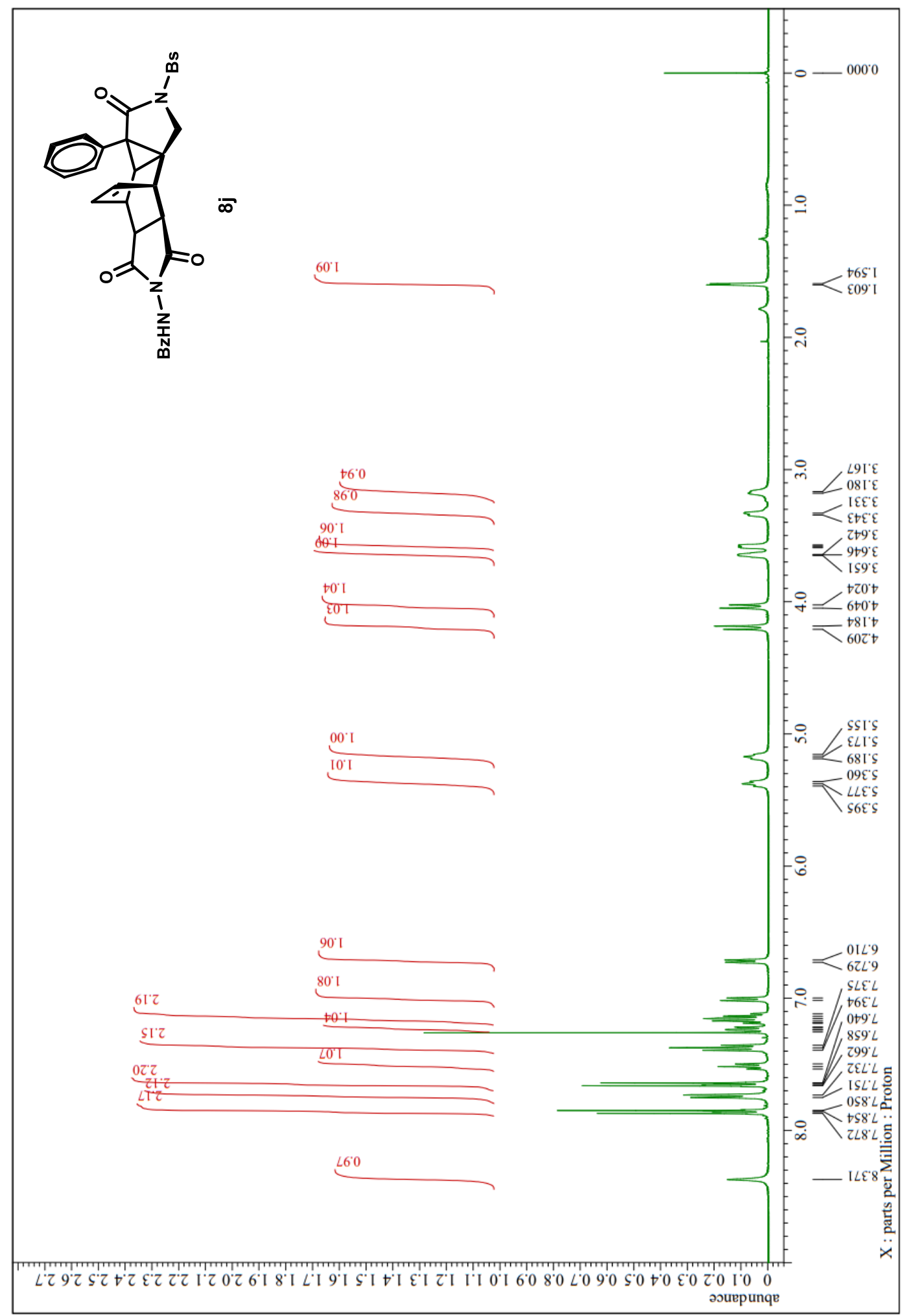


Supporting information

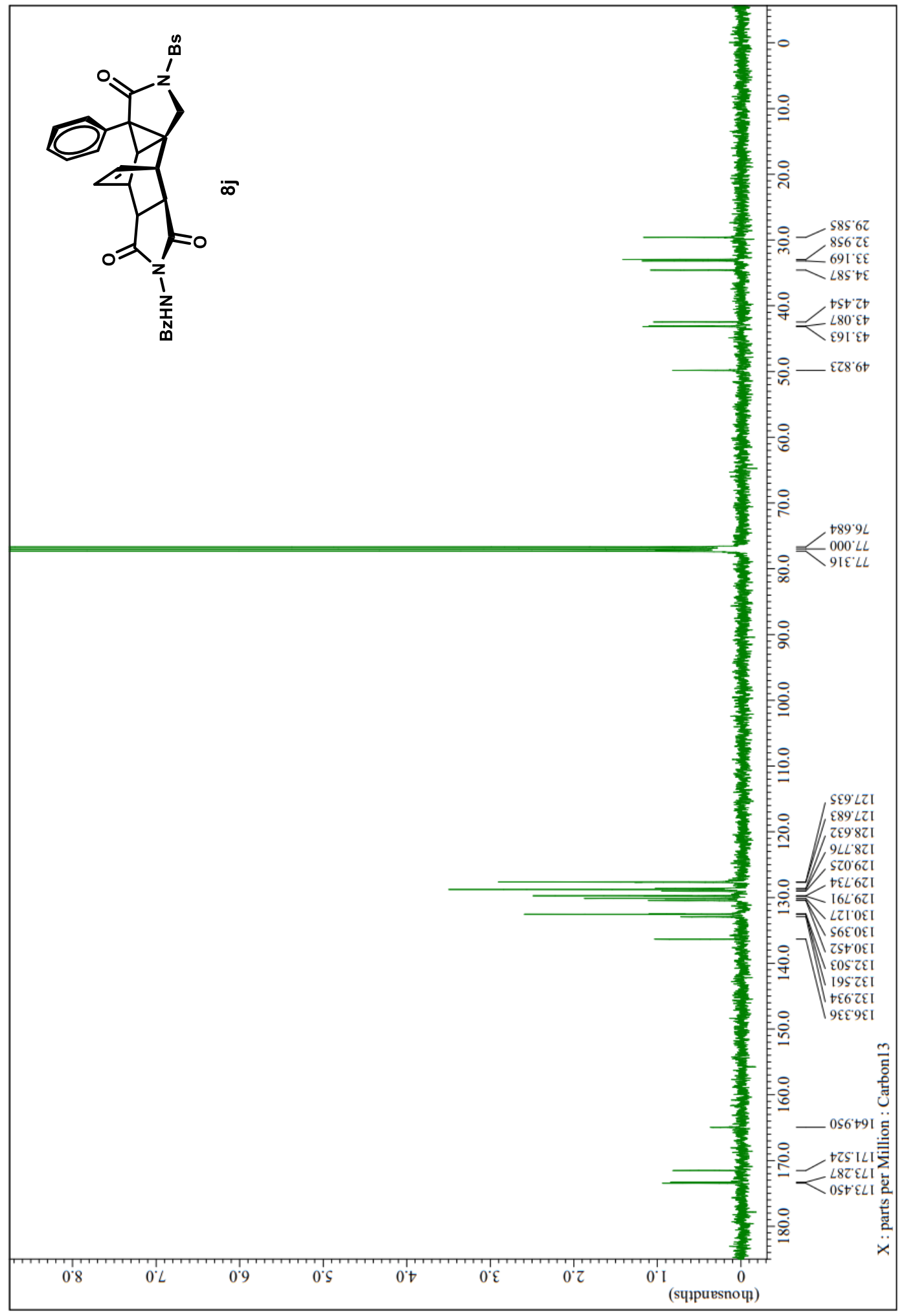


1-((3aR,3bR,4R,7R,7aR)-5-Bromo-7-methyl-3-oxo-3a-phenyl-2-tosyl-

2,3,3a,3b,4,7-hexahydro-1H-benzo[1,3]cyclopropa[1,2-c]pyrrol-4-yl)-4-phenyl1,2,4-triazolidine-3,5-dione (9)

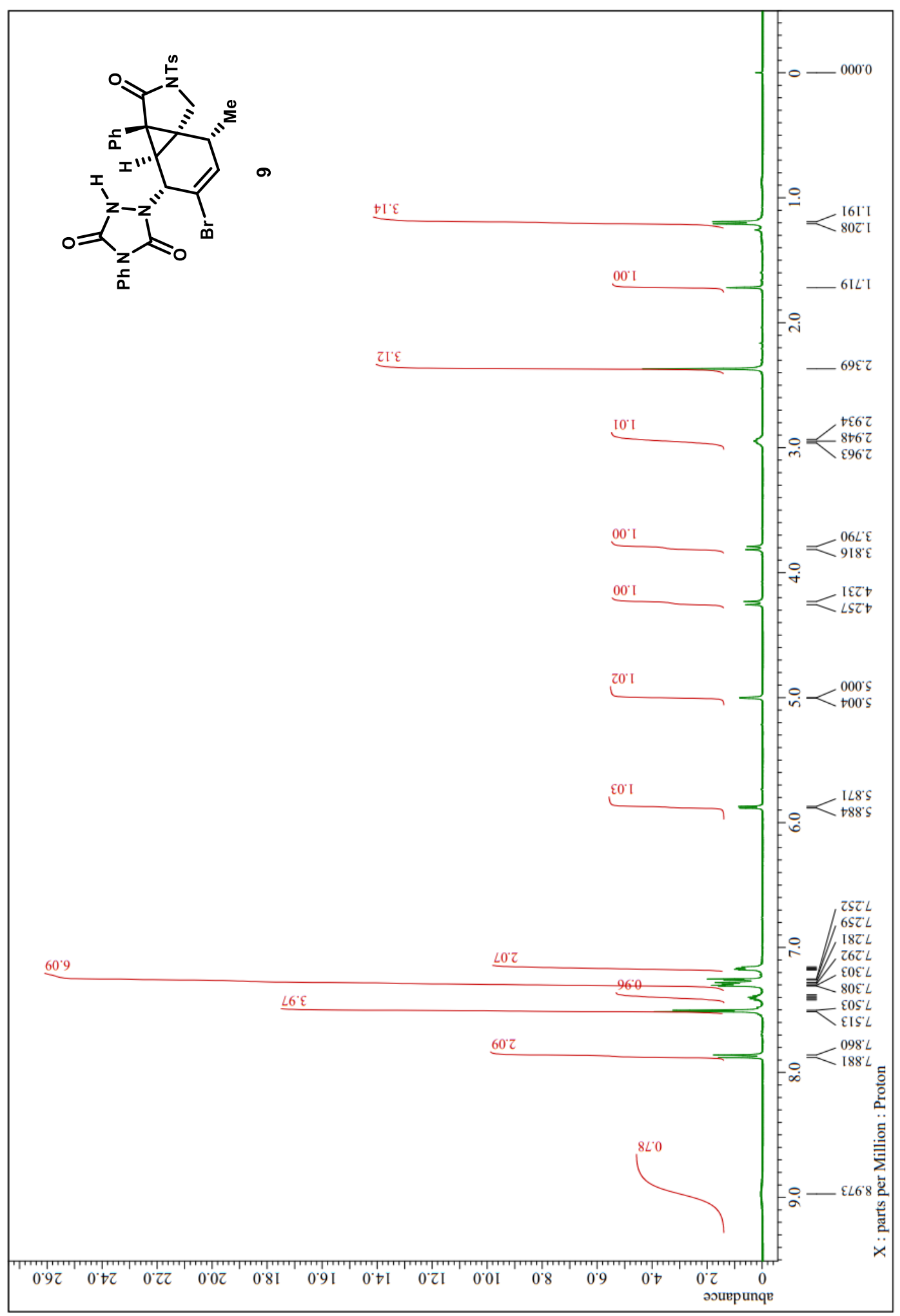


Supporting information

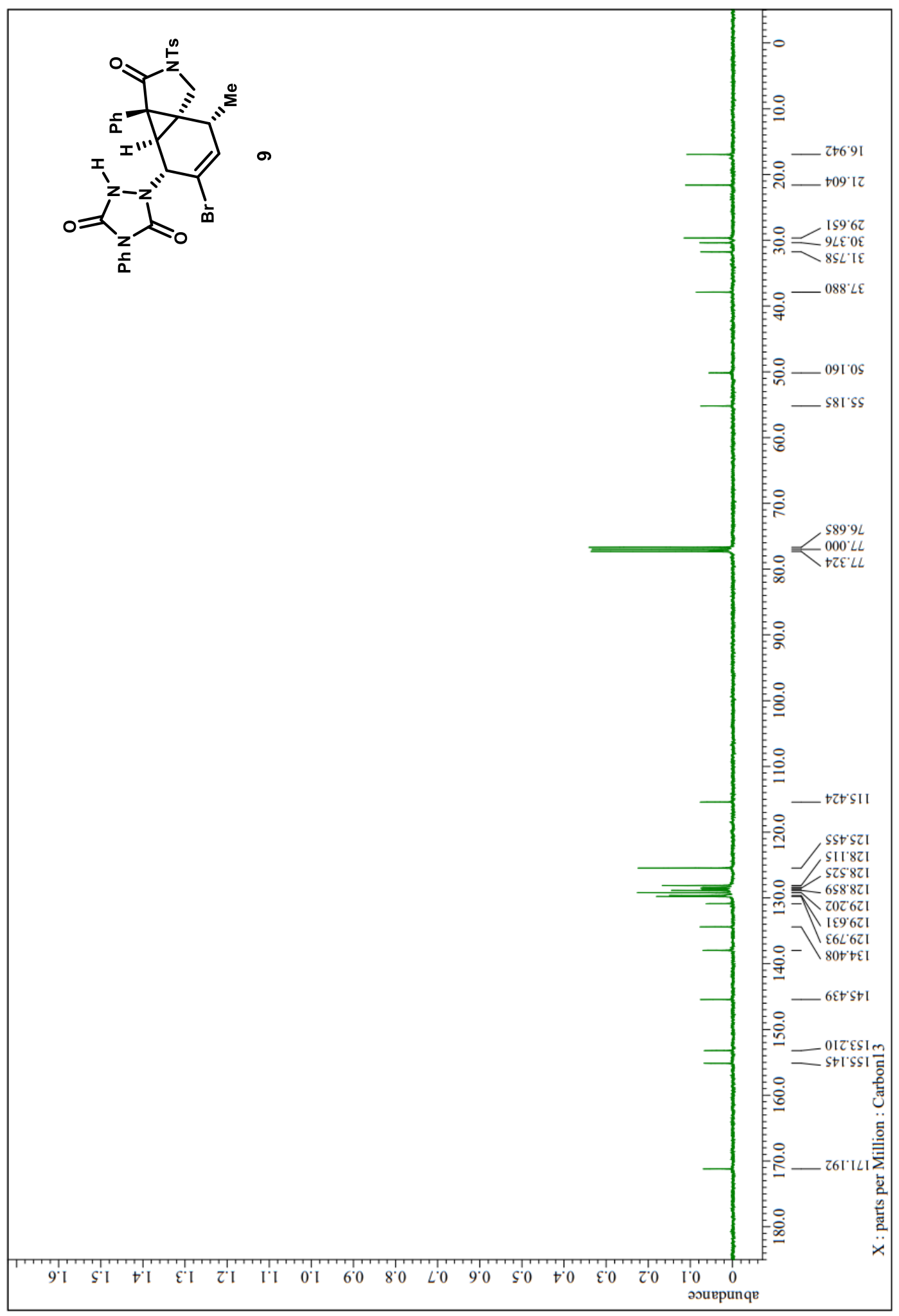


1-((3aR,3b $R, 4 R, 7 R, 7 \mathrm{a} R)$-7-Methyl-3-oxo-3a,5-diphenyl-2-tosyl-2,3,3a,3b,4,7hexahydro-1H-benzo[1,3]cyclopropa[1,2-c]pyrrol-4-yl)-4-phenyl-1,2,4triazolidine-3,5-dione (10)

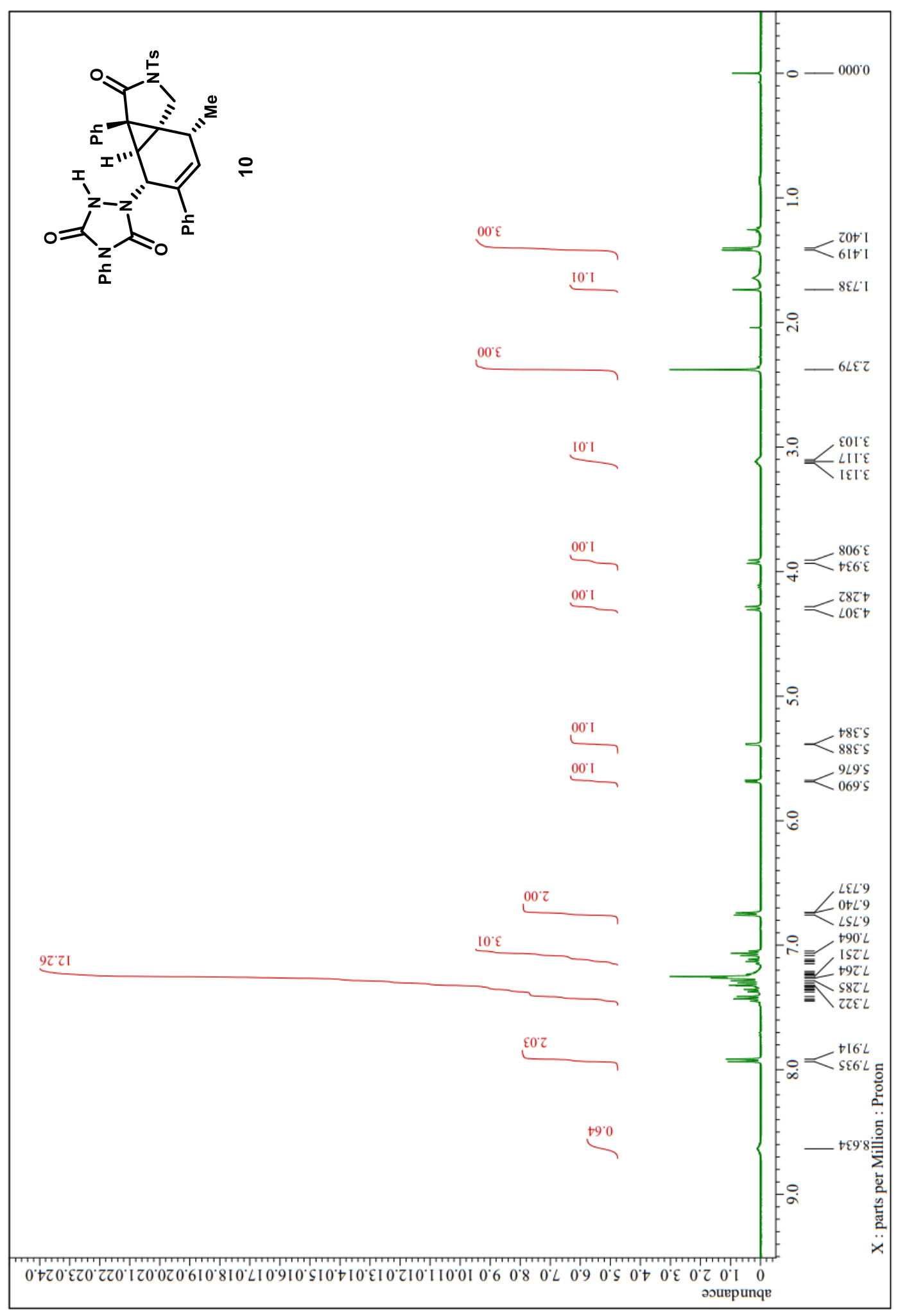


Supporting information

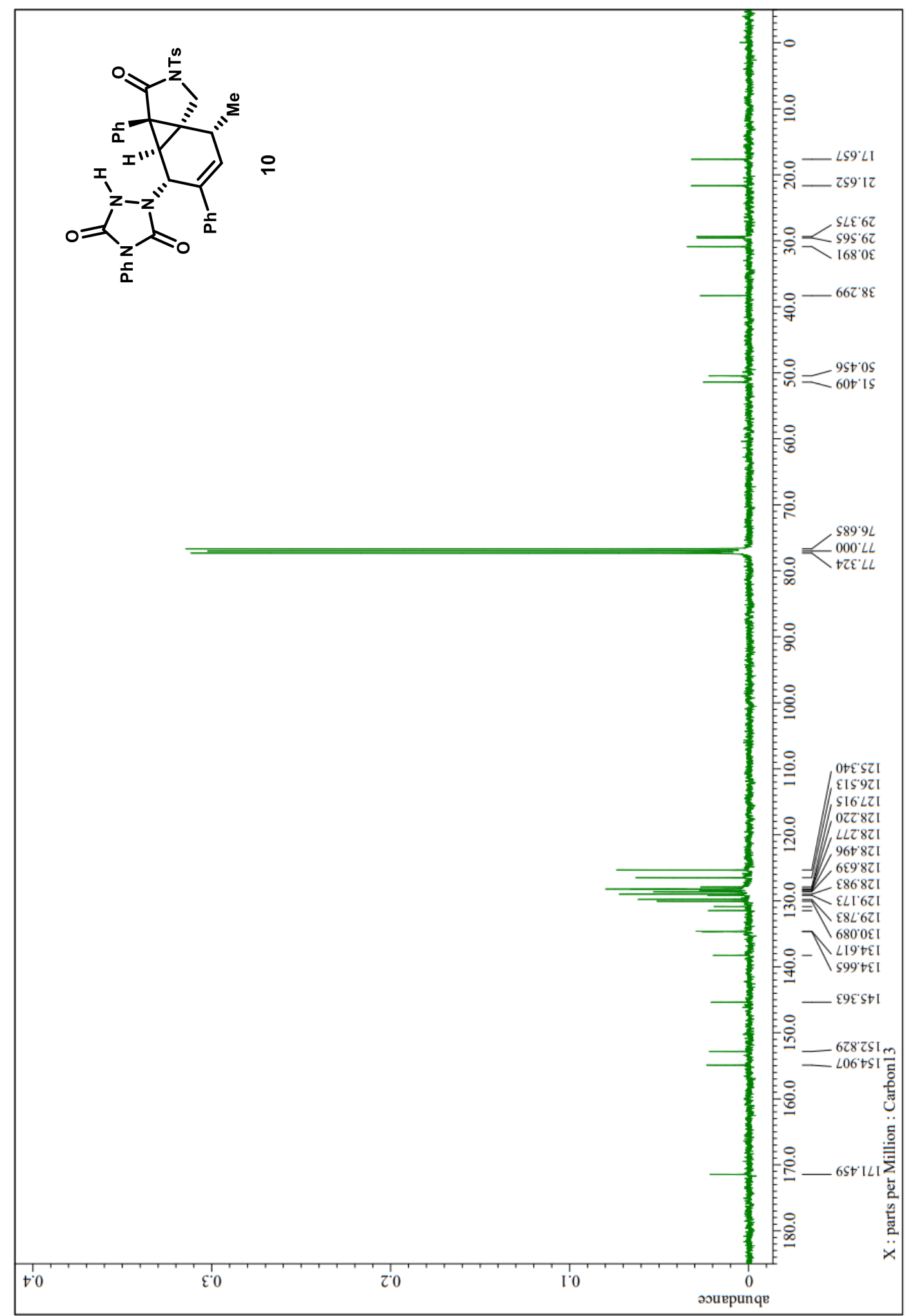


1-((3aR,3bR,4S,5S,7R,7aR)-7-Methyl-3-oxo-3a,5-diphenyl-2-tosyloctahydro-1Hbenzo[1,3]cyclopropa[1,2-c]pyrrol-4-yl)-4-phenyl-1,2,4-triazolidine-3,5-dione (11)

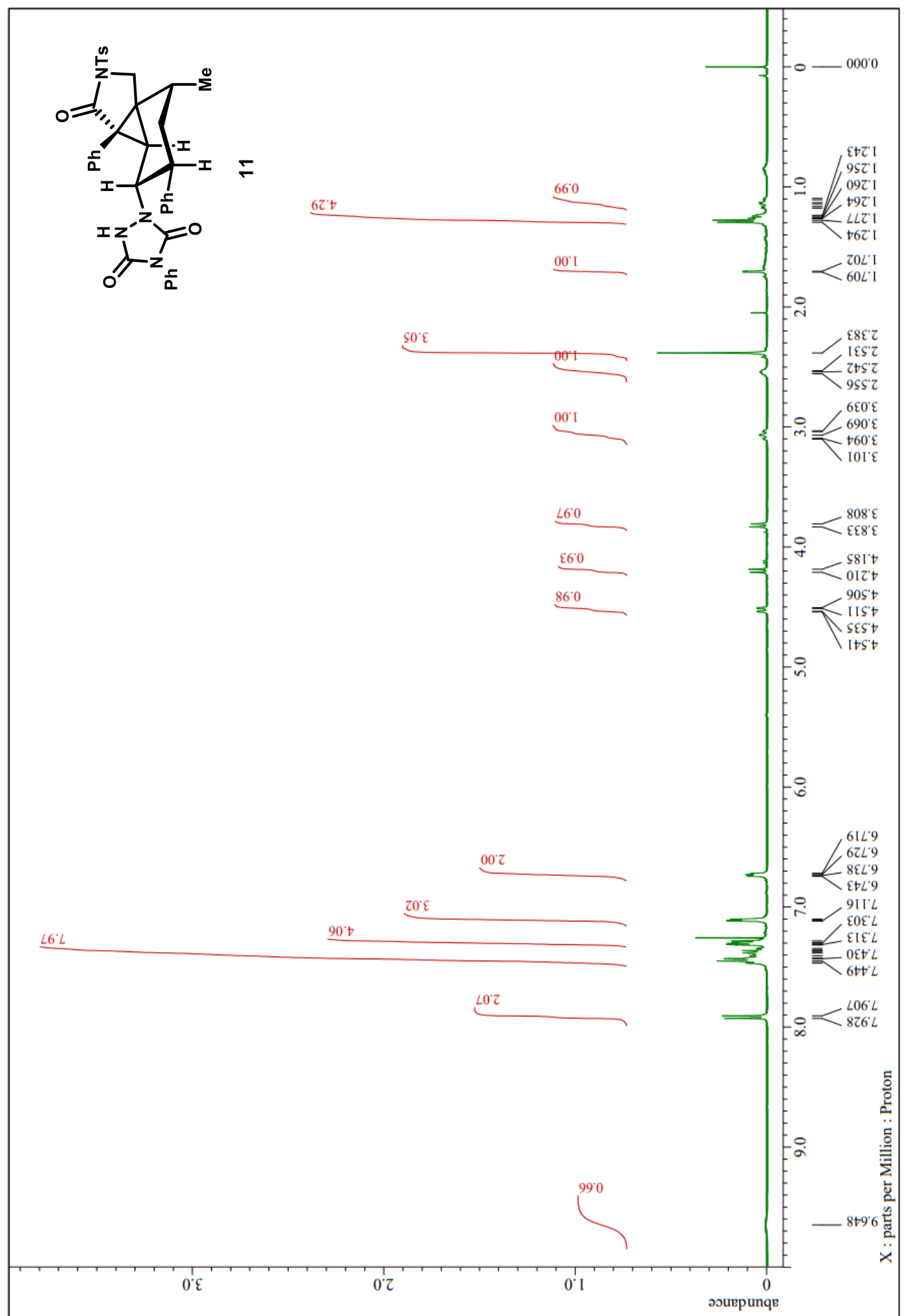


Supporting information

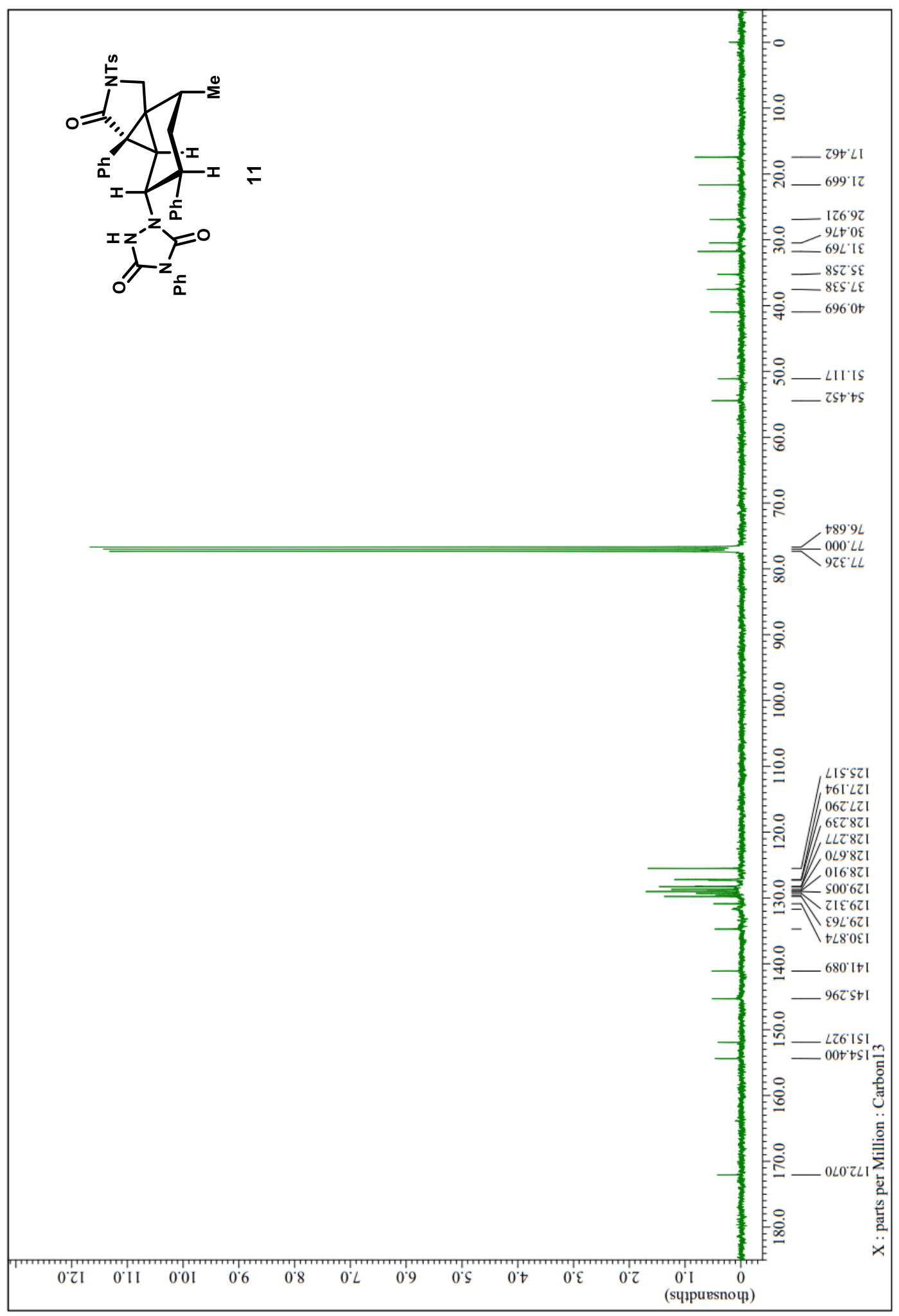


(8R,8aR)-6-Bromo-2-((4-bromophenyl)sulfonyl)-7,8-dihydroxy-8a-phenyl-

3,7,8,8a-tetrahydrocyclohepta[c]pyrrol-1(2H)-one (12)

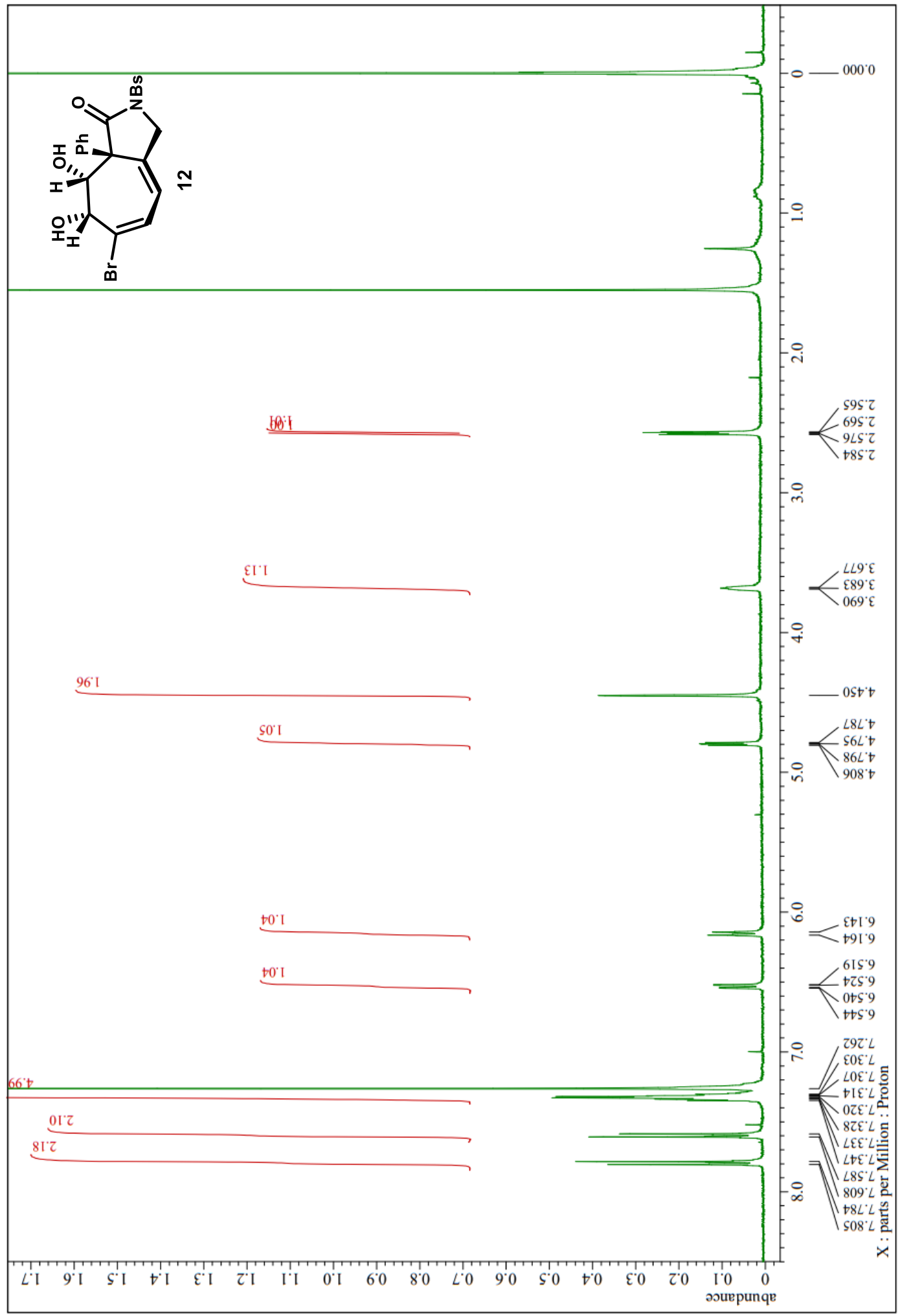


Supporting information

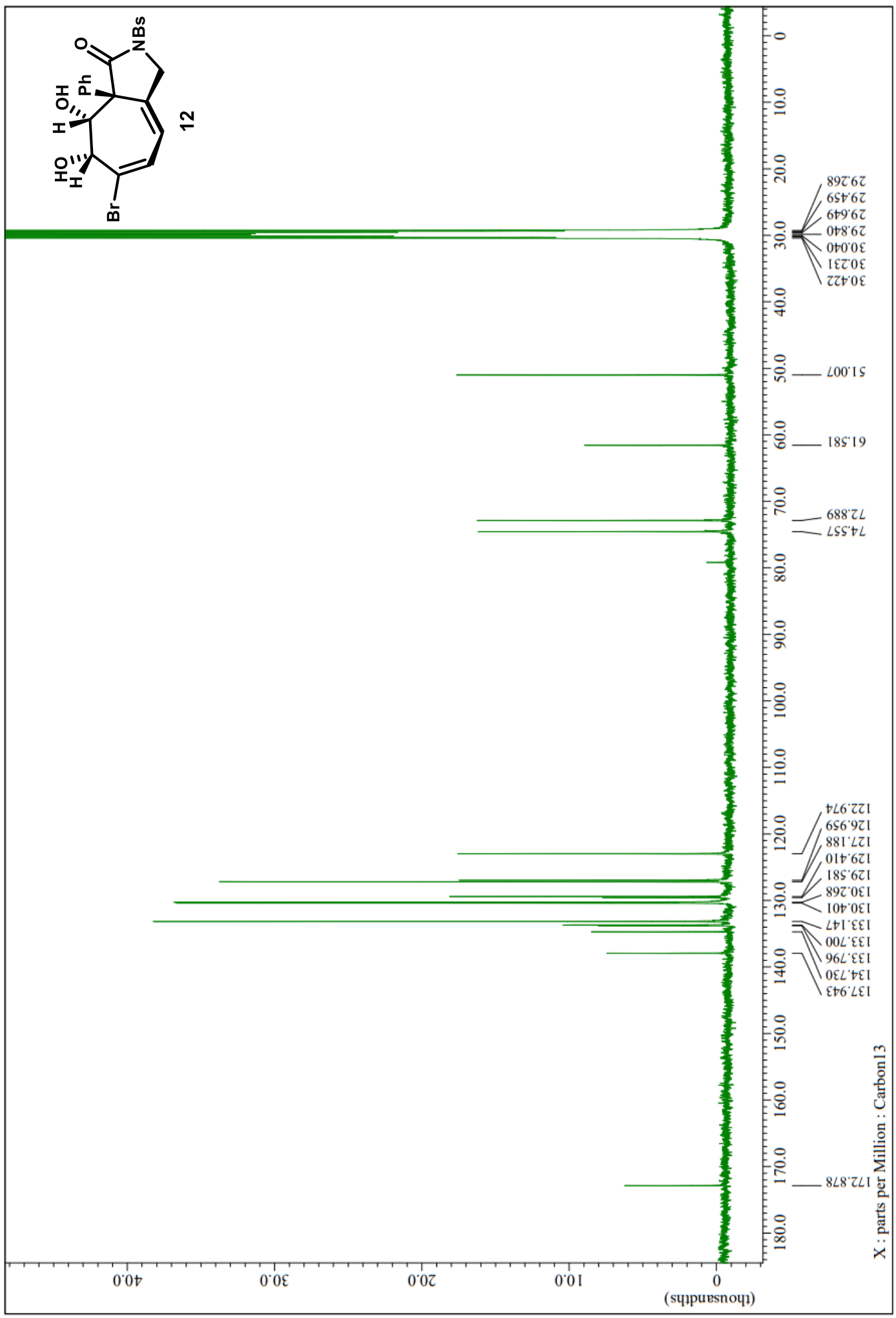




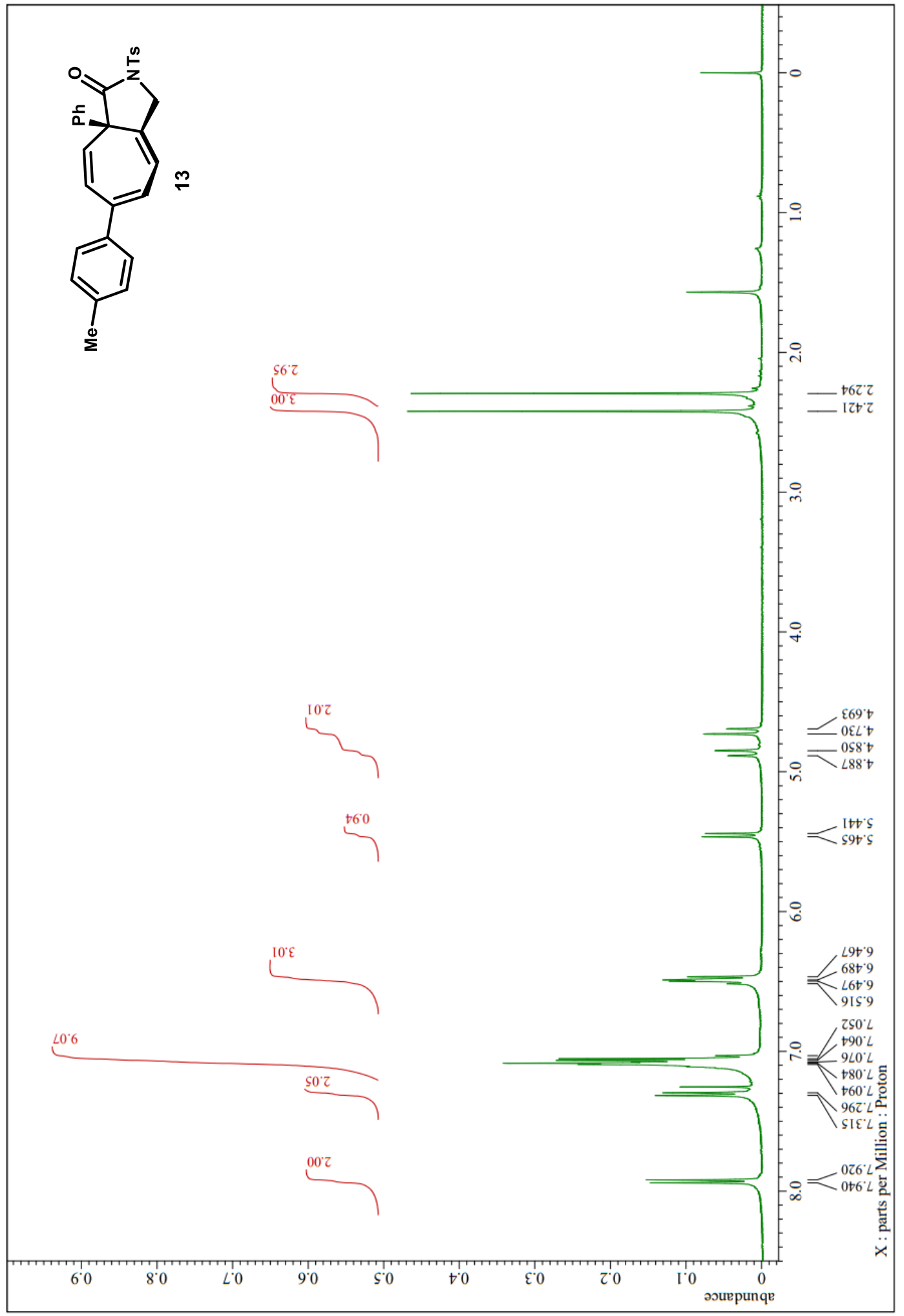


Supporting information

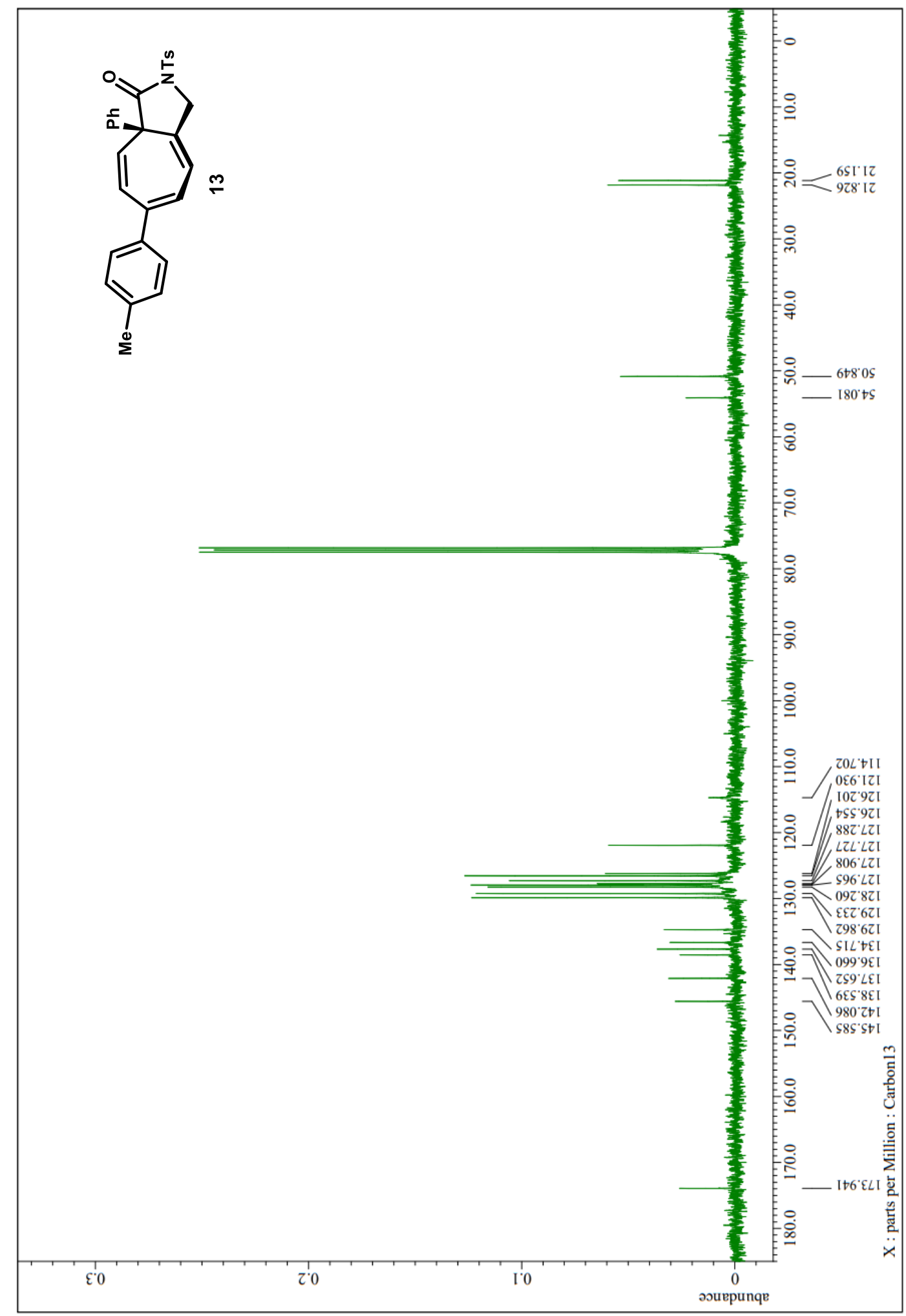


Supporting information

Dimethyl (3bR,4S,7R,7aS)-9-bromo-3-oxo-3a-phenyl-2-tosyl-2,3,3a,3b,4,7hexahydro-1H-4,7-ethenobenzo[1,3]cyclopropa[1,2-c]pyrrole-5,6-dicarboxylate (14)

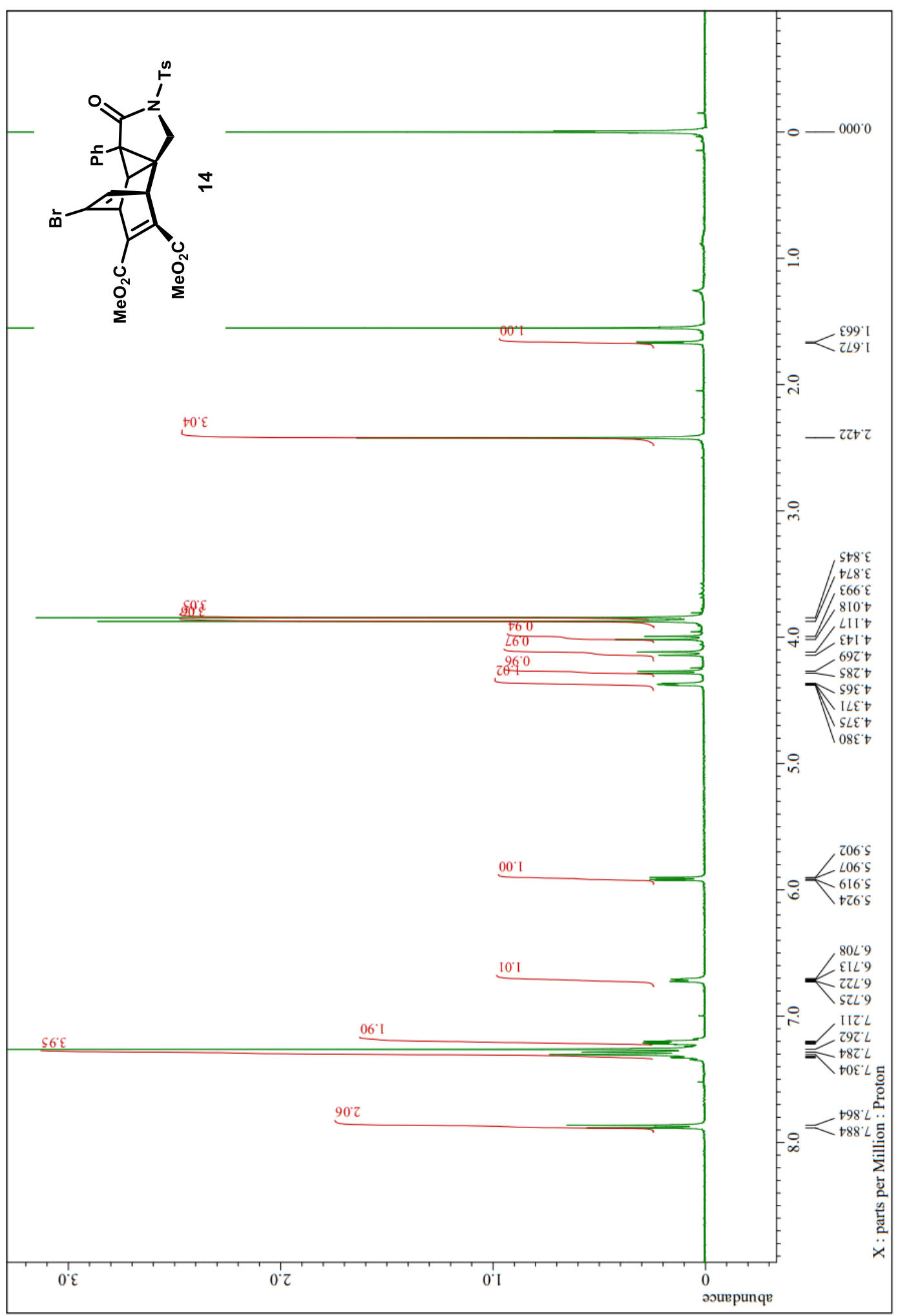


Supporting information

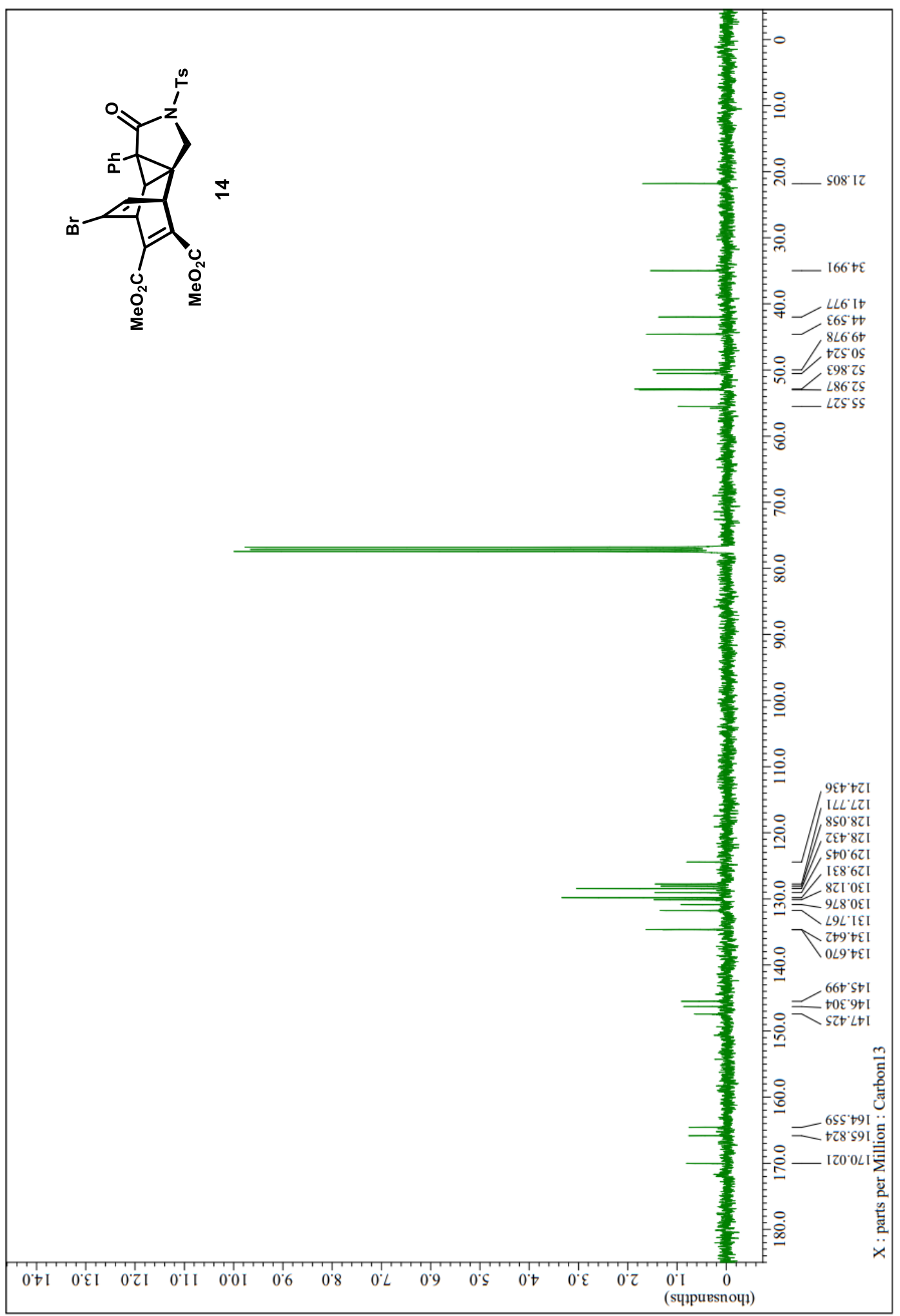




\section{Chiral and Racemic HPLC Traces}

(R)-6-Bromo-2-((4-bromophenyl)sulfonyl)-8a-phenyl-3,8adihydrocyclohepta[c]pyrrol-1(2H)-one (2a)

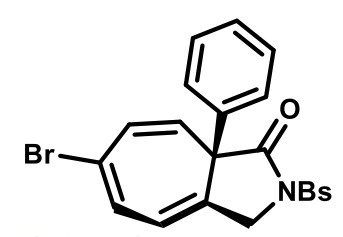

$2 a$

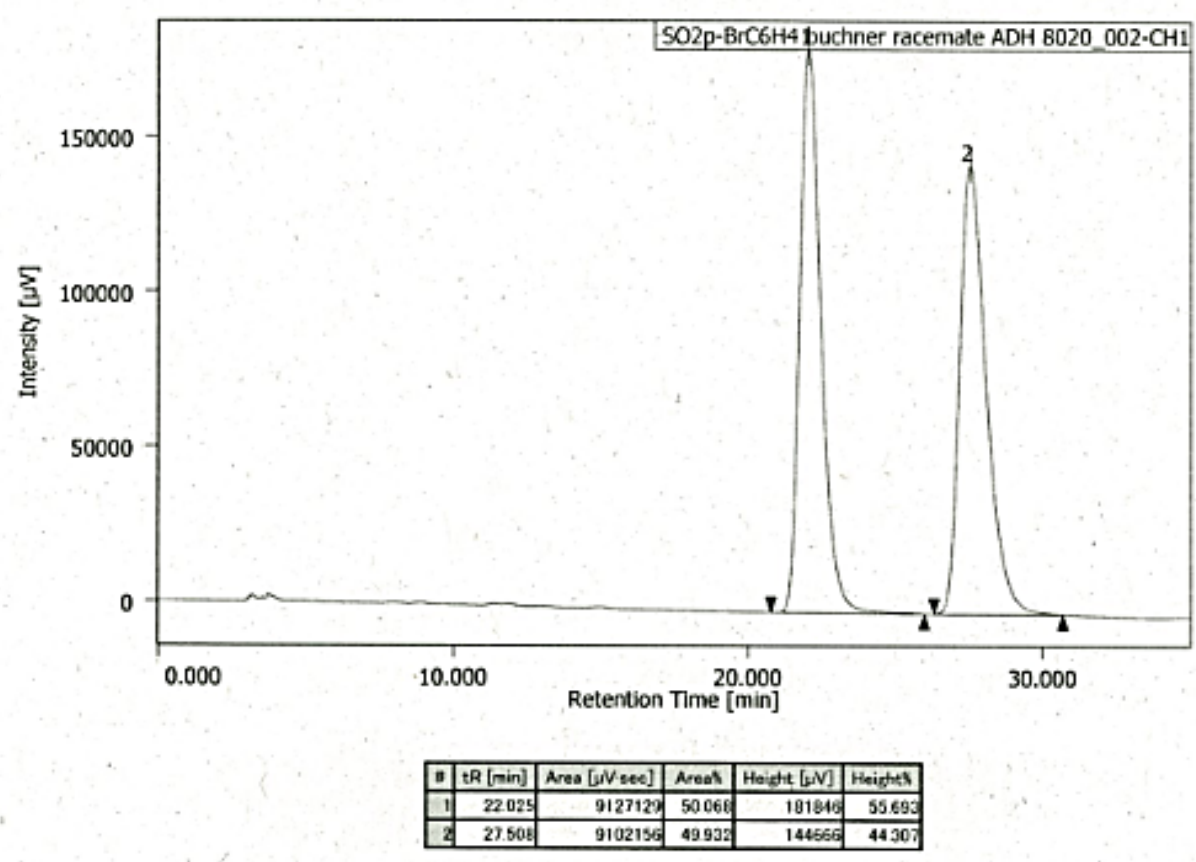

ti.0129 SO2Ph-PBr buchner asym AOHBO20 003 2020/01/29 1502.45

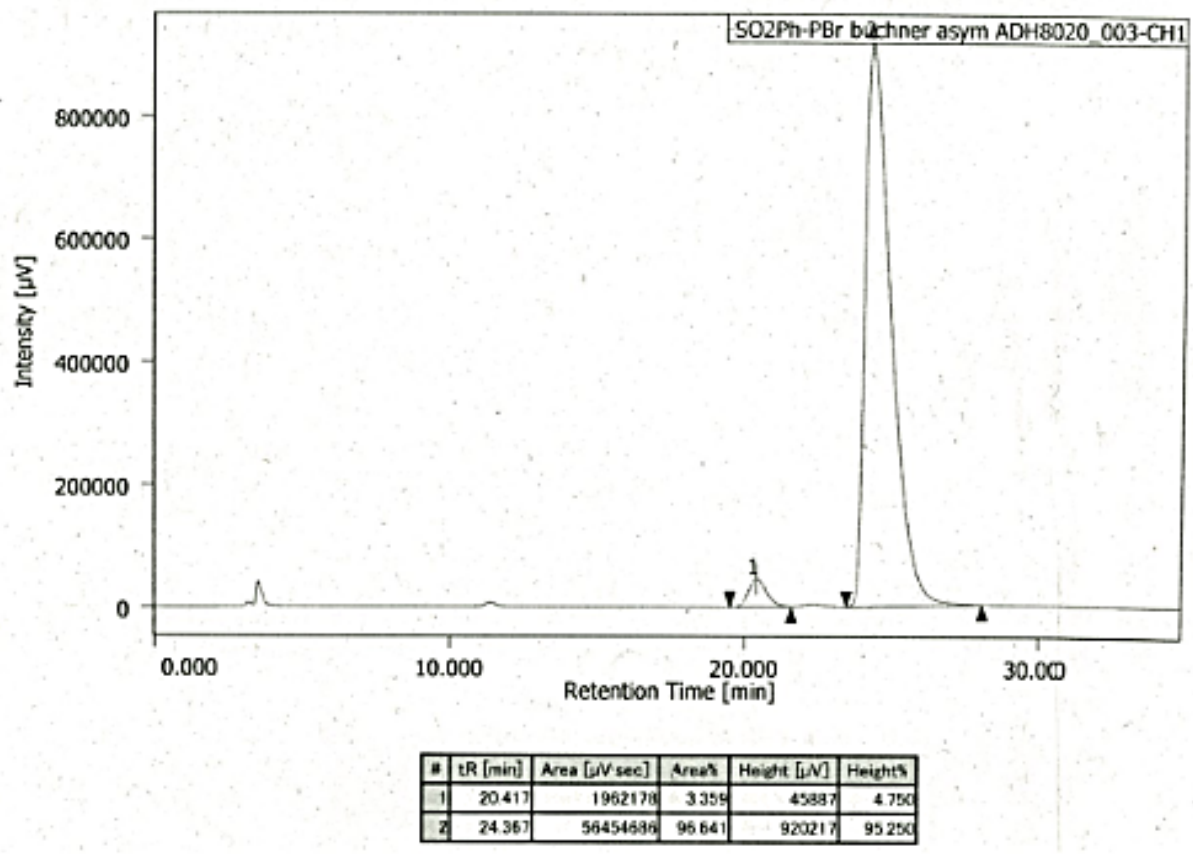


Methyl (R)-4-(6-bromo-2-((4-bromophenyl)sulfonyl)-3-oxo-2,3dihydrocyclohepta[c]pyrrol-3a(1H)-yl)benzoate (2b)

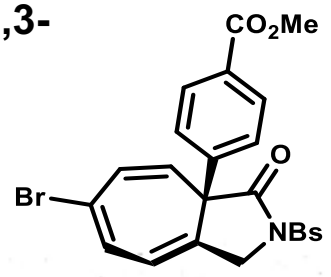

2b
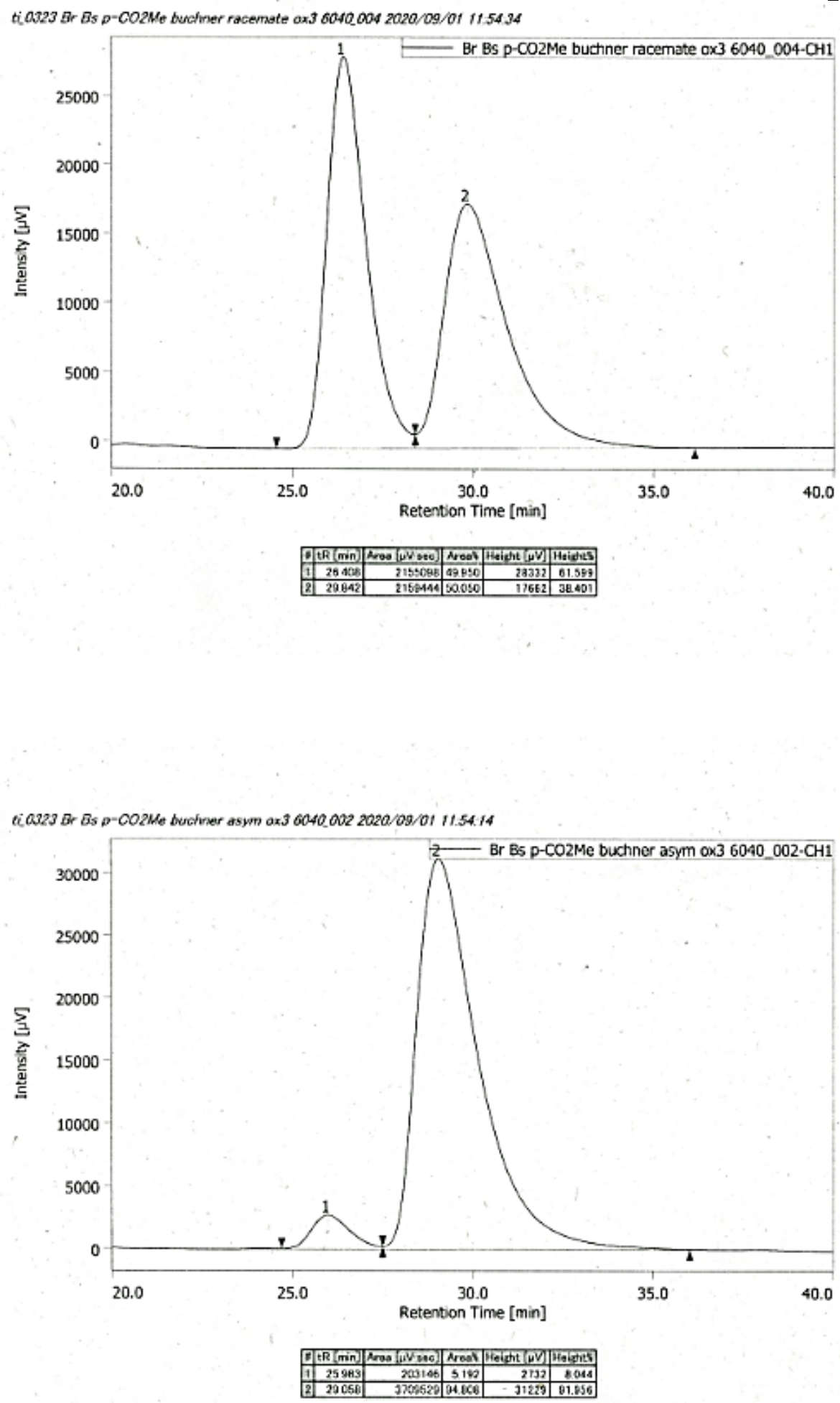
(R)-6-Bromo-8a-(4-bromophenyl)-2-((4-bromophenyl)sulfonyl)-3,8adihydrocyclohepta[c]pyrrol-1(2H)-one (2c)

ti 0312 EnBso-Bor racemate ADH GOSQ 003 2020/02/18 12.40.56
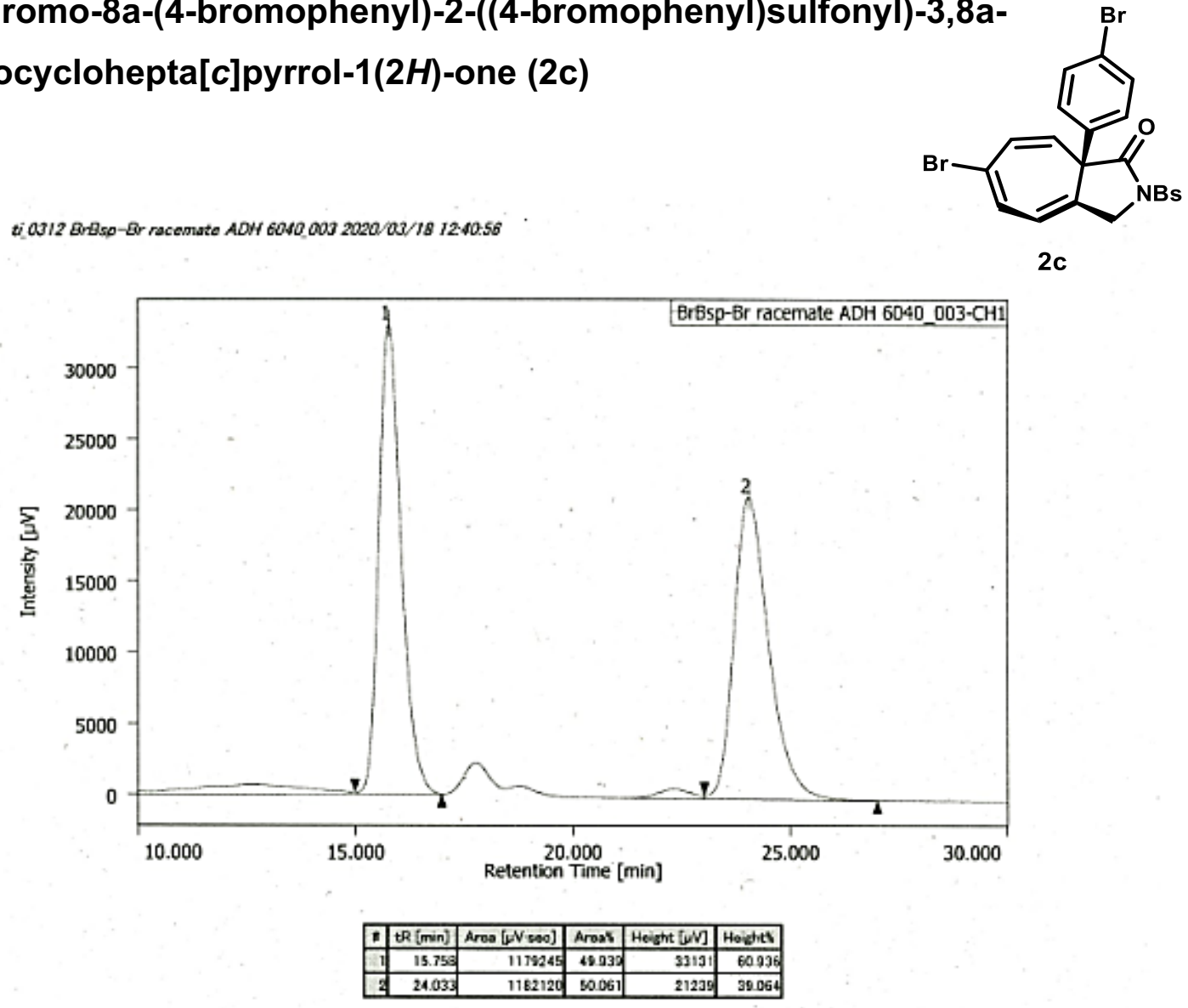

ti O318 Br Bs p-Br buchner racemote ADH 6040 001 2020/03/18 12.41.03

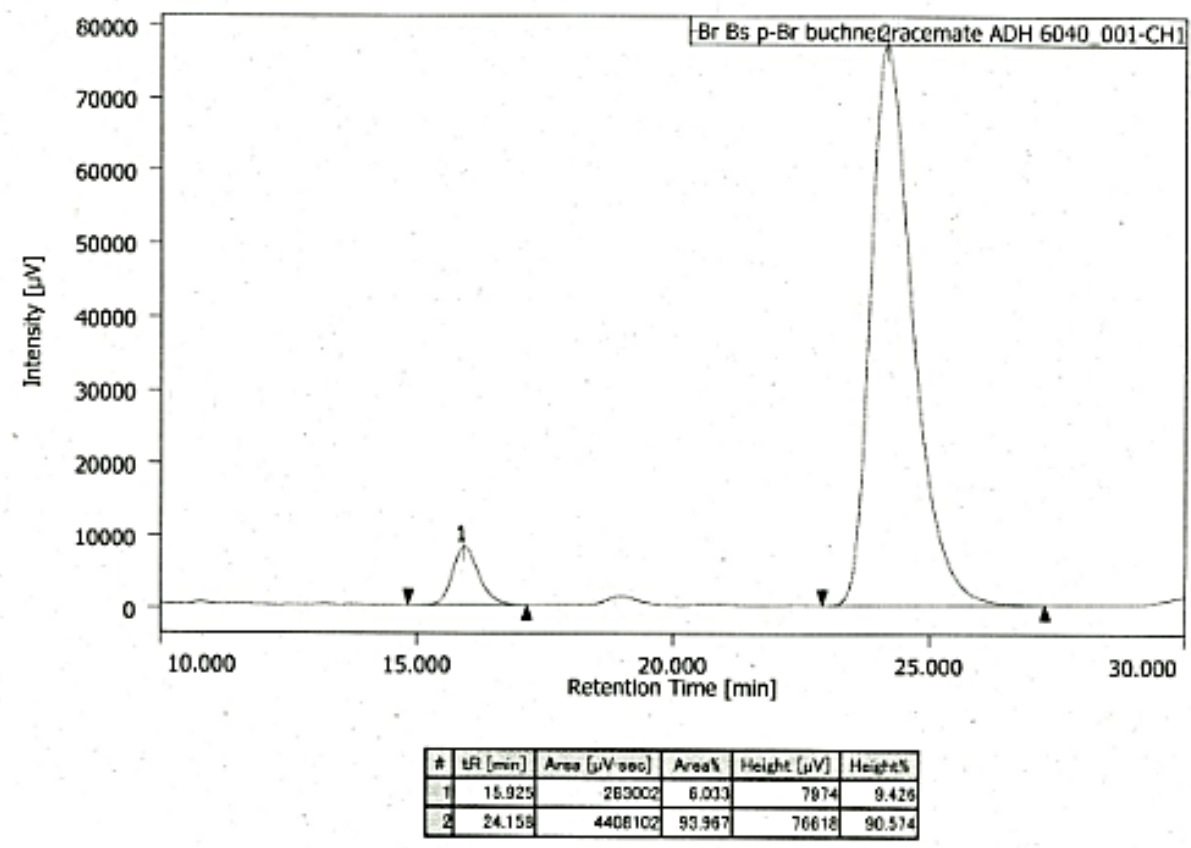


(R)-6-Bromo-2-((4-bromophenyl)sulfonyl)-8a-(3-methoxyphenyl)-3,8adihydrocyclohepta[c]pyrrol-1(2H)-one (2d)

0915 ht-8-14,001 2020/09/15 17:10.55

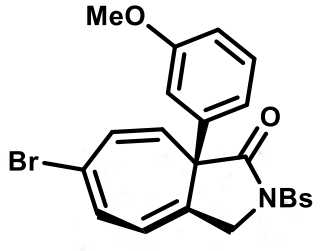

2d

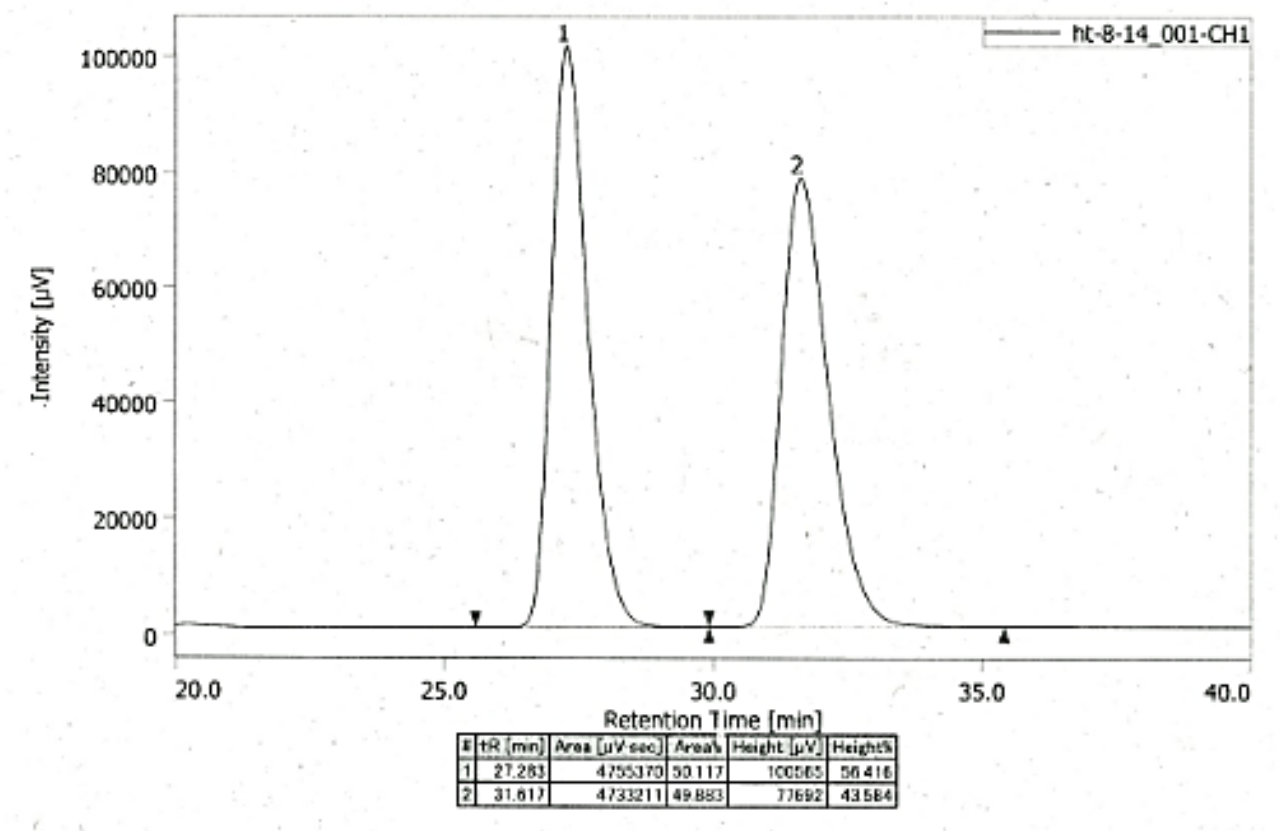

0915 ht-8-16002 2020/09/15 17:10:41

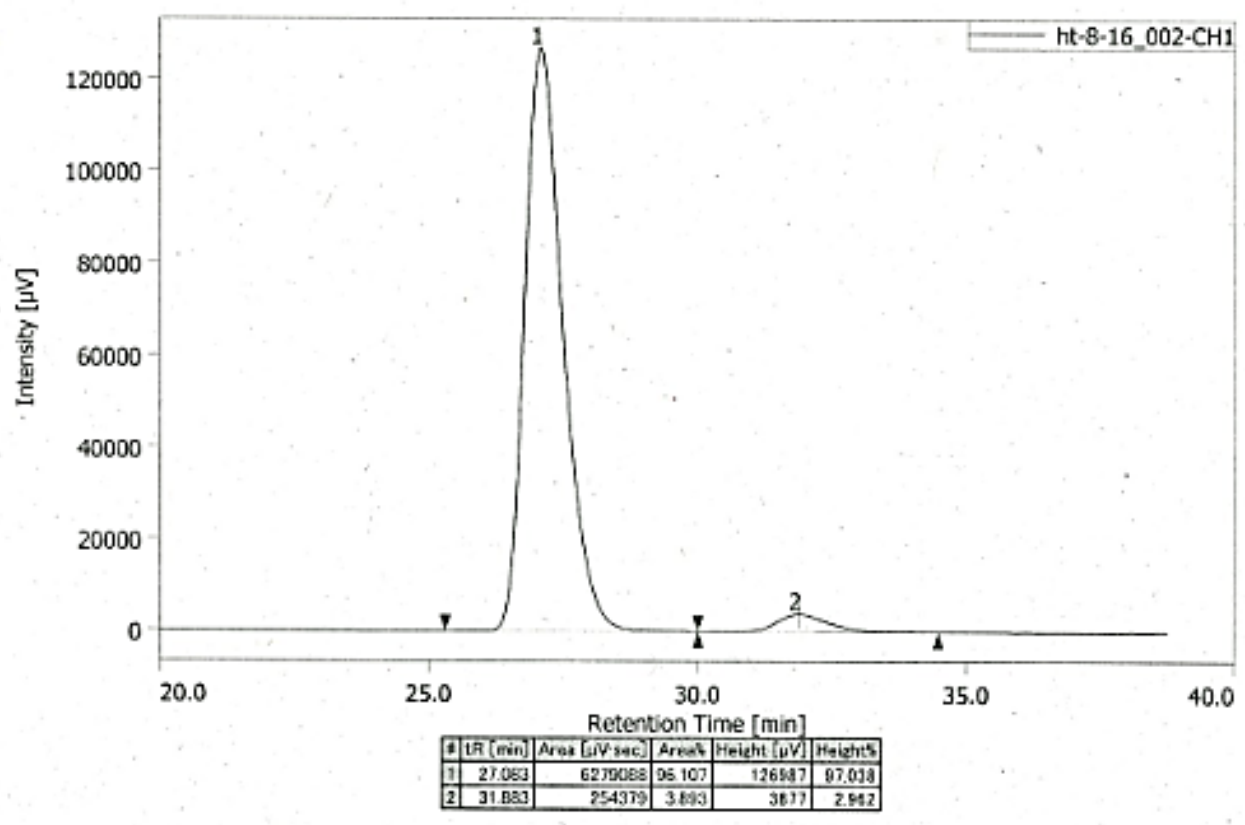


(R)-6-Bromo-8a-(3-methoxyphenyl)-2-tosyl-3,8a-dihydrocyclohepta[c]pyr-rol$1(2 H)$-one (2e)

tj-0127 3-OMe buchner racemate ox3 6010 003 2020 0I/29 22:31:19
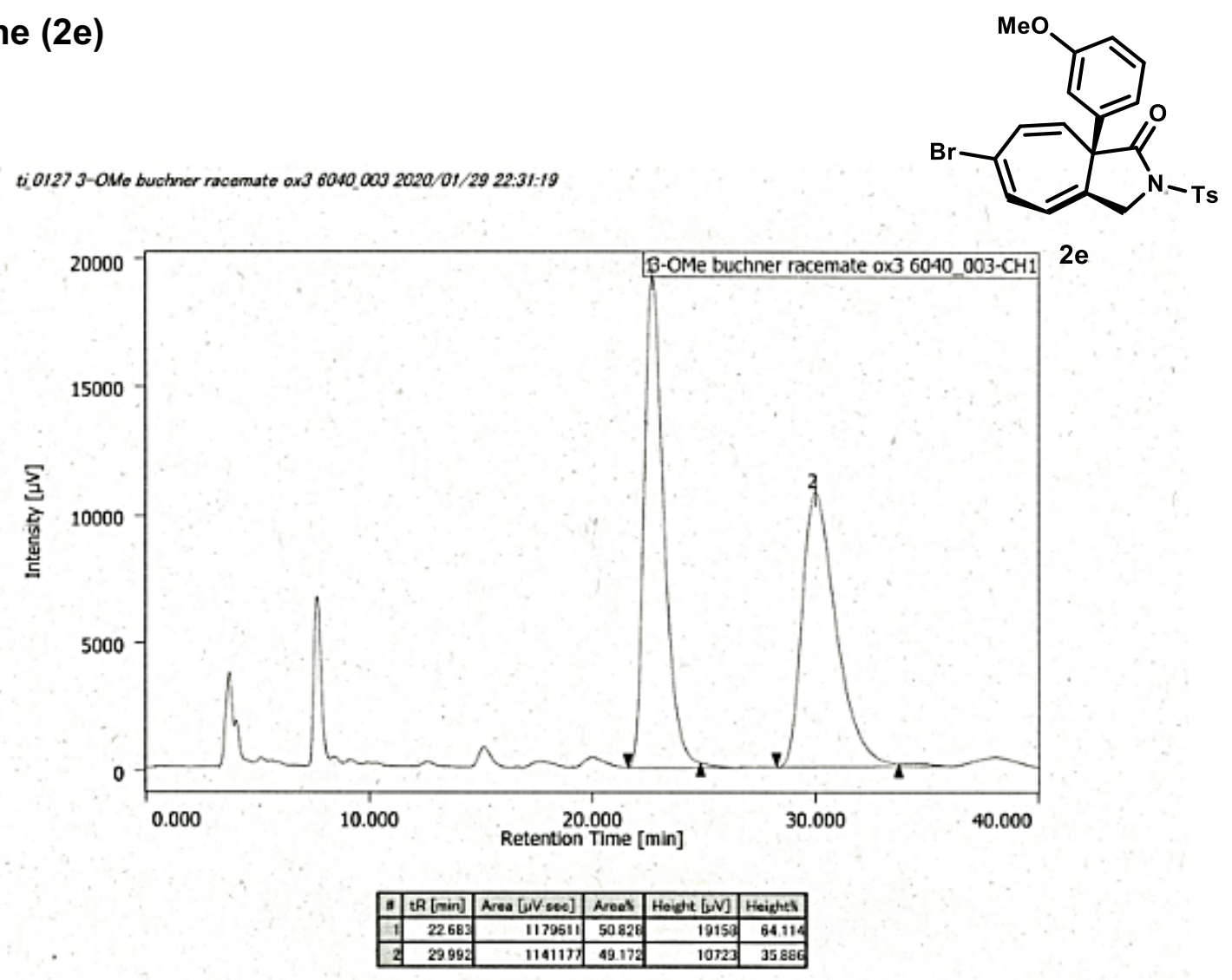

$2 e$

ti.0I29 3-OMe buchner racemate ox36000,006 2020/01/29 22.31:24

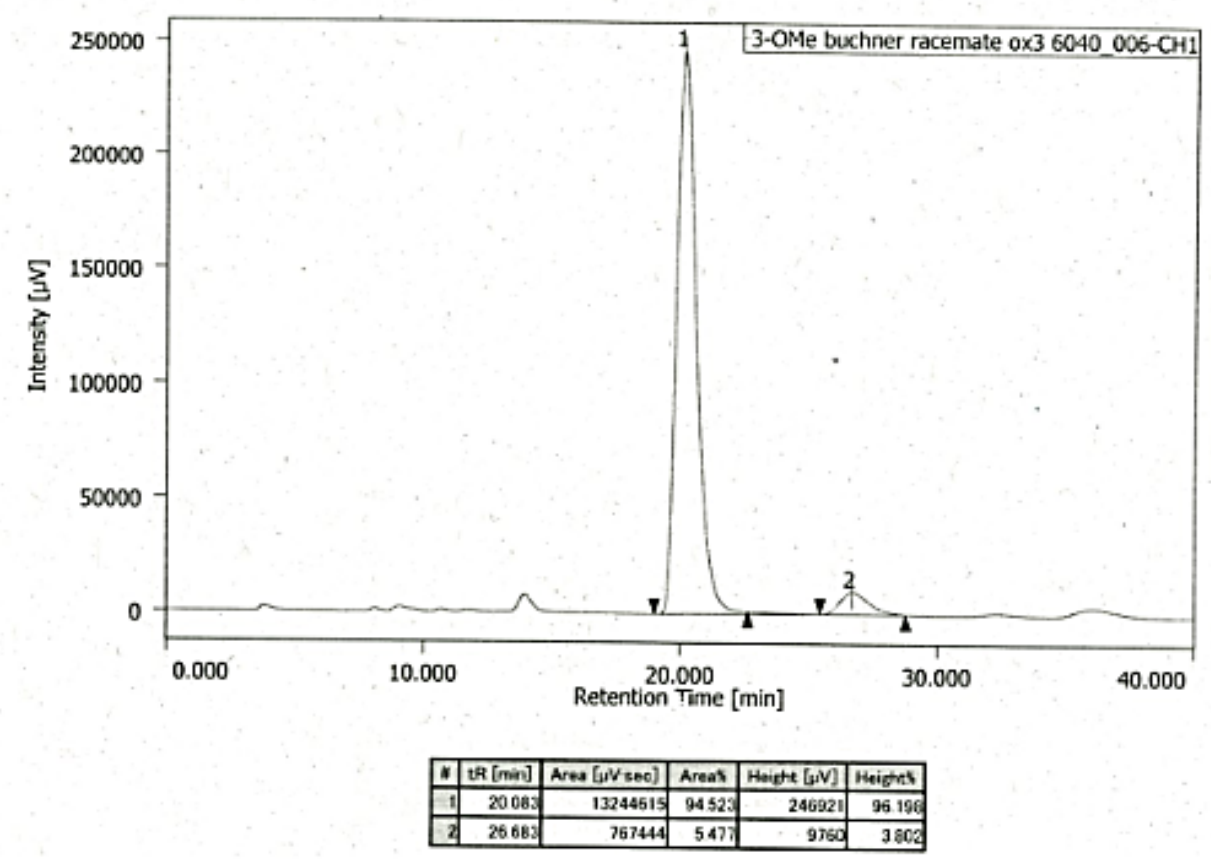


(S)-6-Bromo-2-((4-bromophenyl)sulfonyl)-8a-(2-fluorophenyl)-3,8adihydrocyclohepta[c]pyrrol-1(2H)-one (2f)
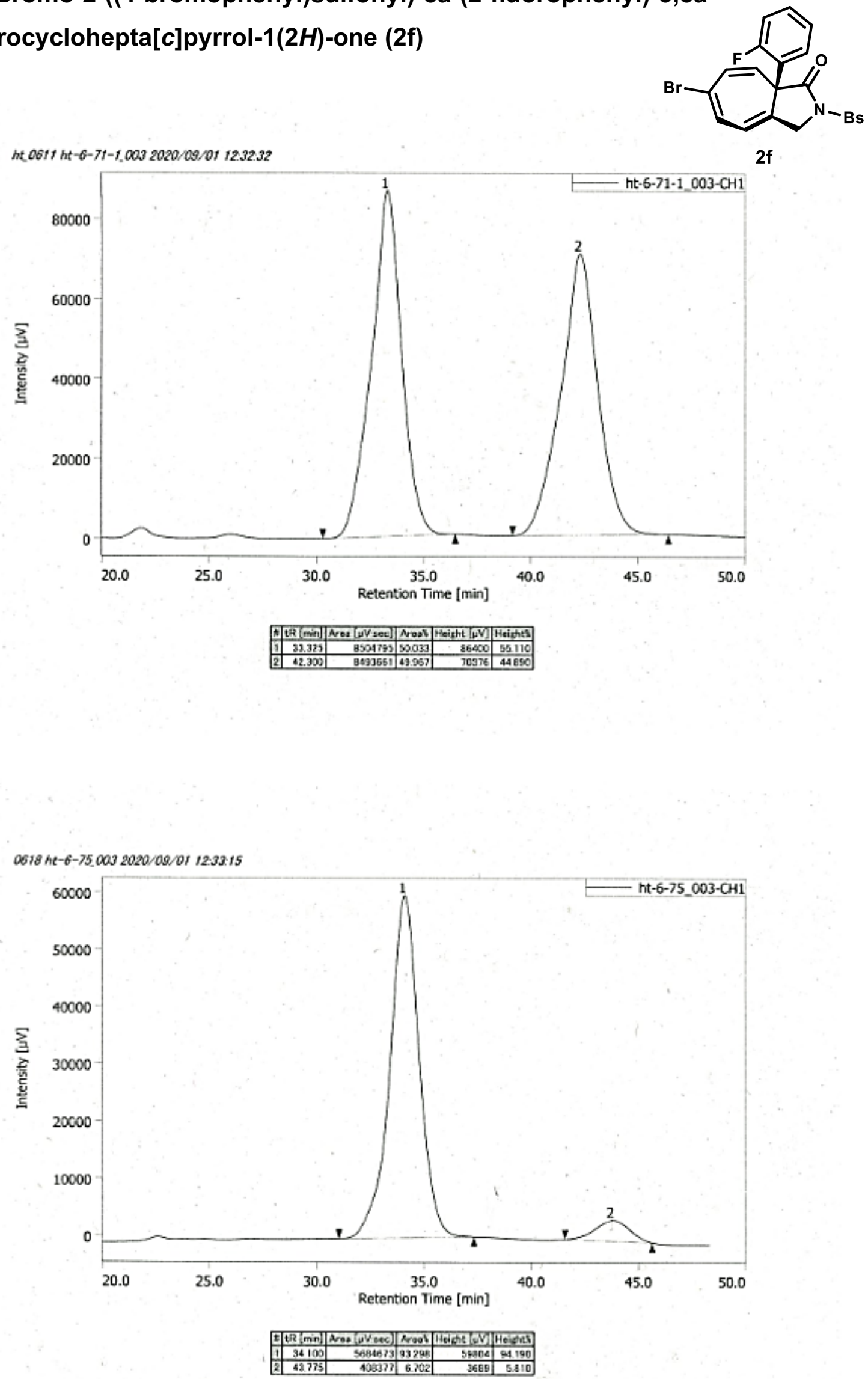
(R)-6-Bromo-8a-(3-bromophenyl)-2-tosyl-3,8a-dihydrocyclohepta[c]pyrrol1(2H)-one (2g)

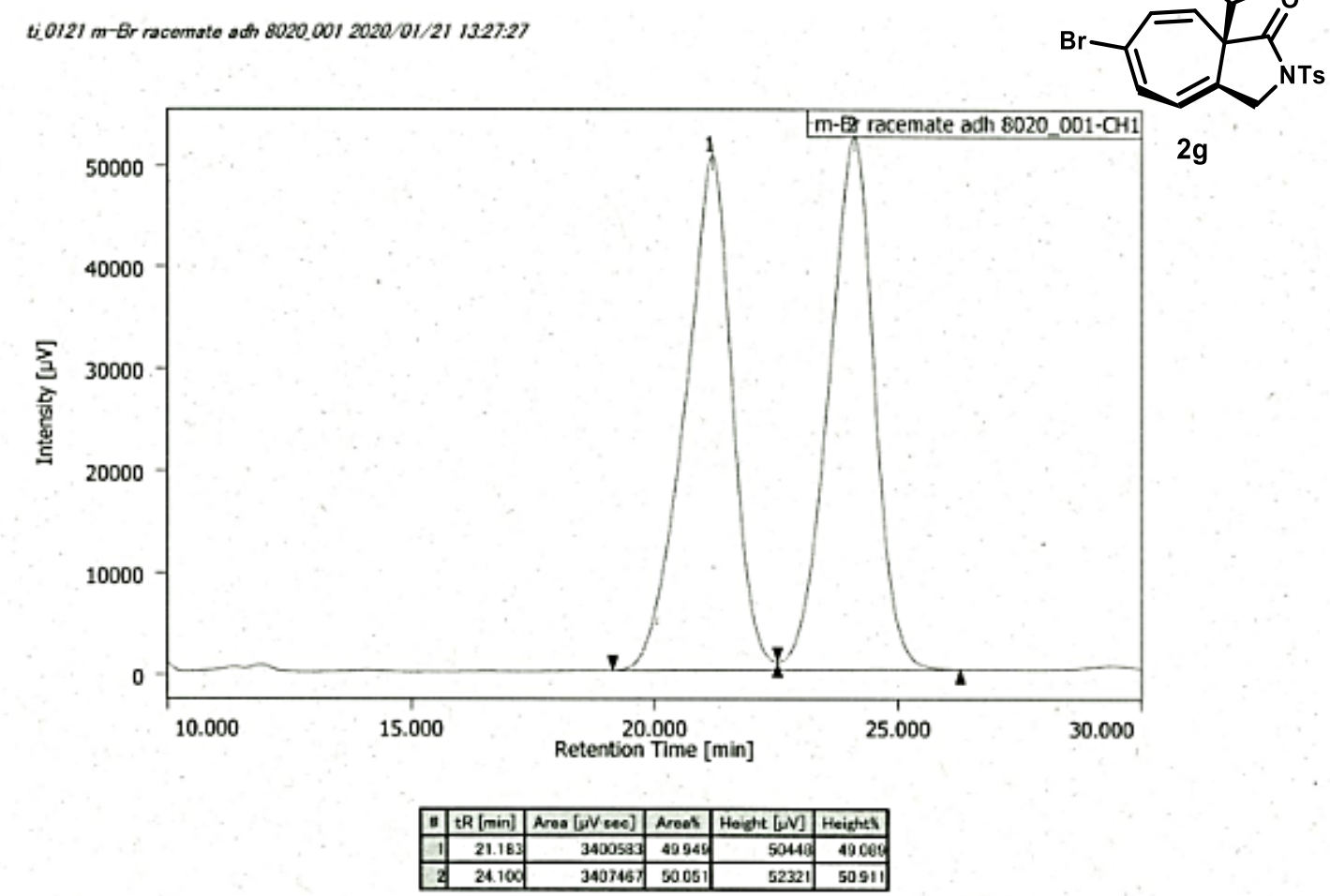

ti.0120 p-Br buchnor asym m-Br 8020 ADH 0032020 OI/21 13228 10

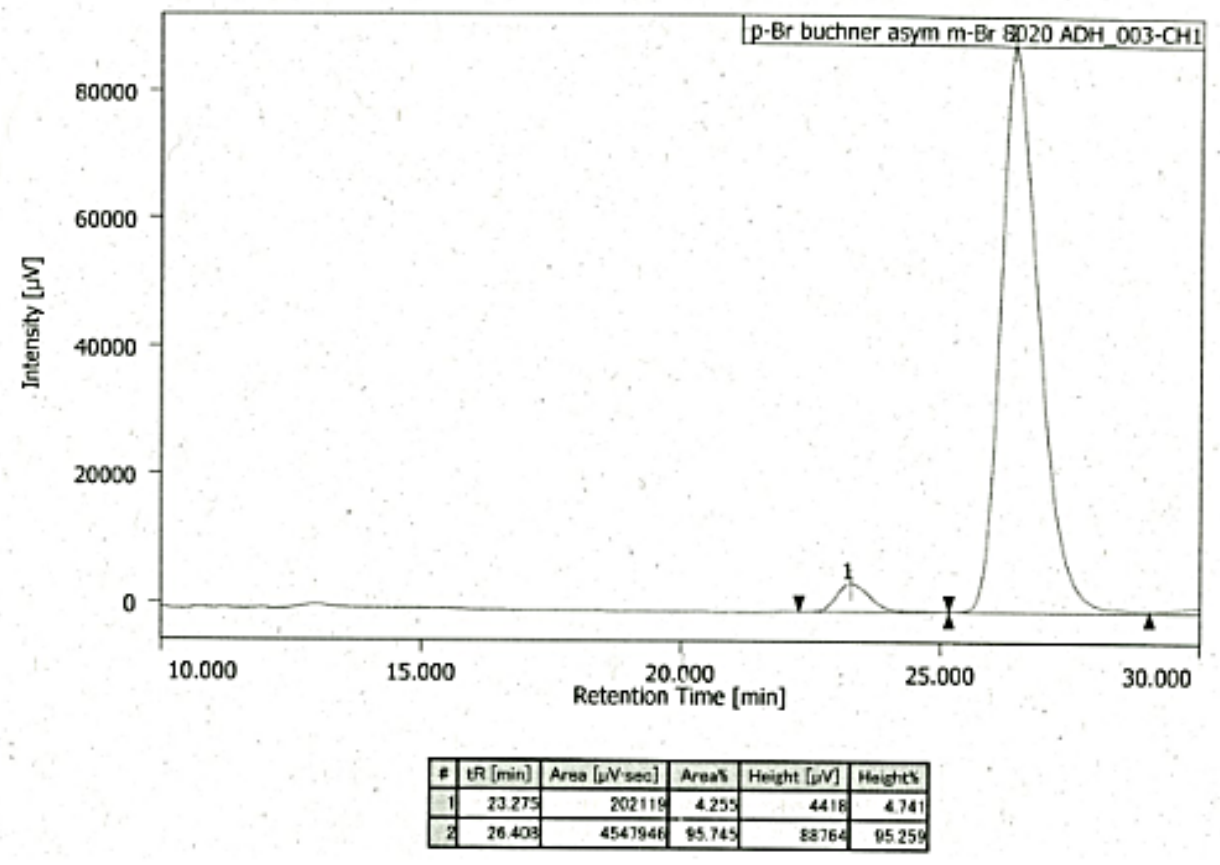


(R)-6-Bromo-8a-phenyl-2-tosyl-3,8a-dihydrocyclohepta[c]pyrrol-1(2H)-one (2h)

ti.0131 Bi H racemote propara ADH 8020 00/ 2020/01/31 1829.57
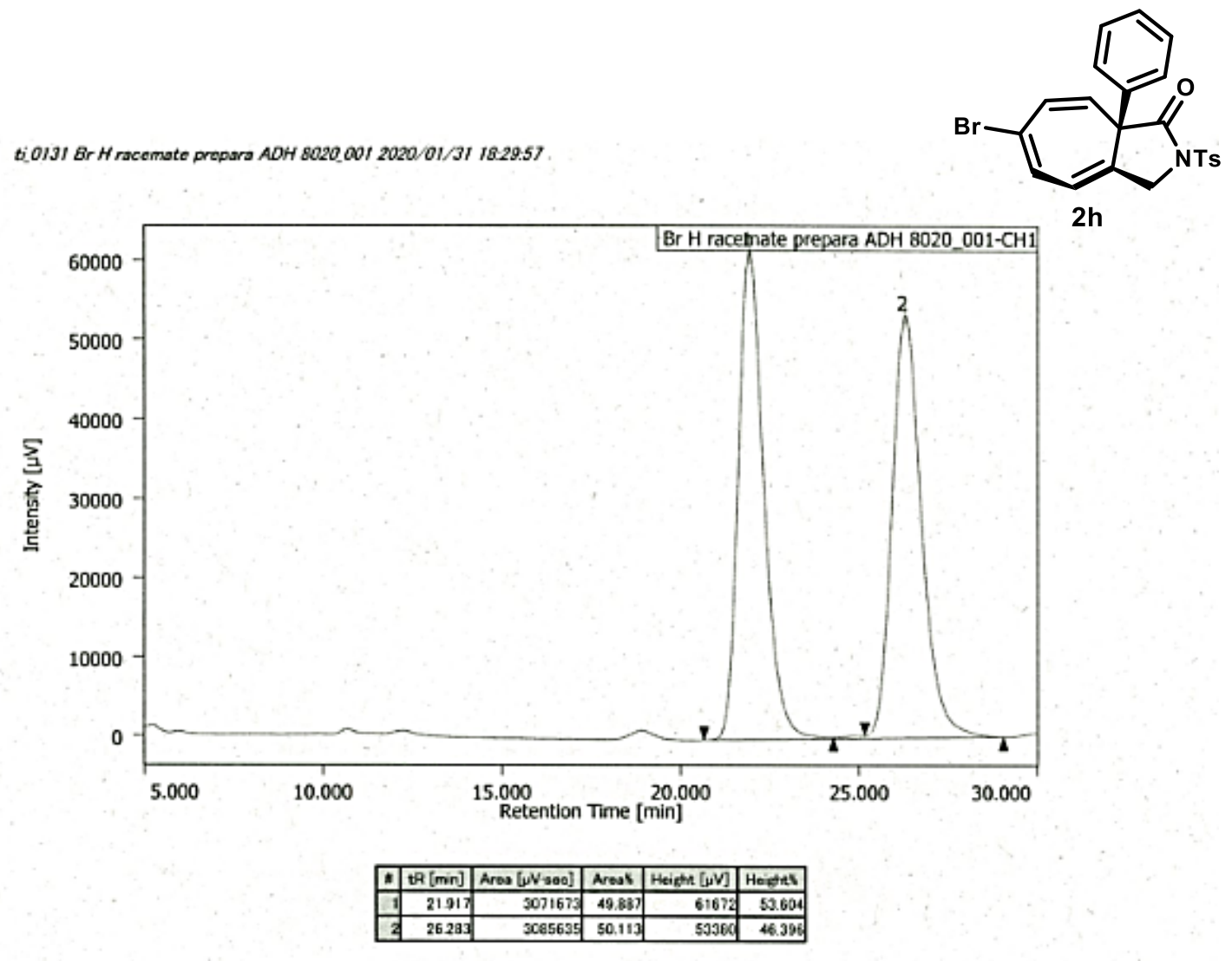

$2 \mathrm{~h}$

ti $0110 \mathrm{TI} 1360 \mathrm{Hasym}$ ach 3020 001 2020/01/31 18.3006

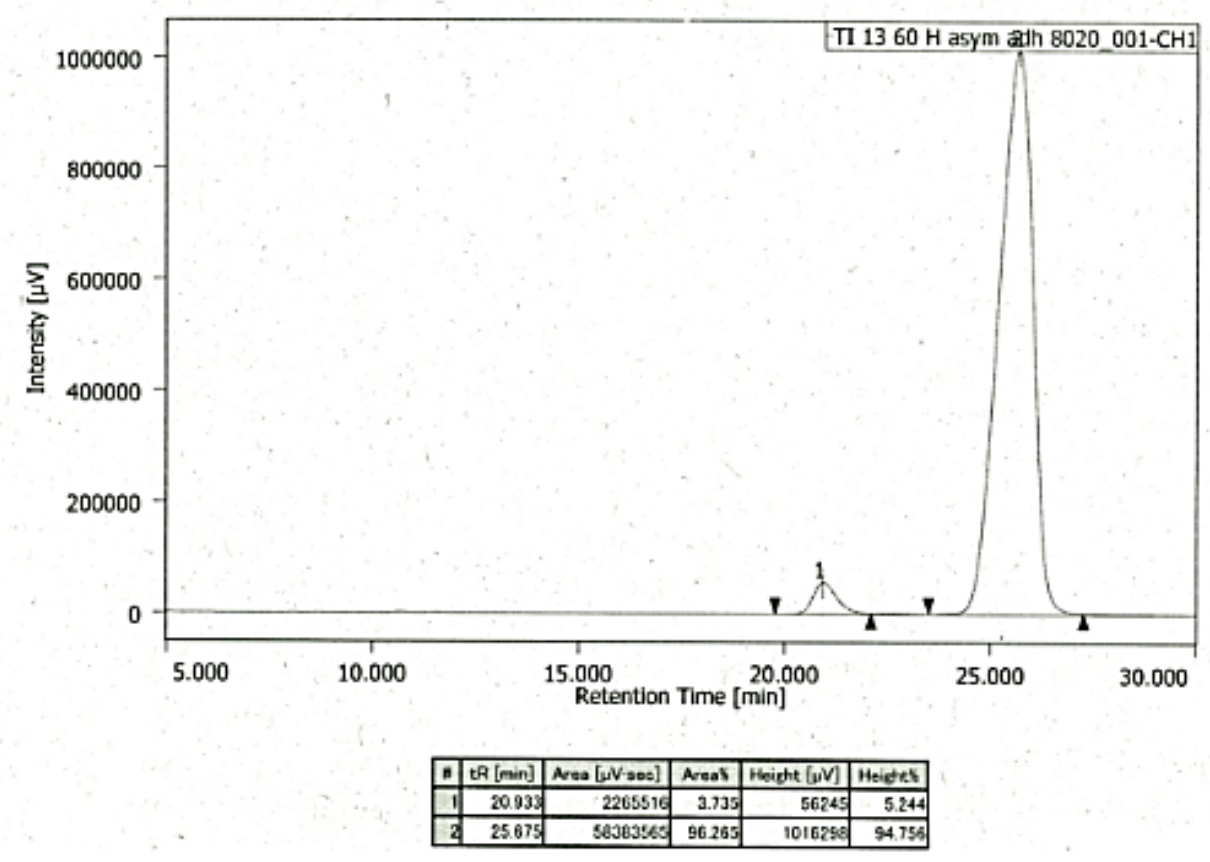


(R)-6-Bromo-2-((4-methoxyphenyl)sulfonyl)-8a-phenyl-

3,8a-dihydrocyclohepta[c]pyrrol-1(2H)-one (2i)

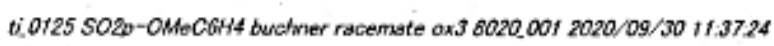
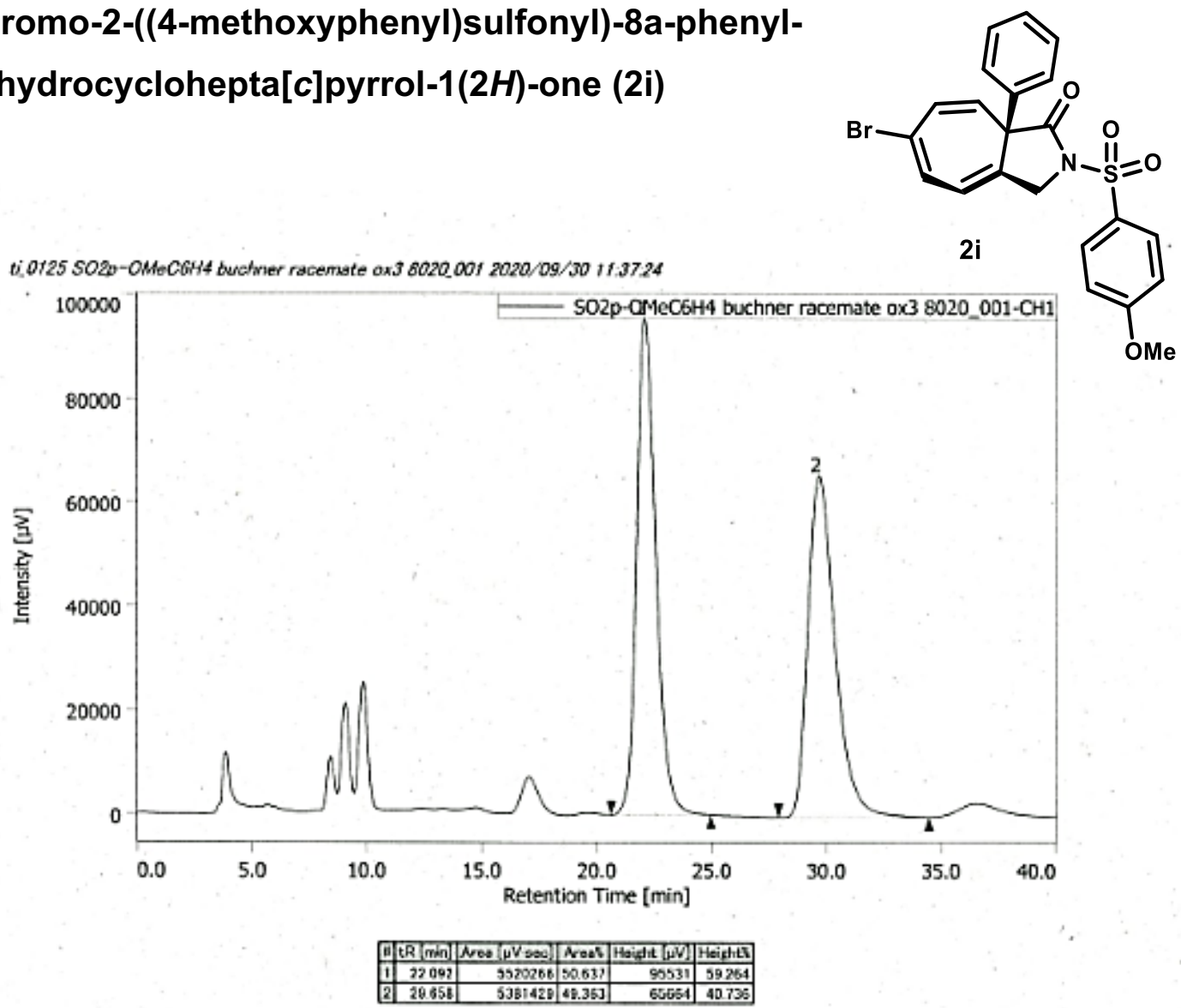

OMe

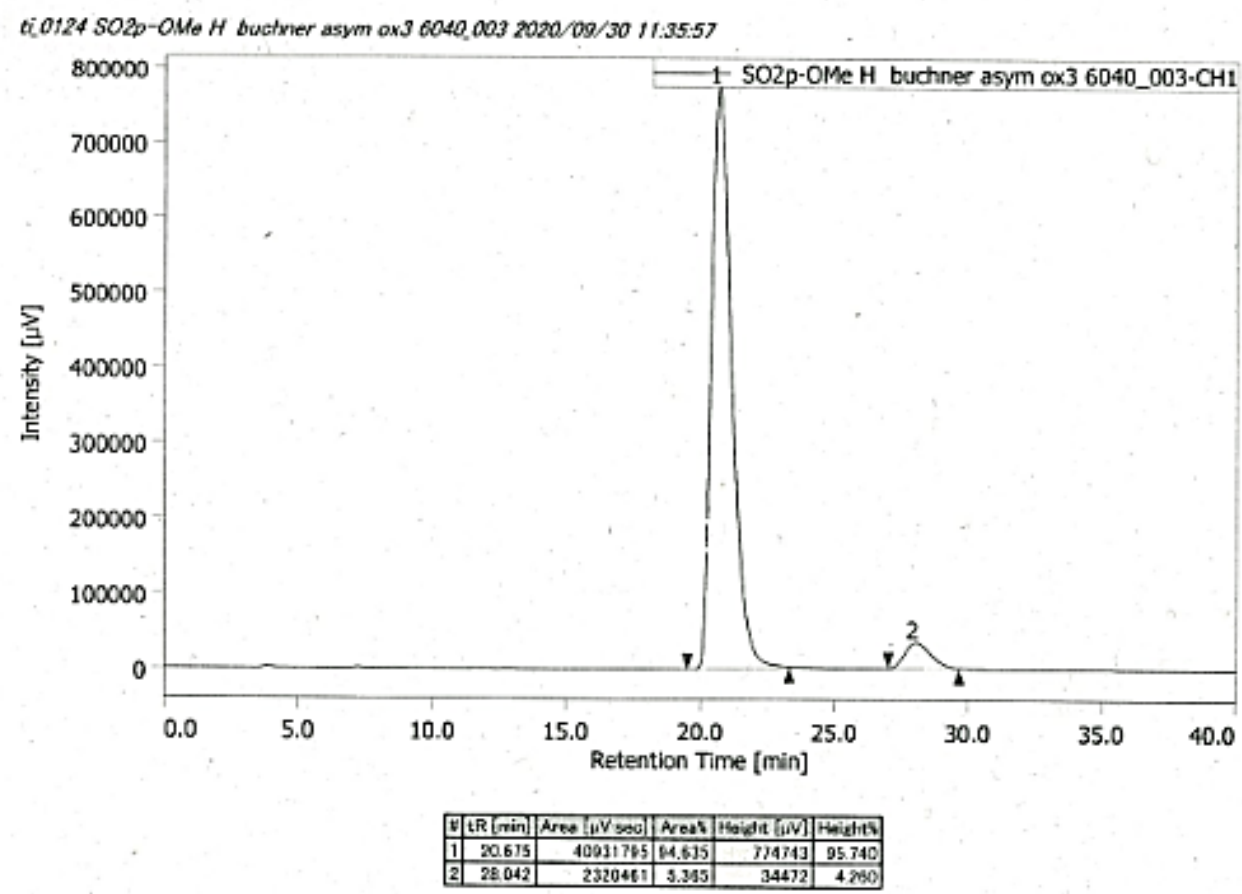


(R)-2-((4-Bromophenyl)sulfonyl)-8a-phenyl-3,8a-dihydrocyclohepta[c]pyrrol$1(2 H)$-one (2j)
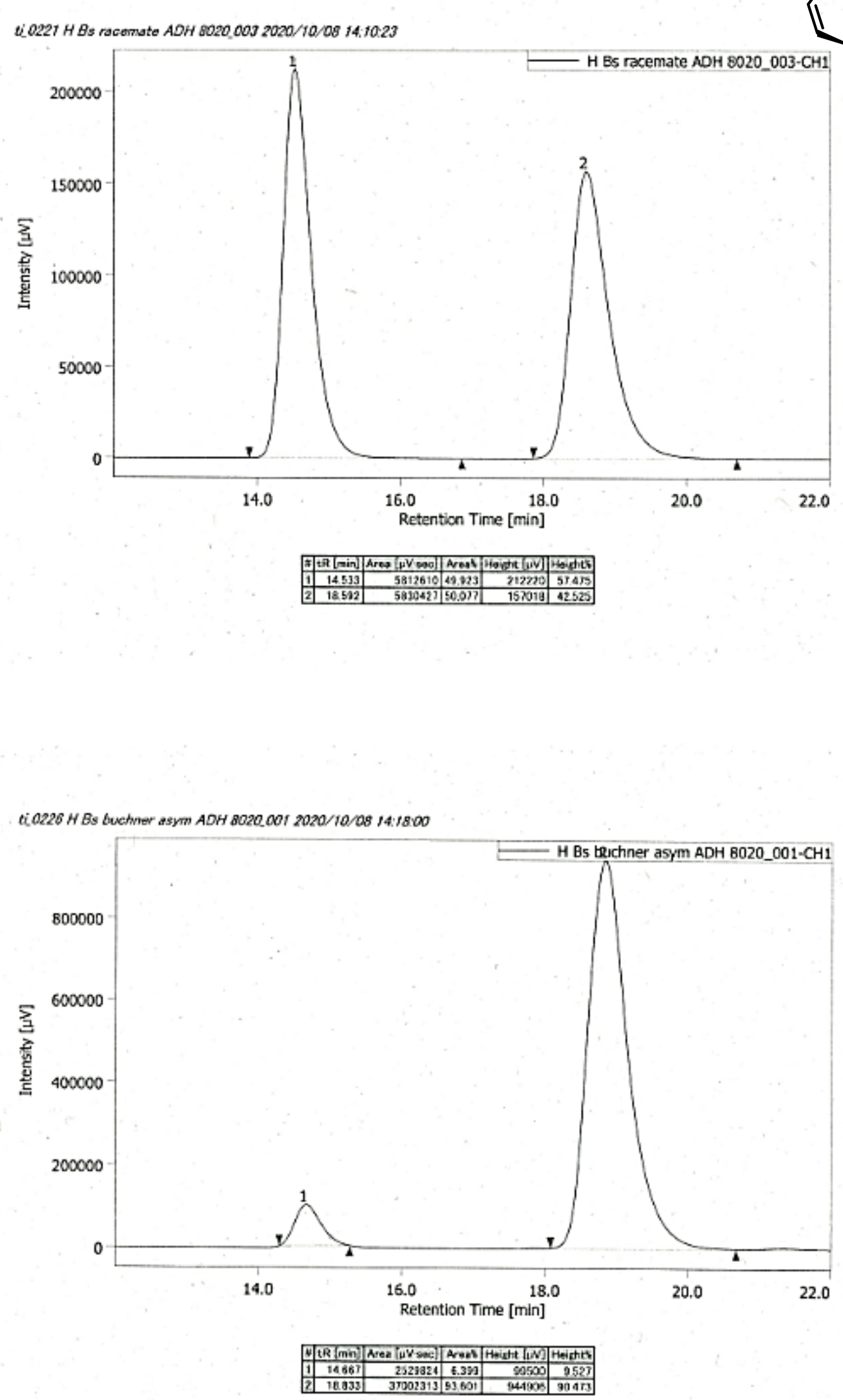


\section{(R)-2-((4-Bromophenyl)sulfonyl)-6-iodo-8a-phenyl-3,8a-}

dihydrocyclohepta[c]pyrrol-1(2H)-one (2k)

ti 0221 4-IH racemate ADH 8020,002 2020/03/78 15:1703
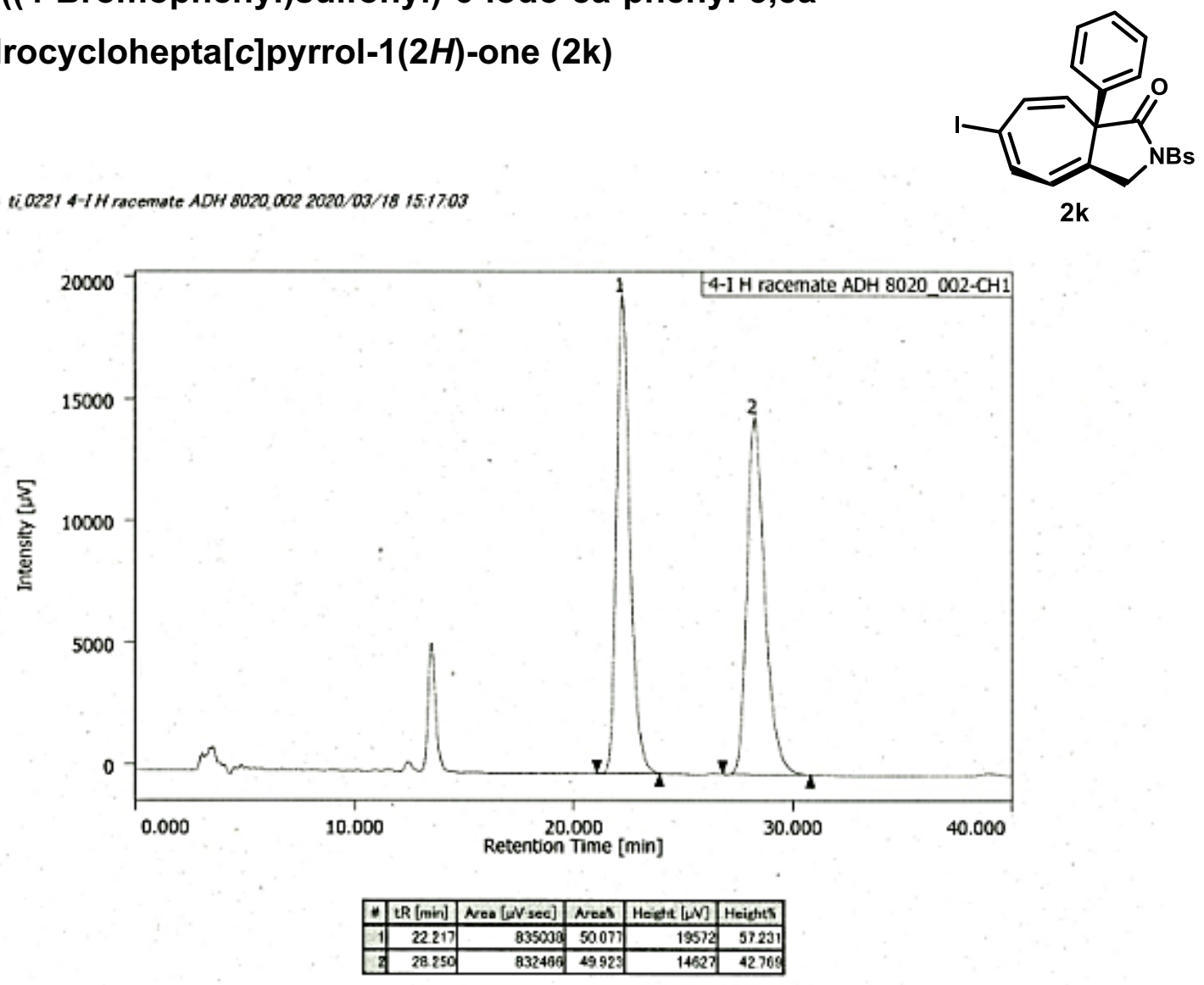

t.0226 p-18s buchner asym ADH 8020 002 2020/03/18 I5:16:56

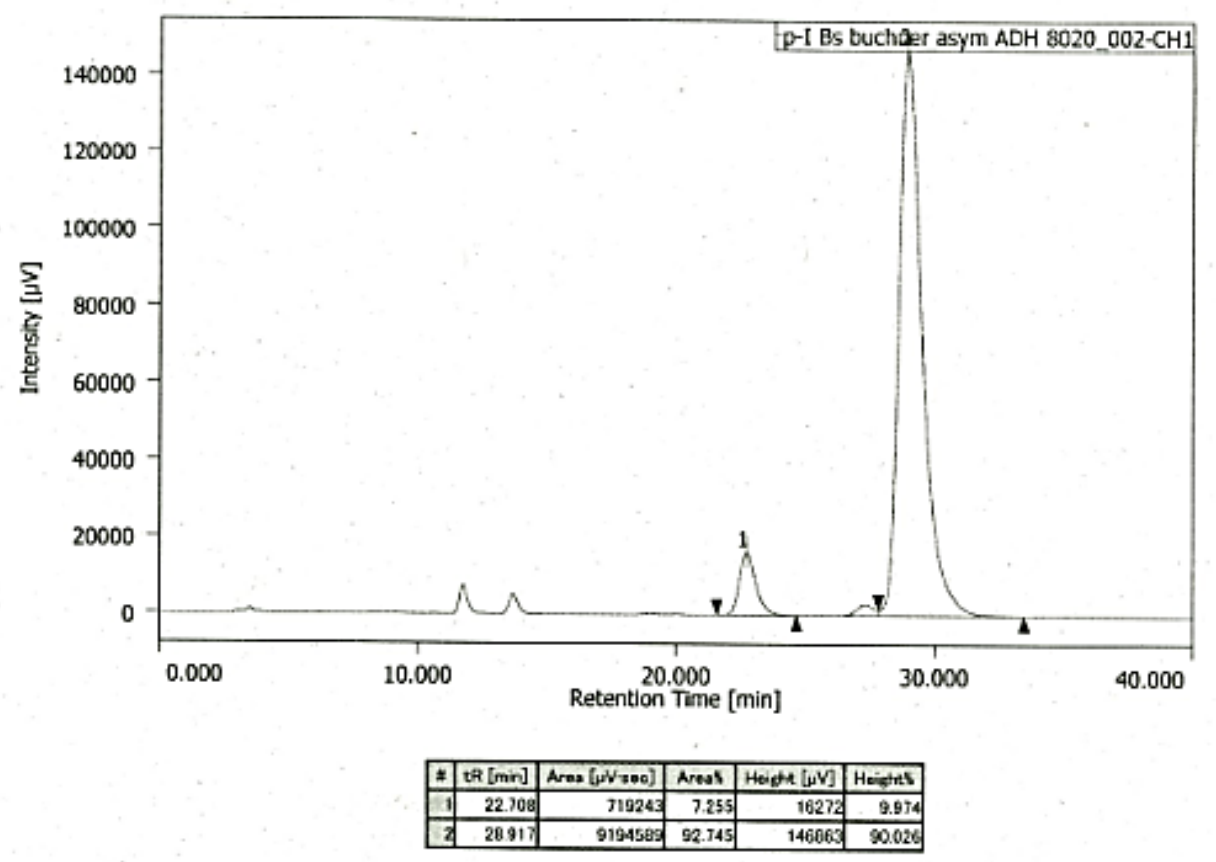


(R)-2-((4-Bromophenyl)sulfonyl)-6-fluoro-8a-phenyl-3,8adihydrocyclohepta[c]pyrrol-1(2H)-one (2I)

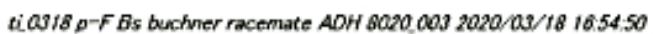

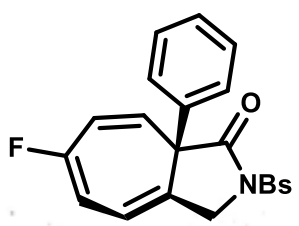

2I

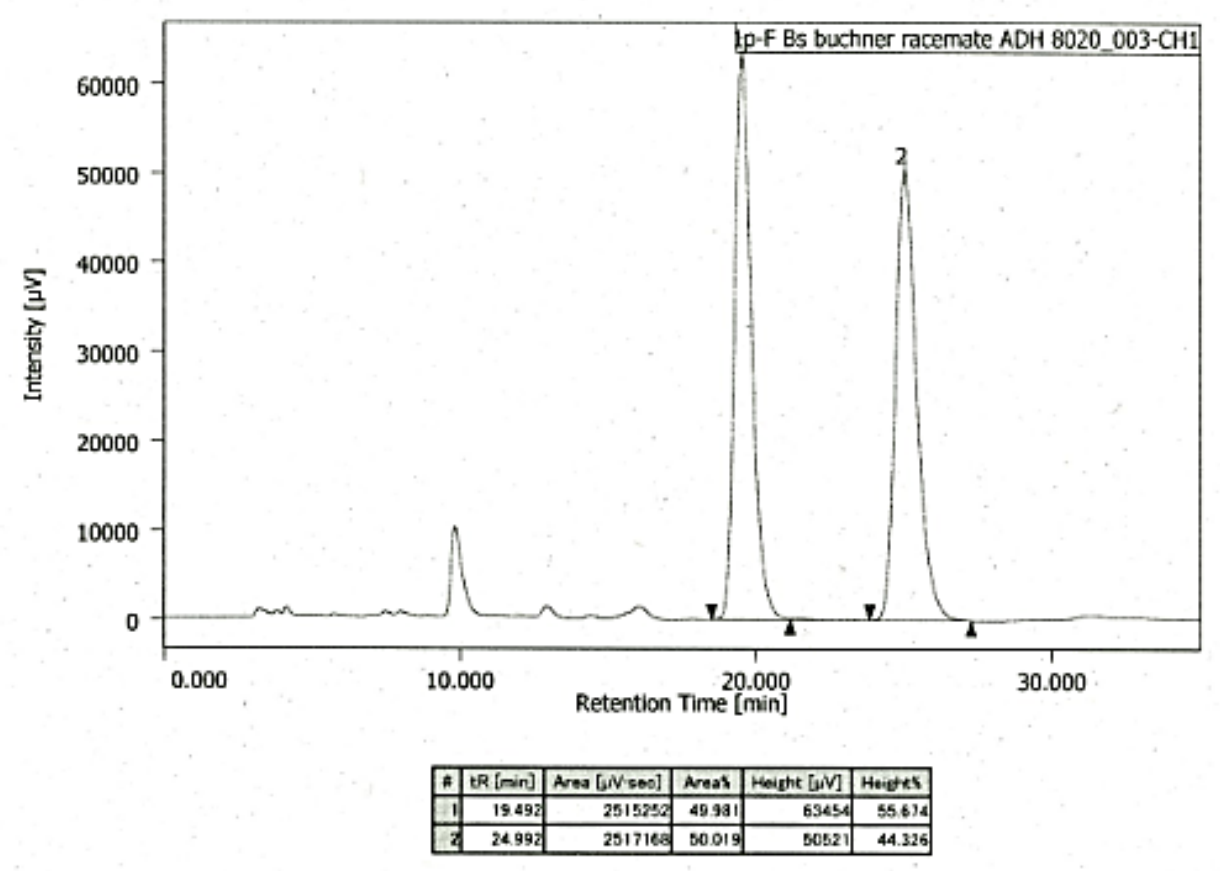

ti asis p-F Bs buchner asym AOH S020,004 2020/03/18 16.54.43

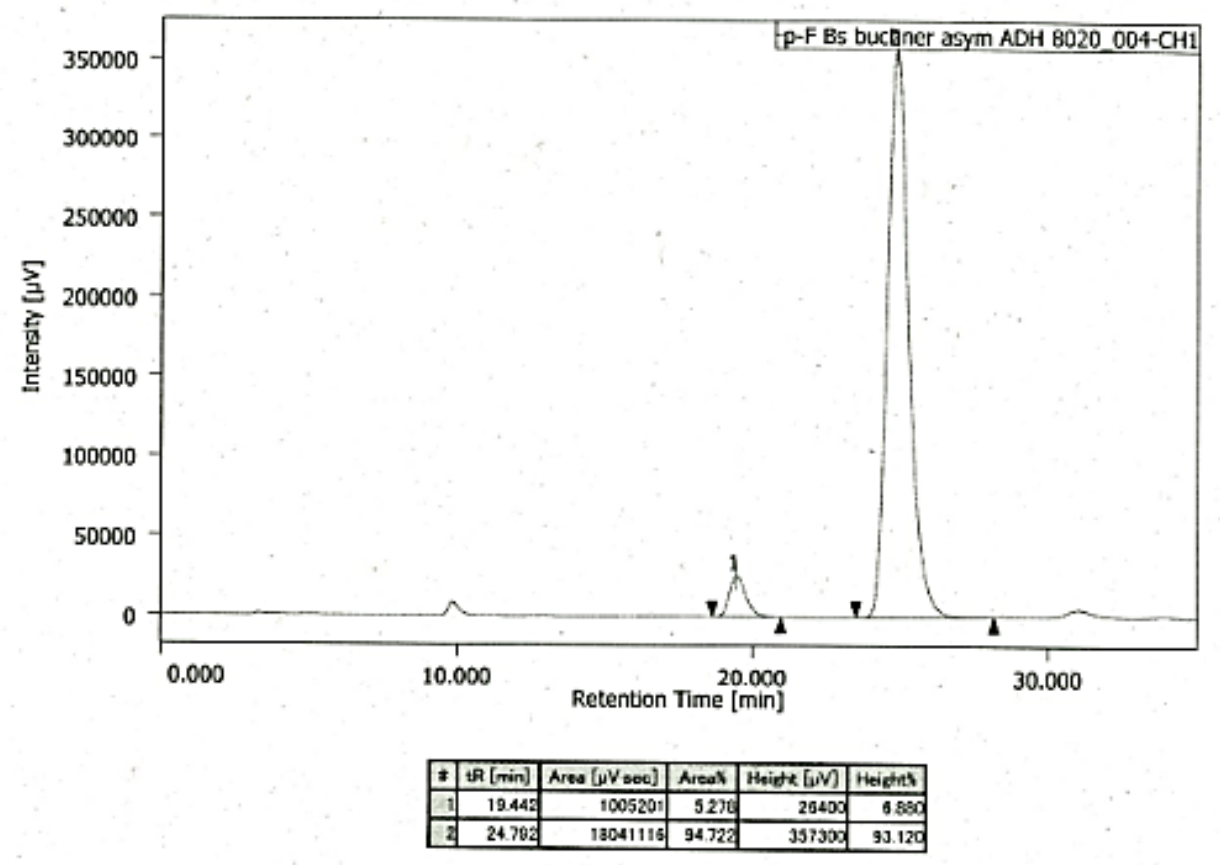


(R)-6-Chloro-8a-phenyl-2-tosyl-3,8a-dihydrocyclohepta[c]pyrrol-1(2H)-on (2m)

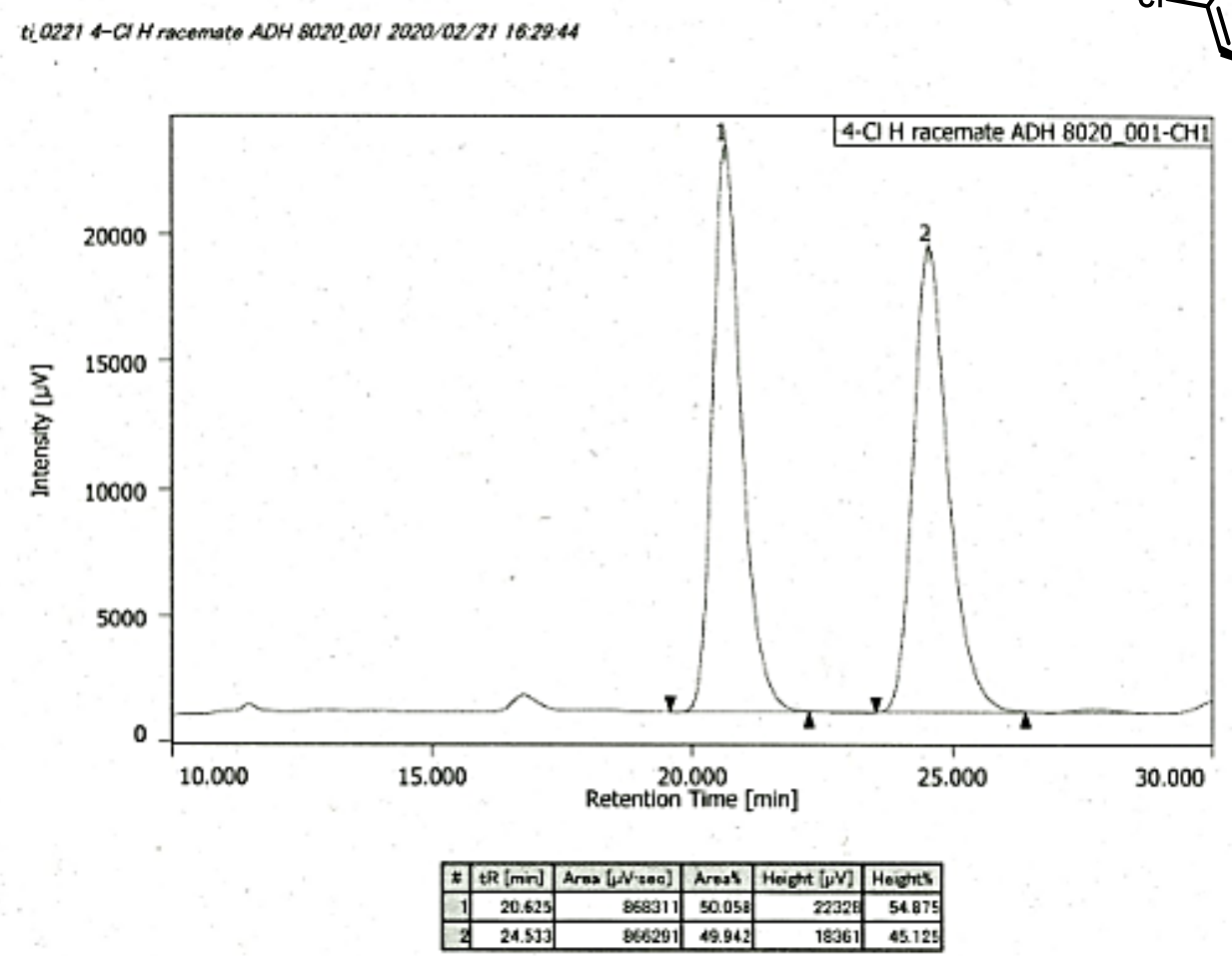

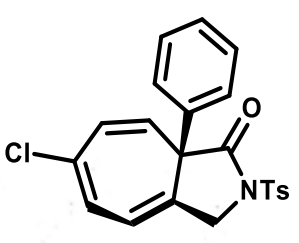

$2 m$

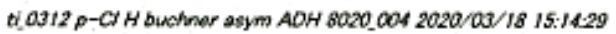

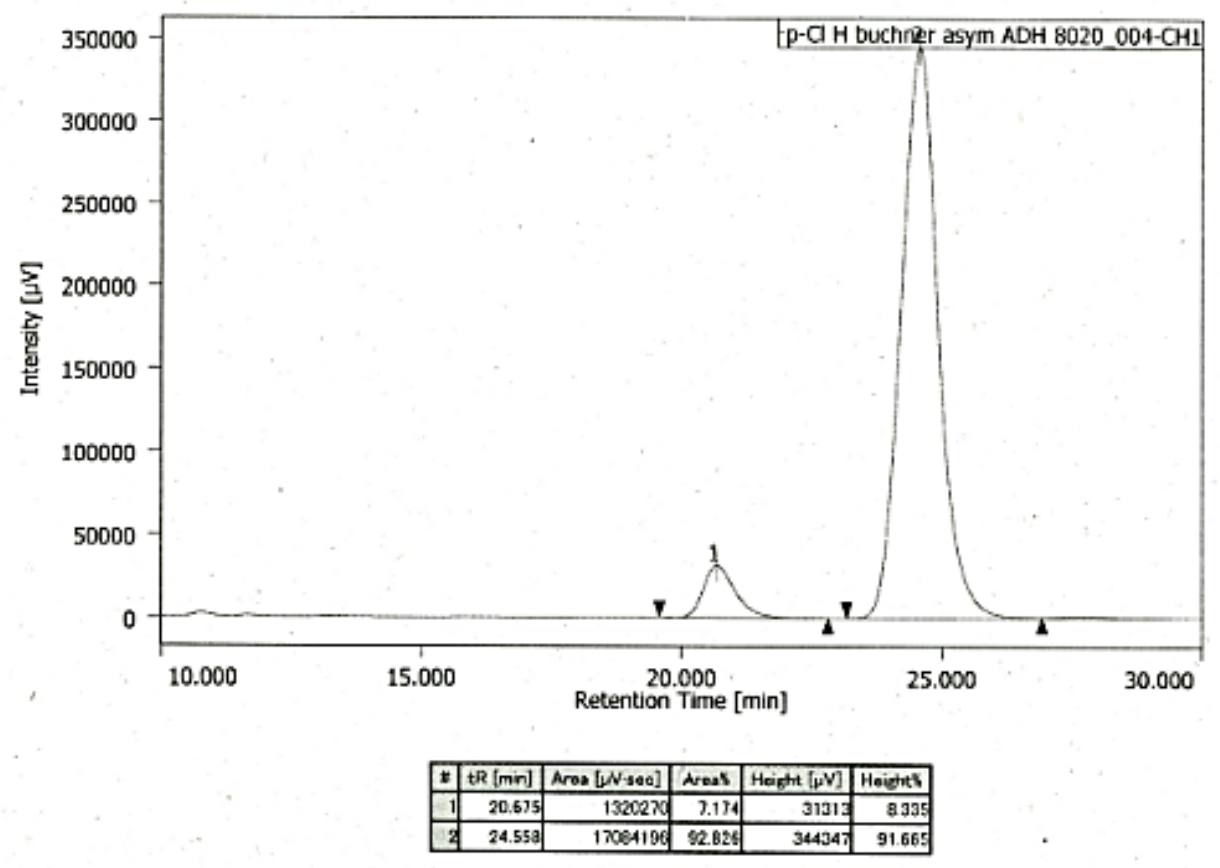


(R)-2-((4-Bromophenyl)sulfonyl)-6-methyl-8a-phenyl-3,8adihydrocyclohepta[c]pyrrol-1(2H)-one (2n)

ti. O310 II MeBs buctner racemute AOH 8020 001 2020/03/18 13.42.25
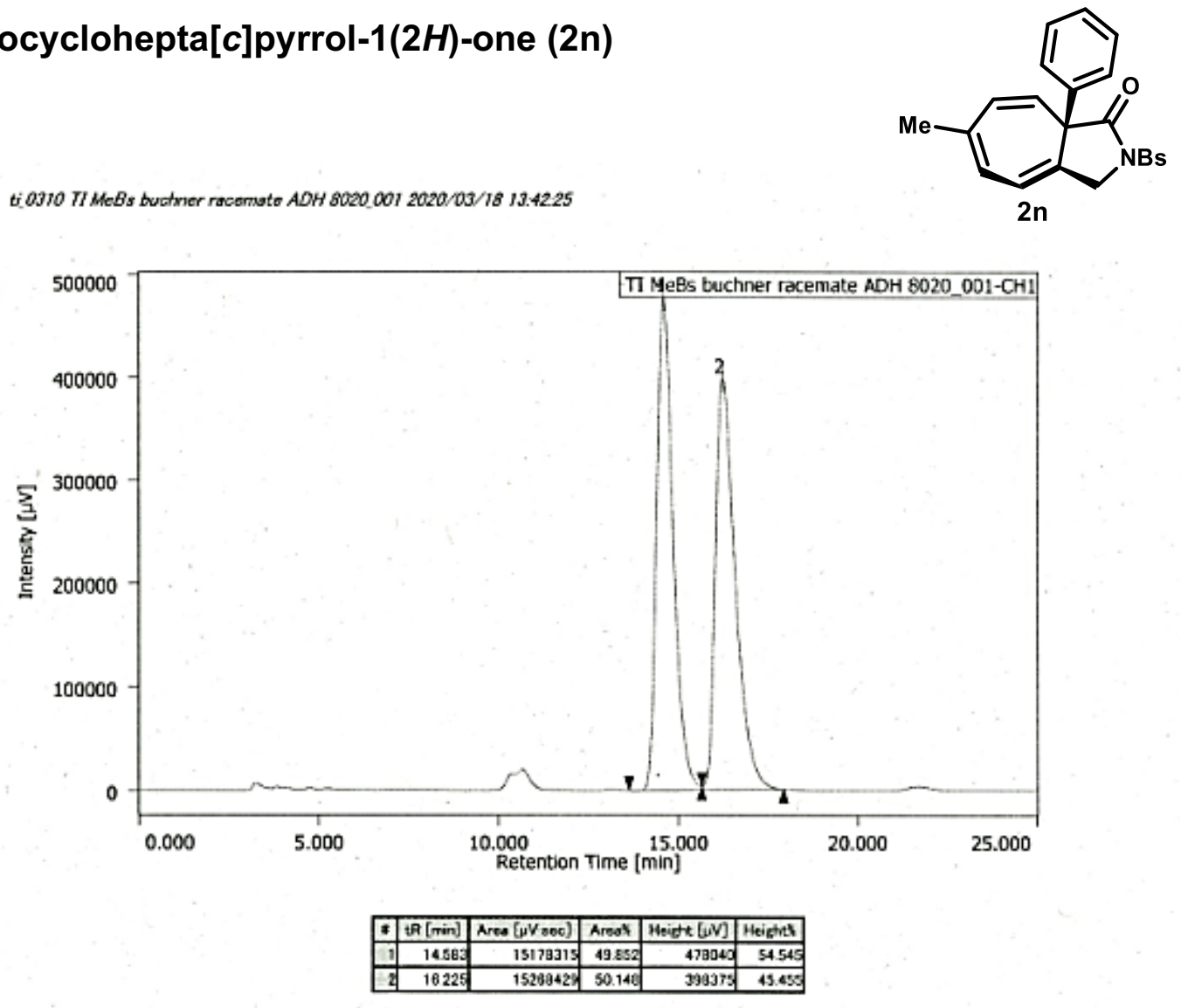

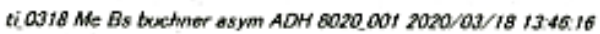

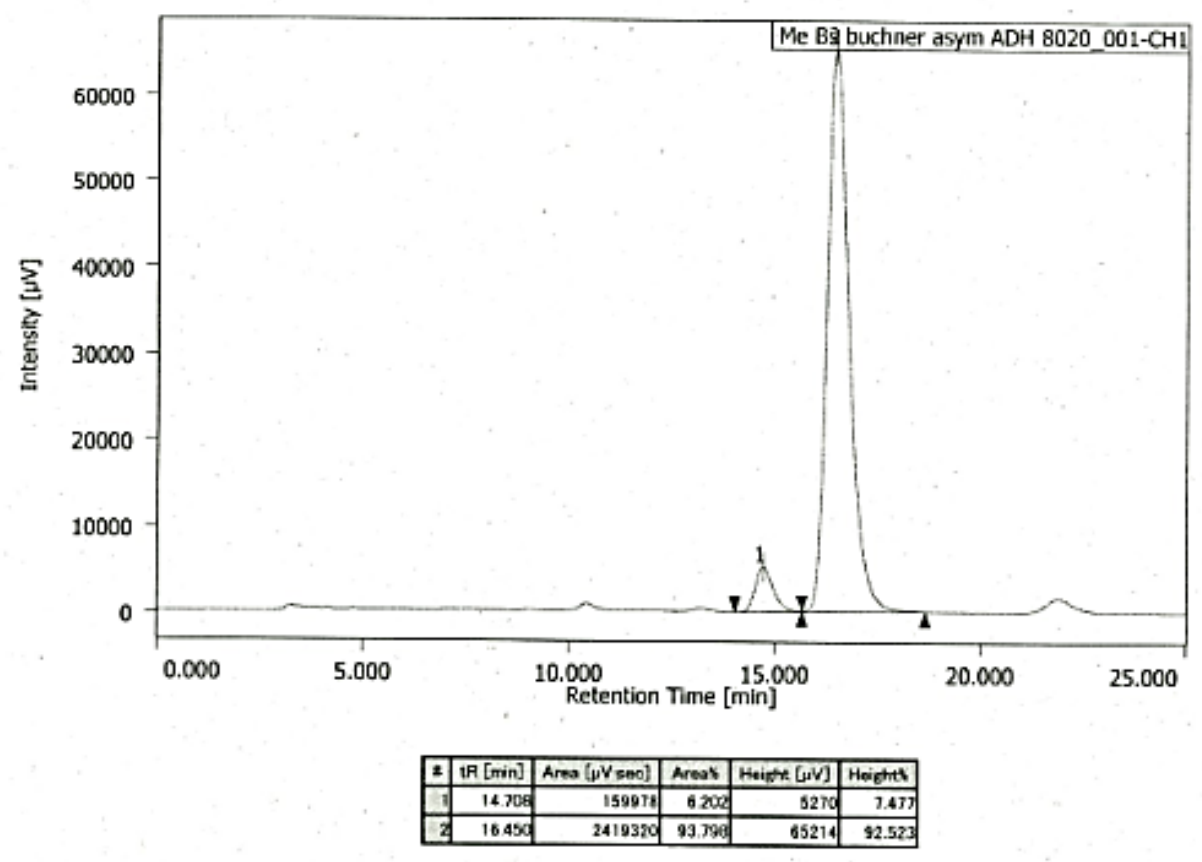


(S)-6-bromo-8a-pentyl-2-tosyl-3,8a-dihydrocyclohepta[c]pyrrol-1(2H)-one (2o)
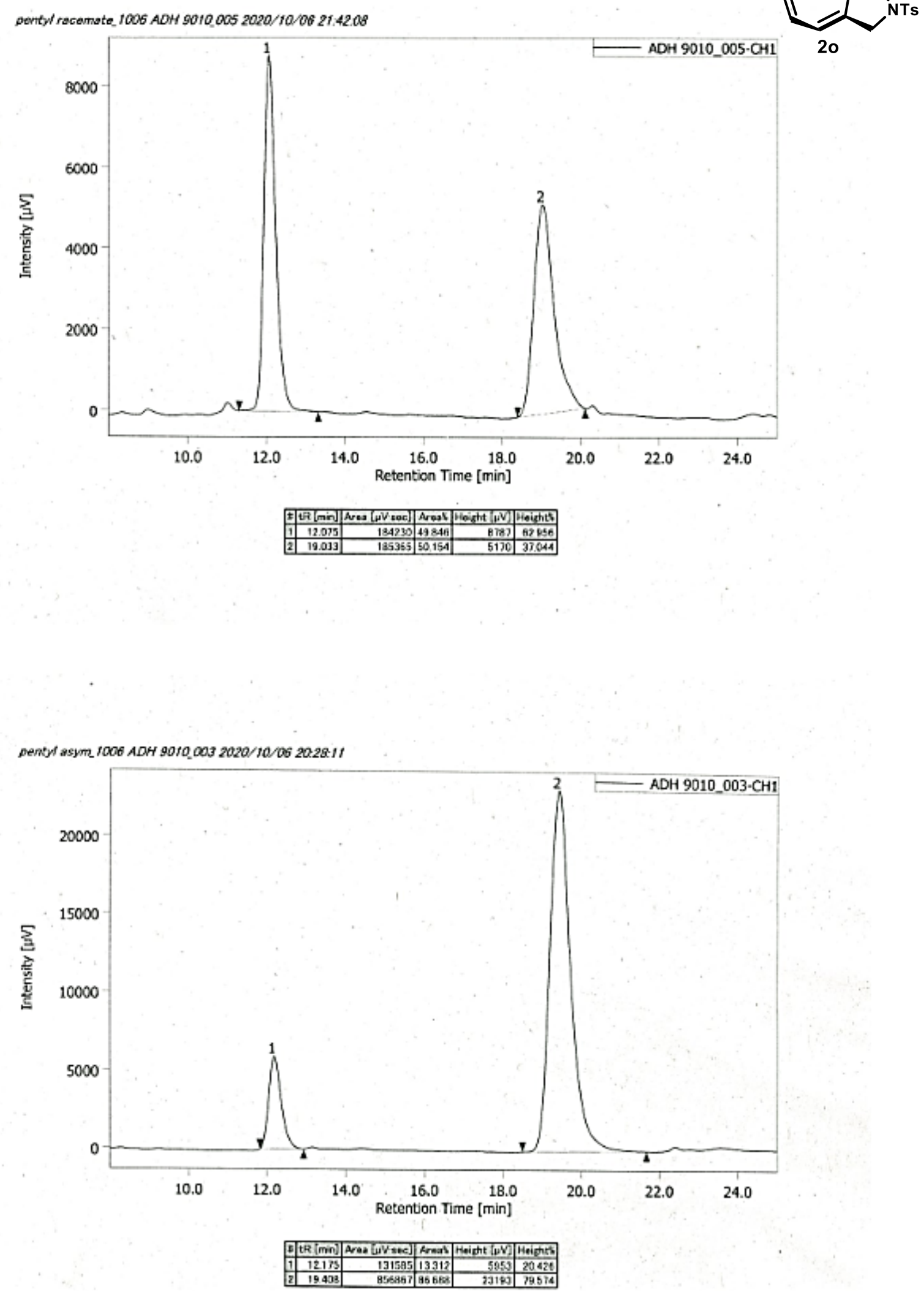
(R)-8a-phenyl-2-tosyl-3,8a-dihydrocyclohepta[c]pyrrol-1(2H)-one (2p)

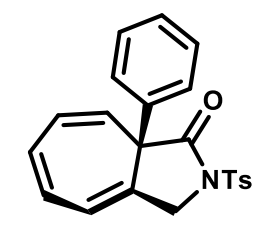

$2 p$
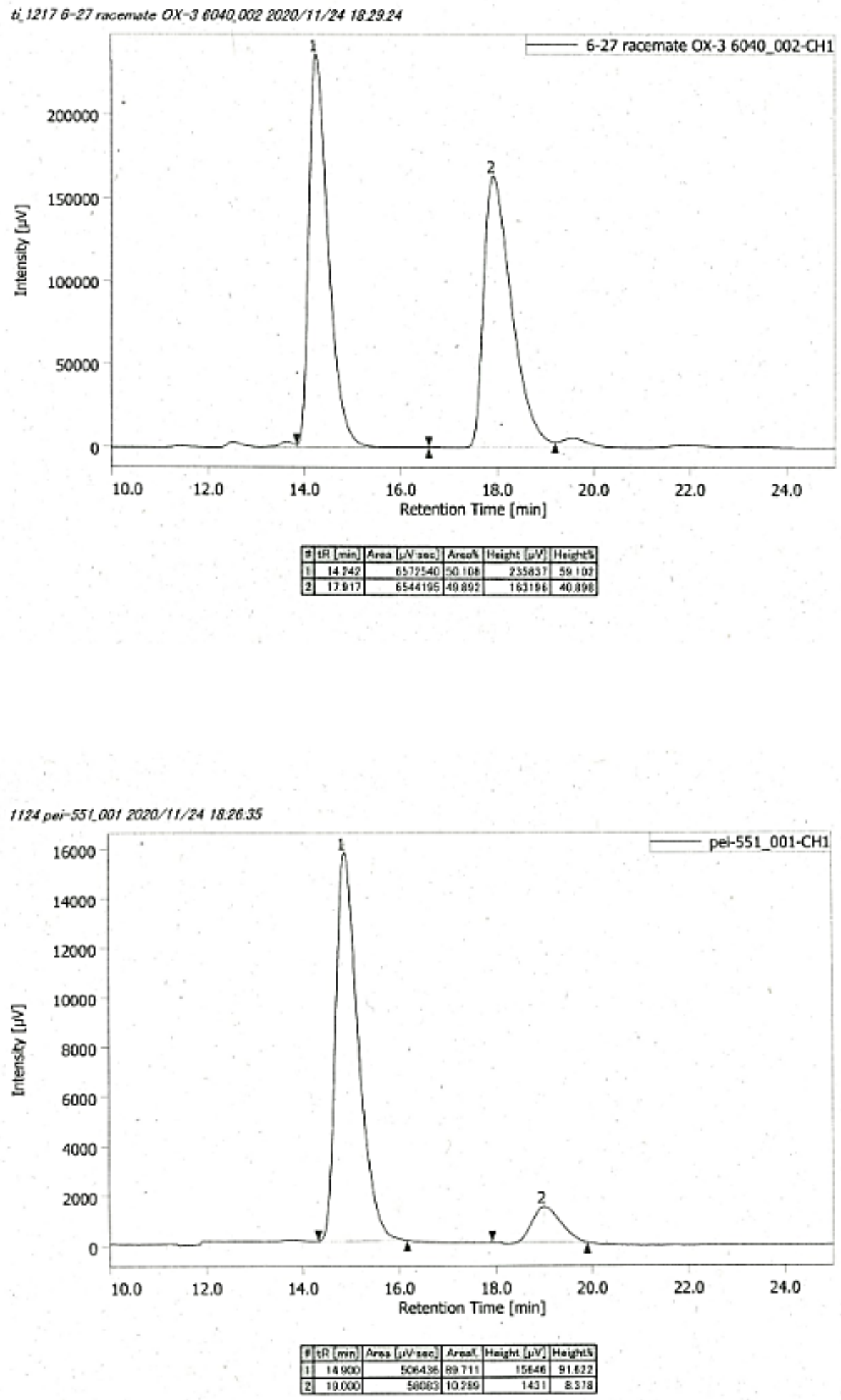
(3bS,4S,10S,10aR)-12-Bromo-2-((4-bromophenyl)sulfonyl)-3a,7-diphenyl-

1,2,3b,4-tetrahydro-6H,10H-4,10-ethenopyrrolo[3',4':1,3]cyclopropa[1,2-

d] $[1,2,4]$ triazolo[1,2-a]pyridazine-3,6,8(3aH,7H)-trione (6a)

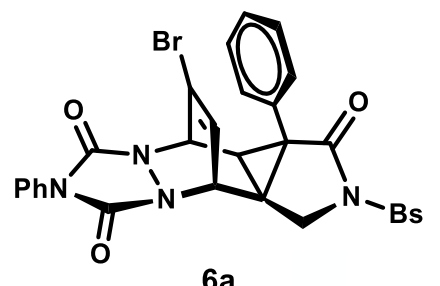

ti. 0123 SO2Pho-Br $4+2$ racemate L43 5050 004 2020/02/01 13:30-13

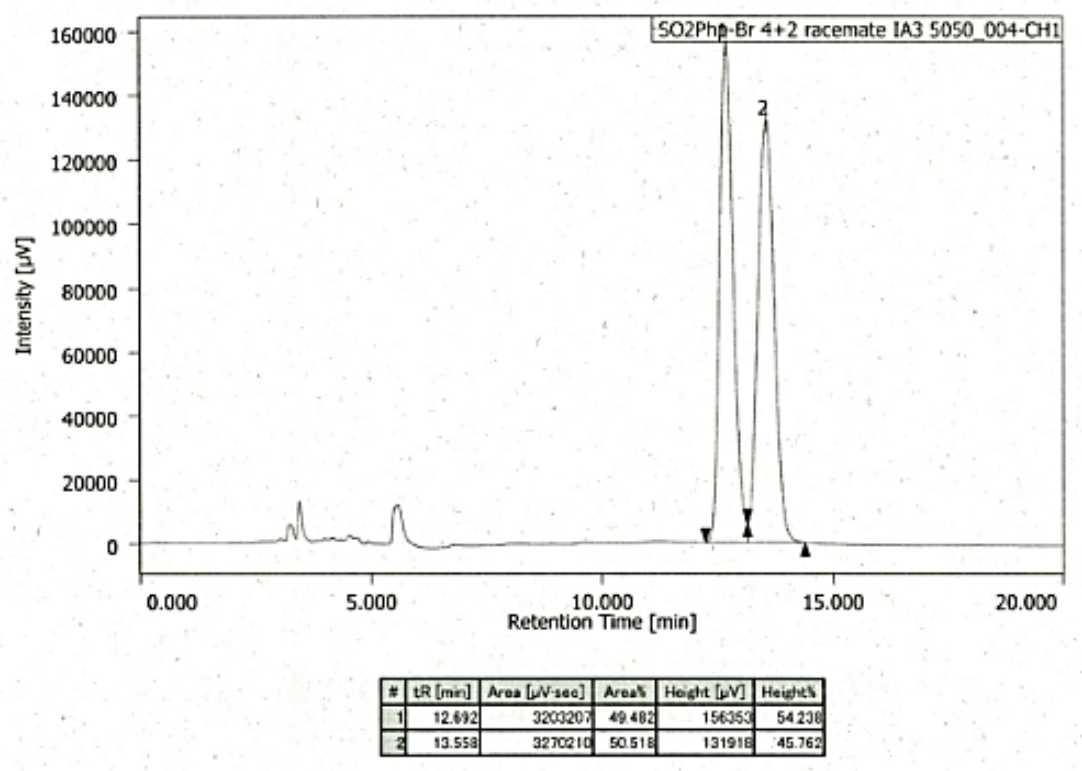

ti:0207 brosyd 4+2 asym LA3 5050.00t 2020 02/07 13:5027

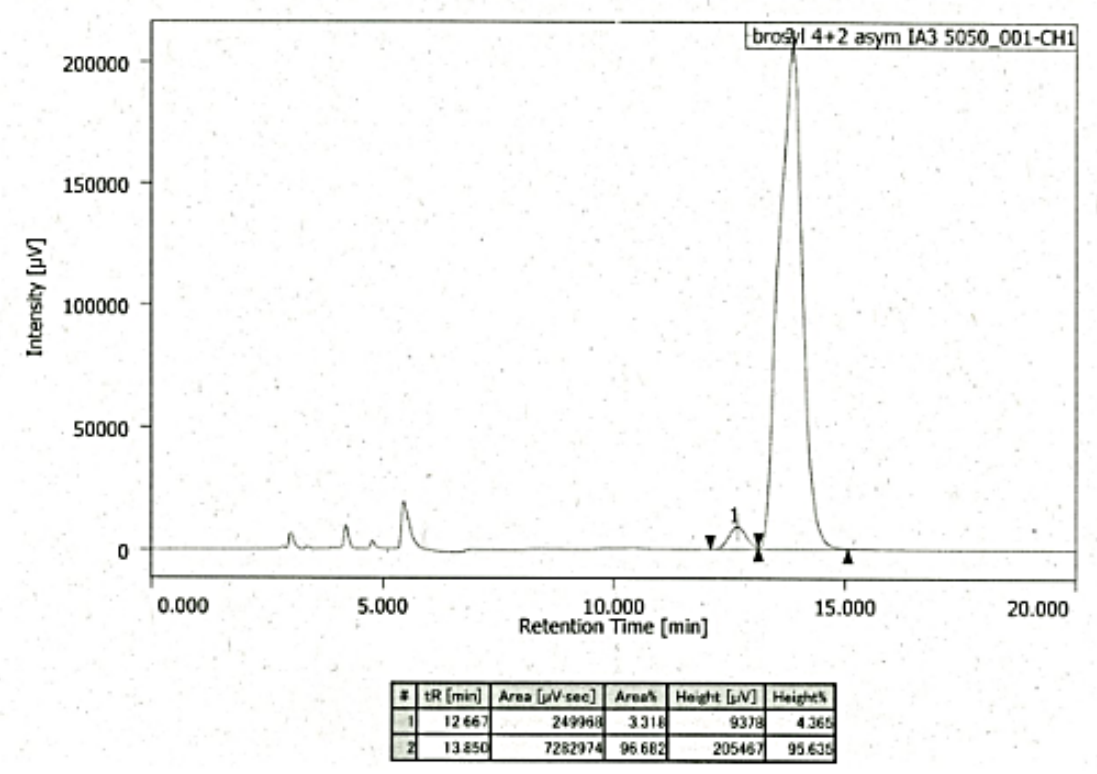


(3bR,4R,10R,10aS)-12-Bromo-2-((4-bromophenyl)sulfonyl)-3a-(4-fluorophenyl)7-phenyl-1,2,3b,4-tetrahydro-6H,10H-4,10ethenopyrrolo[ $\left.3^{\prime}, 44^{\prime}: 1,3\right]$ cyclopropa[1,2-d][1,2,4]triazolo [1,2-a]pyridazine-3,6,8(3aH,7H)-trione (6b)
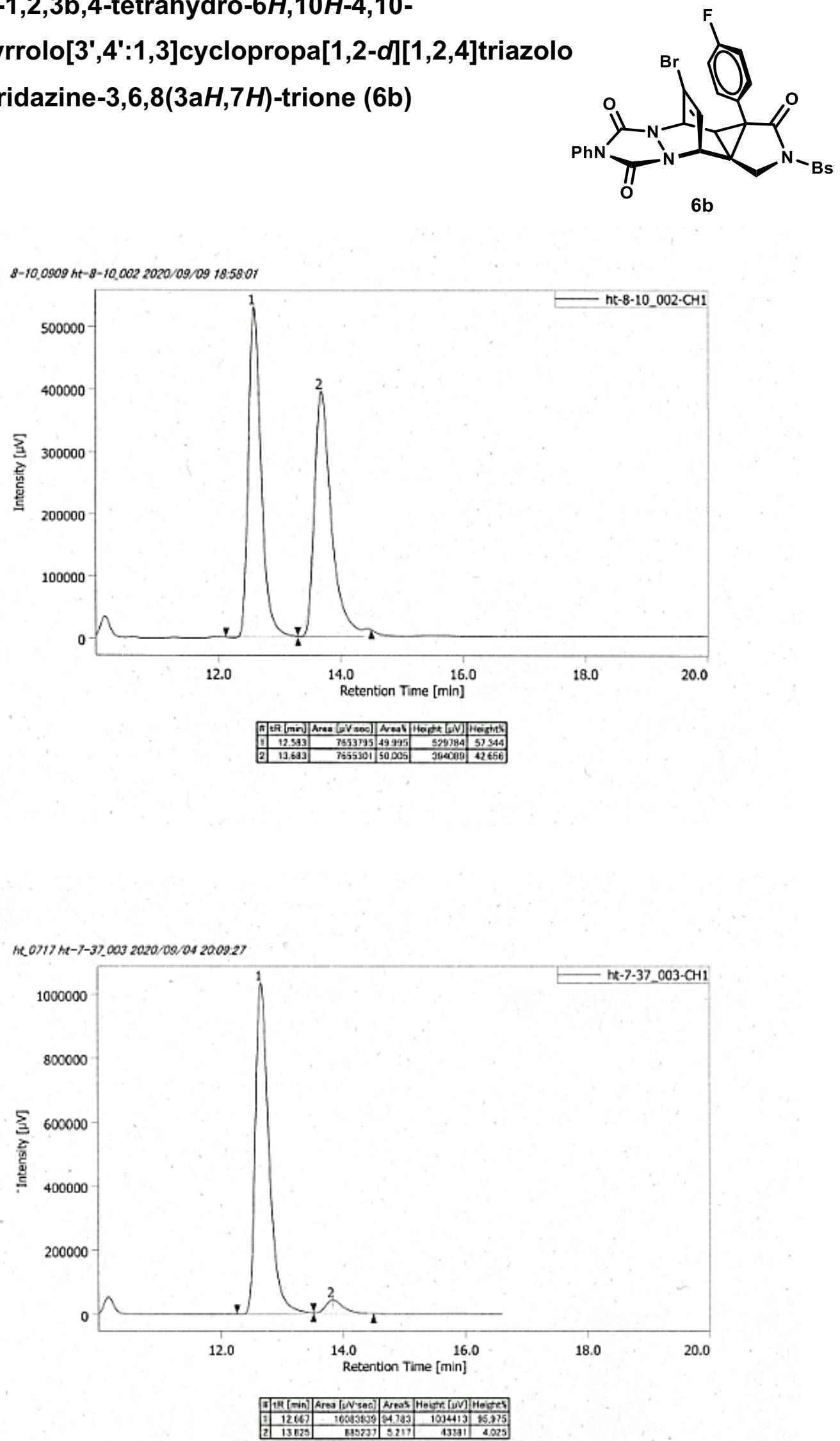
(3bR,4R,10R,10aS)-12-Bromo-3a-(4-bromophenyl)-2-((4-bromophenyl)sulfonyl)7-phenyl-1,2,3b,4-tetrahydro-6H,10H-4,10ethenopyrrolo[ $\left.3^{\prime}, 44^{\prime}: 1,3\right]$ cyclopropa[1,2-d][1,2,4]triazolo [1,2-a]pyridazine-3,6,8(3aH,7H)-trione (6c)
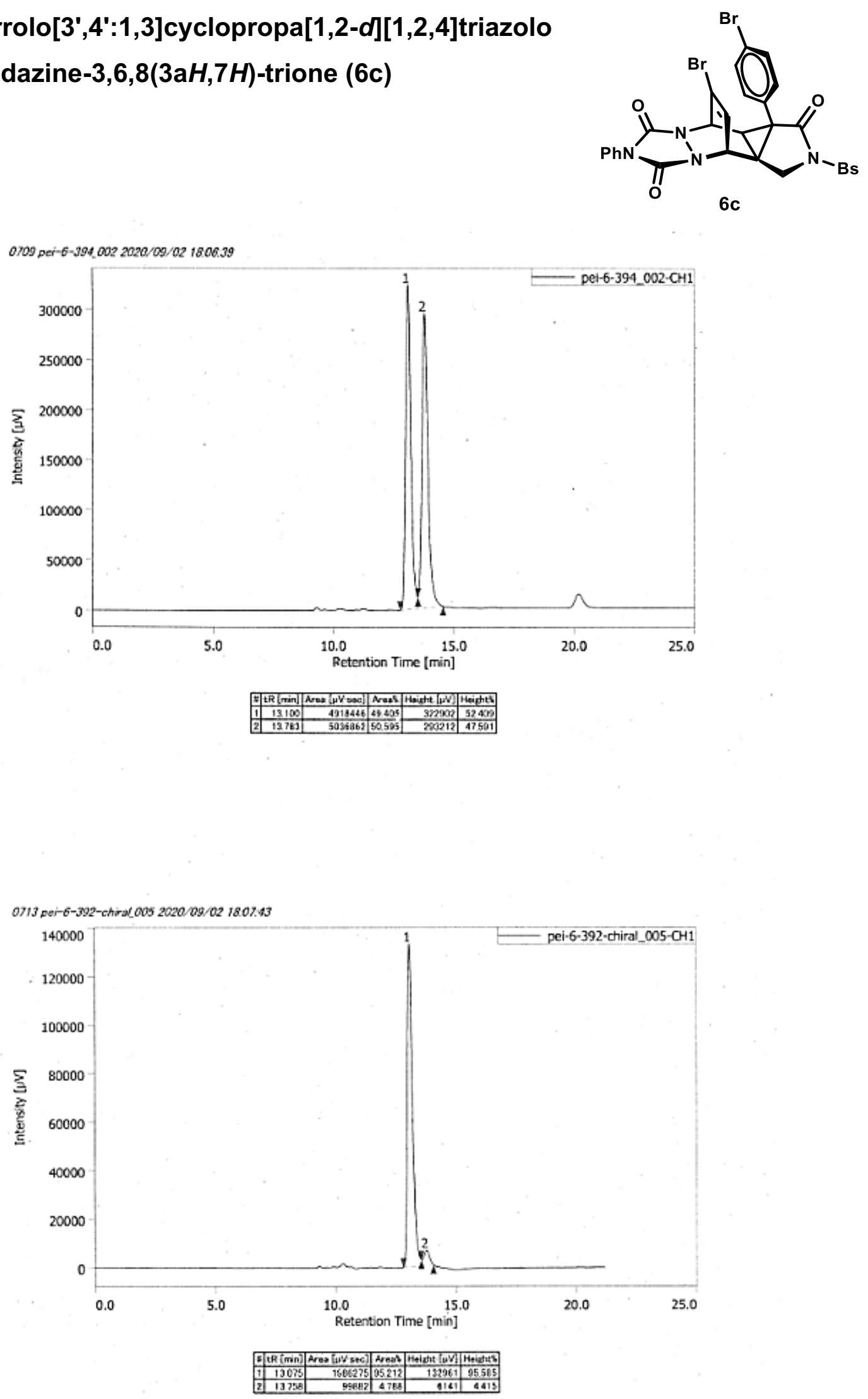
Methyl 4-((3bR,4R,10R,10aS)-12-Bromo-2-((4-bromophenyl)sulfonyl)-3,6,8trioxo-7-phenyl-2,3,3b,4,7,8-hexahydro-6H,10H-4,10ethenopyrrolo[3',4':1,3]cyclopropa[1,2-d][1,2,4]triazolo [1,2-a]pyridazin-3a(1H)-yl)benzoate $(6 \mathrm{~d})$
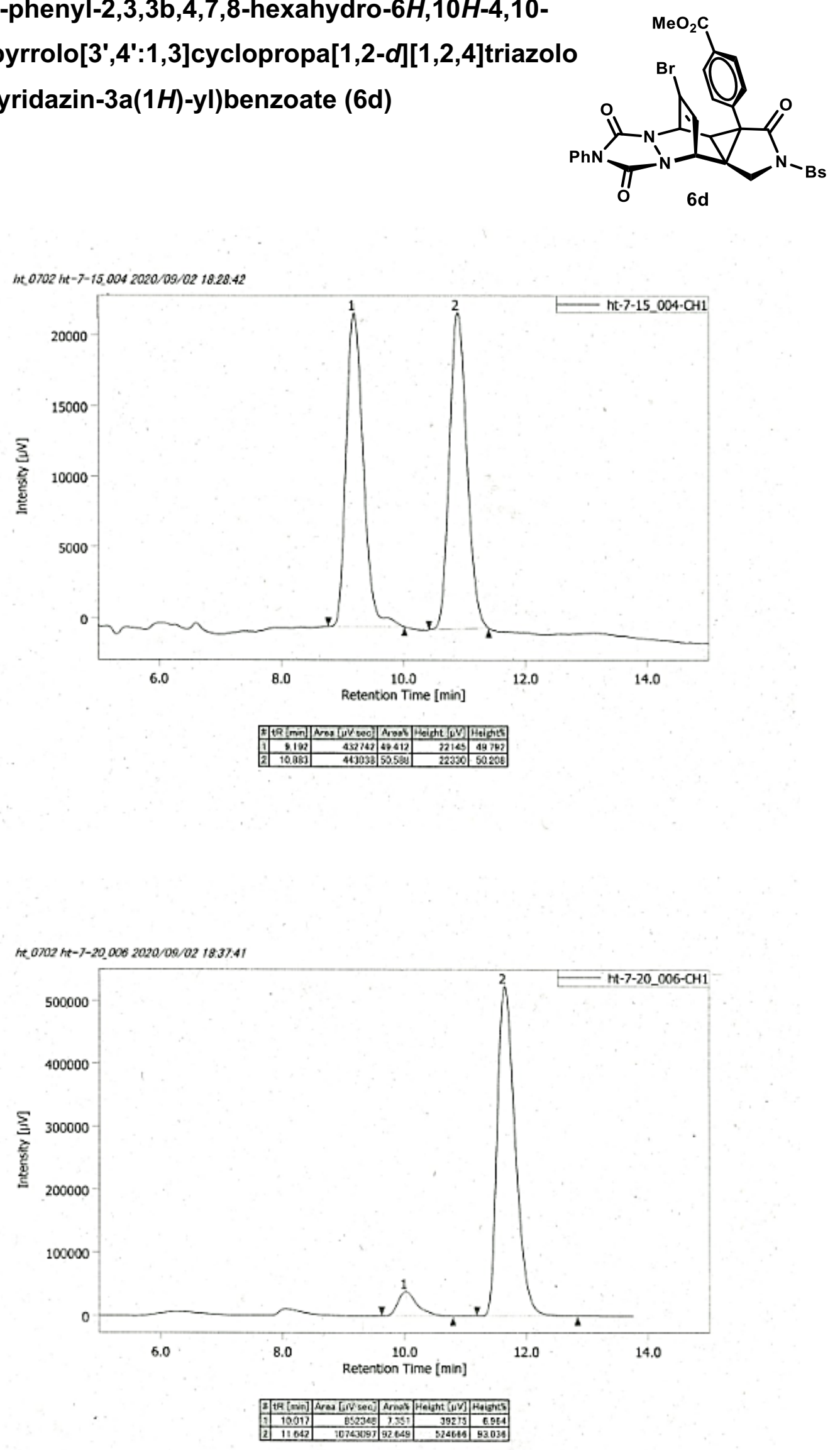
(3bS,4S,10S,10aR)-12-Bromo-3a,7-diphenyl-2-tosyl-1,2,3b,4-tetrahydro-6H,10 H4,10-ethenopyrrolo[3',4':1,3]cyclopropa[1,2-d][1,2,4]triazolo[1,2-a]pyridazine$3,6,8(3 \mathrm{a} H, 7 H)$-trione $(6 \mathrm{e})$

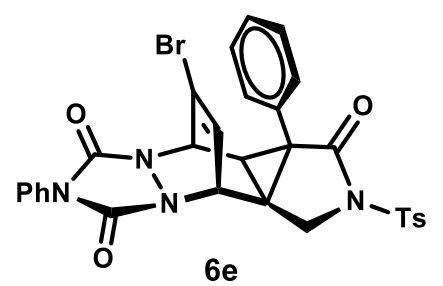

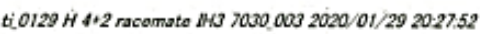

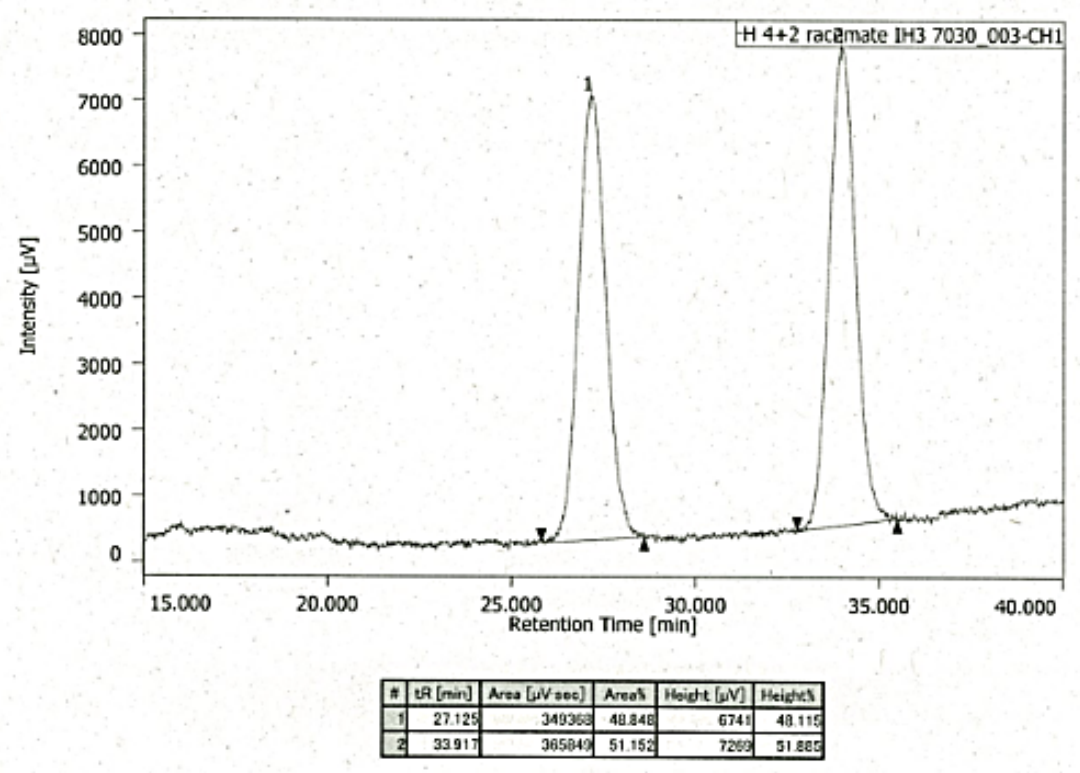

ti. $0129 \mathrm{H} 4+2$ s.5ym $1 \mathrm{H} 337000.0042020101 / 2920.30-10$

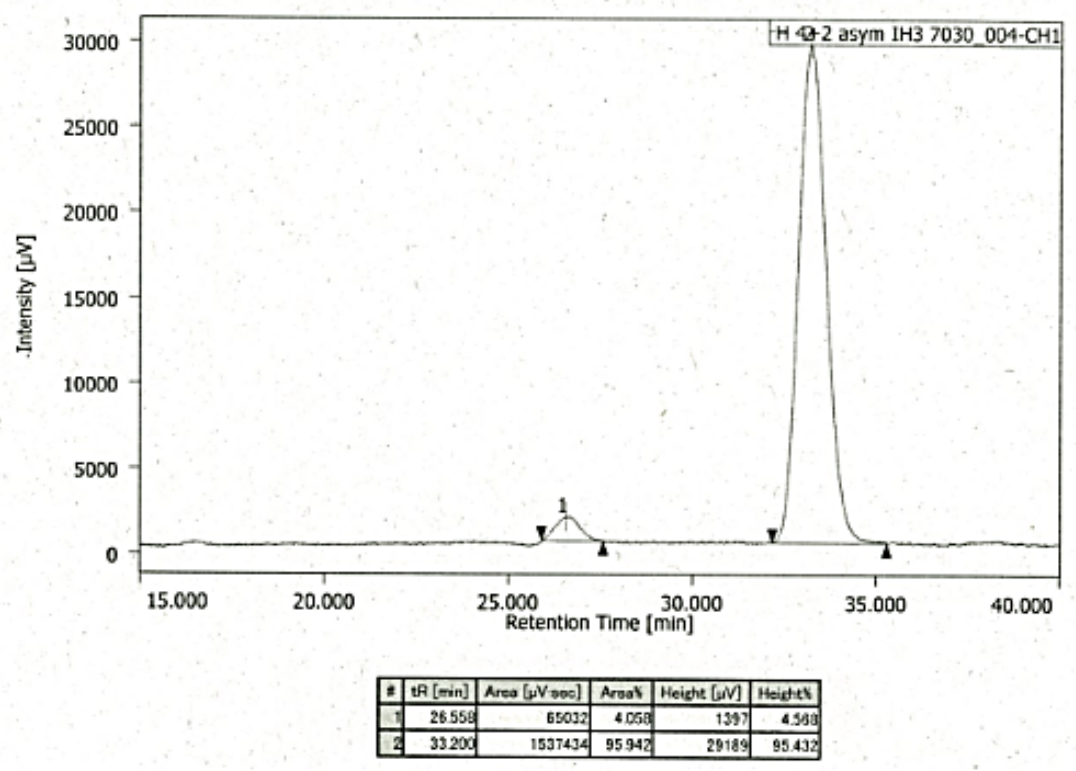


(3bR,4R,10R,10aS)-12-Bromo-2-((4-methoxyphenyl)sulfonyl)-3a,7-diphenyl1,2,3b,4-tetrahydro-6H,10H-4,10-ethenopyrrolo[3',4':1,3]cyclopropa[1,2d] $[1,2,4]$ triazolo[1,2-a]pyridazine-3,6,8(3aH,7H)-trione (6f)

ti. OI28 SOJC6HSp-pOMe H 4+2 racemate LAJ 5050,002 2020/02/03 /3/4:56
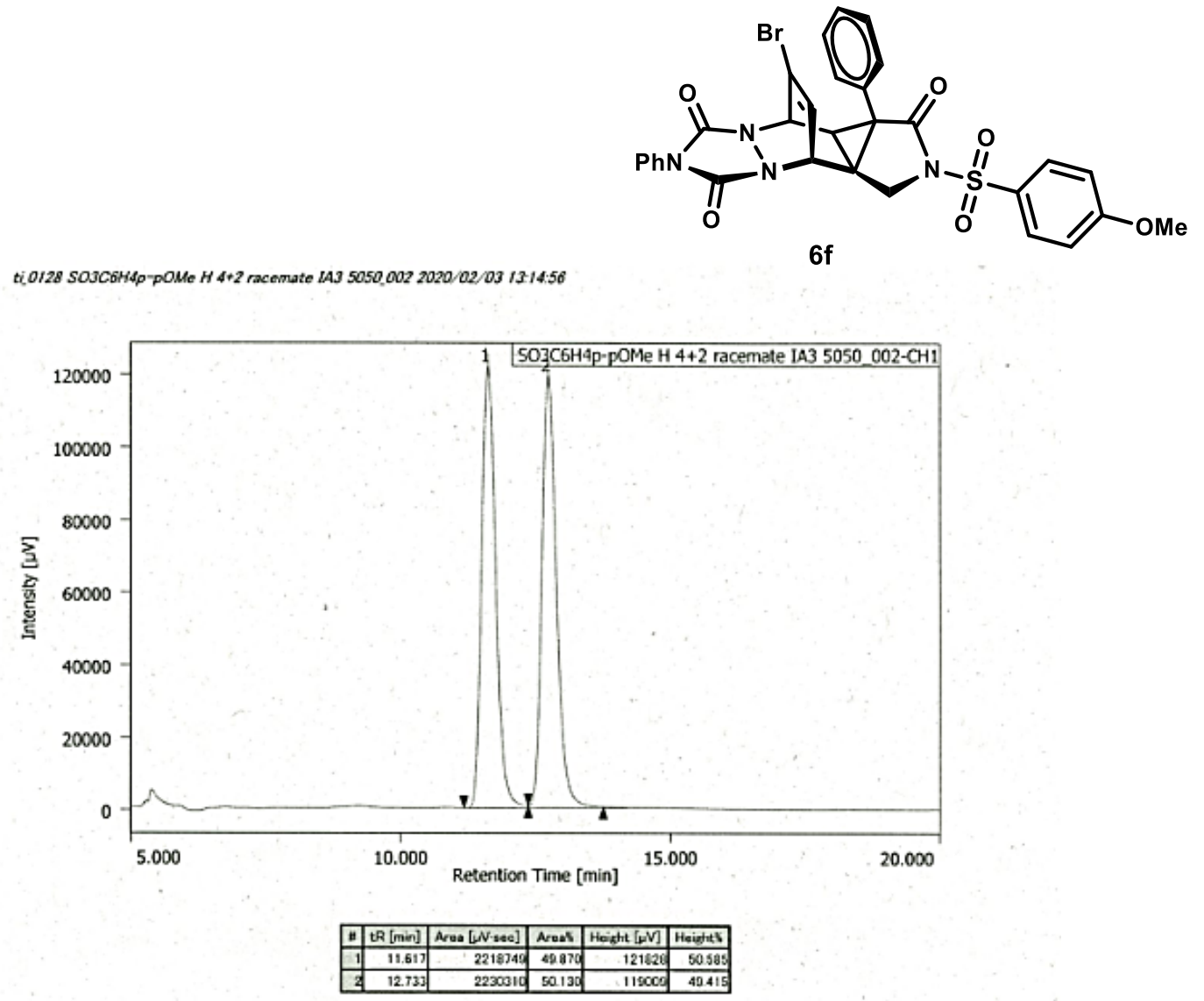

ti. 0203 SO2C6H4p-OMe H 4.2 asym LA3 5050,002 2020/02 03 1314.52

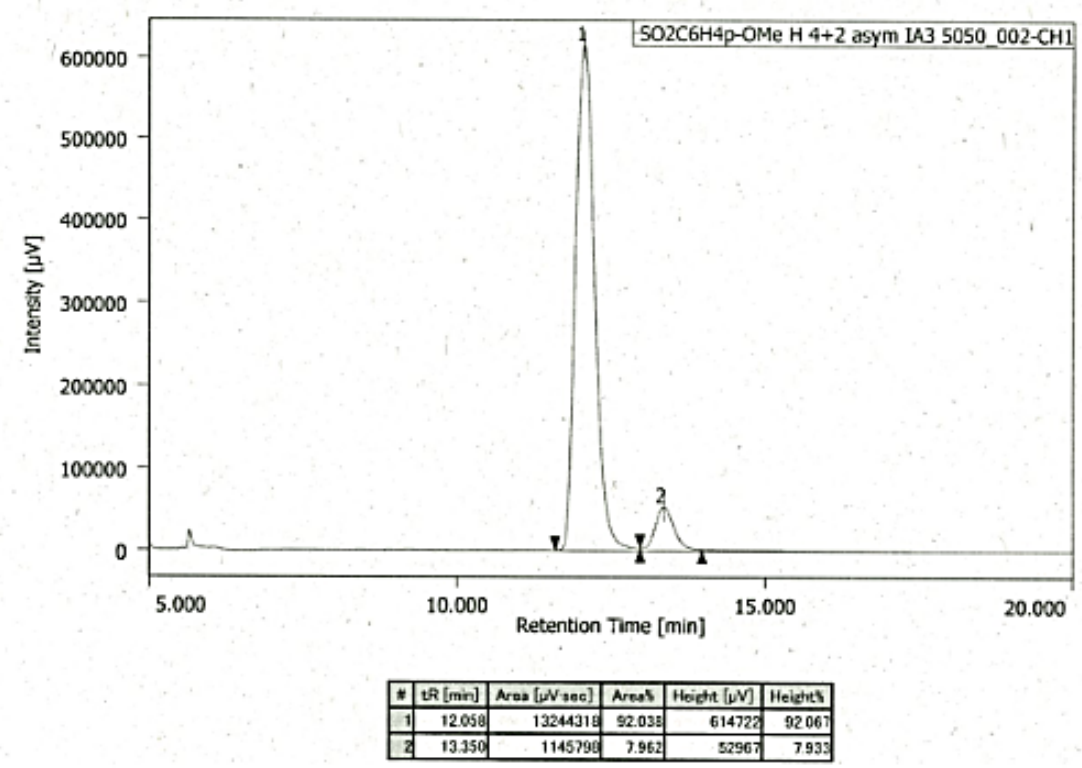


(3bR,4R,10R,10aS)-12-Bromo-3a-(3-bromophenyl)-7-phenyl-2-tosyl-1,2,3b,4tetrahydro-6H,10H-4,10-ethenopyrrolo[3',4':1,3]cyclopropa[1,2d] $[1,2,4]$ triazolo[1,2-a]pyridazine-3,6,8(3aH,7H)-trione $(6 \mathrm{~g})$
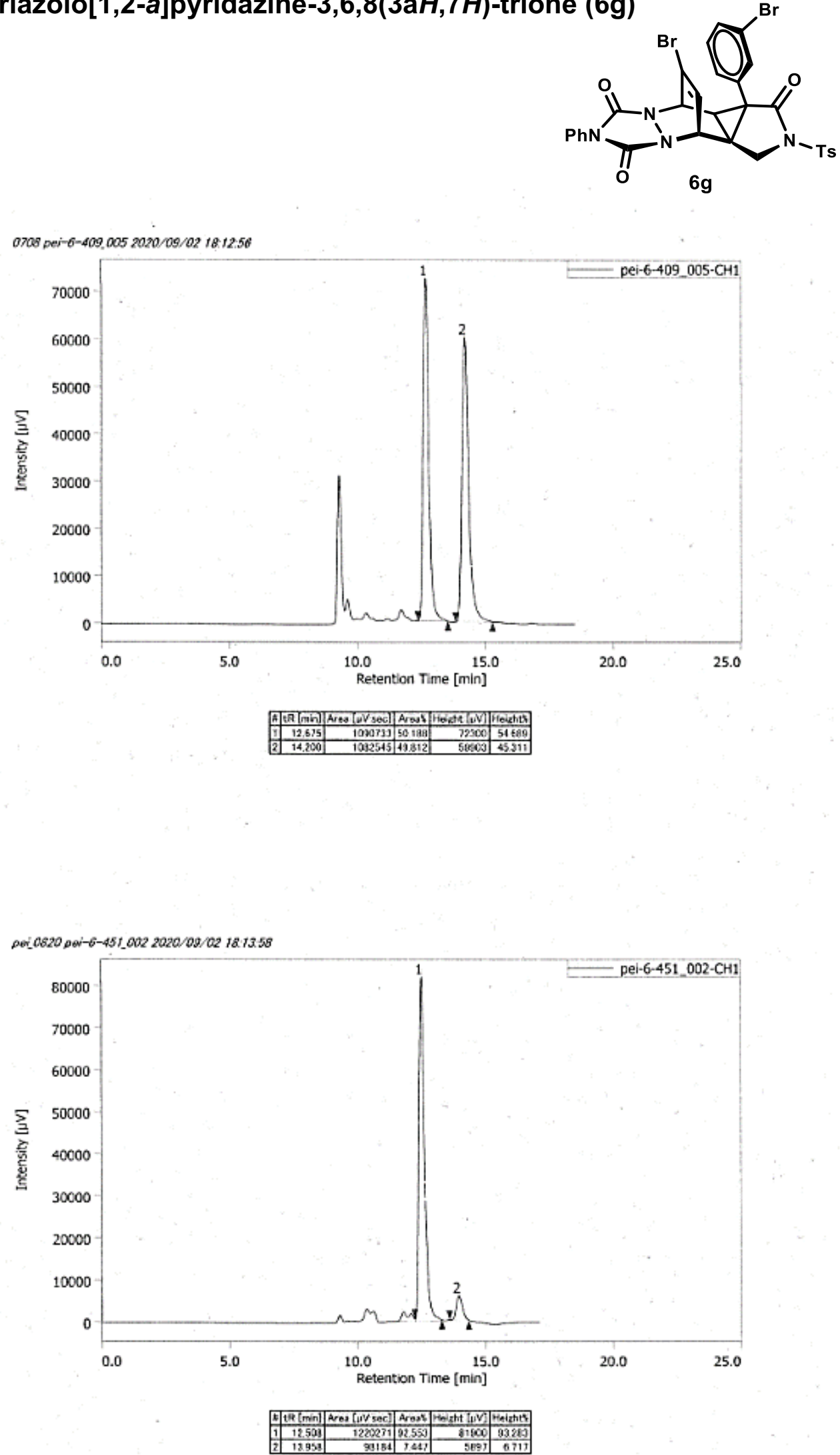
(3bR,4R,10R,10aR)-12-Bromo-2-((4-bromophenyl)sulfonyl)-3a-(3methoxyphenyl)-7-phenyl-1,2,3b,4-tetrahydro-6H,10H-4,10ethenopyrrolo[3',4':1,3]cyclopropa[1,2-d][1,2,4]triazolo[1,2-a]pyridazine3,6,8(3aH,7H)-trione (6h)
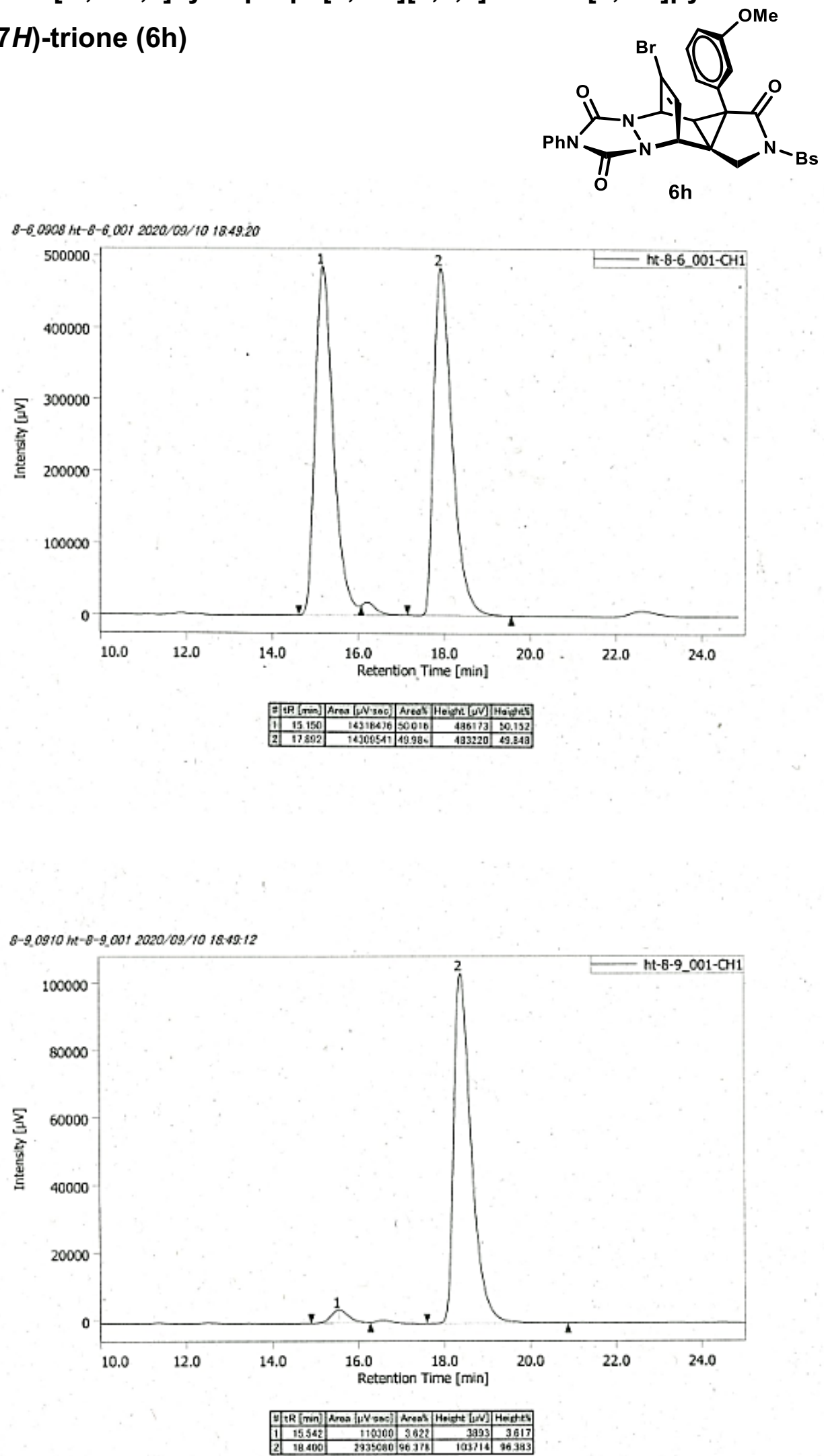
(3bR,4R,10R,10aS)-2-((4-Bromophenyl)sulfonyl)-12-fluoro-3a,7-diphenyl-

1,2,3b,4-tetrahydro-6H,10H-4,10-ethenopyrrolo[3',4':1,3]cyclopropa[1,2-

d] $[1,2,4]$ triazolo[1,2-a]pyridazine-3,6,8(3aH,7H)-trione (6i)
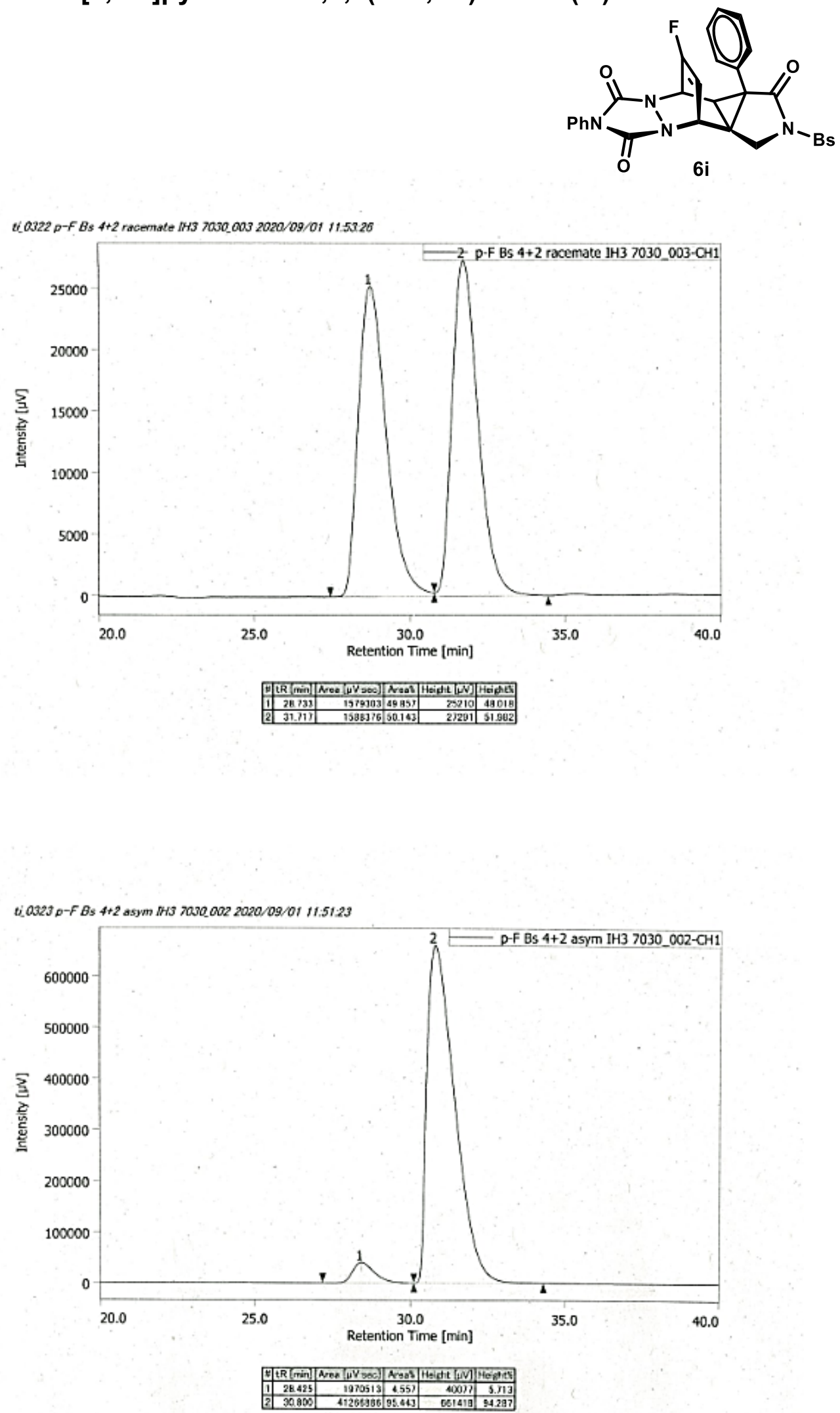
(3bR,4R,10R,10aS)-12-Fluoro-3a,7-diphenyl-2-tosyl-1,2,3b,4-tetrahydro-6H,10H4,10-ethenopyrrolo[3',4':1,3]cyclopropa[1,2-d][1,2,4]triazolo[1,2-a]pyridazine-

\section{3,6,8(3aH,7H)-trione (6j)}

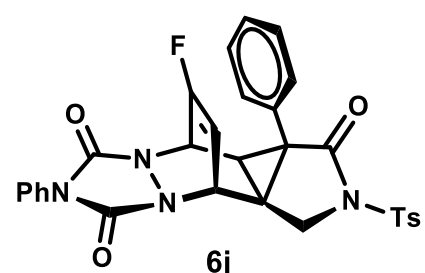

$6 \mathbf{j}$

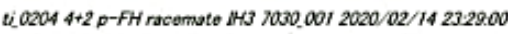

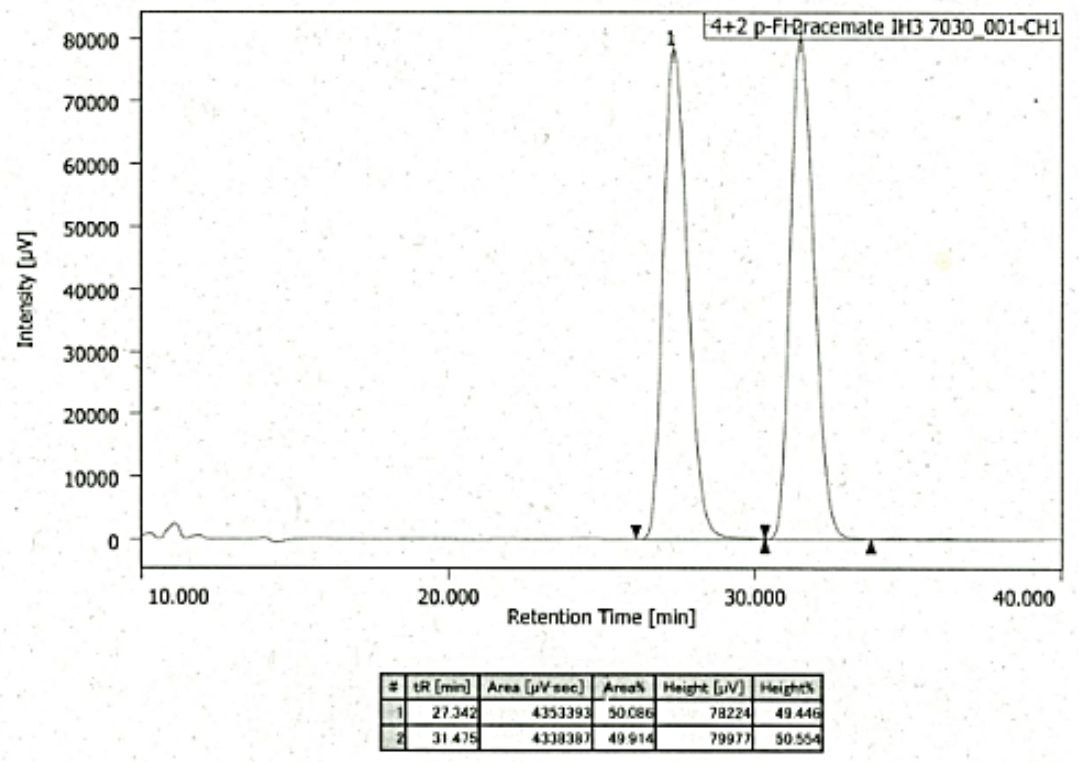

T20214 P-F h asym NH-3 7030,002 2020/02/14 23:29:10

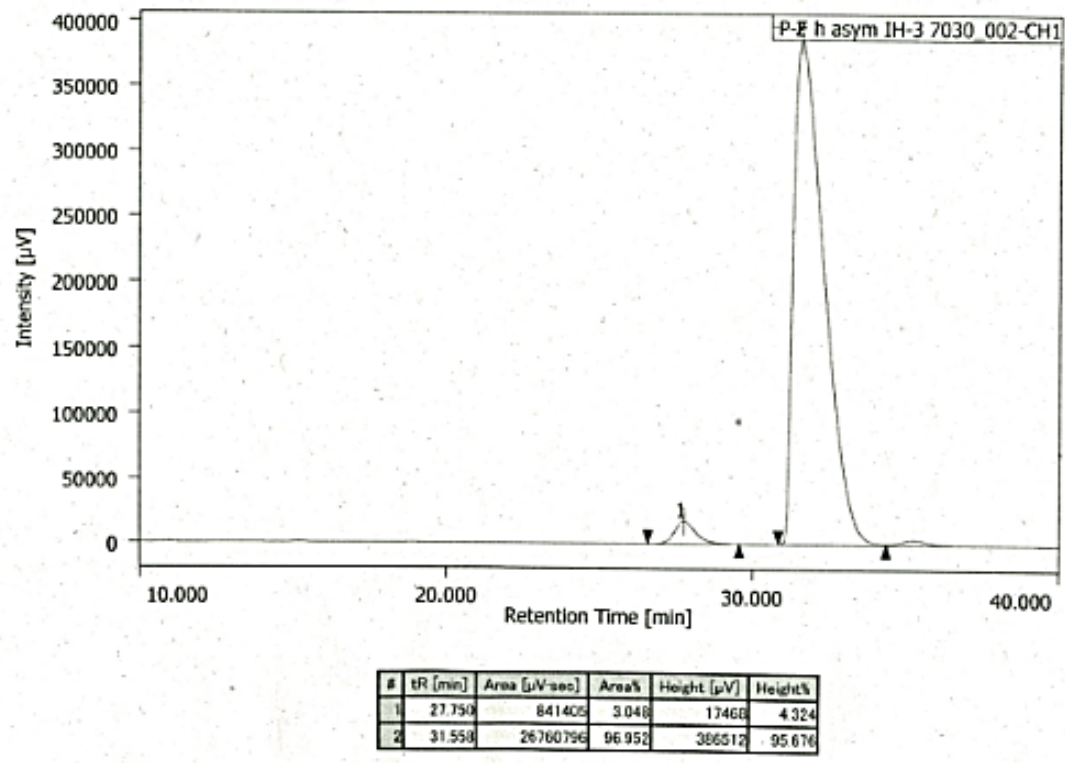


(3bR,4R,10R,10aS)-12-Chloro-3a,7-diphenyl-2-tosyl-1,2,3b,4-tetrahydro-6H,10H4,10-ethenopyrrolo[3',4':1,3]cyclopropa[1,2-o][1,2,4]triazolo[1,2-a]pyridazine3,6,8(3aH,7H)-trione (6k)

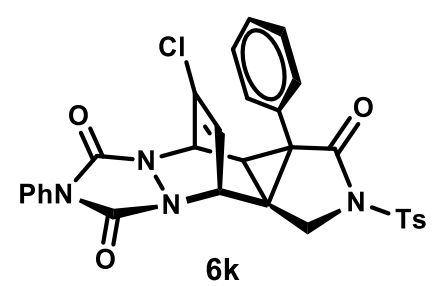

ti.0312p-CA 4+2 racemste LA-35050,002 2020 02/13 1.0028

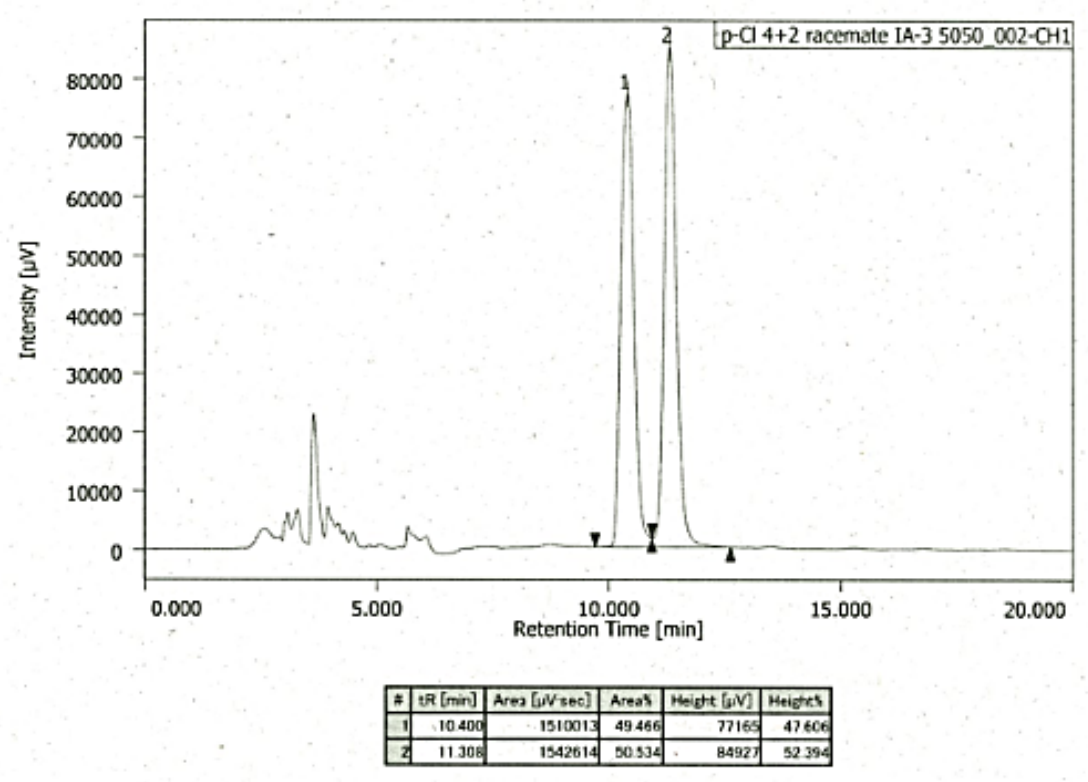

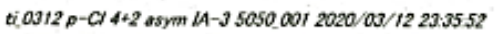

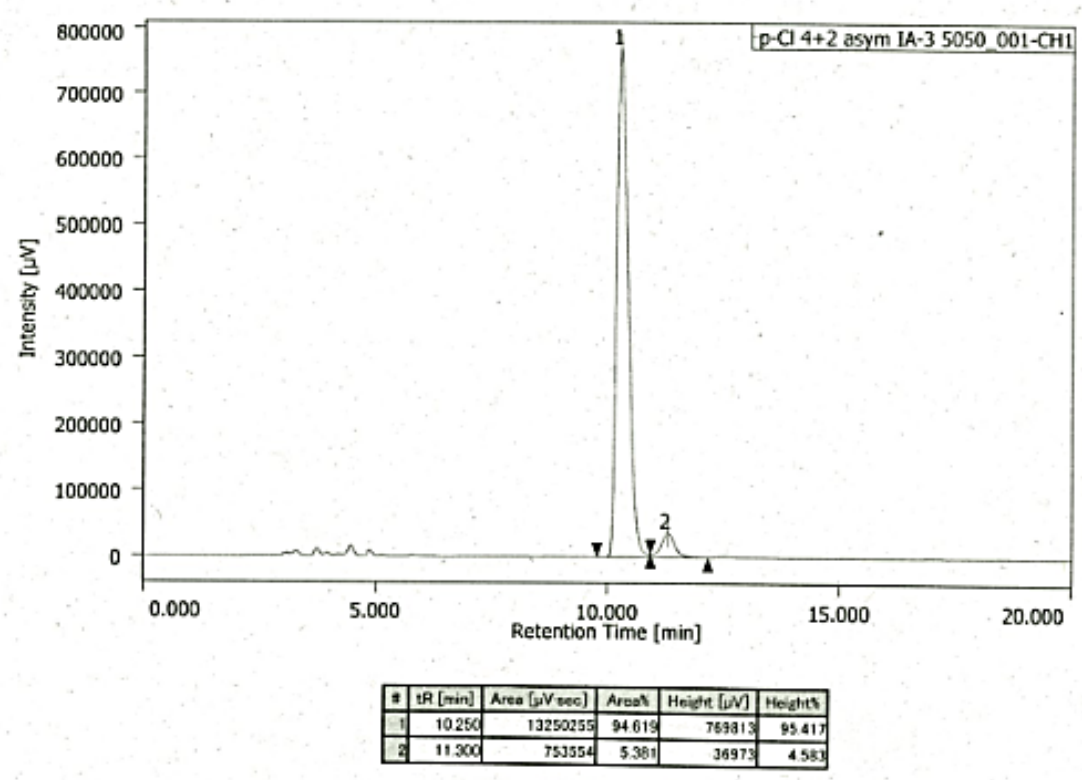


(3bR,4R,10R,10aS)-2-((4-Bromophenyl)sulfonyl)-12-iodo-3a,7-diphenyl-1,2,3b,4tetrahydro-6H,10H-4,10-ethenopyrrolo[3',4':1,3]cyclopropa[1,2d] $[1,2,4]$ triazolo[1,2-a]pyridazine-3,6,8(3aH,7H)-trione (6I)

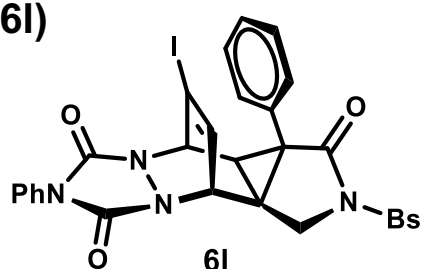

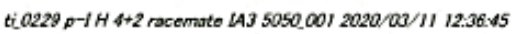

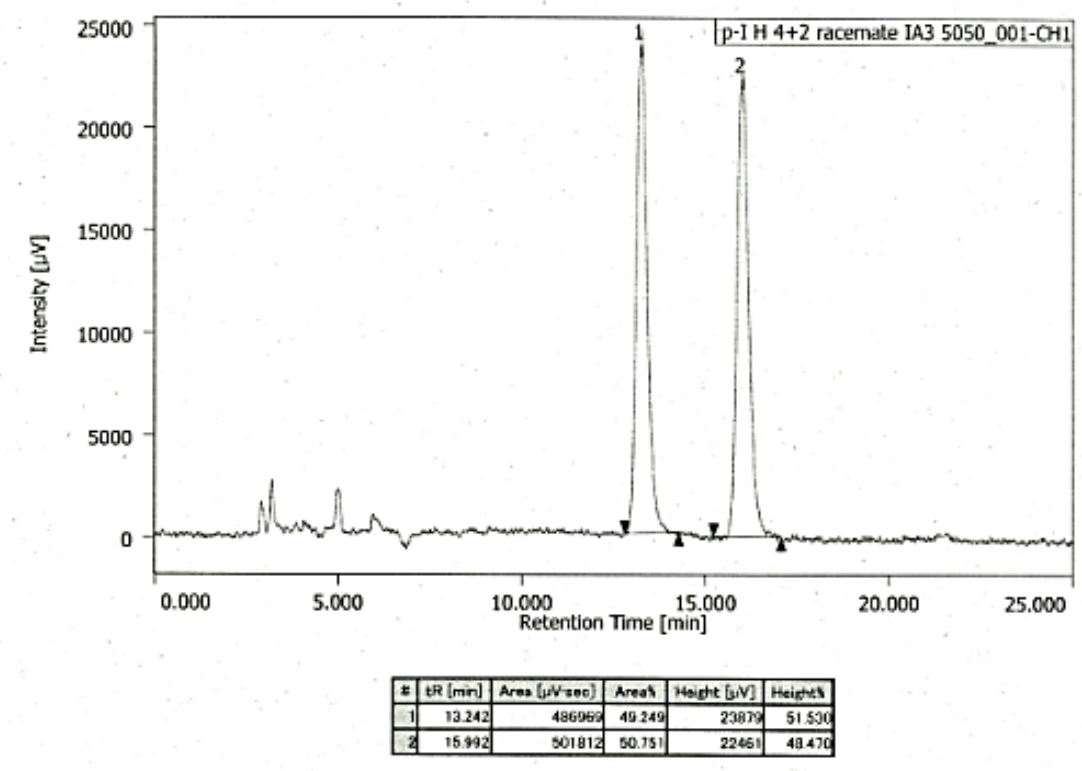

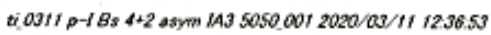

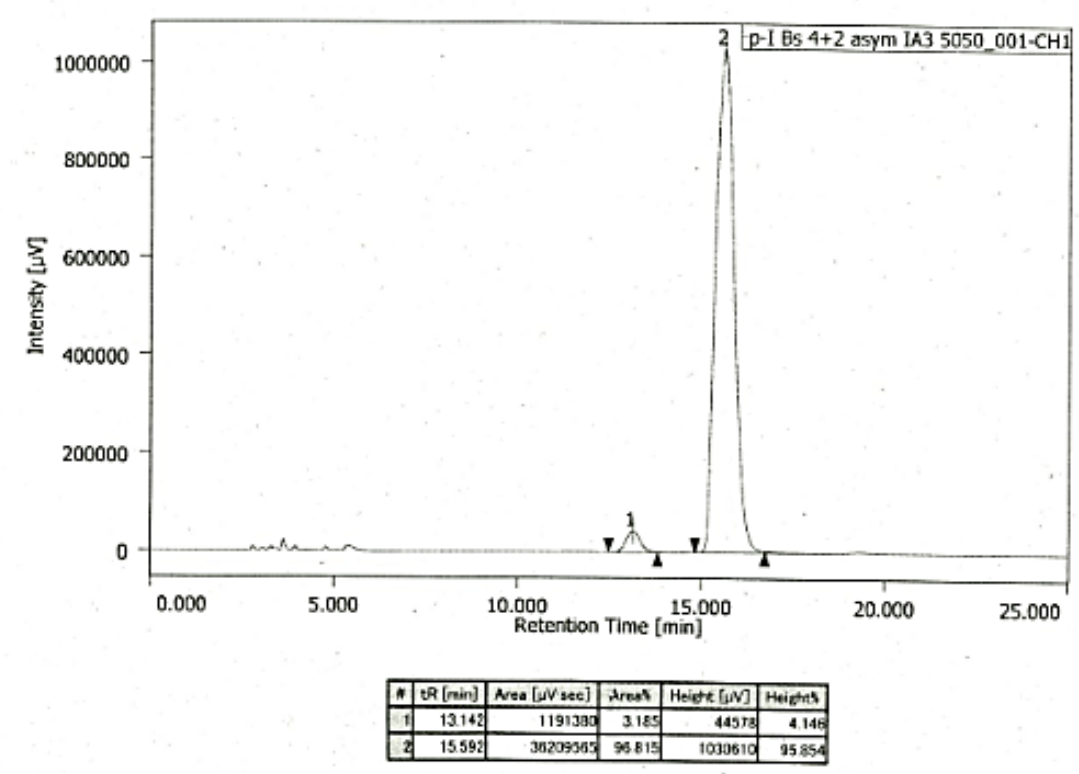


(3bR,4R,10R,10aS)-2-((4-Bromophenyl)sulfonyl)-12-methyl-3a,7-diphenyl-

1,2,3b,4-tetrahydro-6H,10H-4,10-ethenopyrrolo[3',4':1,3]cyclopropa[1,2-

d] $[1,2,4]$ triazolo[1,2-a]pyridazine-3,6,8(3aH,7H)-trione $(6 \mathrm{~m})$
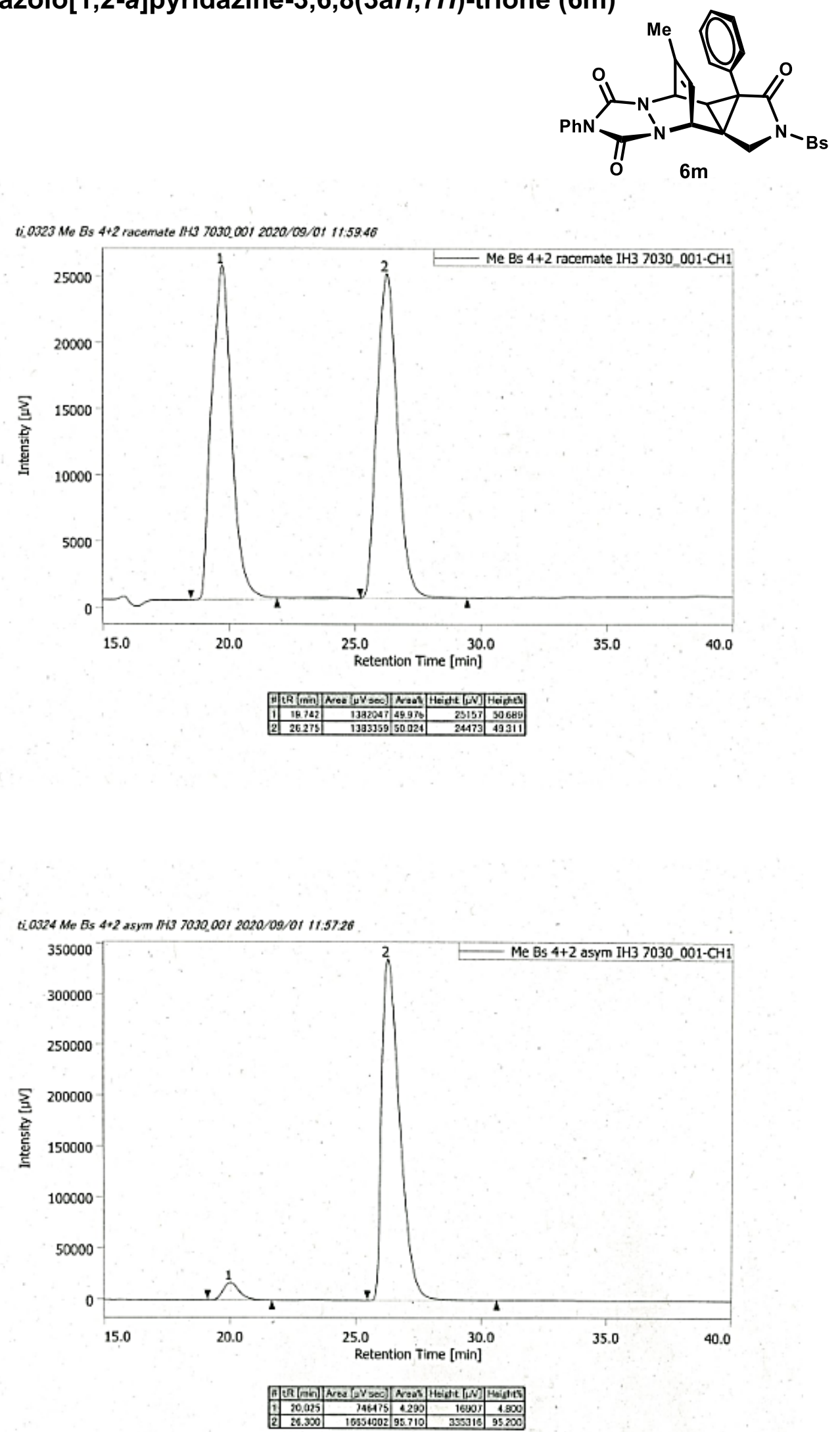
(3bR,4S,10R,10aS)-2-((4-Bromophenyl)sulfonyl)-3a,7-diphenyl-1,2,3b,4tetrahydro-6H,10H-4,10-ethenopyrrolo[3',4':1,3]cyclopropa[1,2d] $[1,2,4]$ triazolo[1,2-a]pyridazine-3,6,8(3aH,7H)-trione $(6 n)$
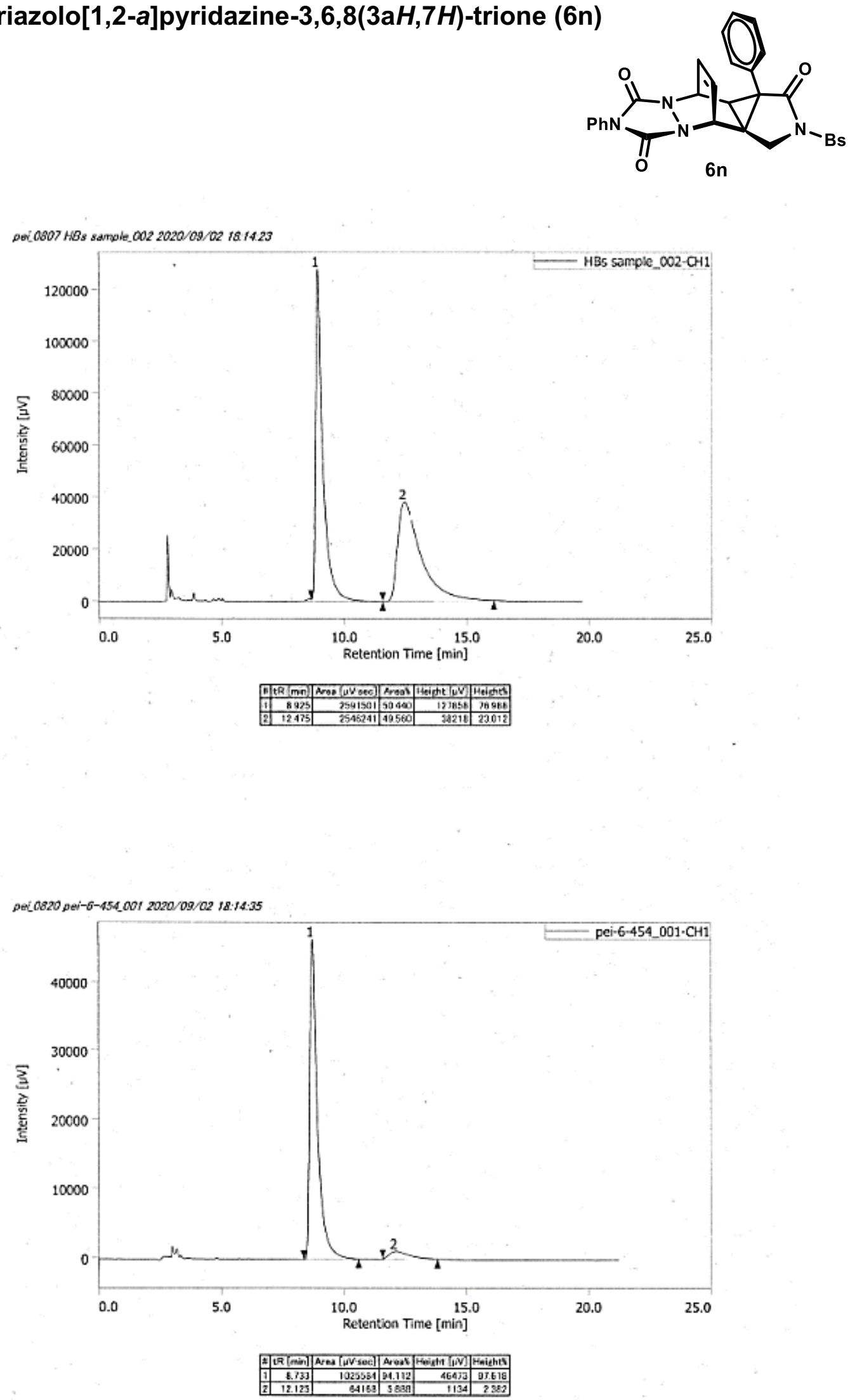
(3bR,4R,10R,10aS)-12-Bromo-2-((4-bromophenyl)sulfonyl)-7-methyl-3a-phenyl1,2,3b,4-tetrahydro-6H,10H-4,10-ethenopyrrolo[3',4':1,3]cyclopropa[1,2d] $[1,2,4]$ triazolo[1,2-a]pyridazine-3,6,8(3aH,7H)-trione (6o)

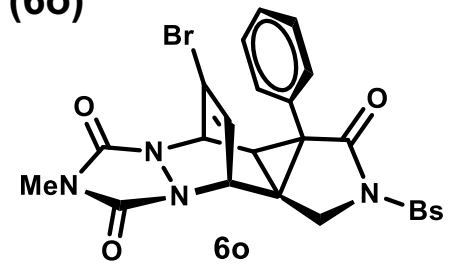
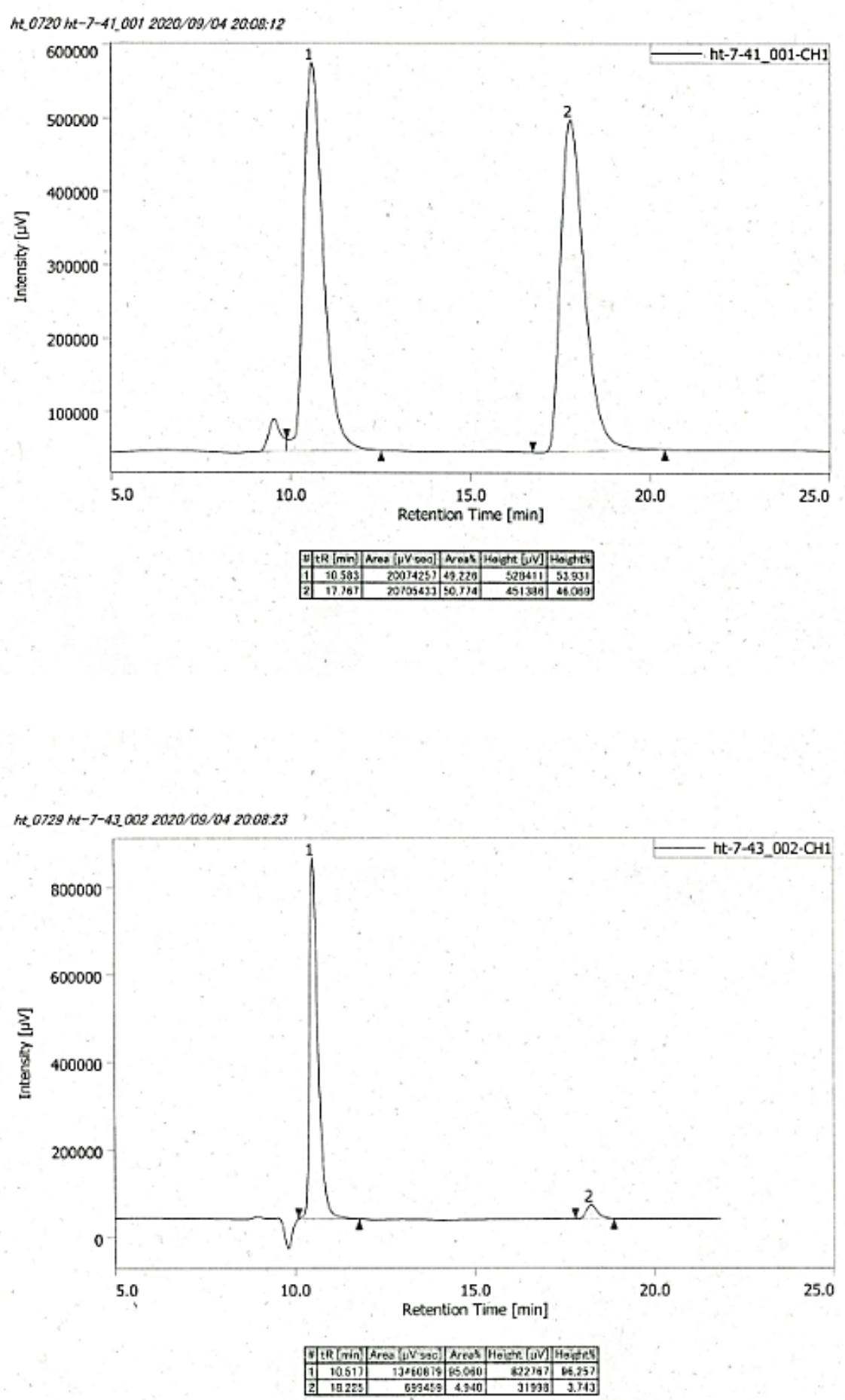
(3bR,4S,7aR,8S,8aR)-2-((4-bromophenyl)sulfonyl)-3a-phenyl-

1,2,3a,3b,4,4a,7a,8-octahydro-7H-4,8-

ethenoisobenzofuro[5',6':1,3]cyclopropa[1,2-c]pyrrole-3,5,7-trione (7j)
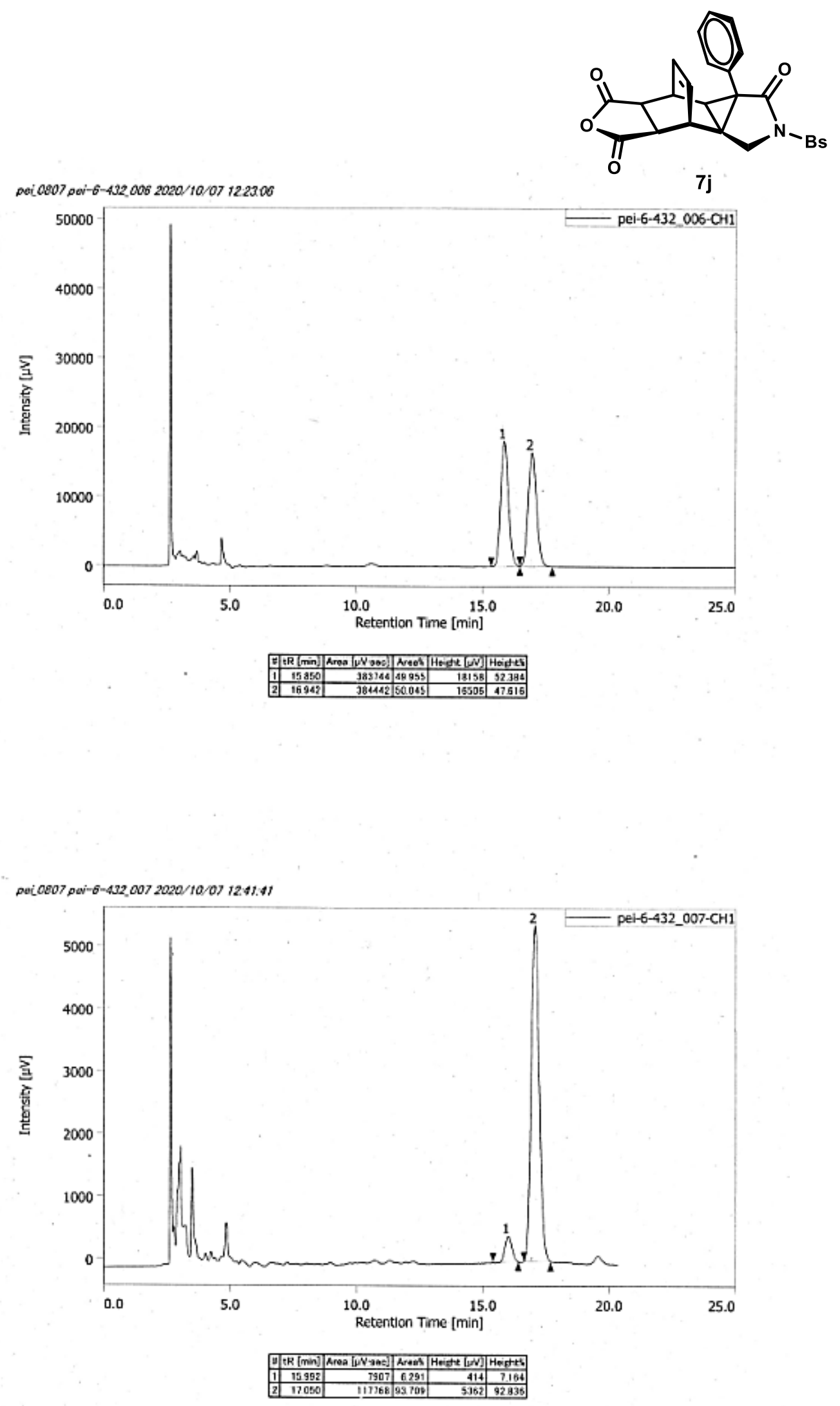
$N$-((3bR,4S,7aR,8R,8aR)-10-Bromo-2-((4-bromophenyl)sulfonyl)-3,5,7-trioxo-3aphenyl-1,2,3,3a,3b,4,4a,5,7a,8-decahydro-4,8-

ethenopyrrolo[3',4':1,3]cyclopropa[1,2-f]isoindol-6(7H)-yl)benzamide (8a)
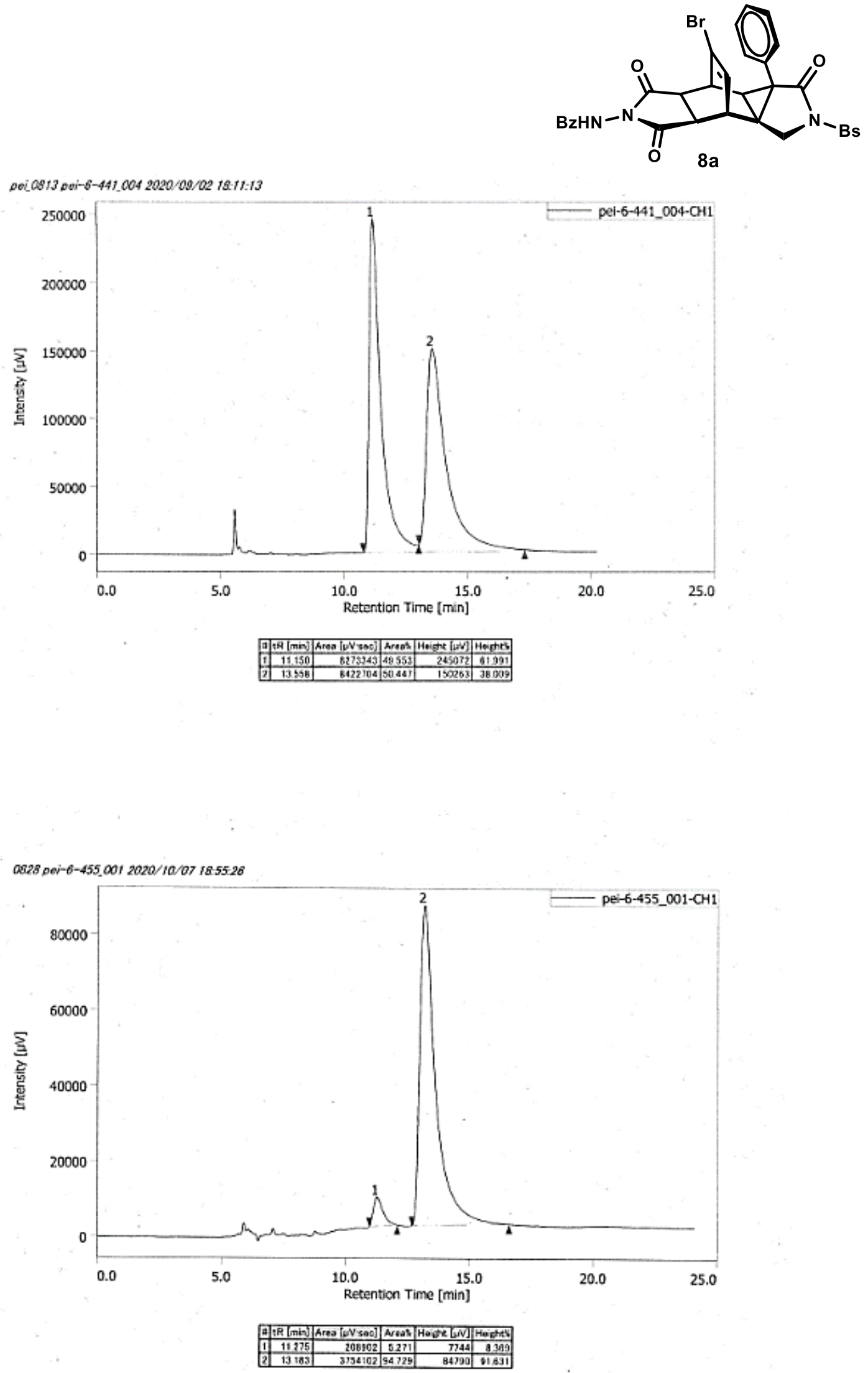


\section{References}

1) Li, L.; Zhou, B.; Wang, Y.-H.; Shu, C.; Pan, Y.-F.; Lu, X.; Ye, L.-W. ZincCatalyzed Alkyne Oxidation/C-H Functionalization: Highly SiteSelective Synthesis of Versatile Isoquinolones and $\beta$-Carbolines. Angew. Chem., Int. Ed. 2015, 54, 8245-8249.

2) Zhao, Y.; Hu, Y.; Wang, C.; Li, X.; Wan, B. Tf 2 NH-Catalyzed Formal [3+2] Cycloaddition of Ynamides with Dioxazoles: A MetalFree Approach to Polysubstituted 4-Aminooxazoles. J. Org. Chem. 2017, 82, 3935-3942.

3) Yang, Y.; Zhang, X.; Liang, Y. Copper-catalyzed coupling of 1,2-dibromo-1styrenes with sulfonamides for the preparation of ynamides. Tetrahedron Lett. 2012, $53,6557-6560$.

4) Gaussian 16, Revision C.01, Frisch, M. J.; Trucks, G. W.; Schlegel, H. B.; Scuseria, G. E.; Robb, M. A.; Cheeseman, J. R.; Scalmani, G.; Barone, V.; Petersson, G. A.; Nakatsuji, H.; Li, X.; Caricato, M.; Marenich, A. V.; Bloino, J.; Janesko, B. G.; Gomperts, R.; Mennucci, B.; Hratchian, H. P.; Ortiz, J. V.; Izmaylov, A. F.; Sonnenberg, J. L.; Williams-Young, D.; Ding, F.; Lipparini, F.; Egidi, F.; Goings, J.; Peng, B.; Petrone, A.; Henderson, T.; Ranasinghe, D.; Zakrzewski, V. G.; Gao, J.; Rega, N.; Zheng, G.; Liang, W.; Hada, M.; Ehara, M.; Toyota, K.; Fukuda, R.; Hasegawa, J.; Ishida, M.; Nakajima, T.; Honda, Y.; Kitao, O.; Nakai, H.; Vreven, T.; Throssell, K.; Montgomery, J. 
A., Jr.; Peralta, J. E.; Ogliaro, F.; Bearpark, M. J.; Heyd, J. J.; Brothers, E. N.; Kudin, K. N.; Staroverov, V. N.; Keith, T. A.; Kobayashi, R.; Normand, J.; Raghavachari, K.; Rendell, A. P.; Burant, J. C.; Iyengar, S. S.; Tomasi, J.; Cossi, M.; Millam, J. M.; Klene, M.; Adamo, C.; Cammi, R.; Ochterski, J. W.; Martin, R. L.; Morokuma, K.; Farkas, O.; Foresman, J. B.; Fox, D. J. Gaussian, Inc., Wallingford CT, 2016.

5) (a) Becke, A. D. Density-functional exchange-energy approximation with correct asymptotic behavior. Phys. Rev. 1988, A38, 3098-3100.

(b) Becke, A. D. A new mixing of Hartree-Fock and local density functional theories. J. Chem. Phys. 1993, 98, 1372-1377.

(c) Becke, A. D. Density-functional thermochemistry. III. The role of exact exchange. J. Chem. Phys. 1993, 98, 5648-5652.

(d) Lee, C; Yang, W.; Parr, R. G. Development of the Colle-Salvetti correlation-energy formula into a functional of the electron density. Phys. Rev. 1988, B37, 785-788.

6) (a) Fukui, K. The Path of Chemical Reactions-the IRC Approach. Acc. Chem. Res. 1981, 14, 363-368.

(b) Ishida, K.; Morokuma, K.; Komornicki, A. The Intrinsic Reaction Coordinate. An ab initio Calculation for $\mathrm{HNC} \rightarrow \mathrm{HCN}$ and $\mathrm{H}^{-}+\mathrm{CH}_{4} \rightarrow \mathrm{CH}_{4}+\mathrm{H}^{-}$. J. Chem. Phys. 1977, 66, 2153-2156.

(c) Gonzalez, C.; Schlegel, H. B. An Improved Algorithm for Reaction Path Following.J. 
Chem. Phys. 1989, 90, 2154-2161.

(d) Gonzalez, C.; Schlegel, H. B. Reaction Path Following in Mass-Weighted Internal Coordinates. J. Phys. Chem. 1990, 94, 5523-5527. 